THE MARKET FOR ELECTRIC VEHICLES IN NEW ZEALAND:

USING STATED CHOICE METHODS TO EVALUATE THE IMPLICATIONS FOR

ELECTRICITY DEMAND AND CARBON EMISSIONS TO 2030

By

Douglas George Clover

A thesis

submitted to the Victoria University of Wellington

in fulfilment of the requirements for the degree of

Doctor of Philosophy

in Environmental Studies

Victoria University of Wellington 



\section{Abstract}

Anthropogenic global climate change caused by the emissions of greenhouse gases (GHGs) from the combustion of fossil fuels is one of the greatest environmental threats faced by society. Electric vehicles (EVs), which use lithium-ion battery technology, have been proposed as a means of reducing GHG emissions produced by light passenger vehicles (LPVs). The ability of this vehicle technology to assist in reducing GHG emissions will depend on the market uptake and the effect that a growing EV fleet has on the GHG emissions produced by the electricity sector.

This thesis is the first use of stated choice methods in New Zealand to develop a vehicle demand model that takes detailed account of car buyers' preferences for EV purchase price, driving range, performance, fuel and battery costs, and charging network availability.

A nationwide stated choice survey of New Zealand car buyers was undertaken in $2010(n=281)$. The data from the survey was used to estimate a mixed multinomial logit discrete choice model, which was linked to a vehicle stock model of the New Zealand LPV fleet developed for this research. These two models were then used to simulate the New Zealand vehicle stock and energy demand, and the LPV fleet's GHG emissions over a twenty year period.

The Electricity Commission's mixed integer programming 'generation expansion model' (GEM) was used to take account of the additional GHG emissions produced by the electricity sector in response to meeting the electricity demand estimates from the vehicle stock model.

The results of this study indicate that, assuming the current state of EV technology and only modest reductions in EV prices over the modelling period, there would be sufficient demand for EVs to reduce, by 2030, the annual GHG emissions produced by the LPV fleet to approximately $80 \%$ of levels emitted in 2010 . Changes in technology or vehicle design that reduce the cost of batteries and the purchase price of EVs would have the greatest impact in increasing the demand for these vehicles, and would further reduce the GHG emissions produced by the LPV fleet. 
The electricity sector modelling indicates that less than $730 \mathrm{MW}$ of additional generation capacity will be required to be built if network operators can prevent EVs from charging during periods of peak demand, but without this capability, up to $4,400 \mathrm{MW}$ of additional generation capacity could be required. The modelling also indicates that a policy environment where the use of coal-fuelled electricity generation is permitted and the price of carbon limited to $\$ 25$ per tonne, the increased electricity sector GHG emissions that would result offset $88 \%$ of the cumulative GHG emission reductions achieved by the introduction of EVs into the LPV fleet. A policy raising the price of carbon to $\$ 100$ per tonne would reduce the offsetting effect to $30 \%$.

EVs are an emerging technology with considerable potential for further development. The results of this study indicate that even at current prices and levels of technological performance, EVs have the capacity to make a significant contribution to New Zealand's efforts to reduce GHG emissions. However, the ability to realise this potential is dependent on vehicle manufacturers' willingness to produce EVs in sufficient quantities and models so that they can fully compete in the market with internal combustion engine vehicles; and on policies that discourage the future use of coal-fuelled electricity generation. 


\section{Acknowledgements}

I would like to acknowledge the support, insights, and enthusiasm of my supervisors Associate Professor Ralph Chapman and Dr Geoff Bertram. I would also like to thank Professor Euan Smith for his assistance in the development of the PHEV electric/non-electric travel model, and Dr Phil Bishop of the Electricity Authority for his generous gift of time and knowledge on all matters related to the GEM.

I would also like to acknowledge Yvonne Matthews who designed the survey website; Dr John Rose, Professor David Hensher and Dr Michel Bliemer for teaching a novice the fundamentals of stated choice methods; and Dr Ric Scarpa for his insights on how a stated choice survey of New Zealand car buyers could be implemented.

This thesis is dedicated to my wife Jennie. Without her love, support, and encouragement I would not have attempted it. 


\section{Table of contents}

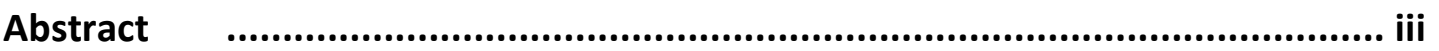

Acknowledgements .................................................................................

Table of contents............................................................................................... vi

List of Figures ....................................................................................................

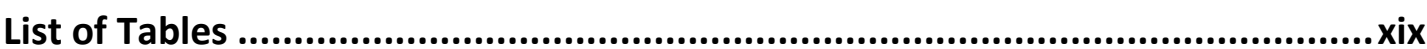

List of abbreviations .......................................................................................xxiii

Chapter 1: Introduction ......................................................................... 1

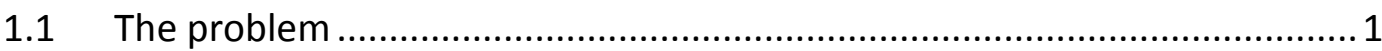

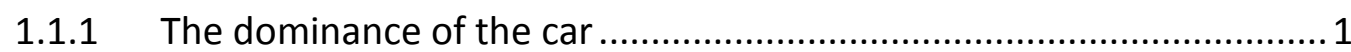

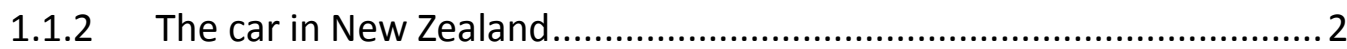

1.1.3 The future of the car: climate change and availability of petroleum ....8

1.2 Addressing the problem: LPVs in a carbon constrained world ..................17

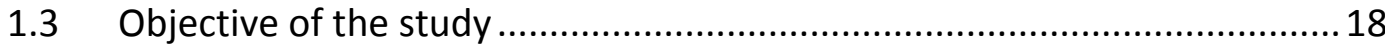

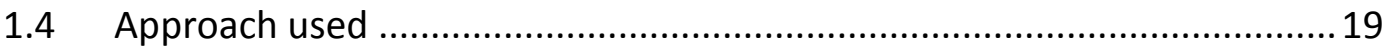

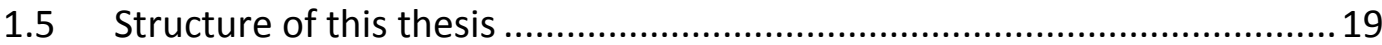

Chapter 2: Review of alternative fuel vehicle technological change studies ..... 21

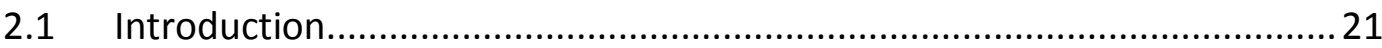

2.2 Replacing the ICEV: a review of modelling methods used.......................22

2.2.1 Diffusion of innovation approaches............................................22

2.2.2 Expert based market penetration scenarios ..................................2 24

2.2.3 Technological and economic replacement .....................................25

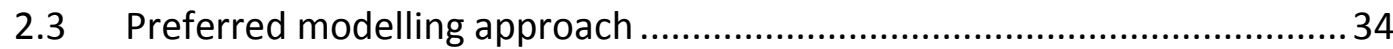

Chapter 3: Theory of discrete choice models and stated choice experiments ... 37

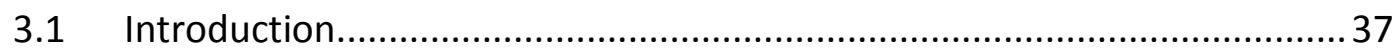

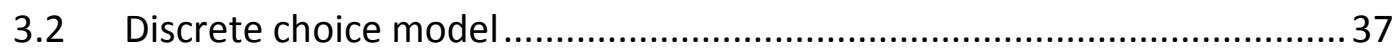

3.2.1 Random utility maximisation: a behavioural model of choice ............37

3.2.2 The multinomial logit (MNL) model .............................................. 40 
3.2.3 Maximum likelihood estimation ..................................................... 41

3.2.4 Limitations of the MNL model .............................................................42

3.2.5 Mixed multinomial logit models ......................................................44

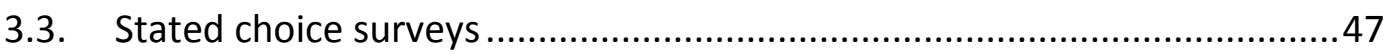

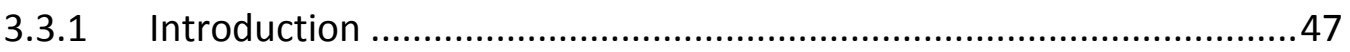

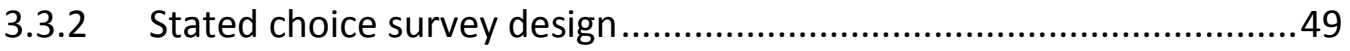

3.3.3 Designing a stated choice experiment .............................................50

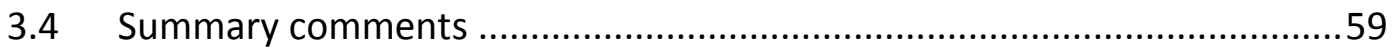

Chapter 4: Light duty passenger vehicle technology: current status and potential .............................................................................. 61

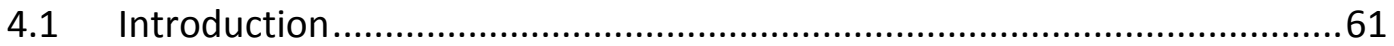

4.2 Non-propulsion measures to reduce GHG emissions ...............................62

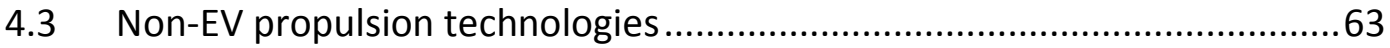

4.3.1 Advanced petrol and diesel ICEVs................................................63

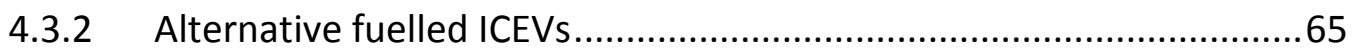

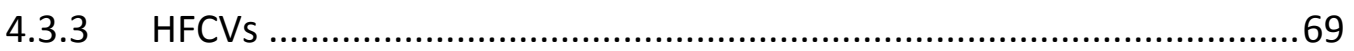

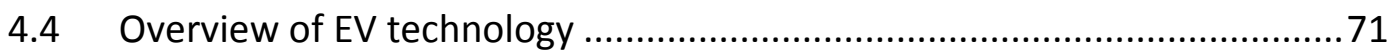

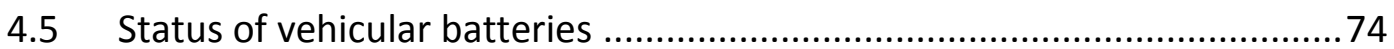

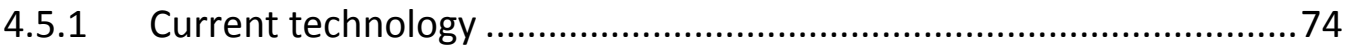

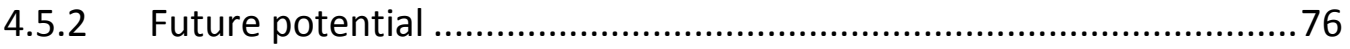

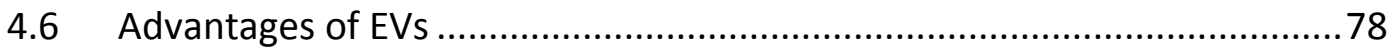

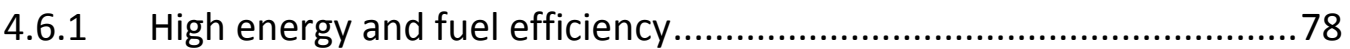

4.6.2 Potential for reducing GHG emissions ............................................ 81

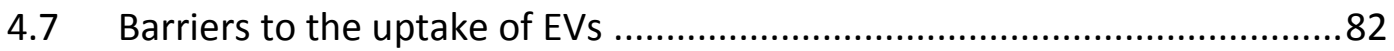

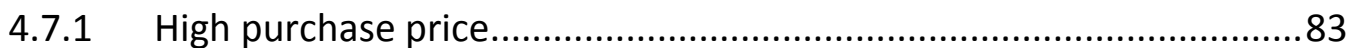

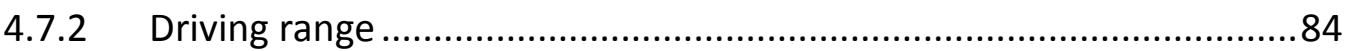


4.7.3 Access to recharging facilities and duration of recharging..................84

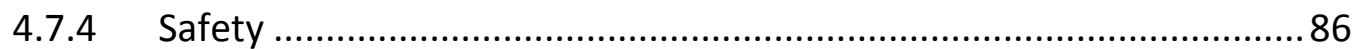

4.7.5 Lack of appreciation of the benefits of EVs ....................................... 86

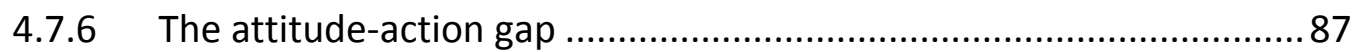

4.7.7 System "lock-in" and resistance from the industry ..........................87

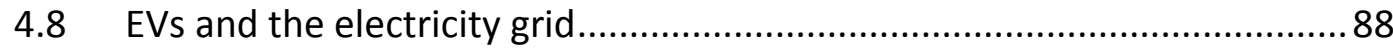

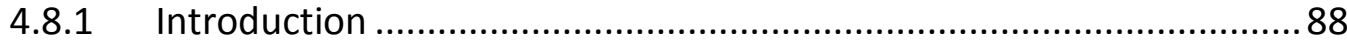

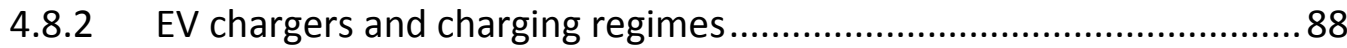

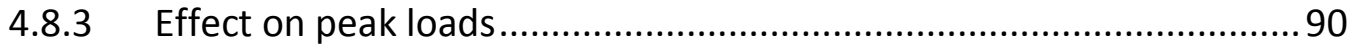

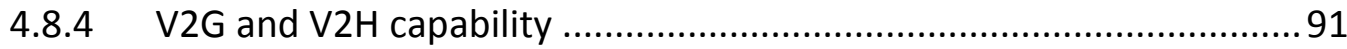

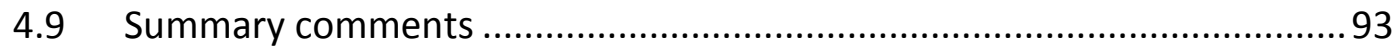

Chapter 5: Methods I: vehicle fleet model..................................................... 95

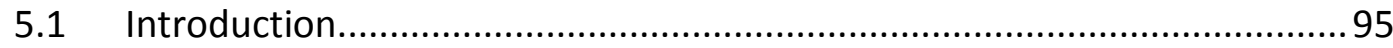

5.2 Stated choice survey design and implementation .................................97

5.2.1. Objective of the stated choice survey ................................................97

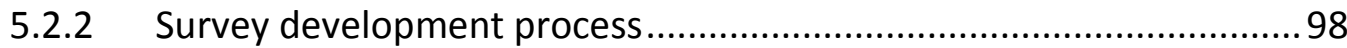

5.2.3 Survey design elements ................................................................ 103

5.2.4 The main survey recruitment process ......................................... 112

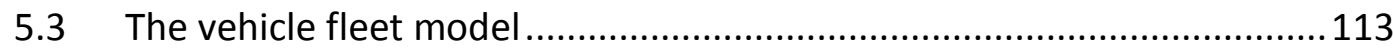

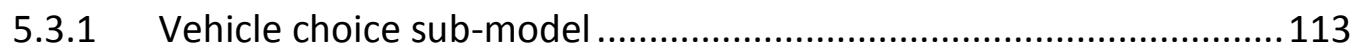

5.3.2 The car ownership sub-model ..................................................... 117

5.3.3 The vehicle use sub-model ......................................................... 132

5.3.4 Energy demand and GHG emissions sub-models .............................. 138

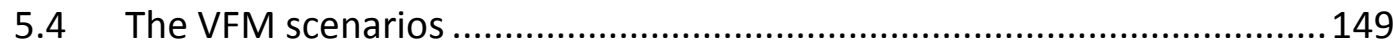

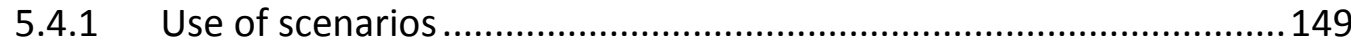

5.4.2 Development of VFM scenarios................................................. 150 
5.4.3 Main dimensions of the VFM scenarios

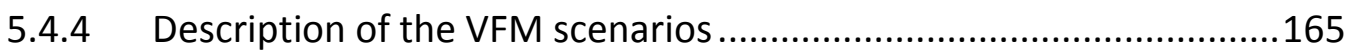

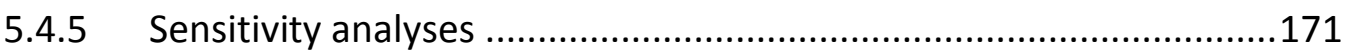

Chapter 6: Methods II: the generation expansion model ............................. 177

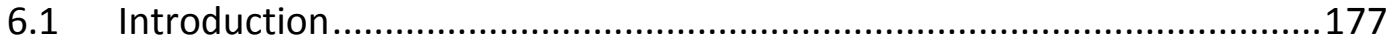

6.2 Overview of the New Zealand electricity sector .................................178

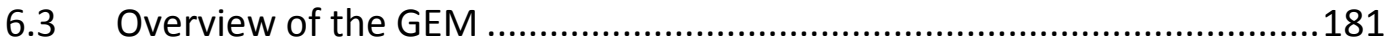

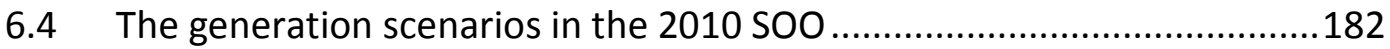

6.4.1 Potential new generation and demand side projects......................185

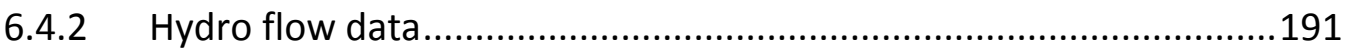

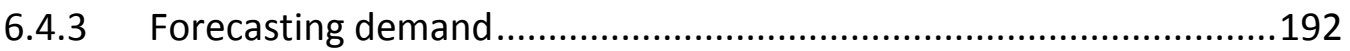

6.4.4 Peak demand constraints and peak demand forecasts ...................193

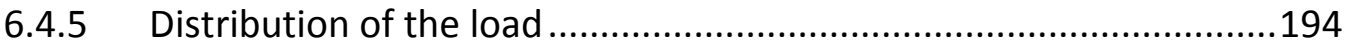

6.4.6 Fossil fuel availability and prices....................................................196

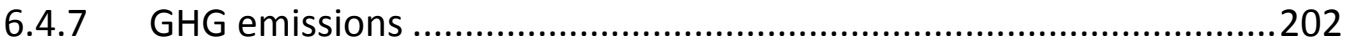

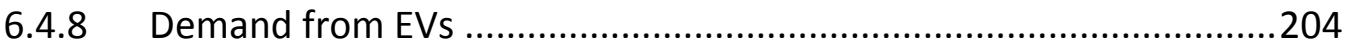

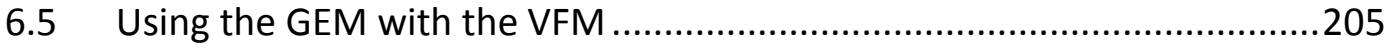

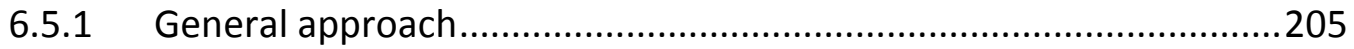

6.5.2 Revision of electricity demand forecasts ......................................209

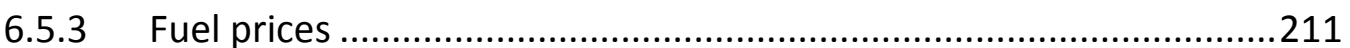

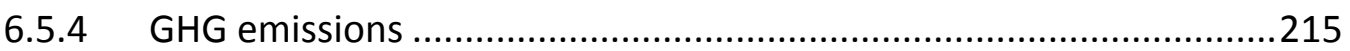

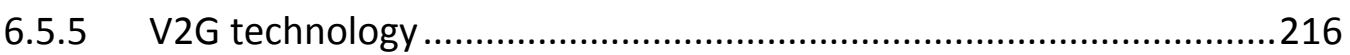

6.5.6 Peak demand and security of supply .........................................216

Chapter 7: Results I: stated choice survey and discrete choice model.............. 221

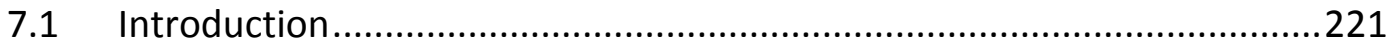

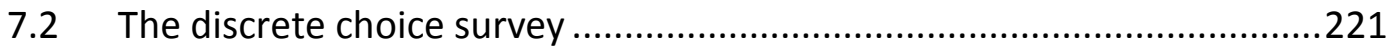




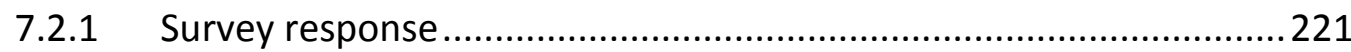

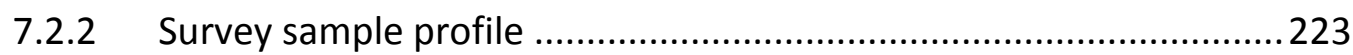

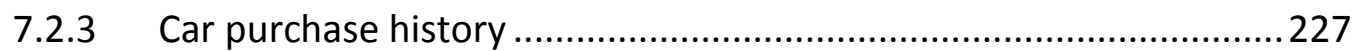

7.2.4 Attitudes to fuel supply and climate change .................................222

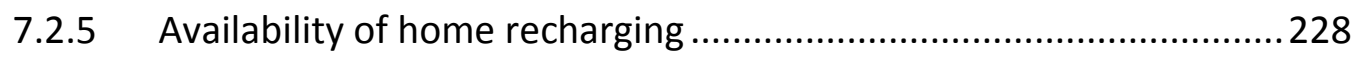

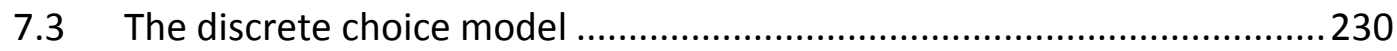

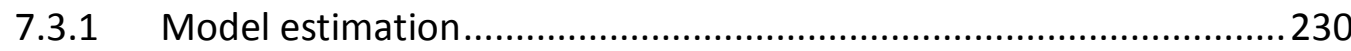

7.3.2 Discrete choice model probability shares and elasticities.................234

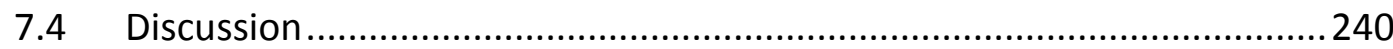

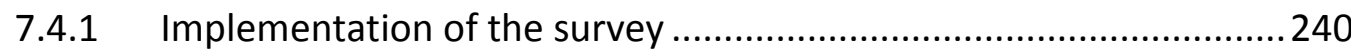

7.4.2 Representativeness of the sample and reweighting .......................240

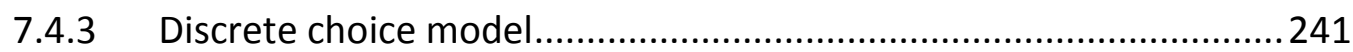

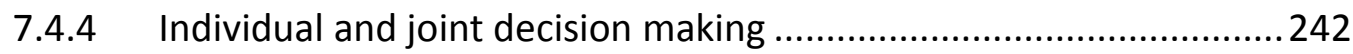

Chapter 8: Results II: composition of the LPV fleet - 2010 to 2030 ................. 243

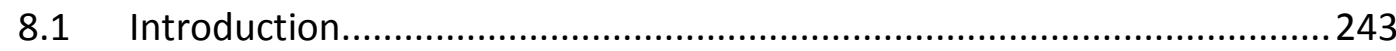

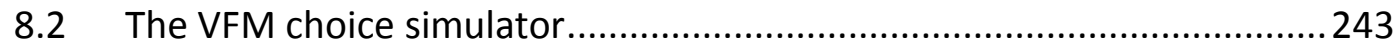

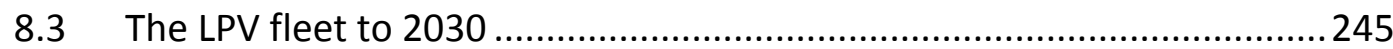

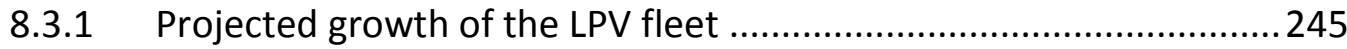

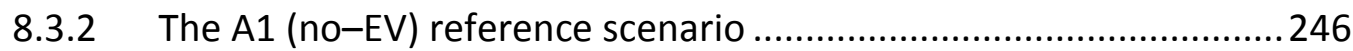

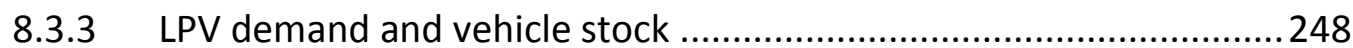

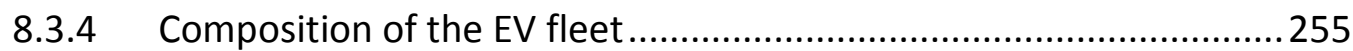

8.3.5 Impact of used imported EVs on demand and vehicle stock ........... 261

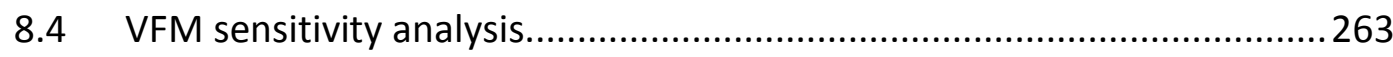

8.4.1 Growth in GDP and rate of vehicle scrapping ...............................263

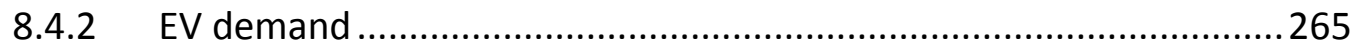

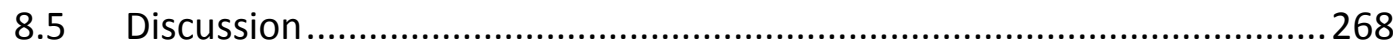


8.5.1 Future composition of the LPV fleet ..............................................268

8.5.2 Impact of possible policy interventions ..........................................270

Chapter 9: Results III: projected energy demand and GHG emissions ............ 273

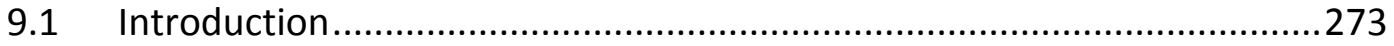

9.2 LPV fleet: projected energy demand and GHG emissions .......................273

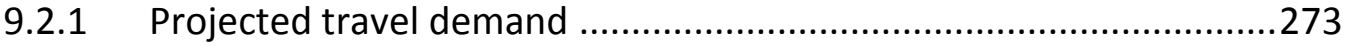

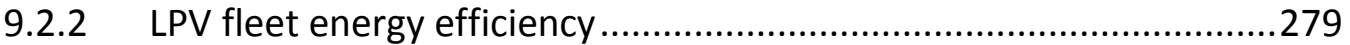

9.2.3 Energy demand forecasts for the LPV fleet ..................................281

9.2.4 Direct GHG emissions from the LPV fleet ....................................286

9.2.5 Sensitivity analysis - electricity demand and GHG emissions ...........287

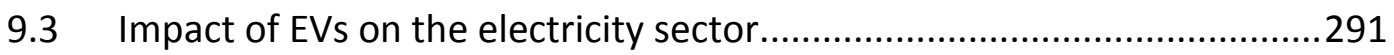

9.3.1 Projected electricity demand and generation ................................291

9.3.2 Generation plant build, retirement, dispatch, and GHG emissions...294

9.3.3 The impact of EVs on installed capacity..........................................314

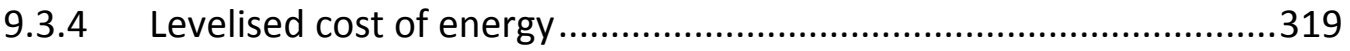

9.4 Combined LPV fleet and electricity sector GHG emissions .....................320

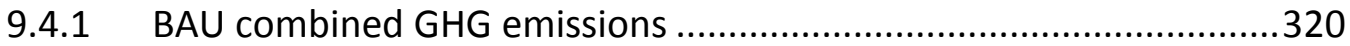

9.4.2 Combined GHG emissions under a low carbon price regime ............321

9.4.3 Combined GHG emissions with a high carbon price .........................322

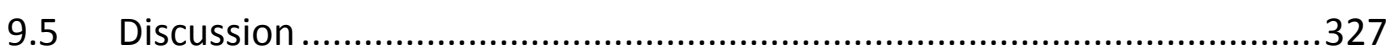

9.5.1 Impact of EVs on LPV fleet energy efficiency ..................................327

9.5.2 Projected electricity demand for the EV fleet .................................328

9.5.3 Impact of EVs on the development of the electricity sector .............329

9.5.4 Impact of EVs on New Zealand's GHG emissions..............................331

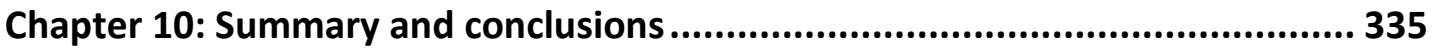

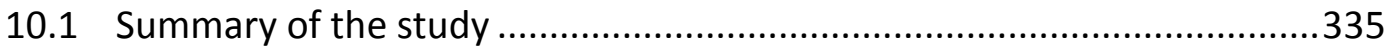




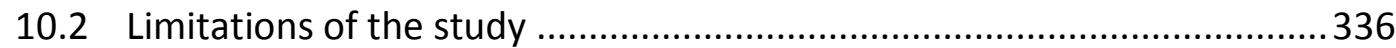

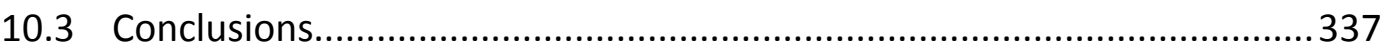

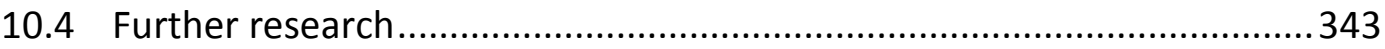

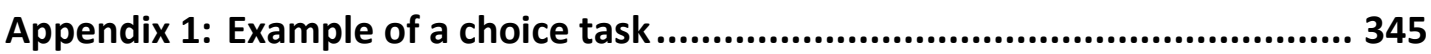

Appendix 2: The supporting questions used in the survey .............................. 347

Appendix 3: Supporting information provided in the survey.......................... 349

Appendix 4: Results of vehicle fleet model choice simulator verification test ... 355

Appendix 5: Method used to estimate PHEV electric and non-electric travel .... 357

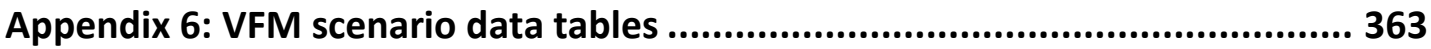

References 


\section{List of Figures}

Figure 1.1: Top twenty vehicle owning nations in 2009: vehicles per 1000 people

Figure 1.2: New Zealand licensed light passenger vehicles: 1962 to $2010 \ldots \ldots \ldots \ldots \ldots . . . . .5$

Figure 1.3: Proportion of household trips by mode - selected countries...................6

Figure 1.4: Composition of the motorised road fleet in 2011 ..................................

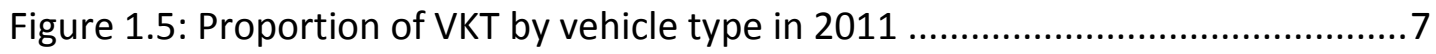

Figure 1.6: 2005 Global GHG emissions by source ............................................

Figure 1.7: $2005 \mathrm{Global}^{\mathrm{CO}_{2}}$ emissions from fuel combustion ...............................10

Figure 1.8: 2009 Gross New Zealand GHG emissions by source.............................10

Figure 1.9: 2009 New Zealand $\mathrm{CO}_{2}$ emissions from fuel combustion........................11

Figure 1.10: New Zealand historic and forecast GHG emissions from road transport: 1990 to 2040 .12

Figure 1.11: Percentage of New Zealand light passenger vehicle fleet by fuel type in 2011 .13

Figure 4.1: Potential vehicle technologies and possible development pathways.....61

Figure 4.2.: Specific energy $(\mathrm{Wh} / \mathrm{kg})$ of various electrochemical cells .78

Figure 4.3: Estimated energy efficiency and fuel cost of alternative fuel vehicle technologies in 2007 and 2035

Figure 4.4: Lifecycle GHG emissions of alternative fuel and vehicle technology pathways in the United States.....

Figure 5.1: Overview of the vehicle fleet model (VFM) .97

Figure 5.2: Cumulative availability profile of used imported EVs. 116

Figure 5.3: New and used vehicles entering the New Zealand light passenger vehicle fleet: 1970 to 2009 . 119

Figure 5.4: Relationship between income per capita and light passenger vehicle ownership in New Zealand: 1962 to 2009

Figure 5.5: New Zealand light passenger vehicle ownership per 1000 people versus income per capita: 1962 to 2009 .125

Figure 5.6: New Zealand light passenger vehicle total ownership: 1962 to 2009...125 
Figure 5.7: The percentage of new vehicles scrapped at a given vehicle age: 2006 to 2009

Figure 5.8: The percentage of used imported vehicles scrapped at a given vehicle age: 2006 to 2009

Figure 5.9: The percentage of new vehicles scrapped at a given vehicle age - Miaou model estimate and average values: 2006 to 2009 data 129

Figure 5.10: The percentage of used imported vehicles scrapped at a given vehicle age - Miaou model estimate and average values: 2006 to 2009 data 129

Figure 5.11: GDP expenditure measure (\% annual change) 130

Figure 5.12: Scrapping rate new vehicles - low, high, and default economic growth

Figure 5.13: Scrapping rate used imported vehicles - low, high, and default economic growth

Figure 5.14: Light petrol fleet fuel consumption (minimum and maximum estimates): 2001 to2010

Figure 5.15: Average petrol and diesel fuel consumption of new cars entering the light passenger vehicle fleet - European test regime

Figure 5.16: Estimated energy efficiencies of CEVs ............................................ 146

Figure 5.17: Estimated energy efficiencies of GEVs and PHEVs ........................... 147

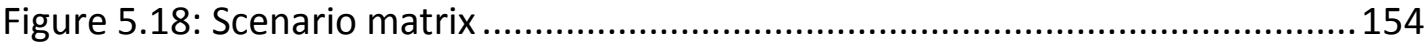

Figure 5.19: GDP 1995/96 prices (\$ millions) .................................................. 172

Figure 6.1: Installed New Zealand generation capacity in 2011 ............................. 178

Figure 6.2: Annual generation and percentage of renewable generation: 1990 to 2011

Figure 6.3: Approximate share of national demand in 2011 180

Figure 6.4: Load duration curve - quarter, low-wind (red), high-wind (green) and medium-wind (blue) sub-blocks

Figure 6.5: 2010 Statement of Opportunities report: coal and lignite price assumptions

Figure 6.6: Electricity Commission domestic gas production projections..... 198

Figure 6.7: 2010 Statement of Opportunities report: gas availability assumptions 199 Figure 6.8: 2010 Statement of Opportunities report: gas price assumptions......... 201 
Figure 6.9: 2010 Statement of Opportunities report: diesel price assumptions.....202

Figure 6.10: 2010 Statement of Opportunities report: carbon prices .203

Figure 6.11: 2010 Statement of Opportunities report: estimated additional electricity demand due to plug-in hybrid electric vehicles 205

Figure 6.12: Projected electricity demand including line losses and excluding demand from EVs.

Figure 6.13: The New Zealand historic retail and industrial price of diesel 212

Figure 7.1: Recruitment response rates

Figure 7.2: Survey method used by respondents

Figure 7.3: Age distribution of the survey sample and New Zealand adult population

Figure 7.4: Household income distribution of the survey sample and New Zealand

Figure 7.5: Gender compared to the New Zealand adult population and New Zealand vehicle licence holders .226

Figure 7.6 Location of respondents

Figure 7.7: Response to - 'Could you recharge an electric vehicle where you live?'

Figure 7.8: Response to - 'Could you recharge an electric vehicle where you live; by location?'.... .230

Table 7.1: Best performing multinomial logit, error components mixed multinomial logit, and random parameters mixed multinomial logit models 233

Figure 7.9: Change in market shares with change in vehicle purchase price (NZ\$) 237 Figure 7.10: Change in market shares with change in fuel cost $(\mathrm{NZ} \$ / 100 \mathrm{~km}) \ldots \ldots .237$ Figure 7.11: Change in market shares with change in replacement battery cost (NZ\$)

Figure 7.12: Change in market shares with change in electric driving range: $\mathrm{km}$ .238

Figure 7.13: Change in market shares with change in time to replacement of battery: years

Figure 7.14: Change in market shares with change in top speed, city electric vehicles only. .239 
Figure 8.1: Projected total light passenger vehicle ownership using default GDP and population assumptions

Figure 8.2: A1 (no-EV) scenario - vehicles entering (sales) and leaving (scrapped) the New Zealand light passenger vehicle fleet

Figure 8.3: Total number of vehicles entering the light passenger vehicle fleet - new and used imports 248

Figure 8.4: Internal combustion engine vehicles entering the light passenger vehicle fleet: 2010 to 2030 249

Figure 8.5: Stock of internal combustion engine vehicles: 2010 to 2030 250 Figure 8.6: EVs as a proportion of vehicles entering the light passenger vehicle fleet

Figure 8.7: Stock of EVs as a percentage of the light passenger vehicle fleet: 2010 to 2030 254

Figure 8.8: Stock of EVs: 2010 to 2030 254

Figure 8.9: Plug-in hybrid electric vehicles as a percentage of EVs entering the light passenger vehicle fleet: 2010 to 2030 256

Figure 8.10: Stock of plug-in hybrid electric vehicles all vehicle fleet model scenarios: 2010 to 2030 257

Figure 8.11: City electric vehicles as a percentage of EVs entering the light passenger vehicle fleet: 2010 to 2030 258

Figure 8.12: Stock of city electric vehicles all vehicle fleet model scenarios: 2010 to 2030 259

Figure 8.13: General purpose electric vehicles as a percentage of EVs entering the light passenger vehicle fleet: 2010 to 2030 . 260

Figure 8.14: Stock of General purpose electric vehicles all vehicle fleet model scenarios: 2010 to 2030 261

Figure 8.15: Proportion of EVs entering the light passenger vehicle fleet as used imports

Figure 8.16: Proportion of new vehicles entering the light passenger vehicle fleet: 2010 to 2030 262

Figure 8.17: Projected total light passenger vehicle ownership low, default, and high GDP growth assumptions 
Figure 9.1: Annual VKT per vehicle for internal combustion engine vehicles: 2010 to 2030

Figure 9.2: Annual VKT for internal combustion engine vehicles - scenarios A2, B3, B4, and C5: 2010 to 2030

Figure 9.3: Light passenger vehicle fleet annual electric powered VKT by vehicle fleet model scenario: 2010 to 2030

Figure 9.4: Light passenger vehicle fleet - total annual VKT by vehicle fleet model scenario: 2010 to 2030

Figure 9.5: Annual VKT of plug-in hybrid electric vehicles using petrol by vehicle fleet model scenario: 2010 to 2030

Figure 9.6: Annual VKT of plug-in hybrid electric vehicles using electricity by vehicle fleet model scenario: 2010 to 2030 .278 Figure 9.7: Annual petroleum demand for the light passenger vehicle fleet: 2010 to 2030 .283

Figure 9.8: Projected total electricity demand including demand from EVs..... .292

Figure 9.9: MDS1 gross and net installed generation and demand side management capacity: 2010 to 2030 .300

Figure 9.10: Proportion of renewable generation, MDS1 - A1 (no-EV), A2, C4, and C5 electricity demand .300

Figure 9.11: Annual GHG emissions, MDS1 - A1 (no-EV), A2, C4, and C5 electricity demand 301

Figure 9.12: MDS3 gross and net installed generation and demand side management capacity: 2010 to 2030 306

Figure 9.13: Proportion of renewable generation, MDS3 - A1 (no-EV), A2 and C4 electricity demand .306

Figure 9.14: Annual GHG emissions, MDS3 - A1 (no-EV), A2, and C4 electricity demand: thousand tonnes $\mathrm{CO}_{2} \mathrm{e}$ 307

Figure 9.15: MDS5 gross and net installed generation and demand side management capacity: 2010 to 2030

Figure 9.16: Proportion of renewable generation, MDS5 - A1 (no-EV) and A2 and C4 electricity demand 
Figure 9.17: Annual GHG emissions, MDS5 - A1 (no-EV), A2 and C4 electricity

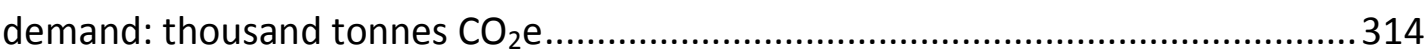
Figure 9.18: Combined annual GHG emissions from the light passenger vehicle fleet

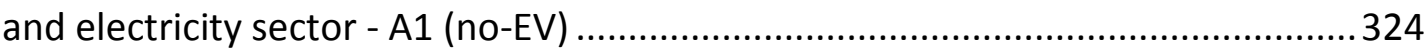
Figure 9.19: Combined annual GHG emissions from the light passenger vehicle fleet and electricity sector - MDS1 324 Figure 9.20: Combined annual GHG emissions from the light passenger vehicle fleet and electricity sector - MDS3 324 Figure 9.21: Combined annual GHG emissions from the light passenger vehicle fleet and electricity sector - MDS5 324

Figure 9.22: Reductions in cumulative GHG emissions from EVs - light passenger vehicle fleet only, and combined light passenger vehicle fleet and electricity sector: 2010 to 2030 327 


\section{List of Tables}

Table 1.1: Measures for reducing GHG emissions from light passenger vehicles .....17

Table 4.1: Estimated future fuel efficiency improvements compared to current

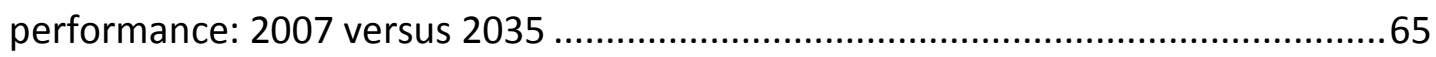

Table 4.2: Plug-in hybrid vehicle operating configurations ...................................73

Table 4.3: Summary of characteristics of lithium-ion batteries .............................77

Table 4.4: TTW and WTW energy efficiencies of alternative fuel and vehicle

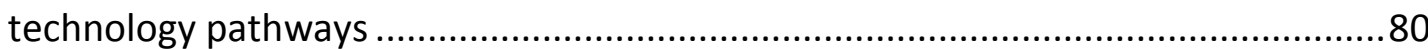

Table 5.1: City and general purpose electric vehicle driving ranges $(\mathrm{km})$..............106

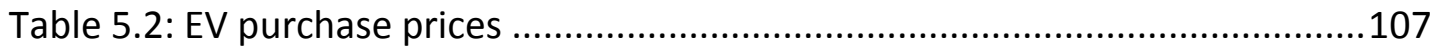

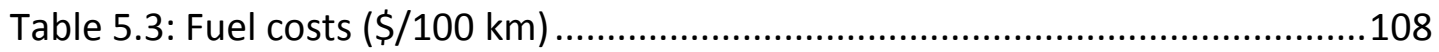

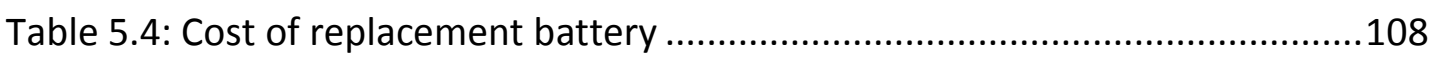

Table 5.5: Time to battery replacement (years) ...................................................109

Table 5.6: Availability of on-street charging facilities ..........................................109

Table 5.7: City electric vehicle top speed ..........................................................110

Table 5.8: Light passenger vehicle ownership model estimation: basic and expanded Gompertz functions

Table 5.9: Elasticity estimates of change in kilometres travelled in response to change in fuel price

Table 5.10: Average annual light passenger vehicle VKT per vehicle: 2001 to 2010

Table 5.11: Results of the regression on change in average annual VKT per vehicle: 2001 to 2010

Table 5.12: Estimated fuel consumption estimate for petrol and diesel cars in 2007

Table 5.13: Estimated fuel consumption estimates for petrol and diesel cars: 2001 to 2009

Table 5.14: Aerodynamic coefficient of drag, frontal area, and equivalent aerodynamic drag values

Table 5.15: Vehicle data

Table 5.16: Key factors in the scenario analysis 
Table 5.17: Overview of the vehicle fleet model scenarios

Table 5.18: Projected residential electric tariffs................................................ 173

Table 5.19: Present Value of road user charges exemption by vehicle type

Table 6.1: Outline of the generation scenarios in the 2010 Statement of

Opportunities report.

Table 6.2: List of committed generation projects used in the 2010 Statement of Opportunities report.

Table 6.3: List of near future generation projects used in the 2010 Statement of

Opportunities report.

Table 6.4: Available generation and demand side projects in the 2010 Statement of

Opportunities report. 191

Table 6.5: Peak contribution factor used in the generation expansion model 194

Table 6.6: Load blocks as used in the generation expansion model 195

Table 6.7: Emission factors used in the generation expansion model 202

Table 6.8: Comparison of input assumptions used in both the Statement of Opportunities report and vehicle fleet model scenarios..... 208

Table 6.9: Forecast model for residential, industrial, and commercial electricity demand 210

Table 6.10: Industrial and retail diesel price relationship model 212

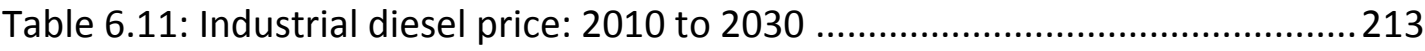

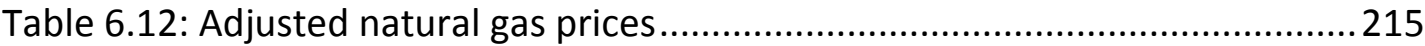

Table 6.13: Estimated peak demand from non EV uses

Table 7.2: Random parameters mixed multinomial logit model elasticities means and standard deviations of attributes choice experiment

Table: 8.1: An example of the output of the choice simulator from scenario B4

Table 8.2: Stock of internal combustion engine vehicles in 2030 by vehicle fleet model scenario 250

Table 8.3: Plug-in hybrid electric vehicles - stock and proportion of EV fleet in 2030

Table 8.4: City electric vehicles - stock and proportion of EV fleet in 2030 258 
Table 8.5: General purpose electric vehicles - stock in 2030 and proportion of EV fleet

Table 8.6: Sensitivity analysis - impact of GDP growth and scrapping rates on the cumulative demand for light passenger vehicles and EVs entering the light passenger vehicle fleet: 2010 to 2030

Table 8.7: Sensitivity analysis - effect of changed assumptions on the cumulative number of EVs entering the light passenger vehicle fleet.

Table 8.8: Comparison of New Zealand EV studies of EV stock in 2030.

Table 9.1: Fleet average energy efficiency values of battery electric vehicles and the electric cycle of plug-in hybrid electric vehicles - selected years

Table 9.2: Fleet average energy efficiency values for internal combustion engine vehicles and the petrol cycle of plug-in hybrid electric vehicles

- selected years

Table 9.3: Light passenger vehicle fleet average energy efficiency by vehicle fleet model scenario - selected years.

Table 9.4: Light passenger vehicle fleet annual electricity demand in 2030 , and total electricity demand in 2030 (GWh)

Table 9.5: Annual petroleum demand for the light passenger vehicle fleet in 2030 and total cumulative petroleum demand (GWh): 2010 to 2030

Table 9.6: Vehicle fleet model scenarios B3 and B4 - sensitivity analysis of petroleum demand with no change in VKT (GWh)

Table 9.7: Total light passenger vehicle fleet petroleum and electricity annual demand in 2030 and cumulative energy demand 2010 to 2030 (GWh)

Table 9.8: Scenario C5 - sensitivity analysis, high and standard EV energy efficiency (GWh): 2010 to 2030 .285

Table 9.9: Estimated light passenger vehicle fleet annual GHG emissions in 2030 and cumulative GHG emissions (thousand tonnes $\mathrm{CO}_{2} \mathrm{e}$ ): 2010 to 2030 286

Table 9.10: Sensitivity analysis - impact of GDP growth and scrapping rates, cumulative electricity demand, cumulative petroleum demand, and GHG emissions: 2010 to 2030 288 
Table 9.11: Sensitivity analysis - effect of changed assumptions on cumulative electricity demand, and GHG emissions, thousand tonnes $\mathrm{CO}_{2} \mathrm{e}$ :

Table 9.12: Sensitivity analysis - effect of changed assumptions of the rate of increase in electricity prices on cumulative electricity demand and GHG emissions: 2010 to 2030 291

Table 9.13: Vehicle fleet model scenarios forecast annual electricity demand in 2030, and cumulative demand (GWh): 2010 to 2030

Table 9.14: Forecast and dispatched electricity demand for MDS1, MDS3 and MDS5 scenarios - annual 2030 and cumulative demand (GWh): 2010 to 2030 ................293

Table 9.15: MDS1: Retirements and new capacity installed (MW) ........................298 Table 9.16: MDS3 medium renewables and no restriction on coal - New capacity installed and retirements (MW) 304 Table 9.17: MDS5 High gas discovery - New capacity installed and retirements (MW)

Table 9.18 Total installed generation and demand side management capacity - fully interruptible $(\mathrm{MW})$ : selected years 316

Table 9.19: Generation and demand side management capacity (MW): selected years

Table 9.20: Levelised cost of energy (c/kWh) 320

Table 9.21: Annual GHG emissions from the light passenger vehicle fleet and electricity sector (thousand tonnes $\mathrm{CO}_{2} \mathrm{e}$ ).

Table 9.22: Cumulative GHG emissions from the light passenger vehicle fleet and electricity sector (thousand tonnes $\mathrm{CO}_{2} \mathrm{e}$ ): 2010 to 2030

Table 9.23: Effect of carbon price and EVs on cumulative GHG emissions from the light passenger vehicle fleet and electricity sector - C4 and C5 model runs (thousand tonnes $\mathrm{CO}_{2} \mathrm{e}$ ): 2010 to 2030 . 326

Table 9.24: EV energy efficiency estimates used in New Zealand studies (Wh/km)

Table 9.25: Comparison of New Zealand EV studies, EV stock and additional electricity demand in 2030 


\title{
List of abbreviations
}

\author{
ABM Agent based models \\ AFV Alternative fuelled vehicle (a vehicle not fuelled by petrol or diesel) \\ AVC Asymptotic Variance Covariance matrix \\ BEV Battery electric vehicles (comprises CEVs and GEVs) \\ BVP The price of the EV excluding the price of the battery \\ CAPI Computer assisted personal interview \\ CCGT Combined cycle gas turbine \\ CCS Carbon capture and storage (sequestration) \\ CD Charge depleting phase of PHEV drive cycle \\ $\mathrm{CdA} \quad$ Equivalent aerodynamic drag \\ CEV City electric vehicle-small EVs designed for urban use \\ $\mathrm{Cl} \quad$ Compression ignition engine \\ CNG Compressed natural gas \\ DSM Demand side management \\ EA Electricity Authority \\ EC Electricity Commission \\ EECA Energy Efficiency and Conservation Authority \\ ECD Efficient choice design \\ ECMNL Error components mixed multinomial logit \\ EV Electric vehicle (comprising both BEVs and PHEVs)
}


E85 internal combustion engine vehicle that can operate on up to $85 \%$ biofuels

GEM The Electricity Commission's Generation expansion model

GEV General purpose electric vehicle - larger EV suitable for family use

GHG Greenhouse gas(es)

GXP Grid exit point.

HEV Hybrid electric vehicle

HFCV Hydrogen fuel cell vehicle

HICEV Hydrogen internal combustion vehicle

HVDC High voltage direct current (the Benmore-Haywards link)

ICE Internal combustion engine

ICEV Internal combustion engine vehicle

IEA International Energy Agency

IIA Independence of irrelevant alternatives

IID Independent and identically distributed

IPCC Intergovernmental Panel on Climate Change

LCOE Levelised cost of energy

LNG Liquefied natural gas

LPG Liquid petroleum gas

LPV Light passenger vehicle (cars, SUVs and MPV less than 3,500 kg)

MED Ministry of Economic Development, now the Ministry of Business Innovation and Employment (New Zealand) 


\begin{tabular}{|c|c|}
\hline MoT & Ministry of Transport (New Zealand) \\
\hline MMNL & Mixed multinomial logit (comprising both ECMNL and RPL models) \\
\hline MNL & Multinomial Logit \\
\hline MPV & Multi-Purpose Vehicle (Minivan) \\
\hline Mtoe & million tonnes of oil equivalent \\
\hline NZTA & New Zealand Transport Agency \\
\hline OCGT & Open cycle gas turbine \\
\hline PHEV & Plug-in hybrid vehicles (use both electricity and petroleum as fuel) \\
\hline RPL & Random parameters mixed multinomial logit \\
\hline RUC & Road User Charges (road taxes paid by non-petrol powered vehicles) \\
\hline RUM & Random utility model \\
\hline SOO & Statement of Opportunities report \\
\hline SI & Spark ignition engine \\
\hline SUV & Sport Utility Vehicle \\
\hline VC & Variance covariance matrix \\
\hline VFM & $\begin{array}{l}\text { Vehicle fleet model (model of the New Zealand LPV fleet comprising the } \\
\text { vehicle choice, vehicle stock, and vehicle use models) }\end{array}$ \\
\hline VKT & Vehicle kilometres travelled (annually) \\
\hline $\mathrm{V} 2 \mathrm{H}$ & Vehicle-to-home \\
\hline V2G & Vehicle-to-grid \\
\hline VUM & Vehicle use model \\
\hline
\end{tabular}




\section{Chapter 1: Introduction}

\subsection{The problem}

\subsubsection{The dominance of the car}

The influential 1963 report "Traffic in Towns" was an early attempt to assess the impact of the development of roads and traffic on the urban environment in the United Kingdom. Even at this time the tension between the utility and convenience afforded by the car and the adverse effects from traffic jams, lack of parking, crashes, and the deterioration of the environment were already apparent (Buchanan et al., 1963). In the report the authors estimated that the size of the United Kingdom vehicle fleet was likely to double in ten years and treble in a little over 20 years and that, by 2010, there would be 30 million cars in the United Kingdom. They concluded that:

The problems of traffic are crowding in upon us with desperate urgency (Buchanan et al., 1963, p. 28)

These projections were remarkably prescient with the United Kingdom car fleet comprising in 201031 million vehicles. The growth in car ownership is a global phenomenon and, in 2010, there were an estimated 707 million cars in operation (Ward's Automotive Group, 2011). As Urry (2006, p. 18) observed the car is:

the predominant global form of 'quasi-private' mobility that subordinates other 'public' mobilities of walking, cycling, travelling by rail and so on.

A possible reason for this dominance is that the car is the only form of transport that permits the numerous social roles of family life, community, leisure, and the pleasure of movement all in one format. Therefore, the car is both immensely flexible and coercive (Sheller and Urry, 2000; Urry, 2004). It is flexible because it allows for travel 24 hours a day, giving freedom from timetables, freedom to stop and enjoy the trip, and freedom to choose the route. But this flexibility has also allowed for the fragmentation of social practices as business, recreational, and retail opportunities that used to be reachable by walking or cycling have become 
gradually separated and often can be practically reached only by the car. In this way, the car is also coercive as people must use cars to accommodate this fragmentation (Sheller and Urry, 2000, p. 744). Those who cannot, or will not, use the car become the disadvantaged and the excluded (Lucas, 2012).

The popularity of the car is not necessarily about rational choice. The car provides pleasure and feeds into some of the deepest emotions such as fear, frustration, euphoria, pain, and envy such that the car becomes "like members of the family" (Sheller, 2003, p. 17).

The car forms the heart of a complex system that encompasses not just vehicle producers and users, but also the petroleum refining and distribution industries, the road-building and maintenance industries, the hospitality industry, and car retail and repair workshops. It permits the development of suburbs, retailing and leisure complexes, and strongly shapes urban design and planning (Urry, 2006). It is, therefore, not surprising that this "car system" has been stable and unchanging through a long period of economic, social, and technological change such that the:

institutional processes are extremely difficult to reverse as billions of agents around the world co-evolved and adapted to it and built their lives around automobility's strange mixture of coercion and flexibility. One would predict that whatever else happens, the current car is here for the foreseeable future..... Urry (2008, p. 266) ${ }^{1}$

It appears that society will not willingly give up the personal flexibility, comfort, and convenience that the car provides.

\subsubsection{The car in New Zealand}

The Ministry of Transport (MoT) defines cars as light passenger vehicles (LPVs). LPVs are passenger vehicles that weigh less than $3,500 \mathrm{~kg}$. This group includes

\footnotetext{
${ }^{1}$ Urry defines automobility as the self-organising system of cars, car-drivers, roads, petroleum supplies, signs, and cultures of mobility (Urry, 2004).
} 
passenger vans, and sport utility vehicles (SUVs). New Zealand has a high level of LPV ownership when compared to other countries ${ }^{2}$ (Ministry of Transport, 2012b).

In 2009, the World Bank ranked New Zealand as having the fifth highest level of ownership per capita for both motor vehicles (light and heavy vehicles) and LPVs (Figure 1.1). The apparent low ownership rate of LPVs in the United States is due to the United States Government defining vans, minivans, SUVs, and pickup trucks as light trucks and not as cars.

Figure 1.1: Top twenty vehicle owning nations in 2009: vehicles per 1000 people

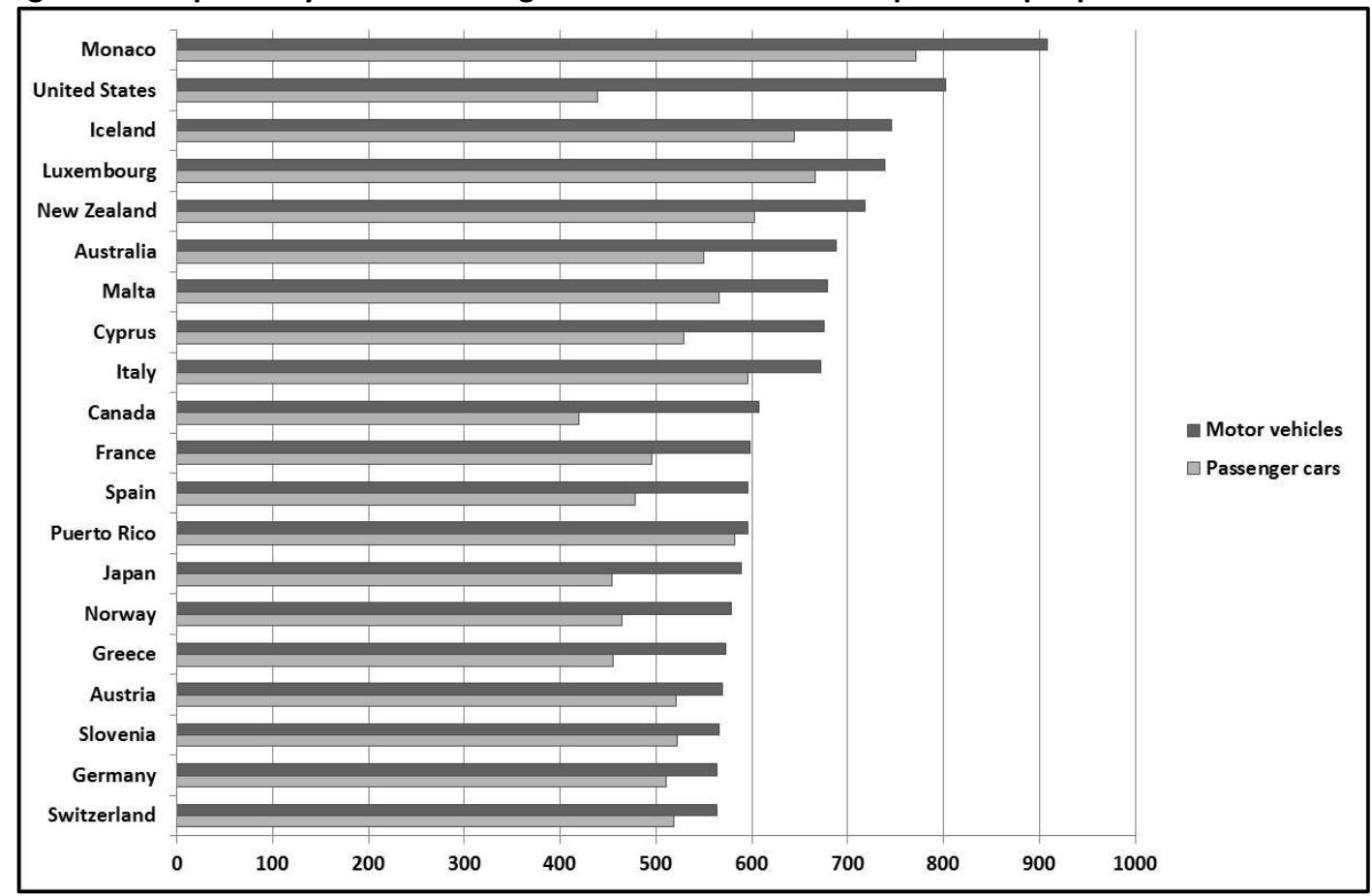

Source: World Bank (2012)

The dominance of the car in New Zealand is in part a function of past government transport policies that have had the effect of promoting the car and making it the default form of personal transport. One of the most influential policy initiatives that helped to promote the car as the dominant form of personal transport in New Zealand was the deregulation of the vehicle industry, which started in the mid1980s and was completed in 1997. This deregulation removed import quotas and reduced import tariffs on vehicle imports. The influx of relatively high quality used

\footnotetext{
${ }^{2}$ Cross country comparisons of levels of LPV ownership are complicated by different definitions of what constitutes an LPV.
} 
vehicles from Japan had the effect of lowering vehicle prices, which meant that the domestic vehicle assembly industry was unable to compete. New Zealand no longer has a car assembly industry (Bollard and Pickford, 1998).

Successive New Zealand Governments have also taken a largely a laissez-faire position on the question of the development of urban forms in New Zealand (Harris, 2007) and this has resulted in a declining role for public transport. Auckland is now one of the most car dependent cities in the world (Mees and Dodson, 2006). Wellington has a higher use of public transport, but is still very car dependent and ranks internationally with cities that have the highest car fuel use per person and lowest urban densities (Newman, 2006). In many rural areas, public transport is virtually non-existent (Rose et al., 2009a).

In 1962, the New Zealand licensed LPV fleet consisted of 582,000 registered vehicles. By 2007, the licensed LPV fleet had increased to 2.42 million vehicles ${ }^{3}$, but from 2008 to 2011, the LPV fleet declined by approximately 20,000 vehicles (Figure 1.2). As a result of the deregulation of vehicle imports, used imports comprised, by 2010, $51.8 \%$ of all LPVs with the remainder either assembled in New Zealand before the cessation of the domestic assembly industry, or brought into the country as new vehicles (Ministry of Transport, 2012b).

\footnotetext{
${ }^{3}$ Licensed vehicles excludes exempt and unlicensed vehicles
} 
Figure 1.2: New Zealand licensed light passenger vehicles: 1962 to 2010

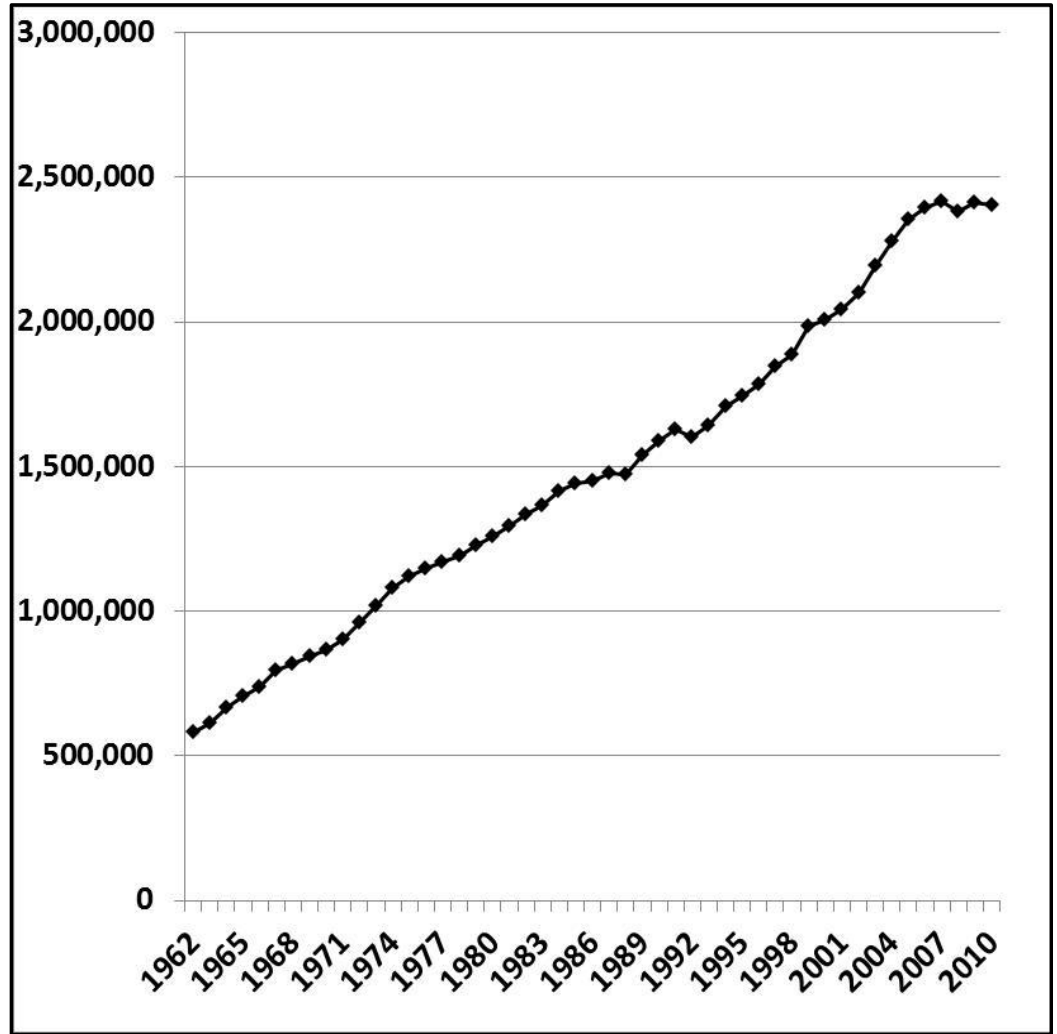

Source: New Zealand Transport Agency (2011)

Data from the MoT's Household Travel Survey shows that New Zealand households are highly car reliant with 78\% of all trips during the period 2008 and 2011 undertaken either as a car driver or a car passenger. This level of car dependence is higher than New South Wales, at 68\%, and Great Britain, at 64\%. Countries with well-developed public transport systems, or strong bicycling cultures such as the Netherlands (48\%), Denmark (57\%), and Sweden (53\%) are less reliant on cars for household transport. New Zealand's use of public transport, at $2.8 \%$ of household trips, was the lowest of the countries reviewed. Sweden, NSW, and Great Britain had the highest use of public transport at $11.7 \%, 11.3 \%$, and $10.0 \%$ respectively. Denmark and the Netherlands, countries with high levels of bicycle use, had lower levels of public transport usage possibly indicating a degree of crossover between these two modes of transport (Figure 1.3). ${ }^{4}$

\footnotetext{
${ }^{4}$ Comparable data could not be found for the United States.
} 


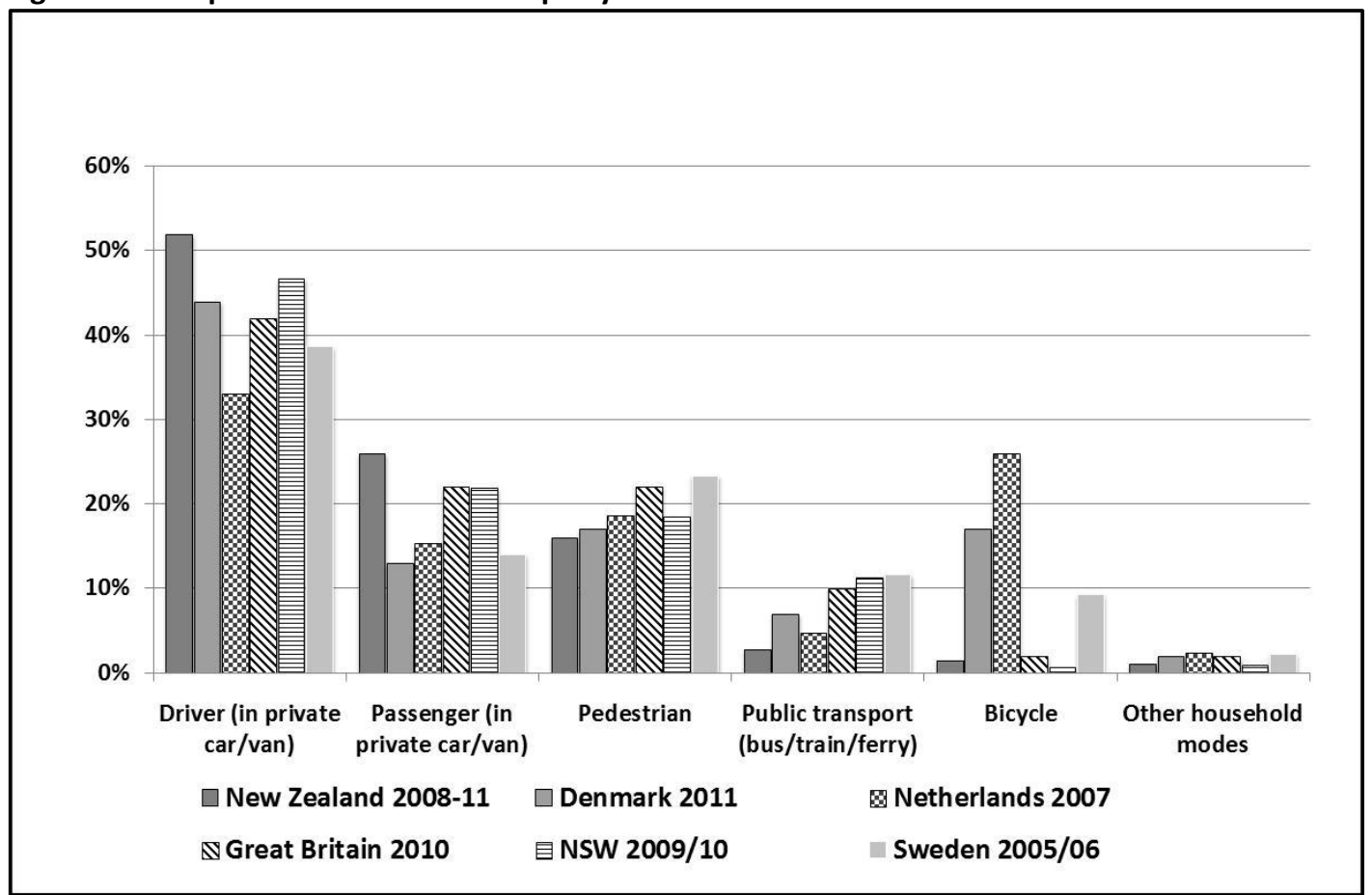

Source: Ministry of Transport (2012a), Technical University of Denmark (2012), Centraal Bureau voor de Statistiek (2012), Swedish Institute for Transport and Communications Analysis (2007), Department for Transport (2011a), Bureau of Transport Statistics (2011)

LPVs not only dominate personal transport in New Zealand, but they are also the largest single class of road vehicle. In 2010, LPVs comprised $80.3 \%$ of all motorised licensed vehicles ${ }^{5}$ and were responsible for $77.9 \%$ of the vehicle kilometres travelled (VKT) by the New Zealand road vehicle fleet (Figures 1.4 and 1.5).

\footnotetext{
${ }^{5}$ The licensed road fleet also includes non-motorised vehicle trailers.
} 
Figure 1.4: Composition of the motorised road fleet in 2011

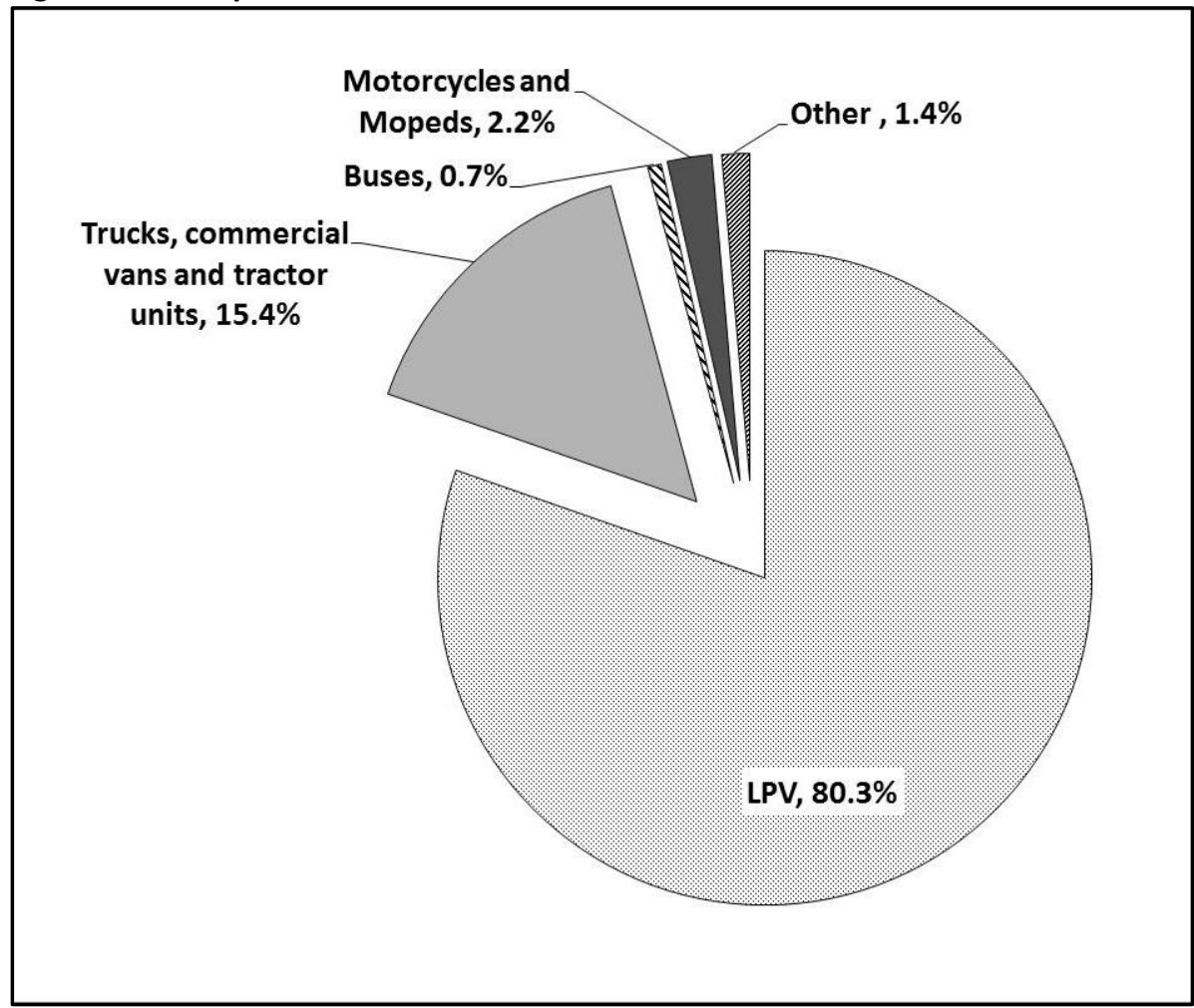

Source: New Zealand Transport Agency (2011)

Figure 1.5: Proportion of VKT by vehicle type in 2011

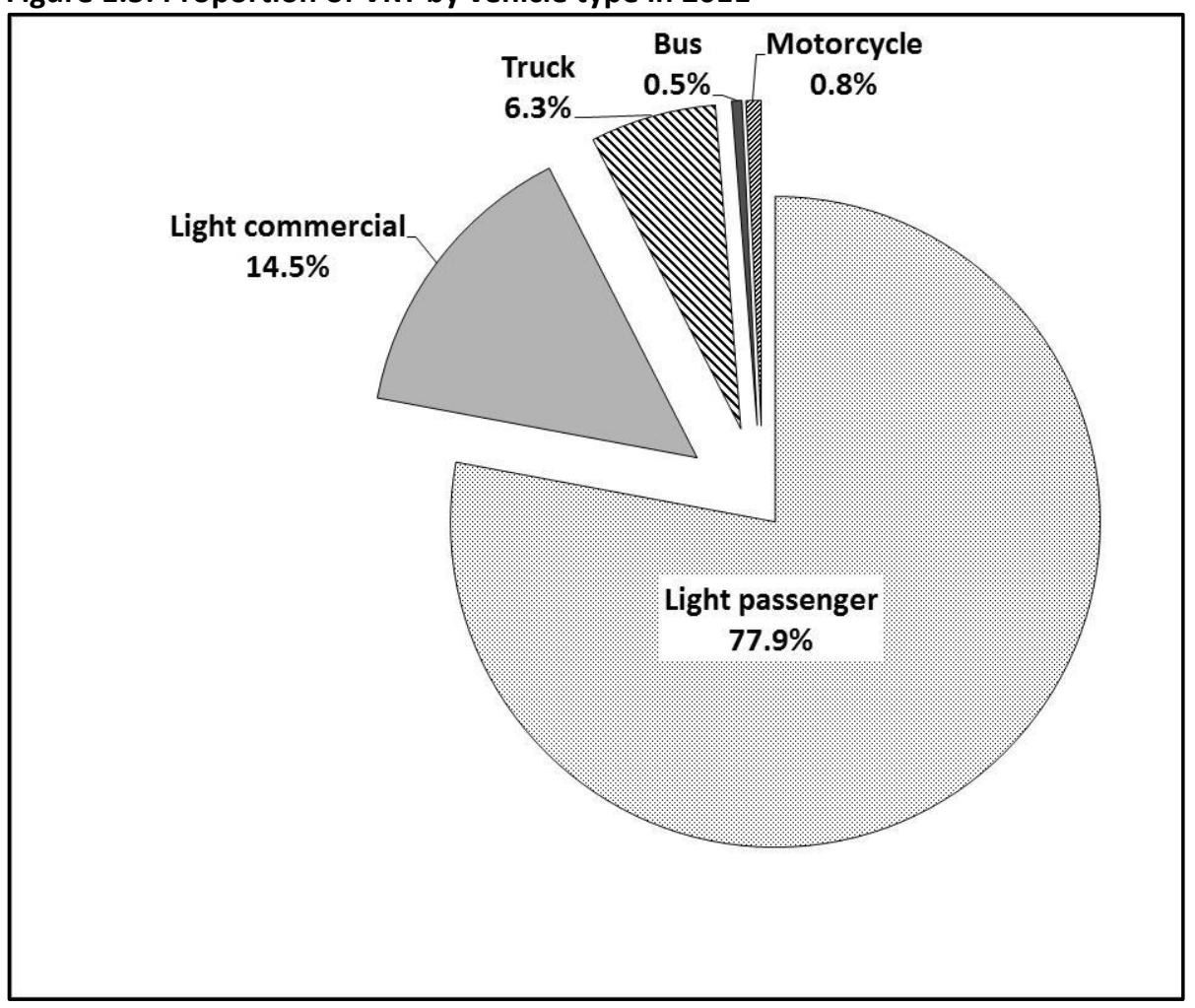

Source: Ministry of Transport (2012d) 


\subsubsection{The future of the car: climate change and availability of petroleum}

The 1963 Buchanan report shows that the problems associated with the prevalence of the car are not new. However, towards the end of the $20^{\text {th }}$ century two issues emerged that have brought into question the sustainability of modern civilisation's continued reliance on the petroleum fuelled car as the dominant form of personal transport. The first issue is the growing threat from anthropogenic global warming and the need to reduce greenhouse gas (GHG) emissions from combustion of fossil fuels, including the combustion of petroleum based transport fuels. The second issue relates to the uncertainties surrounding the future supply and price of petroleum based liquid fuels (Newman, 2009; Urry, 2008).

\section{Road transport's contribution to Greenhouse Gas emissions}

There is very strong scientific evidence that human activities, including the combustion of fossil fuels, are contributing to the warming of the Earth's atmosphere and oceans (IPCC, 2007b, pp. 100-102, 390-393). There is also strong evidence indicating that this warming will result in adverse impacts on: (1) freshwater resources and their management; (2) ecosystems, food, fibre, and forest products; (3) coastal systems and low-lying areas; (4) industry, settlements and society; and (5) human health (IPCC, 2007a).

Because of its almost total reliance on the use of petroleum as a source of energy, the road transport sector is a major source of GHG emissions. At a global level, in 2005 , road transport was estimated to produce $10.7 \%$ of all GHG emissions. Globally, road transport ranks after energy supply, industry, fires, forest clearing etc., and agriculture as a source of GHG emissions (Figure 1.6). In 2005, 60\% of all global GHG emissions were in the form of $\mathrm{CO}_{2}$ emissions from fuel combustion, with road transport responsible for $17 \%$ of all $\mathrm{CO}_{2}$ emissions (Figure 1.7) (International Transport Forum, 2010).

Road transport has a greater role in the production of New Zealand's GHG emissions contributing $18.3 \%$ of gross GHG emissions, which is higher than the 
global average of $10.7 \%$ (Figure 1.6) ${ }^{6}$. After agriculture, road transport is the second highest producer of GHG emissions (Figure 1.8). If the methane and nitrous oxide emissions from the agriculture and waste sectors are excluded, the road transport sector becomes the single largest source of GHG emissions in New Zealand. The relatively low level of GHG emissions from the energy supply sector reflects the high level of renewable electricity generation in New Zealand (Ministry for the Environment, 2011).

Figure 1.6: 2005 Global GHG emissions by source (est. 45,400 $\left.\mathrm{Mt} \mathrm{CO}_{2} \mathrm{e}\right)^{7}$

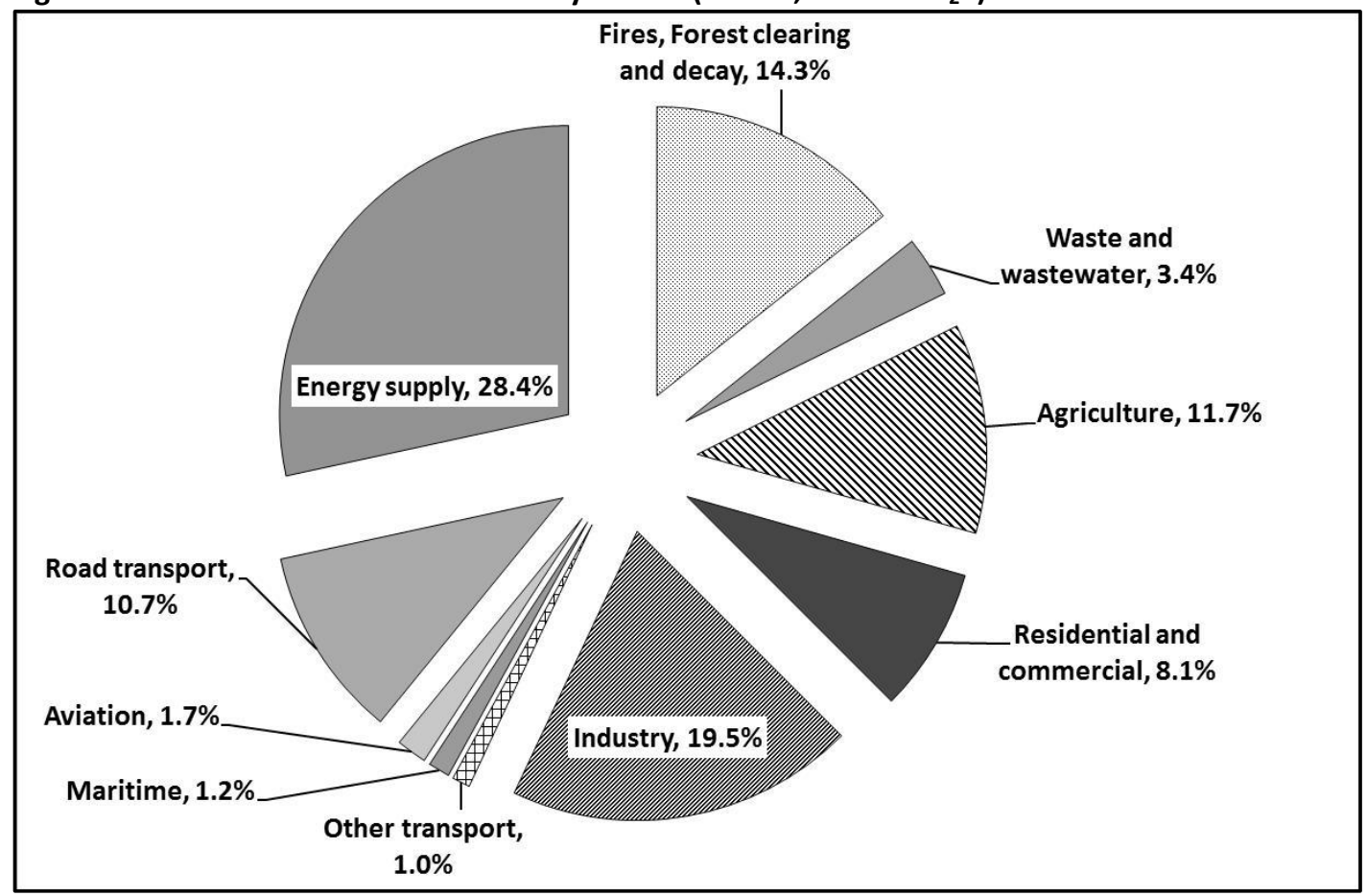

Source: International Transport Forum (2010)

\footnotetext{
${ }^{6}$ The total GHG emissions profile in Figure 1.8 are for New Zealand's gross GHG emissions and exclude the GHG emissions from land use, the effect of land-use change and forestry. In 2009, increased forestry resulted in a net uptake of GHG emissions from this sector.

${ }^{7} \mathrm{CO}_{2} \mathrm{e}$ is carbon dioxide equivalent. This measure is used to compare emissions from various greenhouse gases by converting the non- $\mathrm{CO}_{2}$ greenhouse gases to the equivalent amount of carbon dioxide on the basis of their global-warming potential (GWP).
} 


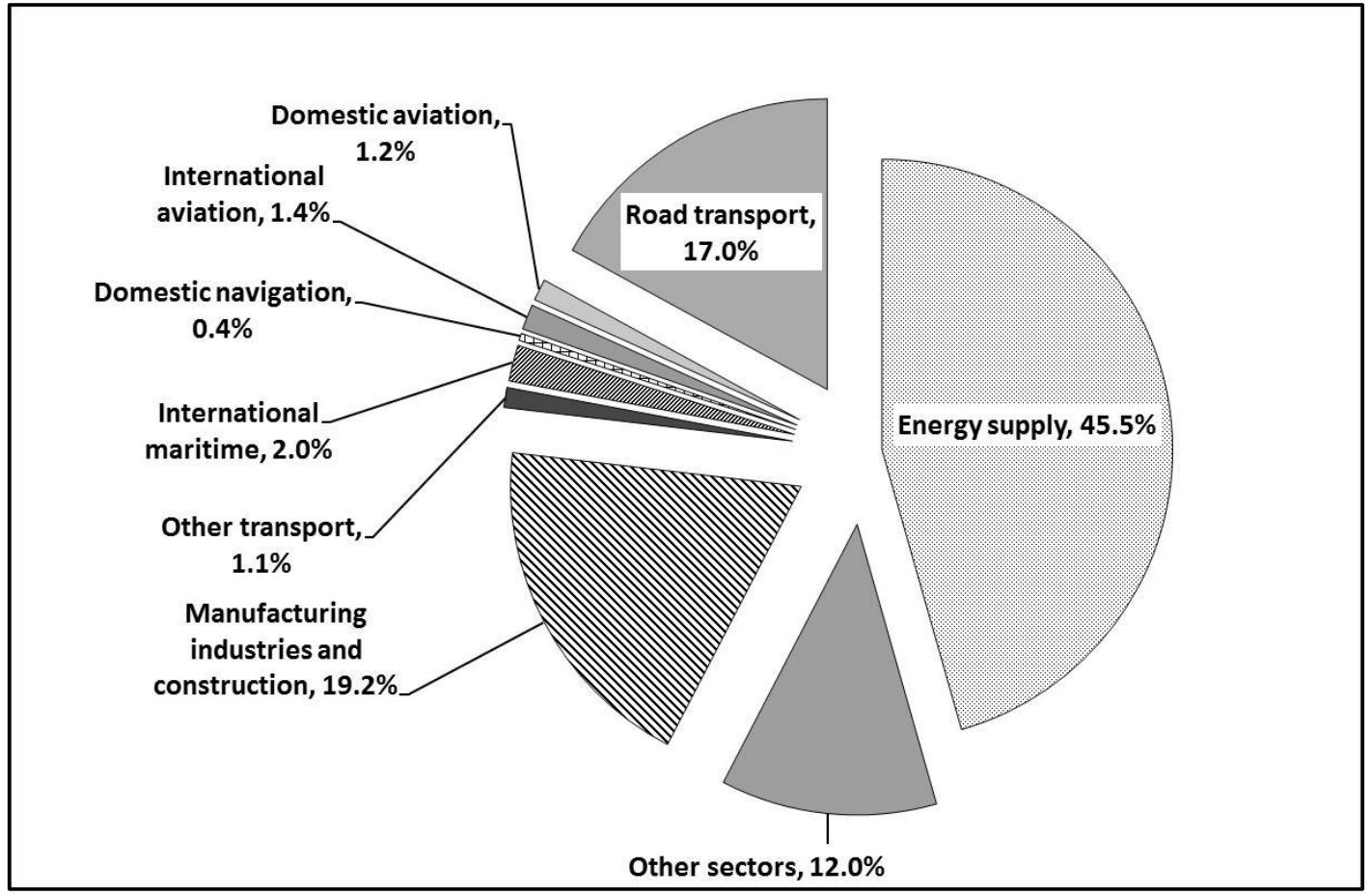

Source: International Transport Forum (2010)

Figure 1.8: 2009 Gross New Zealand GHG emissions by source (est. $67.5 \mathrm{Mt} \mathrm{CO}_{2} \mathrm{e}$ )

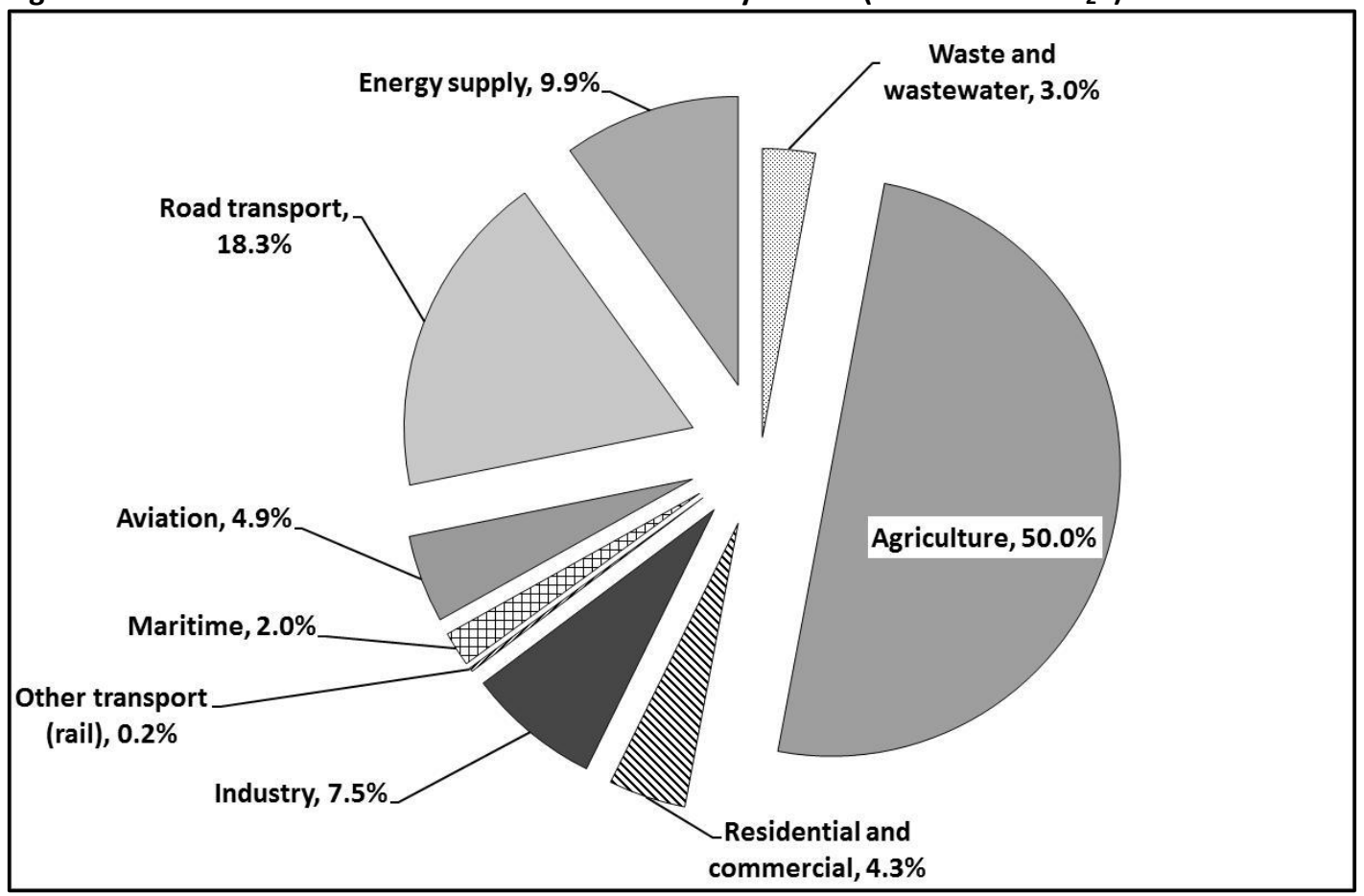

Source: Ministry for the Environment (2011) 


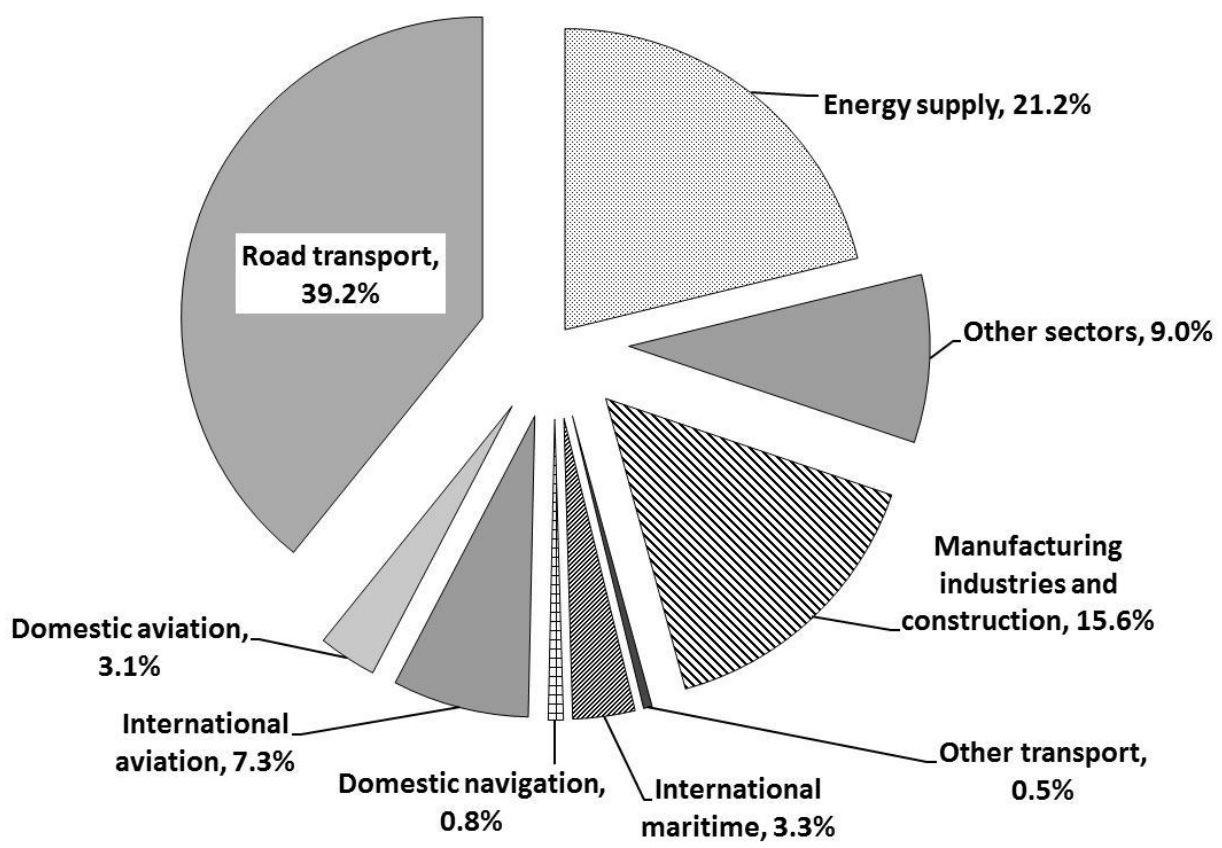

Source: Ministry for the Environment (2011)

Precise estimates of the GHG emissions from the LPV fleet are not available due to difficulties in allocating the amount of diesel fuel used between LPVs, light commercial vehicles, and heavy commercial vehicles (K. Hammond; Ministry of Economic Development, personal communication, November 25, 2010). The MoT provides an estimate that is derived from its own LPV fleet model. They estimate that, in $2009,62.7 \%$ of road transport $\mathrm{CO}_{2}$ emissions were from the LPV fleet, but, by 2010 , this level had increased to $65.5 \%$. These figures should be treated with some caution as estimates of total $\mathrm{CO}_{2}$ emissions from the all road vehicles differ between those produced by the MoT and those produced by the Ministry of Economic Development (MED) and published in New Zealand's national GHG inventory report (Ministry for the Environment, 2011) ${ }^{8}$.

The national inventory data indicates that GHG emissions from road transport increased by $65.9 \%$ from 7.47 million tonnes $\mathrm{CO}_{2} \mathrm{e}$ in 1990 to 12.34 million tonnes $\mathrm{CO}_{2} \mathrm{e}$ in 2011. MED's most recent projections indicate that the GHG emissions from road transport will continue to increase, but not as rapidly as in the past. This view

\footnotetext{
${ }^{8}$ The method used in this study to estimate the GHG emissions from the LPV fleet is described in chapter 5 .
} 
is based on the expectation that the growth of the LPV fleet will be slower due to vehicle ownership per capita approaching saturation levels (Ministry of Economic Development, 2011c). Depending on the future economic growth path, MED considers that, by $2040, \mathrm{GHG}$ emissions from road transport will be between $0.9 \%$ and 29.3\% higher than the levels in 2010 (Figure 1.10). As part of their forecast, MED have incorporated electric vehicles (EVs) into the LPV fleet, but they consider that the demand will be limited and that, by 2030 , they will account for only $5 \%$ of all road transport VKT. This is a more conservative estimate of EV uptake than indicated by the results of this present study, and most of the other New Zealand studies that are discussed in section 8.5.1.

Figure 1.10: New Zealand historic and forecast GHG emissions from road transport: 1990 to 2040

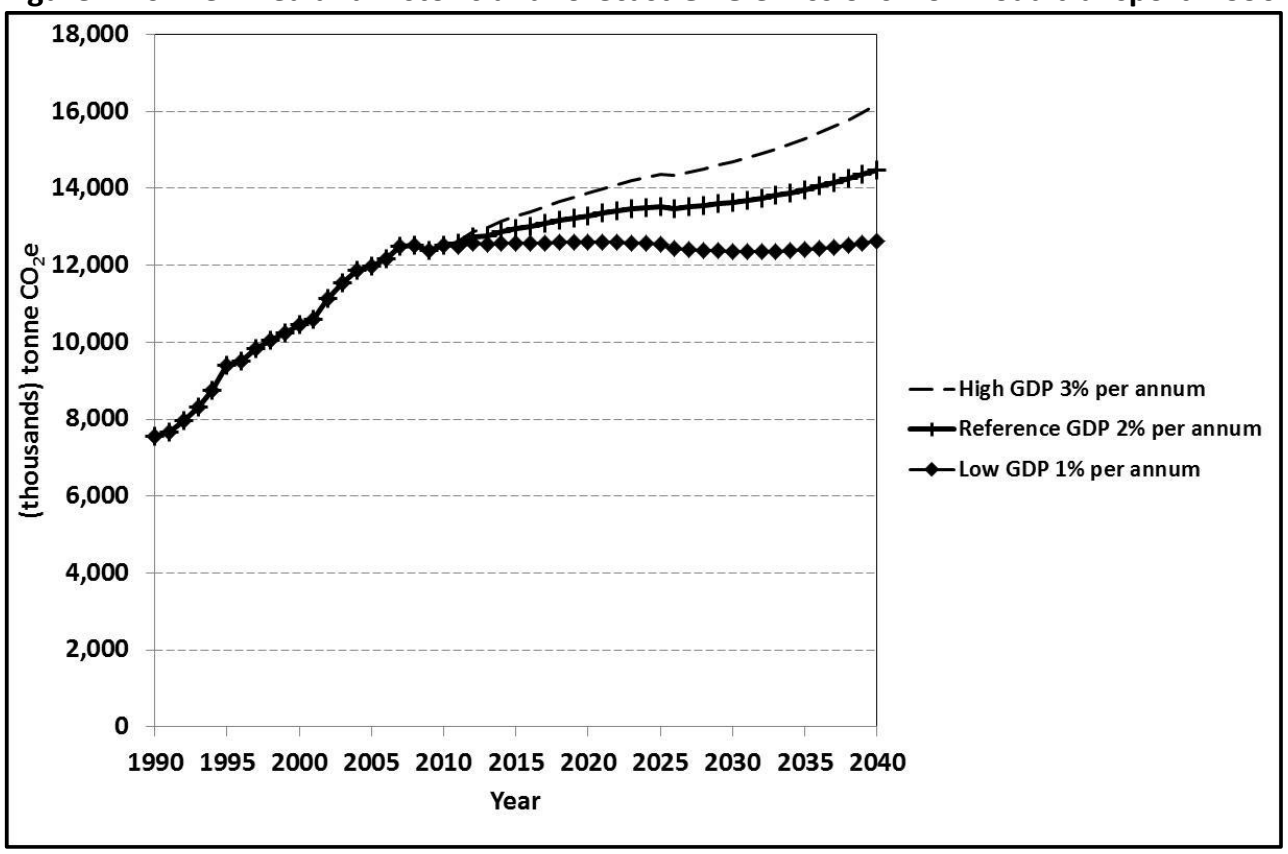

Source: Ministry of Economic Development (2011c)

\section{The importance of petroleum}

If LPVs are the dominant form of personal mobility in New Zealand, then the internal combustion engine vehicle (ICEV) has been the technology that defines the LPV. In 2010, $99.88 \%$ of the LPV fleet was powered by an internal combustion engine (ICE) using either petrol or diesel as fuel. This almost total reliance on petroleum as an energy source for personal mobility increases to $99.92 \%$ if CNG and LPG fuelled ICEVs are also included (Figure 1.11). These proportions remained constant over the period 2005 to 2011 (New Zealand Transport Agency, 2011). 


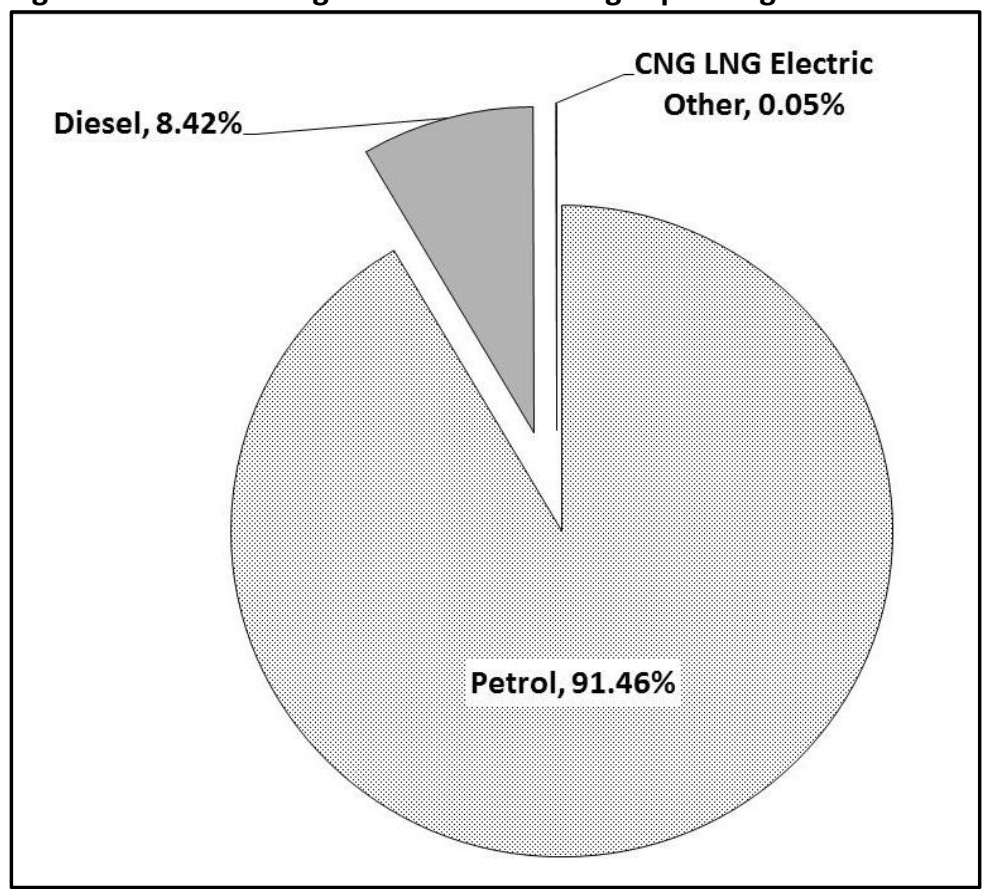

Source: New Zealand Transport Agency (2011)

This high reliance on one type of LPV engine technology and one type of fuel is a global phenomenon. It is estimated that, in $2008,94 \%$ of world's road transport (LPV and heavy vehicles) was fuelled by petrol or diesel, $3 \%$ by natural gas, $2 \%$ from liquid and gaseous biofuels, and 1\% by electricity (Trigg, 2010).

\section{The future availability and cost of petroleum}

In November 2010 Fatih Birol the chief economist of the International Energy Agency (IEA), stated that the age of cheap oil was over and that the best that could now be achieved is to implement policies that would slow the increase of oil prices (Kurczy, 2010). More recently, however, the IEA view has changed in response to the rapid expansion in world output of light tight oil through the use of horizontal drilling and hydraulic fracturing techniques, and the increased production of natural gas liquids and other types of unconventional oil. However, the future of the global supply from these sources remains uncertain as noted in the 2012 World Energy Outlook:

Unconventional resource estimates are less reliable than those of conventional resources, as they have generally been less thoroughly explored 
and studied, and there is less experience of exploiting them (International Energy Agency, 2012, p. 100).

Despite these uncertainties, the IEA remains of the view that the price of oil will continue to rise irrespective of any future developments in oil supply and demand (International Energy Agency, 2012).

However, other commentators still consider these recent developments in the 'oil patch" as unsustainable and the implications of the "peak oil" hypothesis remain a valid concern (Hughes, 2013). The concept of peak oil was first proposed by M. King Hubbert in 1956 when he argued that the production of all finite resources, such as petroleum, will approximately follow the shape of the bell curve, first increasing to a peak and then shifting to an irreversible decline (Hubbert, 1956).

The peak oil model rests on the expectation that, once the easiest fields to find and access are utilised, the increasing demand for oil will have to be met from oil fields that are more expensive to access and, on average, are smaller in size and are of lower quality (Guilford et al., 2011). As the size of the new discoveries has declined in recent decades, the ability to maintain the excess 'swing production' capacity has diminished and new supply has now become 'just in time' to meet growing demand (Campbell and Laherrere, 1998; Hamilton, 2009; Skrebowski, 2011). In recent years, when there has been an outage or disruption to supply, the price must rise rapidly to reduce demand. Murray and King (2012) concluded that global oil production capacity was sufficient to manage such events until 2005.

Those using Hubbert's methods have been criticised for being too pessimistic and for not adequately taking into account improvements in exploration and production technologies. Critics highlight that a number of forecasts using these methods have been proven wrong (IHS Cambridge Energy Research Associates, 2010; Smil, 2006; Hamilton, 2012).

In the face of the uncertainties surrounding the future of global oil supplies, commentators and governments of oil importing nations are framing the issue in terms of energy security (Ministry of Economic Development, 2011b; House of 
Commons: Energy and Climate Change Committee, 2011; Obama, 2011;

Commonwealth of Australia, 2011; Hirsch et al., 2005). EVs are one of the means that have been proposed for reducing reliance on imported petroleum, although this is not a major focus of the Australian, United Kingdom, United States, and New Zealand national energy strategies. Most of the focus is on the development of their nation's domestic energy resources so as to displace imports of petroleum. In the case of New Zealand, the development of oil and coal resources is listed as a key element of the first policy priority in the Government's 2011 Energy Strategy (Ministry of Economic Development, 2011b).

\section{The effect of peak oil on GHG emissions}

The peak oil model, if confirmed, could be taken to imply that there will be rising prices leading to a decline in LPV use, which would result in reduced GHG emissions and less urgency to implement other mitigation measures. Nel and Cooper (2009) have argued that the remaining fossil fuel resources would be sufficient to raise the global temperature by no more than $1^{\circ} \mathrm{C}$ above levels in $2000^{\circ}$. However, their analysis has been criticised for not taking into account the potential non-linear effects associated with climate change, for using a low value for climate sensitivity, and not adequately taking into account the impacts of using unconventional fossil fuels (Zecca and Chiari, 2010).

Work by Kharecha and Hansen (2008) concluded that even if oil resource estimates based on the peak oil theory are correct, it will still be necessary to constrain the use of coal and unconventional fossil fuels to avoid dangerous global warming. Hughes and Rudolph (2011) and Verbruggen and Marchohi (2010) argued that the uncertainties around the climate system, as highlighted by Hansen et al. (2007), and the potential for use of unconventional fossil fuels such as heavy crude and tar sands, warrant the implementation of more proactive GHG emissions reduction policies, and it should not be assumed that resource scarcity will achieve these reductions.

\footnotetext{
${ }^{9} \mathrm{Nel}$ and Cooper (2009) include coal and natural gas resources in their analysis.
} 


\section{The peak car travel phenomenon}

There is emerging evidence that, in a number of cities and regions in the developed world, the amount of VKT by LPVs has plateaued. It has been suggested that these events may indicate that, in developed countries, the demand for travel by car may be approaching saturation (Millard-Ball and Schipper, 2011; Newman and Kenworthy, 2011).

Some have argued that this levelling off in VKT reflects the effects of recent economic conditions, and that once economic growth returns the growth of VKT will resume (Glaister, 2011). This argument does not seem to be supported by data, which shows that VKT ceased to increase in the United States in 2005, in some Australia cities in 2004 (Newman and Kenworthy, 2011), in the United Kingdom in 2000 (Metz, 2012), and in New Zealand in 2007 (Ministry of Transport, 2012b) all before the start of the economic recession in 2008.

The reasons why there might be a changing trend in LPV VKT in these developed countries remain uncertain and a number of possible causes have been proposed. Newman and Kenworthy (2011) argued that the decline in VKT may be in part due to societal changes that are causing a movement of people away from the suburbs back into urban centres. The changes could be in response to the adverse effects of ever increasing commuting times as the result of ongoing urban sprawl. They also argue that another reason could be an aging population whose children have grown and left home are now attracted to living in vibrant urban communities. Other reasons that have been suggested are: (1) improvements in public transport systems in Australia and the United States; (2) the impact of higher fuel prices; (3) the saturation of daily travel needs in the context of current levels of car ownership, public transport availability, and vehicle speed limits (Metz, 2010); (4) increasing income inequality; and (5) declining interest in cars among the young who increasingly use handheld devices and social media for social networking functions that were previously undertaken by car (Cohen, 2012).

So far the plateau in LPV VKT has been only documented in some developed countries and this decline must been seen within the context of growth in 
ownership of all types of road vehicles in India, south Asia, Southeast Asia, and China. For these regions, it is estimated that annual growth rates of road vehicle ownership will be between about 5 and 12\% for the period 2009-2020. As a consequence, the global number of all types of road vehicles, in 2020 , could be $50 \%$ higher than in 2009 (Keshavarzian et al., 2012).

\subsection{Addressing the problem: LPVs in a carbon constrained world}

There is clearly an expectation that LPVs will remain the dominant form of personal mobility for the foreseeable future, and the challenge is how to transform the technology so that it uses less fossil fuel and emits fewer GHGs. The technological approaches to achieving these goals can be classified into: (1) reducing the load on the vehicle; (2) increasing the drive train efficiency; and (3) using less carbon intensive fuels (Table 1.1) ${ }^{10}$ (Kahn Ribeiro et al., 2007; U.S. Department of Transportation, 2010; European Commission, 2011; Bandivadekar et al., 2008; UK Industry Taskforce on Peak Oil \& Energy Security, 2010).

Table 1.1: Measures for reducing GHG emissions from light passenger vehicles

\begin{tabular}{|c|c|}
\hline Measure & Description \\
\hline $\begin{array}{c}\text { (1) Reduce the load on the } \\
\text { vehicle }\end{array}$ & $\begin{array}{c}\text { Reduce vehicle weight and/or size } \\
\text { Reduce aerodynamic and rolling resistance } \\
\text { Reduce auxiliary loads, i.e. air conditioning }\end{array}$ \\
\hline $\begin{array}{c}\text { (2) Increase the efficiency of } \\
\text { the drive train }\end{array}$ & $\begin{array}{r}\text { Improve ICEV energy efficiency- Direct injection diesel } \\
\text { and petrol ICEV, hybrid drive trains } \\
\text { Switch to more energy efficient drive train - EVs, } \\
\text { hydrogen fuel cell vehicles (HFCVs) }\end{array}$ \\
\hline (3) Switch to a less carbon \\
intensive fuel & $\begin{array}{c}\text { Biofuels } \\
\text { Liquid petroleum gas (LPG) }\end{array}$ \\
& $\begin{array}{r}\text { Non-fossil fuel generated hydrogen (ICEV and HFCV) } \\
\text { Non-fossil fuel generated electricity (EVs) }\end{array}$ \\
\hline
\end{tabular}

Adapted from Kahn Ribeiro et al. (2007) and Kromer and Heywood (2007)

\footnotetext{
${ }^{10}$ Other measures to reduce GHG emissions and the demand for petroleum by LPVs involve promoting driver behaviour that has the effect of reducing fuel consumption and promoting measures that decrease the use of LPVs. The measures to reduce the use of LPVs include: (1) shifting travel to other modes, such as public transport, walking, and cycling; (2) changes to land-use planning that result in land use patterns that require less use of LPVs; and (3) promoting the use of telecommunication technologies that reduce the need to travel (Stern, 2006; U.S. Department of Transportation, 2010; European Commission 2011; Ministry of Transport, 2012c; Kahn Ribeiro et al. 2007).
} 
An LPV that does not utilise either petrol (gasoline) or diesel fuel is known as an alternative fuelled vehicle (AFV). There are three types of AFVs that are currently available in the market or that are in the process of being developed: (1) ICEVs that use biofuel, natural gas, liquid petroleum gas, or hydrogen as fuel; (2) HFCVs; and (3) vehicles that use electric energy stored in an electrochemical battery (Kahn Ribeiro et al., 2007; Kromer and Heywood, 2007).

LPVs that use electricity stored in an electrochemical battery are commonly known as EVs and, along with LPVs powered by biofuel, CNG and LPG, are the only AFVs currently available in the market ${ }^{11}$. HFCVs are seen as a technology that will not be ready for wide spread use until at least the late 2020s. Unlike EVs, which can utilise the existing electricity infrastructure as an interim energy distribution system until the development of a network of specialised charging facilities, HFCVs require the development of a new fuelling infrastructure (Ogden et al., 2011; Kalhammer et al., 2007; Leaver and Gillingham, 2010).

EVs are currently available in limited numbers in the New Zealand market. When used in conjunction with renewably generated electricity, EVs open up the opportunity to reduce GHG emissions from the LPV fleet and, at the same time, reduce New Zealand's high reliance on imported petroleum (Duke et al., 2009).

\subsection{Objective of the study}

The extent to which EVs can assist in reducing New Zealand's GHG emissions will be dependent on the rate that they are taken up into the LPV fleet and the mix of fossil fuel and renewable energy used to generate the electricity for EVs (Simpson, 2004; Nguyen et al., 2013; Holdway et al., 2010).

This study addresses these issues by asking the question:

\footnotetext{
${ }^{11}$ Technically HFCVs are also battery EVs, but this technology requires a constant flow of hydrogen and oxygen to operate.
} 
If EVs were available in the New Zealand market, would people buy them, what types would be purchased, and what impact might EVs have on New Zealand's demand for petroleum fuels and GHG emissions?

The objectives of the study are to:

1. Develop a demand model of New Zealand car buyers that can be used to estimate the demand for EVs, taking into account the effects of changing vehicle and fuel prices and improvements in technology.

2. Use the demand model to project the composition of the LPV fleet over a 20 year period.

3. Assess the impact of the changing vehicle stock on the GHG emissions from the LPV fleet.

4. Assess the impact of the increased electricity demand from EVs on GHG emissions produced from electricity generation.

\subsection{Approach used}

This study uses three models to achieve its objectives:

1. The uptake of EVs into the LPV fleet is modelled using a mixed multinomial logit discrete choice model based on data from a nationwide stated choice survey of New Zealand car buyers.

2. A vehicle fleet model (VFM), which incorporates the discrete choice model, is used to estimate the LPV fleet's composition, VKT, fuel consumption, and GHG emissions.

3. The Electricity Authority's mixed integer optimisation generation expansion model (GEM) is used to model the effect of the electricity demand from EVs on the future development of electricity generation plants, and then to estimate the GHG emissions from the electricity sector.

\subsection{Structure of this thesis}

In chapter 2 there is a review of the methods used in previous studies to estimate the demand for EVs and other types of AFV. 
Chapter 3 discusses the theory of discrete choice models, the methods used to design stated choice experiments, and the issues to be considered when implementing a stated choice survey.

Chapter 4 reviews the emerging LPV technologies with an emphasis on EVs. The chapter canvases some of the advantages and barriers facing the acceptance of EVs, and concludes with a discussion of the issues surrounding EV charging and the impact of EVs on electricity grids.

Chapter 5 discusses the methods used to implement the stated choice survey, including the design of the stated choice experiment. The chapter discusses the development of the VFM including the vehicle use sub-model (VUM), which is used to estimate future travel demand by ICEVs and EVs in response to changes in energy prices. Finally, the chapter discusses the development of the scenarios used to specify the values of the inputs used to run the VFM.

Chapter 6 describes the GEM and the methods used to assess the impact of the uptake of EVs on the future development of New Zealand's electricity generation sector.

Chapter 7 discusses the results of the stated choice survey and the output from the discrete choice model.

Chapter 8 presents the VFM's projections of the future composition of the LPV fleet.

Chapter 9 presents the energy demand and GHG emissions projections from the LPV fleet, a discussion of the impacts of EVs on the development of electricity generation in New Zealand, and the estimated GHG emissions from the electricity sector. The chapter concludes with a discussion of the impact of EVs on the combined GHG emissions from the LPV fleet and electricity sector.

Chapter 10 presents the key conclusions, reviews some limitations of the study, and sets out suggestions for further research. 


\section{Chapter 2: Review of alternative fuel vehicle technological change studies}

\subsection{Introduction}

There is an extensive literature in the fields of economics, sociology, marketing, science and technology, and sustainability studies that attempts to explain how technological innovation occurs, how new innovations enter markets, and how new innovations replace existing technologies (Geels, 2004; Markard et al., 2012; Shackley and Green, 2007; Wilson and Grubler, 2011).

There is an emerging view in the literature that the normal process of incremental uncoordinated technological change is insufficient to promote the rapid changes in technology needed to address the pressing environmental issues faced by today's society. This literature focuses on understanding the processes that drive technological change with a view to developing methods to facilitate such change, thereby addressing these environmental issues (Markard et al., 2012; Kemp and Loorbach, 2006). The shift from internal combustion engine vehicle (ICEV) technology to electric vehicle (EV) technology clearly meets the definition of a sustainability transition. Geels (2004) identifies three general approaches to understanding technological change, which he calls the point source, transformational, and replacement approaches.

The point source approaches focus on the emergence and diffusion of new technologies, but do not address how the new technology replaces the old technology. The transformational group of approaches are derived from the sociological literature. This literature focuses on actor groups, their activities, and the rules and routines surrounding the technology, which together form the sociotechnical regime. The socio-technical regime is resistant to change due to sunk investments, existing behavioural patterns, vested interests, infrastructure, subsidies, and regulations (Geels, 2004).

EV technology is currently in the process of entering markets and the focus of this study is on the ability of EVs to compete with and to eventually displace ICEVs 
rather than on their innovation. Geels (2004) defines studies that focus on these elements of the technological change process as replacement approaches. These theories form the basis of the modelling approach used in this study.

A review of past studies of the potential of alternative fuelled vehicle (AFV) technologies to replace ICEV technology also identified studies based on diffusion of innovations theories, and those that used the subjective judgements of experts to develop market penetration scenarios.

This chapter first summarises past studies that have assessed the potential of AFV technologies to replace ICEVs. The chapter concludes with a discussion of the rationale for the modelling approach chosen for use in this study.

\subsection{Replacing the ICEV: a review of modelling methods used}

\subsubsection{Diffusion of innovation approaches}

The diffusion of innovations approach is a popular method used in marketing where the uptake, or diffusion, of a new innovation into the market is explained in terms of the communication over time among the members of a social system (Rogers, 1995, p. 10). Communication allows for the creation and sharing of information about a new technology, which then gives members of the social system some degree of certainty about the new technology. The members of the social system each evaluate the new technology based on the subjective evaluations of their peers. These peers have similar social and personal attributes or already own or have experience with the new technology.

The diffusion of innovations approach sees the members of a social system as falling into different adopter categories: innovators, early adopters, early majority, late majority, and laggards. Innovators are described as "venturesome", while early adopters are described as opinion leaders whose views are seen as an endorsement of the new technology for the later adopters. The early adopters are described in the literature as probably having a more extensive communication network, having more formal education, being less dogmatic with a greater ability to deal with abstract concepts, and being able to cope with uncertainty and risk. They are also 
considered to have a favourable attitude towards science and greater intelligence (Rogers, 1995, p. 273).

The early majority adopters are described as being generally more "deliberate" in their decision making, while late majority adopters will probably hold back until the level of uncertainty associated with the new technology has been eliminated. The laggards may be considered to be suspicious of the new technology and may even reject it completely (Saviak, 2007). In this approach, the rate of diffusion is determined by the following factors:

- whether the attributes of the new technology are perceived as better than the existing technology

- whether the attributes are compatible with the existing values and needs of the adopter

- the ease with which the new technology can be understood

- the degree of difficulty in accessing the new innovation for trialling prior to adoption,

- the degree to which the new technology can be observed being used by others (Wilson and Dowlatabadi, 2007).

Despite its popularity, the diffusion of innovations approach has been criticised for being too linear as a process (Wilson and Dowlatabadi, 2007), for not being readily applicable to a broad range of situations, and not taking into account factors that can limit the diffusion process. It has also been criticised at a practical level for using data from individuals, but then applying this data at the organisational level (Saviak, 2007).

Applying the diffusion of innovations approach usually involves the estimation of a logistic function. Various types of logistic function can be used, but the most popular is the Bass (1969) model. The Bass model defines the shape of the diffusion curve in terms of the number of new adopters and contains three parameters that represent the coefficients for: (1) innovation; (2) imitation; and (3) the total number of adopters or market potential. 
The Bass model was used by Cao and Mokhtarian (2004) to estimate the shape of the diffusion curves for E85 and CNG vehicles. Collantes (2005) used an expanded form of the Bass model (Norton and Bass, 1987) to compare the effects of substitution between hybrid electric vehicles (HEVs) and the, as yet unavailable, hydrogen fuel cell vehicles (HFCVs).

McManus and Senter (2009) modelled the uptake of plug-in hybrid electric vehicles (PHEVs) by using four logistic diffusion models: (1) the Bass model; (2) a logistic model; (3) the Gompertz function; and (4) a modified Bass model. In addition to these models the study also used two models which did not contain any assumptions about the total number of adopters or market potential. The first model was a modified Bass model based on the work of Centrone et al. (2007), which allowed the total number of adopters to vary with births and deaths. The second unconstrained model was a hybrid diffusion of innovations and binominal choice model based on the work of Struben and Sterman (2007). McManus and Senter (2009) found that the market penetration forecasts for PHEVs varied significantly depending on the type of model used and the assumptions that were made about the total number of adopters.

Diffusion models have demonstrated success in accurately forecasting market penetration of products where the product has already entered the market and some market data are available. However, for new products such as EVs, which have either not yet entered the market or are just entering the market, the values of model parameters must be derived either by using values from analogous products, or by using managerial or expert judgements of what would be appropriate values. McManus and Senter (2009) derived their estimates of the parameter values using analogous products, Collantes (2005) used expert judgements, and Cao and Mokhtarian (2004) used a combination of both of these methods.

\subsubsection{Expert based market penetration scenarios}

Market penetration scenario studies have been used extensively in studies of the potential of AFVs in global markets. These studies use the opinions of experts to 
estimate market penetration of new products and cannot be explicitly related to any particular theory of technological change.

The rates of penetration of AFVs in these scenarios are based on subjective assessments, sometimes by the researcher (Dirr, 2008; Baptista et al., 2010; Brady and O'Mahony, 2011) or by eliciting the opinions of experts (Balducci, 2008; Kalhammer et al., 2007; Vyas et al., 1997; International Energy Agency, 2008). The scenarios can be designed to represent the range of feasible market penetration possibilities across a broad range of policy settings (Dirr, 2008; Baptista et al., 2010; Brady and O'Mahony, 2011), or restricted to rates of market penetration that are considered consistent with a particular policy scenario (International Energy Agency, 2008).

The process for eliciting expert opinion varies, but a popular method is the Delphi method (Dalkey and Helmer, 1963) used for example by Vyas et al. (1997), and Balducci (2008). Kalhammer et al. (2007) used a combination of visits and a questionnaire to elicit the opinions of industry experts, while the International Energy Agency (2008) used a process consisting of expert workshops and peer review from its international network of experts.

\subsubsection{Technological and economic replacement}

Technological and economic replacement approaches, based on classical economic theories of technological change, argue new technologies replace older technologies because they perform better, subject to the users' preferences, and have a lower price (Grübler et al., 1999). These models are also based on theories of rational choice and utility maximisation. Individuals and firms are treated as rational actors that will choose a new technology because it will provide more utility than the existing technology (Arrow, 1962).

These approaches also assume that technologies will improve both in performance and in reducing costs due to the effects of learning-by-doing and learning-by-using (Nakićenović, 2002; Grübler and Gritsevskyi, 1997). The theory of learning-by-doing assumes that costs decline with cumulative experience (Arrow, 1962) and this 
decline can be graphed as an experience curve. The cost of a new technology is determined by the formula:

$$
C_{\text {unit }_{T}}=C_{\text {unit }_{1}} *\left(\sum_{s=1}^{S=T} \text { unit }_{S}\right)^{-E}
$$

Where $C_{\text {unit }_{T}}$ is cost of the $\mathrm{T}^{\text {th }}$ unit, $C_{\text {unit }}$ is the initial unit cost, unit $_{S}$ is the $\mathrm{s}^{\text {th }}$ unit produced, and $E$ is the experience parameter (Schwoon, 2006a).

Cumulative production is used as a proxy for cumulative experience. An analysis of historical trends indicates that learning rates in the range of $5 \%$ to $25 \%$ for a doubling in cumulative production can be expected (Leiby and Rubin, 2004; Schwoon, 2006a) $)^{12}$. The use of experience curves has been criticised because of the inability to separate the effects of learning-by-doing on changing costs from those of economies of scale or research and development expenditures (Leiby and Rubin, 2004). Experience curves have also been criticised because they relate to changes in costs, but as prices are determined by supply and demand, the changes in costs may not be reflected in the prices of the new technology. There are also difficulties in using experience curves for forecasting when the curve is based on a limited data set because small variations in the estimated value of the experience parameter can lead to major variations in the estimated rate of learning. In turn, this can have a significant impact on projected success of the new technology (Schwoon, 2006a).

The use of these approaches has been criticised because they assume that user preferences are fixed, so they do not account for changing preferences over time. It is also assumed that new technologies will have to compete directly with old technologies. However, historical analysis shows that rather than directly entering the market, a new technology often emerges in a specialised niche where it has a comparative advantage and then develops from there. These approaches have also been criticised for treating the new and old technologies as completely distinct and competitive. This view does not take account of the possibility that many new technologies are often used to augment the existing technology as an interim

\footnotetext{
${ }^{12}$ IEA (2000) and McDonald \& Schrattenholzer (2001) provide a useful summary of the experience curves for energy technologies.
} 
measure until the time where the new technology improves or the supporting systems and infrastructure develop. An example of this kind of hybridisation is the introduction of the coal fired steam ship, which at first was incorporated into sailing ships (Geels, 2004; Geels et al., 2008) ${ }^{13}$.

The rational choice theory of decision making behaviour that underpins these approaches has been questioned as it is often observed that decision makers act in ways that do not appear to be economically rational (Camerer and Loewenstein, 2004). As a response to this apparent irrational behaviour, the concept of bounded rationality has been developed. This concept holds that rather than implementing a decision making process which will result in the maximum utility, a decision maker often uses decision heuristics which make the decision making process manageable and provide an adequate, if not optimal, answer (Conlisk, 1996; Wilson and Dowlatabadi, 2007).

Replacement approaches of technological change that incorporate bounded rationality have been developed in evolutionary economics. These models recognise that organisations and firms comprise human beings who have limited cognitive capacities and therefore use rules and heuristic routines to make sense of a complex world. Rules and routines are shared within groups, providing a degree of coordination and stability. These rules and routines form a technological regime and focus the activities of firms and organisations in particular directions. This is the basis of technological path dependency and the stability of technological regimes over time (Dosi and Nelson, 1994). The development of technologies is the outcome of a selection process where the variety in routines and research directions across organisations results in different innovation outcomes. Successful innovations are selected by the market, survive, and are further developed (Geels, 2004; Metcalfe, 1994). The approaches based on evolutionary economics are focused on the process of innovation, competition between innovators, and the creation of diversity in technologies and technological systems.

\footnotetext{
${ }^{13}$ The PHEV is potentially another example of this approach to technological development.
} 
These approaches have been criticised as having little to say about the selection process itself, which determines success or failure of technologies, other than to say that it occurs through competition in the market (Geels, 2010).

The literature review indicates that the AFV studies using technological and economic replacement approaches can be categorised as economic cost models, agent based models (ABMs), and discrete choice models.

\section{Economic cost models of replacement}

Economic cost models of replacement have been widely used, often in conjunction with logistic diffusion models, to estimate the market penetration of new technologies (Packey, 1993).

In cost models, the market share of each type of vehicle is determined by a formula of the general form:

$$
M S_{i}=\frac{C_{i}^{-v}}{\sum_{i=1}^{n} C_{i}^{-v}}
$$

Where $M S_{i}$ is the market share of the new technology $i, \mathrm{C}_{\mathrm{i}}$ is the cost of the new technology $i$, and $v$ is a number that reflects penetration ability of the new technology. A larger value of $v$ indicates a greater penetration or attractiveness of the new technology (Jaccard et al., 2004; Christidis et al., 2003; Packey, 1993). The cost of the new technology is the present value of the capital and operating costs over the life of the new technology, or, in some studies, the period of vehicle ownership. The values for $v$ and the discount rate are usually determined by a literature review, the researcher's judgement, meta-analysis, or discrete choice surveys (Jaccard et al., 2004). To reflect the uncertainty surrounding the value of $v$, Christidis et al. (2003) modelled the value by using random draws from a Weibull distribution.

A limitation of the economic cost model is that the rate of penetration of the new technology is solely determined by the capital and operating costs of the new technology and does not take into account any other values that may impact on the uptake of the new technology. Jaccard et al. (2004) attempted to expand the cost 
model by adding a parameter to the cost function that attempted to capture the intangible aspects of the alternative. Baxter et al. (2009) expanded the cost model by first dividing the New Zealand car buying population into groups based on the Rogers typology e.g. innovators, early adopters, etc. Then the cost model was applied to each group, with each group being given a weighting intended to reflect that group's inherent preference for EVs.

\section{Agent based modelling}

A number of recent studies have used $A B M s$ to simulate the market penetration of AFVs. These models are used to simulate the effects of the interactions between car buyers on their purchase decisions (Cui et al., 2011; Eppstein et al., 2011). These models can be extended to also include the effect on car buyers' purchase decisions from their interactions with vehicle manufacturers (Schwoon, 2006b; Zhang et al., 2011), and the Government and fuel producers (Sullivan et al., 2009). In these models, the consumers, manufacturers, and other groups are treated as selforganising automata whose behaviour can be modelled by using deterministic or stochastic functions. The interactions between the automata are simulated by using differential mathematical equations designed to mimic processes such as persuasion, sanctioning, and imitation.

The ABMs used in the AFV studies assume that the consumer agents will attempt to maximise their utility given budget constraints and that the utility derived from a type of car will increase with the increasing uptake of the same type of car by the neighbouring agents within the model. A number of studies have used discrete choice models to estimate consumer utility for the vehicle types in ABMs, but with output adjusted to take account of the neighbourhood effects (Cui et al., 2011; Eppstein et al., 2011).

The power of $A B M s$ is that they can demonstrate interesting emergent and other complex phenomena (Macy and Willer, 2002). ABMs also have the potential to more accurately reflect the complex behaviours that underpin the process of technological change. However, because this real world behaviour is complex and based on psychologies that are both bounded and subjective it is often difficult for 
the modeller to quantify, and validate their models. The complexity demonstrated in $A B M s$ also means that their output is highly sensitive to the initial conditions and to any small variations in the interaction rules as specified by the modeller. As a result, $A B M s$ can demonstrate behaviour that is not observed in the real world (Castle and Crooks, 2006). Zellner (2008) argues that the role for ABMs is not as predictive models, which aim to reproduce patterns in time and space, but to assist in exploring and explaining observed phenomena.

\section{Discrete choice models}

Discrete choice models have been used since the 1979 energy crisis to estimate the demand for AFVs (Train, 1980; Beggs et al., 1981) and this research has resulted in a substantial and methodologically sophisticated literature.

Discrete choice models are based on economic theories of utility maximisation and random utility theory. They bring together insights from the field of psychophysical discrimination (Thurstone, 1927), traditional microeconomic theory of consumer choice, and Lancaster's theory of consumer demand (Louviere et al., 2000, pp. 2-7; Lancaster, 1966). Discrete choice theory is discussed in more detail in chapter 3.

Discrete choice models estimate the probability of a decision maker choosing one product or service out of a set of products or services based on the decision maker's preferences for attributes of the products or services and the personal characteristics of the decision maker. The preferences or taste for the attributes in the model are derived from revealed preference data or, in the case of new products, stated preference data (Hensher et al., 2005, pp. 88-99).

These models are demand models and do not explicitly take into account the effect of constrained product supply on choice behaviour ${ }^{14}$. It is also assumed that, when these models are used for prediction, consumers' preferences will stay constant over the forecasting period (Geels, 2004; Wilson and Dowlatabadi, 2007).

\footnotetext{
${ }^{14}$ It is possible that models based on revealed preference data do take into account the effect of the availability of the product on choice behaviour through the alternative specific constants.
} 
These models have been used, inter alia, in: (1) economics, where they have been used to study labour force participation, residential location, and house tenure status; (2) marketing, where they have been used to study purchase incidence and brand marketing; and (3) transportation studies, where they have been used to study mode choice, destination choice, car ownership, travel demand (Bhat et al., 2008), and car choice (De Jong et al., 2004).

The first use of discrete choice models to study the demand for EVs was by Train (1980). Train's study comprised the development of a multinomial logit (MNL) model based on revealed preference data. The MNL model was then used to predict the household demand for EVs entering the United States market from 1980 to 2025 . The results of this very early model indicated that there would be a low uptake of EVs, comprising less than $3 \%$ of the United States light passenger vehicle (LPV) fleet by 2025 .

Beggs et al. (1981) developed an ordered logit model using survey data to estimate the preferences of United States car buyers. Although the model was not used to project EV uptake, survey respondents placed a very high negative value on the limited driving range of EVs, which the lower running costs did not offset.

Hensher (1982) modelled the uptake of EVs in Sydney based on a three attribute model (EV purchase price, petrol fuel cost, and EV driving range). The focus of this study was the estimation of the elasticities of the various attributes in the model and market shares were not estimated, but the model did indicate that driving range and petrol price were significant factors when choosing an EV, but purchase price was not. This latter result was probably influenced by the range of the purchase price attribute levels used in the survey, which was limited to $30 \%$ lower, or $30 \%$ higher, than a conventional internal combustion engine vehicle (ICEV).

Calfee (1985) developed a discrete choice model using stated preference data from a small sample collected in Berkeley, California. The model's attributes consisted of vehicle price, EV driving range, EV top speed, and operating (fuel) costs. The study found that EVs, with the modest performance of the technology of that time, would 
not gain any significant market share. However, the results of the study indicated that EVs with ranges greater than $240 \mathrm{~km}$ may have a place in the market.

Train (1986, pp. 134-191) developed an approach to estimate both household car purchase and use behaviour that comprised the development of a hierarchy of choice models. The hierarchy operated sequentially by estimating for a household: (1) whether a car would be bought or sold; (2) the number of cars bought or sold; (3) the type of car bought (if two or more cars were bought the choice model was applied to each purchase); and (4) finally the amount of travel undertaken by each car held. The output from the choice models is conditional on the characteristics of the household and took into account the changing character of the household. To apply the model, a number of synthetic households were created that were intended to be representative of the types of 'typical' households in the general population. To model car ownership behaviour at the population level, the survey sample of 'typical' households was reweighted to reflect the proportions of these types of households in the general population. For each time period, changes in each 'typical' household were first estimated, and, then using the updated household characteristics, the car choice behaviour was estimated. Then, the updated household and car ownership characteristics were carried over for use in the next modelling period.

Golob et al. (1996) used the approach developed by Train to model the Californian LPV fleet. They extended the scope of the models to include cars in business fleets. The models comprised a comprehensive set of car holding, transaction and use models that were used to forecast the uptake and use of biofuel cars, natural gas cars, and EVs into the Californian LPV fleet taking into account the effects of the changing structures of households and businesses, and car technology.

Hazard functions were used to simulate the changing structure of the households over time. These functions estimated, for each household type, changes in household income, employment status, education, marital status, ethnicity, fertility and mortality, number of children, and ages of the occupants. A duration model was then used to simulate the age of cars held by the households and the 
probability that they would be replaced. A similar approach was used for fleet operators, but to simulate the changes in the profile of different business types, a separate business forecast model was used.

The discrete choice models used by Golob et al. (1996) in the original models were MNL models. However, later research using the same data found that replacing these models with mixed multinomial logit models resulted in a better overall model performance and more realistic substitution patterns between car alternatives (Brownstone et al., 2000; Brownstone and Train, 1999).

Holding and transaction models are complex. The data requirements for these models are significant because a large amount of data is required on the characteristics of households and businesses. These data are often not available from existing sources and must be collected in addition to the stated preference data on car choices. The data for the Californian study required three survey phases. The first phase was to collect general data on household structure, car ownership, usage, and purchase intentions. The second phase consisted of a questionnaire customised to each household, with more detailed questions on household membership and car usage. This phase also contained two stated choice experiments. The third phase involved a final interview and additional questions about AFVs. For the fleet survey, the researchers had access to the vehicle register of the Californian Department of Motor Vehicles, which allowed them to match cars to fleet owners. This was followed by a two phase survey process similar to the first two phases of the household survey (Golob et al., 1996).

An alternative approach, which is less data intensive, is to: (1) develop econometric models by using aggregate economic data to forecast the demand for new cars; and (2) use the discrete choice model to allocate this demand between different types of car. The output for the discrete choice model can then be used in conjunction with an aggregate car market model to model the changing car stock (De Jong et al., 2004). This type of approach is used by the Oak Ridge National Laboratory in its Transitional Alternative Fuels and Vehicles model (Greene, 2001), the European Commission's TREMOVE model (De Ceuster et al., 2007), and the United States 
Department of Energy's AFV Demand Sector sub-module of the United States National Energy Modeling System (Office of Integrated Analysis and Forecasting, 2009).

Leaver and Gillingham (2010) used a similar approach, but incorporated a conditional logit model into a multi-regional integrated energy dynamic systems model. The conditional logit model comprised one attribute that represented the annualised cost of purchasing and operating the vehicle alternative. The value of this parameter was derived from estimates of the elasticity of new ICEVs. To account for differences in preferences between the AFVs in the model and ICEVs an intrinsic preference parameter was also included in the model. The value of this parameter was adjusted based on the modellers' judgement.

\subsection{Preferred modelling approach}

As a result of reviewing the literature, it was decided to use discrete choice modelling in conjunction with data from a stated choice survey to estimate the uptake of EVs into the New Zealand LPV fleet.

Unlike diffusion of innovation and expert based penetration scenarios, discrete choice models have a strong basis in economic theories of utility maximisation, consistent with traditional microeconomic theory of consumer choice, and Lancastrian consumer theory.

These models also have a number of practical features that make them desirable for use in this study, which are:

1. A long history of use in modelling car choice in transport studies and specifically studies assessing the demand for AFVs.

2. They can be used in conjunction with car ownership models, which provides a feasible alternative to the more resource intensive approach of developing car holding and transaction models.

3. When based on data from a stated choice survey data, the parameter values in the model reflect the preferences of the relevant decision makers for the 
actual product and not those of industry experts or preferences that are derived from what are considered to be closely analogous products.

These models estimate the demand for a product based on the attributes it provides. This provides the flexibility for the modeller to take account of the impact of changes in the values of these attributes on vehicle demand, which is important when modelling EVs where it is expected that there will be significant changes in price and performance over the modelling period. 


\section{Chapter 3: Theory of discrete choice models and stated choice experiments}

\subsection{Introduction}

This chapter discusses the theory underpinning the discrete choice model used in this study and then discusses the design and implementation of stated choice surveys.

\subsection{Discrete choice model}

\subsubsection{Random utility maximisation: a behavioural model of choice}

The theory underpinning discrete choice models can be traced back to Thurstone's Law of Comparative Judgement (Thurstone, 1927). He observed that a person comparing two or more alternatives is likely to respond differently to the alternatives. He called this response, without commenting on the underlying process, the discriminal process. Often when the two alternatives have a small difference between them, and the same person is presented with the same choice on a number of different occasions, there will be different outcomes. This led Thurstone to conclude that the true stimulus associated with an alternative has an element that fluctuates and can be represented probabilistically using a normal distribution. This led to a binary probit model to determine if a person can differentiate two levels of stimulus. Building on this insight, Marschak (1960) applied the concept of response to stimuli to the concept of economic utility and developed a derivation of utility maximisation that contained random elements.

Marschak's theory of random utility assumes that a decision maker acting rationally will choose the alternative that gives the greatest level of utility or satisfaction. Utility is defined in terms of Lancaster's theory of consumer demand that posits consumers seek to acquire the attributes of an alternative rather than the alternative itself (Lancaster, 1966). Utility is described as a latent construct, which cannot be directly measured, comprising two components: (1) a systematic or explainable part; and (2) a random or unexplainable part. It is assumed that these 
two components are independent and additive (Hensher et al., 2005, p. 74). Thus utility takes the form:

$$
U_{n i}=V_{n i}+\varepsilon_{n i}
$$

where $U_{n i}$ is the latent unobservable component, $V_{n i}$ is the systematic component for decision maker $n$ and alternative $i$, and $\varepsilon_{n i}$ is the random component for that decision maker and that alternative.

$V_{n j}$ comprises all the attributes that the researcher observes across all the alternatives, some are the attributes of the alternatives $x_{n j}, \forall_{j}$, and some are the attributes of the decision maker themselves $s_{n}$ denoted as:

$$
V_{n j}=V\left(x_{n j}, s_{n}, \beta\right), \forall_{j}
$$

$\beta$ is a vector of parameters that are either known beforehand or have been estimated (Train, 2003, p. 19).

The random component reflects all the unidentified factors that impact on choices, including Thurstone's insight that as humans are imperfect measurement devices, there will be variability and differences due to the decision maker, which are not necessarily due to differences between the alternatives (Louviere et al., 2010).

The theory assumes that decision makers act rationally when making their decisions. However, due to the presence of the random component in the utility function, all that can be said by the researcher about the decision making process is that decision makers take into account those factors that they consider important at the time of the decision, irrespective of the amount of information that is available to them (Hensher et al., 2005, p. 79). As Train (2003, p. 18) observes:

It is important to note, however, that models derived from utility maximization can also be used to represent decision making that does not entail utility maximization. The derivation assures that the model is consistent with utility maximization; it does not preclude the model from being consistent with other forms of behavior. The models can also be seen 
as simply describing the relation of explanatory variables to the outcome of a choice, without reference to exactly how the choice is made.

The assumption that the decision maker will choose the alternative that provides the most utility implies that alternative $i$ will be chosen if and only if $U_{n, i}>$ $U_{n, j}, \forall_{j} \neq i$. As the researcher does not know $\varepsilon_{n i}, \forall_{j}$ these are treated randomly with a joint density $f\left(\varepsilon_{n}\right)$ and the decision makers' choices are treated probabilistically so that the probability that decision maker $n$ will choose alternative $i$ is:

$$
\begin{aligned}
\operatorname{Prob}_{n i} & =\operatorname{Prob}\left(U_{n i}>U_{n j}, \forall_{j} \neq i\right) \\
& =\operatorname{Prob}\left(V_{n i}+\varepsilon_{n i}>V_{n j}+\varepsilon_{n j}, \forall_{j} \neq i\right) \\
& =\operatorname{Prob}\left(\varepsilon_{n j}-\varepsilon_{n i}<V_{n i}-V_{n j}, \forall_{j} \neq i\right)
\end{aligned}
$$

Thus, the probability of the decision maker choosing alternative $i$ is equal to the probability that the difference in the random utility of alternatives $j$ and $i$ is less than the difference between the systematic utility levels of alternatives $i$ and $j$. Equation (3) is the random utility maximisation model (RUM) (Train, 2003, p. 19). Using the joint probability density function $f\left(\varepsilon_{n}\right)$ the cumulative probability can be rewritten as:

$$
\operatorname{Prob}_{n i}=\int_{\varepsilon} I\left(\varepsilon_{n j}-\varepsilon_{n i}<V_{n i}-V_{n j}, \forall_{j} \neq i\right) f\left(\varepsilon_{n}\right) d \varepsilon_{n}
$$

where $I$ is the indicator function, which is 1 when the expression in the parentheses is true and 0 when it is not. Different discrete choice models assume different distributions of $f\left(\varepsilon_{n}\right)$ and the distribution chosen has different implications for operational tractability. In this study, all the models estimated are logit models, which assume that the distribution of $f\left(\varepsilon_{n}\right)$ are extreme value type 1.

The RUM equation (3) also shows that, in discrete choice models, only the differences in utility between alternatives matter, not absolute utility. Therefore, the parameters in these models can only be estimated where there are differences across the alternatives (Train, 2003, p. 23). 
To operate within the framework of the random utility theory, the set of alternatives in a discrete choice model must be:

- mutually exclusive - choosing one alternative implies not choosing any of the other alternatives

- exhaustive - the choice set must include all possible alternatives

- finite - there must be a limited number of alternatives.

Although the criteria can appear to be restrictive, any constraints can be addressed by the appropriate specification of the alternatives (Train, 2003, pp. 15-18).

\subsubsection{The multinomial logit (MNL) model}

The easiest and most commonly used discrete choice model is the MNL model because its specification of choice probabilities takes a closed form and can be readily interpreted (Train, 2003, p. 38).

The MNL model was developed by McFadden (1974) who built on the work of Luce. Luce made the logit choice model more operationally workable by assuming that the unobserved effects $\varepsilon$ are independently and identically distributed (IID). The behavioural consequence of this assumption is that the MNL model demonstrates the property of independence from irrelevant alternatives (IIA) (Luce, 1959, p 100). IIA means that the ratio of probabilities of choosing one alternative over another is unaffected by the presence or absence of any additional alternatives in the choice set. The practical implications of this assumption are discussed further in section

\subsection{4}

As all logit models assume that the unobserved effects have extreme value type I distributions, the probability density function is:

$$
f\left(\varepsilon_{n j}\right)=e^{-\varepsilon_{n j}} e^{-e^{-\varepsilon_{n j}}}
$$

And the cumulative density function is:

$$
F\left(\varepsilon_{n j}\right)=e^{-e^{-\varepsilon_{n j}}}
$$


When the RUM equation (3) is rearranged to $\operatorname{Prob}_{n i}=\operatorname{Prob}\left(\varepsilon_{n j}<\varepsilon_{n i}+\right.$ $\left.V_{n i}-V_{n j}\right), \forall_{j} \neq i$ and incorporated into equation (6), and taking into account that the $\varepsilon^{\prime}$ s are independent, the cumulative distribution for all $j \neq i$ is the product of the individual cumulative distributions:

$$
\operatorname{Prob}_{n i} \mid \varepsilon_{n i}=\prod_{j \neq i} e^{-e^{-\left(\varepsilon_{n i}+V_{n i}-V_{n j}\right)}}
$$

As $\varepsilon_{n i}$ is not given the choice probability is the integral of $\operatorname{Prob}_{n i} \mid \varepsilon_{n i}$ of all values of $\varepsilon_{n i}$ over the probability density function in equation (4) and is:

$$
\operatorname{Prob}_{n i}=\int\left(\prod_{j \neq i} e^{-e^{-\left(\varepsilon_{n i}+V_{n i}-V_{n j}\right)}}\right) e^{-\varepsilon_{n i}} e^{-e^{-\varepsilon_{n i}}} d \varepsilon_{n i}
$$

This expression can be reduced to the familiar logit probability formula:

$$
\operatorname{Prob}_{n i}=\frac{e^{V_{n i}}}{\sum_{j} e^{V_{n j}}}
$$

In most discrete choice models, $V_{n j}$ is specified to be linear in parameters so that $V_{n j}=\beta^{\prime} x_{n j}$, where $x_{n j}$ is a vector of the observed variables relating to the alternative $j$ (Train, 2003, pp. 38-44).

\subsubsection{Maximum likelihood estimation}

The usual method for estimating the parameters in MNL models is maximum likelihood estimation (Hensher et al., 2005, p. 317). The maximum likelihood method is based on the concept that a sample of observed choices can be generated by different populations of parameter values, but there is a set of population parameters which are more likely to generate the observed sample than any other. The maximum likelihood estimate is the vector of parameter values $\beta$ that gives the greatest probability of obtaining the observed data (Kennedy, 2008, pp. 21-22).

For an MNL model, the probability of person $n$ choosing an alternative $i$ that they were actually observed choosing can be expressed as:

\footnotetext{
${ }^{15}$ See Train (2003) chapter 3 for a detailed explanation of the algebra used to progress from equation (8) to (9).
} 


$$
\prod_{i}\left(\operatorname{Prob}_{n i}\right)^{y_{n i}}
$$

where $\operatorname{Prob}_{n i}$ is the function in equation (9) and $y_{n i}=1$ if the person chose alternative $i$ and zero otherwise, noting that if $y_{n i}=0$, then Prob $b_{n i}$ will equal 1 . If it is assumed that each decision maker's choice is independent of all other decision makers, the probability of each person choosing the alternative that they were observed to choose is:

$$
L(\beta)=\prod_{n=1}^{N} \prod_{i}\left(\operatorname{Prob}_{n i}\right)^{y_{n i}}
$$

where $\beta$ is a vector of parameters. For computational tractability, the log form is used:

$$
L L(\beta)=\sum_{n=1}^{N} \sum_{i} y_{n i} \ln \operatorname{Prob}_{n i}
$$

McFadden showed that $L L(\beta)$ is globally concave for models that have linear-inparameters utility specification and are, therefore, solvable because at the maximum value of the likelihood function the derivative is zero with respect to each of the parameters (McFadden, 1974) ${ }^{16}$.

\subsubsection{Limitations of the MNL model}

The MNL model remains a popular method for estimating choice behaviour due to its ease of computation. However, the MNL model has three features that may result in the researcher considering an alternative type of discrete choice model.

\section{Independence from irrelevant alternatives}

The IID assumption, and the related behavioural assumption of IIA, is often not restrictive, but in cases where the unobserved attributes of utility are not independent, it will lead to biased parameter estimates and added errors in forecasts. In practical terms, the IIA assumption can result in unrealistic substitution

\footnotetext{
${ }^{16}$ For more information on the algorithms used to find the maximum value of the log likelihood function, see chapter 8 of Train (2003). The NLOGIT software used in this project uses the NewtonRaphson algorithm for MNL models and the Broyden-Fletcher-Goldfarb-Shanno (BFGS) algorithm to estimate the mixed logit models.
} 
patterns, especially in the case where a new product, such as electric vehicles (EVs), is introduced into the market.

The IIA behaviour can be demonstrated for two alternatives $i$ and $k$ as the ratio of the logit probabilities:

$$
\frac{\operatorname{Prob}_{n i}}{\operatorname{Prob}_{n k}}=\frac{e^{V_{n i} / \sum_{j}} e^{V_{n j}}}{e^{V_{n k} / \sum_{j}} e^{V_{n j}}}=\frac{e^{V_{n i}}}{e^{V_{n k}}}
$$

As the denominators cancel out, the ratio does not depend on any other alternatives. The practical implications of this outcome are often explained using the blue bus, red bus problem (Train, 2003, pp. 49-51).

\section{Panel data}

Data for discrete choice models often involve repeated observations from the same decision maker. This can occur in economic surveys where the data are collected over a period of time from the same households or firms. It also occurs in stated choice experiments where the survey respondent is asked to make repeated hypothetical choices.

The IID assumption in the MNL model means that the only dynamic effects on decision making that are captured by the model are those in the observed variables. Any dynamic effects that occur between choice tasks that are unobserved are not taken into account. Despite this, it can be expected that there will be unobserved effects that are carried over from one choice to the next, such as the effects of the previous choice itself or decisions based on habit.

\section{Taste variation}

Tastes vary across decision makers because of different personal circumstances, such as income or education. Tastes can also vary because people have different preferences and concerns even if they have the same observed demographic characteristics. MNL models capture only the systematic variations in taste in the observed variables, but not taste variations that occur in the unobserved variables, or that vary randomly (those that cannot be linked to observed characteristics). 
MNL models may capture average tastes reasonably well even when tastes are random, but will not provide information about the distribution of these tastes. This information can be important when forecasting the penetration of new products that appeal to the minority of people, rather than those with average tastes. In such circumstances, other discrete choice model forms should be used (Train, 2003, pp. 46-49).

\subsubsection{Mixed multinomial logit models}

The mixed multinomial logit model (MMNL), or mixed logit model, is a flexible model form that overcomes the three limitations of the MNL model and allows for random taste variation, unrestricted patterns of substitution, and correlation in the unobserved factors. These models were developed in the early 1980s (McFadden and Train, 2000). However, due to their high computational requirements, their use was restricted until the last decade as developments in computer speed, simulation methods, and increased availability of software reduced the barriers to their use (Train, 2003, p. 2).

The term mixed logit reflects the fact that the choice probability is a mixture of logits with a specified mixing distribution. There are a number of forms of MMNL model of which the most widely used is the random parameters mixed multinomial logit (RPL) (Hensher and Greene, 2003). This discussion of MMNL models focuses on the RPL form, followed by a brief review of the error component mixed multinomial logit (ECMNL) model.

\section{RPL}

The RPL model retains the condition that $\varepsilon_{n j}$ for individual $n$, and alternative $j$ is IID, with extreme value I, but introduces a stochastic element into the utility function through the coefficients $\beta_{n}$. The utility of person $n$ from alternative $j$ is:

$$
U_{n j}=\beta_{n}^{\prime} x_{n j}+\varepsilon_{n j}
$$

where $x_{n j}$ are observed variables that relate to the alternative and the decision maker $n ; \beta_{n}$ is a vector of coefficients of these variables that represent $n$ 's tastes, 
which can vary across individuals; and $\varepsilon_{n j}$ is a random term that is an IID extreme value, thus retaining the logit form (Train, 2003, p 141).

The coefficients $\beta_{n}$ vary over the population of decision makers and have a probability density of $f\left(\beta_{n} \mid \theta\right)$, with $\theta$ representing the density function's parameters, such as the mean and covariance. The RPL form allows for the distributions of random coefficients to take a number of forms, including normal, lognormal, and triangular distributions (Hensher and Greene, 2003). The decision maker knows their own $\beta_{n}$ and $\varepsilon_{n j}$ values, but the researcher only observes the $x_{n j}$ 's values, and not the values of $\beta_{n}$ and $\varepsilon_{n j}$. If the researcher could observe $\beta_{n}$, then the choice probability would be standard logit, and the probability conditional on $\beta_{n}$ for alternative $i$ is:

$$
L_{n i}\left(\beta_{n}\right)=\frac{e^{\beta_{n}^{\prime} x_{n i}}}{\sum_{j} e^{\beta_{n}^{\prime} x_{n j}}}
$$

However, $\beta_{n}$ is not known so it must be treated stochastically and the unconditional choice probability is the integral of $L_{n i}\left(\beta_{n}\right)$ over all the values of $\beta_{n}$ is:

$$
\operatorname{Prob}_{n i}=\int\left(\frac{e^{\beta_{n}^{\prime} x_{n i}}}{\sum_{j} e^{\beta_{n}^{\prime} x_{n j}}}\right) f(\beta) d \beta
$$

These models are mixed logit because the choice probability $L_{n i}\left(\beta_{n}\right)$ is a mixture of logits with $f(\beta)$ as the mixing distribution (Hensher and Greene, 2003).

\section{ECMNL}

The MMNL model can also be represented as error components that create correlations among the utility functions of the different alternatives. In this case, utility is specified as:

$$
U_{n i}=\alpha^{\prime} x_{n i}+\mu^{\prime} z_{n i}+\varepsilon_{n i}
$$

Where $x_{n i}$ and $z_{n i}$ are vectors of observed variables relating to the alternative $i, \alpha$ is a vector of fixed coefficients, $\mu$ is a vector of random terms with zero mean, and $\varepsilon_{n i}$ is the logit specification of IID extreme value I. If all the $z_{n i}$ are zero, there is no 
correlation of utility over the alternatives and the model becomes the standard logit and the model is IIA. These random components can be treated as independent or correlated across alternatives (Train, 2003, pp. 143-145).

\section{Substitution patterns}

MMNL models do not exhibit IIA because the denominators of the logit formula are inside the integrals and do not cancel. Therefore, the ratio of mixed logit probabilities $P_{n i} / P_{n j}$ relies on all the data, including the attributes of alternatives other than $i$ and $j$. Substitution patterns between the alternatives are influenced by the researcher's choice of variables and mixing distributions. However, it is important to ensure that the choice of distribution is consistent with the sign of the coefficient (Revelt and Train, 1988).

\section{Panel data}

MMNL models are easily generalised to allow for panel data from stated choice experiments so that the coefficients are constant over the choice situations for each person, but vary between people. In this case, the utility of the alternative $j$ for individual $n$ in choice situation $t$ is:

$$
U_{n j t}=\beta_{n}^{\prime} x_{n j t}+\varepsilon_{n j t}
$$

where $\varepsilon_{n j t}$ is IID extreme value over individuals ( $\left.\mathrm{n}\right)$, the alternatives (j), and choice situations $(\mathrm{t})$. For a sequence of choices, $\mathbf{i}=\left(i_{1}, \ldots i_{T}\right)$ the probability conditional on $\beta$ of making this sequence of choices is the product of the logit formulas:

$$
L_{n \mathbf{i}}\left(\beta_{n}\right)=\prod_{t=1}^{T}\left[\frac{e^{\beta_{n}^{\prime} x_{n i t}}}{\sum_{j} e^{\beta_{n}^{\prime} x_{n j t}}}\right]
$$

The unconditional probability is the integral of this product over all values of $\beta$ :

$$
\operatorname{Prob}_{n \mathbf{i}}=\int L_{n \mathbf{i}}\left(\beta_{n}\right)\left(\beta_{n} \mid \theta\right) d \beta_{n}
$$




\section{Estimation of coefficients in MMNL models}

As already noted, $\theta$ collectively represents parameters of $\beta_{n}$, i.e. the mean and covariance and it is necessary to estimate these parameters. Unlike the probability function in the MNL model, which has a closed form, the integral in the mixed logit probability function (equation 16) prevents the exact estimation of maximum likelihood. However, the probabilities can be approximated by simulation.

Simulation comprises the following process:

1. For a given value of $\theta$, draw a value of $\beta$ and label it $\beta^{r}$ with $r=1$.

2. Calculate the conditional logit formula $L_{n i}\left(\beta^{r}\right)$.

3. Repeat steps 1 and 2 many times, and then average the results giving the simulated probability:

$$
\operatorname{Prob}=\frac{1}{R} \sum_{r=1}^{R} L_{n i}\left(\beta^{r}\right)
$$

where $R$ is the number of draws and $\operatorname{Prob}_{n i}$ is the simulated probability.

The simulated probability is an unbiased estimator of $\operatorname{Prob}_{n i}$ with a number of desirable properties. It is:

- always positive so that the $\log \boldsymbol{P r o b}_{n i}$ is defined. This is necessary for approximating the log likelihood function

- smooth in both the parameters and variables (can be differentiated twice), which facilitates numerical search functions

- sums to one over alternatives, which is necessary for forecasting (Train, 2003, p. 148).

\subsection{Stated choice surveys}

\subsubsection{Introduction}

Stated choice surveys are a special type of stated preference survey used to collect data for discrete choice models (Bateman et al., 2002, p. 249). These surveys are required in situations where the alternatives being studied do not yet exist in the market and there is no information about non-chosen alternatives. Stated choice 
surveys are also required when there is a lack of variability in the market of the attributes that need to be modelled, or where the attributes are correlated (Louviere et al., 2000, pp. 20-25).

The core element of a stated choice survey is the stated choice experiment. The experiment is a series of tasks that require the respondent to make choices between alternative products, or services, in hypothetical situations. The use of stated choice experiments to obtain data for discrete choice models was first proposed by Louviere and Hensher (1983).

Data from stated choice experiments address many of the disadvantages of revealed preference data from the market, but they also have potential limitations in that they can:

- require large and unwieldy questionnaires

- require large samples to obtain statistically significant estimates of the model's parameter values

- result in the possibility of unrealistic choice behaviour by the respondents, known as hypothetical bias

- result in the respondents using choice heuristics that have the effect of invalidating the choice model as a means of estimating willingness to pay (Hensher, 2009).

When suitable revealed preference data is available, it is possible to combine both types of data for use in discrete choice models. This approach has been found to improve the performance of choice models (Louviere et al., 1999; Whitehead et al., 2008; Adamowicz et al., 1994). For new products, where revealed preference data are not available, stated choice data must be used (Hensher et al., 2005, pp. 92-97).

The design of the stated choice survey and the stated choice experiment occur together as an iterative process (Hensher et al., 2005, pp. 100-104). The use of focus groups and pilot surveys are recommended when developing the stated choice survey (Louviere et al., 2000, pp. 255-261). 
The survey design process must take into account the practical constraints facing the researcher, including access to survey tools, available budget, and time constraints (Champ and Welsh, 2006; Mansfield and Pattanayak, 2006).

\subsubsection{Stated choice survey design}

The design of a stated choice survey involves identifying the: (1) study population; (2) sample frame; (3) sampling method; (4) sample size; (5) survey instrument; (6) data collection methods, and (7) survey management methods. The study population is those people whose choices or values are of relevance. The sample frame is the list or database from which these respondents are to be selected, and the sampling method is the process by which those from the sampling frame are selected (Champ and Welsh, 2006).

Rose and Bliemer (2008, pp. 156-157) identify three generic survey instruments suitable for stated choice surveys: (1) paper based questionnaires; (2) computer assisted personal interviews (CAPI); and (3) internet based questionnaires.

Paper based questionnaires can be distributed in person, by mail, or by email. These have the advantage of not requiring technological support and, when undertaken in person, the interviewer can assist with any questions about the survey, provide supporting material, and help keep the respondent on task. However, it is more difficult to randomise the order of the choice tasks than in CAPI or internet surveys. Paper based surveys also require time consuming data entry with the potential for input errors.

CAPI based surveys are considered the best survey instrument for stated choice experiments and involve the respondents completing the questionnaire, which is loaded onto a laptop computer, in the presence of the interviewer. This method has the advantages of: (1) being able to randomise the choice tasks for each response; (2) collecting the data automatically with no subsequent data input errors; and (3) providing personal support during the interview. However, CAPI surveys are expensive, time consuming, and, unless they are well-resourced, are not suitable for a geographically dispersed survey (Champ and Welsh, 2006). 
Internet based surveys have some of the advantages of CAPI surveys in terms of the ease in randomising the choice tasks and no errors during data collection. They also have the advantage of being able to reach a large number of widely dispersed respondents. These surveys can be open to anyone or constrained to a preapproved sample population by using password protection. The disadvantages of internet surveys are that only those with access to, or who are comfortable with using, the internet can be surveyed. This can result in sections of the population being excluded from the survey (Champ and Welsh, 2006; Dillman et al., 2009b, p. 44).

Interviewer-administered telephone surveys are not suitable for surveys as complex as stated choice experiments. They are only suitable if the questionnaire is sent out before the telephone interview. However, the telephone can be used to obtain agreement to participate in the survey, and then the survey can be administered by mail, email, or the internet (Champ and Welsh, 2006). These methods are not mutually exclusive and Dillman et al. (2009a, p. 304) recommends the use of a mixture of survey methods as a means of improving survey response rates.

\subsubsection{Designing a stated choice experiment}

The stated choice experiment design comprises three components: (1) the design of the stated choice tasks; (2) the inclusion and design of supporting questions; and (3) the inclusion and design of any supporting information to assist the respondent.

A critical design feature is to ensure, as far as possible, that the experiment is incentive compatible so that it will elicit truthful responses, which fully reveal the respondent's preferences and minimise the presence of hypothetical bias (Harrison, 2006b).

\section{Design of the stated choice tasks}

A stated choice experiment involves the respondent choosing one of the alternatives in the stated choice task based on the information given about the attributes for each of the alternatives. In most experiments, it is necessary to pool 
the results and each respondent is required to complete a number of different stated choice tasks.

The first step in the design of a stated choice experiment involves identifying all the alternatives that are relevant to the objectives of the study. It is essential that the alternatives are defined so as to ensure that they are compatible with the discrete choice framework, i.e. they are mutually exclusive, comprise all the alternatives available to the sample population, and are finite in number (Train, 2003, p. 15). A decision is required as to whether the alternatives will be labelled or unlabelled. Labelled experiments allow the respondent to bring additional information about the alternatives to the decision making process. In an unlabelled experiment, the respondent makes the choices based solely on the information provided in the experiment. An experiment with labelled attributes is often preferred when the focus is on prediction (Hensher et al., 2005, p. 113).

The next step is to identify the critical attributes for each of the alternatives, the number of attribute levels, and the values that these levels will have in the experiment. It is desirable to have more, rather than fewer, attribute levels to better estimate the utility of an attribute. The consequence of this is that the number of choice tasks required in the experiment rapidly increases. When setting attribute levels, the extreme values should encompass the complete range of values likely to be faced by the respondents, but, at the same time, these should not be so extensive as to be considered unrealistic by the respondent because this can result in the respondent not treating the survey seriously (Hensher et al., 2005, pp. 105-109).

The experimental design of choice tasks involves the manipulation of the attribute levels across the choice tasks in a systematic manner that will allow for the determination of the independent influence of the attributes on choice. The method of manipulation will impact on the ability of the experiment to detect the statistical relationships in the data (Rose and Bliemer, 2008; Rose and Bliemer, 2009). The greater the number of choice sets, the greater the burden on the respondents, which increases the potential for non-responses or poorly considered 
decision making behaviour. The challenge is to design a survey that elicits the most information without creating too much of a burden on the respondent (Johnson et al., 2006).

The standard approach in stated preference surveys is to use full factorial designs that ensure the design will demonstrate orthogonality (Johnson et al., 2006). A design is orthogonal if it demonstrates attribute level balance and all parameters are independently estimated (Bliemer et al., 2011). Attribute level balance occurs if, for each attribute, all attribute levels appear an equal number of times in the survey and there is an even distribution of values so that the respondent is not faced with only high or low values (Rose and Bliemer, 2009).

Orthogonal designs are considered optimal because they ensure that the model to be estimated will not demonstrate multicollinearity and will have smaller variances in the parameter estimates (Carlsson and Martinsson, 2003). However, using full factorial orthogonal designs for a stated choice experiment that has more than a few alternatives and attributes is restrictive because this results in designs with a large number of choice tasks ${ }^{17}$.

In practice, stated choice designs are rarely orthogonal due to the effects of blocking ${ }^{18}$, spoiled returns, the inclusion of covariate variables, and the exclusion, by the researcher, of implausible combinations of attribute values that undermine the reality of a choice task (Rose and Bliemer, 2009).

Efforts to develop stated choice experimental designs that reduce the number of choice tasks that will be faced by the respondents have focused on the development of fractional factorial designs, which still produce a discrete choice

\footnotetext{
${ }^{17}$ The number of choice tasks in an orthogonal design is determined by the formula $\prod_{j=1}^{J} \prod_{k=1}^{K_{j}} L_{k j}$, where $J$ is the number of alternatives with $K_{j}$ attributes, and where attribute $k \in K_{j}$ and has $L_{k j}$ levels. An experiment with a 2 alternative model, each with 1 attribute, and each of these attributes with three levels would require $3^{2}$, or 9 choice tasks. However, a choice experiment with three alternatives, each with three attributes, and each with three attribute levels would result in $3^{9}$ or 19,683 choices tasks (Bliemer et al., 2011). Even with blocking allocating this number of choice tasks would require a very large sample.

${ }^{18}$ Blocking involves dividing the orthogonal design into smaller designs. Each block is not orthogonal in itself, but in combination all the blocks are orthogonal (Bliemer et al., 2011).
} 
model containing statistically significant parameter estimates (Rose and Bliemer, 2009).

Efficient choice designs (ECDs) are not concerned with orthogonality, but aim to result in experimental designs that collect data which generates model parameter estimates with the smallest possible standard errors (Bliemer et al., 2011) ${ }^{19}$. Discrete choice models are non-linear, and model variance and covariance are estimated asymptotically. The asymptotic variance covariance (AVC) matrix is not equivalent to the variance covariance (VC) matrix in linear regression ${ }^{20}$, but is estimated by taking the negative inverse of the expected second derivatives of the log-likelihood function of the model. The standard errors of the model are the roots of the diagonal of the AVC matrix ${ }^{21}$.

Efficient designs require the researcher to take some prior view about the final model's parameter values and the type of discrete choice model that will be specified. This design approach can then be compared to orthogonal experimental designs that implicitly assume no prior knowledge about the parameter values (Rose and Bliemer, 2009; Sandor and Wedel, 2001).

Efficient design has been shown to result in experimental designs that either produce more reliable parameter estimates for a fixed sample size, or reduce the sample size to achieve a specified level of reliability (i.e. 95\% confidence) in the parameter estimates (Bliemer and Rose, 2009; Bliemer et al., 2011).

As the AVC is derived from the log-likelihood function, which varies depending on the type of logit model, the efficient design that minimises the elements of the AVC

\footnotetext{
${ }^{19}$ Two other approaches have been proposed for fractional factorial designs; Optimal Orthogonal Choice Designs, which retain some degree of orthogonality (Street, Burgess, \& Louviere, 2005); and Optimal Choice Probability Designs, which relax the attribute level balance constraint (Rose \& Bliemer, 2009).

${ }^{20}$ The VC matrix, in linear regression takes the form: $V C=\sigma^{2}\left[X^{\prime}, X\right]^{-1}$, where $\sigma^{2}$ is the model variance and $X$ is the matrix of attribute levels in the design.

${ }^{21}$ The AVC matrix means that it is consistent in large samples, or it is representative as an average for small samples when the survey would be repeated many times (Bliemer et al., 2010).
} 
matrix for one type of logit model cannot be assumed to minimise the elements of an AVC matrix of another type of logit model (Rose et al., 2009b).

To implement an efficient design, the form of final discrete choice model must be first defined by identifying:

1. The parameters that will be generic and alternative specific.

2. The format of the parameters (i.e. dummy or effects coded, or other format).

3. Whether interaction effects will be estimated.

4. The likely values (prior values) of the parameters of the final model.

5. The type of model that will be estimated (i.e. Multinomial logit, Nested Multinomial logit, Mixed multinomial logit, Panel mixed multinomial logit) (Rose and Bliemer, 2008).

Items 1 to 3 define the design matrix, while item 4 forms the basis of the ECD method. Item 5 recognises that different model specifications (generic or alternative specific parameters), and different type models, have different types of log-likelihood functions.

There is always a risk that the prior parameter values will be misspecified, which can result in inefficient designs. However, except in cases where there is extreme misspecification, ECD has been shown to result in more efficient designs than using the default assumption of parameters with zero value. Estimates of the prior parameter values can be obtained from similar studies in the literature, economic theory, or from pilot studies (Rose and Bliemer, 2009).

The statistical efficiency of the design is evaluated by comparing the AVC matrices of different experimental designs. Rather than evaluating each element of the AVC matrices independently, summary matrix measures are used. The most common measure of design performance is the D-error, which is derived from the determinant of the AVC matrix by assuming a single respondent and then scaling by the number of parameters in the model. The smaller the D-error the more efficient 
the experiment design and the lowest D-error found is called D-efficient (Bliemer et al., 2011, pp. 91-94).

\section{Supporting questions}

The supporting questions in a stated choice experiment are used to elicit information about a respondent's circumstances, attitudes, behaviour, and the level of knowledge about the study topic. Supporting questions can also be used to elicit opinions about the respondent's views of the design and implementation of the experiment (Krupnick and Adamowicz, 2006). The reasons for including support questions in a stated choice experiment are to:

- provide socio-demographic data for use in weighting the sample so that it conforms with respect to some characteristic of the study population (Hensher et al., 2005, p 226)

- act as covariates in the discrete choice model, which are used to better understand the observed heterogeneity or to have the discrete choice model conform to an economic theory, which can provide the model with a degree of construct validity

- provide information about the error component and variance of the error

- provide information about the decision strategies used by a respondent and to determine whether these change over the course of the experiment

- provide information about the respondent's level of understanding of the choice tasks

- verify whether the choice experiment was considered to be understandable, unbiased, and valid by the respondent (Krupnick and Adamowicz, 2006).

There is a tension between the desire to include supporting questions and the added burden on the respondent. No hard and fast rules are given for determining the correct burden, but it is suggested that focus groups and pilot studies be used to identify whether questions and descriptive text should be included or whether to modify them to improve readability (Krupnick and Adamowicz, 2006). 


\section{Supporting information}

The information provided in the choice matrix of labelled stated choice experiments will always be, by necessity, incomplete, and the researcher will rely on the respondent's knowledge, attitudes, and experience to fill in the gaps. The models then capture the effect of these on choice behaviour through the inclusion of alternative specific constants and error terms in the discrete choice model (Train, 2003, p. 24).

In stated choice experiments, the choice alternatives often comprise products or services that are not yet available and about which there is limited knowledge in the market. Or the experiment may involve making choices about environmental issues that are complex and that involve a large degree of uncertainty. For these types of experiments it is important to incorporate respondents' previous knowledge, but, at the same time, the respondent must understand and correctly process the information in the survey. When faced with incomplete knowledge, respondents will 'fill in the gaps' and this can result in incorrect views or unrealistic expectations about the alternatives. The challenge, when providing information to assist the respondent in their choices, is not to influence their decisions, or provide too much information which could result in respondent fatigue.

This study endeavoured to provide supporting information that was neutral in tone, factually correct, and complete. The information parts of the survey were also pretested, because incorrect or incomplete information can contribute to hypothetical bias (Mathews et al., 2006).

\section{Hypothetical bias}

Hypothetical bias occurs when there are differences in the responses to tasks involving hypothetical economic commitments and the responses made to comparable tasks that involve real economic commitments (Harrison, 2006a; Hensher, 2009).

There is extensive evidence that shows that hypothetical bias can occur in stated preference surveys. Most of the studies that have evaluated hypothetical bias have 
focused its effect on the estimates of total willingness to pay produced using contingent valuation surveys (List and Gallet, 2001; Ajzen et al., 2004; Harrison, 2006a). Most of the studies that have evaluated the presence of hypothetical bias in stated choice surveys have focused on its impact on the estimates of marginal willingness to pay $^{22}$ (Carlsson and Martinsson, 2001; Hensher, 2009).

There have only been a small number of studies that have evaluated the impact of hypothetical bias on the market share estimates produced using data from stated choice surveys. The results of these studies have been mixed. Carlsson and Martinsson (2001) found insignificant differences between the real and hypothetical responses, but Harrison (2006b) concluded that the design of their experiment would result in confounding order effects which invalidate their results. In a study of different beef steak products, Lusk and Schroeder (2004) found that shoppers had a tendency to overstate their propensity to purchase a steak product in the hypothetical situations when compared to shoppers in non-hypothetical situations. Chang et al. (2009) compared the results of using MNL and RPL models for household products using hypothetical and non-hypothetical data. They found that both the MNL and RPL models performed well in predicting market shares when using the non-hypothetical data, but not when using the hypothetical data.

Hensher (2009) questions the conclusions of the latter two studies, because it is common practice in the agricultural, resource, and environmental valuation literature not to re-calibrate the alternative-specific constants even when there are known market shares. The alternative-specific constants in discrete choice models reflect not only the effect of unobserved factors, but also the choice shares in the survey sample. In most cases, the sample shares will not be the same as actual market shares and, in cases where there are known market shares for a product, the alternative-specific constants will need to be recalibrated to reflect the actual market shares (Hensher et al., 2005, pp. 420-426; Train, 2003, p. 37).

\footnotetext{
${ }^{22}$ In the choice modelling literature, marginal willingness to pay refers to the valuation of a specific attribute in the utility function (Hensher, 2009).
} 
There are a number of factors that affect the level of hypothetical bias in a stated choice survey and there is no single technique that will eliminate it (Murphy et al., 2005a). Techniques that have been suggested to reduce hypothetical bias include:

- cheap talk scripts ${ }^{23}$

- an opt-out or no choice option in the choice experiment

- opt-out reminders in the surveys in conjunction with the opt-out alternative

- a reference alternative based on each respondent's actual experience, with the attribute values in the experiment pivoted around the reference alternative (Hensher, 2009).

The evidence of the effectiveness of cheap talk script to reduce hypothetical bias is mixed. List et al. (2006) found that the use of a stated choice experiment survey in conjunction with a cheap talk script resulted in choice behaviour consistent with the real responses. The use of cheap talk scripts in both contingent valuation and choice experiments was found to have some effect in reducing hypothetical bias, but had less effect on experienced or knowledgeable consumers (List et al., 2006; Lusk, 2003; List and Gallet, 2001). Murphy et al. (2005b) found cheap talk scripts to only have a moderate effect on reducing hypothetical bias, and they concluded that a combination of ex ante and ex post methods may be required to address the problem. The length of the cheap talk script may also have an impact on its effectiveness in reducing hypothetical bias (Landry and List, 2007).

There is some evidence that an opt-out, or no choice option, in the stated choice survey is effective in reducing hypothetical bias and that this effectiveness is improved when used in conjunction with an opt-out reminder (Ladenburg et al., 2007). However, the results remain inconclusive (Hensher, 2009).

The use of a reference alternative allows the researcher to frame the decision making process within some existing experience of the survey respondent. This framing is intended to make preference revelation more meaningful for the

\footnotetext{
${ }^{23}$ A cheap talk script is a pre-prepared presentation that thoroughly describes and discusses the tendency of respondents to exaggerate their stated willingness to pay in hypothetical situations Carlsson, \& Martinsson, 2001).
} 
respondent by providing a context (Rose et al., 2008). The reference alternative is also intended to provide more realism in stated choice experiments by ensuring that the alternatives are similar to choices that the respondent has already experienced. This approach has been suggested as a useful approach in choice situations that have a habitual context, such as value of time travel studies (Hensher, 2009).

It appears that there are stated choice specifications that can reduce hypothetical bias in some settings, but there is no evidence that these will be effective in other settings (Harrison, 2006b). At this stage, it is unknown whether there are techniques that can be generalised across all settings and further work is required to better understand the causes of hypothetical bias in stated choice experiments and to develop techniques to mitigate its effects (Harrison, 2006b; Hensher, 2009).

\subsection{Summary comments}

Discrete choice models estimated using data from stated choice surveys have the potential to simulate the demand for products or services that are not yet available in markets. For this study, a MMNL model was chosen as the means of simulating the demand for EVs in the New Zealand market as these logit models produce more realistic patterns of substitution between the alternatives. This is a useful property when using the model to estimate market shares in situation where not all of the alternatives are available ${ }^{24}$.

The stated choice survey in this study was designed using the efficient design method. This approach allowed for the implementation of a relatively complex stated choice experiment using a much smaller survey sample. The implementation of the survey in this study, with resources available, would not have been possible without the use of this method (see section 5.2).

\footnotetext{
${ }^{24}$ In this study, the mixed logit was used with a restricted choice set in two situations. The first situation was to estimate the demand from business car buyers who were assumed only to be interested in new vehicles. The second situation was to estimate the demand from private car buyers during the period when there was a limited supply of used imported EVs from Japan.
} 


\section{Chapter 4: Light duty passenger vehicle technology: current status and potential}

\subsection{Introduction}

The battery based electric vehicle (EV) technology of plug-in hybrid electric vehicles (PHEVs) and full battery EVs (BEVs) are one of a number of light passenger vehicle (LPV) propulsion technologies that are currently being developed with the objective of reducing the greenhouse gas (GHG) emissions produced by the world's vehicle fleets. It is not clear which, if any, will become the dominant technology in the LPV market (Bandivadekar et al., 2008).

The future development of new LPV propulsion technologies is further complicated by the potential for these technologies to be combined into hybrid forms, such as the petrol or diesel hybrid EVs, plug-in petrol or diesel hybrid EVs and potentially hybrid electric and fuel cell vehicles (Bandivadekar et al., 2008). The development pathways by which vehicle technologies may evolve are summarised in Figure 4.1.

Figure 4.1: Potential vehicle technologies and possible development pathways

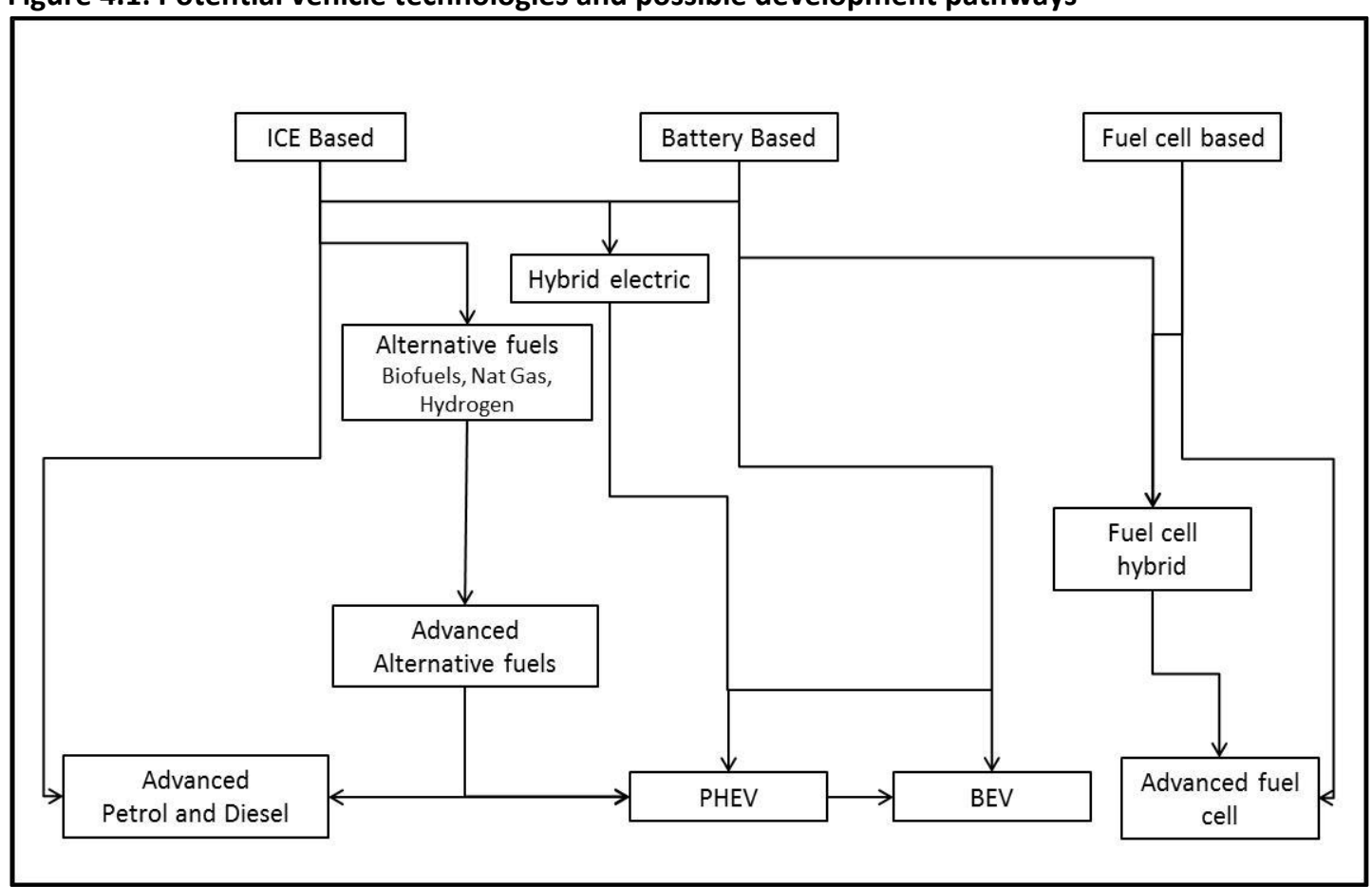

Source: Adapted from Bandivadekar et al. (2008)

This chapter provides a review of the non-propulsive and propulsive technologies currently being developed to reduce the GHGs emitted from LPVs. This chapter first 
discusses the non-propulsion technologies before reviewing the main non-EV LPV technologies being developed. The chapter then focuses on EV technology with a discussion of EV batteries, the main advantages of EVs, and the barriers that are currently preventing the uptake of EVs. The chapter concludes with a discussion of the interaction of EVs with electricity supply systems.

\subsection{Non-propulsion measures to reduce GHG emissions}

In conventional LPVs, the amount of energy reaching the wheels of internal combustion engine vehicles (ICEVs) is estimated to be about $13 \%$ of the energy contained in petroleum fuel. The rest of the energy is lost in heat, noise, and vehicle idling. Of the $13 \%$ of energy that reaches the wheel, $95 \%$ is used to move the vehicle itself. It is estimated that, as most ICEV LPVs are only occupied by the driver, in effect $1 \%$ of the energy contained in the fuel is used for transporting the driver. The vehicle design measures that are intended to reduce the proportion of energy used to move the vehicle involve reducing the weight of the vehicle and reducing the amount of energy lost in friction to the air and road surface (Lovins et al., 2005, pp. 46-47).

Strategic weight reductions are those reductions in vehicle weight that can be achieved while still holding constant both the vehicle's size and performance (Gordon et al., 2006). This approach involves the replacement of traditional steel materials with lighter materials such as aluminium, high strength steel, or polymer composites such as fibreglass or carbon fibre. Further weight reductions can also be achieved by the redesign of vehicle sub-systems and components, and by the development of smaller systems. Studies indicate that a $10 \%$ reduction in vehicle weight can cut fuel consumption by approximately $7 \%$ and that, with aggressive material substitution, reductions of up to $20 \%$ of vehicle weight can be achieved by this approach. Downsizing sub-systems will result in smaller weight savings (Bandivadekar et al., 2008).

Although the aerodynamic design of modern vehicles has improved, further improvements are achievable. It is estimated that changes in vehicle design, which reduce a vehicle's drag coefficient and frontal area in conjunction with low rolling 
resistance tyres, can be expected to reduce fuel consumption in petrol and diesel engines by up to $13 \%$ (Committee on Climate Change, 2008). These measures can also be applied to alternative fuelled vehicles (AFVs). Duke et al. (2009) have suggested that their use in EVs will help to offset the price and performance limitations imposed by current battery technology.

However, the potential of these methods to reduce GHG emissions will be diminished if there is a continuation of the present trend towards vehicles that have higher performance and that are heavier and larger (Plotkin, 2000). At present, the large scale use of lightweight materials is constrained due to the high cost of raw materials and the difficulty in developing manufacturing processes that can ensure consistent quality (Ghassemieh, 2008).

\subsection{Non-EV propulsion technologies}

\subsubsection{Advanced petrol and diesel ICEVs}

The engines used in modern ICEVs can be traced back to Nikolaus Otto's invention of the spark ignition (SI) four stroke engine in 1876, and Rudolf Diesel's development of the compression ignition (CI) engine in 1892 (Eckermann, 2001, p. 21 and 133). These technologies have been significantly improved over the last century, but there is potential for further improvements to reduce their fuel consumption and, as a consequence, GHG emissions (Kromer and Heywood, 2007).

The options for improving the fuel performance of SI engines include the use of turbocharging, direct injection, variable valve lift and timing, engine or cylinder deactivation when stopped or under light loads, variable compression ratios, low friction materials and design features, and smart cooling systems (Bandivadekar et al., 2008).

The performance of the diesel $\mathrm{Cl}$ engine has improved since the 1980s largely through the introduction of the common rail fuel injection technology. This engine technology currently offers a $20-25 \%$ energy efficiency benefit over a comparable petrol SI engine. Diesel technology probably offers less potential for further GHG emissions reductions, but further improvements are possible through 
improvements in valve timing, the use of higher pressure fuel injection systems, improved engine thermal management, and the development of homogeneous charge compression ignition ${ }^{25}$ technology (Bandivadekar et al., 2008; Lave et al., 2010).

Both petrol and diesel ICEVs can be hybridised with electric drive systems to further reduce fuel consumption. Hybrid electric vehicle (HEV) drive systems allow vehicles to use smaller ICEs, while still providing the same performance. These drive systems also facilitate the use of idle stop and regenerative braking technology (Kromer and Heywood, 2007). PHEVs are HEVs that incorporate a battery technology which allows for the recharging of the battery from the electricity grid.

The Massachusetts Institute of Technology's Laboratory for Energy and the Environment has undertaken research to determine the potential improvement in energy efficiency that can be achieved by petroleum based ICEs without decreasing vehicle performance, size, or driving range (Table 4.1). This research used a normally aspirated SI Toyota Camry as the basis for comparison because this vehicle is considered to be an example of a typical mid-sized American family car (Bandivadekar et al., 2008; Kromer and Heywood, 2007; Kromer and Heywood, $2009)^{26}$. Their research indicates that fuel consumption can be reduced from current levels by 35 to $45 \%$ for ICEVs and nearly $60 \%$ if advanced HEV technology is used. The potential fuel consumption saving from PHEVs is more uncertain because the results of the analysis are sensitive to the assumptions that are made about the vehicle's electric driving range and the type of engine management system used (Bandivadekar et al., 2008).

\footnotetext{
${ }^{25}$ Homogeneous charge compression ignition is a $\mathrm{Cl}$ technology that has some features of the $\mathrm{SI}$ engine. This technology can provide engine efficiencies as high as advanced versions of the diesel engine without the associated NOx and PM emissions (U.S. Department of Energy, 2001).

${ }^{26}$ In New Zealand, the Toyota Camry has been in the top 20 of new car sales since 2005 (New Zealand Transport Agency, 2006, 2013).
} 
Table 4.1: Estimated future fuel efficiency improvements compared to current performance: 2007 versus 2035

\begin{tabular}{|c|c|c|}
\hline & \multicolumn{2}{|c|}{$\begin{array}{c}\text { Petroleum consumption } \\
\text { (petrol equivalent) }\end{array}$} \\
\hline Propulsion system & $\begin{array}{c}\text { Relative to current petrol } \\
\text { ICE }\end{array}$ & $\begin{array}{c}\text { Relative to petrol ICE in } \\
2035\end{array}$ \\
\hline Current gasoline ICEV & $1(8.8 \mathrm{~L} / 100 \mathrm{~km})$ & - \\
\hline $\begin{array}{c}\text { Current diesel engine } \\
\text { Current hybrid (HEV) }\end{array}$ & 0.8 & - \\
\hline $\begin{array}{c}\text { Advanced petrol ICEV } \\
\text { (2035) }\end{array}$ & 0.75 & 1 \\
\hline $\begin{array}{c}\text { Advanced diesel engine } \\
\text { (2035) }\end{array}$ & 0.65 & 0.85 \\
\hline $\begin{array}{c}\text { Advanced hybrid (HEV) } \\
\text { (2035) }\end{array}$ & 0.55 & 0.6 \\
\hline PHEV & 0.4 & 0.3 \\
\hline
\end{tabular}

Source: Bandivadekar et al. (2008)

\subsubsection{Alternative fuelled ICEVs}

ICEVs can be operated using fuels other than the conventional petrol or diesel. These vehicles can be operated as fully dedicated AFVs, dual-fuelled (switching from alternative fuel to conventional), or flexi-fuels (using a mixture of both). Alternative fuels that have been used or proposed for use in ICEVs are compressed natural gas (CNG), liquefied petroleum gas (LPG), and various biofuels, primarily methanol, ethanol, and methyl and ethyl esters (biodiesel). ICEVs can also be operated by combusting hydrogen (Kowalewicz and Wojtyniak, 2005).

\section{Natural gas and liquefied petroleum gas}

CNG and LPG LPVs are not a new technology. Fully dedicated and dual fuelled CNG and LPG vehicles have been used in New Zealand since the 1970s. By the late 1980s, New Zealand had approximately 100,000 CNG vehicles, largely through the use of conversion kits, and 50,000 LPG vehicles.

However, the use of these fuels declined as the price of petrol decreased and the Government removed subsidies and imposed excise duties in 1987 (Denne and Colegrave, 2005). By 2011, there were 1,484 registered CNG vehicles and 2,815 LPG vehicles (Ministry of Transport, 2012b). With the decline in numbers of CNG vehicles, the CNG vehicle refuelling infrastructure was also allowed to decline. Unless there is a substantial investment in the distribution network, the potential 
for CNG is limited. The LPG distribution network has also declined, but to a lesser extent because it also used to supply the home barbecue and heating appliance markets (de Pont, 2009).

The GHG emissions from CNG and LPG vehicles are estimated to be up to $13 \%$ lower than diesel and up to $16 \%$ lower than petrol vehicles (Denne and Colegrave, 2005). These estimates do not take into account the impact of fugitive methane emissions from the refuelling network (Wright and Baines, 1990).

The lack of availability of the CNG and LPG refuelling infrastructure is one of the main barriers cited to the uptake of these vehicles. Other barriers are the limited availability of CNG and LPG vehicles for sale, and the higher purchase price (Werpy et al., 2009). In New Zealand, Ford and Holden offer new LPG-fuelled vehicles, but these vehicles cost between NZ\$400-\$1500 more than the equivalent petrol vehicles (de Pont, 2009).

\section{Biofuels}

Biofuel and petroleum fuel blends are already available in New Zealand. Although there are no biofuel mandates or targets in New Zealand, many jurisdictions have either biofuel blending targets or mandates (International Energy Agency, 2011). In 2010, 100 billion litres (volumetric) of biofuels were produced globally or about $3 \%$ of the total global road transport fuel demand (International Energy Agency, 2011).

The development of biofuels, as a major source of transport fuel and means of reducing GHG emissions, faces a number of uncertainties. The biofuels currently being used are known as first generation biofuels, which use natural oils, fruits, and sugars. The main feedstock for first generation biofuels are rapeseed, soybeans, and palm oil for biodiesel; and sugar beets, wheat, corn, and sugarcane for ethanol (Bandivadekar et al., 2008). The feedstock accounts for $45 \%$ to $70 \%$ of total production costs, which means that the price of these biofuels tends to be closely linked to changes in food prices (International Energy Agency, 2011).

First generation biofuels have been criticised for having an adverse impact on food supply and prices through the displacement of these crops from food production to 
biofuels. However, the evidence supporting this argument is mixed (Mitchell, 2008). Some studies indicate that there has been a significant effect on food prices (Collins, 2008; Baier et al., 2009). Other studies indicate that: (1) any impact on food prices was limited to the period between 2004 and 2008; and (2) other factors, including weather and recovering economic growth, have had a greater impact (Trostle et al., 2011; Ajanovic, 2011). Some studies indicate that the impact on food security is context specific, dependent on the crop that is used, how it is grown; the inputs used for the type of land, and the alternative uses of that land, if any (Norgrove, 2010).

It is also uncertain whether the use of biofuels will result in reductions in GHG emissions. A review by Davis et al. (2008) of GHG emissions life cycle analyses of biofuels found that the amount of GHG emissions, when compared to conventional fuels, depending on the feed stock and refining process can vary from $-114 \%{ }^{27}$ to $+93 \%$. The authors acknowledge that this variation is, in part, due to inconsistencies in the assumptions used in the reviewed studies. A study by Scharlemann and Laurance (2008) found that, when factors such as natural resource depletion, damage to human health and ecosystems were included, as well as reductions of GHG emissions, United States corn ethanol, Brazilian sugarcane ethanol, soy diesel, and Malaysian palm-oil diesel have greater environmental costs than petroleum. Biofuels with the lowest environmental costs were derived from residual products, such as bio-waste, recycled cooking oil, and ethanol from grass or wood. Despite the limitations of life-cycle assessment studies, which have been criticised for inconsistent analytical metrics and difficulties in setting of the study boundaries (Davis et al., 2008), there is a general consensus that first generation biofuels need to be replaced by second generation biofuels that will use feedstock sourced from bio-wastes, fast-growing crops grown on marginal land, or the non-edible part of the plant. It is expected that these biofuels will result in greater reductions of GHG emissions and will result in less environmental damage (Committee on Climate Change, 2008; International Energy Agency, 2011).

\footnotetext{
${ }^{27}$ This result implies that all of the fossil fuel emissions would be displaced by the use of biofuels plus an additional $14 \%$ that would be sequestered by the biofuel crop.
} 
The proposed second generation biofuels include lignocellulosic ethanol, biodiesel using gasification and the Fischer-Tropsch process, biodiesel from algae, and biofuels produced using pyrolysis (Naik et al., 2010). The production of these forms of biofuels present technical and economic challenges that, despite significant research efforts, still prevent the transfer of these technologies from the demonstration stage to full commercialisation, which means that the production of these biofuels remains very small (International Energy Agency, 2011; Sims et al., 2010).

Biofuel ethanol can be used in most unmodified vehicles up to blends of $3 \%$, and many models can safely operate on blends of up to $10 \%$. To use higher blends, modifications to the fuel system and engine are required. Specially designed vehicles known as flexible fuelled, or flexfuel, vehicles can operate using blends of up to $85 \%$ (de Pont, 2006).

Biodiesel blends of up to $5 \%$ can be used in unmodified LPVs if they comply with the European standard. Higher blends require modified engines and are only recommended for use by operators of vehicle fleets who have special vehicle management qualifications (Campbell, 2010). In New Zealand, Holden provides two flexfuel models that can operate on up to $85 \%$ ethanol (Sloane, 2012). At this time, biofuel blends of E3 and E10 can be bought from Mobil and Gull stations, and biodiesel can be bought from a small number of specialised local suppliers (Liquid Biofuels Interest Group, 2012).

\section{Hydrogen}

The combustion of hydrogen to drive a vehicle can be traced back to the very start of the $19^{\text {th }}$ century when Francois de Rivaz experimented with an open cylinder engine (Eckermann, 2001, p. 18). The hydrogen internal combustion engine vehicle (HICEV) is being developed in conjunction with the HFCV and is seen as an intermediate step towards full deployment of HFCVs. While there is scope for further development of this technology, the main constraints to the deployment of this technology are the same as for HFCVs in that satisfactory systems of on-board hydrogen storage need to be developed, as well as a hydrogen refuelling 
infrastructure, and the means of producing the hydrogen fuel (Verhelst and Wallner, 2009).

\subsubsection{HFCVs}

Before HFCVs become competitive with ICEVs and EVs, a number of technological challenges must be overcome and system costs reduced. Technical issues comprise increased endurance of the proton exchange membrane materials in the fuel stack, greater power performance, better systems management to control fuel-cell operating conditions, and improved on-board hydrogen storage that provides adequate vehicle range (U.S. Department of Energy, 2009).

The United States Department of Energy has specified that, by the year 2015, fuel cells should be $60 \%$ efficient (they are currently about $55 \%$ efficient), cost US\$30/kW (they currently cost US\$49/kW), and have a useable lifetime of at least 5000 hours, equivalent to $240,000 \mathrm{~km}$ at $50 \mathrm{~km} / \mathrm{h}$ (currently, their average durability is estimated to be 1,700 hours) (U.S. Department of Energy, 2011a; U.S. Department of Energy, 2011b).

There are three generic methods of on-board storage: (1) high pressure compression; (2) cryogenic liquefaction; and (3) materials storage, which involve storing the hydrogen in a chemical form such as a metal hydride. The United States Department of Energy, as part of the FreedomCar programme, estimates that, for a HFCV to travel $500 \mathrm{~km}$, it would be necessary to store 5-13 kg of hydrogen on board the vehicle (U.S. Department of Energy, 2009). To practically store this amount of hydrogen on board a vehicle would require a storage vessel to achieve a gravimetric density of at least 7.5 wt.\% hydrogen and a volumetric density of 70 grams hydrogen/L. Current storage system technologies achieve, on average, gravimetric densities of 5.0 wt.\% hydrogen and volumetric densities of 25 grams hydrogen/L. Progress towards these targets has been slow and as a result, in 2009, the 2015 progress targets were revised downward from $9 \% \mathrm{wt}$ to $5.5 \% \mathrm{wt}$, and from 81 grams hydrogen/L to 40 grams hydrogen/L (U.S. Department of Energy, 2010). 
The production and transport of hydrogen for use in LPV fleets is considered even more challenging than the technological barriers facing HFCVs (Bandivadekar et al., 2008). Hydrogen can be produced by electrolysis using electricity generated from renewable or non-renewable sources, the reformation of natural gas or biomass derived methanol, and the gasification of coal or biomass (Kruger et al., 2003; Smit and Campbell, 2007). Different fuel pathways result in different well-to-wheels GHG emissions, which are discussed further in section 4.6.2.

The development of the refuelling infrastructure is complicated by the need to align its development with the uptake of HFCVs into LPV fleets. Modelling in the United States concluded that a low market penetration of HICEVs and HFCVs would probably result in the development of refuelling stations using on-site steam methane (natural gas) reformers because there would not be enough demand for centrally produced and distributed hydrogen. This modelling concluded that the use of electrolysis or the gasification of biomass as a source of hydrogen, while technically feasible, would be too expensive in the near-term (Ogden and Yang, 2009). The study concluded that the most cost-effective option in the United States would be centralised natural gas reformation.

Smit and Campbell (2007) concluded that, in New Zealand, the production of hydrogen via electrolysis would be the most expensive of the options analysed. However, they concluded that centralised coal gasification would be slightly more cost-effective in New Zealand than natural gas reformation. This result was found to hold even when the additional costs of carbon capture and storage technology were included, which the authors assumed will be technically feasible.

As of 2012, HFCVs had yet to enter global vehicle markets except as part of demonstration projects, at present EVs have an advantage in the market (Eberle et al., 2012). This technology has considerable potential, but significant vehicle technology and refuelling infrastructure challenges remain before these vehicles are ready to enter global markets (Wipke et al., 2012).

The GHG emissions benefits from HFCVs in New Zealand are likely to be less than those from EVs as refuelling of these vehicles is likely to initially occur through the 
use of distributed reformation of natural gas, without carbon sequestration. This configuration would result in more GHG emissions than EVs being recharged from the predominantly renewable New Zealand electricity grid (see section 4.6.2).

\subsection{Overview of EV technology}

The light passenger battery EV is not a new technology and was first developed in the last decade of the $19^{\text {th }}$ century when it competed with ICEVs for the emerging automobile market. The first LPV to exceed $100 \mathrm{~km} / \mathrm{h}$ was an EV in 1899 . By the 1920s, the availability of cheap oil, increasing reliability of ICEs, the development of the self-starter, and the limitations of contemporary battery technology resulted in the decline of EVs. EVs have traditionally been used in areas where quietness and the lack of tailpipe emissions are required, and where a limited driving range is not a factor. Examples are use in warehouses, inside buildings, on golf courses, and as mobility scooters (Larminie and Lowry, 2003, p. 5).

EVs can be divided into BEVs, which rely solely on electricity for motive power, and PHEVs, which partially rely on electricity for motive power and use another source of energy, usually petrol, to supplement the electric drive system.

The key elements of a BEV's drive system are:

- The battery, including the battery management system (BMS). The BMS provides fault detection and controls cell temperature, the depth of discharge, and cell balancing.

- The battery charger, which converts the alternating current from the electricity grid to direct current. Most EVs have an on-board charger suitable for home recharging. For higher voltages and current, a charging station is required.

- Electronic controller, which connects the driving controls to the power converter and BMS. The electronic controller controls the vehicle's speed and direction and provides direction to the BMS so that the battery can be recharged from regenerative braking. 
- Power converter, which controls the flow of power to and from the battery and to the electric motors.

- Electric motor/generator: separate motors and generators can be used to drive individual wheels.

- Transmission system (Larminie and Lowry, 2003).

PHEVs are more complex vehicles because they also incorporate an ICE and electric generator. The hybridisation of the technologies allows a PHEV to operate as a BEV for a limited distance and then, when the battery is almost depleted, operate as a HEV (Gonder and Markel, 2007).

The total driving range of a PHEV is comparable to an ICEV with sufficient electric driving capability to accommodate most of the daily travel undertaken by LPVs, but with the option of using its ICE to accommodate the occasional longer journeys. This facility is seen as a means of overcoming the perceived concern of 'range anxiety', or the fear of being stranded during a journey and being unable to recharge (Tate et al., 2008). The shorter electric driving range requires a smaller and less expensive battery than in a BEV and this can be reflected in the purchase price. The high cost of EV batteries means that a BEV with a comparable driving range to a PHEV would be more expensive, even taking into account the additional costs associated with having an ICE in the PHEV ${ }^{28}$. The smaller battery pack in a PHEV also allows for shorter recharging times (Gremban, 2007; Taylor, 2009).

PHEVs operate using two driving modes: (1) the charge depleting (CD) phase, when the charged battery is being depleted to a predetermined level; and (2) the charge sustaining phase, when the ICE is used to sustain the battery at the predetermined level. PHEVs can be designed as split configuration or series configuration drive trains (Table 4.2).

\footnotetext{
${ }^{28}$ At present the Tesla Model S, with an $85 \mathrm{kWh}$ battery configuration, is the only BEV with a driving range comparable to a PHEV. This vehicle is priced at approximately twice that of the GM Volt and the Toyota Prius PHV PHEVs (Chevrolet.com, 2012; Tesla Motors, 2012; Toyota, 2012)
} 
Table 4.2: Plug-in hybrid vehicle operating configurations

\begin{tabular}{|c|c|}
\hline Spilt configuration & Series configuration \\
\hline $\begin{array}{l}\text { The driving wheels can be powered by the } \\
\text { electric motor, ICE, or a combination of } \\
\text { both. } \\
\text { Used in conjunction with the blended } \\
\text { driving cycle or all-electric range cycle. }\end{array}$ & $\begin{array}{c}\text { The driving wheels are only powered by the } \\
\text { electric motor and the ICE is only used to } \\
\text { charge the battery. } \\
\text { Used in conjunction with the all-electric } \\
\text { range cycle } \\
\text { This configuration is also known as an } \\
\text { extended range EV. }\end{array}$ \\
\hline
\end{tabular}

A PHEV with a blended driving cycle can use its ICE to meet peak power demand during high acceleration or high speed travel in the CD mode. The level of blending can vary from ICE dominant with the electric motor supplementing the ICE, or electric motor dominant with the ICE supplementing the electric motor, during high acceleration or high speed travel (Gonder and Markel, 2007). The blended cycle has the advantage of reducing the peak power demands on the battery and electric motor. Consequently, the size of the battery and electric motor can be reduced, which, in turn, reduces the cost of the vehicle. The blended cycle has the disadvantage of slightly increasing the consumption of petroleum when compared to an all-electric range cycle PHEV (Moawad et al., 2009; Pesaran et al., 2009).

The all-electric range cycle uses only the electric motor during the CD phase, meeting all acceleration requirements. During this phase, the ICE remains inactive. This configuration has the advantage of lower petroleum consumption compared to the blended cycle. The main disadvantage is that the battery and electric motor must be larger to meet the higher peak power demands during acceleration and high speed cruising. The larger battery and electric motor add to the cost of the vehicle (Vyas et al., 2007; Kromer and Heywood, 2007).

The current global status of EVs is changing rapidly with annual sales increasing by $151 \%$ in 2012 to 113,000 vehicles. These sales brought the estimated total global stock of EVs to 180,000 vehicles of which 52,835 units were sold in the United States. The increase in sales in the United States can be attributed to the popularity of PHEVs, which comprised 27,992 of the EV sales. The Electric Vehicle Initiative projects that the global sales of EVs will rapidly increase reaching $6,000,000$ vehicles per year by 2020 , which will bring the global stock of EVs to $20,000,000$ vehicles. However, the selection of EV models available for purchase remains limited. At the 
end of 2012, there were 20 EV models available for sale globally. However, only 6 to $8 \mathrm{EV}$ models were widely available to the general public and only in a limited number of countries (Electric Vehicles Initiative of the Clean Energy Ministerial and International Energy Agency, 2013).

In New Zealand, at the end of 2012, there were two models of BEV and one PHEV model available for sale and sales remain small. However, the trend is encouraging. After a long period where the number of EVs sold was less than 4 per year, since 2009 the number has increased. By 2011, the number of EVs sold increased to 14 and, in 2012, the number of EVs sold increased to 28 (New Zealand Transport Agency, 2013).

\subsection{Status of vehicular batteries}

\subsubsection{Current technology}

The current generation of BEVs and PHEVs utilise lithium-ion batteries (Kalhammer et al., 2007), which are preferred for use in BEVs and PHEVs because they have specific energies twice that of Nickel Cadmium batteries, high nominal cell voltages, and good load characterises with a flat discharge curve offering effective utilisation of the stored energy with a voltage spectrum ranging from 3.7 to $2.8 \mathrm{~V} /$ cell. These batteries are low maintenance because they do not require periodic discharge, with no memory effect ${ }^{29}$, and low self-discharge. They require tighter voltage tolerance during charging and will not tolerate overcharging, so there can be no trickle charge once the battery is at full charge (Buchmann, 2011, p 122).

There is a variety of lithium-ion batteries currently considered suitable for use in EVs depending on the chemical composition of the cathode and anodes. Current lithium-ion batteries use lithium-graphite composition for the anode, except for the lithium titanate oxide, or LTO, battery which uses lithium titanate as the anode material. The most common chemistries used for the cathode are: (1) $\mathrm{LiCoO}_{2}$, which was the first lithium-ion battery developed; (2) $\mathrm{LiMnO}_{2}$ spinel, known as LMO; (3)

\footnotetext{
${ }^{29}$ An effect seen in nickel cadmium batteries where the battery gradually loses energy capacity if repeatedly recharged after only being partially discharged.
} 
$\mathrm{LiFePO}_{4}$, known as the iron phosphate or LFP battery; (4) $\mathrm{LiNiMnCoO}_{2}$, known as the NMC battery; and (5) LiNiCoAl, known as the NCA battery (Burke and Miller, 2009; Buchmann, 2011, p. 49) (Table 4.3).

Despite their advantages, these batteries do not meet all of the United States Advanced Battery Consortium's (USABC) goals, which are seen by the United States automobile industry as the minimum goals required for successful EV commercialisation. The shortcomings relate to energy capacity, in terms of both battery weight (specific density) and volume (energy density), battery cost, and operating temperature range (Table 4.3) (Kalhammer et al., 2007).

Graphite anode lithium-ion batteries currently attain values, at the battery pack level, of 90-150 Wh/kg and 220-330 Wh/L depending on the cathode chemistry. LTO batteries have lower energy densities than lithium-ion batteries that use graphite anodes. Due to their fast charging capability and high charging cycle life, LTO batteries are considered more suitable for organisations operating LPV fleets that can return to a depot with fast charging facilities (Burke and Miller, 2009). In comparison, petrol and diesel fuels have specific density and energy density values of approximately $12,000 \mathrm{Wh} / \mathrm{kg}$ and 9,700 Wh/L. The effect of the low energy densities in lithium-ion batteries is that an EV with a $500 \mathrm{~kg}$ battery can travel about $325 \mathrm{~km}$ whereas an ICEV, assuming average energy efficiency, using $50 \mathrm{~kg}$ of petrol will travel approximately $660 \mathrm{~km}$.

The price of lithium-ion battery cells has declined from over US\$3,000/kWh in 1992 to an average cell price of US\$400/kWh in 2012 and a battery pack, of the order of 30 kWh, would cost approximately US\$21,000 or US\$700/kWh (Element Energy Limited, 2012). For widespread commercialisation of EVs, the USABC of the United States Council for Automotive Research considers that prices of battery packs must decline to at most US\$150 per kWh, in 2012 dollars, or by another 79\% (U.S. Advanced Battery Consortium, 2012).

The operating temperature range of an EV battery is important in regions with extreme climates and is particularly important in the United States. At low temperatures, the power output of the battery declines and high temperatures can 
reduce the life of the battery. To address these limitations, lithium-ion batteries must have thermal regulation, but this adds further to the weight and cost of an EV battery (Srinivasan, 2008).

PHEV batteries have different requirements from BEV batteries. PHEV batteries must be able to provide sufficient power for acceleration and cruising speeds while using a smaller battery. Lithium-ion batteries meet the power requirements for PHEVs currently in the market, but it is considered that further battery development is needed to meet the power, weight, and volume requirements necessary for a mid-size passenger PHEV with a $70 \mathrm{~km}$ all-electric-range and, at the same time, provide assurance that 3,000 to 5,000 "charge-depleting" deep discharge cycles can be achieved (Burke and Miller, 2009; Pesaran et al., 2009).

\subsubsection{Future potential}

Research into advanced lithium-ion batteries is now focused on the development of high voltage cathode materials and the use of silicon anodes. Silicon anodes have shown potential for increased energy density with the potential to have cell level specific energy of up to $800 \mathrm{Wh} / \mathrm{kg}$, but silicon anodes experience rapid capacity decay resulting in reduced cycle life (Lee et al., 2011). An alternative to silicon anodes are silicon-graphite composite anodes, which have cell level specific energy levels of up to $500 \mathrm{Wh} / \mathrm{kg}$, but greater cycling capacity. This technology is expected to be available for use in EVs before 2020 (Amine et al., 2012; Doeff, 2011).

To achieve even higher energy densities will require the use of non-lithium-ion technologies. At present, research is focused on zinc air, lithium sulphide, lithium sulphur, lithium air, and metal air batteries (Figure 4.2). These technologies are at an experimental stage and it is uncertain when, or if, they will be available for use in EVs (Srinivasan, 2008). 
Table 4.3: Summary of characteristics of lithium-ion batteries

\begin{tabular}{|c|c|c|c|c|c|c|c|c|c|c|c|c|}
\hline Specifications & Lead Acid & $\mathrm{NiCd}$ & $\mathrm{NiMH}$ & \multicolumn{8}{|c|}{ Lithium-ion } & \multirow[t]{2}{*}{ Petrol } \\
\hline $\begin{array}{l}\text { Chemistry } \\
\text { (cathode) }\end{array}$ & & & & $\begin{array}{l}\text { Cobalt } \\
\left(\mathrm{LiCOO}_{2}\right)\end{array}$ & $\begin{array}{c}\text { Manganese } \\
\text { Spinel } \\
\left(\mathrm{LiMnO}_{2}\right)\end{array}$ & $\begin{array}{c}\text { LFP } \\
\left(\text { LiFePO }_{4}\right)\end{array}$ & $\left|\begin{array}{c}\mathrm{NMC} \\
\left(\mathrm{LiNiMnCoO}_{2}\right)\end{array}\right|$ & $\begin{array}{c}\text { NCA } \\
\text { (LiNiCoAI) }\end{array}$ & $\begin{array}{c}\text { LTO } \\
\text { Lithium } \\
\text { titanate/ } \\
\text { Manganese } \\
\text { spinel } \\
\end{array}$ & $\begin{array}{c}\text { USABC } \\
\text { Minimum } \\
\text { goal for } \\
\text { commercial- } \\
\text { isation } \\
\end{array}$ & $\begin{array}{c}\text { USABC } \\
\text { Long- } \\
\text { term goal }\end{array}$ & \\
\hline $\begin{array}{l}\text { Specific Energy } \\
\text { Wh/kg } \\
\text { (battery pack) }\end{array}$ & $30-50$ & $45-80$ & $60-120$ & 150 & $100-135$ & $90-120$ & $100-170$ & $100-150$ & $60-70$ & 150 & 200 & 12,000 \\
\hline $\begin{array}{l}\text { Energy density } \\
\text { Wh/L } \\
\text { (battery pack) }\end{array}$ & $60-75$ & $50-150$ & $140-300$ & 330 & 280 & 220 & 260 & 260 & $130-150$ & 230 & 300 & 9,700 \\
\hline Cell voltage & 2 & 1.2 & 1.2 & 3.6 & 3.8 & 3.3 & 3.6 & 3.6 & 2.4 & N/A & & $\mathrm{n} / \mathrm{a}$ \\
\hline \begin{tabular}{|l|} 
Charging cycles \\
$(80 \%$ discharge)
\end{tabular} & $200-300$ & 1,000 & $|300-500|$ & $500-1,000$ & $500-1,000$ & $1,000-2,500$ & $1,000-2,000$ & $\begin{array}{l}1,000- \\
2,000\end{array}$ & $>5,000$ & \multicolumn{2}{|c|}{$>1000$} & $\mathrm{n} / \mathrm{a}$ \\
\hline Fast charge time & $8-16$ hours & 1 hour & $\begin{array}{c}2-4 \\
\text { hours }\end{array}$ & $2-4$ hours & $<1$ hour & $<1$ hour & $<1$ hour & $<1$ hour & $<5 \min$ & $<30 \min$ & & $<5 \min$ \\
\hline $\begin{array}{l}\text { Self-discharge/ } \\
\text { month room } \\
\text { temperature }\end{array}$ & $5 \%$ & $20 \%$ & $30 \%$ & \multicolumn{6}{|c|}{$<10 \%$} & N/A & & $\mathrm{n} / \mathrm{a}$ \\
\hline $\begin{array}{l}\text { Discharge } \\
\text { temperature }\end{array}$ & $\begin{array}{c}-20^{\circ} \mathrm{C} \text { to } \\
50^{\circ} \mathrm{C}\end{array}$ & $-20^{\circ} \mathrm{Ct}$ & to $65^{\circ} \mathrm{C}$ & \multicolumn{6}{|c|}{$-20^{\circ} \mathrm{C}$ to $45^{\circ} \mathrm{C}$} & $\begin{array}{c}-40^{\circ} \mathrm{C} \text { to } \\
50^{\circ} \mathrm{C}\end{array}$ & & $\mathrm{n} / \mathrm{a}$ \\
\hline \multirow{2}{*}{$\begin{array}{l}\text { Safety } \\
\text { requirements }\end{array}$} & \multirow{2}{*}{$\begin{array}{c}\text { Thermally } \\
\text { stable }\end{array}$} & \multirow{2}{*}{\multicolumn{2}{|c|}{\begin{tabular}{|} 
Thermally stable \\
fuse protection \\
common
\end{tabular}}} & $\begin{array}{l}\text { Least } \\
\text { stable }\end{array}$ & $\begin{array}{c}\text { Moderately } \\
\text { stable }\end{array}$ & Stable & $\begin{array}{c}\text { Moderately } \\
\text { stable }\end{array}$ & \begin{tabular}{|c|} 
Moderatel \\
y stable
\end{tabular} & Stable & N/A & & $\mathrm{n} / \mathrm{a}$ \\
\hline & & & & \multicolumn{6}{|c|}{ Protection circuit required } & & & \\
\hline $\begin{array}{l}\text { Cell price } 2011 \\
\text { US\$ / kWh }\end{array}$ & 150 & 500 & 900 & \multicolumn{6}{|c|}{$310-550$} & $\mathrm{~N} / \mathrm{A}$ & N/A & $\mathrm{n} / \mathrm{a}$ \\
\hline Toxicity & Very High & $\begin{array}{l}\text { Very } \\
\text { high }\end{array}$ & Low & \multicolumn{6}{|c|}{ Low } & & & $\begin{array}{l}\text { Very } \\
\text { high }\end{array}$ \\
\hline
\end{tabular}

Sources: Buchmann (2011), Pesaran et al. (2009), Srinivasan (2008), Element Energy Limited (2012). 


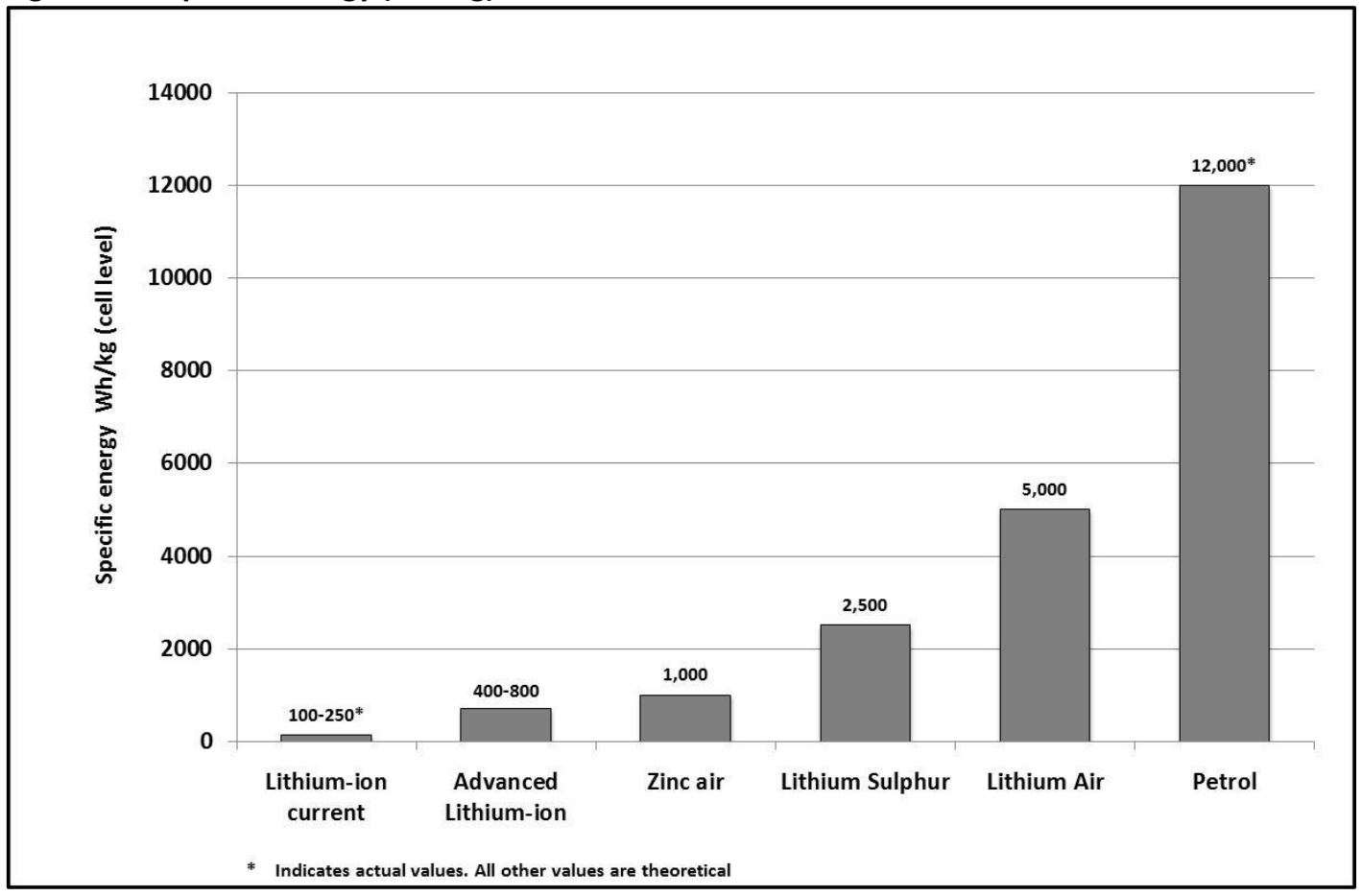

Source: Srinivasan, 2008

The capacity for further reductions in the price of EV grade lithium-ion batteries remains uncertain with contemporary market projections expressing a range of views. More conservative projections assume that the price of lithium-ion battery packs will probably remain within the present range of prices of around US\$700 per kWh, or only decline slightly so that, by 2020 , the price is projected to be US\$400 per kWh (Lux Research, 2012). More optimistic projections indicate faster reductions in lithium-ion battery pack prices. Sankey et al. (2010) project declines to US\$250/kWh by 2020. Hensley et al. (2012) were even more optimistic projecting prices of US\$200/kWh by 2020 and US\$160/kWh by 2025 (in 2011 dollars).

These projections are based on different views about the future cost of materials, rate of improvement in manufacturing processes, effect of increasing economies of scale, and the effect of competitive pressures lowering component prices.

\subsection{Advantages of EVs}

\subsubsection{High energy and fuel efficiency}

The advantages of EVs are directly related to the efficiency of the electric motor and the electrochemical battery relative to other vehicle technologies. Electric 
motors are around 90\% energy efficient and batteries around 85\% energy efficient, which results in Tank-to-Wheel (TTW) energy efficiencies of over $80 \%$. These are much higher than other vehicle technologies, including HFCVs.

When the energy losses associated with the fuel production, transmission, and refuelling stages are included in the analysis, the advantages of EVs are not so clear. Estimates of the well-to-wheel (WTW) energy efficiencies for both BEVs and HFCVs, when the hydrogen is produced by electrolysis, are sensitive to the mix of generation technologies used to produce the electricity. If EVs are fuelled using renewable electricity, they remain the most energy efficient of the LPV alternatives with WTW efficiencies of approximately $65 \%$. If it is assumed that, on average, renewable generation contributes around $75 \%$ of New Zealand's electricity, natural gas $14 \%$, and coal $8 \%$, the WTW declines to about $54 \%$. However, if the electricity is generated solely using thermal power stations, the WTW energy efficiency declines to about $22 \%$, which is not much greater than that of ICEVs and about the same as HFCVs (Table 4.4) (Bossel, 2003; Eberhard and Tarpenning, 2006; Thomas, 2009). 
Table 4.4: TTW and WTW energy efficiencies of alternative fuel and vehicle technology pathways

\begin{tabular}{|c|c|c|c|c|c|c|c|}
\hline & $\begin{array}{l}\text { Generation/ } \\
\text { production } \\
\text { efficiency }\end{array}$ & $\begin{array}{l}\text { Trans- } \\
\text { mission } \\
\text { efficiency }\end{array}$ & $\begin{array}{l}\text { Charging/ } \\
\text { Fuelling } \\
\text { efficiency }\end{array}$ & $\begin{array}{l}\text { Battery } \\
\text { efficiency } \\
\text { Fuel cell } \\
\text { efficiency* }\end{array}$ & $\begin{array}{c}\text { Drive } \\
\text { train } \\
\text { efficiency }\end{array}$ & $\mid \begin{array}{c}\text { TTW } \\
\text { efficiency }\end{array}$ & $\begin{array}{l}\text { WTW } \\
\text { efficiency }\end{array}$ \\
\hline $\begin{array}{c}\text { BEV } \\
\text { renewable } \\
\text { energy }\end{array}$ & $100 \%$ & $91 \%$ & $93 \%$ & $\begin{array}{c}85 \% \\
(94 \% \\
\text { charging } \\
90 \% \\
\text { discharging) }\end{array}$ & $90 \%$ & $81 \%$ & $65 \%$ \\
\hline $\begin{array}{l}\text { BEV thermal } \\
\text { (Huntly coal } \\
\text { turbine) } \\
\text { generation }\end{array}$ & $35 \% \#$ & $91 \%$ & $93 \%$ & $84 \%$ & $90 \%$ & $81 \%$ & $22 \%$ \\
\hline $\begin{array}{c}\text { BEV average } \\
\text { NZ } \\
\text { generation } \\
\text { mix } \\
\end{array}$ & $84 \% \#$ & $91 \%$ & $93 \%$ & $84 \%$ & $90 \%$ & $81 \%$ & $54 \%$ \\
\hline ICE petrol & \multicolumn{3}{|c|}{$81 \%$} & N/A & $17 \%$ & $17 \%$ & $14 \%$ \\
\hline HEV petrol & \multicolumn{3}{|c|}{$81 \%$} & $\mathrm{~N} / \mathrm{A}$ & $30 \%$ & $30 \%$ & $24 \%$ \\
\hline $\begin{array}{c}\text { HFCV } \\
\text { Compressed } \\
\text { gas } \\
\text { (electrolysis } \\
\text { from } \\
\text { renewable } \\
\text { electricity) }\end{array}$ & $63 \%+$ & $90 \% \ddagger$ & $93 \%$ & $50 \%$ & $90 \%$ & $45 \%$ & $24 \%$ \\
\hline $\begin{array}{l}\text { HFCV } \\
\text { Reformation } \\
\text { of natural } \\
\text { gas and } \\
\text { piped } \\
\text { transmission }\end{array}$ & $69 \%+$ & $90 \% \ddagger$ & 93 & $50 \%$ & $90 \%$ & $45 \%$ & $26 \%$ \\
\hline
\end{tabular}

Adapted from: Bossel (2003), Eberhard and Tarpenning (2006), and Thomas (2009)

\# Conversion efficiencies for New Zealand thermal power stations from Wembridge et al. (2009b).

* Includes parasitic energy losses in the battery pack and fuel stack

$+\quad$ includes losses due to hydrogen compression

‡ Estimated value in the United States

The effect of the high TTW efficiency for EVs is lower energy consumption and as a consequence lower fuel running costs (Figure 4.3). The energy efficiency of BEVs and PHEVs varies depending on the size of the vehicle and the driving conditions. However, it is expected that BEVs, PHEVs, and, when they become available, HFCVs 
will be the least expensive vehicles to refuel, even taking into account the projected improvements in the performance of ICEVs.

Figure 4.3: Estimated energy efficiency and fuel cost of alternative fuel vehicle technologies in 2007 and 2035

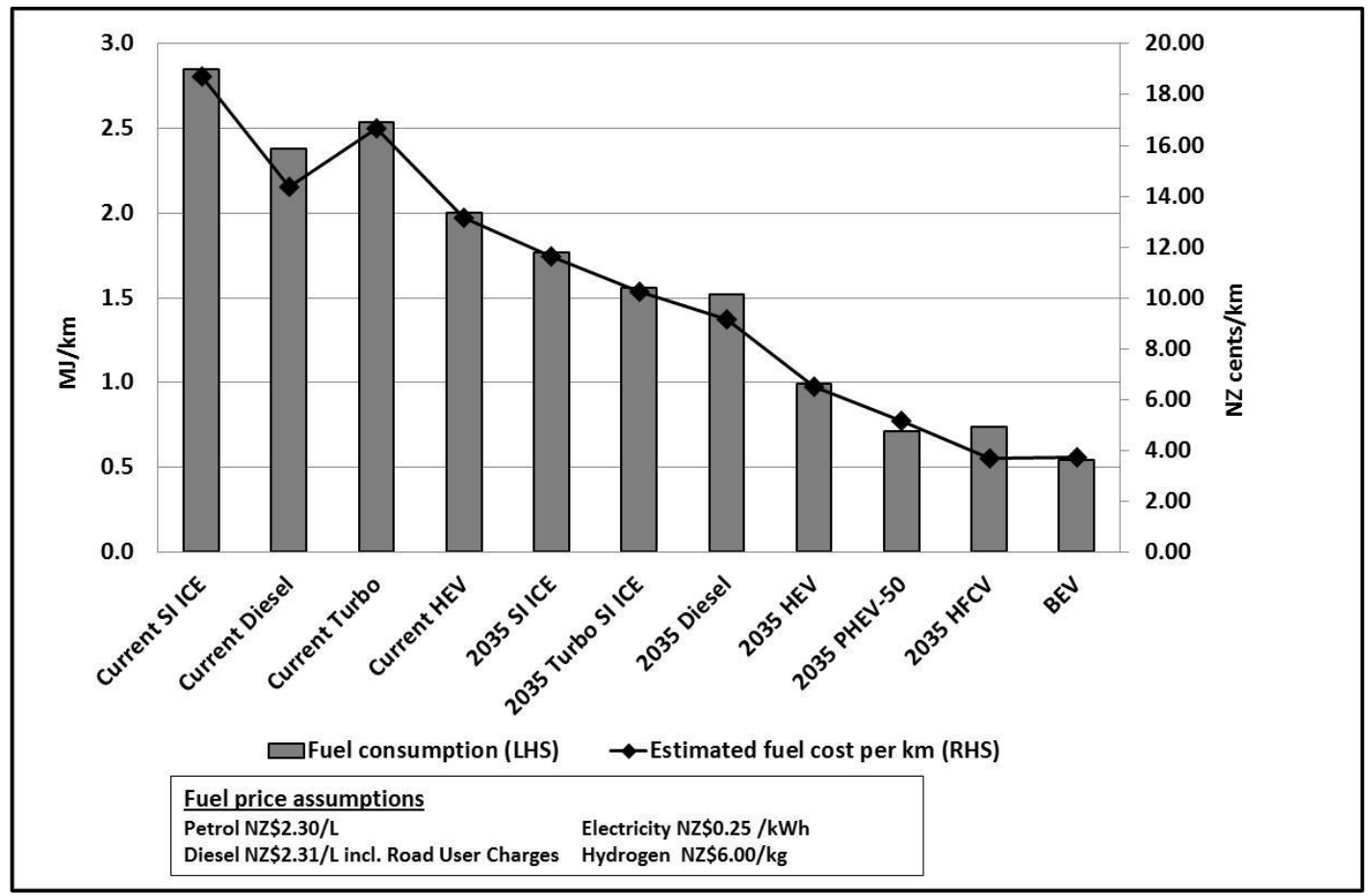

Adapted from: Bandivadekar et al. (2008)

\subsubsection{Potential for reducing GHG emissions}

WTW GHG emissions studies have shown that the type of electricity generation has a significant impact on the reduction of GHG emissions that can be achieved from the use of EVs. When used in conjunction with renewably generated electricity, EVs have the potential to operate without producing any measurable GHG emissions (Ma et al., 2012; Bandivadekar et al., 2008; Nguyen et al., 2013; Samaras and Meisterling, 2008; Simpson, 2004).

Analysis using the United States Department of Energy's Greenhouse Gases, Regulated Emissions, and Energy Use in Transportation (GREET) Model indicates that, under United States conditions ${ }^{30} \mathrm{BEVs}$ are likely to produce $112 \mathrm{~g} \mathrm{CO}_{2} \mathrm{e}$ per kilometre. These estimated GHG emissions are less than the average GHG

\footnotetext{
${ }^{30}$ Between January 2007 and January 2013 approximately 45\% of electricity generated in the United States was from coal and 25\% from natural gas (U.S. Energy Information Administration, 2013).
} 
emissions from current SI ICEVs, but not as low as the estimated GHG emissions from PHEVs. It appears that PHEVs and BEVs have the potential to reduce GHG emissions, when compared to the present LPV technologies, even when the electricity is generated using fossil fuels (Figure 4.4) (Bandivadekar et al., 2008).

Life cycle studies of the GHG emissions associated with the construction of EVs indicate that the production of the larger battery pack in BEVs can result in an additional 4 to 8 tonnes of GHG emissions during the manufacturing process when compared to ICEVs. This estimate is sensitive to the assumptions made about the size of the battery in the BEV and the type of energy used during the production process (Helms et al., 2010; Aguirre et al., 2012).

Figure 4.4: Lifecycle GHG emissions of alternative fuel and vehicle technology pathways in the United States

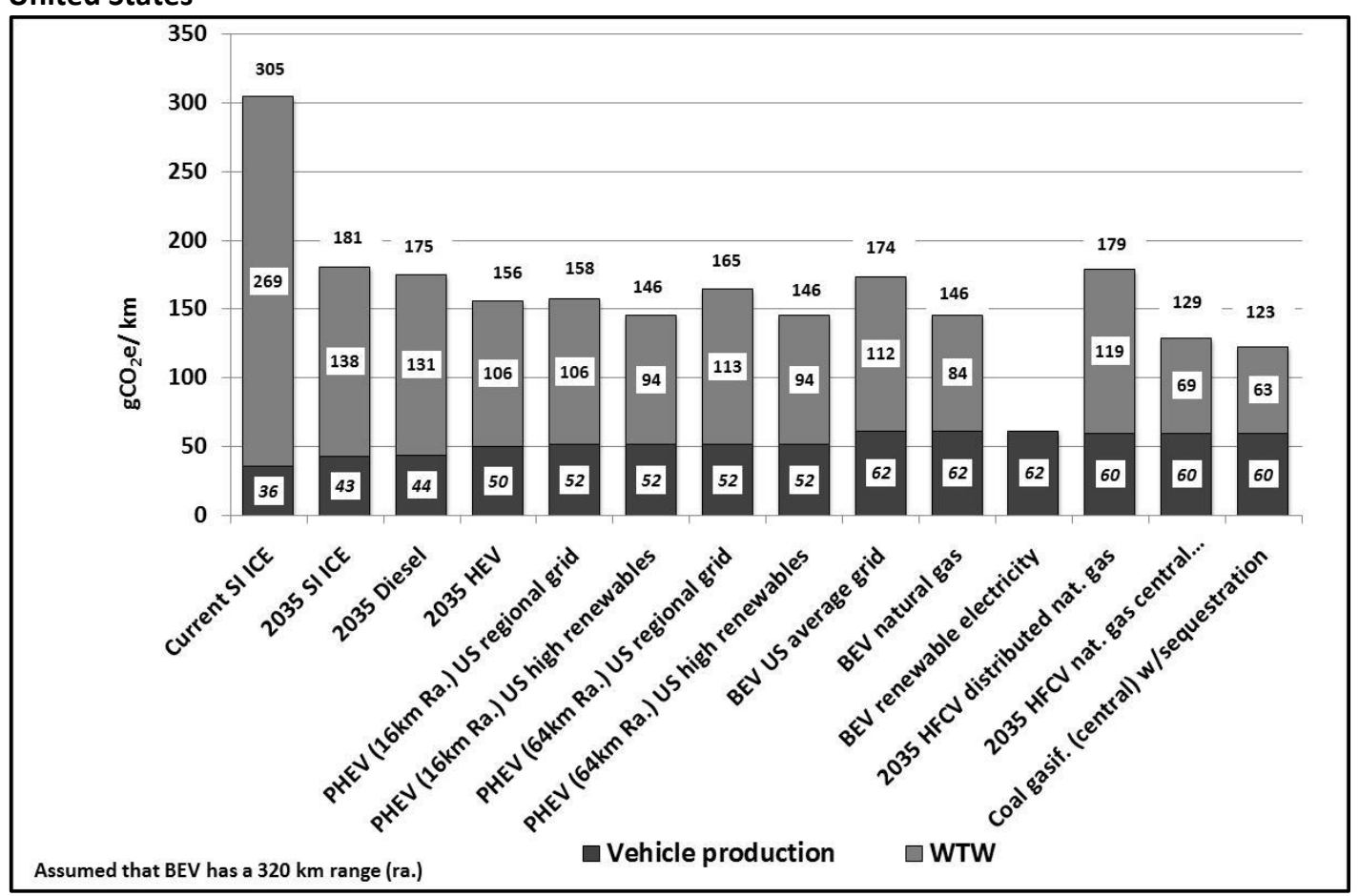

Adapted from: Aguirre et al. (2012), Helms et al. (2010), and Nguyen et al. (2013)

\subsection{Barriers to the uptake of EVs}

The most often cited barriers to the uptake of EVs in LPV fleets are a consequence of the characteristics of contemporary battery technology, which result in limited driving range, long refuelling (recharging) times, and high vehicle purchase price (Tsang et al., 2012; Kromer and Heywood, 2007). Other factors seen as barriers to the use of EVs are vehicle safety, lack of interior space, aesthetics, power and 
performance (Baptista et al., 2012; Baxter et al., 2009), and vehicle manufacturers' reluctance to change (Wells and Nieuwenhuis, 2012).

\subsubsection{High purchase price}

The purchase price of an EV is largely determined by the cost of its battery, which can be up to $50 \%$ of the total cost of the EV (Tsang et al., 2012). Any future decrease in the price of EVs is dependent on the rate and extent of the reduction in the cost of producing complete battery packs (Kromer and Heywood, 2007).

The high cost of lithium-ion batteries can limit the size of the battery that is considered feasible for installation into an EV. However, if the target market does not consider the price of lithium-ion batteries to be a constraint, a larger battery can be installed in EVs. This is a marketing approach of Tesla Motors whose Model S is designed to be a high performance luxury sedan targeted at high income earners and contains either an $85 \mathrm{kWh}$ or $60 \mathrm{kWh}$ battery that provide an EPA rated driving range of $425 \mathrm{~km}$ or $330 \mathrm{~km}$ respectively (U.S. Department of Energy, 2013).

If an EV manufacturer targets the general car buying public, the EV price must be reduced, which can only be done by installing a smaller battery. For example, the Nissan Leaf has a $24 \mathrm{kWh}$ battery, which is rated at $76 \mathrm{~km}$ to $169 \mathrm{~km}$ depending on the driving conditions (Nissan Motor Company, 2012).

A number of approaches have been suggested to reduce the disincentive to purchasing, or using, EVs due to the cost of the battery:

- battery leasing schemes, which are already provided by some manufacturers such as Nissan. There is some research which indicates that these schemes provide a financial advantage over the option of battery ownership (The Independent, 2011)

- reducing the size of the battery required by improving the energy efficiency of EVs. The energy efficiency of EVs can be improved by using lightweight materials and improving EVs' aerodynamics (Williams and Lipman, 2010; Duke et al., 2009) 
- creating resale value for an end of life EV battery. EV batteries retain up to $80 \%$ of their capacity at the end of their useful life in an EV. It has been proposed that a second use market for PHEV and EV batteries could be developed as they would be suitable for use in distributed generation projects such as with domestic solar PV systems, or for use by network operators as distributed electricity storage systems (Neubauer and Pearsan, 2011; Williams, 2011)

- circumventing the need for EV purchase through the development of car sharing schemes. Car sharing schemes are in use in Europe and the United States. Some of these schemes already utilise EVs, such as the Praxiparcs scheme in Paris (Shaheen et al., 1998), the Green Move pilot project in Milan (Lue et al.), and I-GO scheme in Chicago (I-GO, 2012).

\subsubsection{Driving range}

Studies of car buyers' preferences indicate that the driving range is one of the most important considerations when considering the purchase of a BEV (Egbue and Long, 2012; Brownstone et al., 2000; Bunch et al., 1992). This concern does not seem important for those purchasers considering the purchase of a PHEV, with fuel running costs being a more important consideration (Axen and Kurani, 2008).

Research indicates that drivers of BEVs adopt a number of strategies to cope with range limitations. One strategy is to avoid undertaking trips that utilise a significant fraction of a BEV's driving range (Carroll, 2010). More experienced BEV drivers were found to use decision heuristics to manage the range resources of their BEVs so as to avoid stranding, including undertaking a number of supplementary charging events to maintain an energy reserve in the battery (Franke et al., 2012). The same study also found that more experienced drivers did not find the limited driving range a constraining factor because BEVs met most of their daily mobility needs.

\subsubsection{Access to recharging facilities and duration of recharging}

EV owners have the option of being able to recharge the battery at home, but limited access to public recharging facilities has been shown to have an effect on 
the probability of an EV being chosen (Brownstone et al., 2000; Bunch et al., 1992; Melaina and Bremson, 2008).

The duration of recharging has also been found to adversely affect EV purchase behaviour (Egbue and Long, 2012; Cui et al., 2011). Using domestic standard voltage and current to recharge a depleted battery of 20 to $25 \mathrm{kWh}$ would require 6 to 7.5 hours. Lithium-ion graphite anode batteries can be fast charged in less than an hour. The LTO battery can be fast charged in under 5 minutes, but fast charging graphite anode lithium-ion batteries will shorten the cycle life (Buchmann, 2011, p 104). Fast charging involves the use of very high electric voltages and currents that cannot be provided by standard domestic electrical equipment (Thomas, 2009). The extensive use of such equipment in a domestic setting would not be compatible with electricity distribution systems in New Zealand (Duncan et al., 2010).

Deutch and Moniz (2010) concluded that the successful introduction of EVs into the United States LPV fleet would require a widely available and publicly accessible EV recharging system. They argued that for this infrastructure to be successfully developed a number of outstanding issues must first be addressed including: (1) the distribution and extent of the EV recharging system; (2) whether and how to standardise the technology; (3) the extent of control of the time of charging; (4) what regulatory procedures should be applied to the infrastructure providers and users; and (5) who will pay for its development - the public, corporations, or EV users.

Battery swapping has been proposed as an alternative to in-situ battery recharging. Battery recharging can then occur at a more convenient time when electricity prices are low and under conditions that are optimal for battery maintenance. This regime implies that battery ownership is separated from EV ownership and the battery is leased for the period of use. Battery swapping will require standardisation of batteries and the battery housing and connectors on the EVs (Brown et al., 2010). To allow for fast swapping, the battery must be easily accessible for unloading and loading and have a sufficiently comprehensive network to meet its clients' needs (Mak et al., 14 March 2012). As battery swapping is a leasing scheme, it reduces the 
costs of vehicle purchase by avoiding the capital cost of the battery (International Energy Agency, 2011).

\subsubsection{Safety}

Collision safety is a concern primarily for the smaller lightweight BEVs such as neighbourhood EVs or quadricycles such as the G-Wiz (Paine, 2011). Modern BEVs are now designed to meet collision safety standards, but they still carry the stigma associated with the earlier designs (Tsang et al., 2012).

The electrical safety of the batteries is also a concern due to the public's experience of overheating of the $\mathrm{LiCoO}_{2}$ used in information technology equipment. The lithium-ion batteries used in EVs are NCA, LFP and LMO batteries, which have a lower risk of catching fire (Buchmann, 2011, p 51). Battery fires in crash-tested Chevrolet Volt PHEVs resulted in significant adverse publicity in the media, which may have, for a short period of time, adversely affected the sales of these vehicles (Henry, 2011; Keane, 2012).

The low noise levels of EVs are seen as a positive feature by purchasers of EVs, but concerns have been expressed about the risk to pedestrians, especially pedestrians who are visually impaired (Williams, 2012; Holzman, 2011). It has been proposed that noise devices be added to HEVs and EVs to produce sound when they travel at low speeds (Kim et al., 2012).

\subsubsection{Lack of appreciation of the benefits of EVs}

A key benefit of EVs is their low fuel running costs, but research indicates that car owners often have a poor understanding of their fuel costs and put low value on future savings arising from improved fuel economy (Turrentine and Kurani, 2007). A review of the empirical estimates of the discount rate on future fuel savings is inconclusive, with estimates ranging from $<1 \%$ to $400 \%$ (Greene, 2010 ).

Research also indicates that car purchasers may value vehicle performance more than fuel economy (Burge et al., 2007). At present, EVs, with the exception of a few 
brands such as Tesla Motors' Roadster and Model S, have lower 0 to $100 \mathrm{~km} / \mathrm{h}$ rates of acceleration than comparable ICEVs.

\subsubsection{The attitude-action gap}

Research indicates that an individual's concerns about the environment often do not translate into environmentally-supportive behaviours (Kennedy et al., 2009; Mairesse et al., 2012). Similar behaviour has been demonstrated by car purchasers where the level of consumer concern about traffic, air pollution, and climate change is not supported by actual car purchase decisions. This research found that car purchasers used a vehicle's fuel consumption figures as a proxy for both environmental impacts and vehicle costs even when they did not have a clear understanding of the relationship between fuel consumption and GHG emissions (Lane and Potter, 2007).

\subsubsection{System "lock-in" and resistance from the industry}

The car system represents a socio-technical regime that is strongly resistant to change and demonstrates strong "lock-in" characteristics (Schot et al., 1994; Dennis and Urry, 2009, pp. 57-59; Unruh, 2000). The strength of the "lock-in" of the diesel and petrol combustion engine technology is demonstrated by the previous failed attempts to introduce EVs as an alternative in the 1970s (Cowan and Hulten, 1996) and in California in the 1990s as part of the CARB mandate (Hård and Knie, 2001).

Wells and Nieuwenhuis (2012) argue that the profound regime stability demonstrated by the current system of automotive mobility is, in part, due to the dominance of vehicle manufacturers. They identify the current industry business model as a combination of product technology, process technology, and organisational design that defines the mass production process. This model favours the large incumbent organisations and an industry that has large barriers to entry, which often has the effect of preventing radical change. The vehicle manufacturing industry also ensures that there is continuous improvement of existing ICEV technology, reducing the urgency for change to alternative technologies by the development of high efficiency ICEVs. They also can internalise the threat of new 
technologies through corporate capture, for example, the investment in Tesla Motors by Toyota Motors. The presence of institutional isomorphism in the industry reinforces set ways of doing business that favour the existing technology. Finally, the industry uses advertising and the media to reinforce the cultural status of the car along with the associated expectations of performance associated with current car technology.

Sovacool and Hirsh (2009) have also highlighted that industries often use political lobbying to prevent or slow the introduction of regulatory measures that will stimulate the need for technological change. With the exception of Honda and General Motors, the American vehicle manufacturing industry lobbied against California's CARB Mandate. At the same time, a consortium of oil producers made political contributions to candidates for the Californian legislature with the intention of weakening the push to introduce the measures. In addition, both vehicle manufacturers and petroleum companies commissioned reports that argued there were no economic and environmental benefits from introducing EVs into the Californian market. Calef and Goble (2007) state that both of these reports contained errors that undermined the case for EVs.

\subsection{EVs and the electricity grid}

\subsubsection{Introduction}

One of the advantages of EVs is that these vehicles will not require the construction of a completely new refuelling network. However, they will require the existing electricity grid to be expanded. The electricity grid is already pervasive, which means that for many owners they will have the option of refuelling at home. The electricity grid has evolved to meet the demands of its current users and how EVs will ultimately interface with the grid still remains uncertain (Putrus, 2009; Duncan et al., 2010).

\subsubsection{EV chargers and charging regimes}

EV chargers convert alternating current (AC) to direct current (DC) by the use of rectifying and smoothing circuits. EV chargers for lithium-ion batteries must 
carefully regulate peak voltage to prevent battery damage and provide for cut-off once they are fully charged. EV chargers are categorised by the level of the current and voltage that they deliver (Morrow et al., 2008). A level 1 EV charger operates at the United States standard domestic and commercial voltage of $120 \mathrm{~V}$ AC and $15 \mathrm{~A}$ or $20 \mathrm{~A}$. A level $2 \mathrm{EV}$ charger is the preferred primary charging level and delivers $240 \mathrm{~V}$ AC at $15 \mathrm{~A}$ to $40 \mathrm{~A}$. This charger level is consistent with the standard New Zealand domestic voltage subject to some minor household rewiring (Duncan et al., 2010). A level $3 \mathrm{EV}$ charger is a fast charging, off-board (separate from the EV) unit that can typically deliver voltages of $480 \mathrm{~V} \mathrm{AC}$ on a three phase circuit and up to 63 A. To qualify as level 3 an EV charger must be able to achieve at least $50 \%$ battery charge in 10 to 15 minutes (Morrow et al., 2008).

Weiller (2011) identifies six charging regimes based on the: (1) requirements of the EV user; (2) degree of control by the system operator; and (3) capabilities of the charging system. One of the capabilities of the charging system is the level of 'smartness', i.e. the level of control by the system operator of the EV charging activity and whether the flow of electricity between the EV battery and the grid is unidirectional or bidirectional (Lopes, 2009). Using these characteristics, EV charging regimes can be categorised as:

1. Uninterruptible charging: EVs can charge whenever they are within reach of an outlet and the charging occurs immediately upon plugging-in and until fully charged.

2. Time delay charging: The EV owner minimises charging costs by choosing to delay the start of charging until late in the evening.

3. Off-peak charging: Similar to option (2), but in this case it is the system operator, through the use of smart meters with two way communication capability and dedicated electrical circuit for the EV charger who controls the time of charging, and not the EV user.

4. Vehicle to home (V2H) and vehicle-to-grid (V2G): V2H and V2G charging allow for reverse power flow from EVs. In V2H, this energy can be used in the house or business where an EV is plugged in. With V2G charging this concept is expanded, with the grid system operator having remote control 
of the power flow, and the energy stored in an EV's battery can be used to assist in reducing losses in transmission and distribution grids (Weiller, 2011; Kempton and Tomi'c, 2005b; Kempton and Tomi'c, 2005a).

\subsubsection{Effect on peak loads}

Studies that have assessed the effect of the introduction of EVs on electricity generation, transmission, and distribution systems have concluded that additional generation capacity will have to be constructed, but that this demand will be manageable. The critical issue for electricity system operators will be the impact of any uncontrolled charging of EVs during periods of peak loads (Hadley and Tsvetkova, 2009; Kintner-Meyer et al., 2007b; Jenkins et al., 2008; Letendre and Watts, 2009; Kiviluoma and Meibom, 2011; Koyanagi and Uriu, 1997; Perujo and Cuiuffo, 2010; Putrus, 2009). A study undertaken in New Zealand reached similar conclusions. Duncan et al. (2010) estimated that even with a high uptake of EVs, the additional demand would comprise only $8 \%$ of the projected total annual demand in 2039. They also concluded that even modest uncontrolled charging by EVs during a period of very high demand would have adverse impacts on New Zealand's electricity transmission and distribution systems.

Early studies of actual charging behaviour of users of PHEVs in the United States underlined the concerns about uncontrolled EV charging. These studies indicated that unless incentivised, or constrained, the greatest demand from EV charging will occur during a week day between 5 and 10 pm and will peak around 8 pm (Smart et al., 2010; Morrow et al., 2008). Smart et al. (2010) found that up to $80 \%$ of PHEVs studied were plugged in during this period, although not all were charging at that time. However, a more recent study of the charging behaviour of over 3,000 Nissan LEAF and General Motors Volt EVs using the EVSE charging network found that the electricity demand varied depending on the locality. For example, in San Francisco the owners of EVs delayed their charging until after midnight, but in Nashville there was no delay and the demand increased steadily from $4 \mathrm{pm}$ to $8 \mathrm{pm}$. The authors credit the delay in charging in San Francisco to the electric utility offering an experimental time-of-use tariff for low tailpipe emission vehicles. An unintended 
consequence of the delay in charging is that at midnight there was a sudden spike in demand. With a greater number of EVs, this type of spike could possibly present a problem for low voltage distribution networks (Schey et al., 2012).

The New Zealand modelling undertaken by Duncan et al. (2010) found that transmission and distribution operators will face different costs and benefits depending on the capacity to shift EV charging from peak to off-peak periods. They found that, based on projections of 1.8 million EVs in 2039 in New Zealand, if 85\% of charging occurred off-peak, or during the peak demand shoulder periods, and no EV charging occurred during the highest annual peak demand events, there would be a $19 \%$ saving in additional generation system costs over the 30 year modelling period when compared to a scenario where there is a uniform EV charging regime.

\subsubsection{V2G and V2H capability}

The use of EV batteries as a means of developing distributed storage within electricity grids was first proposed by Kempton and Letendre (1997). They noted that, as LPVs sit idle most of the day, batteries in EVs are available for use by system operators for spinning reserve, voltage, and frequency regulation. V2G is achieved by installing communication and control technology that the system operator can use to control the battery charger and the direction of the flow of electricity. Communication can be by radio signal, a cell phone network, direct Internet connection, or by power line carrier (Kempton and Tomi'c, 2005a). These studies indicated that there are economic benefits for system operators to have a V2G capability within their networks.

The present study does not take account of the effect on car buying behaviour of the option to be able to sell grid support services, but modelling by Kempton \& Tomi'c found that, in the United States electricity markets, the revenue stream to owners for the provision of both spinning reserve and regulation was positive, and that this revenue stream may act as an incentive to buy a plug-in vehicle (Kempton and Tomi'c, 2005a; Tomi'c and Kempton, 2007). However, the benefits to EV owners of providing V2G services must take into account the costs associated with any increase in the rate of degradation of the battery due to the extra cycling 
during V2G (Peterson et al., 2010a) and the effect that providing V2G has on the availability of the EV for use by the owner. Peterson et al. (2010b) found that degradation costs are sensitive to the type of lithium-ion battery and that, for more modern lithium-ion batteries with a longer cycle life, the degree of degradation during the V2G regime is not significant. Recent work by Han and Han (2013), which also used longer life lithium-ion batteries, supports this conclusion. Han \& Han found that, in the context of the north-eastern United States transmission system, taking into account battery wear, the revenue to an owner of a PHEV from the provision of grid regulation services would, over the life of the battery, pay for the cost of the battery.

However, studies undertaken in Germany and Sweden indicated that the potential payments from providing ancillary services to electricity system regulation markets would be insufficient to encourage owners to participate in these markets (Andersson et al., 2010; Schill, 2011). Peterson et al. (2010a) concluded that the ancillary services market in California would quickly be saturated by EVs. They estimated that it would require less than $200,000 \mathrm{EV}$ s to provide spinning reserve capability and another 200,000 EVs for system regulation. As of 2011 , the Californian LPV fleet consisted of over 22 million LPVs (Department of Motor Vehicles, 2011). They concluded that most EV owners in California would only have the option of selling into the energy market and that the prices offered in the Californian energy market would be insufficient to offset the costs to the owner from the provision of the V2G service.

Operators of electricity systems are likely to sign contracts with EV owners that specify the minimum time the vehicle must be plugged in and available for use for V2G. These contracts will also specify the amount of battery capacity that can be utilised by the system operator. The system operator will seek to minimise the payment to the EV owner while maximising the time that the vehicle is plugged in and available and the amount of battery capacity that they can use for V2G. From the EV owner's perspective they will seek to maximise the amount of revenue received while minimising the time that the EV must be plugged in and the level of battery depletion. 
In a study by Parsons et al. (2011), a stated choice experiment of car owners was used to determine the level of revenue required to compensate for the inconvenience of making their EV available for V2G. They found that car owners strongly discount the revenue from V2G payments with increasing inconvenience. The authors concluded that, given the level of availability likely to be required by system operators and the current value of $\mathrm{V} 2 \mathrm{G}$ regulatory services, the $\mathrm{V} 2 \mathrm{G}$ payments would be insufficient to get EV owners to sign contracts.

$\mathrm{V} 2 \mathrm{H}$ is an alternative to $\mathrm{V} 2 \mathrm{G}$ where the power is not injected back into the distribution grid, but is used in the home or building where the EV is plugged in. This option still has benefits to the electricity grid if the system operator can shed the household load during periods of high demand. $\mathrm{V} 2 \mathrm{H}$ also provides the house or building occupiers with a backup power supply during blackouts (Tuttle and Baldick, 2012). Both V2H and V2G systems have the potential of being linked to distributed generation. The distributed generation can then be used to charge EVs and to store surplus generation for later use in the home or the grid. This concept is currently being trialled as part of the smart grid scheme on Bornholm Island in Denmark (Jørgensen et al., 2011).

\subsection{Summary comments}

At this time, it is uncertain which type, or combination of types, of LPV technology will emerge as the preferred response to reducing road transport's GHG emissions. The extent of the reduction of GHG emissions will depend, in large part, on the processes used to produce the fuels for these AFVs.

The uptake of AFV technologies is currently constrained by their limited availability, their higher costs and lower performance when compared to equivalent ICEVs, and the need to develop refuelling networks.

EVs have a number of features that make them likely to be one of the preferred AFV technologies. They have high fuel efficiency, which means that, on a per kilometre basis, less fuel will be required to be produced to meet demand. When used in conjunction with electricity generated from renewable fuels or low carbon 
fuels, they have the greatest potential to reduce GHG emissions. While the widespread use of EVs would require the development of specialised refuelling infrastructures, they can utilise the existing electricity grid as a transitional measure. Market announcements also indicate that there will be an increasing range of both BEVs and PHEVs available for sale in global and New Zealand markets.

The emerging EV technology represents a significant risk to the electricity grid if there is uncontrolled charging. Alternatively, through the implementation of $\mathrm{V} 2 \mathrm{H}$ or V2G systems, EVs present a significant opportunity for maximising the potential of existing electricity grids. While the capabilities of EV charging technology will be important, a critical issue will be how the operators of electricity grids interact with the owners and operators of EVs. 


\section{Chapter 5: Methods I: vehicle fleet model}

\subsection{Introduction}

This chapter sets out the methodological choices involved in the modelling undertaken in this study, and the process used to develop the vehicle fleet model (VFM). The VFM was used to simulate over the modelling period 2010 to 2030: (1) the changing composition of the New Zealand light passenger vehicle (LPV) fleet; (2) the amount of travel undertaken by four types of LPV; (3) the amount of energy used by internal combustion engine vehicles (ICEVs) and electric vehicles (EVs); and (4) the greenhouse gas (GHG) emissions produced by the LPV fleet.

The VFM comprises four major components:

1. The VFM scenarios, which set out the assumptions about future economic and population growth, the rate of development of LPV technologies, and the changes in the prices of EVs, fuels and carbon.

2. The vehicle choice sub-model, which contains the mixed multinomial logit model (MMNL).

3. The car ownership sub-model.

4. The vehicle use sub-model (VUM), and energy demand and GHG emissions sub-models ${ }^{31}$.

The car ownership sub-model comprises three elements: (1) the aggregate time series forecast sub-model (Dargay et al., 2007; Conder, 2009); (2) a vehicle stock database; and (3) a scrapping sub-model (De Jong et al., 2004). Figure 5.1 provides an overview of the structure of the VFM.

The VFM operates, in each modelling period, by first removing those vehicles scheduled for scrapping from the stock of LPVs. The revised size of the LPV fleet is

\footnotetext{
${ }^{31}$ The VFM is a spread sheet model using the Excel software. Excel has been used to develop vehicle fleet models by Bandivadekar et al. (2008) and the Ministry of Transport (1998). Models of vehicle fleets are often constructed using commercial software packages. The TREMOVE model uses the GAMS (de Ceuster et al., 2007) software package, the ASTRA model (Schade \& Krail, 2006), and the IPTS transport technologies model (Christidis et al., 2003), use VENSIM software package, and the UNITEC's UniSyD model uses the STELLA software package (Leaver \& Gillingham, 2010).
} 
then compared to the forecast demand for car ownership for that year and the difference between the two estimates represents the number of LPVs required to enter the LPV fleet to meet the forecast level of car ownership. These vehicles are then allocated across the different vehicle types in the vehicle stock database using the vehicle choice model. Lastly, the vehicle stock database is updated ready for the next modelling period.

The MMNL model in the vehicle choice model forms the centrepiece of the VFM and the present study. Section 5.2 contains a discussion of the design and implementation of the nationwide stated choice survey of New Zealand car buyers used to obtain the data used to estimate this model.

In section 5.3, there is a discussion of the design and development of the VFM. That section describes how the vehicle choice sub-model was incorporated into the VFM and the methods used to model vehicle scrapping, travel, and energy use.

In section 5.4, there is a discussion of the VFM scenarios. That section concludes with a discussion of the sensitivity analysis used to assess the effects from changing some of the main input assumptions on the output of the VFM. 


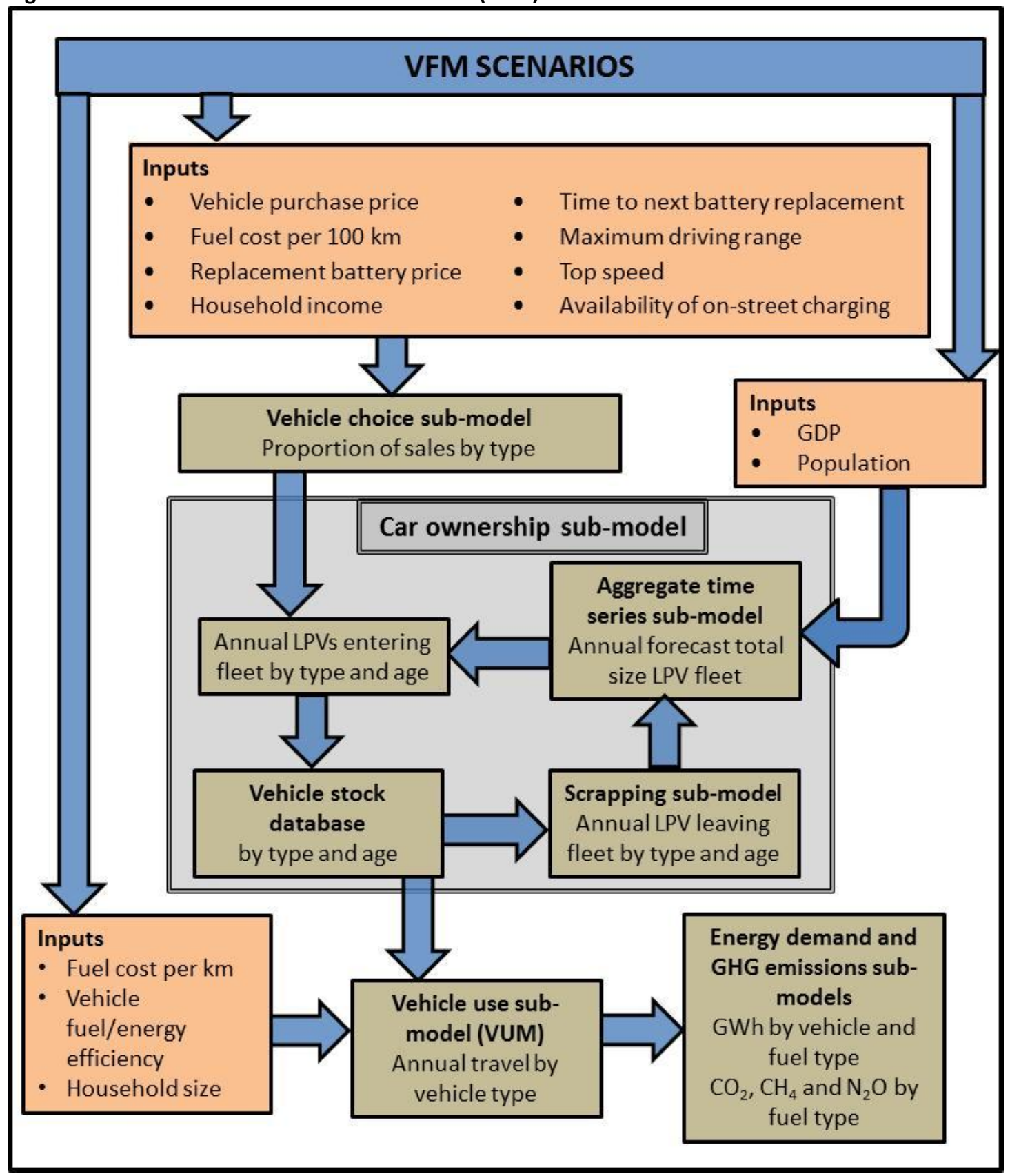

\subsection{Stated choice survey design and implementation}

\subsubsection{Objective of the stated choice survey}

The objective of the stated choice survey was to collect data on the preferences of New Zealand car buyers and to use this data to develop a MMNL model.

The MMNL model's function was to estimate the New Zealand demand for ICEVs and EVs. The design of the stated choice survey had to be a nationwide survey to 
reflect the entire New Zealand car buying population. The survey design also had to accommodate the effect on car buyers' choices of the:

- different characteristics of three generic types of EV

- option of choosing a used imported EV

- expected improvements in EV technology and reductions in the prices of EVs and EV batteries

- changing price of fuels (petroleum and electricity)

- emergence of EV charging facilities.

\subsubsection{Survey development process}

The development of the stated choice survey consisted of:

1. An initial reference group meeting.

2. First survey instrument and choice experiment design.

3. First pilot survey.

4. First revision of the survey and stated choice experiment design.

5. Development of the survey website.

6. Second pilot survey.

7. Second revision of the survey and stated choice experiment design.

8. Implementation of the main survey.

9. Follow-up contact to maximise response.

10. Data processing.

The survey process occurred during the period October 2009 to December 2010.

\section{Reference group}

A reference group meeting comprising seven New Zealand car owners residing in the Wellington region was held to discuss car purchases, EVs, and the issues facing car transport. Recruitment was by sending out invitations to a number of Wellington based email lists. The group comprised four males and three females aged from 35 to the mid-60s. Of the attendees, six had bachelor level degrees and one was a PhD student. Five attendees identified themselves as having a strong 
interest in environmental issues. Two of the male attendees expressed less concern about the environment and indicated that they were attending because they were interested in new vehicle technologies.

The meeting used an open discussion format, but was structured to gain information on the participants':

- last car purchase and the factors they considered when making that purchase

- knowledge of the characteristics of LPVs currently in the market (i.e. prices, fuel running costs, range, and performance)

- expectations of the future development of petrol and diesel LPVs

- knowledge and expectations of EV technology

- concerns about EV technology

- views about the risks from the continued use of petrol and diesel fuelled vehicles

- ranking of the factors identified earlier in the meeting that influence vehicle choice.

The participants also gave feedback on the draft of supporting material to be used in the first pilot survey

\section{First pilot survey}

This survey comprised a personally supervised pen and paper stated choice experiment, carried out with a pilot sample of 11 people, six of whom had previously attended the reference group. The experiment design was a fractional factorial design consisting of a random subset of the full factorial design. The design of this experiment consisted of 22 parameters, excluding constants, all with three attribute levels. The smallest viable design found with attribute level balance contained 30 choice tasks ${ }^{32}$. The order of the alternatives in the choice tasks was randomised to limit the possibility of order effects (Dillman et al., 2009b, p 128).

\footnotetext{
${ }^{32}$ In stated choice experiments the number of choice sets in the experimental design must be equal to or greater than the degree of freedom. The degree of freedom is the number of attributes in the
} 
The ChoiceMetrics NGENE software was used to design all the choice experiments in this study. Due to the small size of the sample, the experiment design was not blocked and the respondents were required to complete all 30 choice tasks.

The supervisor's role during the pilot survey was to elicit feedback from the respondents about the survey, and supporting material, and encourage them to complete all the choice tasks.

The respondents were also asked to complete a feedback form, which collected information about the survey and the attributes they took into account when making their decisions. This information was then used in the development of the second pilot survey.

\section{Second pilot survey}

The second pilot survey provided an opportunity to test the website to be used in the main survey. For this survey, the choice experiment was created using the efficient design method (Bliemer et al., 2011).

Feedback from the first pilot survey indicated that the attributes of "top speed for general purpose electric vehicles (GEVs)", and "EV recharging time" were not considered to be as important as other attributes. To simplify the choice experiment, these attributes were excluded from the design of this survey.

The prior values for the parameters used in the efficient design process were from the best performing multinomial logit ( $\mathbf{M N L}$ ) model estimated using the data from the first pilot survey. The D-error criterion was used to determine the best experiment design as this measure is considered the best when designing choice experiments intended to collect data for discrete choice models that will be used for estimating market shares (Kessels et al., 2006).

The best design was found to have 42 choice tasks and these were blocked into six blocks of seven choice tasks.

experiment, excluding the constants, plus one. To achieve attribute level balance the number of choice sets is some multiple of the number of attribute levels in the design (Bliemer et al., 2011). 
The intention from early in the design process of the VFM was to use a MMNL model for the vehicle choice sub-model (see section 3.4). Using a MMNL model as a design template will ensure the most efficient design (see section 3.3.3). However, this is often impracticable due to computational difficulty. An alternative approach is to use the less computationally intensive MNL model as the design template even though the intention is to use the data collected to estimate a MMNL model. Bliemer and Rose (2010) have shown that this substitution does not result in a significant loss of efficiency in the experimental design.

The second pilot survey was open to anyone interested in participating. An email invitation requesting participation, and feedback on the design of the website and survey instrument was widely distributed. The respondents were encouraged to pass the invitation on to anyone else who might be interested in participating.

The second pilot resulted in 55 completed survey responses and a number of suggestions for improvement of the website design.

\section{Main survey}

The candidates for the main survey were randomly selected from the $2007 \mathrm{New}$ Zealand electoral roll. The survey was conducted over the period 1 June 2010 to 13 October 2010, and 8,000 names and addresses were initially selected from the electoral roll. The names and addresses were then matched to telephone numbers using the White Pages directory website. This process resulted in a pool of 3,262 candidates.

The main survey design had the same specification as the second pilot survey, 42 choice tasks divided into six blocks of seven choice tasks. An example of one of the 42 choice tasks used in the main stated choice survey is provided in Appendix 1.

The "PHEV electric driving range" attribute was dropped from the main survey after the results from both pilot surveys indicated that if the electric driving range was increased PHEVs would be less likely to be chosen.

One reason for obtaining unexpected signs in RPL models is the use of an incorrect distribution form for the random parameter. This explanation could be discounted 
as the same result occurred in all the MNL and error components (ECMNL) models that were estimated.

A more likely reason is that the attribute was being excluded from the cognitive process (Hensher, 2006b). This behaviour seems possible as the first and second pilot surveys contained two driving range attributes for PHEVs: (1) the total driving range, fixed at $500 \mathrm{~km}$; and (2) electric only driving range, which varied between 20 and $100 \mathrm{~km}$. The unexpected sign for the electric only driving range attribute suggests that the respondents were ignoring it, possibly because they did not understand its meaning or because they were focused on the total driving range and fuel running cost attributes.

Feedback from the pilot surveys indicated that some respondents found some of the attribute level combinations unrealistic. Unrealistic, or infeasible, attribute level combinations are a feature of stated choice experiments and often these cannot be completely eliminated from the design. However, to reduce this concern, constraints were imposed on the experiment design that eliminated the occurrence of some of the more unrealistic combinations. Imposing constraints on the design of a choice experiment will result in a decrease in the efficiency of the design (Bliemer et al., 2011). In this case, the loss of design efficiency was considered to be an acceptable trade-off as it increased the realism of the choice tasks and the credibility of the respondents' answers.

The constraints imposed on the design ensured that the choice tasks did not contain a situation where the battery replacement cost for a new EV was greater than the purchase price and, for general purpose EVs, the highest level purchase price $(\$ 160,000)$ always occurred in conjunction with the highest battery replacement price $(\$ 130,000)$ and greatest driving range $(500 \mathrm{~km})$.

When estimating the most efficient design, two different MNL specifications were used as templates. The first model specification was based on the best performing MNL model from the second pilot. This model comprised a mixture of alternative specific and generic parameters. The second model specification comprised only 
generic parameters. The choice experiment design for the main survey was then estimated using the averaging approach suggested by Rose et al. (2009b).

The efficient experiment design process indicated that with a minimum sample size of 250 respondents it would be possible to develop a discrete choice model with statistically significant parameter estimates for all the attributes included in the stated choice experiment.

\subsubsection{Survey design elements}

\section{Survey population}

For this survey, the study population comprised private New Zealand car buyers. Most New Zealand adults will buy a car at some time in their life. The study population was limited to those people intending to buy a new or used car within the next five years for this project in order to make the decision making exercise more realistic for the respondent ${ }^{33}$.

A significant proportion of the new cars entering the LPV fleet are bought by private and public organisations (Kerr, 2009). Due to resource limitations, it was not possible to develop and implement a separate choice experiment for this group of car buyers. To model the car purchase behaviour of this group of buyers, it was assumed that the general car purchasing public's preferences could act as a proxy for these organisations. This was considered a justifiable assumption because these organisations have to onsell their vehicles to the public and they will seek to maximise the resale value. Therefore, the managers of business fleets will take into account the public's car buying preferences when purchasing vehicles.

\section{Choice set specification}

The specification of the choice set used in a choice experiment involves identifying and defining the alternatives for the relevant market segment so that they are consistent with the discrete choice framework. This specification requires that the alternatives be mutually exclusive, exhaustive, and finite in number.

\footnotetext{
${ }^{33}$ I am indebted to Professor Ric Scarpa for this design suggestion.
} 
The first decision made was only to include those EVs that meet vehicle safety standards in this study, which had the effect of excluding many neighbourhood and other types of low-speed $\mathrm{EV}^{34}$.

Reviewing press releases and manufacturers' websites resulted in the identification of three broad classes of EV.

1. City EV (CEV): CEVs are designed for urban travel. They are small and not intended for long distance travel, but can travel at highway speeds. They have capacity for two to four people, with two or four doors, and limited storage space sufficient for shopping. Driving range is sufficient for urban travel, but not for intercity travel.

2. General purpose EV (GEV): GEVs represent a broad class of EVs large enough to act as a family vehicle and with commensurate storage space. These EVs will come in a variety of body shapes (sedan, sport utility vehicle, or multipurpose vehicle). Technological limitations currently restrict their driving range. However, the expectation is that GEVs, with improvements in battery technology, will eventually be suitable for intercity travel and be able to compete directly in the market against petrol or diesel powered family light passenger vehicles (LPVs).

3. Plug-in hybrid EV (PHEV): PHEVs also represent a class of vehicles large enough to act as a family vehicle, and with commensurate storage space and range of vehicle body shapes. When using the internal combustion engine (ICE) a PHEV is suitable for intercity travel and has the same range of functions as an ICEV.

The design of the choice set takes into account that a significant proportion of the LPVs entering the New Zealand LPV fleet will be Japanese used imports (New Zealand Transport Agency, 2011). The expectation is that these imported vehicles will include used EVs and the choice set includes the option of choosing a used import for each type of EV.

\footnotetext{
${ }^{34}$ The United Stated States government identifies neighbourhood EVs as vehicles with a top speed of $50 \mathrm{~km} / \mathrm{h}$, and restricted to roads with posted speed limits less than $70 \mathrm{~km} / \mathrm{h}$.
} 
The fourth type of vehicle included in the stated choice experiment was the ICEV. This vehicle type represents all non-EVs and includes the vehicles that use petrol, diesel, or biofuels. This category also includes hybrid electric vehicles (HEVs) such as the Toyota Prius or Honda Insight, which are not EVs as they ultimately rely only on petroleum for power.

Car buyers have the option of buying, in addition to a new or used imported ICEV, a used ICEV that is already present in the New Zealand LPV fleet. Feedback from the Ministry of Transport, the New Zealand Automobile Association, and the reference group indicated that most New Zealand cars buyers are unconcerned about the pedigree of a used LPV and do not distinguish between a used car already in the LPV fleet and a used vehicle that is entering the LPV fleet for the first time. Based on this feedback, the two types of used ICEV were merged. This specification had the advantage of reducing the choice burden for the respondent (Hensher, 2006a).

The decision was made to combine new and both types of used ICEVs into one ICEV alternative to provide an opt-out alternative that represents the status quo LPV purchase choice. This specification had the potential to reduce the potential for hypothetical bias, but did have implications for when the model was applied to organisational car buyers (see section 5.3.1).

\section{Specification of the attributes}

The selection of the attributes was based on a review of press releases, the discrete choice literature (Bunch et al., 1992; Potoglou and Kanaroglou, 2006), and the feedback received from the reference group and the first and second pilot surveys.

All attributes in the choice tasks were specified using three levels as this is the smallest number of levels that allows for the estimation, with a change in the value of the attribute, of a non-linear relationship in terms of utility (Hensher et al., 2005, pp. 107-108).

\section{Driving range}

The respondents were given information about the driving range of CEVs and GEVs (Table 5.1). The supporting information provided in the survey informed 
respondents that actual driving ranges, in real world situations, are dependent on driving conditions and would likely be less than the values in the stated choice experiment.

At present, most GEVs have driving ranges of around 150 to $200 \mathrm{~km}^{35}$, but there is an expectation among many car drivers that EVs should have longer driving ranges (Giffi et al., 2011) ${ }^{36}$. The attribute levels in the experimental design were specified to accommodate the driving ranges of GEVs in some of the VFM scenarios eventually matching those of ICEVs. This development would result in significantly larger and more expensive batteries (Kromer and Heywood, 2007; Kromer and Heywood, 2009).

The respondents were informed that ICEVs and PHEVs all have maximum driving ranges of at least $500 \mathrm{~km}$.

Table 5.1: City and general purpose electric vehicle driving ranges (km)

\begin{tabular}{|l|c|c|c|}
\hline Vehicle type & Minimum value & Median value & Maximum value \\
\hline New and used CEV & 100 & 150 & 200 \\
\hline New and used GEV & 150 & 325 & 500 \\
\hline
\end{tabular}

\section{Purchase price}

The purchase price of an EV includes the price of its battery. The price of a battery is a function of the battery cell price per kWh, battery fabrication costs, and the battery's energy capacity. The energy capacity of the battery is in turn a function of the vehicle's driving range and energy efficiency. Section 5.4.3 contains a discussion of assumptions used to set the battery prices for this study.

GEVs have the widest range of purchase price attributes because of the effect of the increased driving range assumption and the impact this has on battery costs (Table 5.2).

\footnotetext{
${ }^{35}$ The exception is the prestige Tesla Model S EV, which with the $85 \mathrm{kWh}$ battery option has an EPA certified driving range of $425 \mathrm{~km}$.

${ }^{36}$ The level of expectation varies across countries. In France and the United States, current EV driving ranges would only meet the expectations of $16 \%$ and $25 \%$ of car drivers respectively. In Brazil and Taiwan, these levels increase, respectively, to $42 \%$ and $44 \%$ (Giffi et al., 2011).
} 
Table 5.2: EV purchase prices

\begin{tabular}{|l|c|c|c|}
\hline Vehicle type & Minimum value & Median value & Maximum value \\
\hline New CEV & $\$ 20,000$ & $\$ 45,000$ & $\$ 70,000$ \\
\hline Used CEV & $\$ 5,000$ & $\$ 22,500$ & $\$ 40,000$ \\
\hline New GEV & $\$ 30,000$ & $\$ 95,000$ & $\$ 160,000$ \\
\hline Used GEV & $\$ 7,500$ & $\$ 43,750$ & $\$ 80,000$ \\
\hline New PHEV & $\$ 20,000$ & $\$ 60,000$ & $\$ 100,000$ \\
\hline Used PHEV & $\$ 10,000$ & $\$ 30,000$ & $\$ 50,000$ \\
\hline
\end{tabular}

No values for purchase price were provided to the survey respondents for ICEVs because this alternative comprised all ICEV models and vehicle types and represented a very wide range of prices. The approach used in this stated choice experiment was to inform the respondent that the prices for ICEVs encompassed the range of values currently in the market. The respondent was then asked to make a comparison between the price that they would expect to pay for an ICEV and the purchase prices given for the EV alternatives.

\section{Fuel costs}

Fuel costs were specified in the stated choice experiment in terms of $\$$ per $100 \mathrm{~km}$, rather than cents per kilometre (Table 5.3). The reference group suggested this specification was more understandable and this was confirmed by feedback from the first pilot survey.

This was the only attribute provided for the ICEV alternative in the stated choice experiment. Varying the value of this attribute for ICEVs allowed evaluation of the effects of changes in the running costs of these vehicles on vehicle choice.

Attribute levels were derived using the petroleum and electricity prices, and vehicle energy efficiency assumptions from the VFM scenarios. The maximum attribute values for ICEVs and PHEVs accommodated the possibility of crude oil reaching US\$300 per barrel. 
Table 5.3: Fuel costs $(\$ / 100 \mathrm{~km})$

\begin{tabular}{|l|c|c|c|}
\hline Vehicle type & Minimum value & Median value & Maximum value \\
\hline New and used ICEV & 6 & 33 & 60 \\
\hline New and used CEV & 1 & 4 & 7 \\
\hline New and used GEV & 2 & 9 & 16 \\
\hline New and used PHEV & 2 & 20 & 38 \\
\hline
\end{tabular}

\section{Cost of a replacement battery}

This attribute was included based on feedback from the reference group, which expressed concern about the cost of replacing the battery at the end of its useful life. The attribute levels were derived using the projected range of values of energy consumption, battery manufacturing costs, and expected driving ranges for the period 2010 to 2030 (Table 5.4).

Table 5.4: Cost of replacement battery

\begin{tabular}{|l|c|c|c|}
\hline Vehicle type & Minimum value & Median value & Maximum value \\
\hline CEV & $\$ 4,000$ & $\$ 17,000$ & $\$ 30,000$ \\
\hline GEV & $\$ 10,000$ & $\$ 70,000$ & $\$ 130,000$ \\
\hline PHEV & $\$ 2,500$ & $\$ 8,750$ & $\$ 15,000$ \\
\hline
\end{tabular}

Time to battery replacement

The expected life of a battery depends on how much driving is undertaken and the amount of fast charging undertaken. The assumption used in the choice experiment was that for the present generation of battery technologies, the expected minimum battery life is five years. This assumption is consistent with the eight years, or $100,000 \mathrm{~km}$, warranty provided by the Nissan Motor Company with the battery in the Nissan Leaf. In the United States the average annual travel is $19,000 \mathrm{~km}$ per year, and a vehicle can be expected to travel 100,000 km in five years (Automotive Management Online, 2011).

The minimum attribute level for time to battery replacement for used imports allowed for the possibility that an EV could be imported with its battery already at the end of its useful life and thus require immediate replacement. The maximum 
attribute level for both new and used EVs was set at a level that equated to a battery that can last the life of the EV (Table 5.5).

Table 5.5: Time to battery replacement (years)

\begin{tabular}{|l|c|c|c|}
\hline Vehicle type & Minimum value & Median value & Maximum value \\
\hline New EV & 5 & 12.5 & 20 \\
\hline Used EV & 0 & 10 & 20 \\
\hline
\end{tabular}

\section{Availability of on-street EV charging facilities}

The reference group highlighted that many urban residents may not have access to EV recharging facilities at home. Many urban car owners live in apartments, or flats, without garaging and have to park on the street. The reference group suggested that unless they could recharge while parked on the street near their homes, they would not be interested in buying an EV.

This attribute represented the degree of availability in New Zealand cities and towns of street charging points or posts (Table 5.6). This was the only qualitative and non-continuous attribute in the choice experiment. The choice experiment's supporting material provided an explanation of EV charging posts and the meaning of attribute levels (Appendix 3).

Table 5.6: Availability of on-street charging facilities

\begin{tabular}{|l|r|}
\hline Attribute & Definition \\
\hline None & On-street recharging not available \\
\hline Town centre & $\begin{array}{c}\text { Available in most, if not all, town centres } \\
\text { (i.e. main streets and shopping areas) }\end{array}$ \\
\hline Town centres and inner suburbs & $\begin{array}{r}\text { Available in most, if not all, town centres } \\
\text { and surrounding suburbs. }\end{array}$ \\
\hline
\end{tabular}

\section{Top speed}

This attribute recognised that some CEVs may not be intended for use on open highways and may have limited top speeds. This attribute also acted as a proxy for buyers' preferences with respect to vehicle power (Table 5.7). 
Table 5.7: City electric vehicle top speed

\begin{tabular}{|l|c|c|c|}
\hline Vehicle type & $\begin{array}{c}\text { Minimum value } \\
(\mathrm{km} / \mathrm{h})\end{array}$ & $\begin{array}{c}\text { Median value } \\
(\mathrm{km} / \mathrm{h})\end{array}$ & $\begin{array}{c}\text { Maximum value } \\
(\mathrm{km} / \mathrm{h})\end{array}$ \\
\hline CEV & 70 & 105 & 140 \\
\hline
\end{tabular}

\section{Supporting questions}

The survey included questions to gain information about the respondents':

- socio-demographics (age, gender, income, and locality)

- ability to recharge at home

- car buying history

- views on the impact of climate change, future cost of oil, and government role in reducing GHG emissions from road transport.

A question was also included in the survey to determine whether the respondents were undertaking the survey jointly or on their own. Findings of other choice experiments have found that the preferences expressed by the group are different from those of the individuals in the group. Research also shows that the decision to buy a car is often undertaken by more than one person in the household (Hensher et al., 2008; Hensher et al., 2011).

In an attempt to make the stated choice experiment as realistic as possible, the survey respondents were asked to do the choice experiment jointly if they would normally buy a car with someone else. However, if this was not possible, they were then asked to try and take the other person's or persons' preferences into account when completing the choice experiment. Another question was included to assess the extent to which the respondents complied with this request.

The supporting questions on car buying history, home charging, climate change, cost of oil, and government role were included for use as possible covariates in the discrete choice model. The socio-demographic questions were also included as possible covariates, but were principally included to determine the representativeness of the survey sample.

Appendix 2 contains a copy of the supporting question sheet used in the main survey. 


\section{Supporting information}

The reference group indicated a limited knowledge of EVs and their characteristics. The lack of awareness was particularly noticeable for PHEVs, with most attendees expressing no knowledge about this technology.

For the first pilot survey, a background information sheet on EVs was included. This sheet covered the capabilities of the EV technologies and summary of the possible effects arising from their introduction. Following feedback from the first pilot survey, an expanded version of the information sheet was provided for the second pilot survey. In both pilot surveys, feedback was sought asking whether the information provided was understandable and unbiased. For the main survey, there was only a small amendment to the information sheet. Appendix 3 contains a copy of the supporting information used for the main survey.

\section{Cheap talk reminder}

During the first pilot survey, testing of a 'cheap talk' script found that the respondents were not reading the long script that had been prepared, indicating that unless there was an interviewer present to prompt the respondent, the script would be ignored.

For this survey, a short explanation in the survey instructions was prepared stating:

The main part of the questionnaire takes the form of a series of choice situations (games) where you are asked to make a choice between different types of vehicles taking into account changing vehicle and market characteristics. You are asked to treat these choices like a real vehicle purchase decision; taking into account all the things you normally do when making such an important decision. 
Following this brief explanation, there was a series of short reminders repeated in each choice task in the internet survey, and at the top of each page in the postal survey. This short reminder consisted of the following:

Treat each choice as realistically as possible.

Only make the choice that you think you would make in real life.

\subsubsection{The main survey recruitment process}

Phone recruitment is a recommended approach for use in stated choice surveys (Champ and Welsh, 2006). Phone calls were made during weekday evenings and Sunday afternoons from June to September 2010.

The phone call consisted of an invitation to participate in the survey, subject to the potential respondent meeting the survey inclusion criterion. A potential respondent who expressed an interest in participating was asked:

Are you likely to be buying a new or used car within the next five years?

If they answered "yes", they were given the option of answering the survey by either the internet or by post.

The use of both mail and web survey instruments was in order to maximise the response rate and gain national coverage at a reasonable cost. Using more than one survey instrument has been shown to increase the survey response rate (Dillman et al., 2009b, p. 304) and the provision of the option of a postal survey addressed the risk of not reaching those sections of the community who do not have access to the internet (Dillman et al., 2009a).

Using the randomised pool of 3,262 survey candidates, a total of 2,649 calls were made of which there was no answer to 817. These names were saved in case of the eventuality that the minimum target sample size was not achieved and further calls were required. Section 7.2.1 discusses the survey response rates.

A respondent's selection of the internet option resulted in their receiving the introductory survey information, web address, and personal log-on code by email. 
However, some respondents did not wish to give their email addresses and these people were posted this information.

A follow-up call was made if there was no response after three weeks. The intention was to undertake a second follow-up reminder if required, but this was not done as feedback indicated that another follow-up call could result in alienating the respondents.

\subsection{The vehicle fleet model}

\subsubsection{Vehicle choice sub-model}

The specification of all MNL, ECMNL, and RPL models had the utility functions defined as linear in parameters. Both RPL and ECMNL forms of the MMNL model were estimated using cross-sectional and panel data and alternative specific and generic parameters specifications. Model estimation was undertaken using the NLOGIT 4 software.

The vehicle choice sub-model in the VFM incorporated the best performing of the MMNL models. This sub-model acted as a choice simulator to estimate the market shares of the vehicle alternatives based on the attribute values from the VFM scenarios.

Excel does not have the capability to perform Halton draws for estimating the simulated probabilities (see section 3.2.5) so random draws were used instead. To offset the poorer performance of this form of randomisation, the number of draws was increased from 200 to 5,000.

Even with a large number of random draws, the output from the VFM simulator for each VFM scenario varied slightly across repeated model runs. Therefore, for each VFM scenario, the choice simulator was run 10 times and the mean and variances of the output calculated. Section 5.4 contains a description of the VFM scenarios.

The performance of the choice simulator in the VFM was tested by comparing its output to that of the choice simulator in the NLOGIT software. The simulator was tested using a range of input values with the full choice set and a constrained 
choice set. Using the two-sample t-test it was found that the output from the two simulators was not statistically different at the $5 \%$ level $^{37}$.

The choice simulator, as applied in the VFM, is not used to directly model the choice behaviour of New Zealand car buyers, but that of New Zealand new and used vehicle importers. In New Zealand, with the exception of a small number of direct imports by individuals, vehicle importers determine what new and used LPVs will enter the country in response to demand from car buyers. The New Zealand branches, or local franchises, of the international vehicle manufacturers undertake the importation of new cars, and independent vehicle dealers, vehicle importers, and wholesalers are responsible for the importation of most of the used cars that enter New Zealand.

For this study, the assumption was that the decision making processes used by the vehicle importing industry reflected the preferences of New Zealand car buyers. It is, therefore, valid to use a discrete choice model estimated with data from a survey of New Zealand's car buyers to model the choice behaviour of vehicle importers.

Experience with HEVs indicates that vehicle manufacturers are cautious when introducing new technologies into the market. Therefore, it is likely that the introduction of EVs will occur in a similar manner starting with a limited range of models that are available in limited quantities and in a limited number of markets. Other studies that have assessed the uptake of EVs in New Zealand have attempted to take account of the effect of limited availability based on information provided by industry experts (Electricity Commission, 2010; Baxter et al., 2009; Duncan et al., 2010). In the present study, the focus has been on the effect of car buyers' preferences on the uptake of EVs in New Zealand. It was assumed that from the date of first introduction, in this study 2012, New Zealand car buyers' purchase behaviour would not be constrained by the availability of new EVs, but would be able to select from a full range of EV makes and models.

\footnotetext{
${ }^{37}$ The results of the choice simulator verification test are presented in Appendix 4
} 
The output of the VFM choice simulator was modified to take account of two features of the New Zealand vehicle market. The first modification addressed the fact that most new cars entering the LPV fleet are bought by business and government organisations and that these car buyers are only interested in new vehicles (Kerr, 2009). The second modification was to account for the importation of used EVs from Japan, which would initially only be available in limited quantities.

As discussed in section 5.2.3, the decision was made to combine the new and used ICEV alternatives into a single opt-out alternative so as to simplify the survey design and reduce the likelihood of hypothetical bias. This specification was appropriate for private car buyers who buy both new and used ICEVs, but for organisational buyers, who are only interested in new ICEVs, this model specification would not accurately estimate market shares.

Despite these limitations, applying the discrete choice model to the organisational car buyers' market using a reduced choice set was considered an acceptable provisional measure for the purposes of the present study, but with the caveat that a separate model should be developed.

Japan supplies approximately $95 \%$ of all the used cars that enter the New Zealand LPV fleet (New Zealand Transport Agency, 2011). The availability of used Japanese vehicles in New Zealand is largely a function of the effect of the Japanese vehicle automobile inspection registration system (the Shā-ken test) on Japanese car owners' selling behaviour. In Japan when a vehicle turns 3 years old, it must get an inspection and thereafter be inspected every two years. As a vehicle ages, the expense of the Shā-ken test increases and most Japanese car owners sell their vehicles because of the increasing expense, not the result of a failed test. As a consequence most Japanese used vehicles that enter the New Zealand market are three years or older (Philpot and Shaw, 2006) with the seven to nine year age cohorts forming approximately half of the supply of used petrol imports and $70 \%$ of used diesel imports (Colegrave and Denne, 2006). The expectation is that in future the Shā-ken test will also apply to EVs and determine the age and availability of used EVs entering New Zealand. 
For this study, the assumption was that new EVs would become widely available in the Japanese market at the same time as in New Zealand and as the age of the Japanese EV fleet increased there would be a growing number of used EVs available for export to other countries. This increasing availability of used EVs was simulated in this study by gradually increasing the supply of used EVs based on the average cumulative age profile of used vehicles that entered New Zealand from 2001 to 2009 (Ministry of Transport, 2012b).

Figure 5.2: Cumulative availability profile of used imported EVs

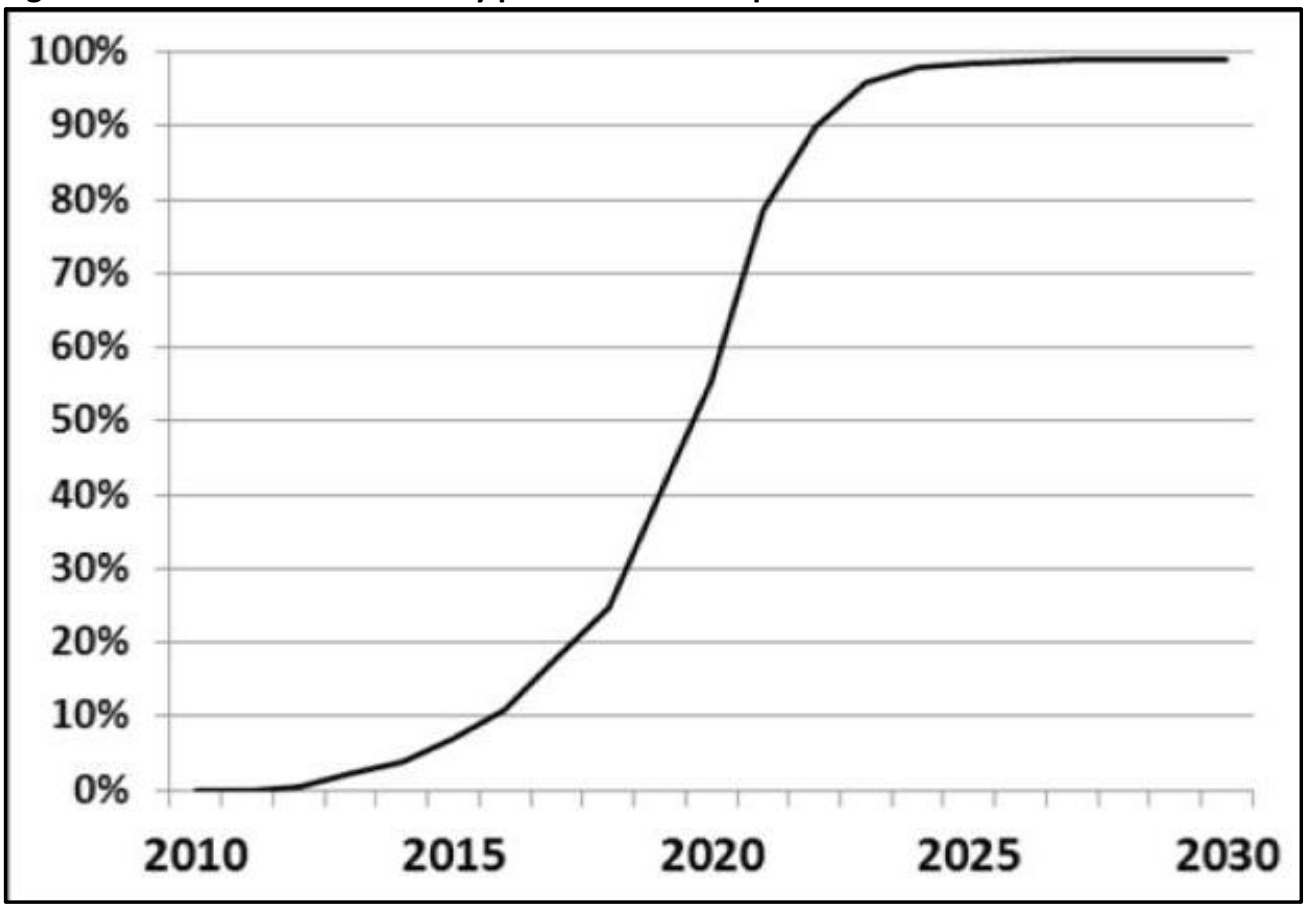

Figure 5.2 shows the projected proportion of the demand for used imported EVs assumed to be available to New Zealand car buyers. For example, in 2015, there is a limited supply meeting only $7 \%$ of the demand for used imported EVs. By 2020 , this proportion rises to $55 \%$ and, by 2025 , as more Japanese owners of EVs sell their cars due to the Shā-ken test, over $98 \%$ of the New Zealand demand for used imported EVs is met.

The application of the choice simulator in each year in the modelling period occurred in three stages.

First stage: simulating the LPV purchases by organisations 
The choice simulator was restricted to ICEVs and new EVs and applied to the forecast demand for new cars by organisations in that year.

Second stage: first round of purchases by private buyers

With all the EV alternatives available, the choice simulator then allocated the forecast demand for new and used LPV demand by private car buyers. However, until a time when EVs imported from Japan were widely available for sale (Figure 5.2), the supply of used EVs was constrained and a proportion of private car buyers were estimated not to be able to buy a used imported EV. This unfulfilled demand was then transferred to the next stage.

Third Stage: second round of purchases by private buyers

The choice simulator allocated the frustrated demand from the second stage using a limited choice set that comprised new and used ICEVs and new EVs.

The aggregated demand from the three stages was entered into the vehicle stock database ready for the next annual period.

\subsubsection{The car ownership sub-model}

The car ownership model in this study is a vehicle stock model (De Jong et al., 2004). These models comprise three components: (1) the vehicle stock in each year by number and vehicle type (stock); (2) the number of vehicles by type entering the LPV fleet each year (sales) and (3) the number of vehicles by type and age of vehicles leaving the LPV fleet each year (scrap). The vehicle stock model is given (De Jong et al., 2004) by:

$$
\operatorname{Stock}_{i}(\mathrm{t})=\operatorname{Stock}_{i}(\mathrm{t}-1)-\operatorname{Scrap}_{i}(\mathrm{t})+\operatorname{Sales}_{i}(\mathrm{t}):
$$

where $i$ is the vehicle type.

The vehicle stock and age profile, sales, and scrap are known in year ( $\mathrm{t}-1)$. To estimate these elements in year $(t)$ it was necessary to forecast at least two elements from which the third component in the equation could then be derived. 
The next sections discuss the approaches used in this study to forecast these elements.

\section{Data sources}

Vehicle import data for new and used imports from 1926 to 2010 were sourced from the New Zealand Transport Agency's New Zealand motor vehicle registration statistics (New Zealand Transport Agency, 2010).

The total number of cars registered in New Zealand for the period 1962-2009 were sourced from Statistics New Zealand's Motor Vehicles Currently Licensed by Type (September quarter) (Statistics New Zealand, 2010b). This database is considered the best representation of active vehicles in the LPV fleet (Conder, 2009).

The Ministry of Transport's New Zealand Vehicle Fleet Statistics 2010 version 2 (Ministry of Transport, 2010c), provides data for LPVs on:

- number and age of new and used vehicles in the LPV fleet; $2000-2009$

- number and age of used vehicles entering the LPV fleet; 2000-2009

- annual vehicle kilometres travelled (VKT) by LPVs; 2001-2009

- scrappage curves for the vehicles leaving the LPV fleet in 2009.

The Ministry of Transport provided fleet scrappage data for the years 2006-2008 (S Badger, Ministry of Transport, personal communication, 18 August 2009).

\section{Method used to forecast LPV sales}

A review of the vehicle sales forecasting literature indicated that econometric models using explanatory variables such as national income per capita, household income, vehicle and fuel prices, construction activity, tourist numbers, value of exports, and the base interest rate have been successfully used in the United States (Carlson and Umble, 1980; Suits, 1958; Shahabuddin, 2009; Christensson, 2008).

Attempts to develop similar forecast models for the sales of new and used imported LPVs in New Zealand using data from the New Zealand Transport Agency (NZTA) (Figure 5.3) were unsuccessful. A variety of structural and time series models were tested, but all models performed poorly. For the structural models no 
explanatory variables were found to be statistically significant across the different model specifications and all performed poorly when tested outside the sample period.

Figure 5.3: New and used vehicles entering the New Zealand light passenger vehicle fleet: 1970 to 2009

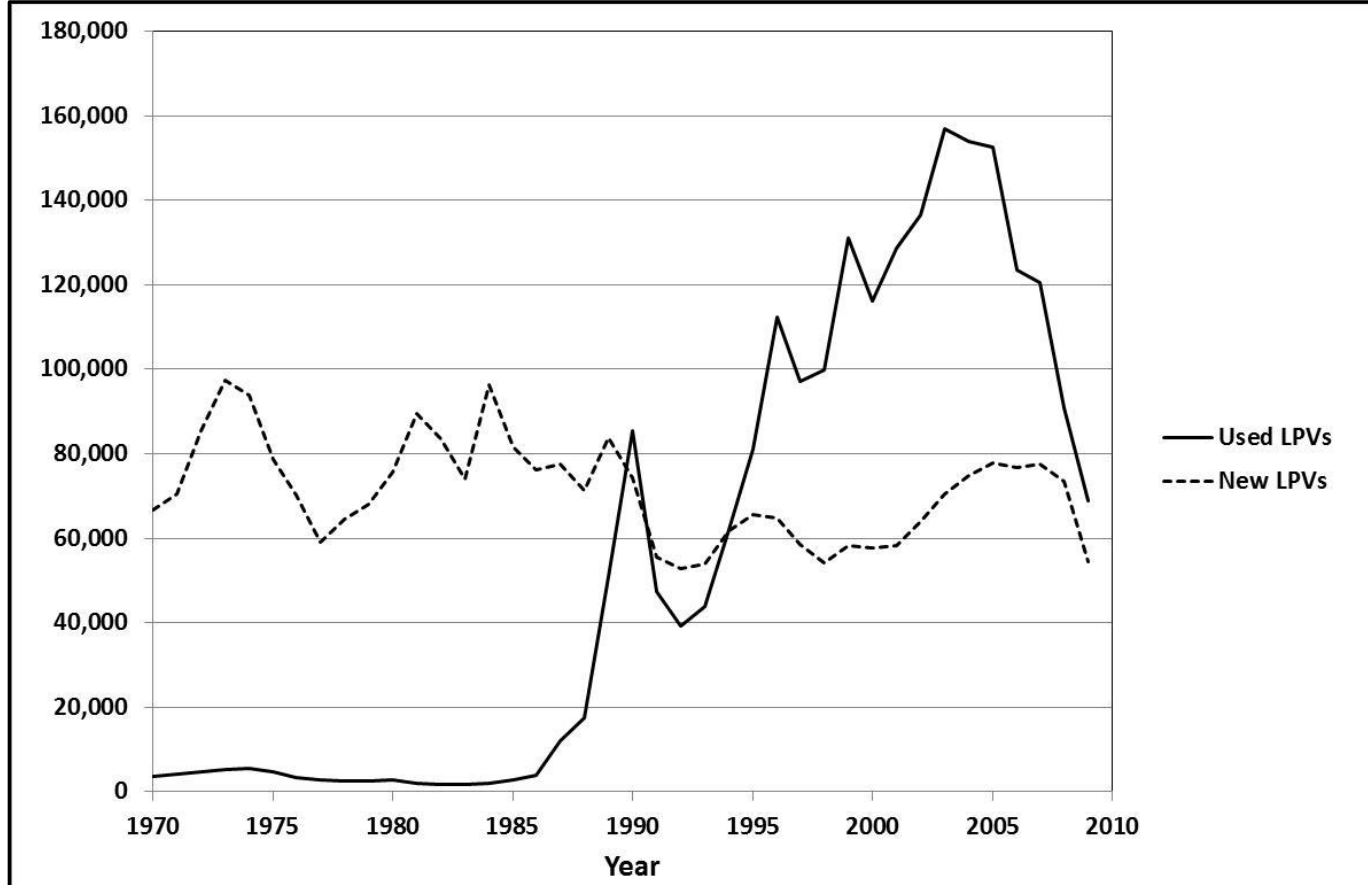

Source: New Zealand Transport Agency (2010)

In the absence of a satisfactory means of directly forecasting new and used LPV sales in New Zealand, the approach used in this study was to forecast the total vehicle stock in year $\mathrm{t}$ and vehicle scrapping in year $\mathrm{t}$ and then derive the total sales in year $\mathrm{t}$ by rearranging the vehicle stock equation to:

$$
\operatorname{Sales}_{i}(\mathrm{t})=\operatorname{Stock}_{i}(\mathrm{t})-\operatorname{Stock}_{i}(\mathrm{t}-1)+\operatorname{Scrap}_{i}(\mathrm{t})
$$

This approach is similar to that used by the European Commission for its TREMOVE model, where total vehicle stock is determined by the number of vehicles needed to meet the forecast travel demand (De Jong et al., 2004; De Ceuster et al., 2007). Greenspan and Cohen (1999) used a similar formulation in their study where they developed models of household car ownership and vehicle scrapping and then derived vehicle sales. 


\section{Forecasting vehicle stock using an aggregate time series model}

Aggregate time series models use a sigmoid-shape function, usually logistic, to model the relationship between income per capita, or GDP, and vehicle ownership per capita. These models are based on diffusion of innovation theories of product uptake, with slow initial uptake followed by a period of accelerated uptake, and then slowing uptake as vehicle ownership approaches saturation (De Jong et al., 2004).

Dargay et al. (2007) have used this type of model to estimate car ownership across a range of countries. These types of models are used in New Zealand by the Ministry of Transport, and some local governments (Conder, 2009; Bone, 2003).

Dargay et al. (2007) found that saturation levels differ between countries due to differences in population density, the population living in urban areas, the availability and reliability of public transport, the role that rail has for moving freight, and the capital invested in roads. They note that countries that invest heavily in roads are likely to have higher vehicle ownership saturation levels (Dargay et al., 2007).

These models have been criticised because they assume that it is possible to forecast the saturation level. Ingram and Liu (1999) found that saturation levels can change over time and it seems to be the case that there is little direct evidence that saturation levels are stationary or that they have a straightforward behavioural interpretation.

Conder (2009) argues that these models are inadequate for forecasting vehicle ownership in New Zealand as he considers that they do not adequately take into account the impact of factors that may cause higher levels of vehicle ownership in the future. He proposes that in New Zealand factors such as changes in age distribution will result in an increased proportion of licensed drivers in the population. He also argues that economic growth will result in a greater proportion of the New Zealand population in high income/high car ownership households. Finally, he argues that the real costs of motoring will continue to decline (both in terms of car purchase and car use). These factors lead him to conclude that there 
will be higher levels of vehicle ownership saturation than current ownership trends indicate.

However, these arguments must be balanced against other influences that would suppress car ownership such as:

- New Zealand has an aging population and adults in the over 65 cohort are often prevented from being licence holders due to a disability (Rose et al., 2009a; Davey, 2007)

- vehicle ownership costs could increase over time due to the decreasing availability of Japanese used vehicle imports resulting from the imposition of stricter emissions standards (Ministry of Transport, 2007)

- the possibility that the future price of transport fuels is likely to be higher due to imposition of policies to reduce GHG emissions

- the future price of transport fuels is also likely to be higher due to the increasing costs now associated with the production of petroleum fuels (International Energy Agency, 2010)

- the emerging evidence that the demand for travel may be peaking in developed countries (see section 1.1.3.).

Due to this uncertainty, it was assumed for this study that the current long-term relationship between vehicle ownership and income, including the current projected saturation level, would be maintained over the duration of the modelling period.

Figure 5.4 shows the historic relationship, from 1962 to 2009, between LPV ownership per capita and income per capita in New Zealand. The graph also shows the effect of events such as the deregulation of used vehicle imports in 1988 offsetting the short-term effects of the 1987 stock market crash and to a lesser extent during the more sustained economic recession of 1991 - 1994. The latest decline in car ownership began in 2006, before the economic recession of 2008 . This decline may be indicative of other market forces coming into effect, which have subsequently been masked by recent economic events (refer to the discussion on peak travel in section 1.1.3). 
Figure 5.4: Relationship between income per capita and light passenger vehicle ownership in New Zealand: 1962 to 2009

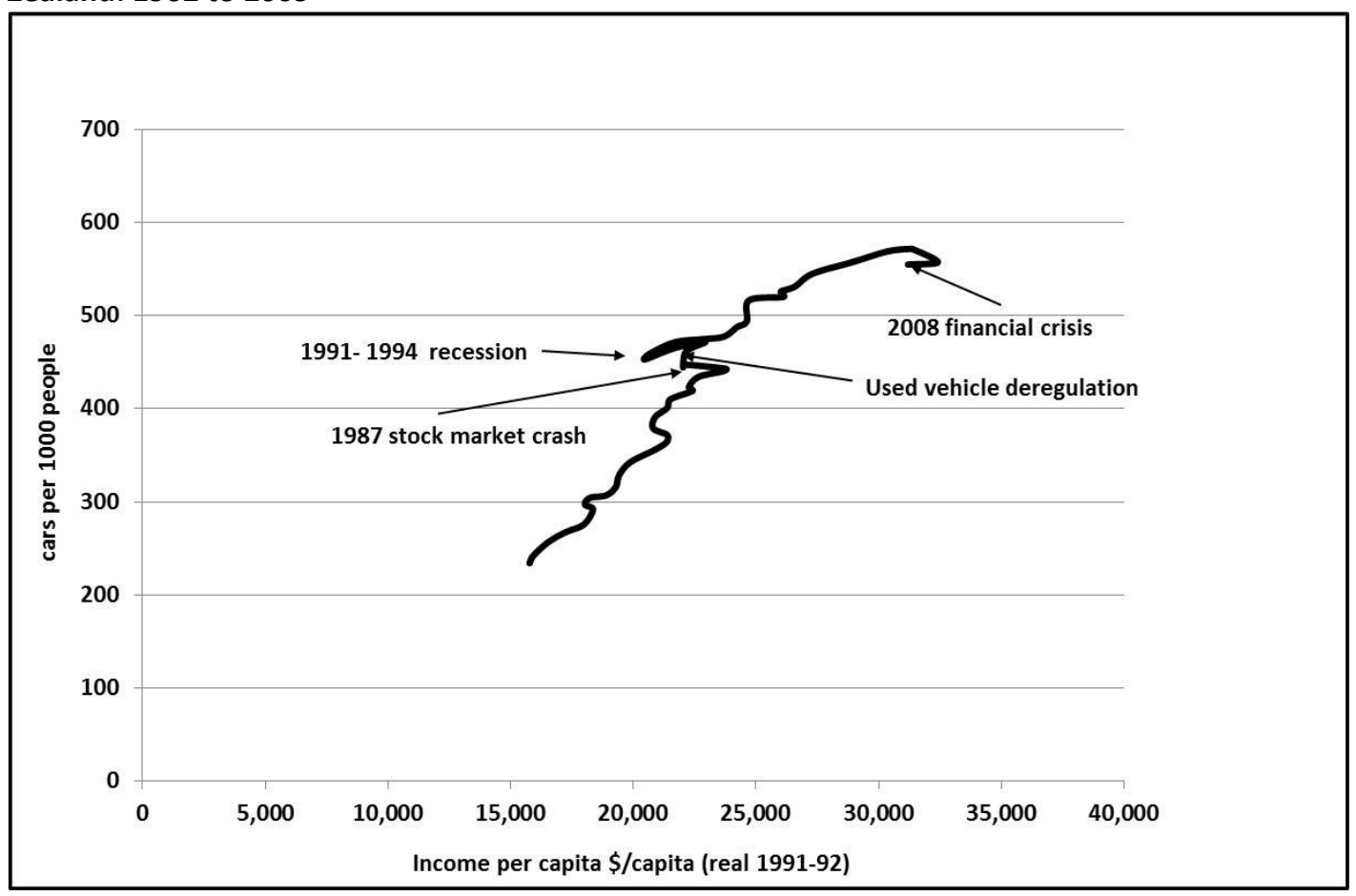

Sources: Chrun (2010), Conder (2009), and Statistics New Zealand (2011)

The approach used to model aggregate vehicle stock is based on the approach used by Dargay et al. (2007) who used the Gompertz version of the logistic function because it is relatively easy to estimate and allows for different curvatures at low and high-income levels.

The Gompertz function takes the form:

$$
V_{t}=\gamma e^{-\alpha e^{-\beta G D P_{t}}}
$$

where $\boldsymbol{\gamma}=$ is the saturation level (cars/1000 people);

$\alpha$ and $\beta$ are parameters that define the shape of the slope;

$\alpha$ defines the displacement along the $\mathrm{x}$ axis and $\beta$ sets the growth rate, or the scaling on the y axis;

GDP = real income per capita and is the independent variable;

$\gamma, \alpha$, and $\beta$ are parameters to be estimated, but $\gamma$ can be set exogenously to take account of the possibility of changes in demographic and geographic characteristics.

The basic Gompertz-form model only takes account of the long-term relationship between income per capita and vehicle ownership. Dargay et al. (2007) suggest that 
the Gompertz function be adjusted to take account of the effect of the slow adjustment in factors such as housing patterns, land use, and demographic change. They introduced a simple partial adjustment factor into the function. This mechanism takes the form:

$$
V_{t}=V_{t-1}+\theta\left(V_{t}^{*}-V_{t-1}\right)
$$

$\theta$ is the speed of adjustment coefficient $(0<\theta<1)$;

$V_{t}^{*}$ is the unconstrained forecast number of vehicles; and

$\mathrm{V}_{\mathrm{t}-1}$ is the number of vehicles in the previous year.

The combined equation then gives the expanded model:

$$
V_{t}=\gamma \theta e^{-\alpha e^{-\beta G D P_{t}}}+(1-\theta) V_{t-1}
$$

In this study, the estimation of both models was with all the parameters left unconstrained, i.e. no assumption was made about the saturation levels.

The models were estimated using a composite time series of New Zealand car ownership from 1962 to 2009, based on data from Conder (2009) for the years 1970 to 2006, data from the NZTA for 1962-1969 (Chrun, 2010) and data from Statistics New Zealand for 2007-2009. The pre 1970 and post 2006 data was adjusted using Conder's method. The data provided by the NZTA were for the second quarter, not the third quarter. The differences between the two quarters were considered not to be significant due to the small number of vehicles involved. Model estimation was by non-linear ordinary least squares and using the LIMDEP 9 econometric software.

Table 5.8 presents the results of the two models. The expanded model with the lag variable had a very good overall fit with an adjusted $R^{2}$ of 0.996 with three of the parameter values significant at the $5 \%$ level, but the $\alpha$ parameter was not significant. The basic unconstrained Gompertz model had a lower overall fit with an adjusted $R^{2}$ of 0.926 but all parameter values were highly significant. These two models indicated that, based on the in-sample long-term relationship, saturation in car ownership would occur somewhere between 615 and 630 cars per 1000 people. 
These saturation levels are lower than the 670 and 750 cars per 1000 people saturation levels projected by Conder (Conder, 2009)

Table 5.8: Light passenger vehicle ownership model estimation: basic and expanded Gompertz functions

\begin{tabular}{|l|c|c|}
\hline & $\begin{array}{c}\text { Basic } \\
\text { model }\end{array}$ & $\begin{array}{c}\text { Expanded } \\
\text { model }\end{array}$ \\
\hline$\gamma$ & $615.910^{* * *}$ & $627.278^{* * *}$ \\
$(p$-value) & $(0.0000)$ & $(0.0000)$ \\
\hline$\alpha$ & $-16.4289 * * *$ & -16.9858 \\
$(p$-value) & $(0.0017)$ & $(0.4639)$ \\
\hline$\beta$ & $-0.173342^{* * *}$ & $-0.20177^{* *}$ \\
$(p$-value) & $(0.0000)$ & $(0.0160)$ \\
\hline$\theta$ & & $0.07915^{* *}$ \\
$(p$-value) & & $(0.0163)$ \\
\hline $\mathbf{R}^{2}$ & 0.926 & 0.996 \\
\hline Adjusted $\mathbf{R}^{2}$ & 0.928 & 0.996 \\
\hline Bayesian information criterion & 6.77870 & 3.99237 \\
\hline
\end{tabular}

Significant at $* * * 1 \%, * * 5 \%$, and $* 10 \%$ level.

Figure 5.5 shows the effect of using the different model specifications to estimate LPV ownership per capita from 1962 to 2009 . Figure 5.6 shows the actual and estimated total number of LPVs using the two models from 1962 to 2009 . The expanded model provides a better fit for the first part of the sample period with the basic model overestimating LPV ownership. Without the lag variable, the basic model was more responsive to changes in income per capita. During the 1991 to 1994 recession, the basic model performed less well as it forecasted a decline in vehicle ownership due to the decline in income per capita. However, there was only a small decline in ownership during this period due to the influx of cheap imported LPVs offsetting the effects of the economic slowdown. Since the end of that recession, the basic model has performed better than the expanded model, which has consistently underestimated LPV ownership.

Both models performed sufficiently well for the purposes of this study, but it was decided to use the basic model in the VFM as it has performed better than the expanded model in the period since vehicle markets were deregulated. 
Figure 5.5: New Zealand light passenger vehicle ownership per 1000 people versus income per capita: 1962 to 2009

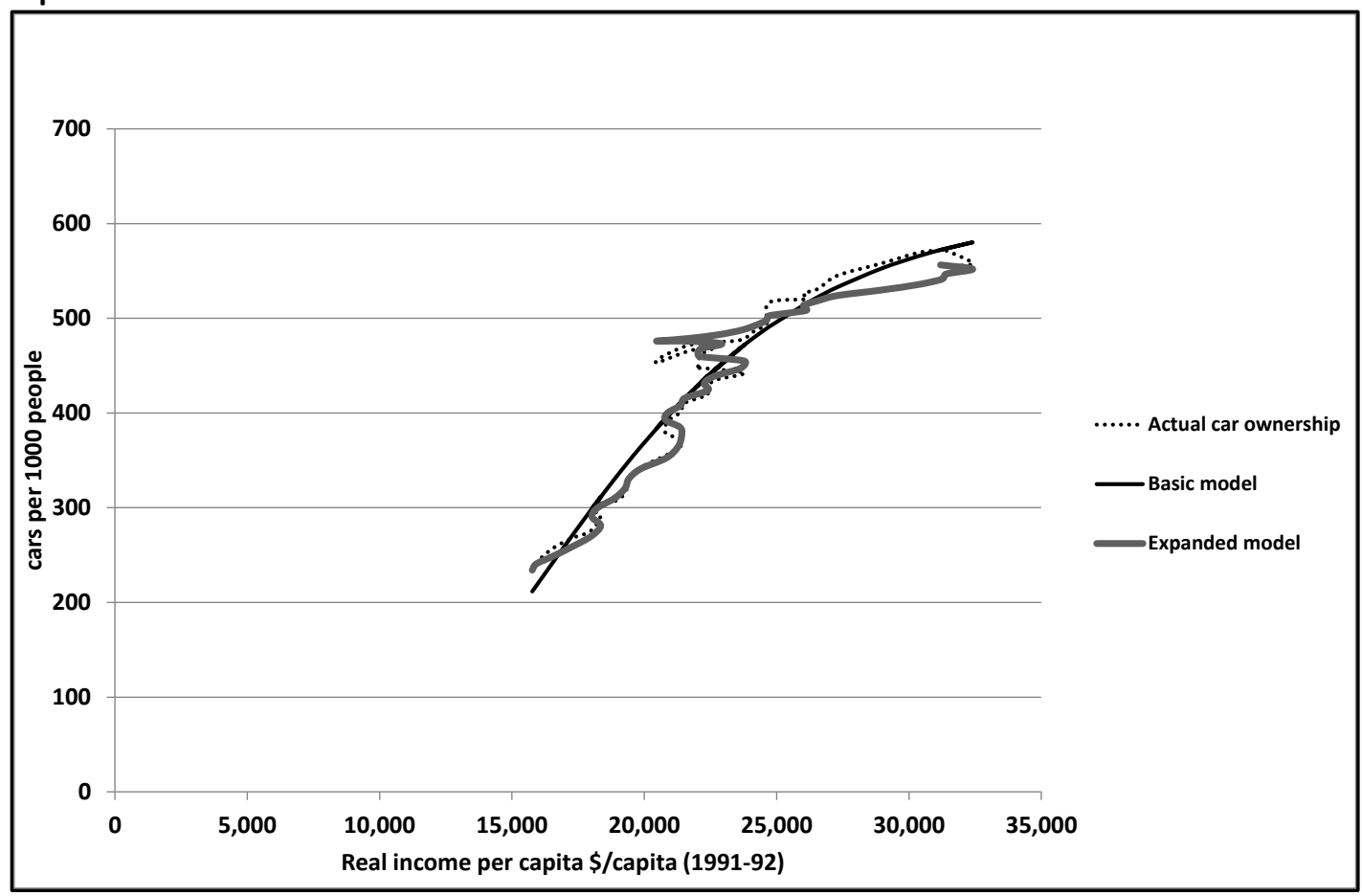

Figure 5.6: New Zealand light passenger vehicle total ownership: 1962 to 2009

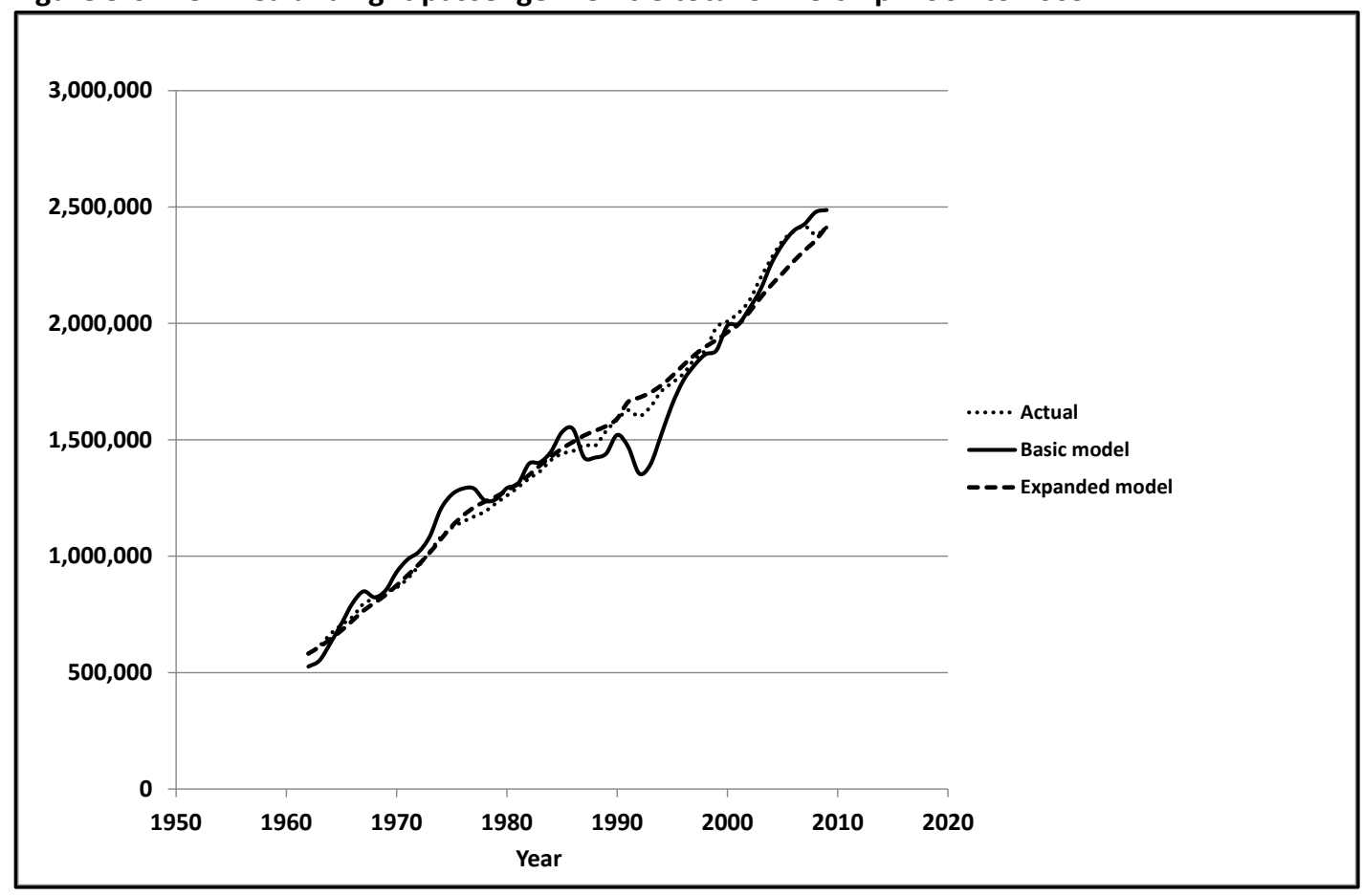

\section{Application of the aggregate car forecast sub-model in the VFM}

Each year the total number of LPV sales (new and used imported vehicles entering the LPV fleet) was derived based on the number of vehicles necessary to achieve the forecast level of vehicle ownership taking into account the size of the vehicle 
stock in the previous year, and the number of vehicles to be scrapped in the current year.

This approach had the practical advantage of reducing the number of exogenous variables to just growth in GDP and population.

The number of vehicle sales, and the number of vehicles being scrapped in the rearranged vehicle stock model had to be derived simultaneously using the iterative calculation capability in Microsoft Excel. This method worked well with the VFM reproducing the initial LPV fleet forecast to within 48 vehicles or $0.002 \%$.

This method cannot forecast the proportion of new and used imported LPV sales. For this study, the assumption was that, on average, new vehicles would constitute $40 \%$ of all LPVs entering the fleet and was based on the average proportion of new LPVs entering the fleet for the period 1989 to 2009 (New Zealand Transport Agency, 2010).

The proportion of new LPVs entering the fleet that were bought by business and government organisations and not private individuals was derived from data provided by the Motor Industry Association for the years 2002 to 2008 (Kerr, 2009). This data shows that, during this period, organisations purchased between $60 \%$ and 64\% of all new LPVs (including sport utility vehicles (SUVs)) entering the country. For this study, the assumption was that, from 2010 to 2030, organisations would purchase $62 \%$ of the new LPVs entering the fleet.

At present, LPV ownership per capita is below the long-term trend as forecast by the basic Gompertz model. Unless constrained in the first forecast year, the model returned the size of the LPV fleet back to the long-term trend in the first forecast period. This resulted in a large influx of new and used imported LPVs. To minimise this effect, the forecast model was constrained to return the size of the LPV fleet back to the long-term trend over a five-year period. During this period, the number of LPVs entering the fleet is slightly less than that projected using the unconstrained model. 


\section{Scrapping sub-model}

All data was sourced from the New Zealand Vehicle Fleet Annual Fleet Statistics (Ministry of Transport, 2010a). These statistics contain information on the:

- age profile of the LPV fleet, available from 2000 to 2009

- age profile of used LPV imports entering the country from 2000, but this information is limited to post 1990 vintage vehicles

- scrapping rates of new and used imported LPVs available for the years 2006 to 2009.

Figures 5.7 and 5.8 show the percentage of new and used imported vehicles scrapped for a given vehicle age in each of the years 2006 to 2009. The data indicate that, with the economic recession in 2009 , the scrapping rates for both new and used imported LPVs declined, with the graphs shifting to the right and the proportion of vehicles scrapped at the age of maximum scrapping decreasing.

Figure 5.7: The percentage of new vehicles scrapped at a given vehicle age: 2006 to 2009

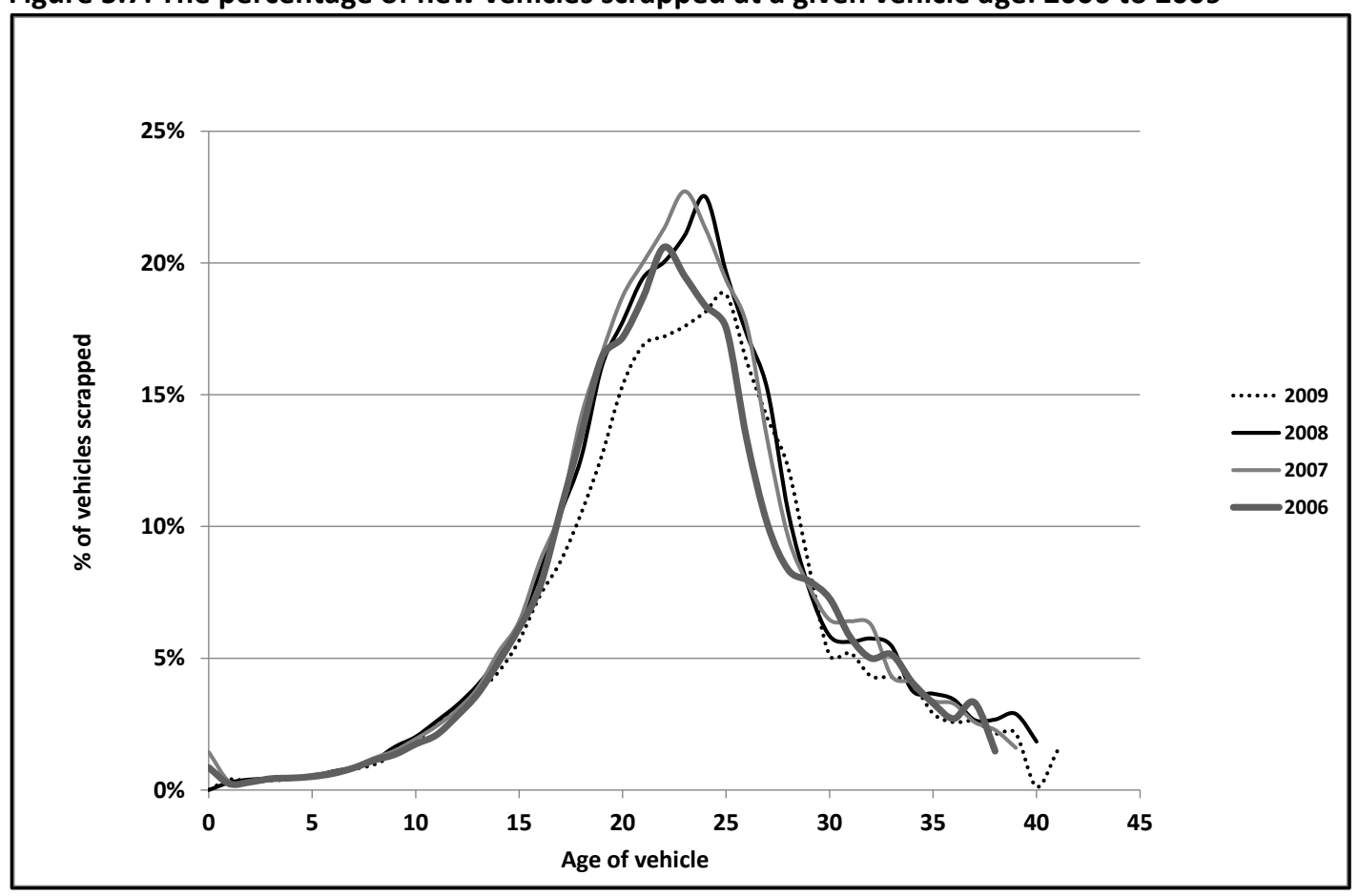

Source: Ministry of Transport (2010a)

The high variability in scrapping rates for the newer used imports (Figure 5.8) was due to the effect of road crashes on a small numbers of vehicles. 


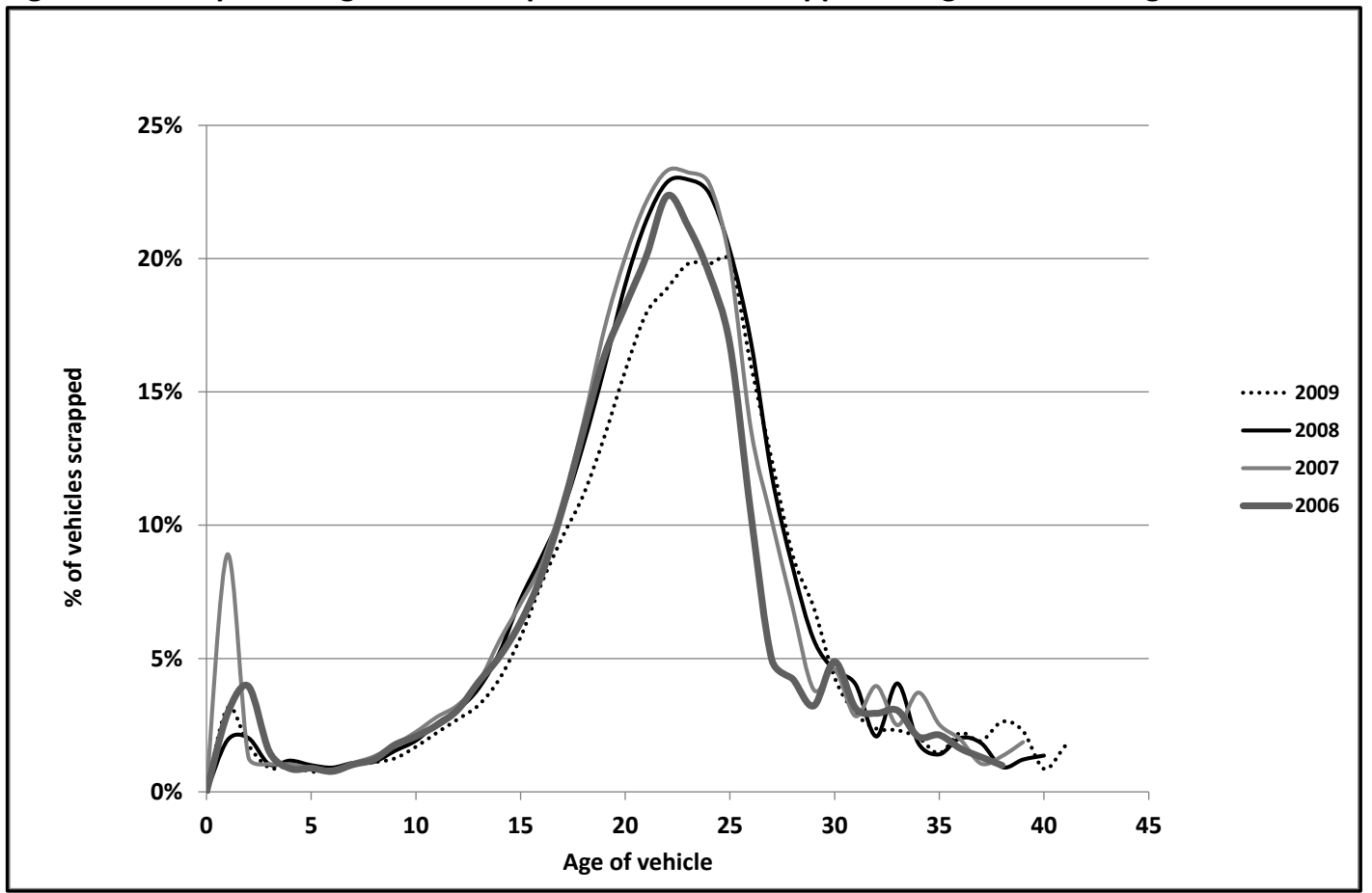

Source: Ministry of Transport (2010a)

Economic scrapping models estimate the probability that vehicle of a specific vintage will leave the LPV fleet. These models require long-term data on the scrapping rates of the different vintages from which a hazard function can then be estimated (Greenspan and Cohen, 1999; Miaou, 1995; Parks, 1977; Golomb and Bunch, 1979).

The development of a hazard function using vehicle age as the explanatory variable was attempted by combining the four years of available scrapping data into four vehicle vintages that had been observed through 38 years. The approach assumed that all of the 38 different vintages had the same level of vehicle durability.

A number of logistic functions were tested, but only the model developed by Miaou (1995) correctly simulated the timing of the maximum rate of scrapping for both new and used imports. However, the model underestimated the rate of maximum scrapping, particularly for used imports (Figures 5.9 and 5.10). 
Figure 5.9: The percentage of new vehicles scrapped at a given vehicle age - Miaou model estimate and average values: 2006 to 2009 data

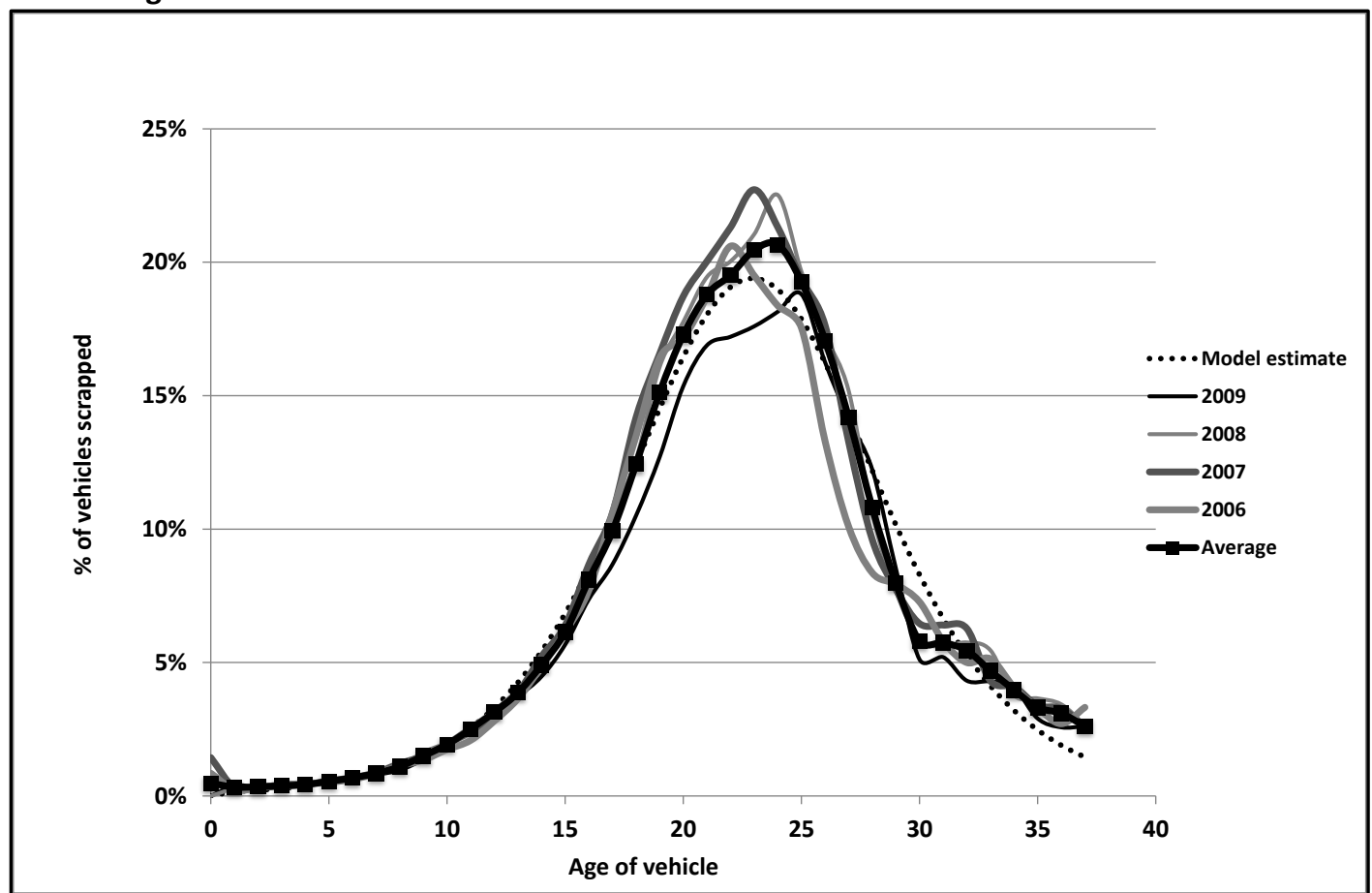

Figure 5.10: The percentage of used imported vehicles scrapped at a given vehicle age - Miaou model estimate and average values: 2006 to 2009 data

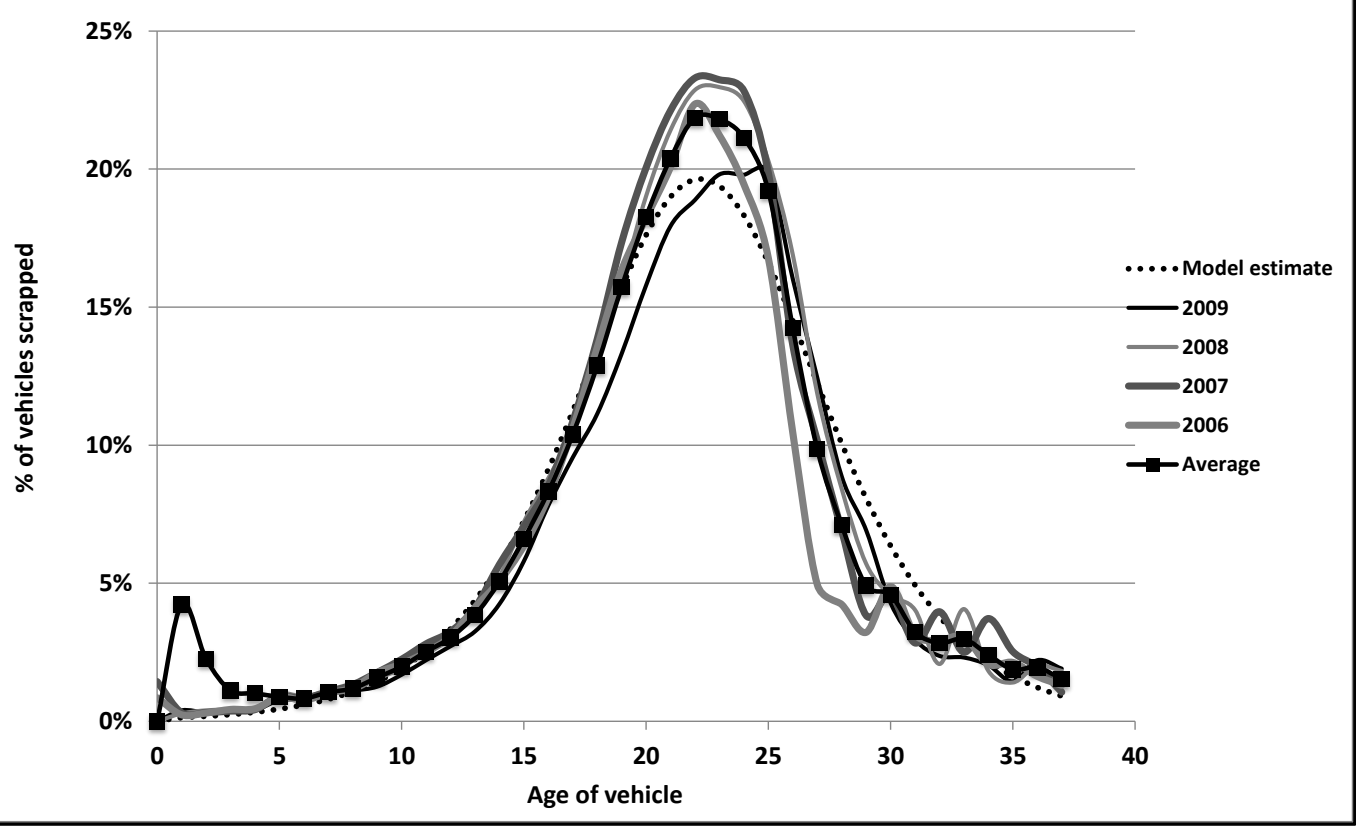


Concerns about the validity of combining 38 separate LPV vintages into one hypothetical vintage as a proxy for long-term scrapping data and the inability of the model to accurately estimate the maximum rate of scrapping resulted in the decision to use the historic average scrapping rate values from 2006 to 2009 . This approach had the advantage of more accurately reflecting peak rates and the rate of scrapping post peak. The four years of available data also captured the effects of strong, weak, and negative annual economic growth on scrapping rates.

By incorporating a sensitivity test into the VFM scenarios, it was possible to assess the effects of different levels of economic growth on scrapping rates. The impact of sustained strong economic conditions was simulated by using the 2007 scrapping rates, as the 2007 economic year was the last year with a long period of positive economic growth. In contrast, 2009 was a period of negative economic growth following a decline in GDP in 2008 and the scrapping rates from this year were used to simulate the effect of sustained low economic growth on the scrapping of LPVs (Figure 5.11).

Figure 5.11: GDP expenditure measure ( $\%$ annual change)

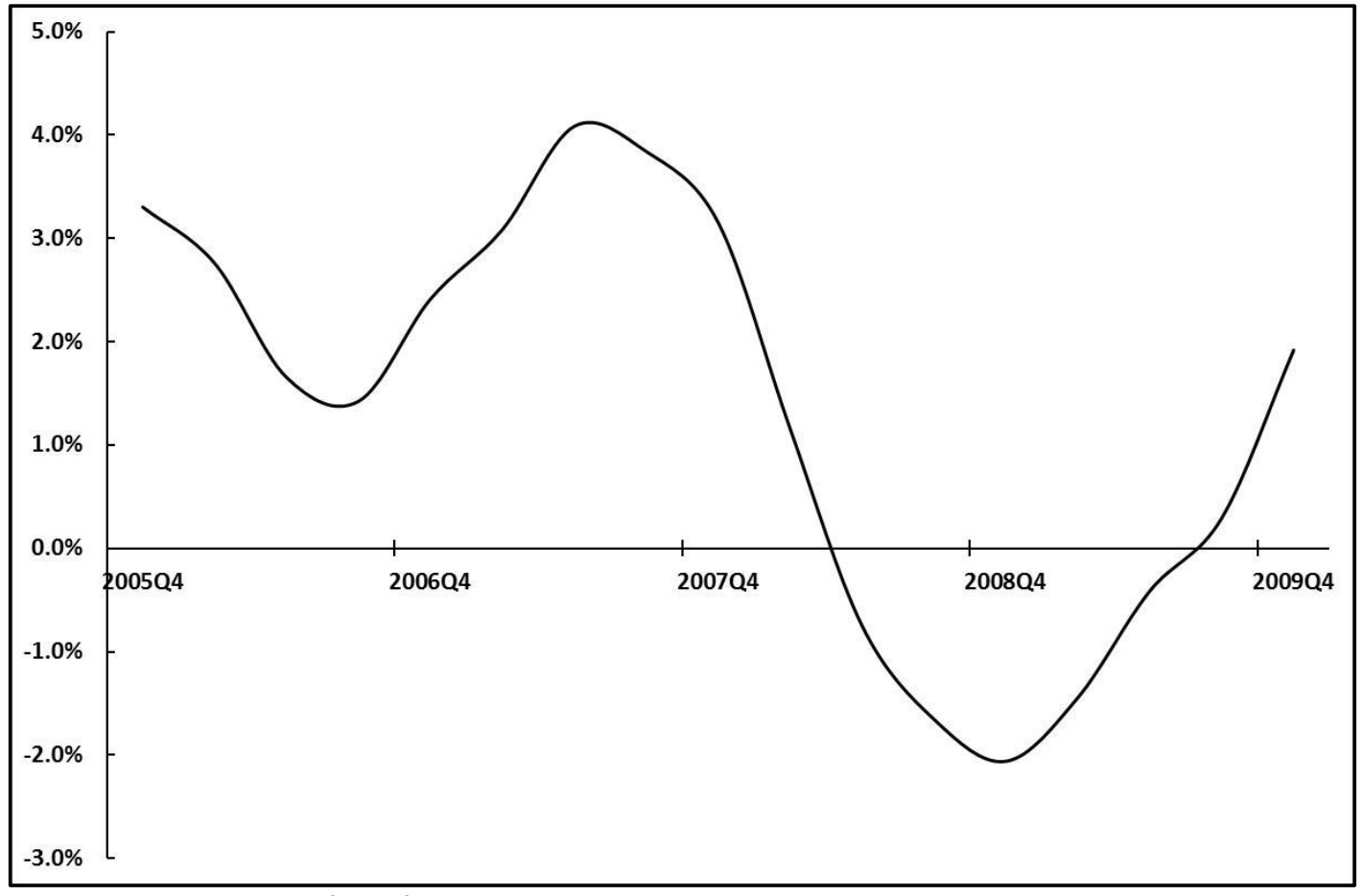

Statistics New Zealand (2011) 
The default scrapping rates for new and used imported LPVs are shown in Figures 5.12 and 5.13. These rates were used in the VFM scenarios that assumed a moderate GDP growth rate.

Figure 5.12: Scrapping rate new vehicles - low, high, and default economic growth

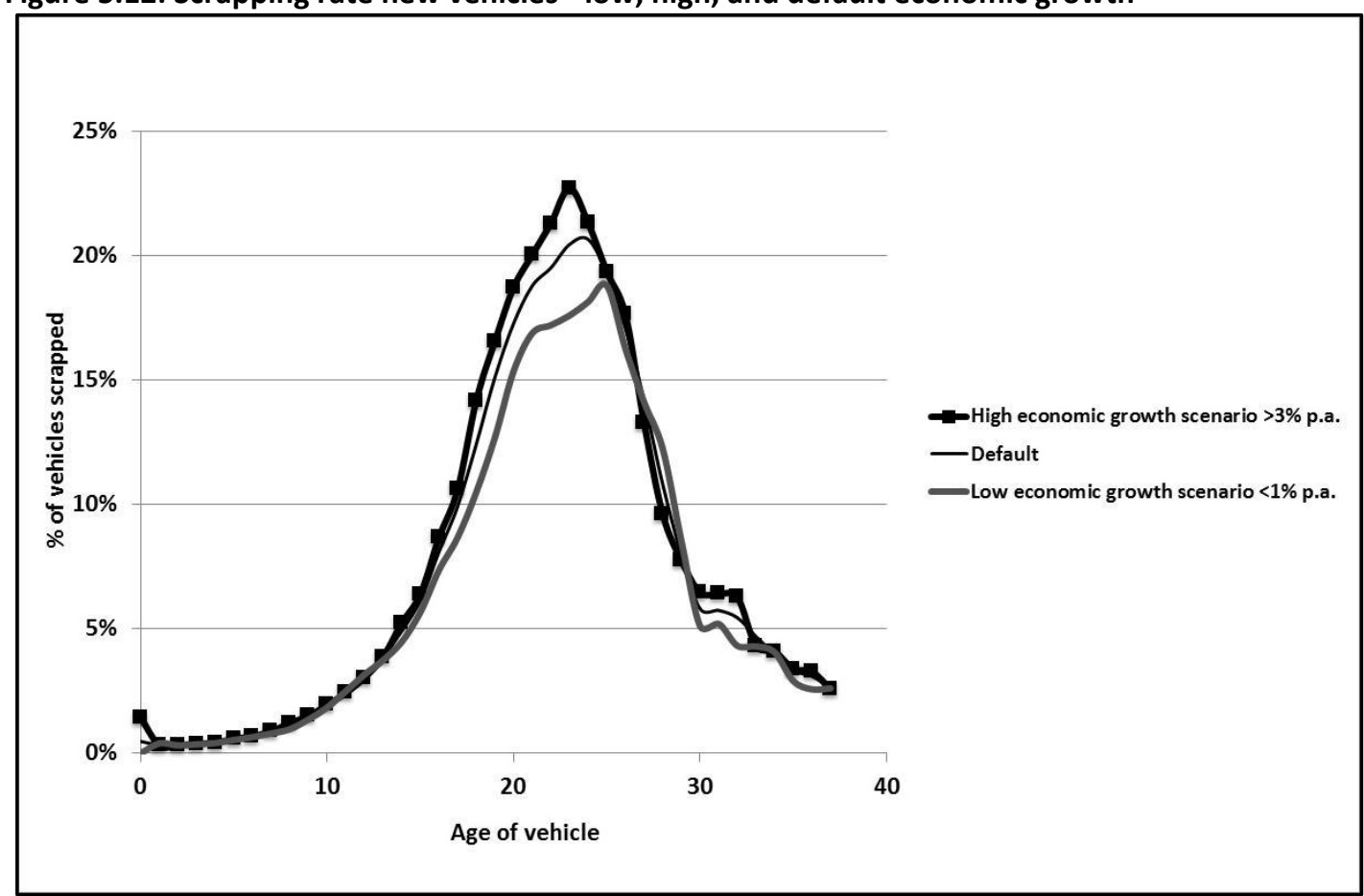

Figure 5.13: Scrapping rate used imported vehicles - low, high, and default economic growth

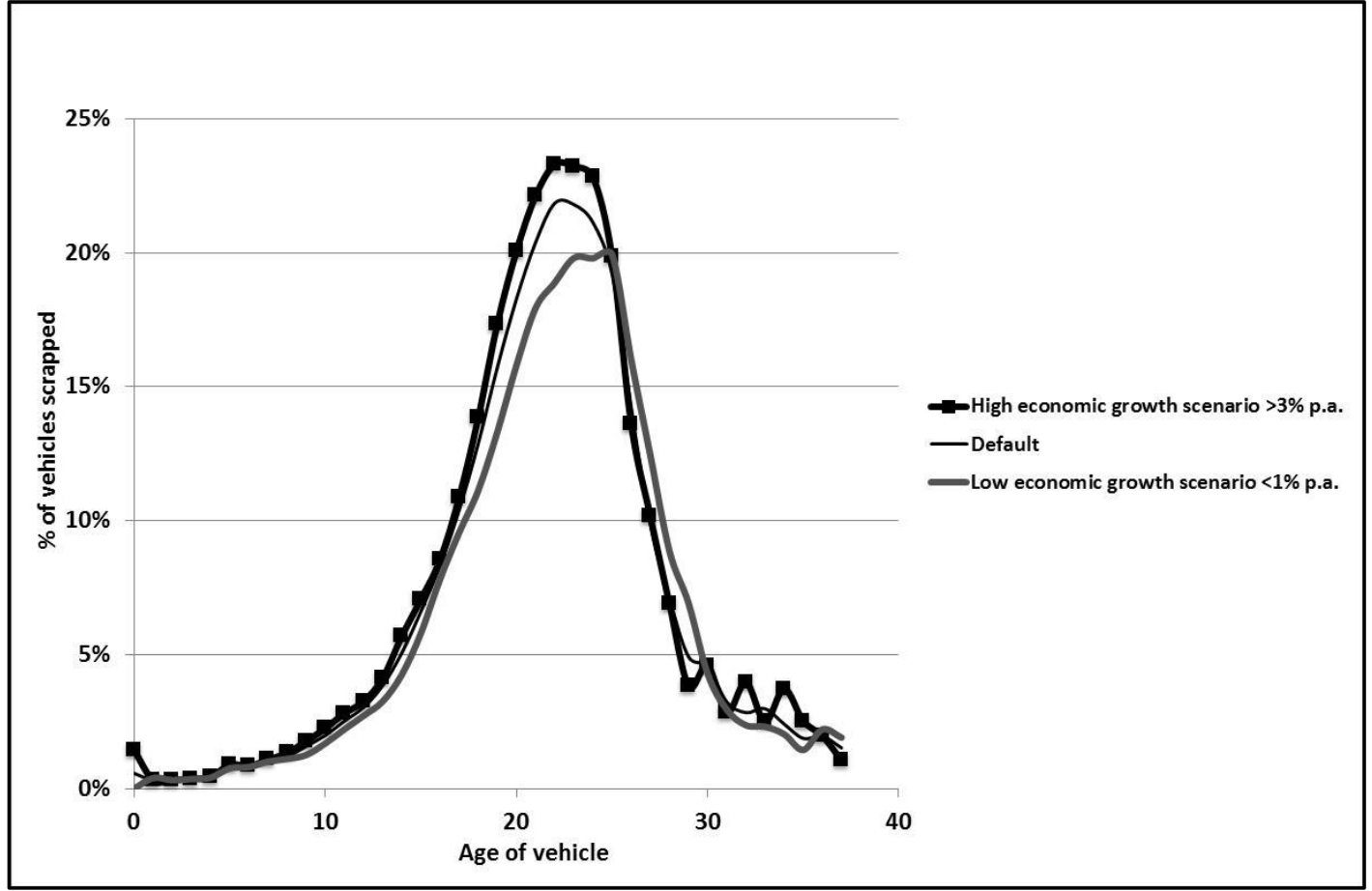




\subsubsection{The vehicle use sub-model}

\section{Fuel costs and travel demand}

Some of the VFM scenarios included the assumption that there would be very high petroleum prices. The intention of these scenarios was to assess the effects of high ICEV fuel running costs on the demand for EVs. However, studies indicate that increasing fuel prices also have an effect on the short and long-term demand for transport fuels and vehicle travel (Goodwin et al., 2004; Graham and Glaister, 2004; Johansson and Schipper, 1997). The vehicle use sub-model (VUM) was included in the VFM to estimate the effects of changes in the price of petroleum and electricity on annual VKT, energy consumption, and GHG emissions (Figure 5.1).

Research undertaken in New Zealand for Land Transport New Zealand ${ }^{38}$ found that changes in petrol prices and GDP had a statistically significant impact on the total annual VKT. This research used weekly data from Transit NZ's State Highway traffic count programme sourced from 108 sites over the period January 2002 to June 2006. These traffic counts excluded traffic on local roads, but the State Highway data was used as a proxy for total annual vehicle kilometres travelled (VKT) (Kennedy and Wallis, 2007).

This study was subsequently reviewed by Donovan et al. (2008) who used a backcasting method to fit a model that included additional explanatory variables. These variables were workforce participation, vehicle ownership, and income. They found that this approach improved the performance of the basic model developed by Kennedy and Wallis.

Table 5.9 summarises the elasticity estimates for kilometres per vehicle and total VKT in response to changes in fuel price.

\footnotetext{
${ }^{38}$ Restructured in 2008 and now the New Zealand Transport Agency (NZTA)
} 
Table 5.9: Elasticity estimates of change in kilometres travelled in response to change in fuel price

\begin{tabular}{|c|c|c|c|c|}
\hline & $\begin{array}{l}\text { Dependent } \\
\text { variable }\end{array}$ & $\begin{array}{l}\text { Short run } \\
\text { elasticity }\end{array}$ & $\begin{array}{l}\text { Long run } \\
\text { elasticity }\end{array}$ & $\begin{array}{l}\text { Total } \\
\text { elasticity }\end{array}$ \\
\hline $\begin{array}{l}\text { Johansson and } \\
\text { Schipper (1997) }\end{array}$ & $\begin{array}{l}\text { Average } \\
\text { vehicle } \\
\mathrm{km} / \text { year }\end{array}$ & - & $\begin{array}{c}-0.35 \text { to }-0.05 \\
\text { (-0.2 Best } \\
\text { guess) }\end{array}$ & - \\
\hline \multirow{3}{*}{$\begin{array}{l}\text { Goodwin, Dargay } \\
\text { et al. }(2004)\end{array}$} & $\begin{array}{c}\text { Vehicle km } \\
\text { (total) }\end{array}$ & $\begin{array}{c}-0.17,-0.05 \\
(-0.10 \text { mean })\end{array}$ & $\begin{array}{c}-0.63,-0.10 \\
(-0.29 \text { mean })\end{array}$ & - \\
\hline & $\mathrm{km} /$ vehicle & $\begin{array}{c}-0.14,-0.06 \\
(-0.10 \text { mean })\end{array}$ & $\begin{array}{c}-0.55,-0.11 \\
(-0.30 \text { mean })\end{array}$ & - \\
\hline & $\begin{array}{l}\text { Static model } \\
\text { results }\end{array}$ & & & $\begin{array}{ll}\text { Pre 1974 } & -0.54 \\
1974-81 & -0.32 \\
\text { Post 1981 } & -0.24\end{array}$ \\
\hline $\begin{array}{l}\text { Graham and } \\
\text { Glaister (2004) }\end{array}$ & $\begin{array}{c}\text { Car km and car } \\
\text { trips }\end{array}$ & -0.16 & $\begin{array}{c}-0.26(\mathrm{~km}) \\
-0.19(\mathrm{car} \\
\text { trips) }\end{array}$ & 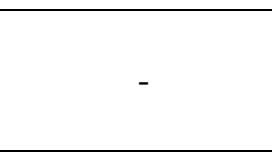 \\
\hline $\begin{array}{l}\text { Kennedy and } \\
\text { Wallis (2007) }\end{array}$ & $\begin{array}{c}\text { Vehicle km } \\
\text { (total) }\end{array}$ & -0.12 & -0.24 & - \\
\hline $\begin{array}{l}\text { Donovan, Genter } \\
\text { et al. (2008) }\end{array}$ & $\begin{array}{l}\text { Vehicle km } \\
\text { (total) }\end{array}$ & - & - & -0.27 \\
\hline
\end{tabular}

The estimation of the VUM used data from the New Zealand Vehicle Fleet Annual Fleet Statistics. These data were considered to more accurately reflect the total amount of travel by LPVs, as it excluded other types of road vehicles and included travel on both state highways and local roads. The limitation of this data source is that it is available only for the period since 2001. The models were initially estimated using data from 2001 to 2009, but they were then re-estimated using data from the August 2011 version of the vehicle fleet statistics so as to include the 2010 data (Ministry of Transport, 2010c).

The use of small samples to estimate regression models is not recommended and the output of the VUM should be treated with caution. Regression analysis using small sets of time series data are often sensitive to the inclusion, or exclusion, of the first and last data points. To test the sensitivity of the VUM, the model was reestimated including and excluding the 2001 and 2010 data points. This test found that the same variables remained statistically significant across the different datasets and the parameter values remained reasonably stable.

Petrol price is used as a proxy for both petrol and diesel powered vehicles and is sourced from the Energy Data File (Ministry of Economic Development, 2010a). To allow for the potential for future significant changes in the average energy 
efficiency of the LPV fleet, the VUM uses fuel costs per kilometre as an explanatory variable rather than fuel cost per litre.

The fuel cost per kilometre for PHEVs takes into account the costs of using both electricity and petrol and are based on the fuel cost per kilometre of a comparable HEV and the fuel cost per kilometre of a comparable GEV. The average annual ratio of electric and non-electric driving by the PHEV fleet was used to derive the combined fuel cost per kilometre for PHEVs.

Travel demand forecasts were undertaken for each of these vehicle classes and it was necessary to estimate the total annual VKT for each vehicle type by forecasting the annual VKT and the number of vehicles of each type.

$$
V K T_{t i}=A V K T_{t i} * N_{t i}
$$

$A V K T_{t i}$ is the forecast average VKT per vehicle in year $t$ for vehicle type $i$; and;

$\mathrm{N}$ is the number of vehicles in category $i$ in year $t$.

Table 5.10 presents data on the average VKT per vehicle for LPVs. The all New Zealand average comprises vehicles that are undertaking urban, peri-urban, and rural driving. It is expected that in the future ICEVs and PHEVs, with their greater range, will be used for urban, peri-urban, and rural driving, but for the foreseeable future both CEVs and GEVs are expected to be limited to urban use. There is no New Zealand data on the average VKT per vehicle travelled by urban vehicles.

In this study, the derivation of the average VKT per vehicle for urban drivers was by scaling the national average by the ratio of the national and urban mean daily travel data. This information was sourced from the household travel survey (Ministry of Transport, 2008). This method indicated that the VKT for urban vehicles was $90 \%$ of the New Zealand average VKT. 
Table 5.10: Average annual light passenger vehicle VKT per vehicle: 2001 to 2010

\begin{tabular}{|l|l|l|}
\hline \multirow{2}{*}{2001} & All New Zealand & Estimated urban \\
\hline 2002 & 12,861 & 11,605 \\
\hline 2003 & 12,981 & 11,713 \\
\hline 2004 & 12,922 & 11,660 \\
\hline 2005 & 12,826 & 11,573 \\
\hline 2006 & 12,510 & 11,288 \\
\hline 2007 & 12,247 & 11,051 \\
\hline 2008 & 12,196 & 11,005 \\
\hline 2009 & 11,959 & 10,791 \\
\hline 2010 & 12,015 & 10,841 \\
\hline
\end{tabular}

Source: Ministry of Transport (2011).

Previous studies have found correlations between VKT and the cost of fuel, the user cost of vehicle travel (which includes fuel costs), GDP, household income, workforce participation, vehicle ownership, household size, number of vehicles per household, vehicle age, population, and population density (Musti and Kockelman, 2009;

Donovan et al., 2008; Kockelman and Zhao, 2000). In urban studies, factors such as urban structure and availability of public transport have also been found to have an impact on VKT (Souche, 2010).

Kennedy and Wallis (2007) found a correlation between changes in the total annual traffic volume and fuel price in the current year, fuel price in the previous year, and GDP. Donovan et al. (2008) in their extended model found correlations between total annual traffic volume and household income, vehicle ownership, and workforce participation in addition to fuel price and GDP.

For this study, fuel cost per kilometre, fuel cost per kilometre in the previous year, GDP, household income, occupants per household, workforce participation, vehicle ownership, number of vehicles per household, average age of vehicles, total population, and ratio of urban population, number of drivers' licences per household, and number of drivers' licences per vehicle were tested as possible explanatory variables. To address the presence of autocorrelation in the residuals, all data was transformed into first differences. Only two variables were found to be correlated to the change in average annual VKT at the $5 \%$ level of significance. 
These variables were the annual change in fuel cost per kilometre and the annual change in household size (Table 5.11).

Table 5.11: Results of the regression on change in average annual VKT per vehicle: 2001 to 2010

\begin{tabular}{|l|c|c|} 
& Coefficient & Elasticities \\
\hline Fuel cost per km & $\begin{array}{c}-50.1848^{* *} \\
(0.0287)\end{array}$ & -0.41 \\
(prob) & $\begin{array}{c}7260.68^{* *} \\
(0.0146)\end{array}$ & 0.53 \\
\hline Household size & 0.79277 & \\
(prob) & 0.76317 & \\
\hline R-squared & 14.2 & \\
\hline Adjusted R-squared & $(0.0002)$ & \\
\hline F[1,6] & 2.47 & \\
(prob) & $(\mathrm{DL}=2.11)$ & \\
\hline Durbin Watson & $2.2583(0.3233)$ & \\
\hline Ljung-Box Q Statistic & & \\
(prob) & & \\
\hline
\end{tabular}

Note: $* * *, * *, *$ Significance at $1 \%, 5 \%, 10 \%$ level.

The Durbin Watson test for autocorrelation was inconclusive, but testing the residuals of the model with the Box-Ljung $Q$ test indicated that autocorrelation was no longer present.

The VUM performed moderately well with an adjusted R-squared value 0.763 .

Demand rebound may occur due to the effects of improved fuel or energy efficiency in reducing vehicle running costs. Greening et al. (2000) identify three types of direct rebound effect: (1) an increase in the number of vehicles in the LPV fleet; (2) an increase in fuel consumption due to demand for larger more powerful vehicles; and (3) an increase in $\mathrm{VKT}^{39}$. They note that the empirical evidence of the rebound effect is from econometric studies that assess the impact on fuel demand and light-duty passenger and commercial vehicle usage. They also note that there is little analysis of the impact of the rebound effect on vehicle numbers or vehicle

\footnotetext{
${ }^{39}$ Greening et al. focus on the direct effects of rebound, but recognise that rebound effects extend to: (1) secondary fuel use effects, such as consumption of other energy services; (2) economy wide effects, such as changes in fuel markets; and (3) transformational effects due to changes in consumers' preferences, social institutions, and production systems.
} 
size ${ }^{40}$. They report that United States data indicate rebound effects, due to improvements in energy efficiency, on fuel consumption and vehicle miles travelled, of between $10 \%$ in the short-run and $20 \%$ to $30 \%$ in the long-run. Small and van Dender (2007) argue that, in the United States, the scale of the rebound effect has declined with increasing income, and the decreasing real fuel cost of driving. They propose that if incomes continue to increase and the real fuel costs of driving continue to decline the rebound effect will continue to decline.

For this study, there was no adjustment made to the estimated travel demand elasticities to account for the possibility of a rebound effect as the result of improvements in energy efficiency. For BEVs, this assumption can be justified because the driving range and battery recharging limitations can be expected to act to suppress the rebound effect. For PHEVs and ICEVs, this assumption cannot be made, and it is possible that for these classes of vehicle the VUM may slightly underestimate total projected VKT.

\section{PHEV fuel consumption}

For this study, it was assumed that PHEVs would use the all-electric drive cycle, where only the electric motor operates during the charge depleting (CD) cycle, or the electric motor dominant blended cycle, where the PHEVs only use the internal combustion engine (ICE) during the CD cycle at times of extreme acceleration (see section 4.4). The Chevrolet Volt uses the electric motor dominant blended cycle.

This approach allows for the separate modelling of the two cycles with a clear distinction between the electric and ICE cycles. Analysis by Moawad et al. (2009) and Pesaran et al. (2009) indicates this approach will slightly underestimate the use of petrol engine for blended cycle PHEVs, but overall there is no significant difference in energy consumption between all electric and blended cycle PHEVs.

The assumption used in this study is that while operating in electric mode, the energy consumption of a PHEV is equivalent to that of a BEV of the same vintage,

\footnotetext{
${ }^{40}$ There is limited evidence on the existence of rebound in terms of increased vehicle size or greater vehicle numbers, but analysis of Swiss car buyers indicates that there is little or no rebound arising from increased fuel efficiency (de Haan et al., 2006).
} 
gross weight, and aerodynamic properties. It was also assumed that when operating in ICE mode, PHEVs have the fuel consumption equivalent to HEVs of the same vintage, weight, and aerodynamic properties.

For this study, a model was developed to determine the proportions of electric and non-electric driving undertaken by a PHEV using data sourced from the annual New Zealand Household Travel Survey covering 2,200 households per year (Ministry of Transport 2010).

Using the New Zealand Household Travel Survey, a cumulative distribution of the daily travel distances was calculated based on data for the period 2003/04 to 2007/08 (inclusive). A log-normal distribution was fitted to the distribution by maximum likelihood estimation. From the log-normal distribution the partial averages for electric and non-electric driving were then derived ${ }^{41}$.

Battery degradation has the effect of reducing the electric driving range and increasing the reliance of the PHEV on the ICE cycle. Consequently, the PHEV fuel/energy efficiency estimates will be sensitive to the declining capacity of the batteries due to aging. The VFM takes account of this effect by assuming a straightline decline in capacity from $100 \%$ to $80 \%$ over the expected life of the battery.

\subsubsection{Energy demand and GHG emissions sub-models}

Vehicle fuel consumption, in ICEVs, refers to the amount of petrol or diesel used to travel a specified distance, usually presented as litres per $100 \mathrm{~km}(\mathrm{~L} / 100 \mathrm{~km})$. Fuel efficiency is the reciprocal of fuel consumption and usually presented as miles per gallon or less commonly kilometres per litre. Energy efficiency, used for EVs, is the equivalent concept to fuel consumption in ICEVs and is the amount of electric energy required to travel a specified distance, usually presented as watt-hours per kilometre $(\mathbf{W h} / \mathbf{k m})$.

\footnotetext{
${ }^{41}$ This model was developed with the assistance of Professor Euan Smith of Victoria University Wellington. A fuller description of the model is provided in Appendix 5.
} 
It is a simple matter to convert $\mathrm{L} / 100 \mathrm{~km}$ to $\mathrm{Wh} / \mathrm{km}$ once the calorific content of the petrol or diesel is known (Mackay, 2009, pp. 29-30).The approach used in the VFM was to measure the energy usage of both ICEVs and EVs in terms of Wh/ $\mathrm{km}$. In this study, energy efficiency figures for ICEVs were the weighted average values for the petrol and diesel vehicle fleet, which were then converted to $\mathrm{Wh} / \mathrm{km}$ using the gross calorific values of $35 \mathrm{MJ} / \mathrm{L}(9.72 \mathrm{kWh} / \mathrm{L})$ for petrol and $38.3 \mathrm{MJ} / \mathrm{L}(10.64$ kWh/L) for automotive gas oil (diesel) (Ministry of Economic Development, 2010a). The VFM estimated the annual demand for energy for eight classes of vehicle. These classes were new and used imported ICEVs, CEVs, GEVs, and PHEVs.

In each year for each class of vehicle, the number of vehicles, and average fuel or energy efficiency for each class of vehicles was calculated.

The technology assumptions in each VFM scenario determined the future changes in average energy efficiency of the ICEV and EVs entering the New Zealand LPV fleet.

In the following sections, there is a discussion of the potential for improvements in the energy efficiency of ICEVs and EVs.

\section{ICEV fuel consumption}

This class comprises petrol and diesel powered LPVs. There is currently no accurate estimate of the average fuel consumption for diesel powered LPVs in the New Zealand LPV fleet. This is due to the difficulty of separating out the amount of diesel used by the LPV fleet from other types of diesel usage. Diesel is widely used for both on- road and off-road transport, and for non-transport purposes. It is thought by the Ministry of Economic Development (MED), now the Ministry of Business, Innovation and Employment, that past estimates of diesel consumption by all forms of road transport have been overestimated by 15 to 20 petajoules (PJ) per year (K. Hammond; Ministry of Economic Development, personal communication, November 25, 2010).

The Ministry of Transport provides information on the average fuel consumption of petrol LPVs and commercial vehicles, motorcycles, and mopeds. 


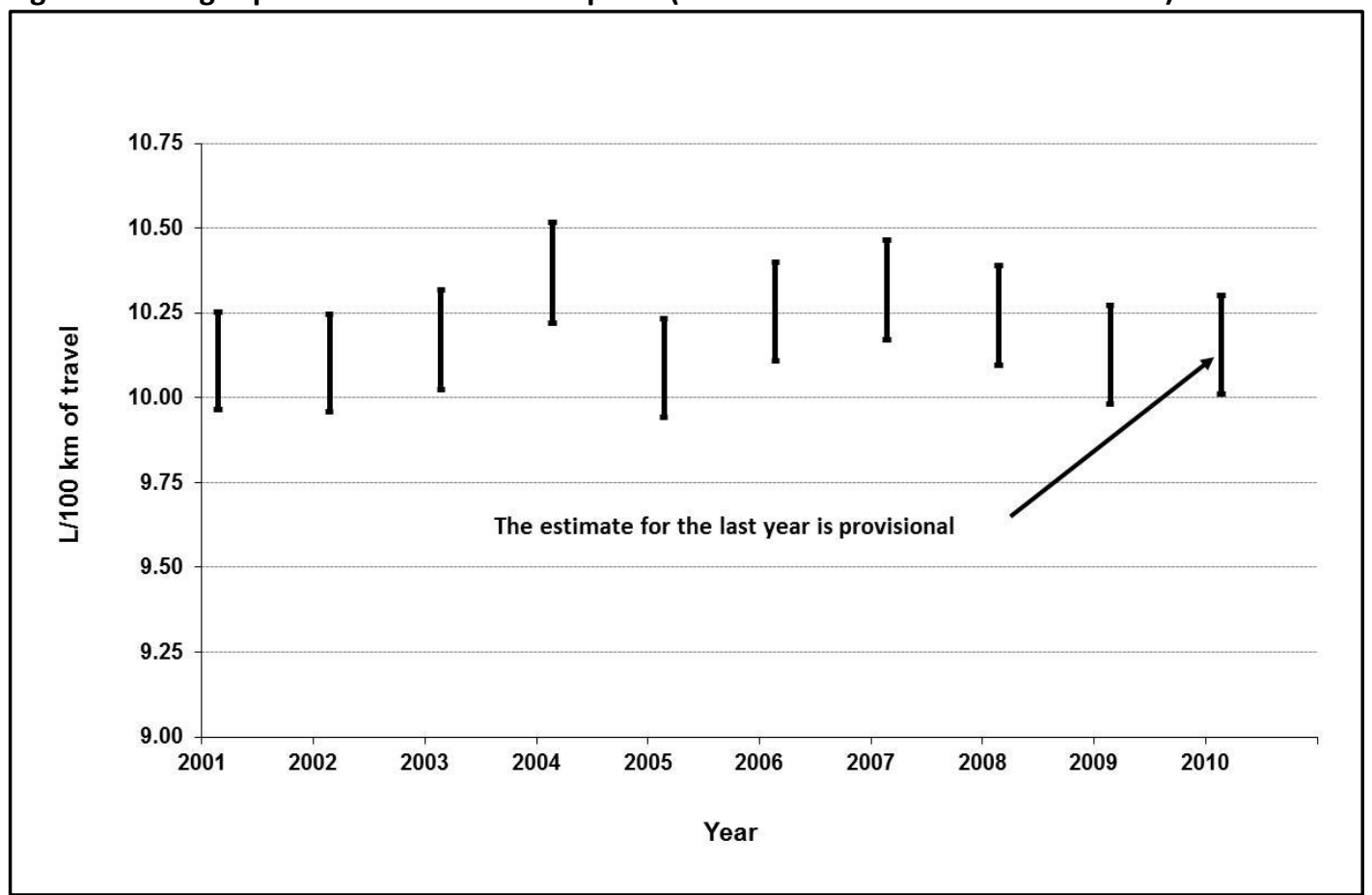

Source: Ministry of Transport (2011)

The estimates in Figure 5.14 of the fuel consumption for the LPV petrol fleet were derived using estimates of the petrol consumed by on-road LPV and the estimated VKT travelled by these vehicles.

The Energy Efficiency and Conservation Authority (EECA) provides in the Energy End Use Database an estimate of the diesel consumption by LPVs in 2007 (Energy Efficiency and Conservation Authority, 2007). Using the EECA data in conjunction with the Ministry of Transport's VKT data for 2007 resulted in an estimated average fuel consumption of $10.1 \mathrm{~L} / 100 \mathrm{~km}$ for petrol LPVs and $12.3 \mathrm{~L} / 100 \mathrm{~km}$ for diesel LPVs.

The Ministry of Transport and EECA's petrol consumption estimates are reasonably consistent and there is some confidence in using this estimate. However, EECA's diesel consumption figures are based on MED data, and for the reasons discussed, the diesel fuel consumption figures should probably be considered an overestimate. For this study, ECCA's diesel fuel consumption estimate for 2007 was revised to take account of MED's view that diesel consumption by road transport has been overestimated by 15 to 20 petajoules (Table 5.12). 
Table 5.12: Estimated fuel consumption estimate for petrol and diesel cars in 2007

\begin{tabular}{|l|c|c|c|}
\hline Energy End Use & $\begin{array}{c}\text { Delivered energy } \\
\text { Cars }\end{array}$ & $\begin{array}{c}\text { VKT } \\
\text { (EECA) }\end{array}$ & $\begin{array}{c}\text { Base year } \\
\text { (2007) } \\
\text { Transport) }\end{array}$ \\
\hline Petrol & 98.64 & $28,279,292,964$ & 10.1 \\
\hline Diesel & 14.99 & $3,195,110,850$ & 12.3 \\
\hline Diesel (revised) & & & 9.6 \\
\hline
\end{tabular}

Source: Energy Efficiency and Conservation Authority (2007), Eng et al. (2008); and Ministry of Transport (2010a)

Figure 5.15 shows data on the average fuel consumption of new light diesel vehicles (both passenger and commercial) entering the New Zealand LPV fleet from 2005 to 2010. During this period, diesel vehicles were, on average, $5 \%$ more efficient than new petrol cars (Ministry of Transport, 2011). All other things being equal, diesel engines are around $20 \%$ more fuel efficient than petrol engines (Bandivadekar et al., 2008). However, the diesel light passenger and commercial vehicles that entered the New Zealand LPV fleet have, on average, engines that are 22-26\% larger than petrol vehicles (Ministry of Transport, 2010a). This has the effect of reducing the efficiency advantage of the diesel vehicle.

Figure 5.15: Average petrol and diesel fuel consumption of new cars entering the light passenger vehicle fleet - European test regime

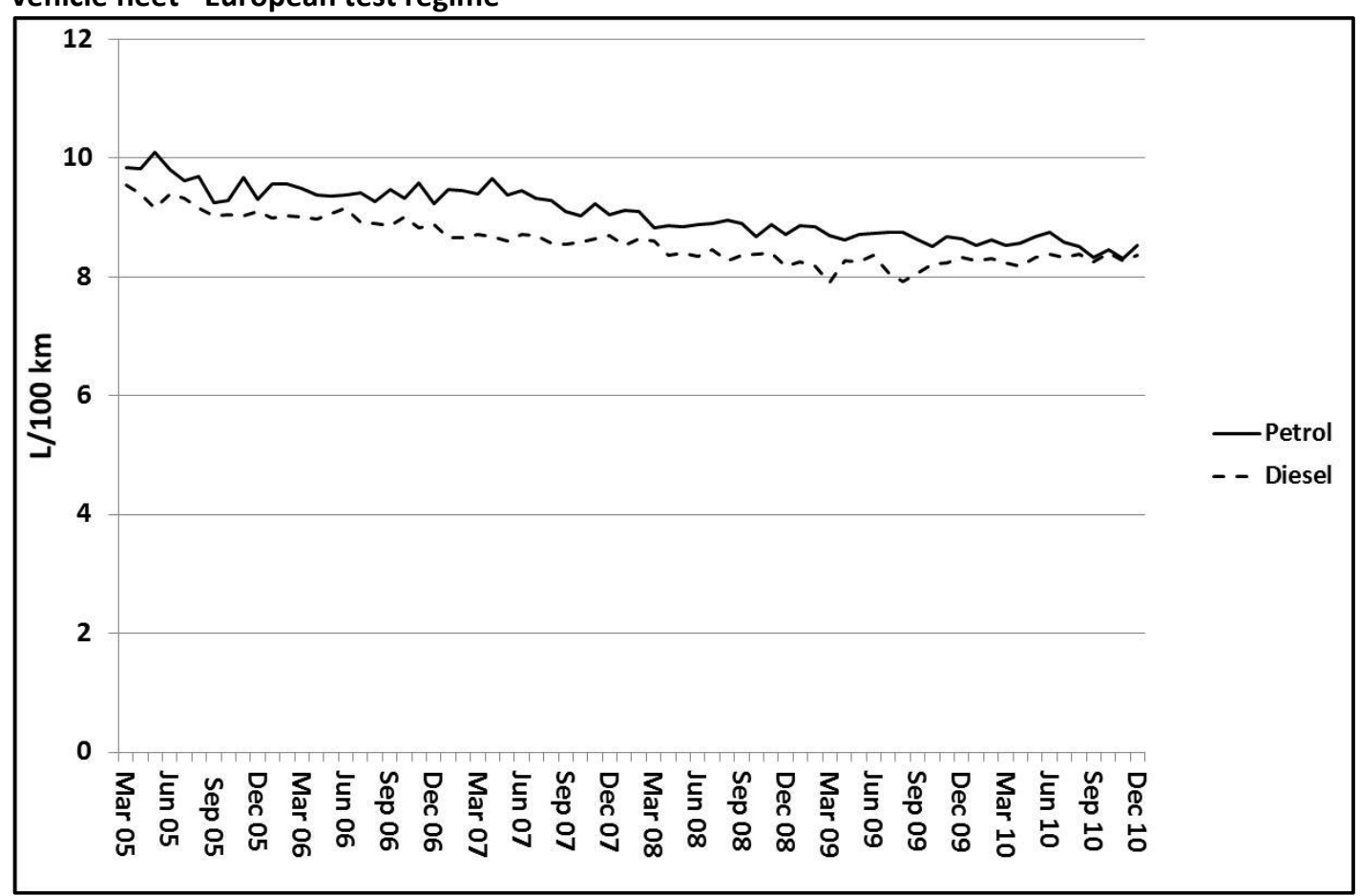

Source: Ministry of Transport (2011) 
The Ministry of Transport data does not separate out passenger from commercial diesel vehicles and it is not possible to determine if there is a difference in the average engine size of these two classes of vehicle. For this study, it was assumed that there is no difference between the average engine size of passenger and commercial diesel vehicles due to the dominance of diesel powered SUVs in the diesel LPV fleet. Using these assumptions, diesel LPVs entering the LPV fleet would be, on average, $5 \%$ more fuel efficient than petrol LPVs of the same vintage (Table 5.13).

Table 5.13: Estimated fuel consumption estimates for petrol and diesel cars: 2001 to 2009

\begin{tabular}{|c|c|c|c|}
\hline $\begin{array}{c}\text { Ministry of Transport data } \\
\text { L/100 km }\end{array}$ & $\begin{array}{c}\text { Diesel } \\
\text { Estimated } \\
\text { L/100 km }\end{array}$ & $\begin{array}{c}\text { Weighted LPV fleet } \\
\text { average } \\
\text { L/100 km }\end{array}$ \\
\hline 2001 & 9.90 & 9.40 & 9.9 \\
\hline 2002 & 9.89 & 9.40 & 9.9 \\
\hline 2003 & 9.96 & 9.46 & 9.9 \\
\hline 2004 & 10.15 & 9.64 & 10.1 \\
\hline 2005 & 9.88 & 9.38 & 9.8 \\
\hline 2006 & 10.04 & 9.54 & 10.0 \\
\hline 2007 & 10.10 & 9.60 & 10.1 \\
\hline 2008 & 10.03 & 9.53 & 10.0 \\
\hline 2009 & 9.92 & 9.42 & 9.9 \\
\hline
\end{tabular}

\section{Energy efficiency of EVs}

The estimates of energy efficiency for EVs and the electric cycle of PHEVs were derived by adapting the method used in Duke et al. (2009).

The power required to propel a vehicle at a specific velocity is defined by the function:

$$
P_{c}=\frac{C_{d} A \rho V^{3}}{2}+M g R R V
$$

$P_{C}$ is the motive power in Watts;

$C_{d}$ is the aerodynamic coefficient of drag;

$A$ is the vertical frontal area of the vehicle $\left(\mathrm{m}^{2}\right)$;

$\rho$ is the air density $\left(1.2 \mathrm{~kg} / \mathrm{m}^{2}\right)$;

$V$ is the speed $(\mathrm{m} / \mathrm{s})$;

$M$ is the vehicle's total mass; 
$g$ is gravity $\left(9.81 \mathrm{~m} / \mathrm{s}^{2}\right)$;

$R R$ is the rolling resistance of the tyres.

This formula assumes that the engine is $100 \%$ efficient. EVs with brushless motors have estimated efficiencies of $90 \%$ and the formula must be adjusted to account for these losses (Duke et al., 2009).

In addition, the power required for auxiliary functions $\left(P_{a}\right)$ such as lights, air conditioning, and sound system must accounted for.

In this study, energy consumption, in $\mathrm{Wh} / \mathrm{km}$, was calculated using the energy usage formula (Duke et al., 2009):

$$
\left(\frac{P_{c}}{\mathrm{E}}+P_{a}\right) / V
$$

where $E$ is the engine efficiency and $V$ is velocity in $\mathrm{km} / \mathrm{h}$.

If driving behaviour, velocity, road conditions, rolling resistance, and gross vehicle weight are held constant, the energy efficiency of a vehicle is determined by a vehicle's vertical frontal area and the aerodynamic coefficient of drag. The standard measure of aerodynamic efficiency is the equivalent aerodynamic $\operatorname{drag}\left(\mathbf{C}_{d} A\right)$ measure, which is derived by multiplying the vehicle's vertical frontal area and aerodynamic coefficient of drag.

Table 5.14 provides examples of the frontal area, aerodynamic coefficient of drag, and $C_{d} A$ values of a selection of EVs and for comparison the 2010 Toyota Prius hybrid vehicle. 
Table 5.14: Aerodynamic coefficient of drag, frontal area, and equivalent aerodynamic drag values

\begin{tabular}{|c|c|c|c|}
\hline & $\mathrm{Cd}$ & A & $\mathrm{CdA}$ \\
\hline \multicolumn{4}{|c|}{ Ultra efficient CEV } \\
\hline Ultracommuter (test prototype) & 0.25 & 1.20 & 0.30 \\
\hline Aptera 2e & 0.15 & 1.9 & 0.29 \\
\hline \multicolumn{4}{|c|}{ CEV } \\
\hline Smart EV (or Smart Ed) & 0.29 & 2.06 & 0.60 \\
\hline Energetique evMe (Mazda 2 conversion) & 0.30 & 2.23 & 0.67 \\
\hline Mitsubishi iMiEV & 0.24 & 1.94 & 0.47 \\
\hline Electric car Corp Citroen C1 EV IE & 0.30 & 2.06 & 0.62 \\
\hline Hyundai Getz (Blade Electron) & 0.50 & 2.13 & 1.07 \\
\hline Micro Vett e500 & 0.33 & 2.06 & 0.67 \\
\hline CEV Average (excluding ultra-efficient EVs) & 0.33 & 2.08 & 0.68 \\
\hline \multicolumn{4}{|c|}{ GEV } \\
\hline Tesla Roadster & 0.35 & 1.63 & 0.57 \\
\hline Tesla Model S & 0.24 & 2.68 & 0.64 \\
\hline Nissan Leaf & 0.29 & 2.27 & 0.66 \\
\hline eBox (Scion conversion) & 0.32 & 2.48 & 0.79 \\
\hline $\begin{array}{c}\text { GEV } \\
\text { Average (excluding Tesla Roadster sports car) }\end{array}$ & 0.31 & 2.38 & 0.74 \\
\hline Prius 2010 Hybrid EV & 0.25 & 2.17 & 0.54 \\
\hline Chevy Volt PHEV & 0.29 & 2.2 & 0.63 \\
\hline
\end{tabular}

Sources: http://www.mayfco.com/tbls.htm, http://www.internetautoguide.com/,

http://carspector.com/, and

http://ecomodder.com/wiki/index.php/Vehicle_Coefficient_of_Drag_List

Using the CdA values in Table 5.14 as a guide, the values used in this study for CEVs, GEVs, and PHEVs were then derived (Table 5.15). In this study, two sets of frontal area and drag coefficient values were used. The standard efficiency values are the default values used in all but one of the VFM scenarios. The standard values represented the current vehicle design having, on average, similar aerodynamic performances to existing ICEVs and the current generation of EVs.

The high efficiency values were based on the insight of Duke et al. (2009) that EVs are capable of significantly higher levels of energy efficiency. These efficiency improvements have the potential to reduce the costs of EVs, and the energy consumed. For this study, it was assumed that in the high efficiency values do not meet the ultra-high efficiency design standard of vehicles such as the Aptera or Ultracommuter, but achieve current best practice as represented by the 2010 Toyota Prius. It was also assumed that the high efficiency vehicles would utilise low rolling resistance tyres. 
Table 5.15: Vehicle data

\begin{tabular}{|l|c|c|c|c|}
\hline & $\begin{array}{c}\text { CEV- } \\
\text { standard } \\
\text { efficiency }\end{array}$ & $\begin{array}{c}\text { CEV - } \\
\text { high } \\
\text { efficiency }\end{array}$ & $\begin{array}{c}\text { GEV and PHEV- } \\
\text { standard } \\
\text { efficiency }\end{array}$ & $\begin{array}{c}\text { GEV and PHEV- } \\
\text { high efficiency }\end{array}$ \\
\hline A & 2.2 & 1.9 & 2.5 & 2.3 \\
\hline Cd & 0.31 & 0.25 & 0.31 & 0.25 \\
\hline RR & 0.014 & 0.008 & 0.014 & 0.008 \\
\hline air density $\left(\mathrm{kg} / \mathrm{m}^{2}\right)$ & 1.2 & 1.2 & 1.2 & 1.2 \\
\hline Passengers and luggage (kg) & 240 & 240 & 400 & 400 \\
\hline auxiliary power (W) & 500 & 250 & 500 & 250 \\
\hline
\end{tabular}

Adapted from: Duke et al. (2009), http://www.mayfco.com/tbls.htm, http://www.internetautoguide.com/, http://carspector.com/, and http://ecomodder.com/wiki/index.php/Vehicle_Coefficient_of_Drag_List

For CEVs, the assumed weight of payload (passengers and luggage) included two $80 \mathrm{~kg}$ occupants and $80 \mathrm{~kg}$ of luggage. For the larger GEV and PHEVs, the assumed payload comprised four $80 \mathrm{~kg}$ occupants and $80 \mathrm{~kg}$ of luggage. These figures are likely to be an overestimate as the average vehicle occupancy in New Zealand is currently 1.65 persons (Ministry of Transport, 2009a).

The power formula assumes that the vehicle is driven at a constant velocity, that the road is flat, and there is no head wind (Duke et al., 2009). Actual driving conditions and driver behaviour will increase the amount of energy consumed. Real world driving experience indicates that, for many EVs, the manufacturer's stated maximum driving range is often not achieved, and actual driving ranges may be only $70 \%-80 \%$ of the stated amount and can, in some cases, be as low as $50 \%$ (Loveday, 2010; Gordon-Bloomfield, 2010; Muller, 2010).

This issue is not unique to EVs as the energy efficiency figures provided by testing agencies are also overstated for ICEVs (Schipper and Tax, 1994; Schipper, 2011) and could be the result of poorly designed dynamometer drive cycles. Schipper (2011) argues that, when converting test data to actual road fuel efficiencies, the test figures should be increased by $19.5 \%$ for the Euro test (the ones reported here) and $33 \%$ for the Japanese test.

In this study, the output of the power formula was modified to take better account of the effect of real world driving conditions. The method used was to estimate the energy consumption of EVs based on the reported performance under real world 
driving conditions and then, using the velocity parameter, the output of the power formula was adjusted to best approximate these real world values. Due to the wide variation in reported performance of EVs, it was assumed that, on average, real world driving ranges are $75 \%$ of the manufacturer's stated range.

It was found that, by holding all variables (except the kerb weight) constant, using the standard aerodynamic values, and assuming a constant velocity of $80 \mathrm{~km} / \mathrm{h}$, the power model provided a reasonable approximation of the energy efficiency estimates derived from the manufacturers' stated values. If real world driving ranges were assumed to reduce driving ranges to $75 \%$ of the manufacturers' stated values, the best approximation using the power formula was achieved by assuming a constant velocity of $105 \mathrm{~km} / \mathrm{h}$.

Figure 5.16 presents the energy efficiency estimates for CEVs and Figure 5.17 shows these for GEVs and PHEVs. The figures also show the effect on vehicle energy efficiency of improving the aerodynamics of the vehicles while maintaining the same driving behaviour and conditions.

Figure 5.16: Estimated energy efficiencies of CEVs

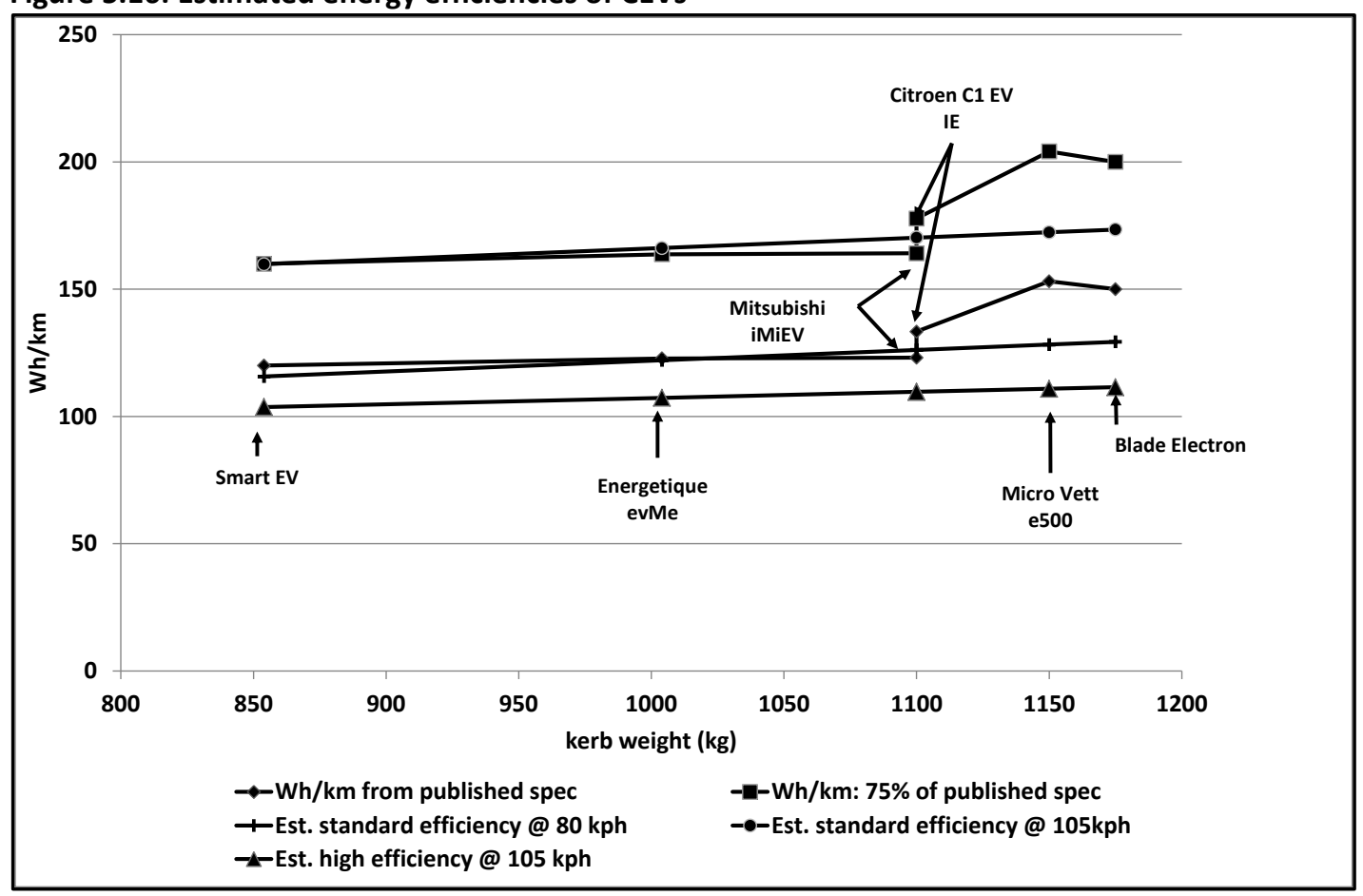


Figure 5.17: Estimated energy efficiencies of GEVs and PHEVs

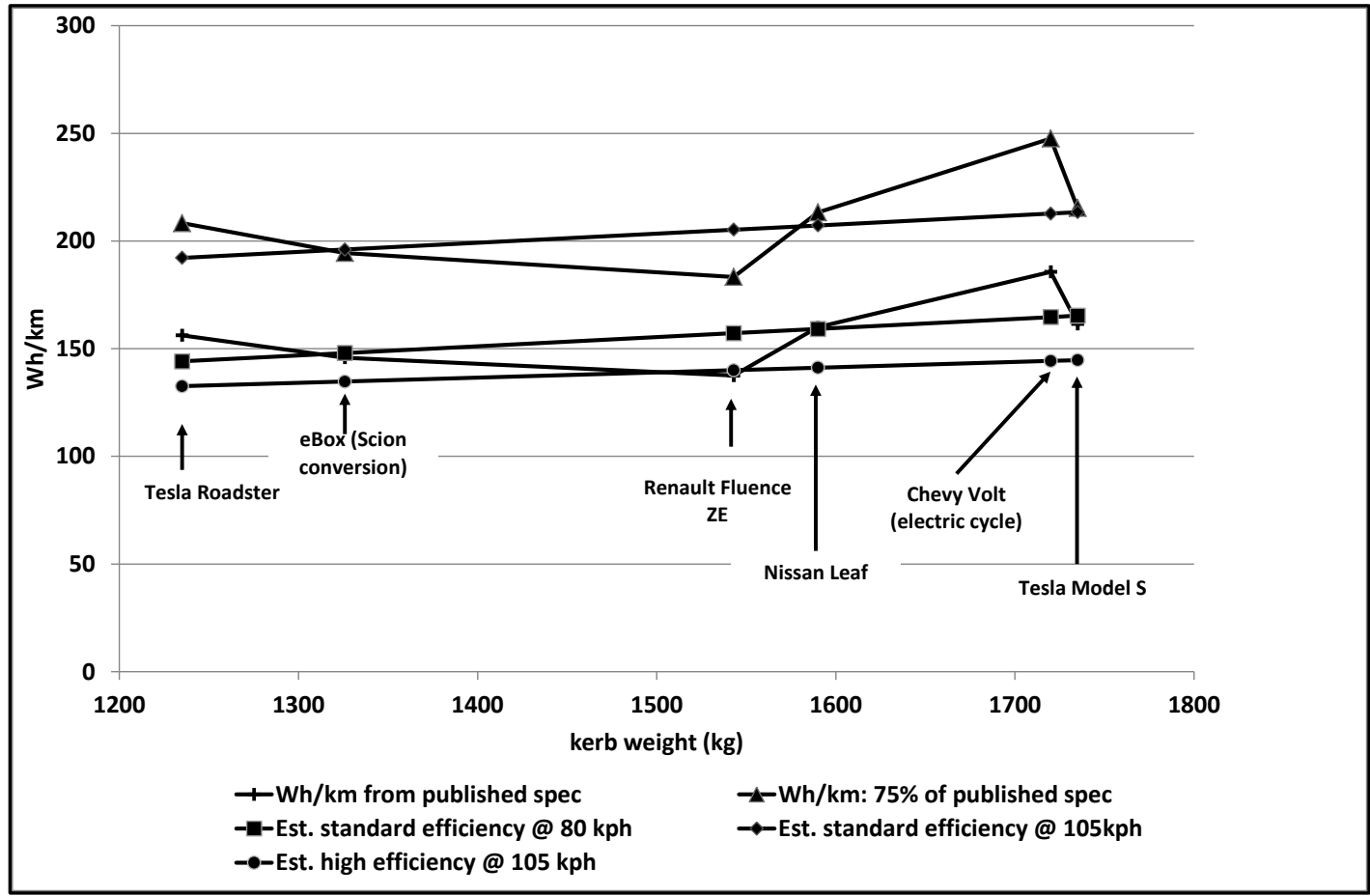

Holding all variables constant (except kerb weight), the energy usage formula can be modelled as a linear function with vehicle kerb weight as the endogenous variable.

The linear functions used in the VFM for the standard and high efficiency scenarios are:

\section{$\underline{\text { Standard efficiency scenarios }}$}

CEV Wh $/ \mathrm{km}=0.0424 *$ Vehicle kerb weight +123.63

GEV and PHEV (electric cycle) Wh/km $=0.0424 *$ Vehicle kerb weight + 139.86

\section{$\underline{\text { High efficiency scenario }}$}

CEV Wh $/ \mathrm{km}=0.0242 *$ Vehicle kerb weight +83.02

GEV and PHEV (electric cycle) Wh/km $=0.0242 *$ Vehicle kerb weight + 102.65

To determine EV energy efficiency per kilometre $(\mathrm{Wh} / \mathrm{km})$ used in the VFM, an estimate of the total vehicle kerb weight taking into account the weight of the 
battery was derived. Battery weight is a function of the battery's total energy content ( $\mathbf{k W h})$, the specific energy of the cell $(\mathbf{k W h} / \mathbf{k g})$, and the additional weight of the control systems and battery structural elements. In turn, the total energy content is a function of the expected driving range under real world driving conditions.

The additional weight due to control systems and structural elements varies considerably, adding between 20 and $60 \%$ onto the final weight of the battery (Kalhammer et al., 2009; Element Energy Limited, 2012). No information has been found to indicate what determines these values, but it was assumed that larger batteries require proportionally fewer control systems and structural elements in relation to the total battery pack weight (Kromer and Heywood, 2007). For this study, it was assumed that: (1) for batteries that were less than $12 \mathrm{kWh}$, there was a 50\% additional weight penalty; (2) for batteries that were between 12 and 15 $\mathrm{kWh}$, there was a $40 \%$ additional weight penalty; and (3) for batteries that were larger than $15 \mathrm{kWh}$, there was a 30\% additional weight penalty.

To test these assumptions, battery sizes were estimated using the same energy capacity figures as the Mitsubishi i-MIEV, Nissan Leaf, and Chevrolet Volt and then compared to the manufacturers' battery weights specifications. This comparison resulted in similar battery pack weights and battery pack specific density values for these vehicles.

For a PHEV battery, adjustments were made to account for usable and non-usable proportions of the battery, and the need to have a battery of sufficient size to meet peak power requirements during the all-electric driving cycle. For PHEV batteries, it was assumed that $60 \%$ of the battery could be used during the CD phase (Pesaran et al., 2009; Moawad et al., 2009).

Due to their smaller batteries, PHEVs require higher power batteries to meet peak power requirements than those in BEVs. However, the trade-off is that high power batteries have lower specific energy values and a larger battery is required to meet the desired driving range. 
For a specified peak kW power output $\theta$, and a battery of size $\alpha \mathrm{kWh}$, the power energy ratio is:

$$
P E=\frac{\theta}{\alpha}
$$

By holding the peak power $\mathrm{kW}$ and driving range fixed, and knowing that the power to energy trade-off can be approximated by a linear function, the adjusted specific energy can be solved using the values given in Kromer and Heywood (2007) with a function taking the form:

Adjusted $\mathrm{kWh}=1.07+-0.182 * \mathrm{PE}$

In this study, it was assumed that standard efficiency PHEVs have a peak power requirement of $120 \mathrm{~kW}$. For the high efficiency PHEVs whose vehicle weight is lower, it was assumed that less power would be required and $100 \mathrm{~kW}$ would be sufficient to meet the user's needs.

To take account of the energy losses that occur during battery charging due to the inefficiencies of battery chargers and batteries it was assumed that the charging cycle of EVs has an efficiency of 82\% (Kintner-Meyer et al., 2007a).

\section{GHG emissions}

The VFM estimates of the direct GHG emissions from the combustion of petroleum by ICEVs and PHEVs were derived using the GHG emission factors for petroleum and diesel in the 2010 Energy Data File (Ministry of Economic Development, 2010a).

\subsection{The VFM scenarios}

\subsubsection{Use of scenarios}

Scenario analysis is a tool that is widely used in business, health, engineering, and environmental studies to explore the possible outcomes from taking, or omitting to take, different actions.

Scenarios are narratives of alternative environments in which to simulate the effect of present day decisions. They are not predictions or strategies, but are hypotheses 
of different futures designed to highlight risks and opportunities of specific strategic issues (Ogilvy and Schwartz, 1998). Building scenarios is a means to explore the possibilities and uncertainties around an issue in a reasonably systematic, rigorous, and policy relevant way (Swart et al., 2004) and to widen perspectives and identify options that may otherwise be missed (Millennium Ecosystem Assessment, 2005, p 36).

Scenario analysis can be quantitative (modelling), qualitative (narrative), or a combination where the narrative scenarios are used to inform the quantitative modelling.

The basis of quantitative scenario analysis is often formal models that use mathematical algorithms and these are widely used in predictive analysis. Swart et al. (2004) considers that this approach is appropriate when algorithms can simulate well-understood systems, but as time and complexity increases, these relationships begin to break down and the usefulness of this approach declines. In such cases, the use of qualitative scenario analysis in conjunction with quantitative analysis is more appropriate.

Qualitative analysis involves the use of narratives that attempt to take account of changes in systems, values, cultural norms, and institutional features. Swart et al. (2004) state that:

Narrative offers texture, richness and insight, while quantitative analysis offers structure, discipline and rigor (p. 141).

The scenarios used in this study to run the VFM comprised elements of both qualitative scenario analysis and quantitative scenario analysis. In this study, the qualitative scenarios provided the framework to develop a range of plausible policy, economic, and technological inputs for use in the VFM.

\subsubsection{Development of VFM scenarios}

The VFM scenarios used in this study were developed using the process proposed by Ogilvy and Schwartz (1998, pp. 57-80). They recommend use of groups to ensure 
inclusion of the widest range of views. For this study, the VFM scenarios were developed using the feedback from the reference group (section 5.2.2) and a review of literature on current and future EV and ICEV technologies.

\section{Objective of the scenario analysis}

The objective of the scenario analysis in this study was to explore, in a systematic, plausible, and manageable fashion, those factors that affect the demand for EVs in New Zealand, and the types and amount of fuel consumed by the LPV fleet.

\section{The main elements or key factors}

Ogilvy \& Schwartz group the key factors in scenario analysis into economic, technological, social, and policy categories. The key factors for this study are summarised in Table 5.16.

Table 5.16: Key factors in the scenario analysis

\begin{tabular}{|c|c|}
\hline Factors & Description \\
\hline Economic factors & $\begin{array}{l}\text { Fuel costs (Price of petrol diesel and electricity) } \\
\text { Price (purchase price, battery replacement price) } \\
\text { Future size of the fleet (vehicle ownership) } \\
\text { Vehicle age, vehicles entering and leaving the fleet }\end{array}$ \\
\hline Technological factors & $\begin{array}{l}\text { Availability of EVs (types of EVs and availability of } \\
\text { used imports) } \\
\text { Technical performance (range, speed, battery life, } \\
\text { energy efficiency) }\end{array}$ \\
\hline Social factors & $\begin{array}{c}\text { Population } \\
\text { Household income } \\
\text { Household size } \\
\text { Vehicle charging behaviour } \\
\text { Endogenous to the model (car buyer preferences } \\
\text { determined by discrete choice model) }\end{array}$ \\
\hline Policy factors & $\begin{array}{c}\text { Price of carbon } \\
\text { Availability of public charging infrastructure } \\
\text { Incentives to develop or buy vehicle technology }\end{array}$ \\
\hline
\end{tabular}

\section{The certainties and uncertainties}

Certainties are those factors that are predetermined and are unlikely to vary significantly across the scenarios. For this study, it was assumed that the following factors would be less likely to change in the future and could be held constant across the scenarios: 
- the relationship between GDP per capita and vehicle ownership per capita

- population growth at $0.8 \%$ per year

- household size (persons per household)

- real household income (income relative to GDP)

- car buyers' purchase preferences (as at 2010).

In addition, a number of simplifying assumptions were made:

- the supply of new EVs would be sufficient to meet global and New Zealand demand from 2012

- the current practice of Japan exporting used vehicles would be extended to EVs

- vehicle occupancy remains at current levels.

The uncertainties modelled in this study comprised the:

- rate of progress in the development of EV battery technology

- rate of decline in EV and battery manufacturing costs and subsequent price reductions

- future rate of increase in the driving range of EVs

- future rate of improvement in the fuel/energy consumption of ICEVs and EVs

- future price of petroleum

- future price of carbon

- extent of public EV charging facilities

- type of vehicle charging behaviour of users of EVs.

For this study, it was assumed that car buyers' preferences will not change over the modelling period. This assumption was made in recognition that it is not easy to predict changes in cultural norms and personal values. However, for the VFM to remain valid it will be necessary to undertake regular stated choice surveys to take better account of these changing values. 


\section{The official future}

The 'official future' is defined as the one that decision makers explicitly or implicitly believe will happen. This future generally assumes that there will be no unexpected changes to the current environment and a continuation of current trends (Ogilvy and Schwartz, 1998). The official future is often known as the reference scenario against which other scenarios are assessed.

In this study, the official future scenario was one where the LPV fleet continued to grow until 2030, but there were no new energy or transport policies introduced beyond those currently in force. In this future, due to increasing exploration costs, petroleum prices continued to increase steadily, but there were no supply constraints resulting in sudden price shocks and the average energy efficiency of ICEVs entering the LPV fleet did not improve. The price on carbon stayed at a relatively low level of $\$ 25$ per tonne of $\mathrm{CO}_{2}$. Without policy support, the availability of EVs remained constrained and the market share of EVs did not increase.

\section{The scenario matrix}

The scenario matrix defines the two most important critical forces or uncertainties of the issue. For this study, the two key uncertainties identified were: (1) the level of support from governments for EVs and other advanced vehicle technologies; and (2) the rate of success in developing EV technology, specifically those research and development breakthroughs that would improve the performance of EV batteries and reduce EV manufacturing costs.

The two axes in Figure 5.18 represent progress in the development of EV technologies and the degree of support for EV and other advanced vehicle technologies. 


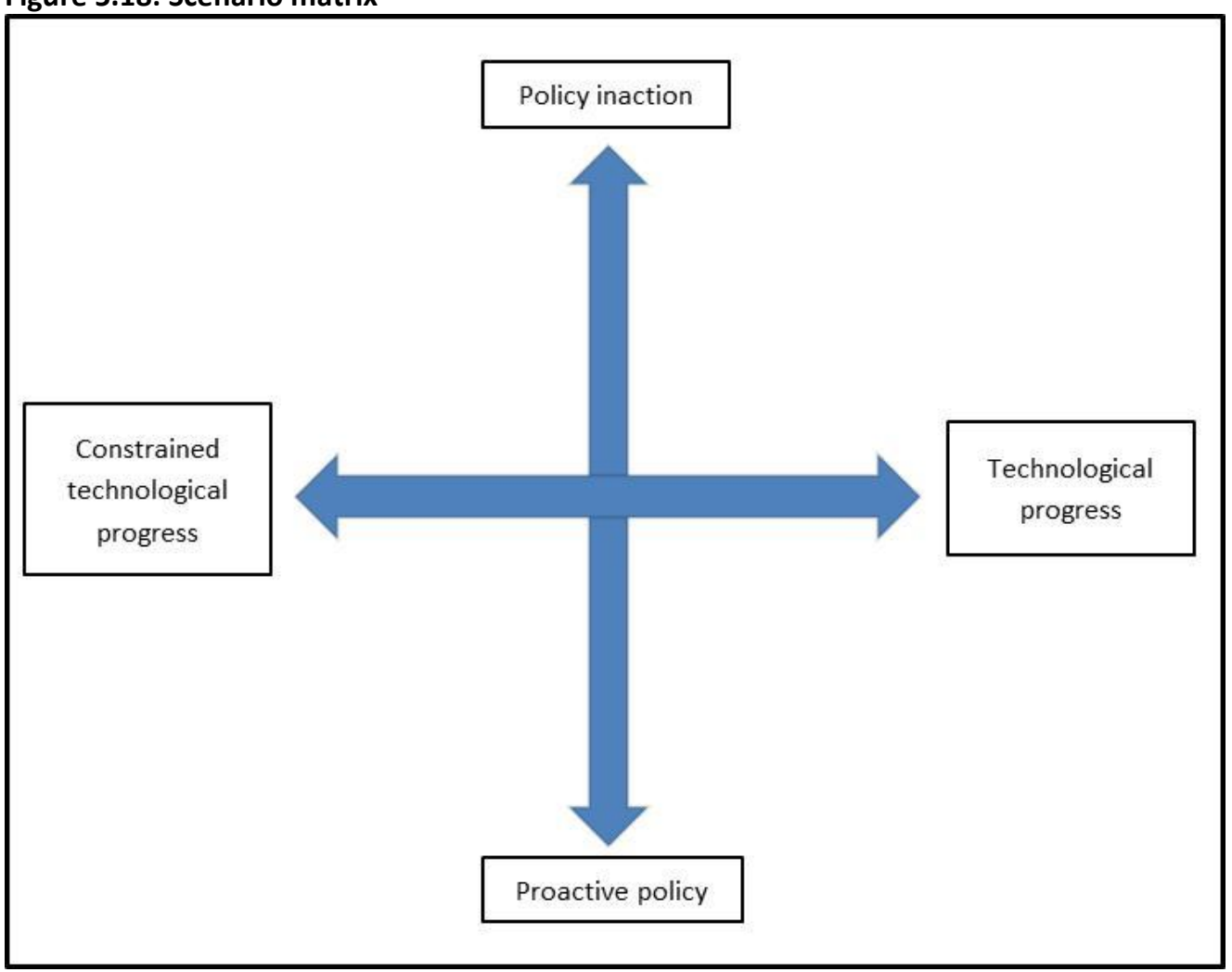

\section{Overview of the VFM scenarios}

The scenarios used in this study were informed by the International Energy Agency's (IEA) World Energy Outlook 2010 (International Energy Agency, 2010) and the New Zealand Energy Outlook 2010 (Ministry of Economic Development, 2010b).

The IEA produces the World Energy Outlook annually. In the 2010 edition, the IEA presented three scenarios. The Current Policies Scenario represents a "business as usual" view of the future. This scenario assumes the continuation of existing and currently implemented policies to address energy demand and GHG emissions. The New Policies Scenario reflects a future where new policies are introduced, but these policies are relatively cautious. The 450 Scenario reflects a future where the policies implemented will be sufficient to limit $\mathrm{CO}_{2} \mathrm{e}$ atmospheric concentrations to $450 \mathrm{ppm}$. This is the level of $\mathrm{CO}_{2} \mathrm{e}$ atmospheric concentration that the IEA regards as sufficient to limit average global temperature to about $2^{\circ} \mathrm{C}$ (International Energy Agency, 2010, p. 53). 
The VFM scenarios in this study were grouped into three scenario families (A, B, and C) informed by the IEA's current policies, new policies, and 450 scenarios. These three different views reflect different levels of policy support towards reducing GHG emissions from the LPV fleet, including the level of support that EVs would receive. In the A family, it was assumed that there was no policy support beyond current levels. In the B family, it was assumed that policy support occurred in response to increasing price of petroleum with the support primarily directed towards improving the fuel efficiency of ICEV based technologies. In the C family, it was assumed that there would be early policy intervention that attempted to reduce GHG emissions from LPVs by promoting the use of EVs and high efficiency ICEVs.

Within each scenario family, the VFM scenarios progress from those that took a relatively conservative view of the rate of improvement of EV technologies to those that envisaged more rapid progress.

In New Zealand, the Ministry of Economic Development (MED) prepared, on a regular basis, an Energy Outlook report. The April 2010 version contained what the MED calls a principal scenario, which has the function of a reference scenario. This scenario was used by the MED to assess the effect of different assumptions of projected growth of GDP, fuel prices, currency exchange rates, and GHG emission prices on the projected demand for energy and GHG emissions. The MED has indicated at that time that further scenarios would be developed to explore the effects of different policy options (Ministry of Economic Development, 2010b).

The MED's Energy Outlook report provided the default GDP, population, currency exchange rates, and electricity price inputs used in the VFM scenarios.

Table 5.17 outlines the overall scheme of the VFM scenarios used in this study and summarises the key features of each scenario. The table indicates those VFM scenarios used for the sensitivity analyses. 
Overview of the vehicle fleet model scenarios

\begin{tabular}{|c|c|c|c|c|c|c|}
\hline & & \multicolumn{2}{|c|}{ No technological progress } & \multicolumn{2}{|c|}{ Technological progress } & \multirow{2}{*}{\begin{tabular}{|c|} 
Tech break-through \\
Rapidly decreasing \\
prices \\
Subsidy on EV \\
prices \\
Drive for EV \\
efficiency
\end{tabular}} \\
\hline & & $\begin{array}{c}\text { No electric vehicles } \\
\text { (reference } \\
\text { scenario) }\end{array}$ & $\begin{array}{c}\text { Steadily decreasing EV } \\
\text { prices }\end{array}$ & $\begin{array}{c}\text { Steadily decreasing } \\
\text { EV prices }\end{array}$ & $\begin{array}{c}\text { Rapidly decreasing EV } \\
\text { prices }\end{array}$ & \\
\hline & Scenario class & 1 & 2 & 3 & 4 & 5 \\
\hline $\begin{array}{l}\text { A Family } \\
\text { Current policies }\end{array}$ & $\begin{array}{l}\text { Moderate growth in } \\
\text { price of oil based } \\
\text { transport fuels to } \\
\text { US\$135 in } 2030 . \\
\$ 25 \text { carbon price }\end{array}$ & $\begin{array}{c}\text { A1 } \\
\text { Sensitivity } \\
\text { Fleet growth/ } \\
\text { scrapping. }\end{array}$ & $\begin{array}{c}\text { A2 } \\
\text { Sensitivity } \\
\text { Fleet growth/ scrapping. } \\
\text { Electricity price. Subsidy. } \\
\text { RUC. } \\
\text { Drive for EV efficiency. }\end{array}$ & A3 & & \\
\hline $\begin{array}{l}\text { B Family } \\
\text { Constrained oil } \\
\text { supply }\end{array}$ & $\begin{array}{l}\text { High and rapid growth in } \\
\text { price of oil based } \\
\text { transport fuels to } \\
\text { US\$250 by } 2015 \text {. } \\
\$ 25 \text { carbon price. }\end{array}$ & & & \begin{tabular}{c|} 
B3 \\
Sensitivity \\
Fleet growth/ \\
scrapping. Electricity \\
price. Subsidy. RUC. \\
Drive for EV \\
efficiency. No EVs.
\end{tabular} & B4 & \\
\hline $\begin{array}{c}\text { C Family } \\
\text { Strong policies }\end{array}$ & $\begin{array}{l}\text { Lower growth in price of } \\
\text { oil based transport fuels } \\
\text { due to measures that } \\
\text { reduce demand } \\
\text { to US\$113 in } 2030 \\
\$ 100 \text { carbon price. }\end{array}$ & & & & \begin{tabular}{|c|} 
C4 \\
Sensitivity \\
Fleet growth/ scrapping. \\
Electricity price. \\
Subsidy. RUC. PHEV \\
Charging. Drive for EV \\
efficiency. \\
No EVs.
\end{tabular} & C5 \\
\hline
\end{tabular}




\subsubsection{Main dimensions of the VFM scenarios}

\section{Population, GDP, vehicle scrapping assumptions}

For all VFM scenarios, it was assumed that New Zealand's resident population would increase by $0.8 \%$ per year, rising from an estimated 4,350,000 in 2009 to $5,142,000$ in 2030 . The basis of this projection was New Zealand Statistics' population series for medium fertility, mortality, and net migration (Statistics New Zealand, 2009).

The default GDP growth assumption used in the scenarios is for growth at $2.4 \%$ per year until 2020 and then $2.2 \%$ per year to 2030 . These values are the same as those used by the MED for the default scenario in the 2010 Energy Outlook (Ministry of Economic Development, 2010b). These assumptions resulted in New Zealand's real GDP, in 1996 prices, increasing from \$137.2 billion in 2009 to \$219.3 billion, in 2030.

The default scrapping assumption (the rate of LPVs leaving the fleet) was that this would occur at the same average rate as for the period 2002 to 2009 (Ministry of Transport, 2010a).

The NZ\$/US\$ exchange rate was assumed to be 0.6 (Ministry of Economic Development, 2010b).

\section{EV availability}

Except for the reference scenario, all VFM scenarios assumed that new EVs would become widely available to car buyers from 2012 and that the current market practice of used imported vehicles from Japan will continue. This assumption means that, over time, supplies of used EVs would become increasingly available for sale in New Zealand (Section 5.3.1). 


\section{Current EV prices}

The VFM scenarios treated the purchase price of EVs as comprising the base vehicle price (BVP) $)^{42}$ and the price of the in-situ battery. The separate treatment of these two elements allowed for different assumptions of the rate of EV and battery development over the modelling period and different rates of depreciation when estimating the price of used imported EVs.

The initial values of the BVPs for the three classes of EV used in this study were derived by subtracting the estimated price of the in-situ battery from the recommended retail price of EVs available in, or about to enter, overseas markets in 2010.

The basis of the battery price estimates used in this study was the price of lithiumion battery cells. In 2009, the prices for battery cells ranged from NZ\$800/kWh to $\mathrm{NZ} \$ 1000 / \mathrm{kWh}$, depending on the battery chemistry and manufacturer. PHEVs and HEVs have smaller battery packs than BEVs and require batteries that have higher power to energy ratios $(\mathrm{W} / \mathrm{Wh})$ to meet peak power requirements necessary for acceleration and high speed cruising (Moawad et al., 2009). HEV batteries have power to energy ratios higher than $30 \mathrm{~W} / \mathrm{Wh}$ and cost significantly more to produce than BEV batteries (Santini, 2010; Kromer and Heywood, 2007).

In this study, PHEVs were assumed to have the minimum electric driving range of $23 \mathrm{~km}$. A PHEV with this driving range would require a battery with a power to energy ratios of less than $20 \mathrm{~W} / \mathrm{Wh}$. Power to energy ratios of this level do not have a price premium that is significantly greater than for BEV batteries (Santini, 2010). In this study, it was assumed that there was no price premium on PHEV batteries.

The fabrication of the battery modules and battery packs adds to the price of the completed battery and these costs vary with different battery designs. In this study, it was assumed that fabrication added approximately $33 \%$ to the final price of BEV batteries.

\footnotetext{
${ }^{42}$ The BVP is the price of the EV excluding the price of the battery.
} 
The fabrication costs constitute a greater proportion of the final battery price for the smaller PHEV batteries (Element Energy Limited, 2012). Using information from Kromer and Heywood (2007), in this study, it was assumed that: (1) for batteries of less than $12 \mathrm{kWh}$, fabrication costs comprise $50 \%$ of the final battery price; (2) for batteries between 12 and $15 \mathrm{kWh}$, fabrication costs comprise $40 \%$ of the final battery price; and (3) for batteries over $15 \mathrm{kWh}$, fabrication costs comprise $33 \%$ of the final battery price.

The Mitsubishi i-MIEV is the most widely available CEV and it is the first CEV produced by a large vehicle manufacturer. Its recommended purchase price, including its battery, varies depending on the location. In Britain, this CEV sold for NZ\$56,500 in 2011 excluding the NZ\$10,000 UK government plug-in car grant (Mitsubishi Motors in the United Kingdom, 2011). In the United States in 2011, the i-MIEV sold for NZ\$33,500 before the NZ\$9,000 federal tax credit (Mitsubishi Motors North America Inc., 2011), and in Australia it sold for NZ\$60,000 (Mitsubishi Motors Australia Limited, 2011). Taking into account the estimated price of the battery, it was assumed that the 2012 BVP for CEVs in New Zealand would be $\mathrm{NZ} \$ 40,000$.

The most widely available general purpose family-sized EV is the Nissan Leaf. The Nissan Leaf is a compact five-door vehicle comparable to the Honda Civic. In the United States, it was priced at NZ\$42,000 in 2011 before the federal tax credit (Nissan North America, 2011). In the United Kingdom, the price in 2011 was NZ\$60,300 before the plug-in grant (Nissan Motor Manufacturing UK, 2011).

Taking into account the purchase price of the Nissan Leaf in the United Kingdom and the estimated price of the battery pack (between NZ\$20,000 and NZ\$24,000), the BVP of this vehicle was estimated to be around NZ\$40,000. This vehicle is a compact family vehicle and the expectation was that a mid-sized family EV would have a higher BVP. In New Zealand, the difference in purchase price between a compact and a mid-sized ICEV range is between NZ\$5,000 and NZ\$10,000. Using these margins as a guide, it was estimated that the current BVP for a midsized EV in the New Zealand market would be NZ\$50,000. 
The Chevrolet Volt is the only mass produced PHEV currently on the market. In the United States, the full retail price is NZ\$48,000 before federal tax credits. In other jurisdictions, the full retail price is higher. In the United Kingdom, the price for these vehicles is NZ\$65,000 before credits (Hendry, 2011). At the time of the study the price of the Volt in New Zealand had yet to be officially announced, but it was estimated at that time it would be approximately $\$ 76,000$ based on an estimate of $\mathrm{NZ} \$ 16,000$ for the battery and a BVP of $\$ 60,000^{43}$.

\section{Future EV prices}

In all the VFM scenarios where it was assumed that EVs would be available for purchase, the expectation was that the prices of EVs and EV batteries would decline over time. The basis of this expectation was past experience with other new technologies and the effects of learning-by-doing (Argote and Epple, 1990; Yelle, 1979) and economies of scale (Spence, 1981; Porter, 1979). The learning curve literature indicates that the rate of decline in the production costs of new technologies varies, but values of $10 \%$ to $25 \%$ for a doubling in cumulative production are typical.

In addition to the effects of learning-by-doing and increasing economies of scale, there is also an expectation that EV prices would decline as the result of ongoing investment in research and development that would lead to technological breakthroughs, which would further reduce manufacturing costs.

The potential for reductions in the BVPs of EVs relative to the purchase price of ICEVs will depend on the type of EV. Due to their additional technical complexity, PHEVs are not expected to ever match the purchase price of an equivalent ICEV and there will always be at least a NZ\$5,000 price premium. However, the BVPs of BEVs could eventually match the purchase price of an equivalent ICEV as the minimum achievable costs of the electric components in a BEV are projected to be at a similar level as the costs of the engine, transmission, and fuel tank in an ICEV (Bandivadekar et al., 2008).

\footnotetext{
43 In late 2012, GM Holden launched the Volt in New Zealand with a retail price of $\$ 85,000$. This price is considerably higher than the Australia recommended purchase price of NZ\$75,500.
} 
Without any significant technological breakthroughs, it was considered that the price of battery cells could decline to $\mathrm{NZ} \$ 300 / \mathrm{kWh}$ and battery packs to NZ\$390/kWh. Recently, the costs of high-energy vehicle grade batteries were declining at the rate of $7.5 \%$ per year (Sankey et al., 2010). If this rate of decline continues, this minimum price level will be reached by 2025 .

The present study took no single view of the effects of learning-by-doing, increasing economies of scale, and research and development on the rate of decline in the prices of EVs and EV batteries. The VFM scenarios present three different views of the future rates of decline in EV prices.

The first view, a 'most conservative' view, assumed that there would be a gradual decline in vehicle production costs, so that by 2030 , the BVP of EVs would reach the minimum level considered achievable for EVs. At this time, the BVP of BEVs would have the same price as the purchase price of an equivalent sized ICEV and PHEVs would be $\$ 5,000$ more expensive. In this view, the price of batteries would continue to decline at the current rate and reach the minimum achievable level in 2025.

The second view projected a more rapid decline in the prices of EVs and batteries. Those VFM scenarios based on this view achieved the same minimum BVP and battery price levels as in the more conservative view, but these levels would be reached in 2020. After 2020, the prices of EVs and batteries would not decline further.

The third view, used in the C5 scenario, represented the effects of early technological breakthroughs on the price of EV batteries. In this scenario, it was assumed that battery cell prices of NZ\$150/kWh and battery pack prices of $\mathrm{NZ} \$ 195 / \mathrm{kWh}$ would be achieved and, as the result of early technological breakthroughs, these price levels would be reached by 2020 .

\section{EV technology}

The VFM scenarios also took account of improvements in battery technology that result in increased EV driving ranges and longer useful battery life. 
The technical specifications in this study were based on different views of the potential of lithium-ion battery technologies. The VFM scenarios in this study contained three different views about the progress of this technology out to 2030 .

The first view conservatively assumed that there would be no significant progress in battery technology. This view assumed that, despite research efforts, the weight and size limitations and useful battery life would remain at current levels. Therefore, EV driving ranges would also remain, on average, at current levels.

The second view assumed steady progress in EV technology over the modelling period so that, by 2030 , lithium-ion battery technologies would meet what are currently considered their practical limits. For this study, it was assumed that the practical limit for advanced lithium-ion battery technologies would be in the order of $300 \mathrm{Wh} / \mathrm{kg}$ at the cell level. This estimate was based on achieving approximately $50 \%$ of the theoretical limit for these types of lithium-ion batteries (Srinivasan, 2008; Global Climate and Energy Project, 2006).

Based on this view, the future driving ranges of BEVs would steadily increase so that, by 2030, CEVs would have a maximum driving range of $200 \mathrm{~km}$, up from the current level of $150 \mathrm{~km}^{44}$, and the electric driving range of PHEVs would increase from $30 \mathrm{~km}$ to $100 \mathrm{~km}$.

It was also assumed that the expectation that GEVs should approach, if not match, the performance of an equivalent ICEV resulted in an assumption that the stated maximum driving range for these vehicles would increase from $180 \mathrm{~km}$ to $500 \mathrm{~km}$ by 2030 .

In the third view used in the technological VFM scenario (C5), it was assumed that incentives introduced by the world's governments would result in increased support for the research into EV technologies and that this investment would be successful in accelerating the development of advanced lithium-ion batteries. These

\footnotetext{
${ }^{44}$ Maximum driving range is the range that the vehicle can be expected to reach under ideal driving conditions with conservative driving behaviour. The VFM assumes that, under real driving conditions, the actual range is $75 \%$ of the maximum range.
} 
breakthroughs in battery technology would result in bringing high energy, long life batteries onto the market by 2020 rather than 2030 .

\section{Progress in ICEV technology}

The assumption in some VFM scenarios was that the energy efficiency of new ICEVs (petrol and diesel) would improve to the highest levels thought to be technically achievable. The potential for petrol and diesel powered vehicles to further improve their energy efficiency and reduce GHG emissions has been explored in a number of recent studies (Bandivadekar et al., 2008; Eads, 2011; Kromer and Heywood, 2009). The consensus in these studies is that, even without reducing vehicle size and power, further energy efficiency improvements are technically possible in ICEVs. These can be achieved by shifting to advanced petrol and diesel engines, hybridising ICEs, improvements in aerodynamic efficiency, and reduction in average vehicle weight.

For these VFM scenarios, it was assumed that, in real world driving conditions, new petrol vehicles entering the LPV fleet could, on average, achieve fuel consumption figures of $5.0 \mathrm{~L} / 100 \mathrm{~km}$, and for diesel vehicles $4.7 \mathrm{~L} / 100 \mathrm{~km}$.

The assumption used in all the VFM scenarios was that diesel vehicles would stay at $10 \%$ of the LPV fleet ${ }^{45}$.

In these VFM scenarios, it was assumed that the improvements in ICE technology would also be incorporated into the ICE drive trains used in PHEVs. The fuel consumption of a Chevrolet Volt, when using its ICE, and operating as an HEV, is 6.4 $\mathrm{L} / 100 \mathrm{~km}$. This figure represents the current fuel consumption of the first generation of PHEVs, when operating in HEV mode. Bandivadekar et al. (2008) estimate the current fuel consumption of HEVs is $75 \%$ that of the current generation of ICEVs, but could be reduced to $40 \%$. This level of improvement, on a fleet average basis, equates to approximately fuel consumption of $4.0 \mathrm{~L} / 100 \mathrm{~km}$ and represents the best performance considered achievable by a PHEV. Diesel powered

\footnotetext{
${ }^{45}$ Currently diesel fuelled LPVs comprise $8 \%$ of all the LPVs entering the fleet, a reduction from the maximum level of 11\% in 2003 (Ministry of Transport, 2010a).
} 
HEVs and PHEVs have yet to come on the market, but the expectation is that these vehicles will be slightly more efficient than petrol powered HEVs or PHEVs. It was assumed that, on average, these vehicles would have an initial fuel consumption of $6.1 \mathrm{~L} / 100 \mathrm{~km}$, but that, over time, the diesel efficiency advantage would diminish and, by 2030, it would be at similar levels to petrol powered HEVs or PHEVs (Bandivadekar et al., 2008).

\section{EV infrastructure}

The discrete choice model is able to take account of the availability of roadside EV charging on vehicle choice. In the C family of VFM scenarios (Table 5.17), it was assumed that there would be a progressive introduction of EV street charging infrastructure into New Zealand cities and major towns.

\section{Treatment of used imported EVs}

The price and technical specifications of used imported EVs entering New Zealand are based on the estimated average age of the vehicle at time of importation (Ministry of Transport, 2009b). The estimated age uses the age distribution of used imported LPVs that entered New Zealand from 2005 to 2009 (see section 5.3.1). Due to the variety of ages and types of used imported vehicles that enter the market it is difficult to estimate the average prices of these vehicles and the used imported vehicle industry does not collect data on the prices of used imported vehicles as they enter the country (P. King, The New Zealand Automobile Association, 21 May, 2009).

Therefore, for this study the price of used imported EVs entering the market was the depreciated BVP of the EVs and in-situ battery.

The rate of depreciation for used imported vehicles was based on information from the Automobile Association of New Zealand, The Dog and Lemon Guide (Matthew and Robertson, 2010, pp. 76-77), and Customs NZ (New Zealand Customs Service, 2011).

For this study, the assumed depreciation on the BVP of an EV in the first year was $35 \%$. From two to five years, the assumed depreciation was $20 \%$ per year, and 
thereafter $5 \%$ per year. The estimates using this method were compared to the prices for comparable used vehicles on the Red Book website to confirm that the assumptions produced reasonable results (The RedBook, 2010).

The value of the batteries in the used imported EVs was estimated using straightline depreciation. It was also assumed that there would be no second-hand market for EV batteries and that an EV battery would have zero value at the end of its life as an EV battery.

\section{Treatment of replacement batteries}

In this study, it was assumed that a car buyer taking account of the cost of the replacement battery at the time of a car purchase bases their decision on current replacement prices, rather than anticipating the future price of replacement batteries.

\section{Residential electricity prices}

The default growth rate in the price of residential electricity was assumed to be $1.1 \%$ per year, based on MED's medium price assumption used in the 2010 Energy Outlook.

The tariffs offered by the electricity retailers were reviewed and it was found that where night tariffs were offered, these tariffs are approximately half of the anytime rate. For this study, it was assumed the night tariff was half that of the day tariff.

\subsubsection{Description of the VFM scenarios}

This section sets out the story lines used to develop each of the scenarios outlined in Table 5.17. The input values for each of the VFM scenarios are set out in Appendix 6.

\section{A: Current policies scenario family}

In this family of VFM scenarios, the world's governments introduce no new policies to address climate change or to reduce energy demand. Without international leadership, the New Zealand Government does not move beyond the current policy 
setting other than to raise the price on carbon to NZ\$25 in 2012. This has the effect of imposing a carbon charge of approximately $6 \mathrm{c} / \mathrm{L}^{46}$.

In these scenarios, the global demand for oil is projected to rise from current levels of around 4,000 Mtoe to 4,550 Mtoe in 2030. Despite increasing demand, oil industry investments are sufficient to find and develop new conventional and nonconventional sources of oil to meet this demand. Developing these resources is more costly and the price of oil increases to US\$135 per barrel (2009 prices), by 2030. These price projections were based on the IEA's Current Policies scenario (International Energy Agency, 2010). Using the method in Donovan et al. (2008), a crude oil price of US\$135 per barrel is equivalent to a New Zealand retail price for petrol of $\$ 2.90$, including GST and a 6 c/L carbon charge.

In New Zealand, with no change in the policy setting the Government's support for EVs remains limited to maintaining the existing exemption from Road User Charges (RUC) and public charging infrastructure remains limited with daytime charging available to those with access to charging facilities at workplaces. As a result, 95\% of EV charging occurs at home after $11 \mathrm{pm}$ on the night tariff.

With no policy in place to encourage energy saving, the overall energy efficiency of the ICEV fleet does not improve.

\section{A1 EVs remain niche vehicles (reference scenario)}

This VFM scenario explored a business as usual view of the LPV fleet where EVs are only available in a very limited range of makes and models, or conversions of ICEVs, and reflects the market situation as of 2010 .

\section{A2 No improvement in EV technology, prices decline steadily}

This VFM scenario explored the possibility that EV technology does not progress beyond current levels, but due to increasing production, the price of EVs and EV batteries steadily declines. The BVP of both BEVs and PHEVs achieve their

\footnotetext{
${ }^{46}$ The development of this family of scenarios occurred before the New Zealand Government elected in late 2012 not to set a second commitment period target for New Zealand under the Kyoto Protocol.
} 
projected minimum prices relative to the equivalent sized ICEVs by 2030 . The price of lithium-ion battery cells continues to decline at current rates and achieves the expected minimum price level by 2025 .

\section{A3 Steady development of EV technology, steady decline in EV prices}

This VFM scenario has the same decline path for BVPs and battery cell prices as scenario A2. However, in this scenario there is progress in the development of lithium-ion battery technology, and, by 2030, the expected practical limits of this type of technology are achieved.

\section{B: $\quad$ Responding to oil shocks}

This family of VFM scenarios explored the impact of an oil-constrained future on vehicle choice and vehicle use. In these scenarios, the price of oil reaches very high levels relatively quickly, sharply increasing the fuel running costs of ICEVs and to a lesser extent PHEVs.

In these VFM scenarios, it was assumed that the effect of rapidly declining production in the major oil producing regions and political disruptions results in the price of oil surging from just over US\$100 per barrel in 2012 to US\$300 per barrel in 2015 (2009 prices). After this time, the market adjusts with reduced global demand for oil and the oil price declines slightly to US\$250 per barrel by 2022 . The price of oil then stays around this level until at least 2030.

This VFM scenario was based on the high oil price projection used in Donovan et al. (2008), but used higher values to reflect the higher oil prices in effect during 2010.

In these scenarios, the world's governments do not introduce new policies to address climate change and the price of carbon stays at a low level (NZ\$25 per tonne). The policy focus in these scenarios is to reduce the reliance on oil by improving the energy efficiency of the petrol and diesel LPV fleet. EVs are considered by policy makers to be a marginal technology and not a viable means of contributing to the goal of rapidly reducing the reliance on oil. In this future, the governments of major vehicle markets impose policies, which, by 2030 , would require that ICEVs be, on average, twice as fuel efficient as at present. 
The efficiency improvements in ICEV technology spill over into the ICEs used in PHEVs and ICE fuel consumption in PHEVs declines to 4 L/100 km by 2030 (Bandivadekar et al., 2008).

As in the A family of VFM scenarios, the New Zealand Government is assumed not to support the introduction of EVs and there is no development of EV street charging infrastructure. As a result, $95 \%$ of vehicle charging occurs at home on the night rate.

B3 Steady development of EV technology, steady decline in EV prices

This VFM scenario assumed that, due to increasing demand, EV technology progresses and EV prices decline.

The EV technological, BVP, and battery cell price parameters are the same as in scenario A3. However, due to the high crude oil prices and policies to improve the energy efficiency of ICEVs, the fuel running costs of ICEV and PHEVs differ from those in scenario A3 (See Appendix 6; Table A6.8).

\section{B4 Steady development of EV technology, faster decline in EV prices}

This VFM scenario has the same parameters for EV technology, BVP, and battery cell price as in the $\mathrm{A} 3$ and $\mathrm{B} 3$ scenarios. The petroleum price path is the same as for scenario B3.

This VFM scenario explored the impact on vehicle choice of the effect of a faster decline in BVP and battery cell prices. It was assumed that due to the increased demand for EV caused by the high price of crude oil, BVPs of BEVs and PHEVs achieve their projected minimum prices earlier. BVPs and battery cell prices reach the projected minimum levels by 2020. After this time, they stay at the same level.

\section{C: Action on climate change}

The basis for this family of VFM scenarios was that, in this future, there is widespread agreement by the world's governments to mitigate climate change and there is recognition that road transport's reliance on fossil fuels should be reduced. 
This concern leads to the introduction of a portfolio of policies intended to reduce the demand for energy and specifically the demand for fossil fuels.

These policies involve the world's governments putting in place the same stringent measures on ICEVs as in the B family of VFM scenarios, but these come into force in 2012. The effect of these policies is that the demand for petroleum declines slightly from 4,000 Mtoe to 3,950 Mtoe by 2030. In 2030, the price of oil is assumed to be US\$113 (2009 prices) per barrel. These policies have the result of avoiding severe constraints on oil supply over the period 2012 to 2030.

This group of VFM scenarios was based on the IEA's 450 scenario, but used the slightly more conservative view of the future price of crude oil from the IEA's New Policies scenario.

As part of New Zealand's response to these initiatives, the Government puts in place a higher price on carbon, of NZ\$100 per tonne in 2012 equivalent to a carbon charge of approximately $23 \mathrm{c} / \mathrm{L}$. The Government also implements measures to ensure that new ICEVs entering the LPV fleet meet the same energy efficiency standards as implemented in other countries.

The New Zealand Government working with local government implements a programme for progressively installing street-located EV charging facilities starting in major cities and then moving to provincial towns. By 2015, the central business districts of all major New Zealand cities have these facilities. By 2020, these street charging facilities are available in the central business districts of all major towns and the inner suburbs of major cities.

Due to the availability of these street EV charging facilities, the amount of daytime charging of BEVs increases to $30 \%$ with some users of PHEVs using these facilities to undertake a top-up recharge during the travelling day to extend the amount of driving using electric power.

\section{C4 Steady development of EV technology, faster decline in EV prices}

This scenario had the same parameters for EV technological, BVP, and battery cell price as in scenario B4. 
Even with the higher carbon charge, fuel costs per kilometre for ICEVs entering the country decline due to improving energy efficiency (see Appendix 6; Table A6.13). The improved energy efficiency of the ICEs in PHEVs also contributes, in conjunction with the greater electric driving range, to the reduction in fuel running costs of these vehicles (see Appendix 6; Table A6.14).

On average, $30 \%$ of PHEV users undertake a secondary top-up recharge, which reduces the average fuel running costs of PHEVs.

\section{C5 Technological and market breakthrough}

This VFM scenario explored the situation where a number of policy initiatives and technological breakthroughs have the effect of significantly lowering the purchase price and running costs of EVs.

In this VFM scenario, technological breakthroughs result in bringing low cost, high energy, long life batteries onto the market ten years earlier than in the other scenarios. The technological breakthroughs reduce the price of battery cells to NZ\$150 by 2020.

In this VFM scenario, concerns about the potential impact of a large numbers of EVs on the security of electricity systems stimulate the world's governments to introduce policies in 2012 that require manufacturers to improve the energy efficiency of EVs.

These policies require that, on average, EVs have aerodynamic performance comparable to that of the best ICEVs, low rolling resistance tyres are mandatory, and that the on-board power requirements for air conditioning and other auxiliary functions meet current best practice.

These measures also require that, on average, the kerb weight for EVs, excluding the weight of the battery, is $20 \%$ lower than the current generation of EVs. Manufacturers achieve these weight reductions through the use of lightweight materials and reductions in the average size of the EVs produced (Bandivadekar et al., 2008; Duke et al., 2009). 
A consequence of this policy is that EVs can operate using smaller batteries to travel the same distance and this has the effect of further reducing the purchase price of EVs and costs of replacement batteries.

To promote EVs in New Zealand, the Government is assumed to introduce a $20 \%$ subsidy on the purchase price of new and used EVs entering the country from 2012 to 2030 . The subsidy is capped at the value of $\$ 7,500$ for new EVs and $\$ 3,750$ for used imported EVs.

\subsubsection{Sensitivity analyses}

To test the sensitivity of the output of the VFM, a sensitivity analysis using scenarios $A 2, B 3$, and C4 as a representative subset of the VFM scenarios was undertaken. This analysis tested the sensitivity of the output of the VFM to changes in the assumptions used for determining:

- the future growth in the size of the LPV fleet

- the rate at which vehicles leave the LPV fleet

- the growth rate of the price of residential electricity

- the removal of EVs' exemption from paying RUC

- the impact of different levels of top-up charging by PHEV owners (scenario C4)

- the impact of introducing a subsidy for EV purchases

- factors that affect the energy efficiency of EVs.

\section{Growth in GDP and vehicle scrapping rates}

This sensitivity analysis assessed the impact of changing the default GDP growth and scrapping rate assumptions.

The assumption used for the lower economic growth and scrapping rate sensitivity analysis was that GDP growth would (rather than the default assumption of $2.4 \%$ per year to 2020 and then $2.2 \%$ per year: see section 5.4 .3 ) be $0.9 \%$ per year to 2020 , and then $0.7 \%$ per year to 2030 . This growth projection was based on the low economic growth sensitivity case used by the Ministry for Economic Development 
in the 2010 Energy Outlook (Ministry of Economic Development, 2010b). This sensitivity analysis also assumed that future average scrapping rates would remain at the levels experienced in 2009 when there was a period of low GDP growth $0.1 \%$ per year).

For the higher economic growth and scrapping rate sensitivity analysis, the assumption was that GDP growth would be $3.9 \%$ to 2020 , and then $3.7 \%$ to 2030 . The basis of this growth projection was the high growth projection from the 2010 Energy Outlook. In this study, the high economic growth also resulted in a higher rate of vehicle scrapping similar to those that occurred in 2006, when annual real GDP growth was 3.3\% per year. The GDP projections used for the default and low and high sensitivities are shown in Figure 5.19.

Figure 5.19: GDP 1995/96 prices (\$ millions)

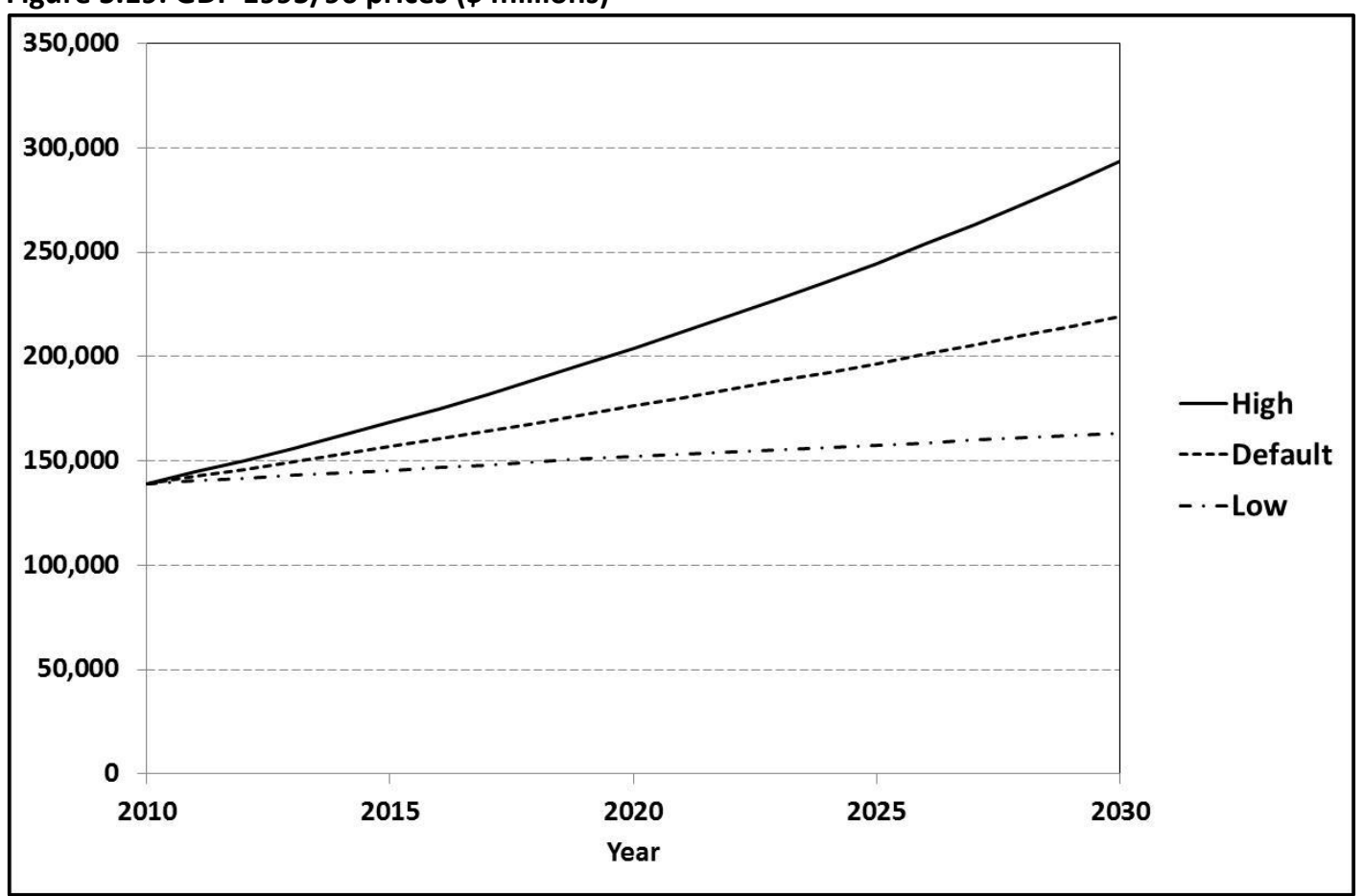

Residential price of electricity

To assess the sensitivity of the VFM to different growth in the price of electricity assumption of the analysis the model was run using low and high rate growth rates. The basis of the high growth electricity price path was the assumption that residential price increases would continue at the historic rate of $2.9 \%$ per year (Ministry of Economic Development, 2010a). The basis of the low electricity price 
path, of $0.75 \%$ per year, was the assumption that, under low economic growth conditions, residential electricity prices would grow at the same rate as the low wholesale electricity price path in the Energy Outlook 2010 (Ministry of Economic Development, 2010b).

Table 5.18: Projected residential electric tariffs

\begin{tabular}{|l|c|c|c|c|c|c|}
\hline Year & \multicolumn{2}{|c|}{$\begin{array}{c}\text { Default tariff sensitivity } \\
\text { case } \\
\end{array}$} & $\begin{array}{c}\text { Low tariff sensitivity } \\
\text { (1.1\% per year growth) }\end{array}$ & \multicolumn{2}{|c|}{$\begin{array}{c}\text { High tariff sensitivity } \\
\text { case } \mathbf{0 . 7 5 \%} \text { per year) }\end{array}$} \\
\cline { 2 - 7 } & $\begin{array}{c}\text { Day } \\
\text { c/kWh }\end{array}$ & $\begin{array}{c}\text { Night } \\
\text { c/kWh }\end{array}$ & $\begin{array}{c}\text { Day } \\
\text { c/kWh }\end{array}$ & $\begin{array}{c}\text { Night } \\
\text { c/kWh }\end{array}$ & $\begin{array}{c}\text { Day } \\
\text { c/kWh }\end{array}$ & $\begin{array}{c}\text { Night } \\
\text { c/kWh }\end{array}$ \\
\hline 2010 & 25.0 & 12.5 & 25.0 & 12.5 & 25.0 & 12.5 \\
\hline 2015 & 26.4 & 13.2 & 26.0 & 13.0 & 28.8 & 14.4 \\
\hline 2020 & 27.9 & 13.9 & 26.9 & 13.5 & 33.3 & 16.6 \\
\hline 2025 & 29.5 & 14.7 & 28.0 & 14.0 & 38.4 & 19.2 \\
\hline 2030 & 31.1 & 15.6 & 29.0 & 14.5 & 44.3 & 22.1 \\
\hline
\end{tabular}

\section{Removal of RUC exemption}

At present, vehicles that are wholly or partially powered by electricity are exempt from RUC, but this exemption will be reassessed in 2013 (Ministry of Transport, 2010b).

The default assumption used in the VFM scenarios was that the RUC exemption would stay in force throughout the modelling period. The VFM could not directly take account of the effect of removing the RUC exemption on vehicle choice. To simulate the effect of its removal, the value of the exemption was capitalised into the purchase price of the EVs.

RUC for light vehicles varies depending on vehicle weight, but for LPVs under a maximum gross weight of $2000 \mathrm{~kg}$, the charge is NZ\$44.31/1000 km. To calculate the present value of all RUC payments, assumptions were made about the period of vehicle ownership, the personal discount rate, and the amount of annual travel.

The average life of LPVs in New Zealand is 20 years (Ministry of Transport, 2009b), but there is no data on the average period of car ownership. The New Zealand Transport Agency does provide data on the number of changes in ownership of cars in a year. Using data from 2010, and dividing the size of the LPV fleet by the number 
of changes in car ownership for that year gives an estimate of the average period of car ownership of 3.5 years (Chrun, 2010). This figure is not considered a reliable estimate due to the large number of transactions undertaken by registered and unregistered vehicle traders. Vehicle traders buy and sell a large number of vehicles, which they then hold for relatively short periods. On balance, it was assumed that a more realistic estimate of the average period of car ownership in New Zealand was 10 years.

In this study, a discount rate of $8 \%$ was used based on the New Zealand public sector discount rate (The Treasury, 2010). It was assumed that car buyer would estimate the annual cost of RUC using the contemporary level of travel demand.

The amount of RUC that would be paid by the owner of a PHEV, absent of the exemption, would depend on the type of fuel used in the ICE. If it is petrol, RUC need only cover the distance travelled on electric power as the levy for using petrol occurred at the pump. If the PHEV used diesel, then without the exemption RUC is charged on all the distance travelled. The assumption used in this study was that $90 \%$ of PHEVs used petrol.

Table 5.19: Present Value of road user charges exemption by vehicle type

\begin{tabular}{|c|c|c|c|c|c|}
\hline \multirow{2}{*}{ Scenario } & \multicolumn{2}{|c|}{ A2, B3, C4 } & A2 & B3 & C4 \\
\hline & $\begin{array}{c}\text { CEV Present } \\
\text { Value }\end{array}$ & $\begin{array}{c}\text { \$ Present } \\
\text { Value }\end{array}$ & $\begin{array}{c}\text { \$ Present } \\
\text { Value }\end{array}$ & $\begin{array}{c}\text { \$ Present } \\
\text { Value }\end{array}$ & $\begin{array}{c}\text { \$ Present } \\
\text { Value }\end{array}$ \\
\hline \hline 2010 & 0 & 0 & 0 & 0 & 0 \\
\hline 2011 & 0 & 0 & 0 & 0 & 0 \\
\hline 2012 & 0 & 0 & 0 & 0 & 0 \\
\hline 2013 & 3470 & 3470 & 3770 & 1620 & 1620 \\
\hline 2015 & 3460 & 3460 & 3760 & 1640 & 1670 \\
\hline 2020 & 3460 & 3460 & 3750 & 2150 & 2420 \\
\hline 2025 & 3460 & 3460 & 3740 & 2560 & 2760 \\
\hline 2026 & 3460 & 3460 & 3740 & 2620 & 2820 \\
\hline 2030 & 3450 & 3450 & 3730 & 2840 & 3020 \\
\hline
\end{tabular}

\section{PHEV charging behaviour}

To test the effect of the top-up charging event on petrol and electricity consumption two sensitivity analyses were undertaken using scenario C4. One 
analysis assumed that no top-up recharging occurred and the other assumed that all users of PHEVs would undertake a secondary top-up charging event.

\section{Street charging availability}

Using scenarios A2 and B3, a sensitivity analysis to assess the effect of the progressive introduction of street charging facilities in these VFM scenarios was undertaken.

As scenario C4 already incorporated this assumption, the sensitivity analysis assessed the effect of removing the street charging facilities.

\section{Imposition of energy efficiency standards on EVs}

To test the sensitivity of the output of the VFM model to the aerodynamics and weight, assumptions used to estimate the energy efficiency of the EVs, VFM scenarios $\mathrm{A} 2, \mathrm{~B} 3$, and $\mathrm{C} 4$ were run using the same high efficiency assumptions as in scenario C5.

\section{Subsidy on purchase price}

Subsidies delivered through grants and tax incentives to promote the uptake of EVs are currently available in a number of countries, including the United States and countries in the European Community (Inland Revenue Service, 2009; European Automobile Manufacturers' Association, 2011; Department for Transport, 2011b). This sensitivity analysis comprised testing the output of the VFM by applying the assumed subsidy used in scenario $\mathrm{C} 5$ on the purchase price of new and used EVs entering the New Zealand LPV fleet to VFM scenarios A2, B3, and C4. 


\section{Chapter 6: Methods II: the generation expansion model}

\subsection{Introduction}

The Electricity Commission's (EC) generation expansion model (GEM) is used in this study to assess the effect of the increased demand for electricity, due to the introduction of EVs, on the future development and operation of the electricity sector. In particular, the GEM allows for the estimation of the additional generation capacity that would be needed and the additional GHG emissions that would be produced by the electricity sector resulting from the introduction of EVs into the New Zealand LPV fleet.

The GEM was developed by the EC from 2006 to 2010 to implement its functions at that time as the regulator of the electricity sector. One of these functions was to authorise electricity transmission pricing, contracting, and investment (Electricity Commission, 2010).

As a means of engaging with the electricity industry, user groups, and interested members of the public, the EC prepared a series of Statement of Opportunities reports (SOO). The last SOO prepared by the EC was published in September 2010 (Electricity Commission, 2010).

The EC stated that the objectives of the SOO were to:

11. Act as an input to Transpower's grid planning activities by:

- forecasting whether the grid would meet the grid reliability standards

- identifying opportunities for economic investment

- developing formal proposals for grid investment

- managing and operating grid assets.

12. Provide a common information source for interested parties.

13. Inform, in an indicative fashion, the consideration of Transpower's Grid Upgrade Plan, (Electricity Commission, 2010).

On 1 November 2010 following a Government review, the EC was restructured and became the Electricity Authority (EA). As a result of the restructuring of the EC, the 
development of future SOOs was moved to the Ministry of Economic Development (MED), now the Ministry of Business, Innovation and Enterprise, and the function of approving Transpower's grid upgrade plans was transferred to the Commerce Commission (Ministry of Economic Development: Electricity Technical Advisory Group, 2009; New Zealand Government, 1986). As part of the restructuring, the responsibility for the maintenance, and further development, of the GEM was also transferred to MED (Wembridge, 2011).

\subsection{Overview of the New Zealand electricity sector}

The five major generation companies produce $90 \%$ of New Zealand's electricity, with the remaining $10 \%$ produced by onsite industrial cogeneration plants and locally owned hydro. As of 2011, total installed generation capacity was 9,751 MW, with hydro generation being the largest component at 5,252 MW (Figure 6.1) (Ministry of Economic Development, 2012).

Figure 6.1: Installed New Zealand generation capacity in 2011

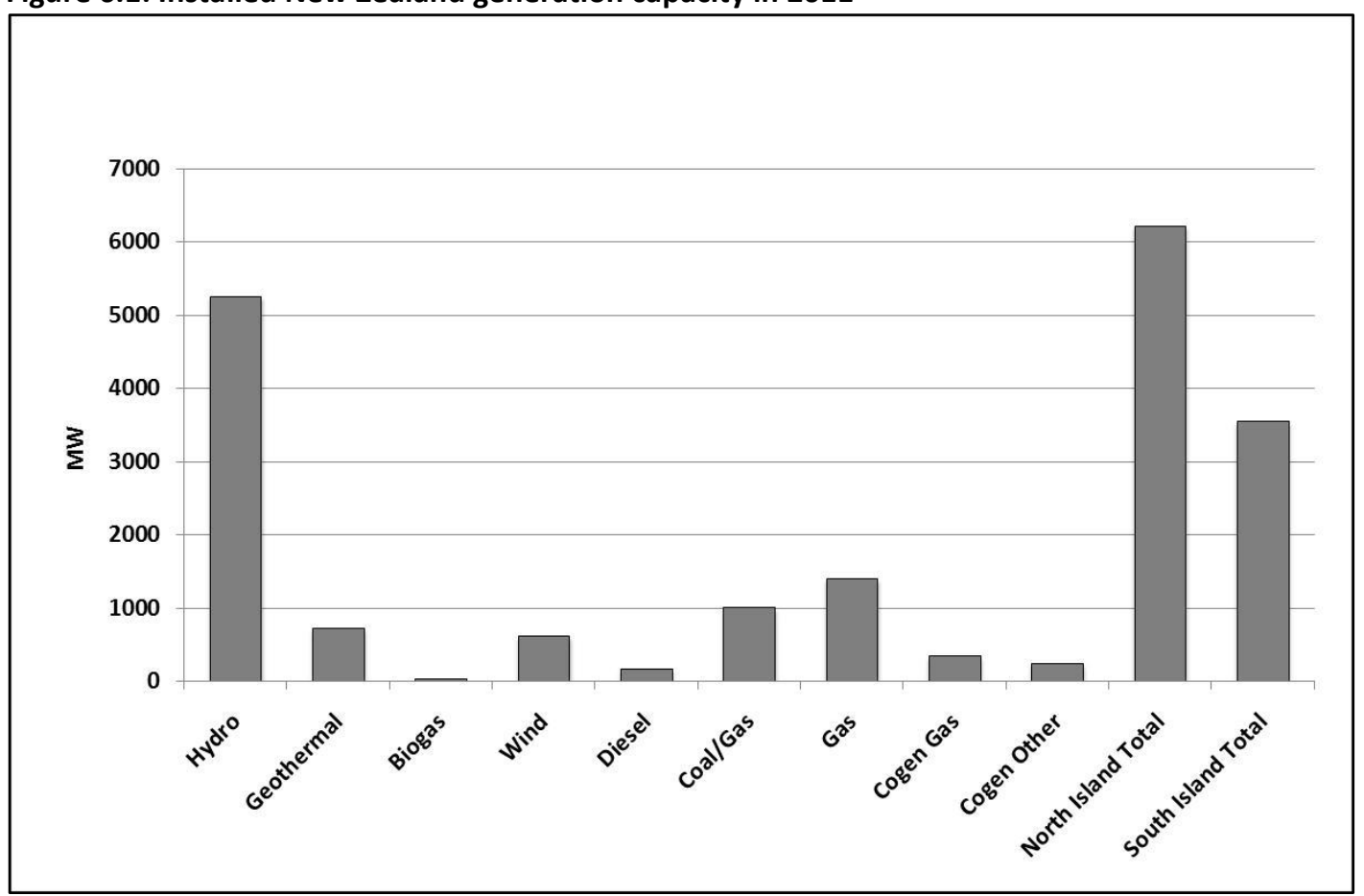

Source: Ministry of Economic Development (2012)

In 2011 , renewable generation comprised $67.9 \%$ of the installed capacity and $76 \%$ of the electricity generated. However, the proportion of renewable electricity has declined from approximately $90 \%$ in 1980 . Since 1999, $600 \mathrm{MW}$ of wind power, and 
$320 \mathrm{MW}$ of new geothermal capacity, have been commissioned. This investment was responsible for the slight increase in the proportion of renewable electricity generated during the period 2008 to 2011 (Figure 6.2) (New Zealand Geothermal Association, 2012; New Zealand Wind Energy Association, 2012).

A key feature of New Zealand's electricity generation assets is that a large proportion of the hydro capacity, comprising $46 \%$ in 2011 , is located in the Waitaki and Clutha catchments in the South Island. These catchments are mainly snow fed and susceptible to variations in precipitation that can result in low hydro flows during some winters. Since 1990, low flows have occurred in 1992, 2001, and 2008. To offset the shortfall in supply from hydro generation, greater use is made of gas thermal generation, and since the conversion of the Huntly station from gas to coal, coal fuelled thermal generation (Figure 6.2) (Bertram and Clover, 2009).

The total amount of electricity generated increased by $37.1 \%$ between 1990 and 2011 from 31,500 GWh to $43,100 \mathrm{GWh}^{47}$.

Figure 6.2: Annual generation and percentage of renewable generation: 1990 to 2011

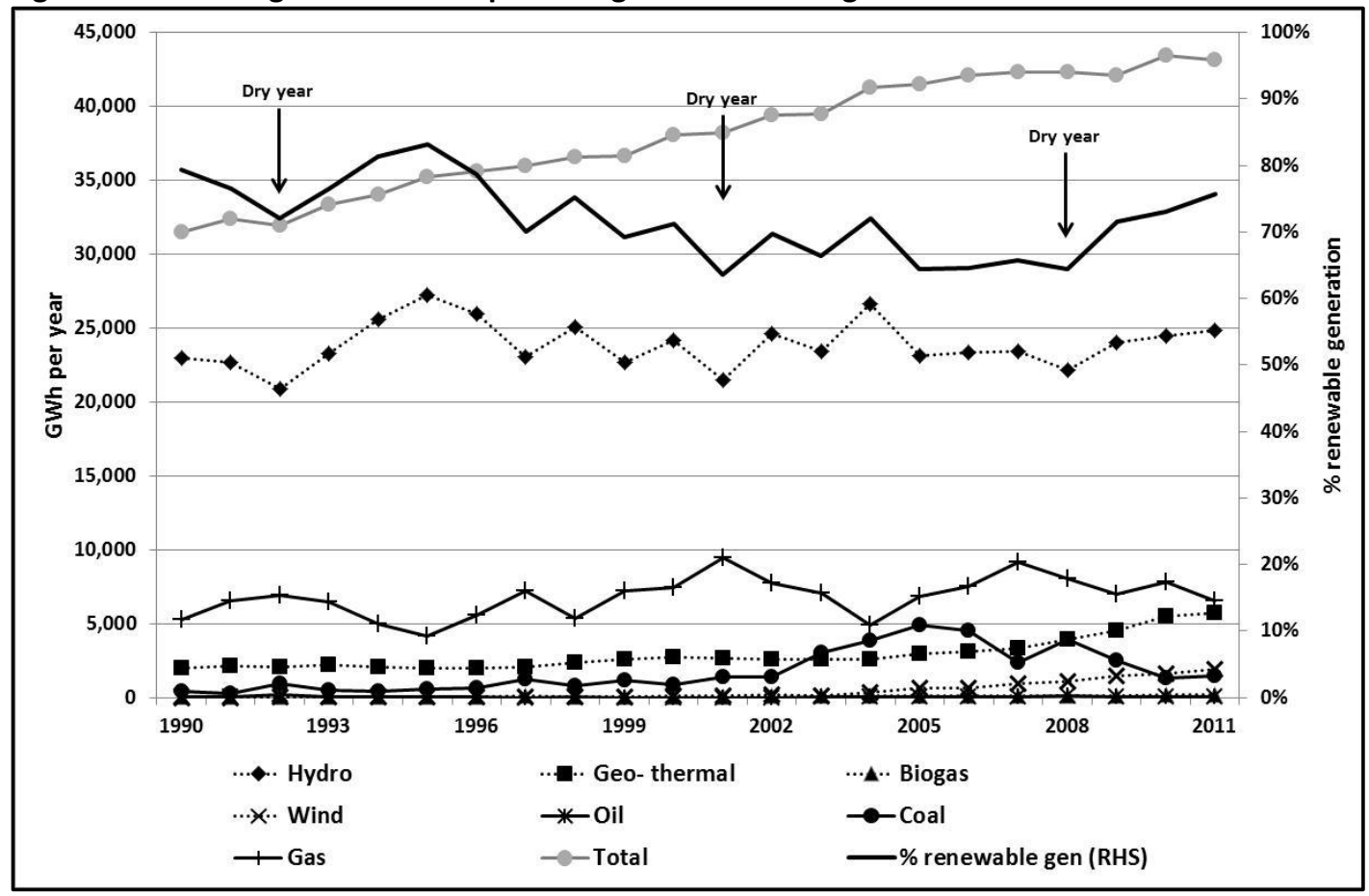

Source: Ministry of Economic Development (2012)

\footnotetext{
${ }^{47}$ This is a provisional figure.
} 
Although approximately $40 \%$ of electricity is generated in the South Island, two thirds of New Zealand's electricity demand is located in the North Island. If the demand from the Tiwai Point aluminium smelter is excluded, this proportion increases to 77\% (Ministry of Economic Development, 2012). Tiwai Point is a special case as it is almost entirely supplied by the Manapouri power station. In 2011, 90\% of the smelter's total demand was met from Manapouri. Although there is interconnection with the national grid, the Manapouri-Tiwai Point system operates, to a large extent, in isolation from the rest of the national grid (Figure 6.3) (Bertram, 2006; Transpower, 2012).

Figure 6.3: Approximate share of national demand in 2011

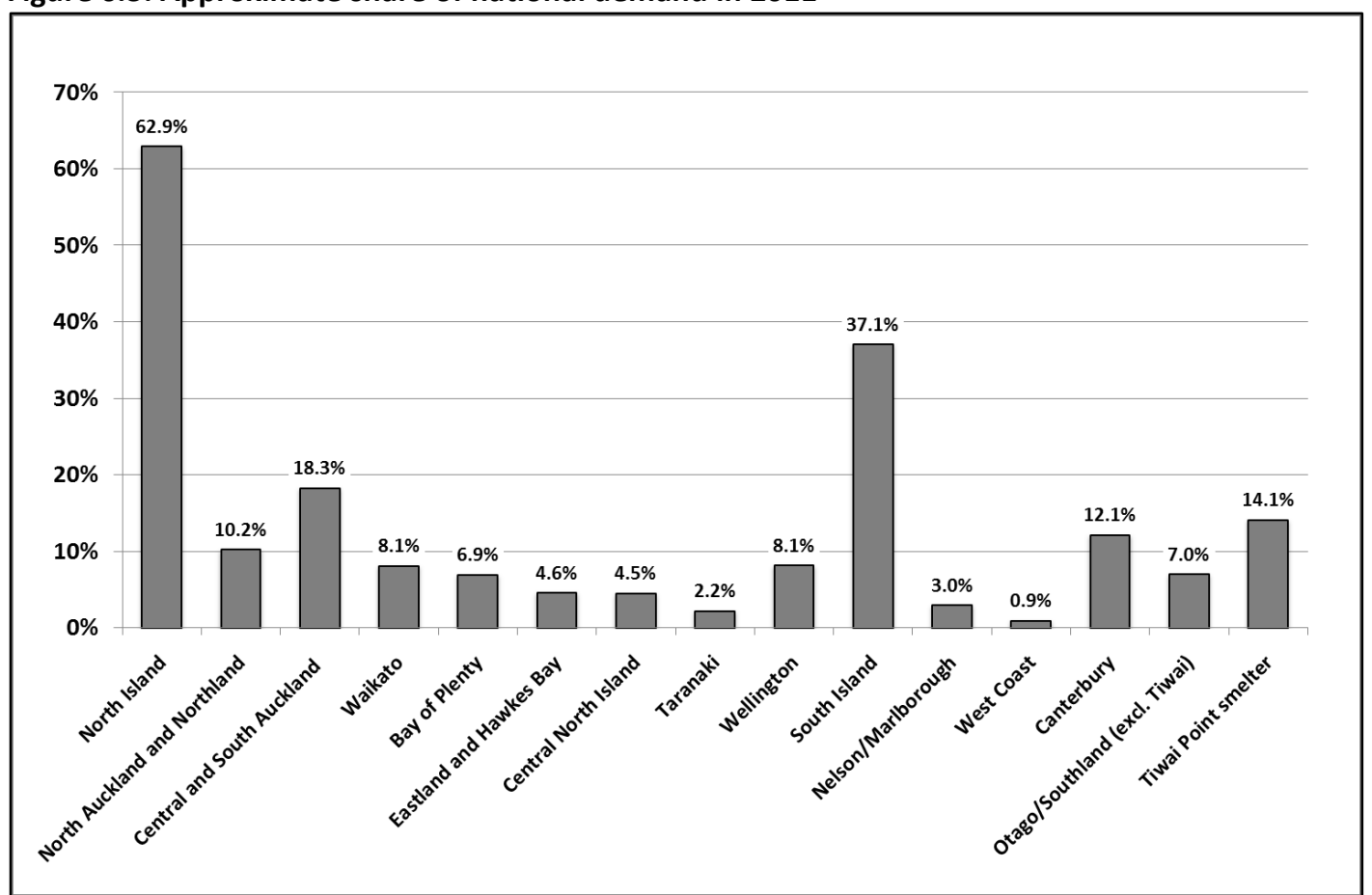

Source: Ministry of Economic Development (2012) and Electricity Authority (2012)

New Zealand's transmission grid transports electricity from the major generation plants to the local distribution networks and a small number of large industrial sites. The transmission grid operated by Transpower comprises four types of transmission line. The backbone of the transmission grid is the $350 \mathrm{kV}$ high voltage direct current (HVDC) link that extends from the Benmore station in the South island to the Haywards substation near Wellington. The HVDC link is used to 
balance generation and demand in the two islands ${ }^{48}$. The rest of the transmission grid consists of $220 \mathrm{kV} \mathrm{AC}$ and $110 \mathrm{kV}$ AC lines supplemented by a small number of 55 kV AC and 66 kV AC spur lines (Electricity Authority, 2011; Transpower, 2012).

The local distribution networks are operated by 29 regionally based companies that utilise a variety of distribution lines ranging from the $230 \mathrm{~V} / 440 \mathrm{~V}$ low voltage distribution networks up to $110 \mathrm{kV}$ feeder lines. However, most common feeder lines voltages are 11 kV AC and 33 kV AC (Electricity Authority, 2011; Electricity Network Association, 2012).

\subsection{Overview of the GEM}

The GEM was designed by the EC to determine, in response to forecast electricity demand, the optimal timing of the commissioning and refurbishment of generation plant and transmission equipment, and, taking into account the merit order and the availability of individual plant simulate an optimal dispatch pattern.

The GEM's objective function is to minimise total weighted average system costs for new generation and plant refurbishment while also meeting a number of constraints. These constraints are to:

- satisfy a fixed load in each load block of each time period within each year

- satisfy peak load security constraints

- satisfy the specified reserves cover

- ensure that the capital costs of a plant are incurred in the same year that the new plant is built

- satisfy energy constraints arising from the limited availability of hydro inflows across different load blocks (the key issue for defining the load block is the availability of wind)

- $\quad$ satisfy the transmission constraints of the HVDC link ${ }^{49}$ (Bishop, 2007).

\footnotetext{
${ }^{48}$ The HVDC is currently being upgraded, as of May 2013 it could transfer $1000 \mathrm{MW}$ in both a northerly and southerly direction and by the end of 2013 the northern flow will be increased further to 1,200 MW (Transpower, 2013).

${ }^{49}$ This list is from the EC's programmers notes within the main GAMs batch file.
} 
Using the optimal commissioning, refurbishment, and dispatch plan, the model can then estimate the consumption of fossil fuel and geothermal fluid and, based on accepted GHG emissions factors, the GHG emissions $\left(\mathrm{CO}_{2} \mathrm{e}\right)$ from the electricity sector.

The GEM is a mixed integer programme model and is operated using the General Algebraic Modelling System (GAMS) software in conjunction with a Mixed Integer/Linear programming solver. These types of linear programming models have variables that can be specified as either real numbers or integers (Chinneck, 2010; Kalvelagen, 2004). This capability is useful in a situation where variables such as generation capacity for specific projects occur in discrete amounts.

Until 2011, as part of its engagement with the electricity industry, user groups, and interested members of the public, the EC freely distributed the GEM and associated input data with the stated aim of promoting transparency and allowing for the exploration of alternative scenarios.

\subsection{The generation scenarios in the 2010 SOO}

To operate the GEM, data is required on:

- the availability of future generation, and demand side, projects ${ }^{50}$

- technical specifications of the projects (type of technology, nearest substation, size, and earliest commissioning date)

- technical specification of the technologies (base or peak load, expected refurbishment life, capacity factors, and depreciation rates)

- the industry discount rate

- capital and operating costs of the projects

- future energy demand at the national, regional, and grid exit point (GXP) ${ }^{51}$ level

\footnotetext{
${ }^{50}$ Availability in this context means the likelihood that that the project could be implemented given different economic, environmental, political, and cultural circumstances.

${ }^{51}$ A GXP is where the local line company takes electricity from the transmission grid. The version of the GEM used in this study (1.5.11) models 192 GXPs.
} 
- future peak loads at the national, regional, and GXP level

- future fuel prices and carbon prices

- load profiles at the various GXPs

- transmission charges.

The approach used by the EC for the 2010 SOO was to develop, in consultation with the industry, five generation scenarios that represented what were considered to be the range of possible development futures for the electricity sector. The assumptions underpinning these generation scenarios were then used to specify the values of the inputs used in the GEM. Table 6.1 shows the main features and assumptions of the five generation scenarios developed for the 2010 SOO.

Many of the inputs were common to all the generation scenarios in the 2010 SOO and some were scenario specific. The key inputs required to operate the GEM are:

- choice of discount rate ${ }^{52}$

- the type and total quantity of new generation and demand side projects available to be modelled

- hydrological data

- availability and price of coal, gas, and diesel; and the price on carbon

- future demand for electricity

- future peak load demand

- electricity demand from EVs (Electricity Commission, 2010).

Many of the assumptions and input values used in the 2010 SO were also used in this study. In the following sections, there is a brief discussion of the key inputs, and the assumptions used by the EC. In section 6.5 , there is a discussion of how some of the inputs used for the SOO were adjusted to align them with the assumptions used in the VFM scenarios.

\footnotetext{
52 It was assumed by the EC that investors in both generation and transmission would require a posttax real weighted average cost of capital (WACC) of $8 \%$.
} 
Table 6.1: Outline of the generation scenarios in the 2010 Statement of Opportunities report

\begin{tabular}{|c|c|c|c|c|c|}
\hline $\begin{array}{l}\text { Scenario } \\
\text { Code }\end{array}$ & MDS1 & MDS2 & MDS3 & MDS4 & MDS5 \\
\hline $\begin{array}{l}\text { Scenario } \\
\text { name }\end{array}$ & $\begin{array}{c}\text { Sustainable } \\
\text { path }\end{array}$ & $\begin{array}{l}\text { South Island } \\
\text { Wind }\end{array}$ & $\begin{array}{c}\text { Medium } \\
\text { Renewables }\end{array}$ & Coal & $\begin{array}{l}\text { High gas } \\
\text { discovery }\end{array}$ \\
\hline $\begin{array}{l}\text { Scenario } \\
\text { summary }\end{array}$ & $\begin{array}{l}\text { Restriction } \\
\text { on new base } \\
\text { load coal- } \\
\text { and gas-fired } \\
\text { stations. Coal } \\
\text { and gas CCS } \\
\text { is available } \\
\text { after } 2030 .\end{array}$ & $\begin{array}{l}\text { Restriction } \\
\text { on new base } \\
\text { load coal- } \\
\text { and gas-fired } \\
\text { stations. Coal } \\
\text { and gas CCS } \\
\text { is available } \\
\text { after } 2030 .\end{array}$ & $\begin{array}{l}\text { Restriction } \\
\text { on new coal- } \\
\text { fired plants } \\
\text { until 2019, } \\
\text { gas scarcity } \\
2020-2030 .\end{array}$ & $\begin{array}{c}\text { Restriction } \\
\text { on coal-fired } \\
\text { plants until } \\
2017 \text {, gas } \\
\text { scarcity } \\
2020-2030 .\end{array}$ & $\begin{array}{c}\text { Restriction } \\
\text { continues on } \\
\text { coal until } \\
2019, \text { though } \\
\text { CCGTs can be } \\
\text { built after } \\
2015 \text {. }\end{array}$ \\
\hline \multirow[t]{7}{*}{$\begin{array}{l}\text { Scenario } \\
\text { specification }\end{array}$} & \multirow[t]{2}{*}{$\begin{array}{c}\text { Focus on } \\
\text { renewables. } \\
\text { High access } \\
\text { to hydro } \\
\text { sites. } \\
\text { Low gas } \\
\text { discovery and } \\
\text { high gas } \\
\text { prices }\end{array}$} & \multirow[t]{2}{*}{$\begin{array}{c}\text { Greater } \\
\text { access to } \\
\text { wind sites } \\
\text { than in MDS1 } \\
\text { specifically in } \\
\text { the lower } \\
\text { South Island, } \\
\text { but less } \\
\text { access to } \\
\text { hydro sites }\end{array}$} & $\begin{array}{l}\text { Renewables } \\
\text { sites } \\
\text { accessible in } \\
\text { both Islands } \\
\text { but hydro } \\
\text { access not to } \\
\text { the extent as } \\
\text { MDS1. }\end{array}$ & 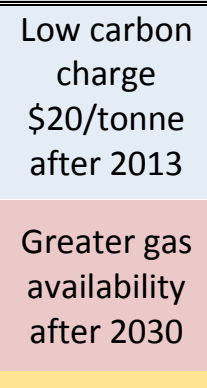 & $\begin{array}{l}\text { Major new } \\
\text { gas } \\
\text { discoveries } \\
\text { keep gas } \\
\text { prices lower } \\
\text { than the } \\
\text { other } \\
\text { scenarios }\end{array}$ \\
\hline & & & $\begin{array}{c}\text { Carbon } \\
\text { charge rising }\end{array}$ & $\begin{array}{c}\text { Little DSM } \\
\text { and EVs }\end{array}$ & $\begin{array}{c}\text { Less DSM } \\
\text { than MDS1 }\end{array}$ \\
\hline & $\begin{array}{c}\text { Carbon } \\
\text { charge of } \\
\$ 60 / \text { tonne by } \\
2018\end{array}$ & $\begin{array}{c}\text { Carbon } \\
\text { charge rising } \\
\text { to } \$ 50 / \text { tonne } \\
\text { by } 2018 .\end{array}$ & \multirow{4}{*}{$\begin{array}{l}\text { Little DSM } \\
\text { and no } \\
\text { uptake of EVs } \\
\text { or carbon } \\
\text { capture } \\
\text { Gas shortage } \\
\text { between } \\
2020 \text { and } \\
2030 \text { (Higher } \\
\text { gas Prices) }\end{array}$} & $\begin{array}{l}\text { uptake is } \\
\text { rapid after } \\
2020 \text { and no } \\
\text { carbon } \\
\text { capture. }\end{array}$ & $\begin{array}{l}\text { and no } \\
\text { uptake of EVs } \\
\text { or carbon } \\
\text { capture }\end{array}$ \\
\hline & $\begin{array}{l}\text { Significant } \\
\text { demand side } \\
\text { management }\end{array}$ & $\begin{array}{l}\text { Less access } \\
\text { to } \\
\text { geothermal }\end{array}$ & & & \multirow{4}{*}{$\begin{array}{c}\text { Carbon } \\
\text { charge rising } \\
\text { to } \$ 40 / \text { tonne } \\
\text { by } 2018 .\end{array}$} \\
\hline & uptake. & $\begin{array}{c}\text { than MDS1 } \\
\text { due } \\
\text { consenting }\end{array}$ & & \multirow{3}{*}{$\begin{array}{c}\text { Very few } \\
\text { hydro } \\
\text { schemes can } \\
\text { obtain } \\
\text { resource } \\
\text { consents. }\end{array}$} & \\
\hline & $\begin{array}{c}\text { Solar, } \\
\text { marine, } \\
\text { carbon } \\
\text { capture, and }\end{array}$ & issues & & & \\
\hline & $\begin{array}{l}\text { Vehicle to } \\
\text { Grid (V2G) } \\
\text { technologies } \\
\text { become } \\
\text { available } \\
\text { after } 2030 .\end{array}$ & $\begin{array}{l}\text { Little DSM } \\
\text { and no } \\
\text { uptake of EVs } \\
\text { or carbon } \\
\text { capture }\end{array}$ & $\begin{array}{l}\text { Tiwai Point } \\
\text { decommissio } \\
\text { ned from mid } \\
2020 \mathrm{~s}\end{array}$ & & \\
\hline
\end{tabular}




\subsubsection{Potential new generation and demand side projects}

The GEM solves each generation scenario by selecting from a menu of potential generation and demand side projects (Interruptible load and voluntary demand side reduction in response to price). The menu of projects was developed by the Electricity Commission who compiled a list of existing, committed, and other possible future projects along with estimates of project capacity, capital and fuel costs, plant performance, depreciation, and occurrence frequency. This list was derived using a variety of sources:

- The Transmission to Enable Renewables project (Hume and Chiu, 2008).

- Other commissioned reports on possible future generation projects (Parsons Brinckerhoff Associates Ltd, 2006).

- Publicly available information such as newspaper articles and generator websites.

- Industry publications such as annual reports.

- Discussion with stakeholders (Electricity Commission, 2010).

The other possible future projects were then divided into those that were near future projects and those that were prospective projects. The former group of projects are those that were expected to be developed in the next few years. The latter group was considered to be much more uncertain in terms of project characteristics and timing. This group also included a number of emerging generation and demand side technologies.

The list did not include projects of less than $10 \mathrm{MW}$ and distributed generation projects. The focus of the SOO was the impact of generation developments on future transmission investment. Distributed generation had either no effect on, or reduced the demand for, increased transmission capacity. The EC assumed that the energy demand forecasts implicitly included the effect of investments in distributed generation. It was also assumed by the EC that distributed generation capacity would grow at the historical rate, although no reason was given for this assumption (Electricity Commission, 2010). 


\section{Committed projects}

The committed projects listed in Table 6.2 were those that, at the time of the development of the SOO generation scenarios, the electricity generators had indicated to the EC that they had already begun developing or would definitely be proceeding with. These projects were included in all the SOO generation scenarios.

Table 6.2: List of committed generation projects used in the $\mathbf{2 0 1 0}$ Statement of Opportunities report

\begin{tabular}{|l|c|c|c|c|c|}
\hline Year & Name & Type & Owner & Island & $\begin{array}{c}\text { Nameplate } \\
\text { MW }\end{array}$ \\
\hline 2010 & Stratford Peaker & Thermal & $\begin{array}{c}\text { Contact } \\
\text { Energy }\end{array}$ & North & 200 \\
\hline 2011 & Te Rere Hau 4 & Wind & $\begin{array}{c}\text { NZ } \\
\text { Windfarms }\end{array}$ & North & 15 \\
\hline 2012 & $\begin{array}{c}\text { Te Uku } \\
\text { (change of use of } \\
\text { existing hydro) }\end{array}$ & Hydro & $\begin{array}{c}\text { Meridian } \\
\text { Energy }\end{array}$ & North & 64 \\
\hline 2013 & Te Mihi & Geothermal & $\begin{array}{c}\text { Contact } \\
\text { Energy }\end{array}$ & South & 160 \\
\hline
\end{tabular}

\section{Near future generation}

At the time of developing the generation scenarios for the 2010 SOO, a number of projects were identified that were considered to be highly likely, but not committed to being developed in the near term. These projects were allocated to those generation scenarios that were most consistent with the development of that type of technology and given a likely earliest development date. The EC acknowledged that this allocation was somewhat arbitrary. Since 2010, some of these projects have been postponed. 
Table 6.3: List of near future generation projects used in the 2010 Statement of Opportunities report

\begin{tabular}{|c|c|c|c|c|c|c|}
\hline Scenario code & & MDS1 & MDS2 & MDS3 & MDS4 & MDS5 \\
\hline Project title & Description & $\begin{array}{l}\text { Sustain- } \\
\text { able path }\end{array}$ & $\begin{array}{l}\text { South } \\
\text { Island } \\
\text { Wind } \\
\end{array}$ & \begin{tabular}{|c|} 
Medium \\
Renew- \\
ables
\end{tabular} & Coal & $\begin{array}{l}\text { High gas } \\
\text { discovery }\end{array}$ \\
\hline Arnold & $\begin{array}{c}\text { Trustpower's proposed } \\
46 \text { MW hydro project } \\
\text { (South Island) }\end{array}$ & 2014 & & & & \\
\hline Central Wind & $\begin{array}{c}\text { Meridian Energy's } \\
\text { proposed } 120 \mathrm{MW} \text { wind } \\
\text { project } \\
\text { (North Island) }\end{array}$ & 2013 & & 2014 & & 2013 \\
\hline $\begin{array}{c}\text { Hawea } \\
\text { Control Gate }\end{array}$ & $\begin{array}{c}\text { Contact Energy's } 17 \text { MW } \\
\text { retrofit hydro project } \\
\text { (South Island) }\end{array}$ & & 2013 & & 2013 & \\
\hline $\begin{array}{l}\text { Kaiwera } \\
\text { Downs }\end{array}$ & $\begin{array}{c}\text { Trustpower's } 240 \mathrm{MW} \\
\text { wind farm } \\
\text { (South Island) }\end{array}$ & 2015 & 2015 & & & \\
\hline Mahinerangi & $\begin{array}{c}\text { Trustpower's } 200 \mathrm{MW} \\
\text { wind farm } \\
\text { (South Island) }\end{array}$ & & 2014 & & & \\
\hline Manapouri & $\begin{array}{l}\text { Meridian Energy's } 90 \text { MW } \\
\text { Hydro Tailrace amended } \\
\text { discharge project } \\
\text { (South Island) }\end{array}$ & 2011 & 2011 & 2011 & & \\
\hline Mill Creek & $\begin{array}{c}\text { Meridian Energy's } \\
\text { proposed } 70 \mathrm{MW} \text { wind } \\
\text { project } \\
\text { (North Island) }\end{array}$ & & & 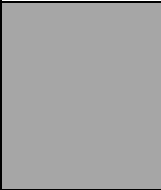 & 2015 & \\
\hline Mokihinui* & $\begin{array}{c}\text { Meridian Energy's } \\
85 \text { MW hydro project } \\
\text { (South Island) }\end{array}$ & & & 2015 & & \\
\hline Ngatamariki & $\begin{array}{c}\text { Mighty River Power } \\
82 \text { MW geothermal } \\
\text { project } \\
\text { (North Island) }\end{array}$ & 2013 & 2013 & 2013 & & 2013 \\
\hline
\end{tabular}


Table 6.3: (cont.)

\begin{tabular}{|c|c|c|c|c|c|c|}
\hline $\begin{array}{l}\text { Scenario } \\
\text { code }\end{array}$ & & MDS1 & MDS2 & MDS3 & MDS4 & MDS5 \\
\hline Project title & Description & $\begin{array}{l}\text { Sustain } \\
\text {-able } \\
\text { path }\end{array}$ & $\begin{array}{l}\text { South } \\
\text { Island } \\
\text { Wind }\end{array}$ & $\begin{array}{l}\text { Medium } \\
\text { Renew- } \\
\text { ables }\end{array}$ & Coal & $\begin{array}{l}\text { High gas } \\
\text { discovery }\end{array}$ \\
\hline $\begin{array}{c}\text { Project Hayes } \\
\text { Stage } 1^{*}\end{array}$ & $\begin{array}{c}150 \text { MW Stage } 1 \text { of } \\
\text { Meridian Energy's } \\
\text { proposed wind project } \\
\text { (South Island) }\end{array}$ & & 2013 & & & \\
\hline $\begin{array}{c}\text { Project Hayes } \\
\text { Stage } 2^{*}\end{array}$ & $\begin{array}{c}160 \text { MW Stage } 2 \text { of } \\
\text { Meridian Energy's } \\
\text { proposed wind project } \\
\text { (South Island) }\end{array}$ & & 2015 & & & \\
\hline $\begin{array}{c}\text { Project Hayes } \\
\text { Stage 3* }\end{array}$ & $\begin{array}{c}160 \text { MW Stage } 3 \text { of } \\
\text { Meridian Energy's } \\
\text { proposed Wind Project in } \\
\text { the South Island } \\
\text { (South Island) }\end{array}$ & & 2016 & & & \\
\hline $\begin{array}{c}\text { Taranaki Co- } \\
\text { generation }\end{array}$ & $\begin{array}{c}50 \text { MW co-generation } \\
\text { proposal } \\
\text { (North Island) }\end{array}$ & & & & & 2015 \\
\hline Te Mihi & $\begin{array}{l}\text { Replacing Wairakei } \\
\text { geothermal with } \\
\text { equivalent capacity (a } \\
\text { further upgrade may } \\
\text { follow) } \\
\text { (North Island) }\end{array}$ & 2013 & 2013 & 2013 & 2013 & \\
\hline Titiokura & $\begin{array}{c}\text { Unison's, now Meridian's, } \\
48 \text { MW proposed wind } \\
\text { project } \\
\text { (North Island) }\end{array}$ & & 2013 & 2012 & & \\
\hline Wairau* & $\begin{array}{c}\text { Trustpower's } 73 \mathrm{MW} \\
\text { hydro project } \\
\text { (South Island) }\end{array}$ & 2013 & 2014 & 2014 & & \\
\hline
\end{tabular}

Postponement announced since 2010

\section{Prospective generation and demand side projects}

The prospective generation and demand side projects varied in terms of development status. Some had received consent under the Resource Management Act 1991 or were proceeding through the consent process. Other projects were proposed by generators, but they had not yet begun the consent process.

Other projects were proposed, but at that time it was uncertain whether they were being investigated or whether they were technically, economically, or politically practicable. These prospective projects comprised: 
- $150 \mathrm{MW}$ of interruptible load (DSM)

- up to 1,300 MW demand side response (DSM)

- 40 possible wind projects ranging from 10 to $540 \mathrm{MW}$

- 12 geothermal projects in the central North Island totalling over 1,000 MW

- over 55 hydro projects distributed throughout New Zealand ranging in size from $10 \mathrm{MW}$ to $340 \mathrm{MW}$ and totalling over $3750 \mathrm{MW}$

- six generic marine projects totalling over $250 \mathrm{MW}$

- nine co-generation projects totalling approximately $450 \mathrm{MW}$

- eight gas-fired CCGT plants totalling near 3,000 MW and 12 gas fired peakers totalling $1250 \mathrm{MW}$ located in Taranaki or the Waikato

- 24 diesel fired thermal peakers totalling approximately 1,500 MW and six $40 \mathrm{MW}$ reciprocating diesel engine generators

- six generic solar plants each up to $50 \mathrm{MW}$

- seven black coal plants each in the 300 to $400 \mathrm{MW}$ range and nominally located at Glenbrook, Taranaki, Christchurch, Tauranga, Northland, and in the Waikato

- two $400 \mathrm{MW}$ lignite plants located in Southland and Otago

- post 2030 , seven coal, gas, or lignite plants with carbon capture and sequestration totalling $2,820 \mathrm{MW}$

- post 2030, 1,000 MW of vehicle to grid (V2G) demand side potential.

Many of these projects were listed as generic with no specific name or developer. This may have been due to the confidentiality agreements with the potential developers, or so that the EC could have the flexibility to include emerging technologies in the generation scenarios (Electricity Commission, 2010). The confidentiality requirement may also have been due to the concern that it was likely that many of these projects would face strong opposition from local communities and other interested parties. 


\section{Allocation of projects to generation scenarios}

The list of potential generation and demand side management (DSM) projects was allocated to each generation scenario in a manner consistent with the future envisaged in that scenario.

Table 6.4 sets out the total potential MW capacity, by project type, available for use by the GEM in each generation scenario. From the list of potential projects, the GEM selects the mix that achieves the model's objective function subject to the various constraints. In many cases, not all the potential generation available in the generation scenario is used by the GEM.

All the generation scenarios contained the potential to install large amounts of gas and diesel peakers which were used by the GEM to provide backup for intermittent renewable generation, primarily wind plant.

The small amount of wave, tidal and solar capacity in the generation scenarios is a reflection of the uncertainty about potential of these technologies. However, in recognition that marine technology is developing rapidly the EC did include six of the most prospective of the marine projects in the list of available projects. Of these six projects, three wave projects were considered suitable for development before 2030.

The EC also considered that grid connected photovoltaic generation and large scale solar plants would become increasingly cost effective over the modelling period. Without specifying the type of technology they included in the list of projects two tranches of $100 \mathrm{MW}$ of solar generation that become available to the GEM in 2015 and 2025. 
Table 6.4: Available generation and demand side projects in the 2010 Statement of Opportunities report

\begin{tabular}{|c|c|c|c|c|c|}
\hline \multicolumn{6}{|l|}{ Potential MW } \\
\hline & $\begin{array}{c}\text { Sustainable } \\
\text { path } \\
\text { MDS1 }\end{array}$ & $\begin{array}{l}\text { South } \\
\text { Island } \\
\text { Wind } \\
\text { MDS2 }\end{array}$ & $\begin{array}{c}\text { Medium } \\
\text { Renewables } \\
\text { MDS3 }\end{array}$ & $\begin{array}{c}\text { Coal } \\
\text { MDS4 }\end{array}$ & $\begin{array}{l}\text { High gas } \\
\text { discovery } \\
\text { MDS5 }\end{array}$ \\
\hline Coal & 0 & 0 & 2,220 & 2,220 & 2,220 \\
\hline Lignite & 0 & 0 & 0 & 800 & 800 \\
\hline $\begin{array}{l}\text { Combined cycle gas } \\
\text { turbine }\end{array}$ & 0 & 0 & 407 & 1,677 & 2,467 \\
\hline Cogeneration, gas-fired & 135 & 135 & 135 & 135 & 135 \\
\hline $\begin{array}{l}\text { Peaker, diesel-fired } \\
\text { OCGT }\end{array}$ & 1,520 & 1,520 & 1,520 & 1,520 & 1,520 \\
\hline $\begin{array}{l}\text { Peaker, fast-start gas- } \\
\text { fired peaker }\end{array}$ & 610 & 1,120 & 1,440 & 1,440 & 1,440 \\
\hline $\begin{array}{l}\text { Reciprocating engine } \\
\text { diesel }\end{array}$ & 240 & 240 & 240 & 240 & 240 \\
\hline Geothermal & 1,065 & 830 & 1,065 & 1,065 & 830 \\
\hline $\begin{array}{l}\text { Cogeneration, biomass- } \\
\text { fired }\end{array}$ & 157 & 157 & 157 & 157 & 157 \\
\hline Cogeneration, other & 80 & 80 & 80 & 80 & 80 \\
\hline Hydro, pumped storage & 600 & 300 & 900 & 300 & 900 \\
\hline Hydro, peaking & 1,348 & 1,198 & 728 & 482 & 901 \\
\hline Hydro, run of river & 1,306 & 1,147 & 436 & 119 & 436 \\
\hline Wind & 2,627 & 4,381 & 4,381 & 3,703 & 4,333 \\
\hline Wave & 114 & 114 & 114 & 114 & 114 \\
\hline Solar & 200 & 200 & 200 & 200 & 200 \\
\hline $\begin{array}{l}\text { Price-responsive load } \\
\text { curtailment }\end{array}$ & 1,000 & 300 & 300 & 300 & 300 \\
\hline Interruptible load & 150 & 150 & 150 & 150 & 150 \\
\hline CCS Gas (post 2030) & 820 & 820 & 820 & 820 & 820 \\
\hline CCS Lignite (post 2030) & 400 & 400 & 400 & 400 & 400 \\
\hline CCS Coal (post 2030) & 1600 & 1600 & 1600 & 1600 & 1600 \\
\hline $\begin{array}{l}\text { Vehicle to Grid (post } \\
\text { 2030) }\end{array}$ & 1000 & 0 & 0 & 0 & 0 \\
\hline Total & 14,972 & 14,692 & 17,293 & 17,522 & 20,043 \\
\hline
\end{tabular}

\subsubsection{Hydro flow data}

The GEM incorporates historical hydrological data from 1932 to 2007 . This data is used by the GEM to estimate the future variability of hydro inflows.

The GEM first estimates the build schedule using $97 \%$ of the average of the hydro inflows from 1932 to 2007 . The build schedule is then re-optimised taking into account the effect of five different hydro inflow sequences that range from very wet to very dry. The three moderate inflows are given more weight than the two 
extreme cases. In this way, the GEM modellers have attempted to simulate the decision faced by investors who do not want to build plant that may be unused during 'wet years'. To incorporate the stochastic uncertainty due to varying hydro inflows, the dispatch simulations are then run a number of times using the hydro inflows from 1932 to 2007 with the final dispatch being the average of all the dispatch runs (Electricity Commission, 2010).

The GEM does not take into account the effect of changing hydrological patterns, due to climate change, on generators' investment perception of risk around future dispatch of generating plant.

\subsubsection{Forecasting demand}

\section{National electricity demand}

The EC forecasts for national level electricity demand were based on three separate models: (1) residential; (2) commercial and industrial; and (3) heavy industry, which comprised the Tiwai Point aluminium smelter. Energy demand forecasts were undertaken at both the national and regional levels (Kirtlan, 2008; Kirtlan, 2009).

At the national level, the EC's forecasts for the residential, and the commercial and industrial sectors were estimated using regression analysis.

For national residential demand the EC found the best specification was a log model, with GDP/capita, number of households/capita, and electricity price as explanatory variables and demand/capita as the dependent variable.

The econometric model developed for the commercial and industrial sector was specified as a levels model with only GDP as an explanatory variable.

Demand from heavy industry represents the demand from one consumer, which is the Tiwai Point aluminium smelter. This demand was assumed to be constant through the modelling period based on annual demand in 2009. 


\section{Treatment of energy efficiency}

These forecasts assumed that future improvement in energy efficiency would continue at historical rates. Therefore, the generation scenarios did not take into account the effect of any policies that would result in significant improvements in energy efficiency.

\section{Regional and GXP energy demand forecasts}

A lack of historical data prevented the development of regional level energy forecast models using econometric methods. Regional forecasts were derived by apportioning residential demand down to the local network area level was based on network level population forecasts using data from Statistics New Zealand (Kirtlan, 2008), and then scaling these forecasts so that the aggregate of these forecasts matched the total demand forecast from the national level residential forecast.

For regional commercial and industrial energy demand, the apportionment was based on regional GDP growth forecasts. These regional GDP forecasts were provided by NZIER (Kirtlan, 2008).

\subsubsection{Peak demand constraints and peak demand forecasts}

The peak load capacity constraints in the GEM require that modelled electricity generation capacity must meet the cold year winter peak demand for each island, while still retaining enough reserve to cover the failure of the single largest generation unit or one HVDC pole. This constraint must be met in all years, including dry hydro years (Electricity Commission, 2010).

For each technology, the GEM allocates a peak contribution factor that indicates the amount of peak power that type of technology can provide (Table 6.5). For thermal, co-generation, geothermal, and hydro backed by storage, these factors were based on the performance of existing plant. For new run-of-river hydro, wind, and marine, the estimates of peak contribution factors were derived from EC simulations. 
Table 6.5: Peak contribution factor used in the generation expansion model

\begin{tabular}{|l|c|}
\hline Technology & Peak contribution factor \\
\hline Thermal (various) & 0.95 \\
\hline Co-generation & 0.60 \\
\hline Geothermal & 0.90 \\
\hline New hydro backed by storage & 0.95 \\
\hline New run-of-river hydro & 0.65 \\
\hline Wind & 0.20 \\
\hline Marine & 0.30 \\
\hline
\end{tabular}

Source: Electricity Commission (2010)

The peak demand forecasts used in GEM are "prudent after diversity maximum demand" (prudent ADMD) peak loads at the national, regional, and GXP levels.

Peak load is defined as the maximum load for a half hour period in any given year. Prudent peak forecasts are projections of the half hourly peak demand by transmission region that have a $10 \%$ chance of being exceeded. ADMD takes account of the fact that the maximum peak that occurs at any distribution node is very rarely as much as the sum of all the loads linked to that node.

The forecasts were developed using peak load data, at the GXP level, for the years 1997 to 2007 . The data was sourced from the Centralised Dataset ${ }^{53}$.

The EC first developed forecasts, based on recent historical trends, of annual region peaks, regional prudent peaks, and the mean expected peak for each GXP. Using these forecasts, diversity factors for each GXP were estimated and prudent ADMD forecasts derived.

For more detail on the methods used by the EC to develop these forecast models refer to Kirtlan (2008), Kirtlan (2009) and Bull (2008).

\subsubsection{Distribution of the load}

The forecast annual energy demand was then distributed across a load duration curve (LDC), which represents the percentage of time that a load will be exceeded.

\footnotetext{
${ }^{53}$ The Electricity Authority is required by the Electricity Industry Participation Code 2010 (Code) to maintain the Centralised Dataset, which provides data on the electricity system to any interested party. The dataset includes information on the demand at each GXP and Grid input point http://www.ea.govt.nz/industry/modelling/cds/
} 
The shape of the LDC was estimated by aggregating all the historical LDC data at 192 GXPs. To make the modelling of load manageable, the time periods in the LDC were grouped into a number of load blocks. The number of blocks has varied with different versions of the GEM. In the version of the GEM used in this study, six quarterly load blocks were used. The three highest load blocks were then subdivided into no wind and wind sub-blocks to take account of the variability of this generation resource. During a low wind period, the output from wind generation was assumed to be half the average output (Electricity Commission, 2010). The load blocks and the annual average percentage of time that demand occurs in each block or sub-block are given in Table 6.6. An example of an LDC produced by the GEM is given in Figure 6.4.

Table 6.6: Load blocks as used in the generation expansion model

\begin{tabular}{|l|c|c|}
\hline Load block code & Load block name & $\%$ of annual half hours \\
\hline b1 & Low wind top block & $0.068 \%$ \\
\hline b1w & Windy top block & $0.205 \%$ \\
\hline b2 & Low wind second block & $0.822 \%$ \\
\hline b2w & Windy second block & $2.466 \%$ \\
\hline b31 & Low wind third block & $2.740 \%$ \\
\hline b3w & Windy third block & $8.219 \%$ \\
\hline b4 & Fourth block & $47.123 \%$ \\
\hline b5 & Fifth block & $27.397 \%$ \\
\hline b6 & Sixth/last block & $10.959 \%$ \\
\hline
\end{tabular}


Figure 6.4: Load duration curve - quarter, low-wind (red), high-wind (green) and medium-wind (blue) sub-blocks

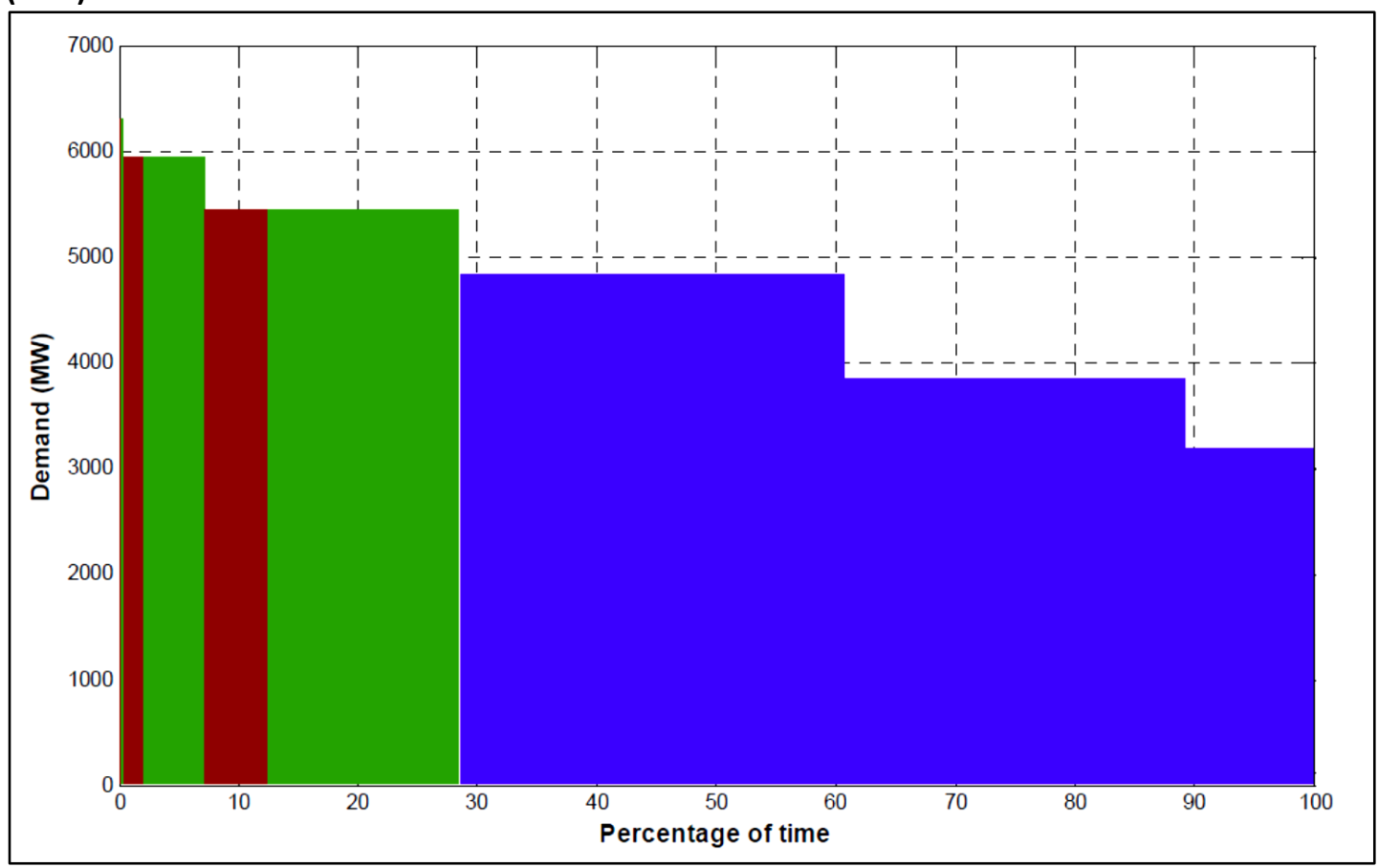

Source: Electricity Commission (2010)

\subsubsection{Fossil fuel availability and prices}

The EC identified the future availability and price of thermal fuels as one of the key drivers of the generation scenarios in the 2010 SOO.

\section{Coal}

In the SOO, the EC's view on the future availability of coal in New Zealand was that between 2010 and 2050 there will be an unlimited supply and prices will increase from \$4 per GJ in 2010 to \$6 per GJ in 2030. The availability of New Zealand lignite for use in electricity generation was also considered to be unconstrained over the modelling period and prices will increase from \$2 per GJ in 2010 to \$3 per GJ in 2030 (Figure 6.5) (Electricity Commission, 2010; Wembridge et al., 2009a). 


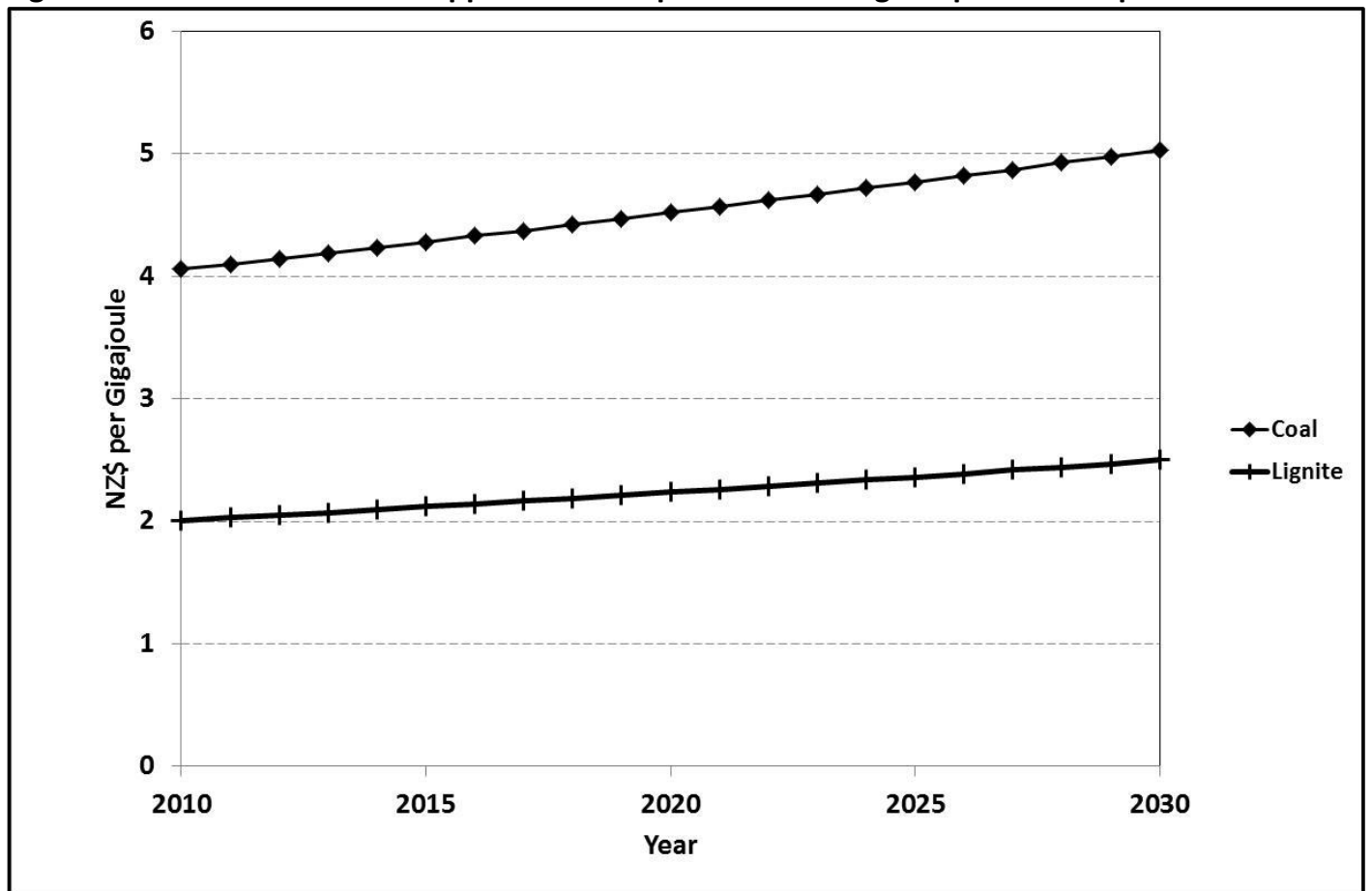

Source: Bishop (2010)

\section{Natural gas}

The EC's analysis concluded that the future availability of natural gas for electricity generation will depend upon three factors: (1) the depletion of currently producing gas fields; (2) the rate and size of future domestic discoveries; and (3) the degree to which New Zealand's gas market becomes linked to international natural gas markets through the importation or, in the case of very high domestic gas discoveries, exportation of gas as liquefied natural gas (LNG) (Hemery, 2009).

The EC's gas supply forecast model estimates of the timing and field size of future gas discoveries were based on historical discovery rates. Therefore, on this basis future domestic discoveries were assumed to occur every two years. The size of the future discoveries was divided into small (5-280 PJ), equivalent to a Rimu or Tariki field, medium (400-1600 PJ), equivalent to the Kapuni or Pohokura fields, and large (2600-4000 PJ) equivalent to the Maui field. The EC's initial assumption was that these different sized fields will be discovered with the same frequency as in the past ( $85 \%$ small field, $10 \%$ medium, and $5 \%$ large). 
This assumption was then reassessed by the EC and the following approach was used to estimate the frequency of future gas discoveries.

For each field size category, the possible production profiles were estimated using a model derived from the production data of existing fields. The probabilities of different future production profiles were then estimated using a Monte Carlo analysis. The Monte Carlo analysis was then run using different likelihoods for finding medium ( $8 \%$ to $12 \%$ ) and large fields (1\% to $5 \%$ ). It was found that the initial value of $5 \%$ for large fields resulted in too large a production forecast and the probability of finding small, medium, and large fields was then revised to $85 \%, 12 \%$ and $3 \%$.

The results from this simulation were sorted into the $10^{\text {th }}, 30^{\text {th }}, 50^{\text {th }}, 70^{\text {th }}$, and $90^{\text {th }}$, percentiles representing very low, low, medium, high, and very high gas production forecasts (Hemery, 2009) (Figure 6.6).

Figure 6.6: Electricity Commission domestic gas production projections

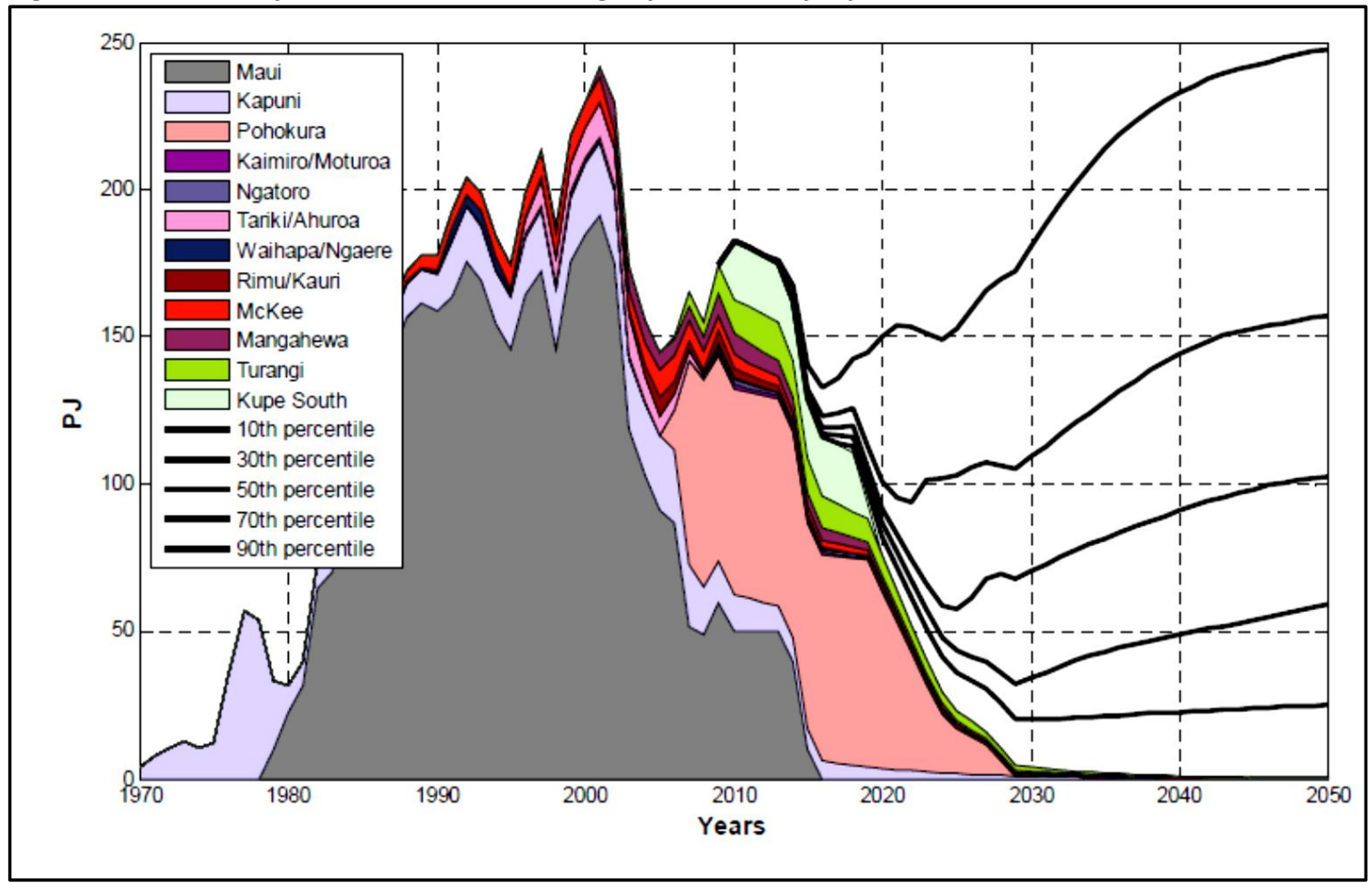

Source: Electricity Commission (2010)

The amount of gas available for electricity generation was estimated as the residual left after the demand from the residential, commercial, and industrial sectors. The EC assumed that the non-electricity gas demand would grow at $1 \%$ per year. 
In the MDS1 and MDS2 scenarios, it was assumed that there would be insufficient domestic gas discoveries $\left(10^{\text {th }}, 30^{\text {th }}, 50^{\text {th }}\right.$ percentiles) to meet New Zealand's total demand. In these generation scenarios, the EC assumed that a LNG import terminal would be completed by 2020 and that, after this time, gas availability can be considered to be unconstrained.

For the MDS3 and MDS4 scenarios, the EC assumed that gas discoveries would follow the $70^{\text {th }}$ percentile forecast and this would have the effect of producing sufficient gas supplies to meet New Zealand's total demand. However, it was also assumed that the production from these discoveries would not come on-stream until after 2030 and gas supplies would be scarce from 2020 to 2030.

In the MDS5 scenario, the EC assumed that gas discoveries would follow the $90^{\text {th }}$ percentile and there would be a high availability of gas in New Zealand (Figure 6.7).

Figure 6.7: 2010 Statement of Opportunities report: gas availability assumptions

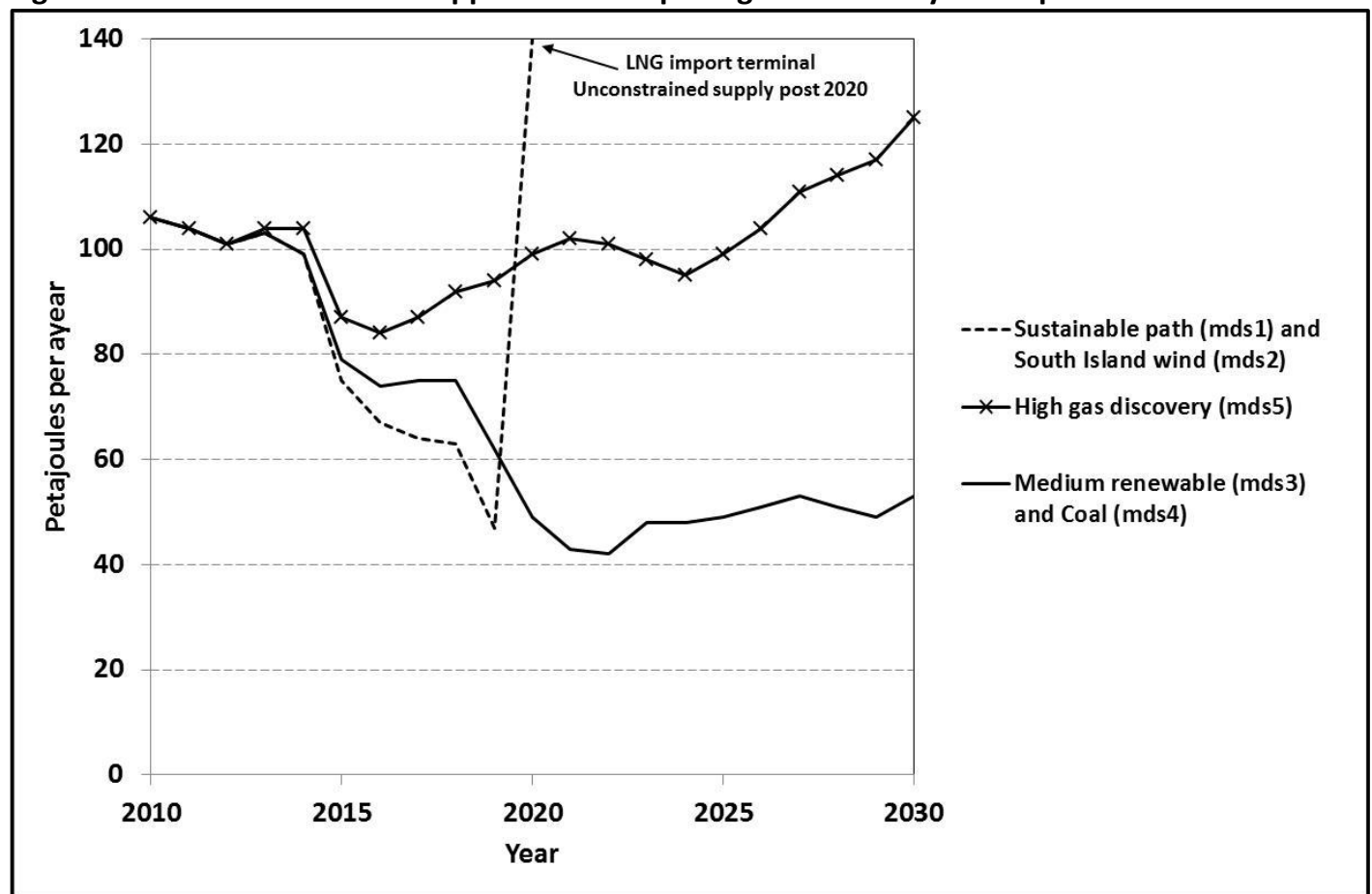

Source: Bishop (2010)

For its gas price projections, the EC used two methods to estimate future gas prices. For short time periods, the EC assumed that the price would follow the historical relationship in New Zealand between the quarterly gas price and quarterly 
production $^{54}$. To take account of contractual and other market constraints on the movement of prices during the short term, the EC put in place a floor and ceiling price (NZ\$3/GJ and NZ\$13/GJ) (Hemery, 2009).

In the longer term for the MDS1 and MDS2 scenarios, where New Zealand production cannot meet domestic demand, the EC assumed that from 2020 LNG would be imported. In these generation scenarios, the domestic price of gas was assumed to trend over the first five years of LNG importation to the international price of gas. For the MDS1 scenario, the EC assumed that the international price would be NZ\$25/GJ. For the MDS2 scenario, the EC assumed that the international price would be NZ\$19/GJ (Electricity Commission, 2010).

The MDS3 and MDS4 scenarios were based on the high gas discovery scenario, which would be sufficient to meet domestic demand, but would not be large enough to result in the construction of a LNG export facility. Therefore, the price of domestic gas would remain decoupled from the international LNG markets. Scarcity of supply during the 2020 s would drive the price up to $N Z \$ 13 / G$, but by 2030 , new supplies would result in a levelling off of prices.

In the MDS5 scenario as originally specified, it was assumed that the increased availability of domestic gas would result in the development of a New Zealand LNG export facility and that this would have the effect of linking New Zealand's domestic gas prices to those in international markets (Hemery, 2009). However, this assumption was modified for the SOO analysis and it was assumed in the MDS5 scenario that there would be no exports of LNG and that the large discovery would result in keeping the domestic price of gas below NZ\$8.50/GJ (Figure 6.8).

\footnotetext{
${ }^{54}$ The price production relationship was modelled using the formula: $y=a+b e^{-(k x)}$

where

y price $\mathrm{NZ \$} / \mathrm{GJ}$

$\mathrm{x}$ the quarterly production in $\mathrm{PJ}$

$a=3, b=110$, and $k=0.09$
} 
The reason for changing the export assumption was not discussed in the 2010 SOO. It is possible that this change was due to a submission on the draft SOO (Electricity Commission, 2009) by the University of Auckland Business School Energy Centre.

The School argued that the development of an export LNG industry in New Zealand would be unlikely due to the dominant position of Qatar and New Zealand's distance from important markets ${ }^{55}$.

Figure 6.8: 2010 Statement of Opportunities report: gas price assumptions

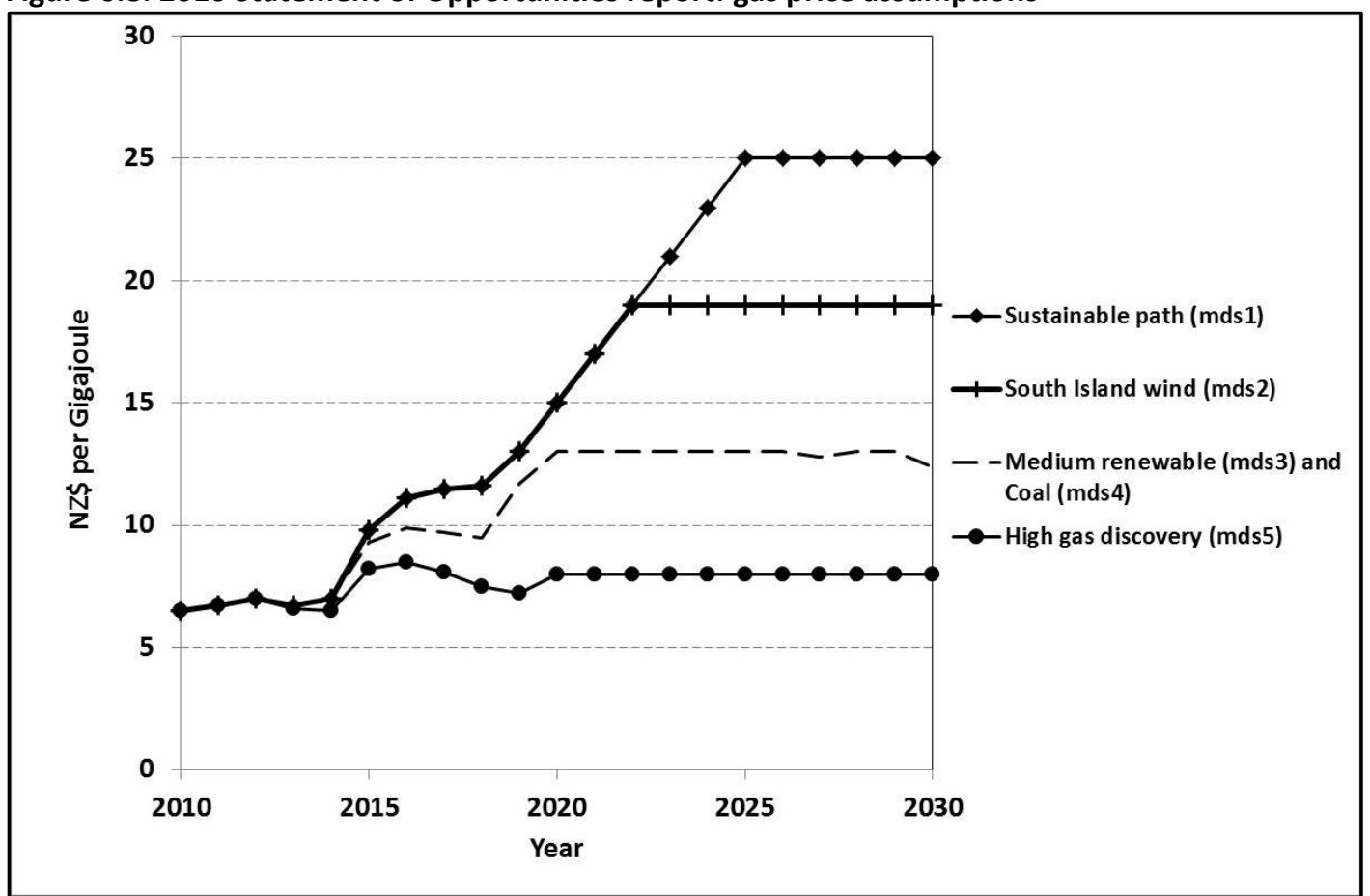

Source: Bishop (2010)

\section{Diesel}

Since oil is used only in small quantities for electricity generation, oil prices had little direct impact on the generation scenarios. The EC considered that supplies of diesel would be unconstrained and prices for all generation scenarios would stay at $\$ 25 / G J$, except for scenario MDS1 where the price would rise to NZ\$35/GJ by 2020 (Figure 6.9).

\footnotetext{
${ }^{55}$ It should be noted that the University of Auckland Business School Energy Centre submission also argued that LNG imports would be unlikely as well. However the EC has not appeared to support this view and did not adjust the MDS1 and MDS2 scenarios (Electricity Commission, 2009).
} 


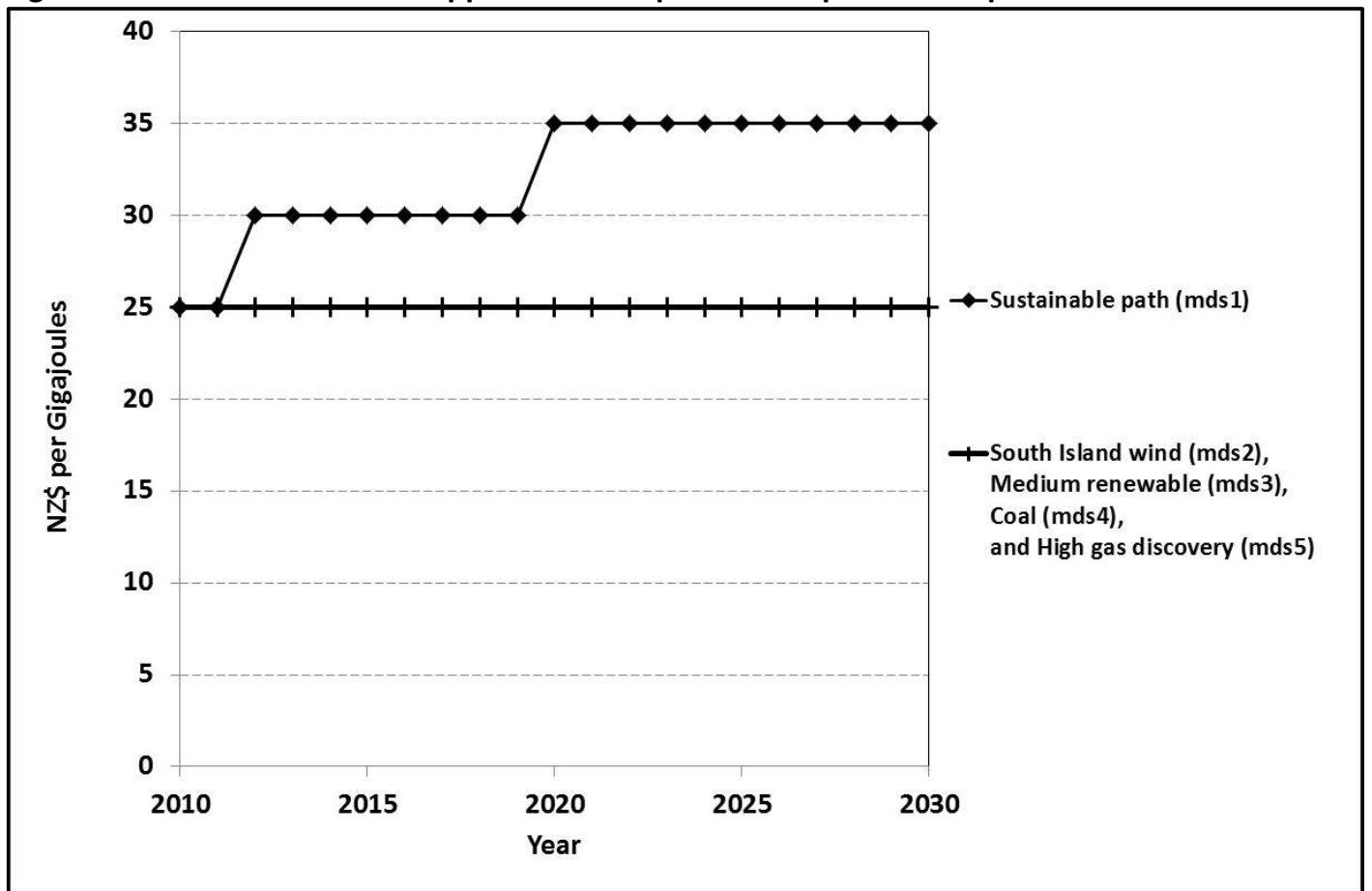

Source: Bishop (2010)

\subsubsection{GHG emissions}

\section{GHG emission factors}

The GEM estimates the impact of any carbon price that is imposed on the variable costs of generating $(\$ / M W h)$ by multiplying the carbon charge by the emission factor and each plant's designated heat rate (GJ/GWh). Table 6.7 gives the $\mathrm{CO}_{2} \mathrm{e}$ emission factors used in the GEM (P Bishop, Electricity Authority, personal communication, 29 August 2011).

The GHG emissions factors were based on information provided by the MED and Ministry for the Environment. The EC then confirmed their validity with these organisations (P Bishop, Electricity Authority, personal communication, 22 June 2011).

Table 6.7: Emission factors used in the generation expansion model

\begin{tabular}{|l|c|}
\hline Fuel & $\mathrm{t} \mathrm{CO}_{2} \mathrm{e} / \mathrm{PJ}$ \\
\hline Coal & 91,200 \\
\hline Lignite & 95,200 \\
\hline Gas & 52,800 \\
\hline Diesel & 73,000 \\
\hline Geothermal & 8,333 \\
\hline
\end{tabular}




\section{Carbon price}

All generation scenarios have a price on carbon, but the price varies across the scenarios after 2013 . The carbon prices, in $\$ \mathrm{NZ} /$ tonne $\mathrm{CO}_{2} \mathrm{e}$, that were used in the 2010 SOO are shown in Figure 6.10.

Figure 6.10: 2010 Statement of Opportunities report: carbon prices

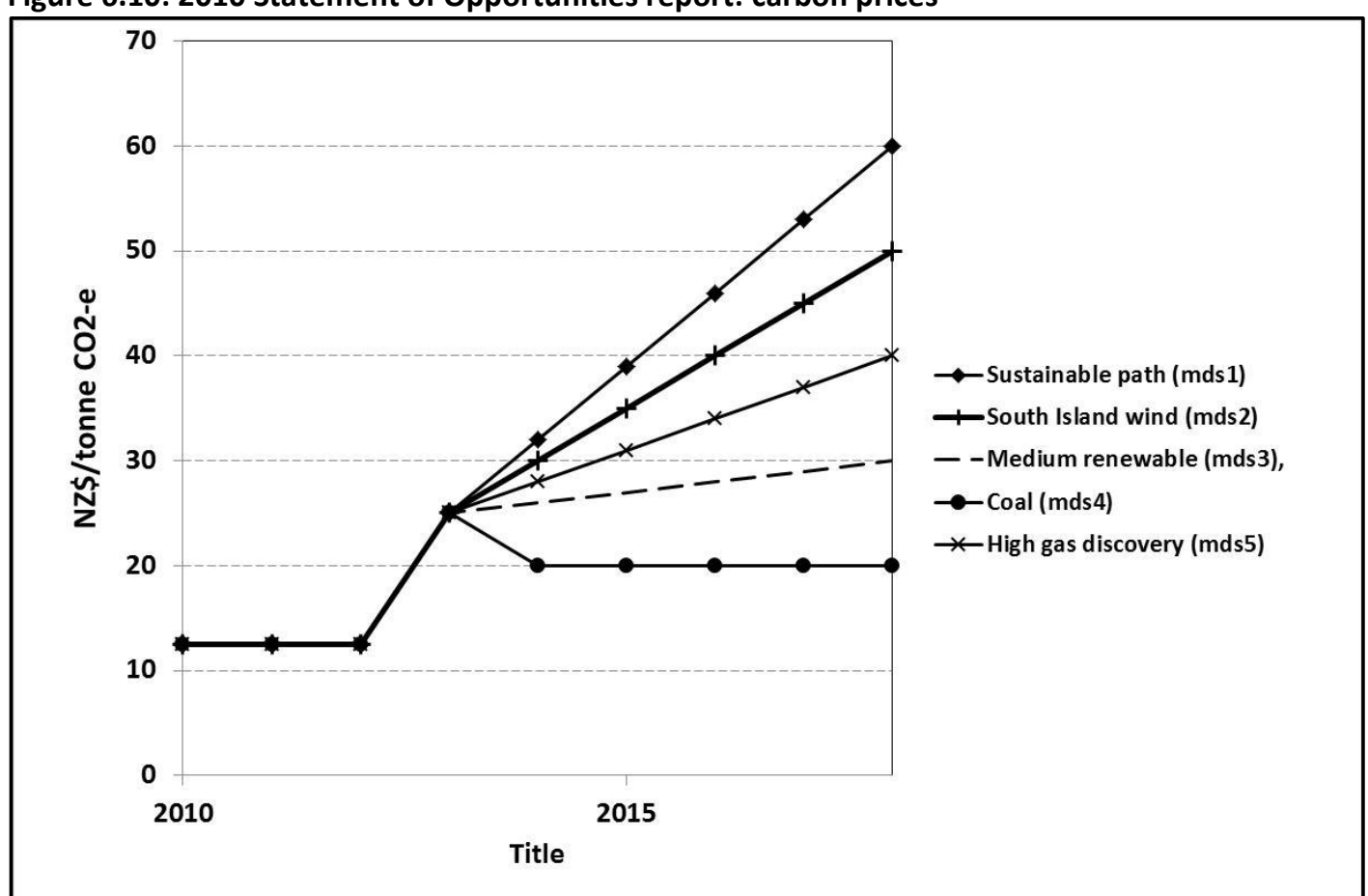

Source: Bishop (2010)

The EC found in its modelling that prices above $\mathrm{NZ} \$ 60 /$ tonne $\mathrm{CO}_{2} \mathrm{e}$ had little impact on the merit order of the technologies used.

The GEM has an imposed limit on the amount of wind power in the electricity system of $20 \%$. This constraint was based on concerns about the effect of wind on grid stability (Electricity Commission, 2010). The New Zealand Wind Energy Association questioned the validity of this assumption and the EC indicated in the SOO that this constraint will be reassessed in future modelling (Electricity Commission, 2009; Electricity Commission, 2010).

The EC found that when carbon prices are set above $\mathrm{NZ} \$ 100 /$ tonne $\mathrm{CO}_{2} \mathrm{e}$, the amount of wind power in the system becomes limited by the $20 \%$ constraint. 


\subsubsection{Demand from EVs}

The EC assumed in the MDS1 and MDS4 generation scenarios that EVs will be an increasing part of the New Zealand LPV fleet.

The EC prepared and presented its projections of the uptake of EVs in New Zealand and their impact on the electricity grid at the Grid Planning Assumptions Workshop held in February 2008 (Hemery and Smith, 2008).

The EC's projections of EVs were based on the assumption that:

- all EVs would be treated as PHEVs

- the car fleet would increase from approximately 2.4 million in 2010 to 3.2 million in 2030

- the electric driving range of PHEVs would increase from approximately $60 \mathrm{~km}$ in 2010 to over $150 \mathrm{~km}$ in 2030

- PHEVs would number approximately 1 million vehicles in 2030

- PHEVs would travel approximately 10 billion kilometres annually in 2030.

The projections were subsequently amended and presented on 31 March 2008. No documentation could be found explaining this revision, but it may have been in response to feedback received at the workshop.

The revised projections used in the 2010 SOO analysis were that:

- the definition of car fleet included light duty commercial vehicles with the fleet size projections adjusted accordingly: to approximately 3 million vehicles in 2010 and 4 million vehicles in 2030.

- the energy efficiency of PHEVs was expected to improve from $125 \mathrm{Wh} / \mathrm{km}$ in 2010 to $118 \mathrm{Wh} / \mathrm{km}$ in 2030.

- PHEVs would number approximately 400,000 vehicles in 2030.

- PHEVs would travel approximately 5 billion kilometres annually in 2030 (Hemery and Smith, 2008).

Using these latter assumptions, the EC re-estimated the additional demand for electricity arising from PHEVs for use in the 2010 SOO (Figure 6.11). 
Figure 6.11: 2010 Statement of Opportunities report: estimated additional electricity demand due to plug-in hybrid electric vehicles

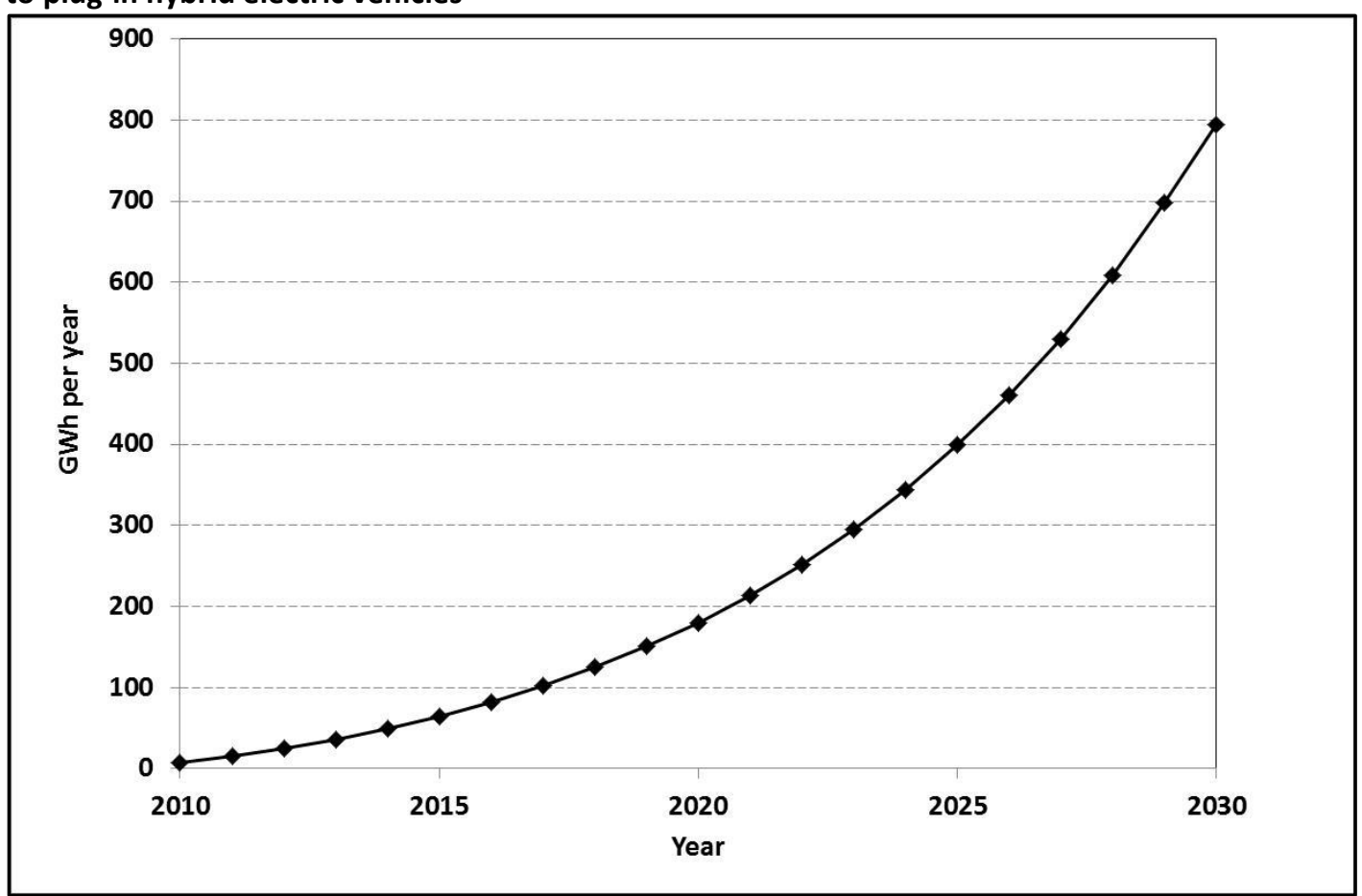

Source: Bishop (2010)

\subsection{Using the GEM with the VFM}

\subsubsection{General approach}

When developing the generation scenarios for the present study, it was decided not to modify many of the assumptions used by the EC in its generation scenarios for the SOO. This decision was made because these assumptions had already been extensively peer reviewed by industry and experts as part of the development of the SOO.

For this study, the MDS1, MDS3, and MDS5 generation scenarios were used as these scenarios were considered to fully represent the range of possible futures for the development of electricity generation in New Zealand. The MDS1 scenario represents a future where there is potential to develop a wide range of new hydro and wind generation sites, but no use of coal and lignite resources. The MDS3 scenario represents a future where there is still considerable potential to develop wind sites, but the potential to develop new hydro sites is more limited. In this future, there are no restrictions on the use of coal. The MDS5 scenario represents a 
future where efforts to discover new gas resources are successful and large quantities of gas are available for use in electricity generation.

The MDS2 scenario was excluded because it was considered to be a variation of the MDS1 scenario and was developed by the EC to assess the impact of large-scale South Island wind development on the transmission system. The MDS4 scenario was excluded because it was considered to be a variation of the MDS3 scenario, but focusing more on the use of fossil fuels.

In this study, the following scenario inputs used in the SOO were not changed: (1) the future costs and availability and timing of generation and demand side projects; (2) the availability of natural gas; (3) the rate of WACC; and (4) the $20 \%$ the energy wind constraint ${ }^{56}$.

For the generation scenarios used in this study, it was necessary to modify the EC's generation scenarios and electricity demand projections to align them with the input assumptions used in the VFM scenario for GDP growth, future crude oil prices, and the future price of carbon. The different input assumptions used for the SOO and this study are presented in Table 6.8. Population growth did not need to be adjusted as the same assumptions had been used in both studies.

The outputs from VFM scenarios A2, C4, and C5 were chosen for inclusion in modelling with the GEM. Scenarios $\mathrm{A} 2$ and $\mathrm{C} 4$ were chosen because they represent the lower and upper boundaries of the range of uptake of EVs in New Zealand under conditions where there is no breakthrough in battery technology or very high oil prices.

The GEM was then run using the electricity demand from scenario $\mathrm{C} 5$, but only in conjunction with the MDS1 scenario. The model run contained the most optimistic assumptions about the development of fuel efficient ICEV and EV technology and modelled a situation where battery technological breakthroughs occurred and

\footnotetext{
${ }^{56}$ The $20 \%$ wind energy constraint is considered to be a level that can be incorporated in the electricity system with a minimum of difficulty. Additional levels are technically possible but incur additional economic costs. This issue remains an area of on-going research by electricity utilities (Electricity Commission, 2010).
} 
there were changes to EV design that would result in further reductions in vehicle and battery prices. This scenario resulted in the greatest uptake of EVs of all the VFM scenarios and was treated as the upper limit for EV uptake. The MDS1 scenario reflected a future where extensive policies were used to promote renewable generation. This combination of VFM and generation scenarios represented a future where there was coordinated action and a forceful set of measures across both the transport and electricity sectors to reduce GHG emissions.

It was assumed in the generation scenarios that the future price of carbon would be at the same level assumed in the VFM scenarios. For the GEM model runs using electricity demand projections from scenario $A 2$, the carbon price was assumed to be $\$ 25$ per tonne $\mathrm{CO}_{2} \mathrm{e}$. For the GEM model runs based on VFM scenarios $\mathrm{C} 4$ and $\mathrm{C} 5$, the carbon price was assumed to be $\$ 100$ per tonne $\mathrm{CO}_{2} \mathrm{e}$. 
Table 6.8: Comparison of input assumptions used in both the Statement of Opportunities report and vehicle fleet model scenarios

\begin{tabular}{|c|c|c|c|c|c|c|}
\hline \multirow[t]{2}{*}{ Input variable } & \multirow[t]{2}{*}{ Year } & \multicolumn{3}{|c|}{ SOO } & \multicolumn{2}{|c|}{ VFM } \\
\hline & & & & & $\mathrm{A} 2$ & $\begin{array}{c}\mathrm{C} 4 \text { and } \\
\text { C5 }\end{array}$ \\
\hline \multirow{14}{*}{$\begin{array}{l}\text { GDP } \\
\% \text { growth per year }\end{array}$} & 2010 & \multicolumn{3}{|c|}{$-1.51 \%$} & $2.4 \%$ & $2.4 \%$ \\
\hline & 2011 & \multicolumn{3}{|c|}{$4.38 \%$} & $2.4 \%$ & $2.4 \%$ \\
\hline & 2012 & \multicolumn{3}{|c|}{$3.31 \%$} & $2.4 \%$ & $2.4 \%$ \\
\hline & 2013 & \multicolumn{3}{|c|}{$3.88 \%$} & $2.4 \%$ & $2.4 \%$ \\
\hline & 2014 & \multicolumn{3}{|c|}{$3.31 \%$} & $2.4 \%$ & $2.4 \%$ \\
\hline & 2015 & \multicolumn{3}{|c|}{$2.86 \%$} & $2.4 \%$ & $2.4 \%$ \\
\hline & 2016 & \multicolumn{3}{|c|}{$2.69 \%$} & $2.4 \%$ & $2.4 \%$ \\
\hline & 2017 & \multicolumn{3}{|c|}{$2.10 \%$} & $2.4 \%$ & $2.4 \%$ \\
\hline & 2018 & \multicolumn{3}{|c|}{$2.12 \%$} & $2.4 \%$ & $2.4 \%$ \\
\hline & 2019 & \multicolumn{3}{|c|}{$1.96 \%$} & $2.4 \%$ & $2.4 \%$ \\
\hline & 2020 & \multicolumn{3}{|c|}{$1.96 \%$} & $2.2 \%$ & $2.2 \%$ \\
\hline & 2022 & \multicolumn{3}{|c|}{$1.88 \%$} & $2.2 \%$ & $2.2 \%$ \\
\hline & 2027 & \multicolumn{3}{|c|}{$1.76 \%$} & $2.2 \%$ & $2.2 \%$ \\
\hline & 2030 & \multicolumn{3}{|c|}{$1.76 \%$} & $2.2 \%$ & $2.2 \%$ \\
\hline \multirow{13}{*}{$\begin{array}{l}\text { Crude Oil } \\
\text { US\$ per barrel }\end{array}$} & 2010 & \multicolumn{3}{|c|}{63} & 100 & 100 \\
\hline & 2012 & \multicolumn{3}{|c|}{75} & 103 & 101 \\
\hline & 2013 & \multicolumn{3}{|c|}{75} & 105 & 102 \\
\hline & 2014 & \multicolumn{3}{|c|}{75} & 106 & 102 \\
\hline & 2015 & \multicolumn{3}{|c|}{75} & 108 & 103 \\
\hline & 2017 & \multicolumn{3}{|c|}{75} & 111 & 104 \\
\hline & 2019 & & 75 & & 114 & 106 \\
\hline & 2021 & & 90 & & 118 & 107 \\
\hline & 2024 & & 90 & & 123 & 109 \\
\hline & 2026 & & 90 & & 127 & 110 \\
\hline & 2027 & & 90 & & 129 & 111 \\
\hline & 2029 & & 90 & & 133 & 112 \\
\hline & 2030 & & 90 & & 135 & 113 \\
\hline & & mds1 & mds3 & mds5 & & \\
\hline & 2010 & 12.5 & 12.5 & 12.5 & 12.5 & 12.5 \\
\hline & 2011 & 12.5 & 12.5 & 12.5 & 12.5 & 12.5 \\
\hline & 2012 & 12.5 & 12.5 & 12.5 & 25.0 & 100.0 \\
\hline & 2013 & 25.0 & 25.0 & 25.0 & 25.0 & 100.0 \\
\hline Price of carbon & 2014 & 32.0 & 26.0 & 28.0 & 25.0 & 100.0 \\
\hline NZS per tonne & 2015 & 39.0 & 27.0 & 31.0 & 25.0 & 100.0 \\
\hline & 2016 & 46.0 & 28.0 & 34.0 & 25.0 & 100.0 \\
\hline & 2017 & 53.0 & 29.0 & 37.0 & 25.0 & 100.0 \\
\hline & 2018 & 60.0 & 30.0 & 40.0 & 25.0 & 100.0 \\
\hline & 2030 & 60.0 & 30.0 & 40.0 & 25.0 & 100.0 \\
\hline
\end{tabular}




\subsubsection{Revision of electricity demand forecasts}

The electricity demand forecast models for the residential, industrial, and commercial sectors were re-estimated using historical data provided by the $\mathrm{EC}^{57}$ and the VFM scenario input assumptions.

The revised models were estimated using LIMDEP software.

Input variables tested for inclusion in the forecast models were:

- GDP (real 1995/96)

- population

- number of households

- residential electricity price (real price excluding GST)

- line losses

- shortage (years in which electricity conservation campaigns occurred) ${ }^{58}$.

Models were specified with input variables in levels, logs, de-trended and, in first differences.

The Ljung-Box statistic indicated the presence of autocorrelation in the residuals of the residential, and commercial and industrial models. The models were then reestimated using feasible generalised least squares (FGLS) in conjunction with the Prais-Winsten estimator (Greene, 2002, p. 273).The residential models were estimated using data from 1974 to 2008 and data from 1986 to 2008 for the industrial and commercial model (Kirtlan, 2008; Kirtlan, 2009; New Zealand Institute of Economic Research, 2009).

Using ordinary least squares (OLS) the EC found that a log-log model performed the best for the forecasting residential electricity demand and that the residential retail price was a statistically significant explanatory variable. Using FGLS a log-log model

\footnotetext{
${ }^{57}$ Energy forecast input assumptions http://www.ea.govt.nz/industry/monitoring/forecasting/demand-forecasting/.

${ }^{58}$ The shortage variable is a dummy that removes from the regression results those years in which "shortages" have occurred. This is done to ensure that demand is not biased downwards due to extraordinary circumstances (Kirtlan, 2008).
} 
was also found to be the best performing model, but residential retail price was not found to be a statistically significant (Table 6.9).

The best performing model for the industrial and commercial sector used untransformed data and resulted in the shortage variable being found to be statistically significant (Table 6.9).

Table 6.9: Forecast model for residential, industrial, and commercial electricity demand

\begin{tabular}{|l|c||c|c|}
\hline \multicolumn{2}{|l|}{ Domestic demand (log model) } & \multicolumn{2}{c|}{$\begin{array}{c}\text { Industrial and commercial } \\
\text { demand }\end{array}$} \\
\hline Variable & $\begin{array}{c}\text { Coefficient } \\
(\text { prob) }\end{array}$ & Variable & $\begin{array}{c}\text { Coefficient } \\
\text { (prob) }\end{array}$ \\
\hline \hline Constant & $\begin{array}{c}-11.0667 * * * \\
(0.0000)\end{array}$ & Constant & $\begin{array}{c}4056.24 * * \\
(0.0472)\end{array}$ \\
\hline Log (GDP/Population) & $\begin{array}{c}8.76915 * * * \\
(0.0000)\end{array}$ & GDP & $\begin{array}{c}0.16162^{* * *} \\
(0.000)\end{array}$ \\
\hline Log (Number of households/Population) & $\begin{array}{c}14.6543 * * * \\
(0.0001)\end{array}$ & Shortage & $\begin{array}{c}-561.342 * * * \\
(0.0015)\end{array}$ \\
\hline RHO & $\begin{array}{c}0.53061 * * * \\
(0.003)\end{array}$ & RHO & $\begin{array}{c}0.90193 * * * \\
(0.000)\end{array}$ \\
\hline R-squared & 0.95463 & R-squared & 0.94805 \\
\hline Adjusted R-squared & 0.95179 & $\begin{array}{c}\text { Adjusted R- } \\
\text { squared }\end{array}$ & 0.9431 \\
\hline Durbin-Watson & 2.00 & $\begin{array}{c}\text { Durbin- } \\
\text { Watson }\end{array}$ & 1.81 \\
\hline
\end{tabular}

For the heavy industry sector (Tiwai Point aluminium smelter), the EC assumed that the demand would continue at the current maximum level of 5,331 GWh per year for the entire modelling period.

In the SOO, the MDS3 'Medium Renewables' scenario contained the assumption that, from 2022, Tiwai Point would begin to close, reducing demand steadily over six years. This assumption has not been used. In this study, it was assumed that the demand from Tiwai Point would be constant out to 2030 in all three generation scenarios used.

Figure 6.12 shows the historic demand from 1980 to 2008, including line losses, from the EC forecasting input file, and the demand forecast using the revised model developed for this study. The revised model indicates that demand increased from $41,650 \mathrm{GWh}$ in 2008 to $43,100 \mathrm{GWh}$ in 2010 . Using the GDP growth projections 
from the VFM scenarios, electricity demand was projected to increase, without the introduction of EVs, to $60,300 \mathrm{GWh}$ by 2030 . This demand projection was not intended as a fully realistic forecast of New Zealand's future electricity demand as it did not take into account the effects that the current economic recession would have on electricity demand. The forecast was intended for use as a baseline projection that represented a future where electricity demand would remain unaffected by the introduction of EVs into New Zealand, against which the effects of the introduction of EVs on the electricity system could be assessed.

Figure 6.12: Projected electricity demand including line losses and excluding demand from EVs

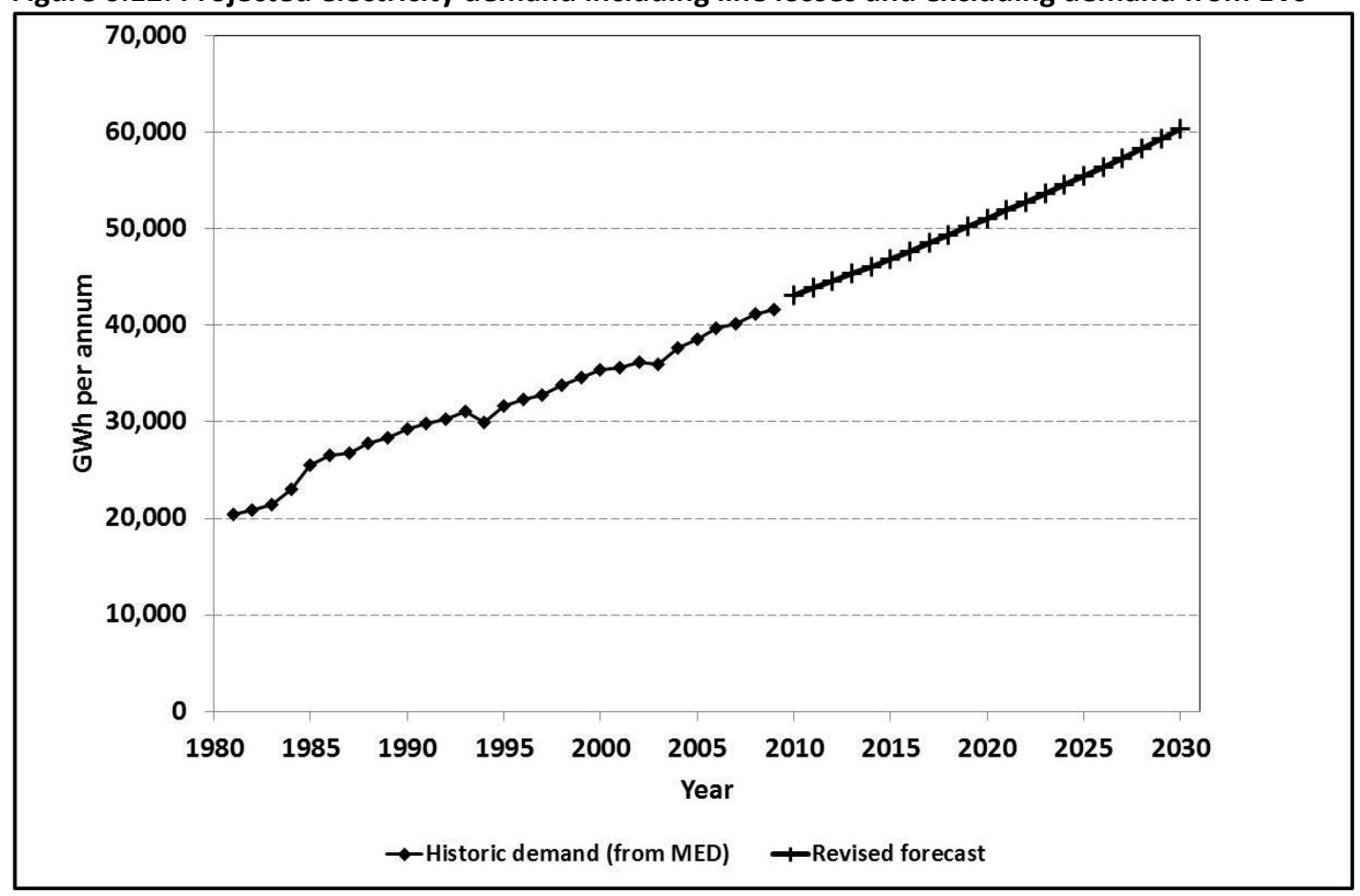

\subsubsection{Fuel prices}

\section{Diesel prices}

The projections of the future price of crude oil used in the GEM were aligned with those used in the VFM scenarios. Historically, the industrial price, excluding GST, of diesel has tracked that of the retail price, including GST (Figure 6.13) (Ministry of Economic Development, 2012). 
Figure 6.13: The New Zealand historic retail and industrial price of diesel

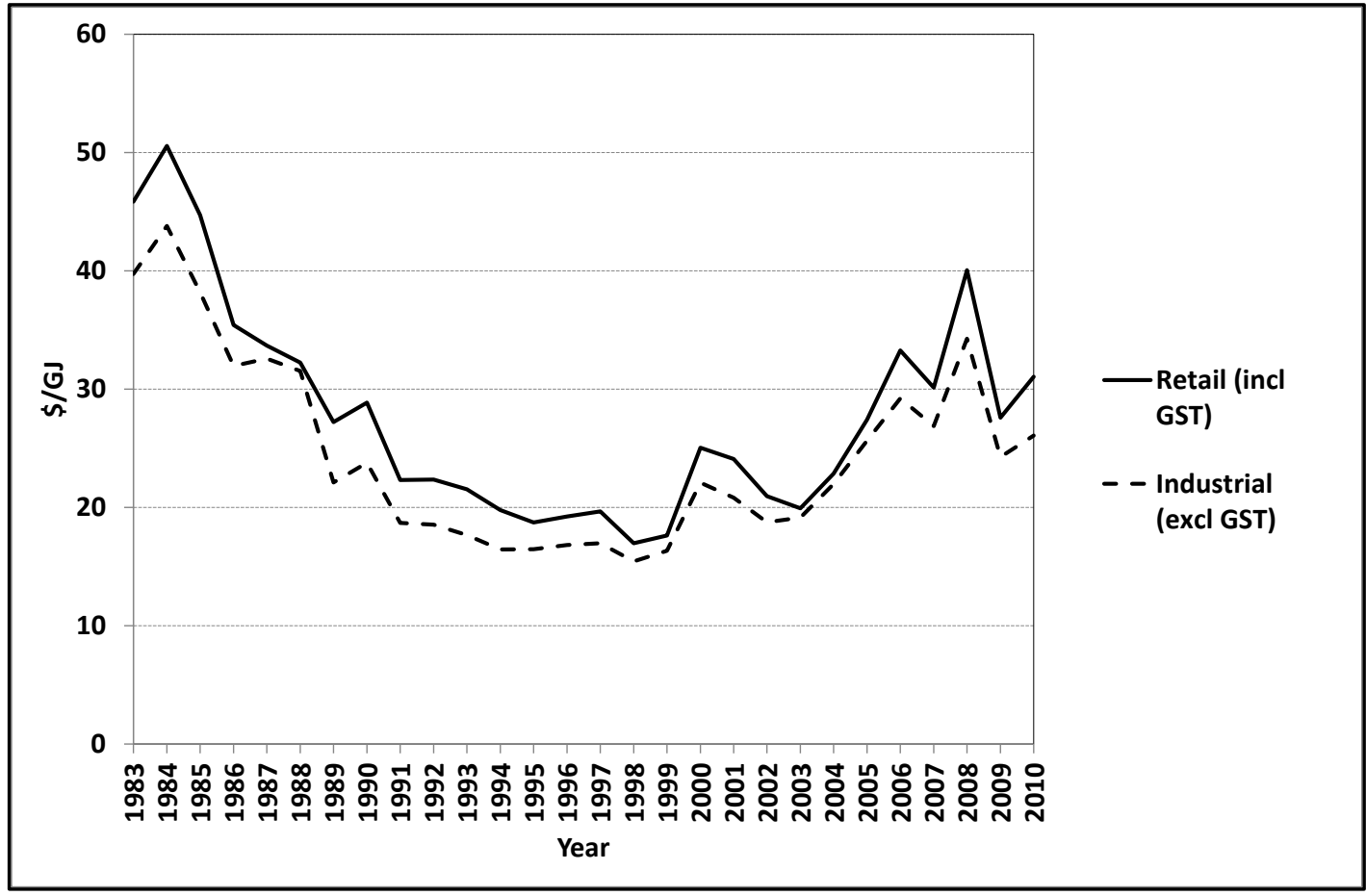

The industrial price of diesel was aligned to the retail diesel price using an OLS model with the data specified in first differences. Diesel price data from 1983 to 2010 was used to estimate the model. Transforming the data into first differences was found to remove the autocorrelation that was present (Table 6.10).

Table 6.10: Industrial and retail diesel price relationship model

\begin{tabular}{|l|c|}
\hline Industrial price of diesel (excl. GST) (1 & \multicolumn{2}{|c|}{ st Difference) } \\
\hline Variable (1 & $\begin{array}{c}\text { Coefficient } \\
\text { (prob) }\end{array}$ \\
\hline Retail Price of Diesel (incl GST) (Prob) & $0.79831^{* * *}$ \\
\hline R-squared & $(0.000)$ \\
\hline Durbin-Watson & 0.90521 \\
\hline
\end{tabular}

Using this model and the retail price of diesel from the A and C VFM scenarios, the industrial price of diesel was estimated (Table 6.11). 
Table 6.11: Industrial diesel price: 2010 to 2030

\begin{tabular}{|c|c|c|c|c|c|}
\hline & $\begin{array}{c}\text { A Group of } \\
\text { VFM } \\
\text { scenarios }\end{array}$ & $\begin{array}{c}\text { C Group of } \\
\text { VFM } \\
\text { scenarios }\end{array}$ & $\begin{array}{c}\text { A Group of } \\
\text { VFM } \\
\text { scenarios }\end{array}$ & $\begin{array}{c}\text { C Group of } \\
\text { VFM } \\
\text { scenarios }\end{array}$ \\
\hline 2010 & \multicolumn{2}{|c|}{ \$/GJ } & & \multicolumn{2}{c|}{ \$/GJ } \\
\hline 2011 & 40 & 40 & 2021 & 46 & 42 \\
\hline 2012 & 40 & 40 & 2022 & 46 & 42 \\
\hline 2013 & 41 & 40 & 2023 & 47 & 43 \\
\hline 2014 & 42 & 41 & 2024 & 47 & 43 \\
\hline 2015 & 42 & 41 & 2025 & 48 & 43 \\
\hline 2016 & 43 & 41 & 2026 & 48 & 43 \\
\hline 2017 & 43 & 41 & 2027 & 49 & 43 \\
\hline 2018 & 44 & 41 & 2028 & 50 & 44 \\
\hline 2019 & 45 & 42 & 2029 & 50 & 44 \\
\hline 2020 & 45 & 42 & 2030 & 51 & 44 \\
\hline
\end{tabular}

\section{Natural gas prices}

Historically natural gas has been traded in regional and intra-continental markets, but the international trade in natural gas, in the form of LNG, has increased significantly in recent years (Cohen et al., 2011).

Trends in international natural gas prices tend to be aligned with trends in international crude oil prices. The reasons for this vary. In Europe and Asia, many gas contracts are benchmarked against the price of crude oil, but with floor and ceiling prices to control volatility. In the United States where there is a liquid natural gas spot market, the prices of the two commodities are not as strongly linked (Ramberg, 2010). A number of studies looking at United States gas markets still show a price linkage between crude oil and natural gas in the United States, but this relationship varies over time (Brown and Yucel, 2007; Villar and Joutz, 2006). Villar and Joutz found that, in United States markets, the price in crude oil markets influenced the price of natural gas, but the price of natural gas was not found to influence the price of crude oil.

This study used the same assumptions about the future availability of natural gas in New Zealand as used by the EC in the SOO. In the MDS1 scenario used in the SOO, the EC assumed that the importation of LNG would occur after 2020 and the average domestic price of natural gas would start to move towards the 
international price of LNG. The EC assumed that, by 2025 , the domestic natural gas price would follow the international LNG price. The same assumption was used in this study. For the MDS1 scenario the projected gas price from this time was adjusted to reflect the international crude oil prices projections used in the VFM scenarios $^{59}$.

To estimate the price of LNG, the method developed by Eng (2008) was used. This method estimates the price of LNG, in New Zealand currency, in relation to international crude oil prices using the formula:

Price NZ\$ $=(0.1374 *(\mathrm{WTI}-1)+0.94787) * \mathrm{NZ \$}: \mathrm{US}+1.50 * \mathrm{NZ \$}: \mathrm{US}(0)$ per GJ where:

- WTI is the price of West Texas intermediate crude oil in US\$

- NZ\$:US\$ is the exchange rate (assumed to be 0.65 )

- $1.50 * \mathrm{NZ}: \mathrm{US} \$(0)$ is the New Zealand storage and regasification tariff ${ }^{60}$.

The adjusted gas prices are given in Table 6.12.

\footnotetext{
${ }^{59}$ The proposal to develop an LNG importation plant in New Zealand remains uncertain (Richie, 2009). The effects of a constrained gas supply in the MDS1 scenario would impact on the retirement schedule of existing gas thermal plants and the extent that gas peaker plant could be used as a backup to wind generation.

${ }^{60}$ The estimate of regasification tariff was sourced from the Centre of Advanced Engineering (2004).
} 
Table 6.12: Adjusted natural gas prices

\begin{tabular}{|c|c|c|c|c|}
\hline & Scenario A & Scenario C & \multicolumn{2}{|c|}{ Scenarios A and C } \\
\hline & \multicolumn{4}{|c|}{$\$ / G J$} \\
\hline & MDS1 & MDS1 & MDS3 & MDS5 \\
\hline 2010 & 7 & 7 & 7 & 7 \\
\hline 2011 & 7 & 7 & 7 & 7 \\
\hline 2012 & 7 & 7 & 7 & 7 \\
\hline 2013 & 7 & 7 & 7 & 7 \\
\hline 2014 & 7 & 7 & 7 & 7 \\
\hline 2015 & 10 & 10 & 9 & 8 \\
\hline 2016 & 11 & 11 & 10 & 9 \\
\hline 2017 & 12 & 12 & 10 & 8 \\
\hline 2018 & 12 & 12 & 10 & 7 \\
\hline 2019 & 13 & 13 & 12 & 7 \\
\hline 2020 & 15 & 15 & 13 & 8 \\
\hline 2021 & 18 & 17 & 13 & 8 \\
\hline 2022 & 21 & 20 & 13 & 8 \\
\hline 2023 & 24 & 22 & 13 & 8 \\
\hline 2024 & 27 & 24 & 13 & 8 \\
\hline 2025 & 30 & 27 & 13 & 8 \\
\hline 2026 & 30 & 27 & 13 & 8 \\
\hline 2027 & 31 & 27 & 13 & 8 \\
\hline 2028 & 31 & 27 & 13 & 8 \\
\hline 2029 & 32 & 27 & 13 & 8 \\
\hline 2030 & 32 & 27 & 12 & 8 \\
\hline
\end{tabular}

\subsubsection{GHG emissions}

\section{Carbon prices}

The carbon prices in the GEM were adjusted to align them with those used in the VFM scenarios. For GEM model runs using the output from the VFM A1 (no-EV) and A2 scenarios, the price of carbon was assumed to be $\$ 25 /$ tonne. For scenarios C4 and $\mathrm{C} 5$, the price of carbon was assumed to be $\$ 100 /$ tonne (Table 6.8 ).

\section{Carbon capture and storage}

The generation scenarios included the option of up to $2820 \mathrm{MW}$ of coal, lignite, and gas generation that incorporated carbon capture and storage technology (CCS) (Bishop, 2010). The EC did not see this technology becoming available in New Zealand before 2030, which is outside the period of this study (Electricity Authority, 2010). 
The current understanding of the potential of large scale CCS in New Zealand is at an early stage. Work by the MED is currently focused on developing a legislative and regulatory framework in case CCS is deployed in New Zealand. GNS Science is also undertaking work to develop an understanding of New Zealand's capacity to store $\mathrm{CO}_{2}$ in geological structures (Ministry of Economic Development, 2011a).

Due to the lack of knowledge about the potential of this technology in New Zealand, it was decided for the generation scenarios used in this study not to alter the EC's assumption; i.e. it is maintained that this technology is not available during the VFM modelling period.

\subsubsection{V2G technology}

The MDS1 scenario allowed for the possibility that, in conjunction with EVs being introduced into New Zealand, there would be V2G technology available from 2030. In this generation scenario, up to $1000 \mathrm{MW}$ of V2G capacity could be developed.

The literature that assesses benefits and costs of V2G from the perspective of EV owners has only just begun to emerge. At this time, it is uncertain whether EV owners will be interested in providing V2G services (see section 4.8.4). For this study, it was decided not to include this technology in the generation scenarios before 2030 .

\subsubsection{Peak demand and security of supply}

The GEM models system security by requiring the model to find a solution taking into account a forecast of the annual maximum peak demand in conjunction with single outage of a component of the core electricity system, either generation or transmission, which is known as n-1 security.

The additional demand from EVs will have an effect on security of the supply system. Estimates of the additional peak demand from EVs, over and above the peak demand caused by other uses, are sensitive to assumptions of the:

- number of EVs that are plugged in and drawing current during periods of annual maximum peak demand 
- amount of vehicle charging can be delayed, or shifted, until after periods of peak demand

- power capacity of the battery chargers (Weiller, 2011).

For this study, the additional electricity demand from EVs was allocated proportionally across the nine load blocks for each annual quarter. While the demand in each load block has increased, the shape of the load curve remains unchanged. This approach assumed that, on average, in each three month period, approximately $85 \%$ of EV charging would occur in the three lowest demand load blocks, a further $13 \%$ would occur in the next three load blocks, and $1 \%$ of charging would occur in the three highest demand load blocks.

This allocation of demand implies that there are some incentives in place to encourage EV owners to do most of their charging during off-peak periods and not during the peak periods, but these measures do not ensure that there will be no charging occurring during times of peak demand.

Even with such incentives, there is still potential for charging behaviour to vary on a day-to-day basis and it is possible there could be a large demand from EVs that coincides with periods of the very highest demand or a system security event.

To address the risk from EVs to the system security system, operators can instigate measures that prevent vehicle owners from charging during these periods, or they can ensure that there is sufficient peak generation capacity available to meet the probable demand from EVs that might occur during a security event.

To assess the impact of EVs on peak demand and system security, three charging options were used:

1. Fully interruptible: system operators have the ability to switch off $100 \%$ of EVs that are charging at times of very high peak demand or during a system security event (the default assumption).

2. Partially interruptible: System operators have the ability to control half of the EV fleet. Assuming that the maximum number of EVs plugged in at any 
one time is $80 \%$ of the EV fleet, this implies that up to $40 \%$ of EVs owners could be charging during a system security event.

3. Uninterruptible: System operators have no ability to prevent EV owners from charging during periods of maximum peak demand. It was assumed that the worst case scenario is a maximum of $80 \%$ of the EV fleet plugged in and drawing current at the same time as the period of peak system demand.

Home charging stations in New Zealand will be Level 2 chargers. New Zealand has $230 \mathrm{~V}$ domestic supply with either $15 \mathrm{~A}$ or, for fixed wired appliances, $32 \mathrm{~A}$ (Energy Safety Service, 2004). Duncan et al. (2010) note that the low voltage networks are designed for an average load per connection at peak demand of, after diversity, 5 kVA and that this will place a limitation on the capacity of EVs chargers.

For this study, the impact of using 20 Amp charging units providing 5 kVA was assessed. It was assumed that the charging units would deliver a continuous rating of $80 \%$ of maximum rated power resulting in power outputs of 4 kVA (Parks et al., 2007; Weiller, 2011).

For this study, the projected annual maximum peak demand, excluding the additional demand from EVs, was assumed to be the same as that used in the high load growth forecasts used in the 2010 SOO. Table 6.13 shows the National and North Island high load growth peak demand forecasts used in the 2010 SOO. These forecasts form the baseline peak capacity before any additional peak demand due to the presence of EVs. 
Table 6.13: Estimated peak demand from non EV uses

\begin{tabular}{|l|c|c|c|c|c|}
\hline & NZ Peak Demand (MW) & $\begin{array}{c}\text { North } \\
\text { Island } \\
\text { Peak } \\
\text { Demand } \\
\text { (MW) }\end{array}$ & $\begin{array}{c}\text { North } \\
\text { NZ Peak } \\
\text { Demand } \\
\text { (MW) }\end{array}$ & $\begin{array}{c}\text { Island } \\
\text { Peak } \\
\text { Demand } \\
\text { (MW) }\end{array}$ \\
\hline 2012 & & & 2021 & 9,447 & 6,664 \\
\hline 2013 & 7,838 & 5,302 & 2022 & 9,625 & 6,795 \\
\hline 2014 & 8,048 & 5,465 & 2023 & 9,792 & 7,030 \\
\hline 2015 & 8,249 & 5,602 & 2024 & 9,974 & 7,101 \\
\hline 2016 & 8,439 & 5,762 & 2025 & 10,157 & 7,348 \\
\hline 2017 & 8,601 & 5,887 & 2026 & 10,330 & 7,460 \\
\hline 2018 & 8,773 & 6,090 & 2027 & 10,509 & 7,487 \\
\hline 2019 & 8,944 & 6,224 & 2028 & 10,689 & 7,571 \\
\hline 2020 & 9,111 & 6,394 & 2029 & 10,872 & 7,864 \\
\hline
\end{tabular}

The estimated additional peak demand under fully and partially interruptible loads from EVs for each of the VFM scenarios is presented in section 9.3.3.

Where the generation project database in the GEM provides insufficient peak generation to meet the potential additional load from EVs, it was assumed that additional peak capacity would be delivered through the installation of diesel-fired open cycle gas turbines. It was also assumed that access to diesel fuel would not be constrained during the modelling period. These plants were chosen as they have the advantage of being modular and able to be located close to centres of demand as required. The use of diesel peakers also means that no assumptions had to be made about the future availability of natural gas, hydro, and geothermal generation resources. 


\section{Chapter 7: Results I: stated choice survey and discrete choice model}

\subsection{Introduction}

This chapter comprises two sections. The first section presents the results of the discrete choice survey, including the survey response rates and information on the demographic profile of the survey sample. This section also presents the responses to the questions on: (1) car purchasing history; (2) the level of concern about the future price of oil; (3) the need to reduce GHG emissions from road transport; and (4) the availability of EV charging at home.

The second section presents the results of the best performing multinomial logit (MNL), error component mixed multinomial logit (ECMNL) and random parameters mixed multinomial logit (RPL) models. The section also presents the estimated market shares and point elasticities estimates from the best performing RPL model.

\subsection{The discrete choice survey}

\subsubsection{Survey response}

During the phone recruitment phase, 1,642 potential candidates were contacted. Of these, 946 did not meet the qualifying criterion of intending to buy a car in the next five years, and a further 290 refused to participate in the survey. After the initial contact, 406 people agreed to complete the survey. During the follow-up calls, 38 people indicated that they had changed their mind and would not be participating. In total, 284 surveys responses were received, but three postal responses were incomplete and could not be used for model estimation.

The overall response rate of all those contacted by phone was $17.2 \%$, but the response rate of those contacted, and who qualified for this study, was $40.5 \%$. Of those who initially agreed to participate in the survey, the response rate was $69.5 \%$ (Figure 7.1). 


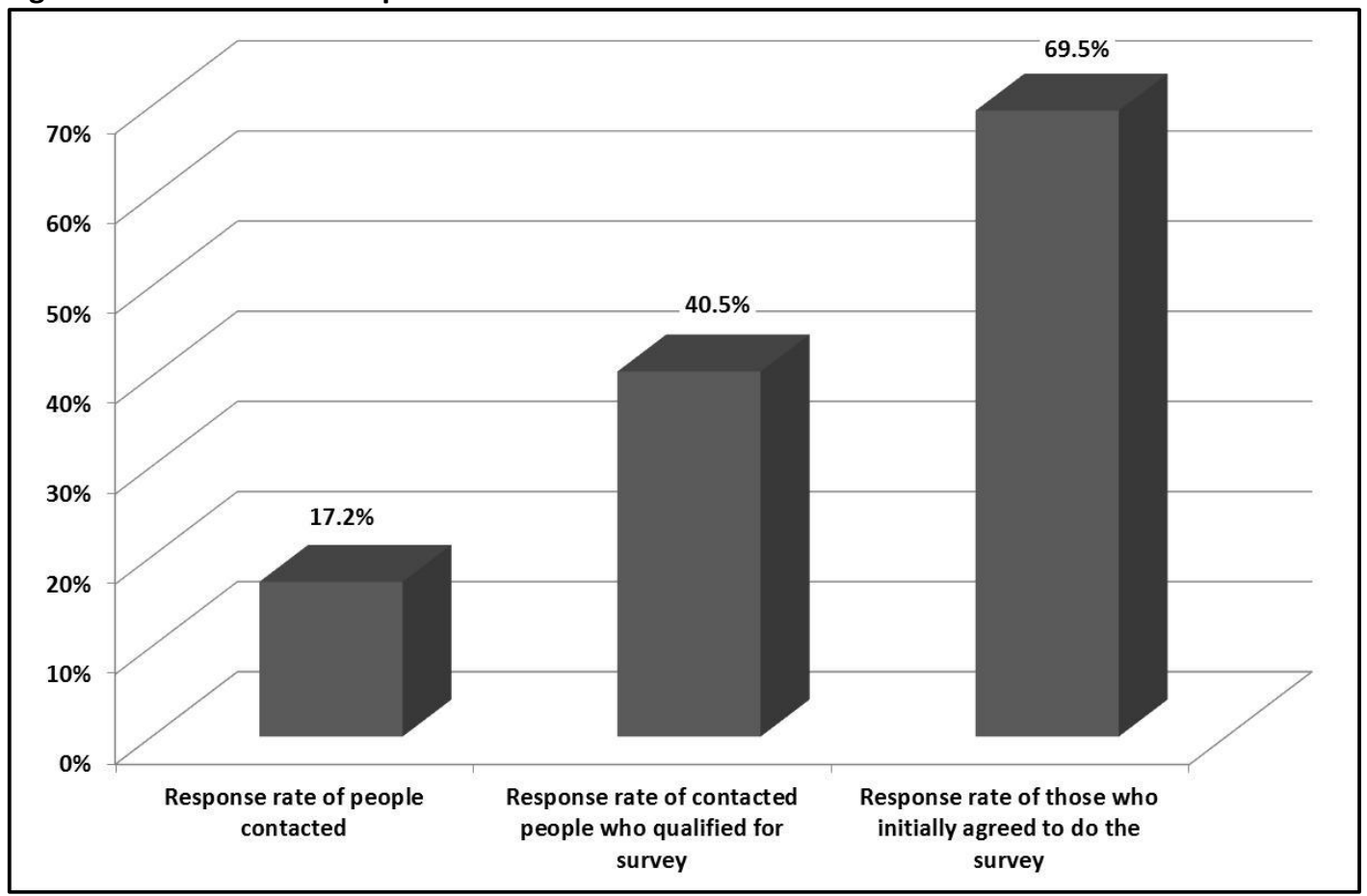

Of the 406 people who initially agreed to participate in the survey, 157 indicated a preference to respond by postal survey and 247 indicated a preference to respond through the website. Those people who expressed a preference to use the website had a response rate of $66 \%$ (163), compared to those who chose to complete the survey by post with a response rate of $75.8 \%$ (119) (Figure 7.2).

\section{Figure 7.2: Survey method used by respondents}

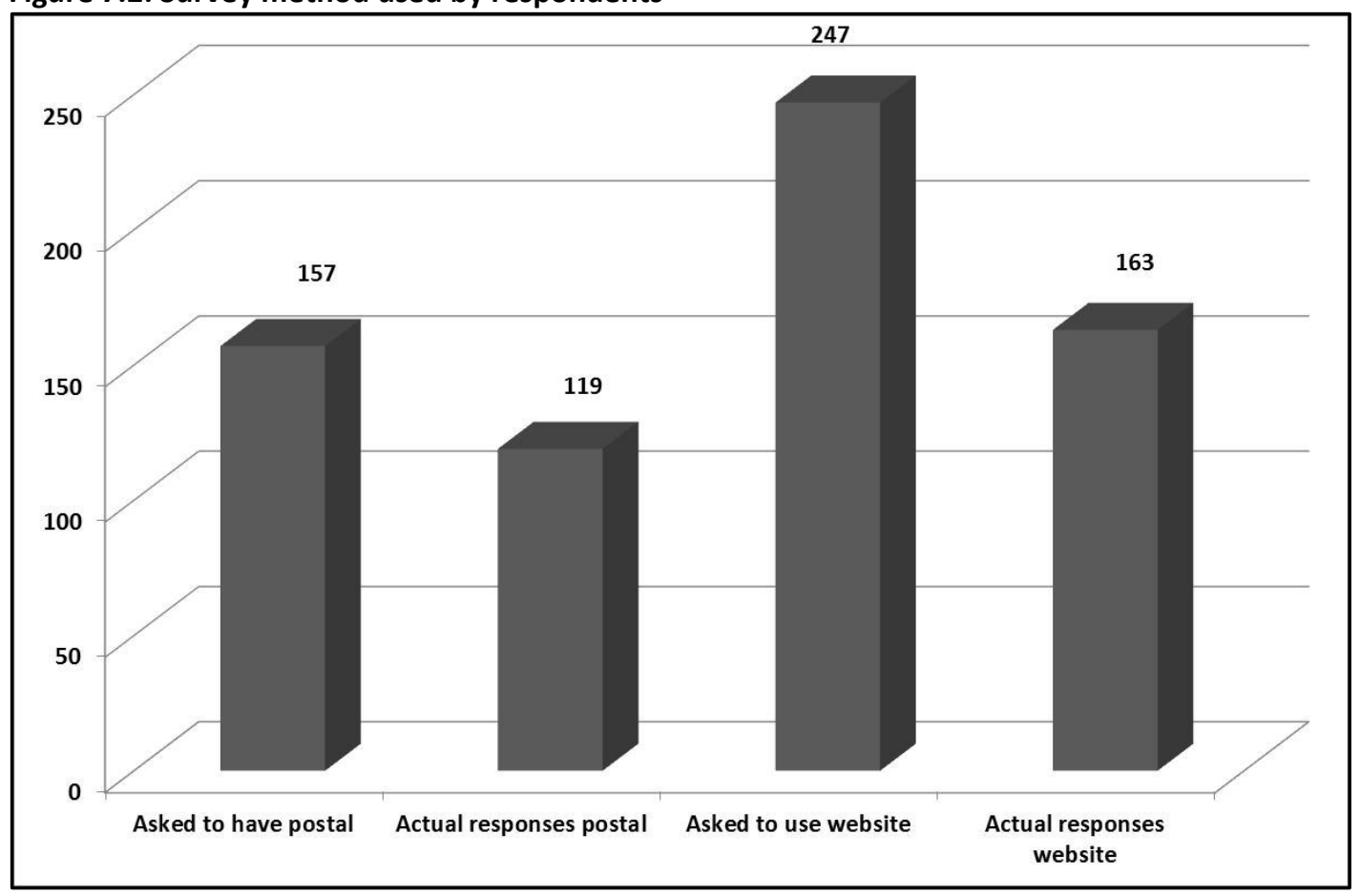


Analysis of the postal and web respondents indicates that those who chose to respond by website were, on average, slightly younger (50 years of age) than those who chose to use the postal method ( 55 years of age). Those who chose to use the website were also more likely to live in urban areas (78\%) compared to postal $(63 \%)^{61}$.

\subsubsection{Survey sample profile}

People between 50 and 79 years of age comprised $73.4 \%$ of the sample. Compared with the 2006 census data this group appears to be overrepresented in the sample, as they comprise only $37.8 \%$ of New Zealand's adult population. In contrast, those aged between 20 and 39 years comprised $4.6 \%$ of the survey, but represented $37.4 \%$ of New Zealand's adult population (Figure 7.3).

The low representation of those less than 40 years old in the survey sample could be a function of the eligibility criterion - younger people were less likely to consider buying a car during the 'next five years'. Without data on the age profile of New Zealand car buyers it was not possible to confirm whether the survey sample was biased.

However, the low representation of younger respondents is a widespread concern in surveys that rely on directory listings or random digit dialling for recruitment. This is due to the increasing use of mobile phones, especially by young adults, as these phones are likely to be unlisted (Keeter et al., 2007; Gray and Suntheralingam, 2007). Another possible contributing factor to the low response by young adults is the use of the electoral rolls to collect the initial list of names and addresses in this survey. Electoral rolls are updated on a three year cycle, but young adults are more likely to move residence (Statistics New Zealand, 2006a) and it is therefore more likely that the details in the electoral rolls for this group of voters will be outdated.

\footnotetext{
${ }^{61}$ During the follow-up call, 17 of the potential respondents who had chosen the website option and received the survey material by email indicated that they had accidentally deleted the email. The email was subsequently re-sent. One respondent who chose the postal survey required another copy as the first copy had not been received.
} 


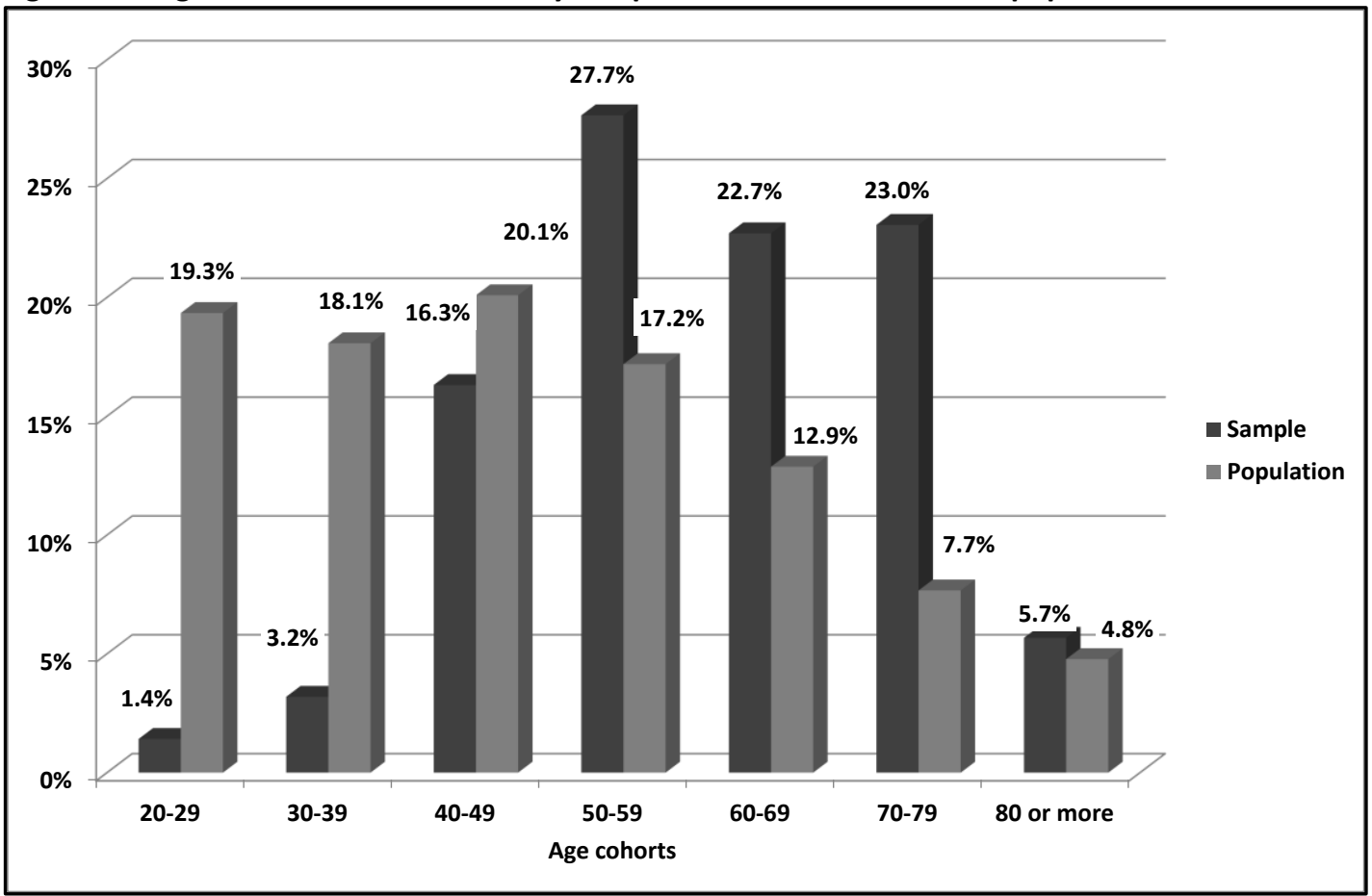

Sources: Statistics New Zealand (2006b)

The household income distribution of the sample is weighted towards households with incomes over $\$ 40,000$ per year, with a stronger representation of households with incomes above $\$ 80,000$ per year, which comprised $49.1 \%$ of the sample. Data from the Statistics NZ Household Economic Survey indicated that, in 2010, these income groups comprised $37 \%$ of all households. It is clear that the sample had a low representation of households with incomes below $\$ 40,000$ per year, comprising $16.0 \%$ of the sample, while, in 2010 , they represented $30 \%$ of all New Zealand households (Figure 7.4). 
Figure 7.4: Household income distribution of the survey sample and New Zealand

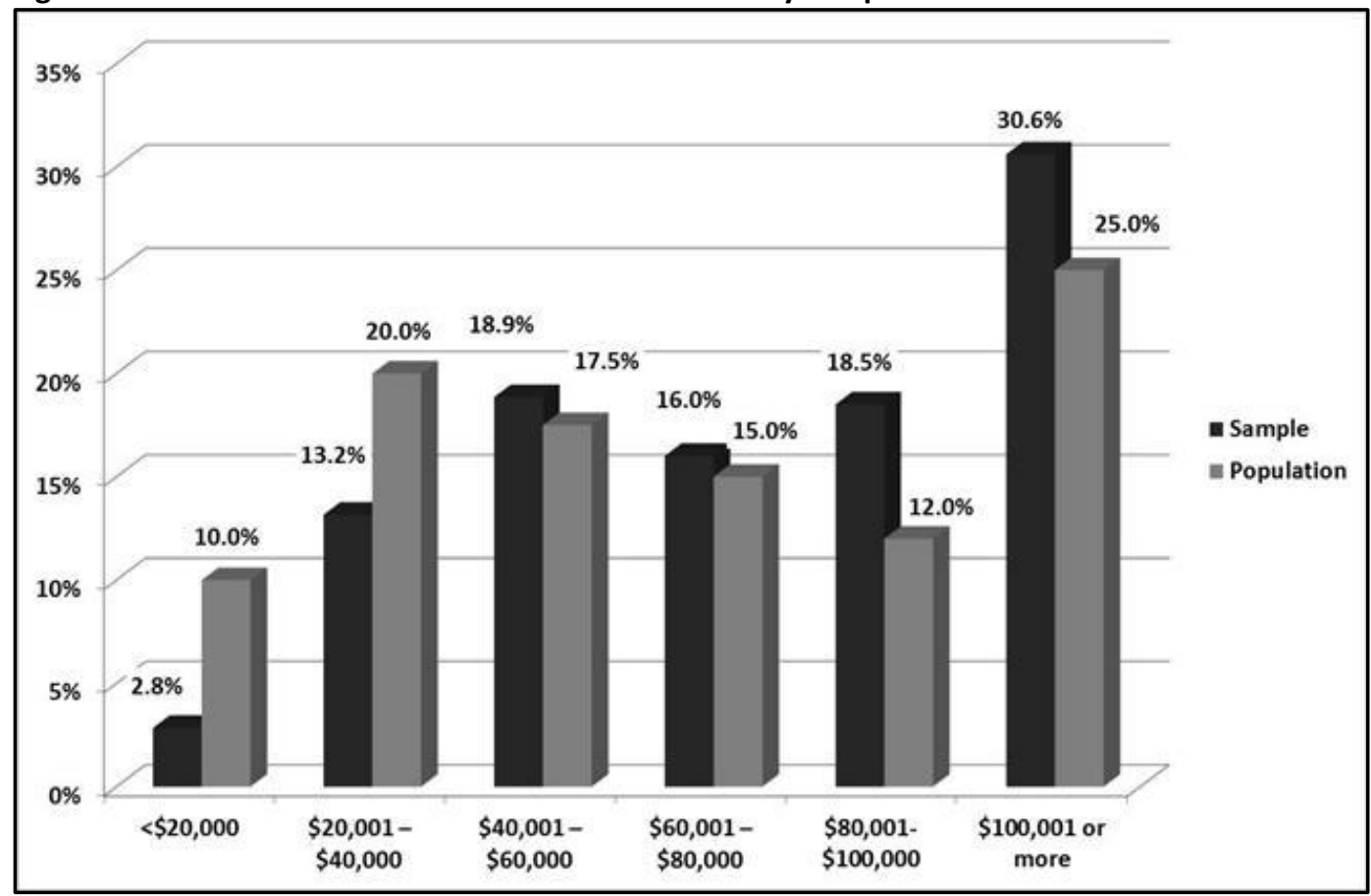

Source: Statistics New Zealand (2010a)

The sample comprised $58.3 \%$ male and $41.3 \%$ female, which when compared to the 2006 census data indicates that the sample may over-represent adult males who comprise $48.4 \%$ of the population and $52.5 \%$ of class 1 vehicle licence holders (Figure 7.5). 
Figure 7.5: Gender compared to the New Zealand adult population and New Zealand vehicle licence holders

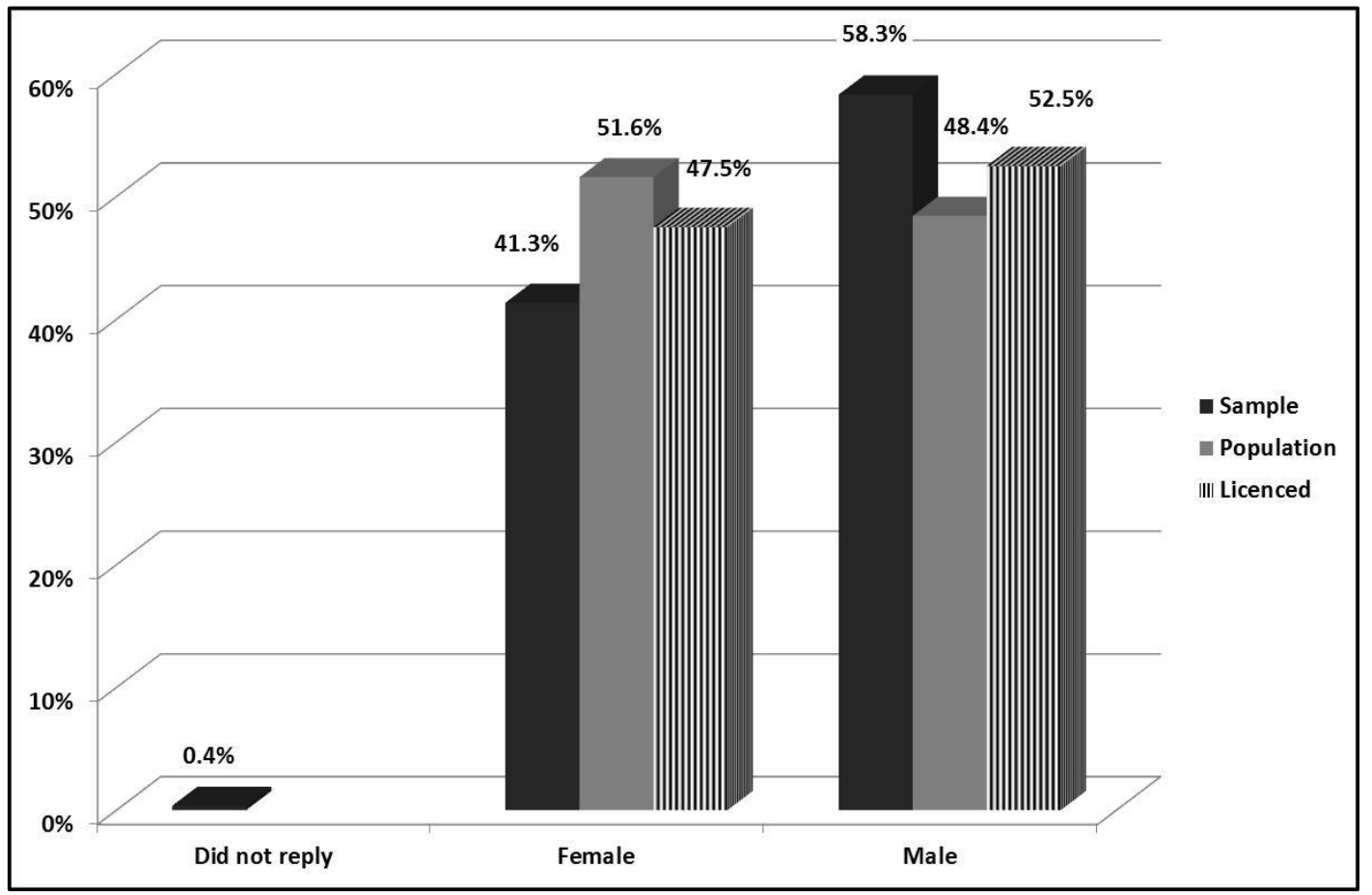

Sources: Statistics New Zealand (2006b), and New Zealand Transport Agency, personal communication, September 23, 2010.

The data from the survey sample indicated that $51.2 \%$ of the respondents considered that they live in urban areas (over 30,000 population), $19.1 \%$ in secondary urban areas, and $29.3 \%$ in rural areas. When compared to data from the 2006 census, there appears to be an overrepresentation of respondents from rural and secondary urban areas (Figure 7.6). 


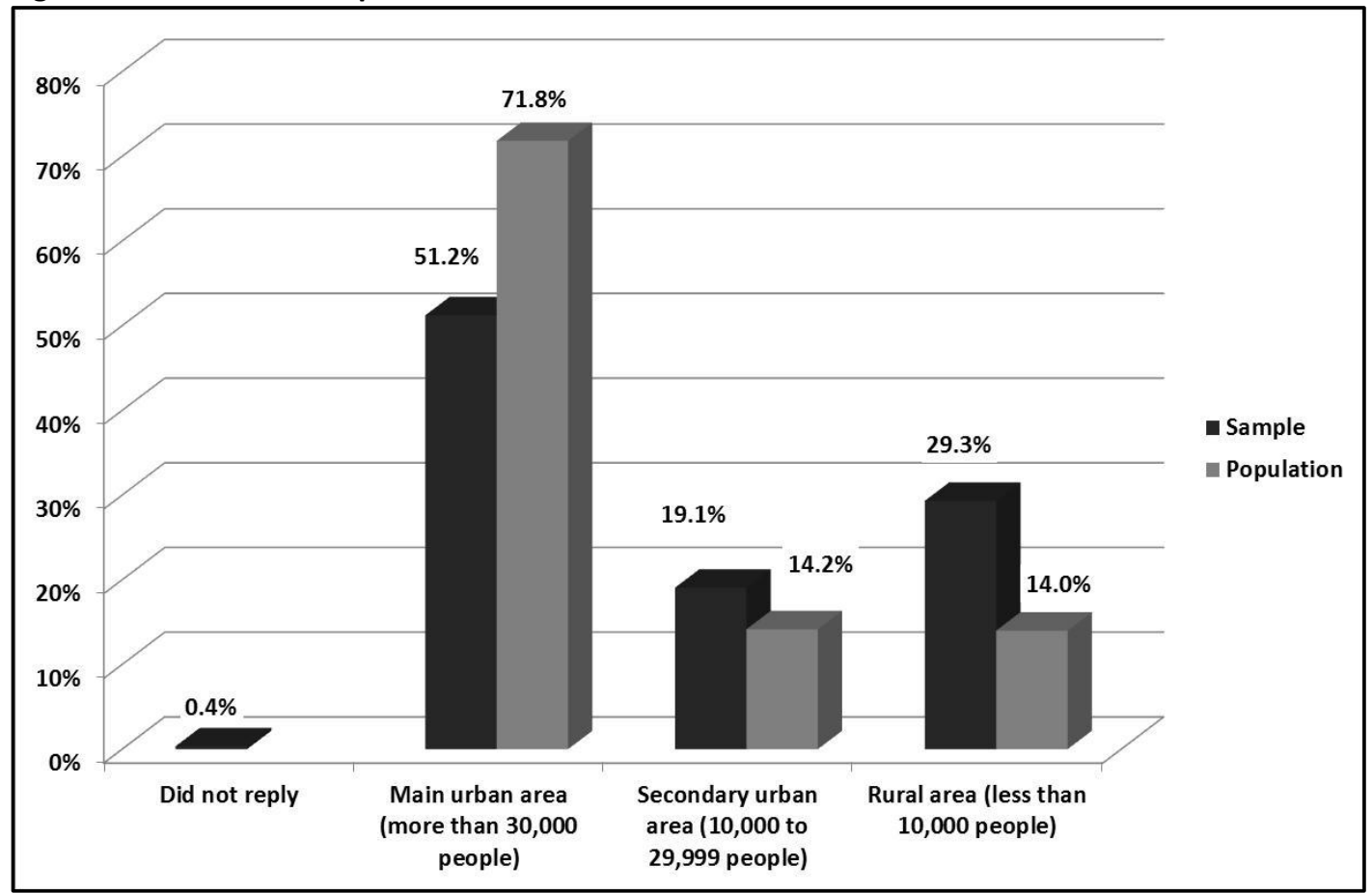

Source: Statistics New Zealand (2006b)

\subsubsection{Car purchase history}

Of those people in the survey sample who owned a car at the time of the survey, $22.4 \%$ had a new car. Of the remaining $77.6 \%$ of the sample who owned a used car, 43.5\% owned used cars that entered New Zealand as new and $52 \%$ as used imports. Of the used car owners, $2.5 \%$ did not know whether their car had entered the country as a new or used vehicle.

\subsubsection{Attitudes to fuel supply and climate change}

The survey indicated that a majority of the respondents were of the view that "The era of cheap oil is over", with $84 \%$ either supporting or strongly supporting this view and $9.9 \%$ disagreeing with the statement. No one in the survey sample strongly disagreed with the statement. However, $6 \%$ of the sample replied that they did not know.

The survey also indicated that a majority of the respondents were of the view that "there is an urgent need for policies to reduce greenhouse gas (GHG) emissions from road transport in New Zealand" with $60.3 \%$ either agreeing or strongly agreeing with the statement (50.7\% and $9.6 \%$ respectively). Those taking the 
opposing view consisted of $29 \%$ of the sample (24.1\% disagreeing and $5 \%$ strongly disagreeing). The "do not knows" comprised $10.6 \%$ of the sample.

The question seeking to gauge views as to whether the Government should influence car purchases as a means of reducing GHG emissions resulted in $60.3 \%$ either agreeing or strongly agreeing that the government should not be involved ${ }^{62}$ and $29.6 \%$ either disagreeing or strongly disagreeing. The results indicated that, while there was about two-to-one support for reducing GHG emissions from road transport, there was no similar support for the Government directly implementing measures that influence car purchase behaviour.

Further analysis found that 15 of the respondents who disagreed with the need for policies to reduce GHG emissions from road transport also supported the idea of the Government taking action to reduce these GHG emissions. This seems to be an inconsistent result, which probably indicates that the negative phrasing of the question on the Government's role may have been confusing to some of the respondents. Therefore, the response to this question should be treated with caution.

\subsubsection{Availability of home recharging}

The home recharging question was included in the survey to determine whether this factor would have an effect on the choice of an electric vehicle (EV). During the development of the survey, it became apparent that many people could not answer this question without specialist advice. The question was framed to find out whether the respondent was of the opinion that they could recharge an EV at their home and the results may not reflect the actual capability of these households to undertake the recharging of EVs.

The survey indicated that $66.7 \%$ of the respondents believed that they would be able to recharge an EV at their home, and $8.2 \%$ said that they could not. However,

\footnotetext{
${ }^{62}$ The question asked "In aiming to reduce greenhouse gas emissions, the Government should not try to influence the purchase decisions of private car buyers?"
} 
$25.2 \%$ of the respondents indicated that they did not know if they could recharge an EV at their home (Figure 7.7).

Analysis by residential location resulted in $70.3 \%$ of urban residents being confident that they could recharge at their home compared to $62.7 \%$ of rural residents. The proportion who did not know if they could recharge at their home was relatively constant across the locations with $26.2 \%$ of urban residents, $25.9 \%$ of semi urban residents, and $22.9 \%$ of rural residents (Figure 7.8 ).

Analysis by gender indicated different views about the ability to recharge an EV at their home. Of the males, $77.0 \%$ indicated that they thought they could recharge at their home, while $6.1 \%$ indicated that they could not. Of the females, $52.1 \%$ indicated that they could recharge at their home, and $11.1 \%$ indicated that they could not. The level of uncertainty about the ability to recharge at their home was twice as high for females, at $36.8 \%$, as for males, at $17 \%$.

Figure 7.7: Response to - ‘Could you recharge an electric vehicle where you live?’

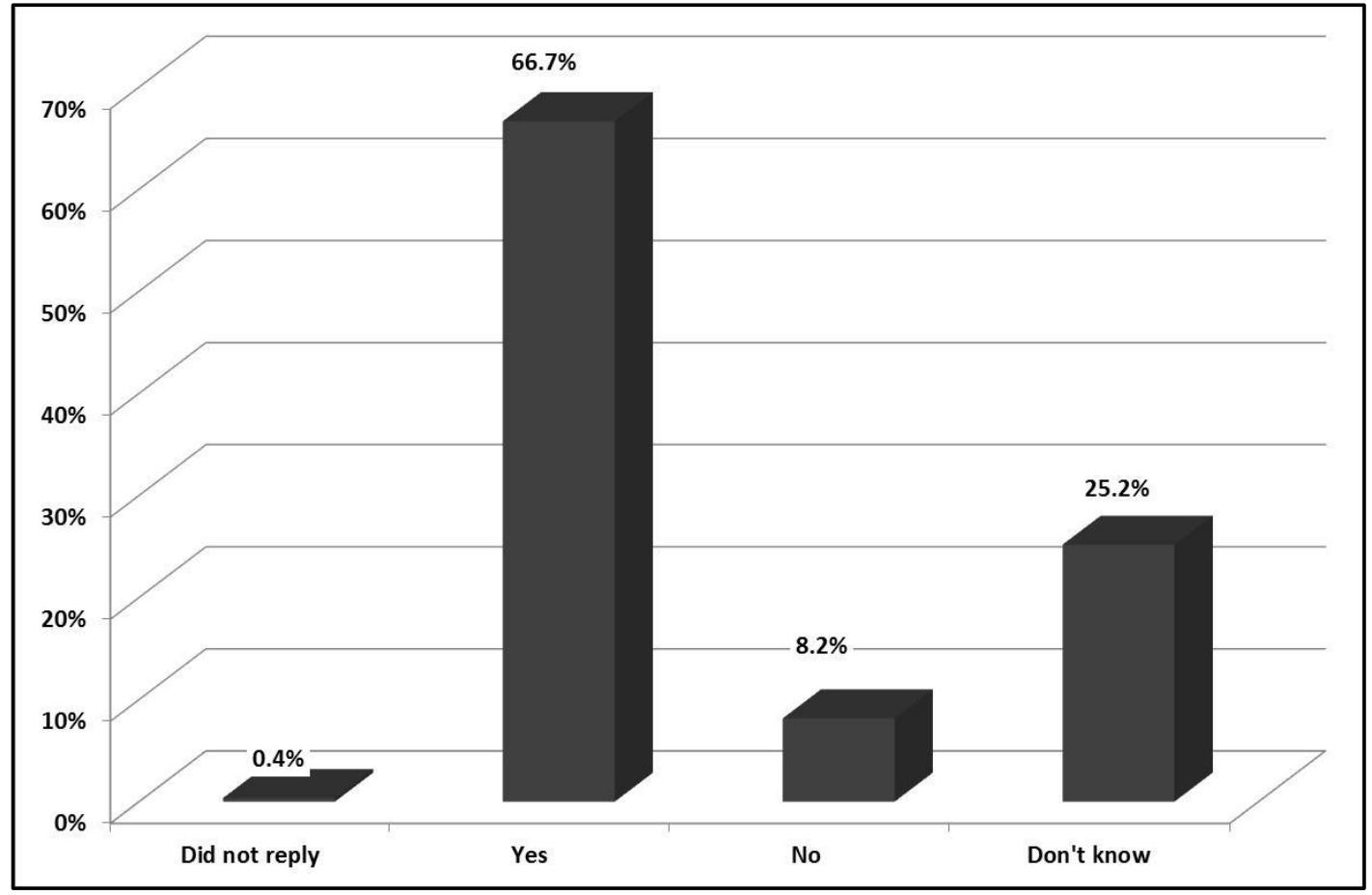




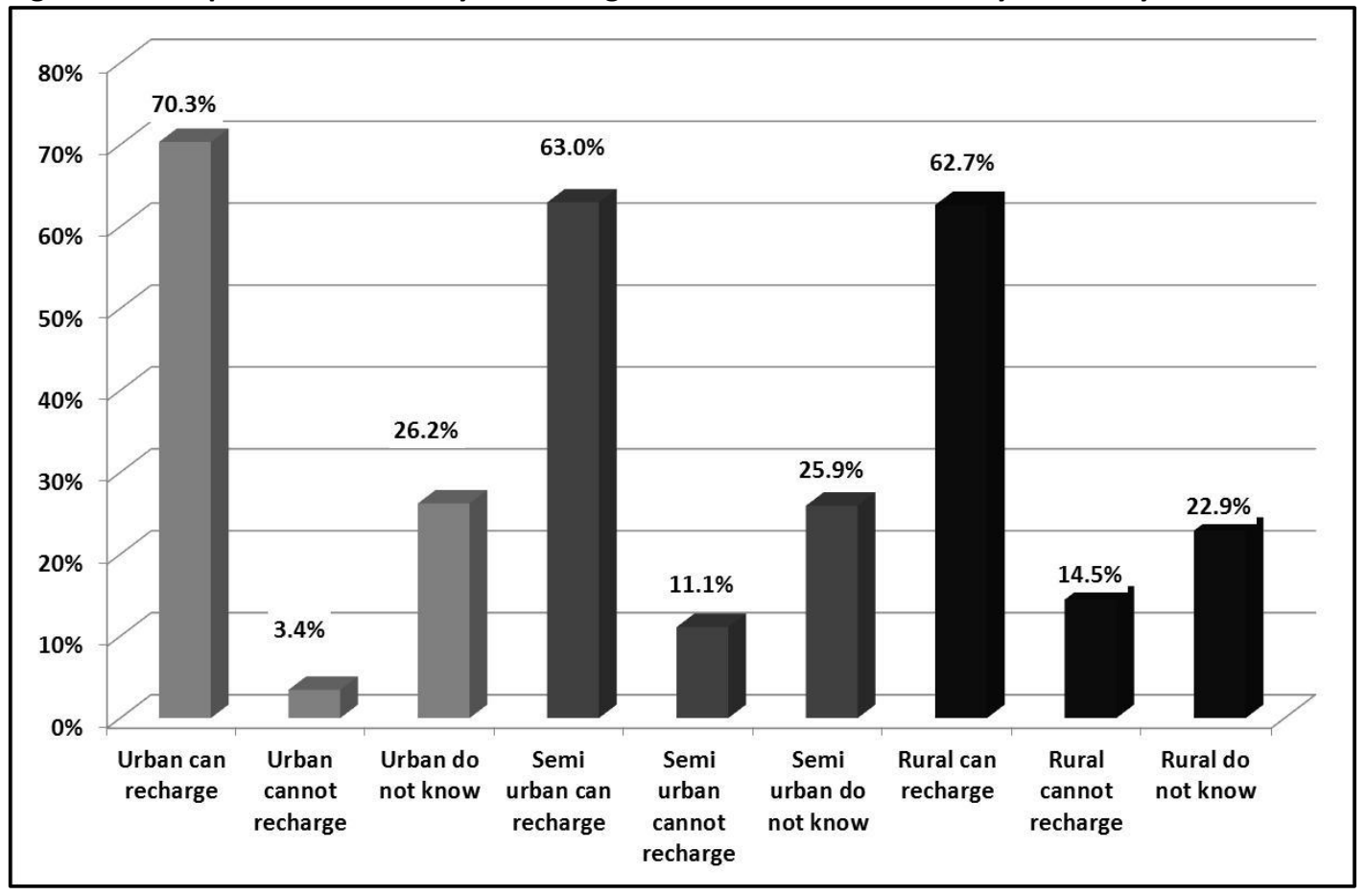

\subsection{The discrete choice model}

\subsubsection{Model estimation}

Both the MNL and the RPL models performed best with the models specified using generic attribute parameters. The ECMNL model performed best with a mixture of alternative specific and generic parameters. Both the ECMNL and RPL models using a panel data specification significantly outperformed the same type of logit models using cross-sectional data.

During the estimation process, a number of socio-demographic variables were also included in the model specifications to determine whether they would have an effect on choice behaviour. The variables tested were household income, gender, age, locality (urban, secondary urban, and rural), the ability to recharge at home, views on the future price of fuel, and the level of concern about the need to reduce GHG emissions from road transport.

All RPL model specifications were first estimated using triangular distributions, and then compared to the same models using normal distributions. Models with triangular distributions consistently performed better. All mixed multinomial logit 
(MMNL) models were estimated using 200 Halton draws. Using Halton draws has been proven superior to the use of random draws (Train, 1999) ${ }^{63}$.

Model selection consisted of choosing the best overall model using the likelihood ratio test, Akaike information criterion and Bayesian information criterion, while also ensuring that all the attributes included in the model were statistically significant at the $5 \%$ level (Greene, 2002). McFadden pseudo $\mathrm{R}^{2}$ measures were also calculated using an 'alternative specific constants (ASC) only' specification for the base model (Hensher et al., 2005, p. 337; Koppelman and Bhat, 2006).

As no data could be found on the socio-demographics of New Zealand car buyers, rather than assuming that under or over sampling had occurred, it was decided not to reweight the survey sample data before model estimation (Hensher et al., 2005, p. 418).

The ASCs in the models were not recalibrated as the design of the choice experiment contained only one alternative present in the market (ICEV) at the time of model estimation. ASC recalibration requires at least two existing market alternatives and it was decided to assume that the market shares in the sample accurately reflect the future market shares ${ }^{64}$.

The use of efficient designs resulted in all the attributes included in the design process having statistically significant parameter estimates (Table 7.1). The covariates were not included in the efficient survey design process and, when tested, only some of these variables resulted in statistically significant parameter estimates.

The parameter estimates of the attributes for all the discrete choice models demonstrated the expected signs. The best performing discrete choice model was a panel RPL model with generic parameters. This RPL model had an adjusted

\footnotetext{
${ }^{63}$ Halton sequences are a quasi-random number sequence that are deterministic, but appear random. When used for the mixing distributions in mixed logit models they result in more evenly spread draws for each observation and the simulated probabilities vary less over the observations, relative to those calculated with random draws (Train, 1999).

${ }^{64}$ I am thankful to Dr John Rose for his advice on this matter.
} 
McFadden pseudo $\mathrm{R}^{2}$ value of 0.329 , and the lowest Akaike information criterion and Bayesian information criterion measures. This model specification was chosen for incorporation into the choice simulator in the VFM.

This RPL model included only one covariate - household income - in the utility function specification for used imported EVs. The negative parameter value for this variable indicated that, as household incomes increased, the probability of choosing a used imported EV would decline. When tested, it was found that including the respondents' views about the future of cheap oil resulted in a statistically significant parameter estimate, but its inclusion in the RPL model reduced the overall performance of the model. For reasons of simplicity, it was decided not to include this variable in the version of the model used in the VFM.

The inclusion of other covariate variables improved the performance of the MNL and ECMNL models. The incorporation of the 'cheap oil is over' and climate change questions into the MNL and ECMNL model specifications improved their performance. The parameter estimates indicate that those people who more strongly held the view that "the era of cheap oil is over" and that "there is a need to reduce GHG emissions from road transport" were more likely to buy an $\mathrm{EV}^{65}$.

The best performing ECMNL model contained latent random effects associated with the internal combustion engine vehicle (ICEV), city electric vehicle (CEV), and plugin hybrid electric vehicle (PHEV) alternatives, and with the random components correlated between new and used CEVs, and new and used PHEVs (Table 7.1).

\footnotetext{
${ }^{65}$ The coding of both covariates indicated that agreement with the statement gave a negative value and disagreement a positive value. As the level of disagreement increased, the value of the attribute increased. A negative parameter value indicated that as the level of disagreement with the statements increased, the probability of choosing that alternative decreased.
} 
Table 7.1: Best performing multinomial logit, error components mixed multinomial logit, and random parameters mixed multinomial logit models

\begin{tabular}{|c|c|c|c|c|c|c|}
\hline \multirow[t]{2}{*}{ Variable } & \multicolumn{2}{|l|}{ MNL } & \multicolumn{2}{|c|}{ Panel ECMNL } & \multicolumn{2}{|c|}{$\begin{array}{c}\text { Panel RPL } \\
\text { (used in VFM) }\end{array}$} \\
\hline & Coefficient & t-stat & Coefficient & t-stat & Coefficient & t-stat \\
\hline Constant ICEV & & & 0.267 & -0.8 & $-0.919 * * *$ & -3.85 \\
\hline Constant new CEV & $-2.597 * * *$ & -8.49 & $-2.344 * * *$ & -4.83 & $-3.769 * * *$ & -8.98 \\
\hline Constant used CEV & $-2.592^{* * *}$ & -8.89 & $-2.662 * * *$ & -5.65 & $-3.479 * * *$ & -8.74 \\
\hline Constant new GEV & $-1.312^{* * *}$ & -5.28 & $-1.447 * * *$ & -3.52 & $-1.299 * * *$ & -5.32 \\
\hline Constant used GEV & $-1.134 * * *$ & -5.39 & $-1.977^{* * *}$ & -5.28 & $-1.431 * * *$ & -5.76 \\
\hline Constant new PHEV & $-0.415 * * *$ & -2.67 & $0.397^{*}$ & 1.84 & -0.1203 & -1.00 \\
\hline Constant used PHEV & -0.177 & -1.50 & & & & \\
\hline Purchase price (all EVs) & $-3.648 \mathrm{E}-05^{* * *}$ & -20.85 & & & $-5.444 \mathrm{E}-05 * * *$ & -14.92 \\
\hline Purchase price (new CEV) & & & $-5.383 \mathrm{E}-05^{* * *}$ & -6.83 & & \\
\hline Purchase price (used CEV) & & & $-4.479 \mathrm{E}-05^{* * *}$ & -4.86 & & \\
\hline Purchase price (new GEV) & & & $-4.181 \mathrm{E}-05^{* * *}$ & -7.90 & & \\
\hline Purchase price (used GEV) & & & $-3.286 \mathrm{E}-05^{* * *}$ & -5.13 & & \\
\hline Purchase price (new PHEV) & & & $-4.879 \mathrm{E}-05^{* * *}$ & -14.79 & & \\
\hline Purchase price (used PHEV) & & & $-3.873 \mathrm{E}-05^{* * *}$ & -8.48 & & \\
\hline Driving range (CEV and GEV) & $0.00219 * * *$ & 4.62 & $0.00164 * * *$ & 2.81 & $0.0022 * * *$ & 3.84 \\
\hline Fuel cost (all) & $-0.0273 * * *$ & -17.36 & & & $-0.0534 * * *$ & -14.08 \\
\hline Fuel cost (ICEV) & & & $-0.0444 * * *$ & -11.51 & & \\
\hline Fuel cost (CEV and GEV) & & & $-0.0568 * * *$ & -3.98 & & \\
\hline Fuel cost (PHEV) & & & $-0.0434 * * *$ & -11.73 & & \\
\hline Battery replac. cost (all EVs) & $-3.578 \mathrm{E}-05^{* * *}$ & -10.63 & & & $-8.325 \mathrm{E}-05^{* * *}$ & -9.75 \\
\hline Battery replac. cost (CEV) & & & $-6.057 \mathrm{E}-05^{* * *}$ & -6.96 & & \\
\hline Battery replac. cost (GEV) & & & $-2.366 \mathrm{E}-05^{* * *}$ & -6.87 & & \\
\hline Battery replac. cost (PHEV) & & & $-8.692 \mathrm{E}-05^{* * *}$ & -7.40 & & \\
\hline Time to batt. replac. (all EVs) & $0.0227^{* * *}$ & 5.77 & $0.0305^{* * *}$ & 6.06 & $0.0321 * * *$ & 5.75 \\
\hline Avail. of street recharging & $0.252 * * *$ & 6.52 & $0.311^{* * *}$ & 6.90 & $0.3303^{* * *}$ & 6.30 \\
\hline Top speed (CEV) & $0.00491^{* *}$ & 2.45 & $0.00628 * * *$ & 2.63 & $0.00886 * * *$ & 3.15 \\
\hline \multicolumn{7}{|c|}{ Covariates } \\
\hline HH income (used EVs only) & $-0.0586 * * *$ & -4.41 & $-0.0807 * * *$ & -5.17 & $-0.08076 * * *$ & -3.72 \\
\hline Cheap oil is over(new CEV) & $-0.423 * * *$ & -4.41 & & & & \\
\hline Cheap oil is over(used CEV) & $-0.3042 * * *$ & -3.78 & & & & \\
\hline Cheap oil is over(new GEV) & $-0.259 * * *$ & -2.68 & & & & \\
\hline Cheap oil is over(new PHEV) & $-0.329 * * *$ & -4.60 & & & & \\
\hline Cheap oil is over(used PHEV) & $-0.133^{* *}$ & -2.42 & & & & \\
\hline Cheap oil is over(all EVs) & & & $-0.5889 * * *$ & -3.82 & & \\
\hline $\begin{array}{l}\text { Reduce GHG emissions (used } \\
\text { CEV) }\end{array}$ & $-0.184 * * *$ & -2.72 & & & & \\
\hline $\begin{array}{l}\text { Reduce GHG emissions (used } \\
\text { PHEV) }\end{array}$ & $-0.155^{* * *}$ & -3.49 & & & & \\
\hline $\begin{array}{l}\text { Reduce GHG emissions (all } \\
\text { EVs) }\end{array}$ & & & $-0.5107 * * *$ & -3.62 & & \\
\hline Location of owner (used CEV) & $-0.328 * * *$ & -3.06 & & & & \\
\hline Location of owner (new PHEV) & $-0.2749 * * *$ & -2.87 & & & & \\
\hline $\begin{array}{l}\text { Location of owner (used } \\
\text { PHEV) }\end{array}$ & $-0.249 * * *$ & -3.46 & & & & \\
\hline
\end{tabular}


Table 7.1: (cont.)

\begin{tabular}{|c|c|c|c|c|c|c|}
\hline \multirow[t]{2}{*}{ Variable } & \multicolumn{2}{|c|}{ MNL } & \multicolumn{2}{|c|}{ Panel ECMNL } & \multicolumn{2}{|c|}{$\begin{array}{c}\text { Panel RPL } \\
\text { (used in VFM) }\end{array}$} \\
\hline & Coefficient & t-stat & Coefficient & t-stat & Coefficient & t-stat \\
\hline \multicolumn{7}{|c|}{ ECMNL model standard deviations of latent random effects } \\
\hline Sigma E01 (ICEV) & & & $-3.191 * * *$ & -13.28 & & \\
\hline Sigma E02 (CEVs) & & & $1.43007^{* * *}$ & 7.77 & & \\
\hline Sigma E03 (PHEVs) & & & $0.938 * * *$ & 7.12 & & \\
\hline \multicolumn{7}{|c|}{ RPL model distributions of random parameters -Limits of triangular distributions } \\
\hline ICEV constant & & & & & $8.129 * * *$ & 10.79 \\
\hline CEV new constant & & & & & $4.3089 * * *$ & 9.77 \\
\hline CEV used constant & & & & & $4.3089 * * *$ & 9.77 \\
\hline Purchase price (all EVs) & & & & & $6.372 \mathrm{E}-05^{* * *}$ & 8.61 \\
\hline Fuel cost (All) & & & & & $0.0942 * * *$ & 10.66 \\
\hline Battery repl. cost (All EVs) & & & & & $0.00012 * * *$ & 8.38 \\
\hline Time to battery repl. (all EVs) & & & & & $0.0688^{* * *}$ & 3.30 \\
\hline \multicolumn{7}{|c|}{ Model summary statistics } \\
\hline Parameters (K) & 24 & & 28 & & 21 & \\
\hline Log likelihood & -2573.80 & & -2129.644 & & -2092.402 & \\
\hline Akaike information criterion & 2.641 & & 2.194 & & 2.149 & \\
\hline Bayesian information criterion & 2.7095 & & 2.273 & & 2.2085 & \\
\hline McFadden $\mathrm{R}^{2}$ & 0.183 & & 0.324 & & 0.336 & \\
\hline Adjusted McFadden $\mathrm{R}^{2}$ & 0.173 & & 0.314 & & 0.329 & \\
\hline
\end{tabular}

$* * * 1 \%, * * 5 \%, * 10 \%$ significance level

\subsubsection{Discrete choice model probability shares and elasticities}

Due to the logit transformation, the behavioural interpretation of the parameters of discrete choice models, beyond that of the sign, is difficult (Hensher et al., 2005, p. 383). To assist in evaluating the performance of the RPL model the average direct point elasticities were estimated (Table 7.2). To evaluate further the performance of the RPL model, the market shares were calculated by changing the value of one attribute of one alternative while the values of all other attributes were held constant at their default values. The default values were the average values from the stated choice experiment (see Table 7.2). Charting the changing market shares provided a visual means of the checking the overall performance of the RPL model (Figures 7.9 to 7.14$)$.

The probability weighted direct point elasticities (Hensher et al., 2005, p. 384) indicated that market shares for all EVs were most sensitive to changes in the purchase price and by implication, the reduction in battery costs. The elasticities also indicated that CEVs with reduced top speed, and by implication lower 
performance, would not be as successful as CEVs with top speeds and performance comparable to ICEVs. The effect of improvements in the driving range of GEVs had more of an impact on the market share of these vehicles than increases in the driving range of CEVs had on the demand for CEVs.

Table 7.2: Random parameters mixed multinomial logit model elasticities, means, and standard deviations of attributes choice experiment

\begin{tabular}{|c|c|c|c|c|c|}
\hline Attribute & Elasticity & Mean & $\begin{array}{l}\text { Standard } \\
\text { Deviation }\end{array}$ & Mean & $\begin{array}{l}\text { Standard } \\
\text { Deviation }\end{array}$ \\
\hline & $\begin{array}{c}\text { Direct point } \\
\text { elasticity } \\
\text { averaged } \\
\text { over } \\
\text { observations }\end{array}$ & \multicolumn{2}{|c|}{$\begin{array}{l}\text { Of all } 1967 \\
\text { observations }\end{array}$} & \multicolumn{2}{|c|}{$\begin{array}{l}\text { Of those that chose } \\
\text { that vehicle type }\end{array}$} \\
\hline \multicolumn{4}{|c|}{ ICEV } & \multicolumn{2}{|c|}{$\begin{array}{c}857 \text { observations } \\
\text { chose ICEV }\end{array}$} \\
\hline Fuel Cost $(\$ / 100 \mathrm{~km})$ & -0.3024 & 32.465 & 22.075 & 27.613 & 22.659 \\
\hline \multicolumn{4}{|c|}{ New City EV } & \multicolumn{2}{|c|}{$\begin{array}{l}105 \text { observations } \\
\text { chose new CEV }\end{array}$} \\
\hline Fuel Cost $(\$ / 100 \mathrm{~km})$ & -0.1019 & 4.018 & 2.443 & 2.771 & 2.415 \\
\hline Purchase price (\$) & -0.9866 & $52,155.60$ & $17,395.40$ & $33,333.30$ & $16,650.60$ \\
\hline Electric driving range $(\mathrm{km})$ & 0.1856 & 150.203 & 40.752 & 155.238 & 48 \\
\hline Replacement battery cost (\$) & -0.3432 & $14,997.50$ & $10,733.90$ & $8,085.70$ & $8,704.70$ \\
\hline Time to battery replacement (years) & 0.2702 & 12.501 & 6.101 & 15 & 6.873 \\
\hline $\begin{array}{l}\text { Availability of street charging (None } \\
-1 \text {, Town } 0 \text {, Town and Sub 1) }\end{array}$ & $\mathrm{n} / \mathrm{a}$ & -0.002 & 0.815 & 0.114 & 0.824 \\
\hline Top speed $(\mathrm{km} / \mathrm{h})$ & 0.5433 & 105.48 & 28.555 & 105.333 & 34.49 \\
\hline \multicolumn{4}{|c|}{ Used City EV } & \multicolumn{2}{|c|}{$\begin{array}{l}152 \text { observations } \\
\text { chose used CEV }\end{array}$} \\
\hline Fuel Cost $(\$ / 100 \mathrm{~km})$ & -0.1208 & 4.092 & 2.453 & 4.099 & 2.749 \\
\hline Purchase price (\$) & -0.6855 & $27,384.30$ & 12,189 & $23,421.10$ & $12,048.80$ \\
\hline Electric driving range $(\mathrm{km})$ & 0.1883 & 149.644 & 40.813 & 156.579 & 43.704 \\
\hline Replacement battery cost (\$) & -0.4965 & $16,828.20$ & $10,594.10$ & $11,868.40$ & $10,164.70$ \\
\hline Time to battery replacement (years) & 0.1918 & 8.693 & 8.328 & 9.737 & 9.346 \\
\hline $\begin{array}{l}\text { Availability of street charging (None } \\
-1 \text {, Town } 0 \text {, Town and Sub 1) }\end{array}$ & $\mathrm{n} / \mathrm{a}$ & -0.011 & 0.816 & 0.079 & 0.81 \\
\hline Top speed $(\mathrm{km} / \mathrm{h})$ & 0.5167 & 104.982 & 28.362 & 111.447 & 31.05 \\
\hline \multicolumn{4}{|c|}{ New General purpose EV } & \multicolumn{2}{|c|}{$\begin{array}{l}100 \text { observations } \\
\text { chose new GEV }\end{array}$} \\
\hline Fuel Cost (\$/100 km) & -0.2602 & 8.982 & 5.721 & 7.04 & 6.606 \\
\hline Purchase price (\$) & -1.099 & $87,994.40$ & $44,540.80$ & 38,450 & $21,969.80$ \\
\hline Electric driving range (km) & 0.4762 & 349.911 & 145.237 & 353 & 156.762 \\
\hline Replacement battery cost (\$) & -0.508 & $41,540.40$ & $39,089.50$ & 11,200 & $8,442.30$ \\
\hline Time to battery replacement (years) & 0.3283 & 12.509 & 6.124 & 12.725 & 7.42 \\
\hline $\begin{array}{l}\text { Availability of street charging (None } \\
-1 \text {, Town } 0 \text {, Town and Sub 1) }\end{array}$ & $\mathrm{n} / \mathrm{a}$ & -0.014 & 0.817 & 0.24 & 0.78 \\
\hline
\end{tabular}


Table 7.2: (cont.)

\begin{tabular}{|c|c|c|c|c|c|}
\hline Attribute & Elasticity & Mean & $\begin{array}{l}\text { Standard } \\
\text { Deviation }\end{array}$ & Mean & $\begin{array}{l}\text { Standard } \\
\text { Deviation }\end{array}$ \\
\hline & $\begin{array}{c}\text { Direct point } \\
\text { elasticity } \\
\text { averaged } \\
\text { over } \\
\text { observations }\end{array}$ & \multicolumn{2}{|c|}{$\begin{array}{c}\text { Of all } 1967 \\
\text { observations }\end{array}$} & \multicolumn{2}{|c|}{$\begin{array}{l}\text { Of those that chose } \\
\text { that vehicle type }\end{array}$} \\
\hline \multicolumn{4}{|c|}{ Used General purpose EV } & \multicolumn{2}{|c|}{$\begin{array}{l}104 \text { observations } \\
\text { chose used GEV }\end{array}$} \\
\hline Fuel Cost $(\$ / 100 \mathrm{~km})$ & -0.1828 & 9.128 & 5.673 & 4.49 & 5.154 \\
\hline Purchase price (\$) & -0.8009 & \begin{tabular}{|l|}
$54,733.70$ \\
\end{tabular} & $25,323.20$ & $30,853.40$ & $21,970.80$ \\
\hline Electric driving range $(\mathrm{km})$ & 0.4142 & 348.399 & 145.114 & 314.904 & 168.644 \\
\hline Replacement battery cost (\$) & -0.4415 & $53,528.20$ & $41,658.90$ & $24,423.10$ & $33,956.80$ \\
\hline Time to battery replacement (years) & 0.173 & 8.62 & 8.31 & 8.269 & 9.496 \\
\hline $\begin{array}{l}\text { Availability of street charging (None } \\
-1 \text {, Town } 0 \text {, Town and Sub 1) }\end{array}$ & $\mathrm{n} / \mathrm{a}$ & 0.005 & 0.822 & 0.202 & 0.781 \\
\hline \multicolumn{4}{|c|}{ New Plug-in Hybrid EV } & \multicolumn{2}{|c|}{$\begin{array}{l}224 \text { observations } \\
\text { chose new PHEV }\end{array}$} \\
\hline Fuel Cost $(\$ / 100 \mathrm{~km})$ & -0.3258 & 19.899 & 14.608 & 14.054 & 16.061 \\
\hline Purchase price (\$) & -0.9232 & 60,000 & $32,825.60$ & $34,642.90$ & $25,408.90$ \\
\hline Replacement battery cost (\$) & -0.3647 & $8,759.50$ & $5,107.60$ & $8,052.50$ & $5,892.40$ \\
\hline Time to battery replacement (years) & 0.243 & 12.52 & 6.117 & 11.864 & 7.092 \\
\hline $\begin{array}{l}\text { Availability of street charging (None } \\
-1 \text {, Town } 0 \text {, Town and Sub 1) }\end{array}$ & $\mathrm{n} / \mathrm{a}$ & -0.004 & 0.815 & 0.174 & 0.826 \\
\hline \multicolumn{4}{|c|}{ Used Plug-in Hybrid EV } & \multicolumn{2}{|c|}{$\begin{array}{l}425 \text { observations } \\
\text { chose used PHEV }\end{array}$} \\
\hline Fuel Cost $(\$ / 100 \mathrm{~km})$ & -0.315 & 20.156 & 14.732 & 12.546 & 14.446 \\
\hline Purchase price (\$) & -0.6316 & 29,756 & $16,161.10$ & $27,082.40$ & $16,737.60$ \\
\hline Replacement battery cost (\$) & -0.3052 & 8,715 & $5,115.30$ & $8,088.20$ & $5,380.50$ \\
\hline Time to battery replacement (years) & 0.1532 & 10.015 & 8.129 & 10.494 & 9.059 \\
\hline $\begin{array}{l}\text { Availability of street charging (None } \\
-1 \text {, Town } 0 \text {, Town and Sub 1) }\end{array}$ & $\mathrm{n} / \mathrm{a}$ & -0.004 & 0.813 & 0.296 & 0.822 \\
\hline
\end{tabular}


Figure 7.9: Change in market shares with change in vehicle purchase price (NZ\$)

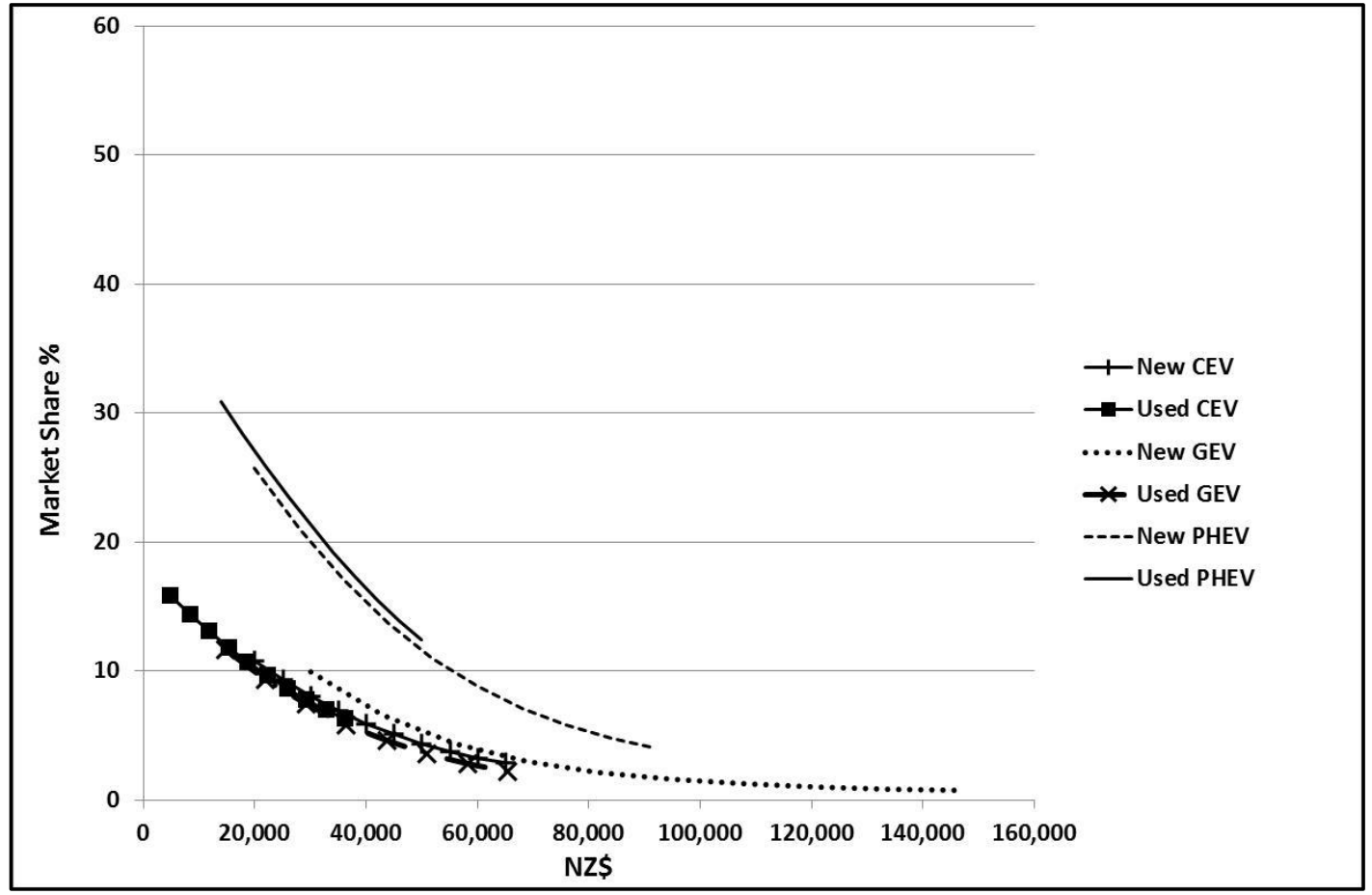

Figure 7.10: Change in market shares with change in fuel cost (NZ\$/100 km)

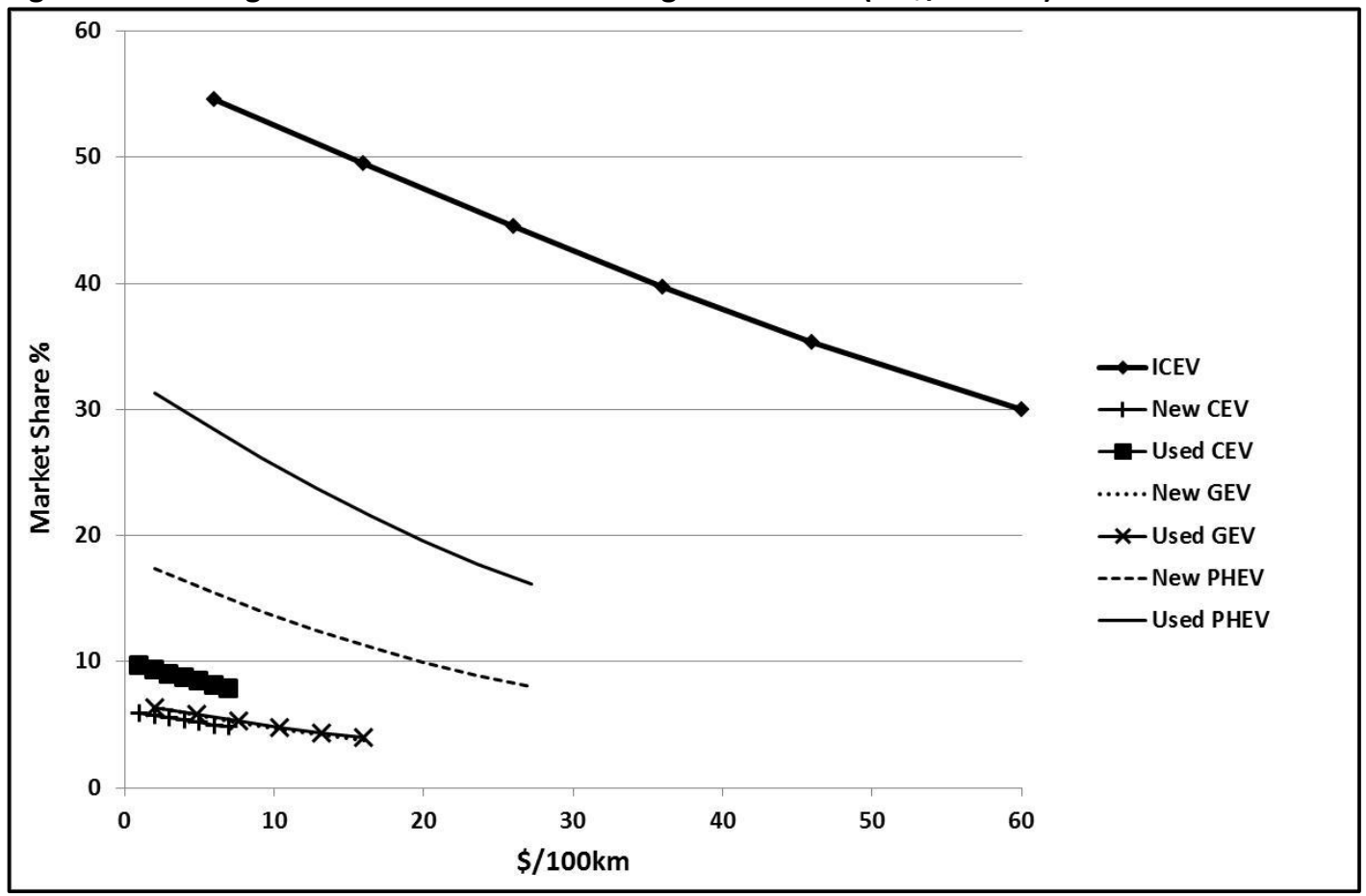


Figure 7.11: Change in market shares with change in replacement battery cost (NZ\$)

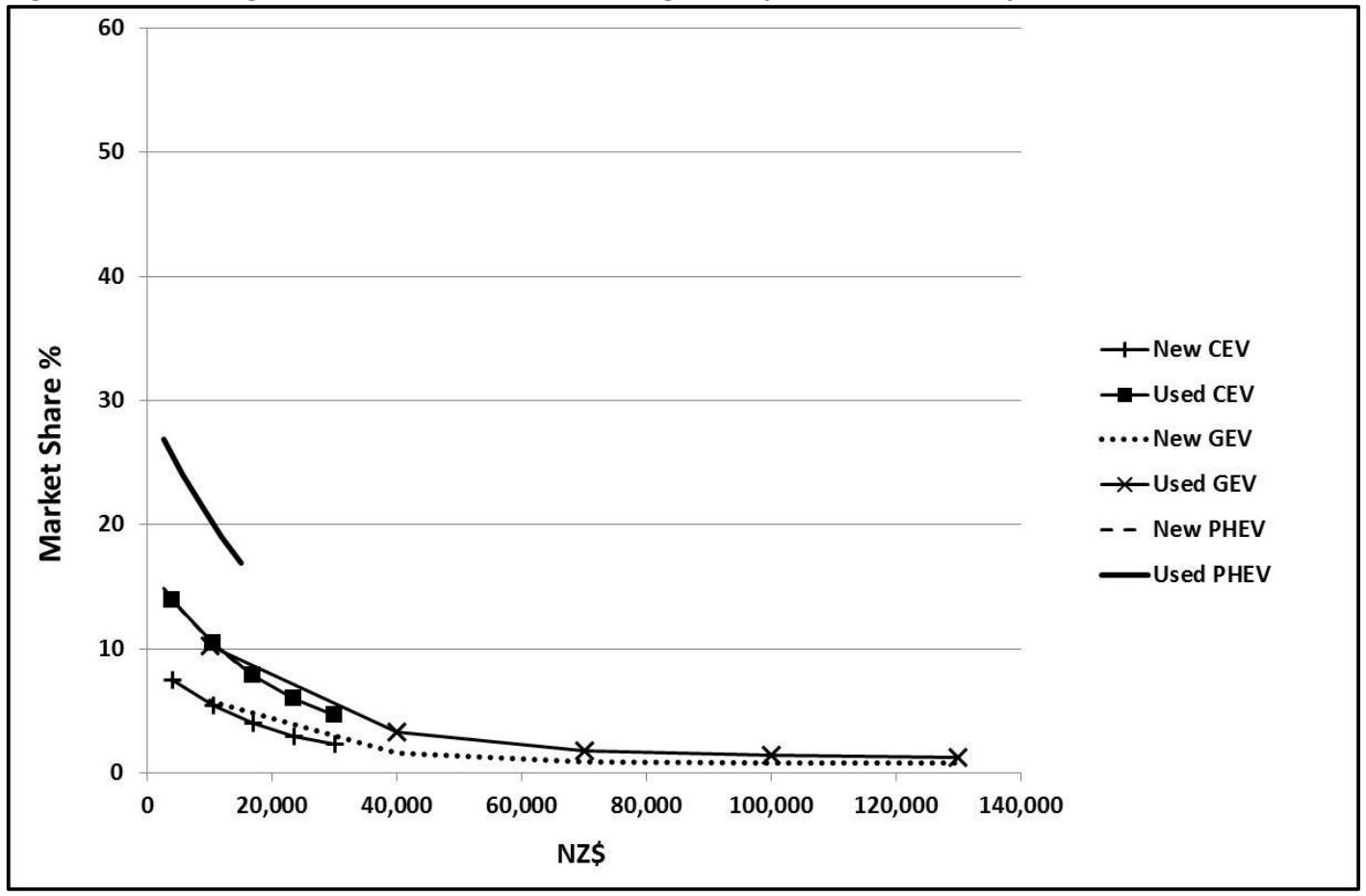

Figure 7.12: Change in market shares with change in electric driving range: $\mathbf{k m}$

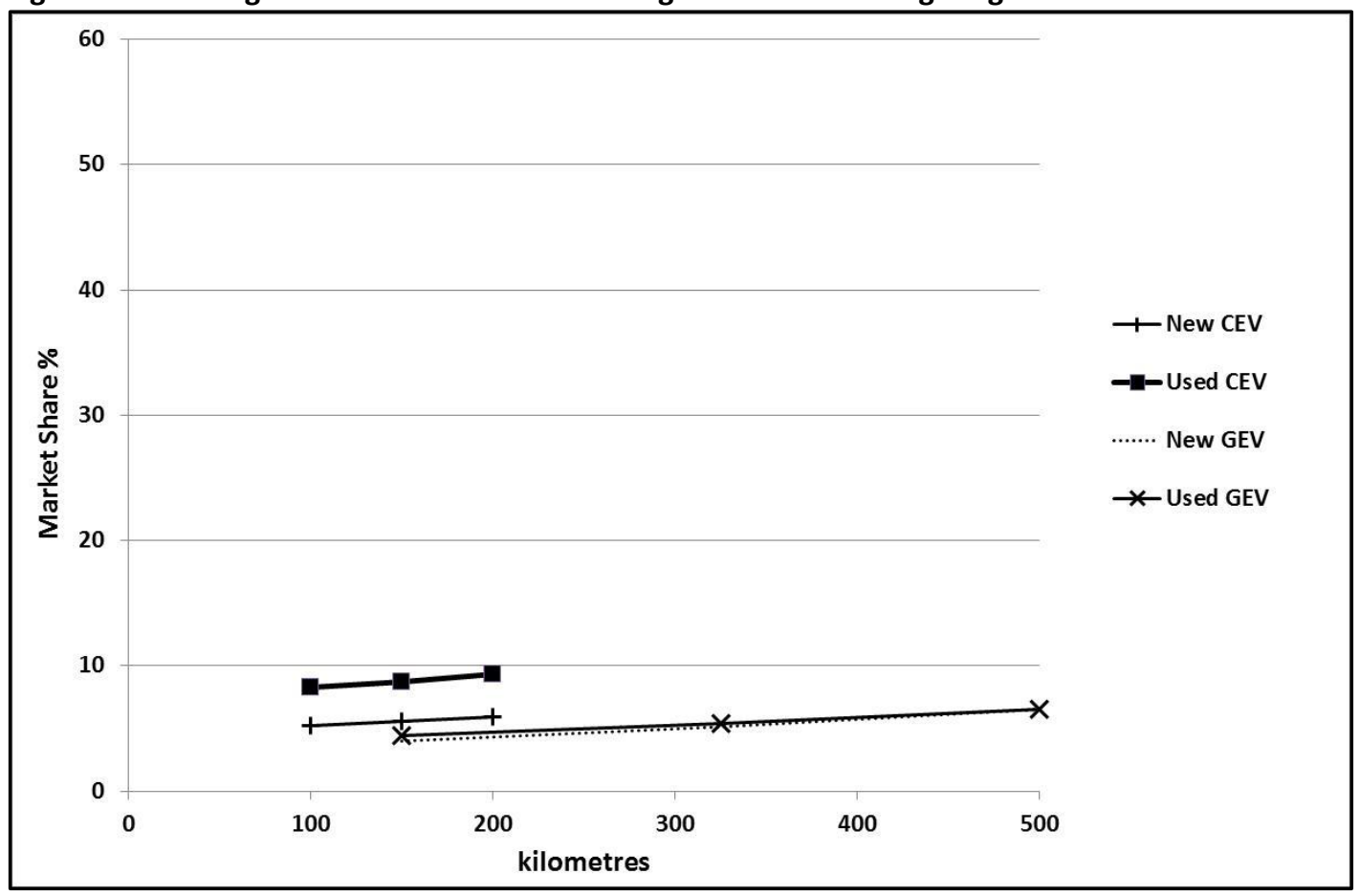


Figure 7.13: Change in market shares with change in time to replacement of battery: years

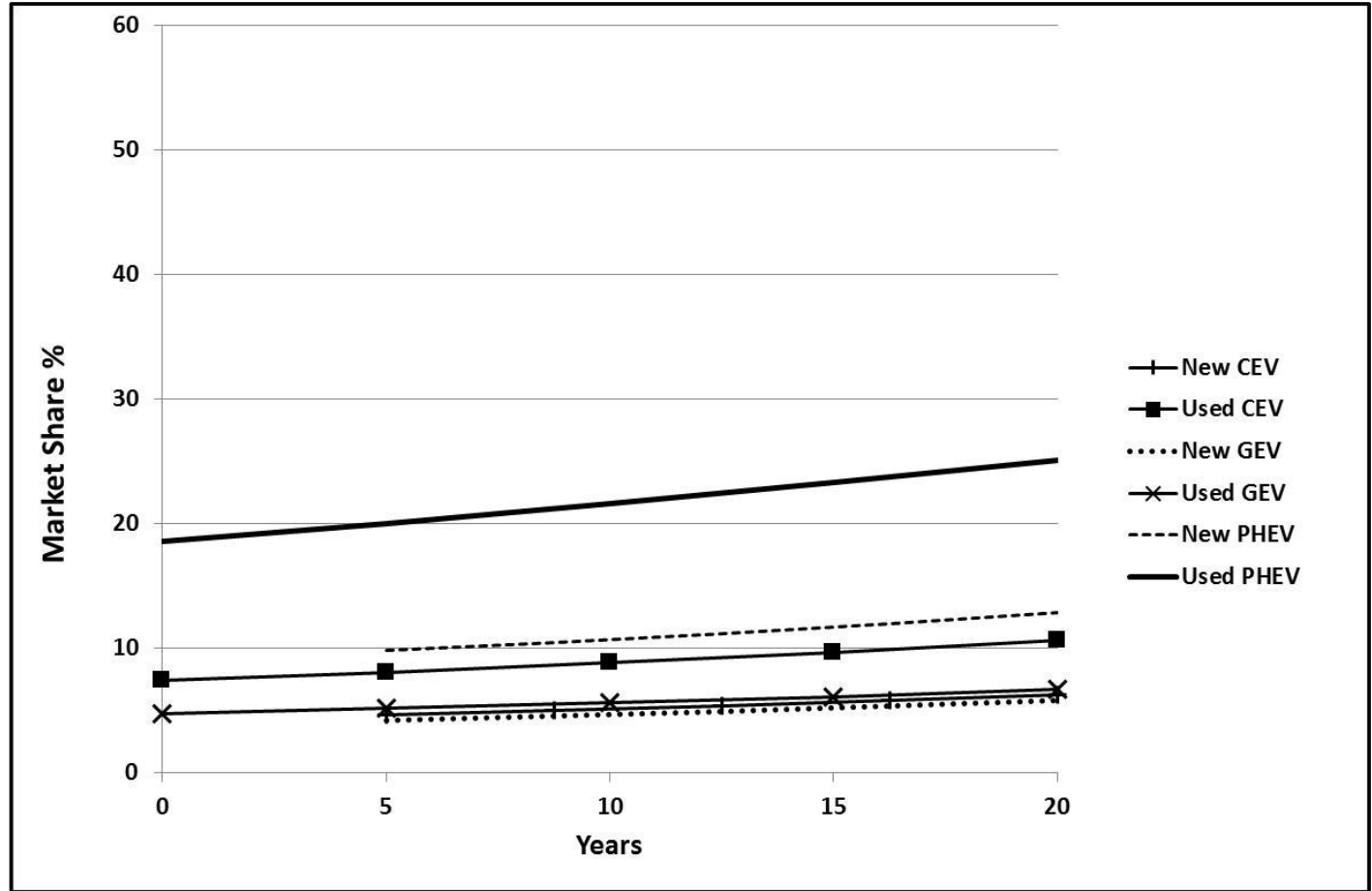

Figure 7.14: Change in market shares with change in top speed, city electric vehicles only

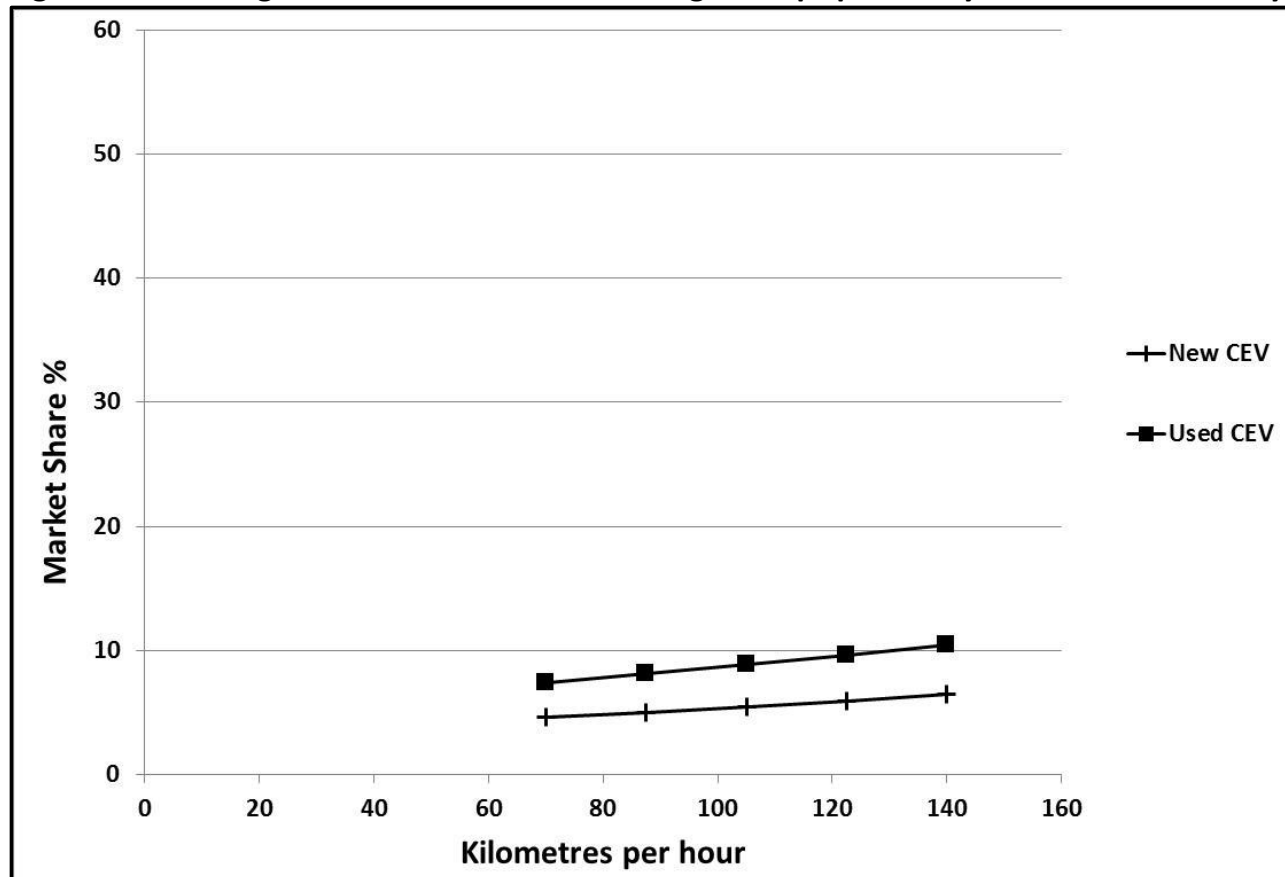




\subsection{Discussion}

\subsubsection{Implementation of the survey}

Until recently, the development and implementation of stated choice surveys has been restricted to specialists with access to significant monetary or data resources. The development of survey design methods, including efficient designs, and survey design software such as NGENE, allowed for the implementation of the relatively complex stated choice experiment in this study.

Providing the option of completing the survey using either postal and web based methods was found to increase the initial acceptance rate during the recruitment phase. However, the use of email as a means of providing the background material and consent forms may not be as effective in securing the respondent's attention as providing these materials in hard copy by the post.

A consideration when using the internet is privacy. During this survey, nine people indicated that even though they had the internet they preferred the postal option. The stated reasons for this choice were concerns about email spam and internet privacy.

\subsubsection{Representativeness of the sample and reweighting}

The apparent underrepresentation of younger car buyers in the sample is a potential concern. However, because there is no socio-demographic data on New Zealand car buyers, the representativeness of the survey sample cannot be determined. It is possible that the low response from young adults and low-income households was a reflection, at least in part, of the actual age and income distribution of New Zealand car buyers.

Rather than assume that the age and income distributions of car buyers were the same as that of the general population, it was decided not to reweight the survey sample data before model estimation.

Models estimated using the sub-sample of respondents aged below 40 years of age indicated that this sub group of the survey sample were, on average, less likely to 
choose an EV. This result seems counter to the view that younger people are more open to innovation. However, this group also had a lower household income. Moreover, these results are not robust as all these sub-models estimated had statistically insignificant parameter estimates.

\subsubsection{Discrete choice model}

The MMNL models were estimated using both cross-sectional and panel data. The MMNL models using the panel specification performed better than the models using the cross-sectional specification. Both panel ECMNL and RPL logit models performed well, with adjusted McFadden pseudo $\mathrm{R}^{2}$ values of over 0.3 . Hensher et al. (2005, pp. 338-339) state that values above 0.3 for a discrete choice model are indicative of an acceptable model fit.

Both the RPL and ECMNL models were considered acceptable for inclusion into the VFM; the RPL model was chosen due to its better overall performance.

The ASCs in the MNL, ECMNL, and RPL models all indicated that once the effect of the observed attributes were taken into account, there was little difference between the utility provided by a used vehicle and a new one.

Covariates that represented the characteristics of the decision maker can be expected to impact on the decision making process. Covariates can be included in the efficient design process, but when this was done it significantly increased the minimum required sample size. Increasing the minimum sample size would have imposed additional costs and time constraints and the decision was made to exclude covariates from the efficient design process. Despite these compromises in the design of the survey, the ECMNL model's estimated parameters (Table 7.1) strongly indicated that household income, attitudes about the future price of oil, and the need to act on climate change would have an impact on the choice of an EV.

There was an expectation that a respondent's ability to recharge an EV at their residence would influence the decision to choose an EV. However, across all the 
MNL, ECMNL, and RPL model specifications tested, this variable was not found to be significant.

\subsubsection{Individual and joint decision making}

In an attempt to make the decision making process as close as possible to that used in real life, respondents were asked to complete the survey with the person they would buy a car with in real life. If unable to comply with this request, they were asked to take that person's preferences into account when completing the choice tasks. This request had limited success, as even though the results indicated that $79.5 \%$ of females, and $60.7 \%$ of males, would make the purchase decision in conjunction with someone else in real life, only $10.3 \%$ of females and $6.0 \%$ of males did the survey together.

There was a greater compliance by these respondents with the request that if they could not complete the survey with the other person, they should try to take account of other person's preferences when completing the choice experiment. In this case, $50.9 \%$ of females and $39.7 \%$ of males said they complied with this request. 


\section{Chapter 8: Results II: composition of the LPV fleet - 2010 to 2030}

\subsection{Introduction}

This chapter discusses the performance of: (1) the choice simulator in the vehicle fleet model (VFM) (Section 8.2); and (2) the VFM's internal combustion engine vehicle (ICEV) and electric vehicle (EV) demand projections and the vehicle stock projections for the different VFM scenarios (section 8.3). Section 8.4 presents the results of the sensitivity analysis on these projections.

Section 8.5 contains a discussion of how the projections of EV demand in this study compare to three other New Zealand studies. This section concludes with a discussion of the insights provided by the sensitivity analysis of the relative effectiveness of promoting the demand for EVs in New Zealand through the provision of public charging facilities, purchase subsidies, and the current exemption from the Road User Charge (RUC).

\subsection{The VFM choice simulator}

The choice simulator, incorporating the random parameters multinomial logit (RPL) model, when used in the VFM introduced a small stochastic element to the VFM's output. To estimate mean values of the VFM's outputs, each of the VFM scenarios described in section 5.4.4 was run 10 times. Using the test developed by Bowman and Shenton, the distributions of the 10 model runs were tested and found to be normally distributed allowing for the calculation of confidence intervals and comparison of the scenarios using the T-test.

The level of uncertainty in the output of the VFM varies depending on the size of the market share for each vehicle type. As the market share increased, the level of uncertainty decreased. The tables in this chapter include the $95 \%$ confidence intervals for the VFM output. The figures do not show the confidence intervals due to the small scale.

Table 8.1 shows an example of the output of the choice simulator using scenario B4. This VFM scenario assumed that there would be very high oil prices and a rapid 
decline in the price of EVs and batteries. This VFM scenario resulted in the highest level of uncertainty in the output of all the scenarios.

The table sets out the estimates of the market shares for the year 2012, the assumed first year in which EVs would become widely available for sale, and for the years 2020 and 2030. The table shows the output from the choice simulator when: (1) both new and used EVs were available for sale; (2) there were no used imported EVs available for sale; and (3) only new EVs were available for sale. The table also presents the market shares produced by the VFM after the three-stage process described in section 5.3.1.

The effect of the three-stage application of the choice simulator was that during the period of restricted supply of used imported EVs, the VFM simulated increased demand for ICEVs. The relaxation of the supply constraint on used imported EVs resulted in the output of the VFM choice simulator converging to the output produced by the choice simulator in the NLOGIT software package. 
Table: 8.1: An example of the output of the choice simulator from scenario B4

\begin{tabular}{|c|c|c|c|c|c|c|}
\hline & \multicolumn{2}{|c|}{ All EVs options available } & \multicolumn{2}{|c|}{$\begin{array}{l}\text { ICEV vehicles and new } \\
\text { EVs only available }\end{array}$} & \multicolumn{2}{|c|}{$\begin{array}{c}\text { VFM market shares } \\
\text { output }\end{array}$} \\
\hline & $\begin{array}{l}\text { Market } \\
\text { share } \\
\end{array}$ & \pm & $\begin{array}{l}\text { Market } \\
\text { share } \\
\end{array}$ & \pm & $\begin{array}{l}\text { Market } \\
\text { share } \\
\end{array}$ & \pm \\
\hline \multicolumn{7}{|l|}{ ICEV } \\
\hline 2012 & $58.88 \%$ & $0.41 \%$ & $71.10 \%$ & $0.34 \%$ & $77.62 \%$ & $0.38 \%$ \\
\hline 2020 & $32.76 \%$ & $0.47 \%$ & $47.92 \%$ & $0.52 \%$ & $44.69 \%$ & $0.47 \%$ \\
\hline 2030 & $37.61 \%$ & $0.49 \%$ & $55.34 \%$ & $0.49 \%$ & $42.53 \%$ & $0.46 \%$ \\
\hline \multicolumn{7}{|c|}{ New City EV } \\
\hline 2012 & $2.77 \%$ & $0.09 \%$ & $5.89 \%$ & $0.15 \%$ & $4.79 \%$ & $0.06 \%$ \\
\hline 2020 & $8.33 \%$ & $0.15 \%$ & $19.99 \%$ & $0.27 \%$ & $14.23 \%$ & $0.12 \%$ \\
\hline 2030 & $7.00 \%$ & $0.14 \%$ & $17.83 \%$ & $0.28 \%$ & $9.63 \%$ & $0.07 \%$ \\
\hline \multicolumn{7}{|c|}{ Used City EV } \\
\hline 2012 & $7.33 \%$ & $0.09 \%$ & NA & NA & $0.02 \%$ & $0.00 \%$ \\
\hline 2020 & $13.46 \%$ & $0.11 \%$ & NA & NA & $5.48 \%$ & $0.08 \%$ \\
\hline 2030 & $13.61 \%$ & $0.12 \%$ & NA & NA & $9.91 \%$ & $0.14 \%$ \\
\hline \multicolumn{7}{|c|}{ New General purpose EV } \\
\hline 2012 & $1.07 \%$ & $0.03 \%$ & $2.80 \%$ & $0.06 \%$ & $2.15 \%$ & $0.05 \%$ \\
\hline 2020 & $2.09 \%$ & $0.05 \%$ & $6.23 \%$ & $0.11 \%$ & $4.21 \%$ & $0.07 \%$ \\
\hline 2030 & $1.19 \%$ & $0.03 \%$ & $3.32 \%$ & $0.07 \%$ & $1.80 \%$ & $0.04 \%$ \\
\hline \multicolumn{7}{|c|}{ Used General purpose EV } \\
\hline 2012 & $1.92 \%$ & $0.05 \%$ & NA & NA & $0.01 \%$ & $0.00 \%$ \\
\hline 2020 & $9.32 \%$ & $0.15 \%$ & NA & NA & $3.84 \%$ & $0.03 \%$ \\
\hline 2030 & $4.43 \%$ & $0.08 \%$ & NA & NA & $3.28 \%$ & $0.03 \%$ \\
\hline \multicolumn{7}{|c|}{ New Plug-in hybrid EV } \\
\hline 2012 & $7.81 \%$ & $0.09 \%$ & $20.20 \%$ & $0.20 \%$ & $15.34 \%$ & $0.34 \%$ \\
\hline 2020 & $7.95 \%$ & $0.14 \%$ & $25.86 \%$ & $0.38 \%$ & $16.79 \%$ & $0.28 \%$ \\
\hline 2030 & $7.06 \%$ & $0.10 \%$ & $23.51 \%$ & $0.27 \%$ & $11.41 \%$ & $0.20 \%$ \\
\hline \multicolumn{7}{|c|}{ Used Plug-in hybrid EV } \\
\hline 2012 & $20.23 \%$ & $0.13 \%$ & NA & NA & $0.07 \%$ & $0.00 \%$ \\
\hline 2020 & $26.09 \%$ & $0.14 \%$ & NA & NA & $10.76 \%$ & $0.10 \%$ \\
\hline 2030 & $29.10 \%$ & $0.10 \%$ & NA & NA & $21.44 \%$ & $0.19 \%$ \\
\hline
\end{tabular}

$\pm \quad 95 \%$ confidence intervals

\subsection{The LPV fleet to 2030}

\subsubsection{Projected growth of the LPV fleet}

Using the default GDP growth assumptions (Section 5.4.3) in the aggregate time series vehicle forecast model resulted in the projected ownership of light passenger vehicles (LPVs) in New Zealand increasing from 562.3 per 1000 people, in 2010, to 609.7 per 1000 people, in 2030 . The default population projection equated this increase in vehicle ownership to the size of the LPV fleet increasing by $25.9 \%$ from 2,488,800 vehicles in 2010 to 3,135,400 vehicles in 2030 (Figure 8.1). 
Figure 8.1: Projected total light passenger vehicle ownership using default GDP and population assumptions

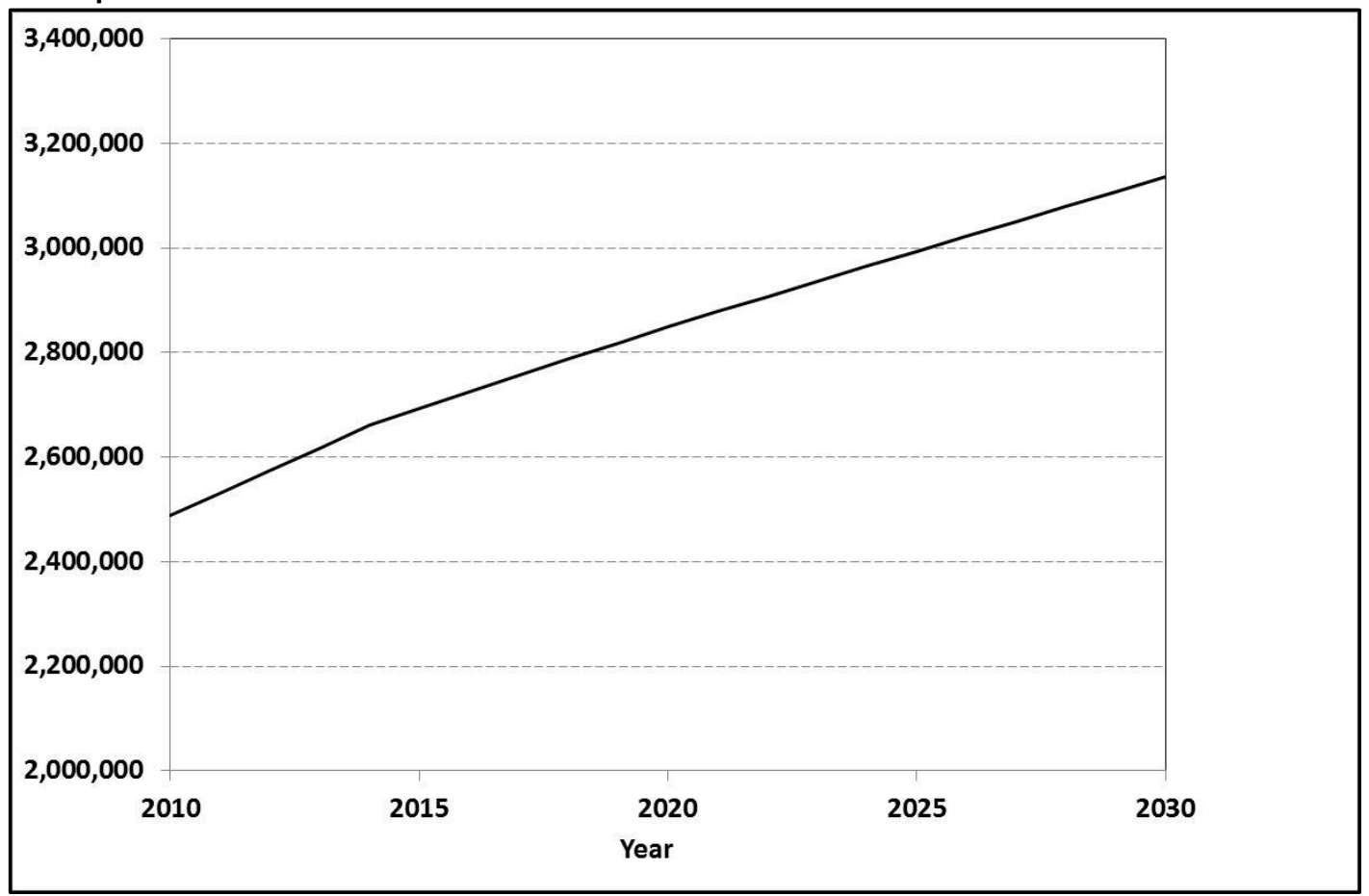

\subsubsection{The A1 (no-EV) reference scenario}

Scenario A1 (no-EV) represented the no surprises or the business as usual (BAU) future and acted as the reference scenario. In this future, the availability of EVs remains limited in New Zealand and overseas markets. The average energy efficiency of the LPVs entering the LPV fleet remains at current levels, and vehicle scrapping continues at the same average rate as during the period 2006 to 2009. In this future, the price of petrol continues to increase so that, in real terms, it increases from $\$ 2.30 / \mathrm{L}$ in 2010 to $\$ 2.87 / \mathrm{L}$ in 2030 .

In this VFM scenario, the projected growth of the LPV fleet and vehicle scrapping result in an estimated 4,257,700 new and used imported LPVs entering the LPV fleet and 3,568,250 leaving the LPV fleet over the modelling period (Figure 8.2). The number of vehicles leaving the LPV fleet annually is projected to increase from 142,500 vehicles in 2010 to 171,300 in 2016 and then to decline to 168,700 by 2020. This surge in scrapping is due to the large number of used LPVs imported during the mid-1990s reaching the age where vehicles would have the highest probability of leaving the LPV fleet. After 2020, the number of vehicles leaving the 
LPV fleet increases steadily and, by 2030 , the number has reached 184,100 vehicles per year.

LPV ownership, in 2009, was below the long-term trend as estimated by the aggregate time series sub-model. Rather than have a large influx of LPVs enter the LPV fleet in the first modelling year, the size of the LPV fleet was returned to the long-term trend over a five-year period.

Figure 8.2: A1 (no-EV) scenario - vehicles entering (sales) and leaving (scrapped) the New Zealand light passenger vehicle fleet

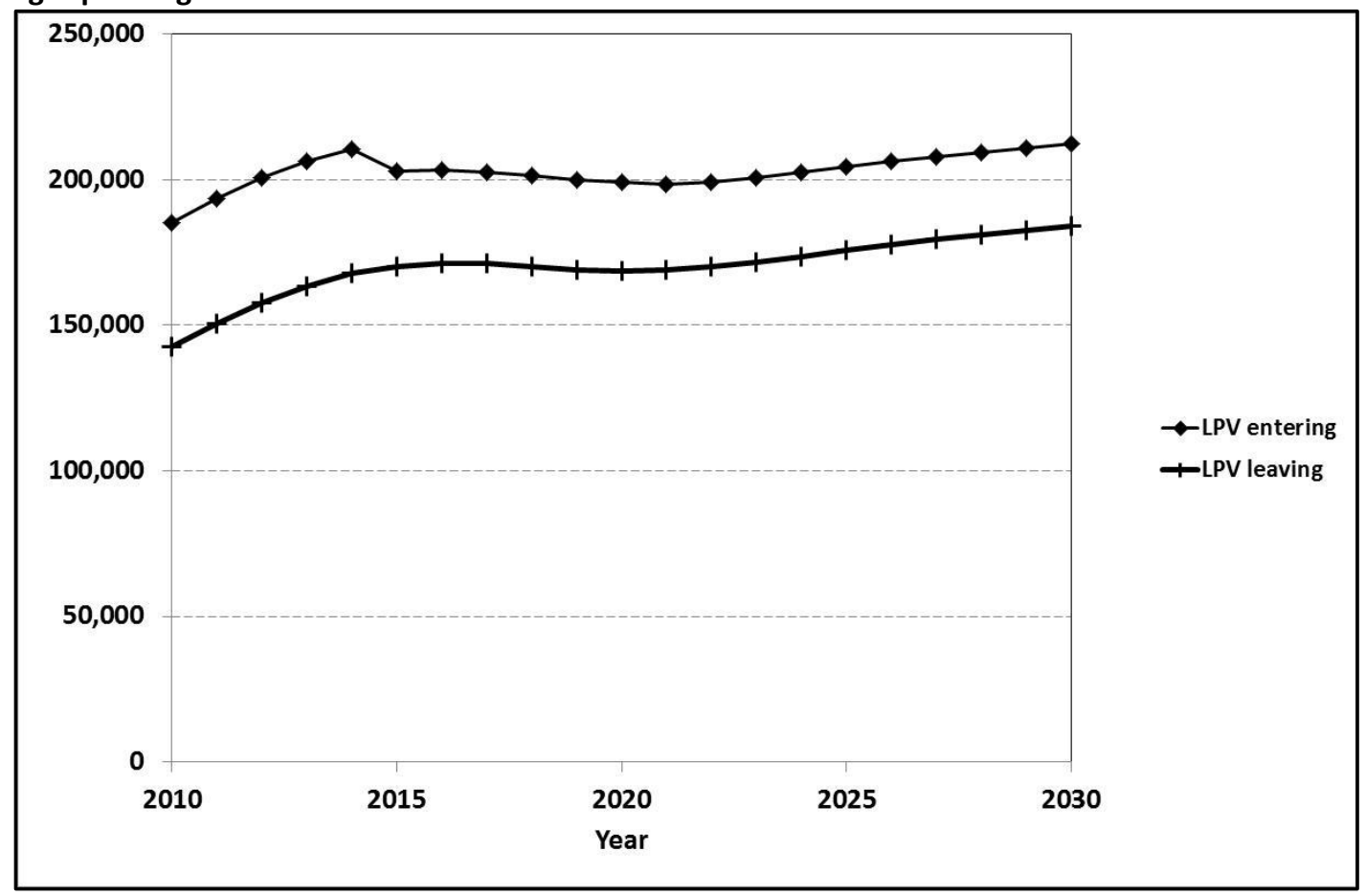

Using the default growth projections for the LPV fleet, the projected total LPV fleet vehicle kilometres travelled (VKT) increased from 30,487 million kilometres per year, in 2010 , to 37,154 million kilometres per year in 2030 . The vehicle use model (VUM) in the VFM estimated that, for this VFM scenario, the effect of the slowly increasing fuel costs over the modelling period would result in a slight decline in the average VKT per vehicle from 12,250 kilometres per year in 2010 to 11,850 kilometres per year in $2030^{66}$.

\footnotetext{
${ }^{66}$ In 2011, due to weak GDP growth of $1.3 \%$ per year and an annual increase in price of fuel of $11.9 \%$ actual LPV VKT declined by $0.62 \%$. The assumption in scenario A1 (no-EV) was that, during this period, annual GDP growth would increase by $2.4 \%$ and fuel by $1.0 \%$. Consequently, the VFM projected a 1.6\% increase in VKT from 2010 and 2011.
} 
In this VFM scenario, the total amount of energy used by the LPV fleet annually increases from the 2010 estimate of $29,532 \mathrm{GWh}(106.3 \mathrm{PJ})$ to $35,989 \mathrm{GWh}$ (129.6 PJ) in 2030. The cumulative energy demand over the modelling period in the reference scenario is $694,954 \mathrm{GWh}(2,501.8 \mathrm{PJ})$. Based on the assumptions in this VFM scenario, the annual greenhouse gas (GHG) emissions from the LPV fleet increase by $22 \%$ from the estimated $7,216,000$ tonnes $\mathrm{CO}_{2} \mathrm{e}$ in 2010 to $8,794,193$ tonnes $\mathrm{CO}_{2} \mathrm{e}$ in 2030, and the cumulative GHG emissions over the modelling period are estimated to be $169,819,154$ tonnes $\mathrm{CO}_{2} \mathrm{e}$.

\subsubsection{LPV demand and vehicle stock}

The default growth projection for the size of the LPV fleet in section 8.3.1 applies to all the VFM scenarios. However, different vehicle choice behaviour across the VFM scenarios has an impact on the age profile of the LPVs entering the LPV fleet. In VFM scenarios that have a higher demand for EVs, the average age of the LPVs that enter the LPV fleet is lower due to the constrained supply of used EVs transferring some of the demand to new EVs. The lower age of the LPVs entering the LPV fleet delays vehicle scrapping and fewer new and used imported LPVs are required to enter the LPV fleet (Figure 8.3).

Figure 8.3: Total number of vehicles entering the light passenger vehicle fleet - new and used imports

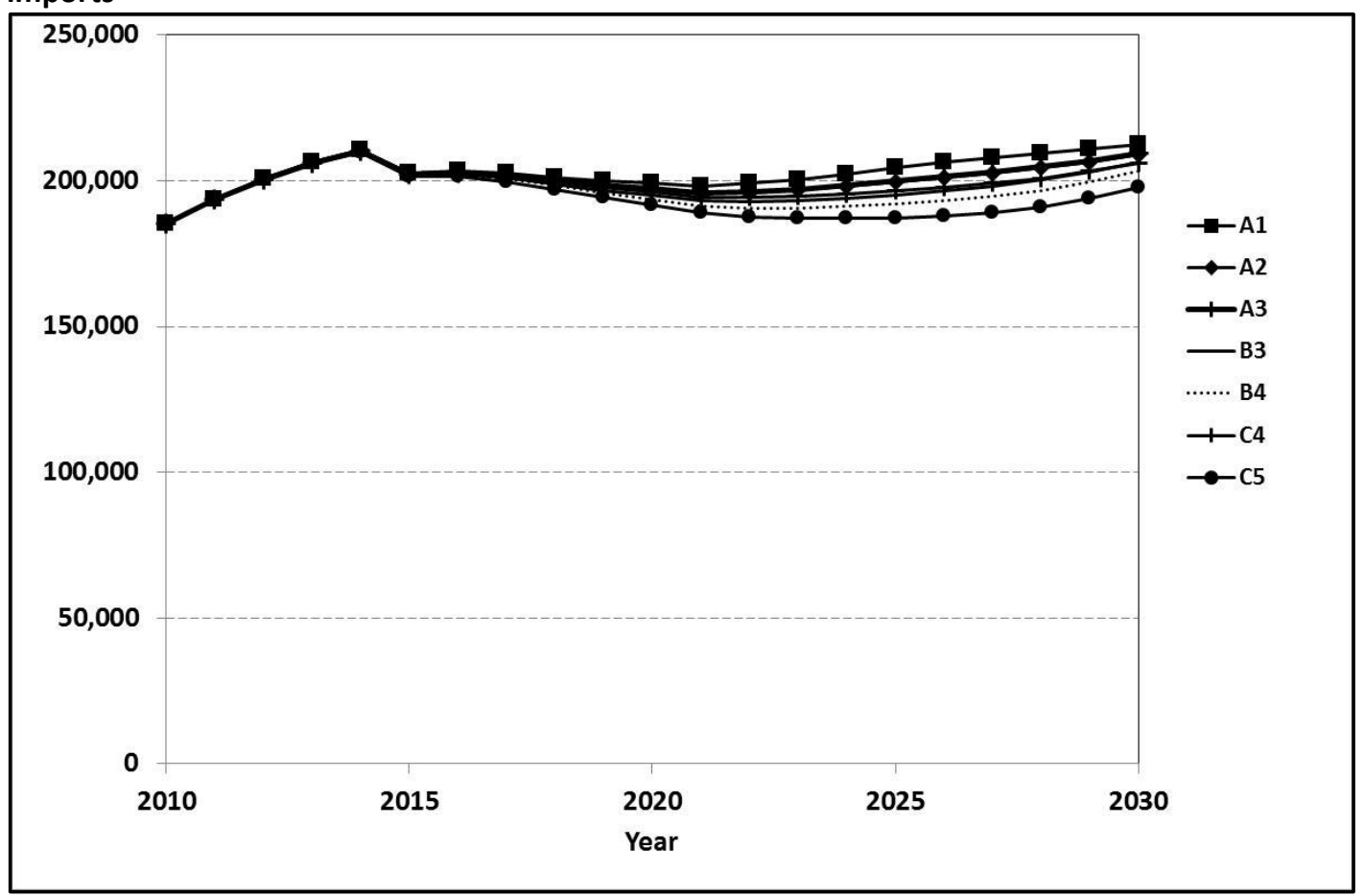




\section{ICEVs}

The number of ICEVs projected to enter the LPV fleet annually in VFM scenarios A2, A3, B3, B4, and C4 declines from 194,000 vehicles in 2011, to between $86,200 \pm 600$ and $91,700 \pm 200$ vehicles in 2030 . This level of demand for ICEVs is approximately $40 \%$ lower than the demand in the $\mathrm{A} 1$ (no-EV) reference scenario. In scenario $\mathrm{C} 5$, where it was assumed that there were technological changes that significantly reduced the final purchase price that could be achieved by EVs, the number of ICEVs entering the LPV fleet declines to $61,000 \pm 300$ in 2030 , which is approximately $28 \%$ of the forecast demand in $\mathrm{A} 1$ (no-EV) reference scenario (Figure 8.4).

Figure 8.4: Internal combustion engine vehicles entering the light passenger vehicle fleet: 2010 to 2030

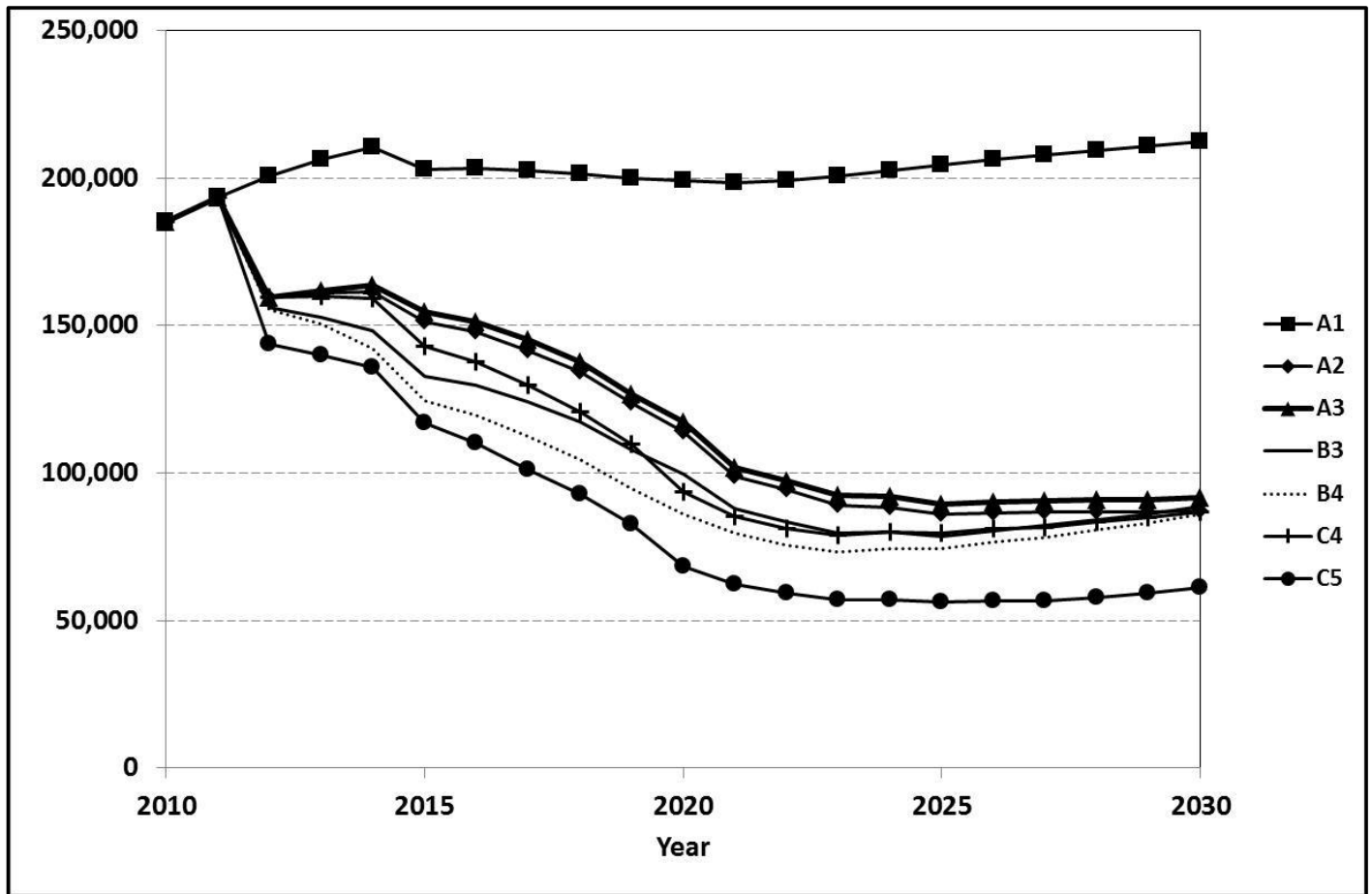

The reduction in demand for ICEVs results in ICEVs comprising, in 2030, between $48.8 \% \pm 0.5 \%$ and $56.7 \% \pm 0.5 \%$ of the LPV fleet in VFM scenarios $A 2, A 3, B 3, B 4$, and $\mathrm{C} 4$, and $41.7 \% \pm 0.3 \%$ in the technological breakthrough scenario C5 (Table 8.2 and Figure 8.5). 
Table 8.2: Stock of internal combustion engine vehicles in 2030 by vehicle fleet model scenario

\begin{tabular}{|c|c|c|c|c|c|c|c|}
\hline & A1 & A2 & A3 & B3 & B4 & C4 & C5 \\
\hline Stock in 2030 & $3,135,370$ & $1,728,619$ & $1,777,043$ & $1,615,828$ & $1,531,333$ & $1,621,424$ & $1,307,477$ \\
\hline \pm & $\mathrm{n} / \mathrm{a}$ & 6,359 & 9,172 & 9,835 & 8,009 & 7,614 & 3,722 \\
\hline $\begin{array}{c}\text { Proportion of } \\
\text { LPV fleet in } \\
2030\end{array}$ & $100 \%$ & $55.1 \%$ & $56.7 \%$ & $51.5 \%$ & $48.8 \%$ & $51.7 \%$ & $41.7 \%$ \\
\hline \pm & $\mathrm{n} / \mathrm{a}$ & $0.4 \%$ & $0.5 \%$ & $0.6 \%$ & $0.5 \%$ & $0.5 \%$ & $0.3 \%$ \\
\hline
\end{tabular}

$\pm$

95\% confidence intervals

Figure 8.5: Stock of internal combustion engine vehicles: 2010 to 2030

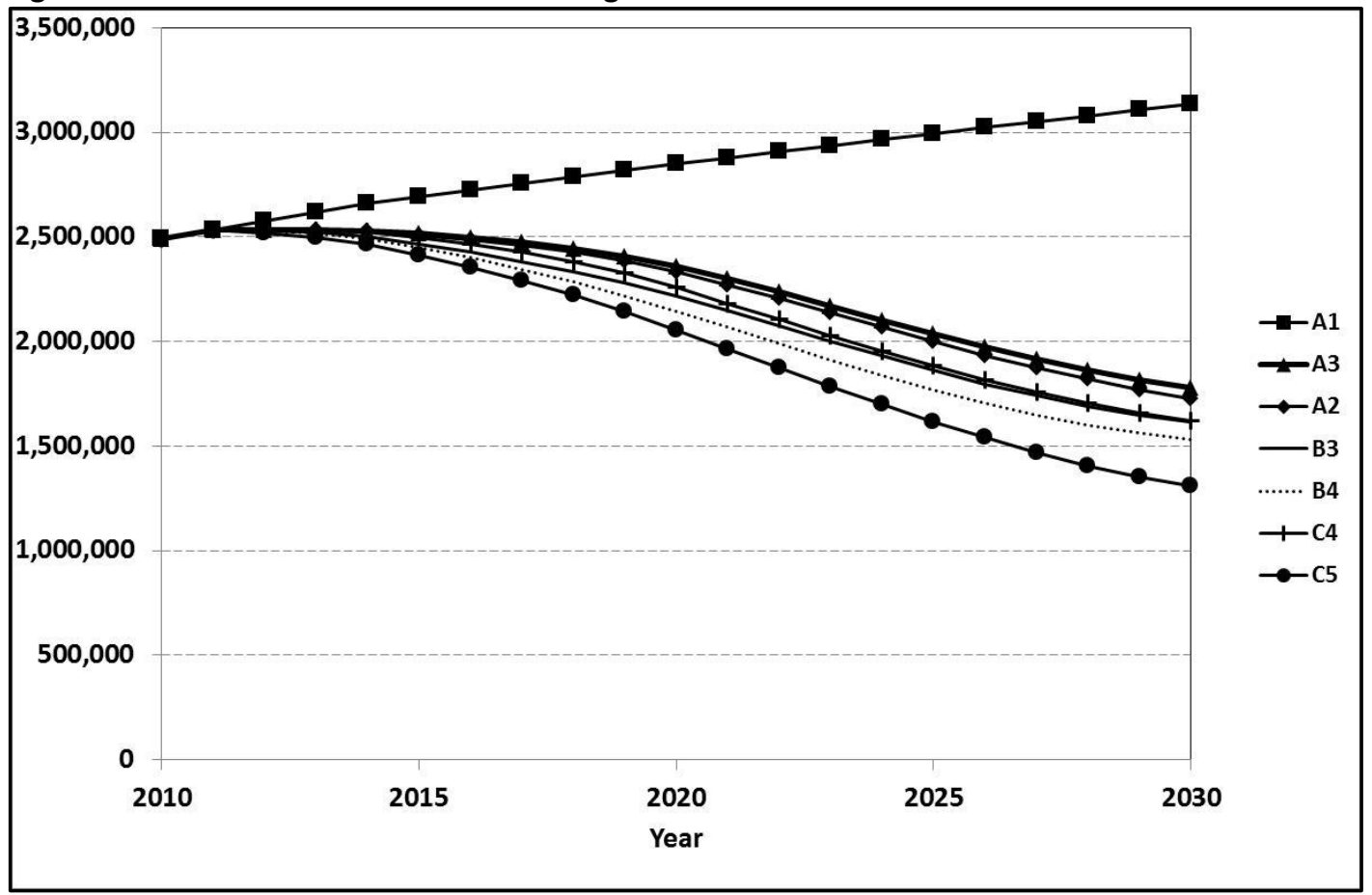

EVs

The choice simulator in the VFM projected that if new EVs became widely available for sale in 2012 and if EV prices and vehicle performance reflect current market conditions, EVs would comprise $20.3 \% \pm 0.1 \%$ to $22.5 \pm 0.2 \%$ of the vehicles entering the LPV fleet. If the price on carbon was $\$ 25$ per tonne, the projected demand in the first year of introduction would be between $20.3 \% \pm 0.1 \%$ and $20.4 \% \pm 0.2 \%$ (scenarios $A 2$ and $A 3$ ). If a higher carbon charge of $\$ 100$ per tonne was assumed to be in effect, the proportion of EVs entering the LPV fleet in the first year of introduction would increase to $20.6 \% \pm 0.2 \%$ (scenario C4). The effect on EV demand of increased petrol prices would be greater. In scenarios B3 and B4, the assumption was that petrol prices would be $35 \%$ higher than prices in 2010 . This 
assumption had the effect of increasing the demand for EVs in the first year of introduction to between $22.2 \% \pm 0.3 \%$ and $22.5 \% \pm 0.2 \%$.

To increase the demand for EVs further in the first year of introduction, it would be necessary to reduce the purchase price of EVs. In scenario C5, the EVs contained smaller and less expensive batteries due to their energy efficient design. With a $22 \%$ to $23 \%$ price reduction for battery electric vehicles (BEVs), and a $15 \%$ reduction for plug-in hybrid electric vehicles (PHEVs), the proportion of EVs that entered the LPV fleet in the first year would increase to $28.0 \% \pm 0.1 \%$ (Figure 8.6).

With the exception of scenario C5, all the scenarios assumed that EV technology would reach the same final point of development sometime within the modelling period and only the rate of improvement varied across the scenarios. After that time, the purchase price of new EVs would remain constant relative to ICEVs and any decline in the average price of EVs that entered the LPV fleet would be due to the effect of used imported EVs. For the VFM scenarios that assumed a steady development of EV technology, the final level would be achieved by 2030 (scenarios A3 and B3). For the rapid development scenarios, the final level would be achieved by 2020 (scenarios B4 and C4). With these assumptions, the VFM estimated that the maximum proportion of EVs that entered the LPV fleet would be approximately $62 \%$, with the final levels, in 2030 , varying between $56.2 \% \pm 0.3 \%$ and $58.3 \pm 0.2 \%$. The differences between VFM scenarios were due to different projections of the price of used imported EVs that entered the LPV fleet, and fuel running costs for ICEVs.

The assumption in breakthrough scenario $\mathrm{C} 5$ was that there would be a rapid development of EV technologies and that the cost of battery cells would decline to half the price assumed in the other scenarios. This assumption, in conjunction with the impact of the EV energy efficient design assumption, resulted in lower EV purchase prices relative to the price of ICEVs. Lowering the projected purchase price of EVs further was the assumption in this VFM scenario that the Government would also provide EV purchase subsidies. Together, these assumptions had the 
effect of increasing the projected maximum level of EV uptake to $69.2 \% \pm 0.2 \%$, which was achieved by 2025 (Figure 8.6).

The assumption in scenarios B3, B4, C4, and C5 was that, over time, improvements in ICEV technology would reduce the fuel running costs of these vehicles. This improvement in energy efficiency resulted in some recovery in their market share in these VFM scenarios. This effect could be seen in scenarios B3 and B4 in Figure 8.6, but was also present to a lesser extent in scenarios C4 and C5. In scenarios A2 and A3, it was assumed that there would be no improvement in the energy efficiency of ICEVs and in this VFM scenario, these vehicles continued to lose market share throughout the modelling period.

Figure 8.6: EVs as a proportion of vehicles entering the light passenger vehicle fleet

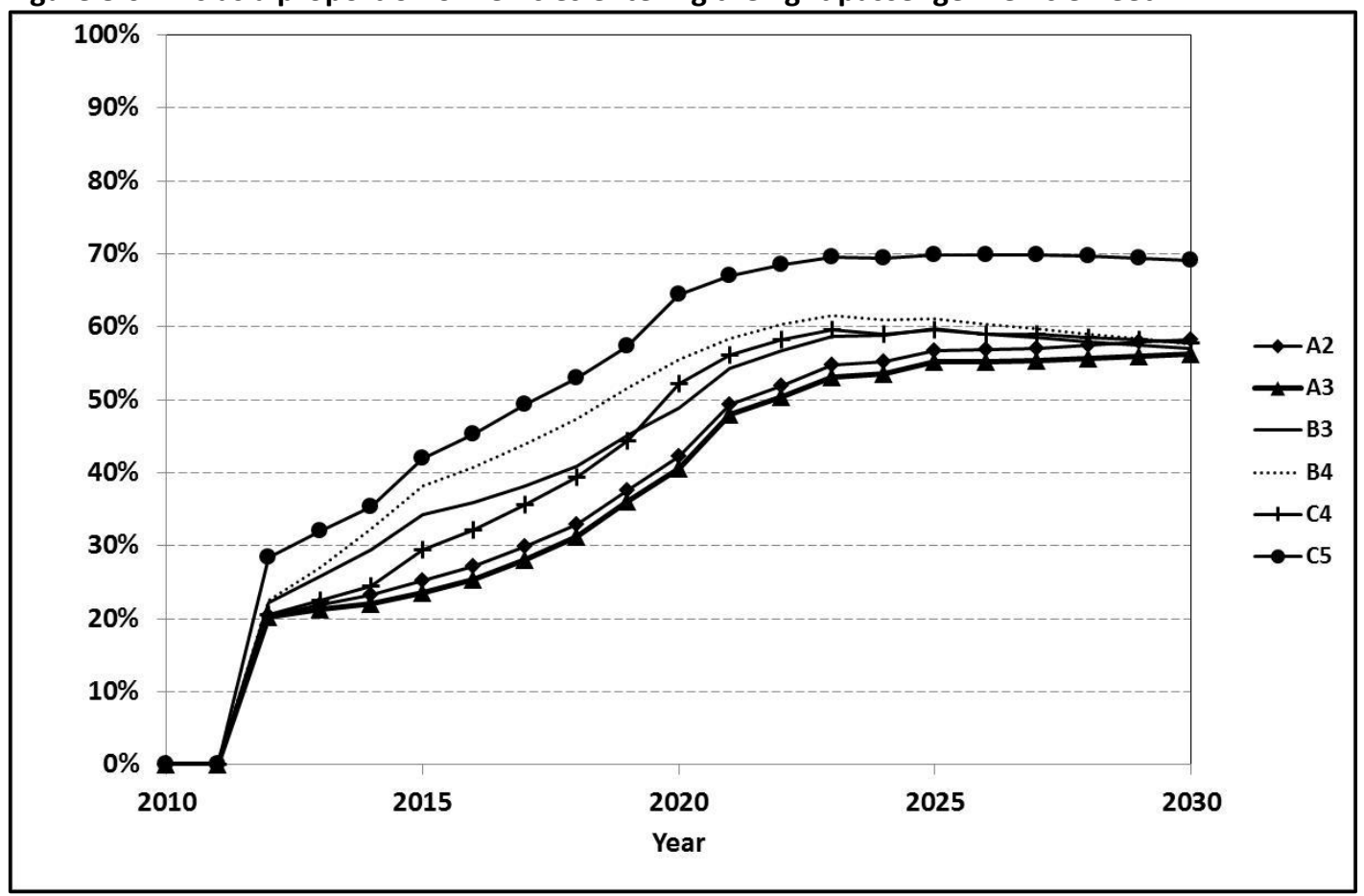

The effect of introducing EVs on the stock of LPVs is that, by 2030, it was estimated that EVs would constitute between $43.3 \% \pm 0.3$ and $58.3 \% \pm 0.2$ of the LPV fleet (Figure 8.7 ) or $1,358,327 \pm 8,244$ to $1,827,893 \pm 5,411$ vehicles (Figure 8.8 ) depending on the VFM scenario.

Scenarios A2 and A3 were the most conservative of the VFM scenarios, reflecting futures where there were no rapid increases in the price of petroleum, technological breakthroughs, or government intervention. In these VFM scenarios, 
EVs were projected by 2030 to comprise $43.3 \% \pm 0.3$ of the LPV fleet in scenario $A 3$ and $44.9 \% \pm 0.2$ in scenario $\mathrm{A} 2$.

Of all the VFM scenarios, scenario A3 had the lowest demand for EVs. This was due to the assumptions that there would be a slow decline in the price of EV technologies, but, at the same time, there would be an increase in the driving ranges of GEVs. Together, these two assumptions had the effect of significantly increasing the purchase price of GEVs. The increased purchase price of GEVs resulted in demand being transferred to PHEVs and ICEVs and an overall reduction in the total demand for EVs (Figures 8.3, 8.9 and 8.13).

The high uptake of EVs in scenarios B3 and B4 was due to the effect of high crude oil prices increasing the running costs of ICEVs. In these VFM scenarios, EVs comprised between $48.5 \% \pm 0.3$ and $51.2 \% \pm 0.3$ of the LPV fleet in 2030.

Scenario C4 did not have the assumption of high petroleum price used in the B scenarios, but it was assumed that there would be support for EVs that promoted the development of EV technology and the availability of public street recharging facilities. At the same time, it was assumed that there would be policy measures that improved the energy efficiency of ICEVs entering the LPV fleet, and that the price of carbon would increase to $\$ 100$ per tonne. These assumptions resulted in a similar uptake of EVs to that in scenario B3 with the stock of EVs reaching $48.3 \%$ \pm 0.3 of the LPV fleet in 2030 .

The price reduction assumptions used in scenario $\mathrm{C} 5$ resulted in the highest uptake of EVs with $58.3 \% \pm 0.2$ of the LPV fleet being EVs in 2030 (Figure 8.7).

Sensitivity analysis undertaken of scenario C5 indicated that the assumption that battery cell prices would decline to NZ\$150 per kWh, rather than the NZ\$300 per $\mathrm{kWh}$, had the greatest impact on the projected rate of uptake of EVs. This assumption had the effect of increasing the projected stock of EVs, in 2030, by $10.2 \% \pm 0.1 \%$ relative to the same VFM scenario using the standard battery cell price assumption. The assumption of energy efficient EV design had the next greatest impact on EV uptake increasing the stock of EVs in 2030 by $8.4 \% \pm 0.1$. Provision by 
the Government of a $20 \%$ purchase subsidy, capped at $\$ 7,500$ for new EVs and $\$ 3,750$ for used imported EVs, was found to increase the stock of EVs in 2030 by $6.1 \% \pm 0.1 \%{ }^{67}$.

Figure 8.7: Stock of EVs as a percentage of the light passenger vehicle fleet: 2010 to 2030

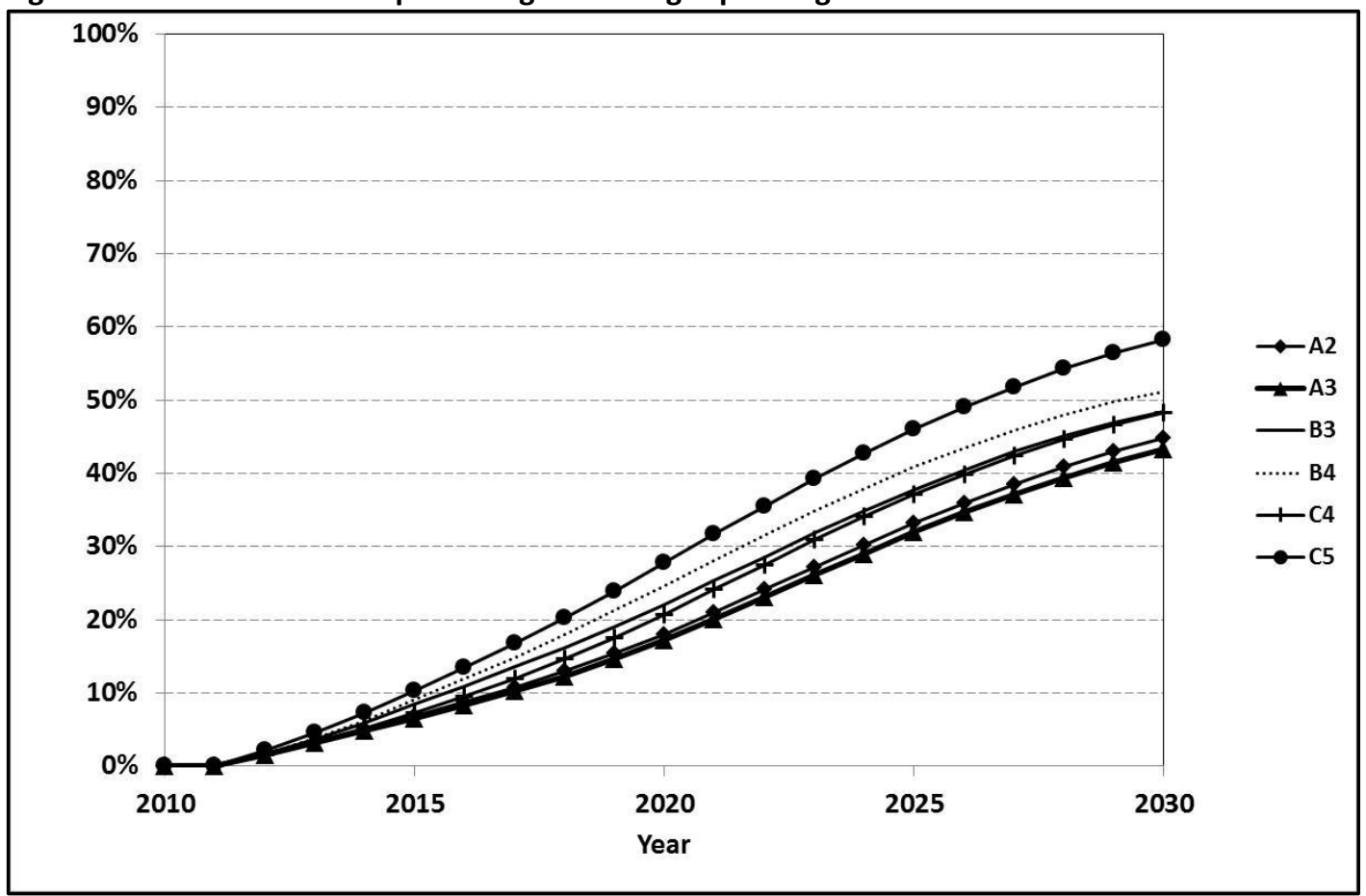

Figure 8.8: Stock of EVs: 2010 to 2030

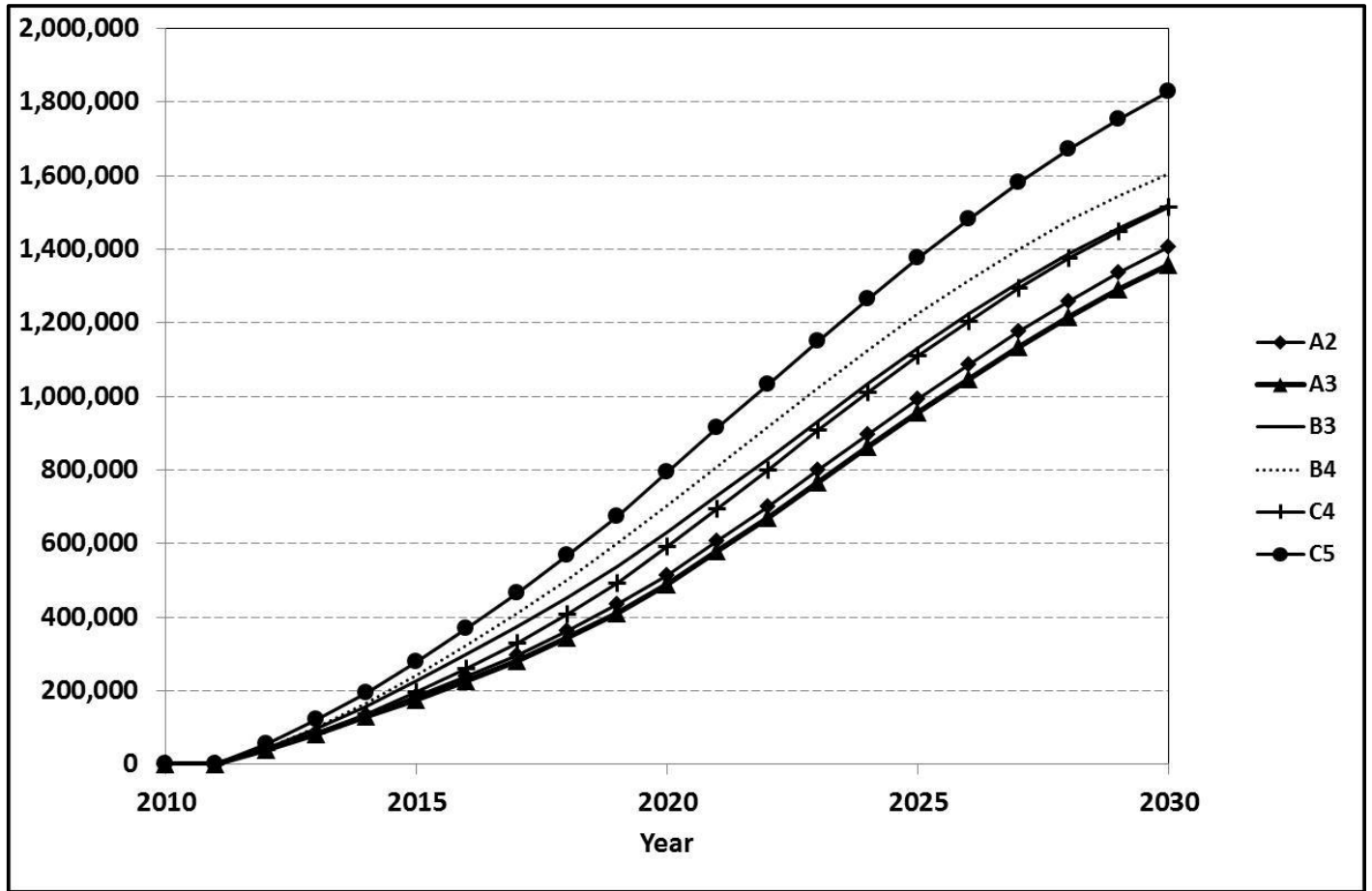

${ }^{67}$ For a discussion of the assumptions in scenario $\mathrm{C} 5$, see section 5.5.4. 


\subsubsection{Composition of the EV fleet}

\section{PHEV demand and vehicle stock}

PHEVs were the most popular EVs across all the VFM scenarios. In the VFM scenarios with the standard EV technological assumptions, they constituted at least $49.5 \% \pm 1.2 \%$ of all EVs that entered the LPV fleet annually. Even in scenario C5, which contained assumptions that had the effect of reducing the projected price of BEVs to the lowest level of all the VFM scenarios, PHEVs were still the most popular type of EV and comprised at least $46.3 \% \pm 0.6 \%$ of the annual EV demand.

The discrete choice model did not include an attribute for the PHEV electric driving range, but the demand for PHEVs was sensitive to the effect that increasing electric driving range had on PHEV fuel running costs.

In scenarios B3, B4, C4, and C5, it was assumed that the energy efficiency of the internal combustion engines (ICEs) in PHEVs would improve along with those of ICEVs. The improved energy efficiency of the PHEVs' ICE further reduced the fuel running costs and further increased the demand for PHEVs. In these VFM scenarios, with increasing energy efficiency PHEVs recovered some of the market share that was lost in scenarios $A 2$ and $A 3$ to the other types of EV (Figure 8.9). 
Figure 8.9: Plug-in hybrid electric vehicles as a percentage of EVs entering the light passenger vehicle fleet: $\mathbf{2 0 1 0}$ to $\mathbf{2 0 3 0}$

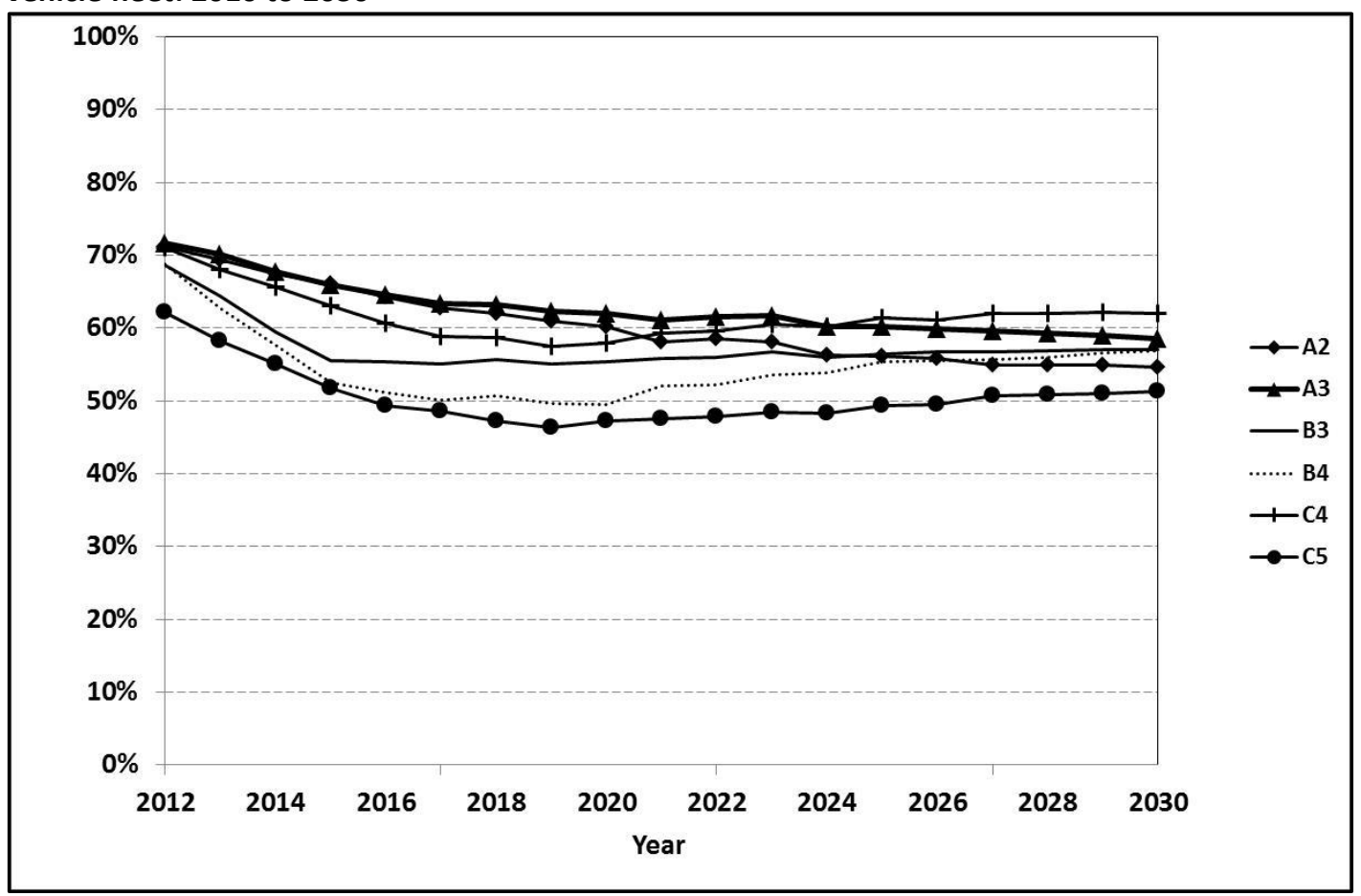

Scenario C4 had the largest uptake of PHEVs of all the VFM scenarios due to lower PHEV fuel running costs in this scenario (Table 8.3 and Figure 8.10). The lower PHEV fuel running costs were a function of the lower petroleum price assumption and the assumption that, with the availability of public charging, 30\% of PHEV users would take the opportunity to undertake an additional recharging event during the day to extend the amount of driving undertaken using inexpensive electric power.

Table 8.3: Plug-in hybrid electric vehicles - stock and proportion of EV fleet in $\mathbf{2 0 3 0}$

\begin{tabular}{|c|c|c|c|c|c|c|}
\hline VFM scenario & A2 & A3 & B3 & B4 & C4 & C5 \\
\hline Stock in 2030 & 815,429 & 827,924 & 858,664 & 865,554 & 918,600 & 903,918 \\
\hline \pm & 4,267 & 6,047 & 7,653 & 6,021 & 7,347 & 2,496 \\
\hline Proportion of EV fleet in 2030 & $58.0 \%$ & $61.0 \%$ & $56.5 \%$ & $54.0 \%$ & $60.7 \%$ & $49.5 \%$ \\
\hline \pm & $0.4 \%$ & $0.6 \%$ & $0.6 \%$ & $0.5 \%$ & $0.6 \%$ & $0.2 \%$ \\
\hline
\end{tabular}

$\pm \quad 95 \%$ confidence intervals 
Figure 8.10: Stock of plug-in hybrid electric vehicles all vehicle fleet model scenarios: 2010 to 2030

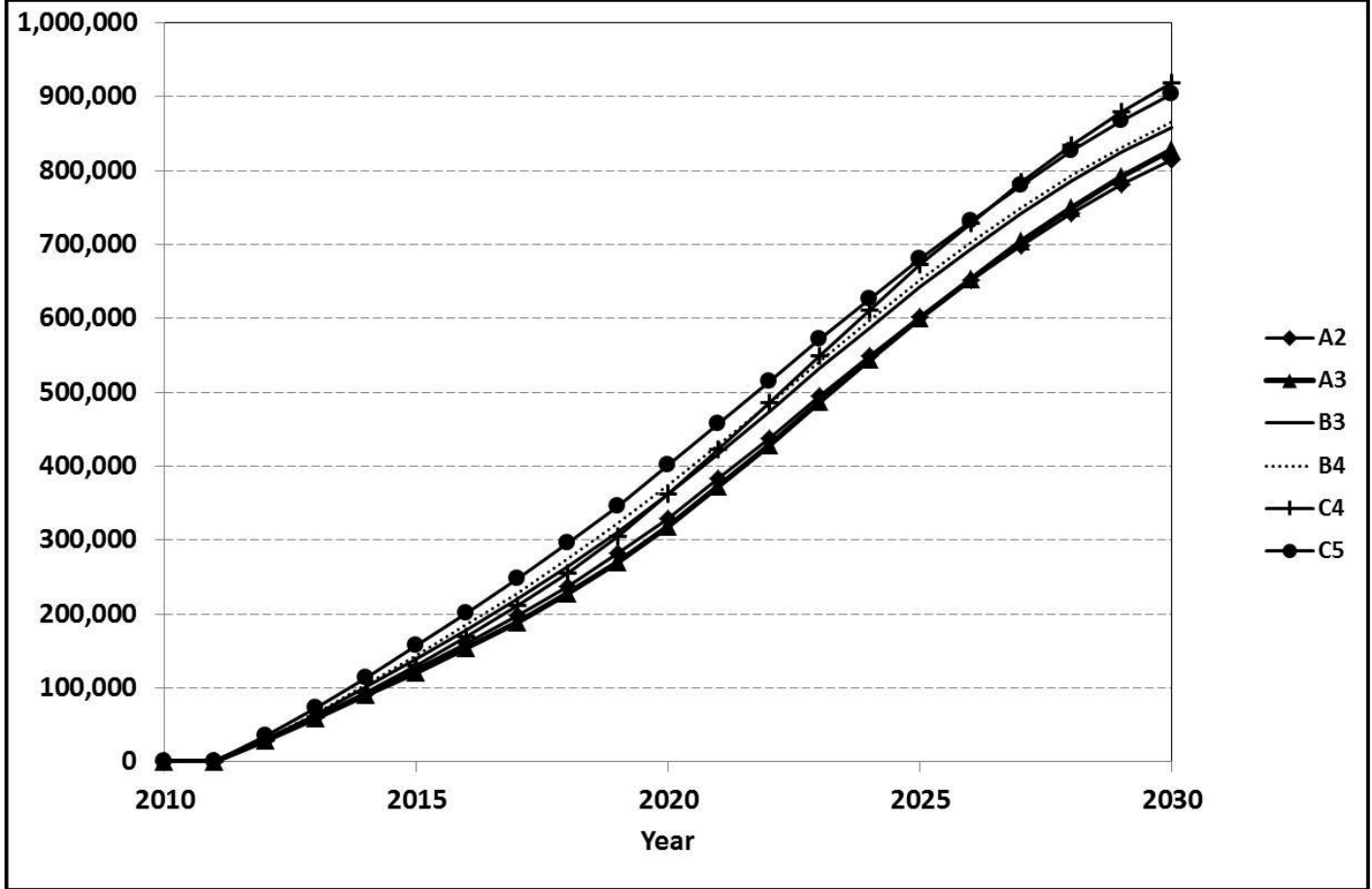

CEV demand and vehicle stock

After PHEVs, CEVs were the next most popular EVs across all the VFM scenarios. In the $B$ and $C$ scenarios, the fuel running costs of PHEVs strongly influenced the demand for CEVs. In these VFM scenarios, the demand for CEVs was highest during the period 2012 to 2015, when the fuel running costs of the PHEVs that entered the LPV fleet were at their highest. Over time, improving ICE energy efficiency, increasing electric driving ranges of PHEVs, and in the B scenarios declining petroleum prices, reduced the PHEVs' fuel running costs and CEVs lose market share to the PHEV. This decline in the CEV market share did not happen in scenario A2 because in that VFM scenario the running cost of PHEVs increased steadily throughout the modelling period (Figure 8.11).

The steadily increasing demand for CEVs in scenario $\mathrm{A} 3$ was the result not of reducing demand for PHEVs, but of the transfer of demand from GEVs to CEVs. 
Figure 8.11: City electric vehicles as a percentage of EVs entering the light passenger vehicle fleet: 2010 to 2030

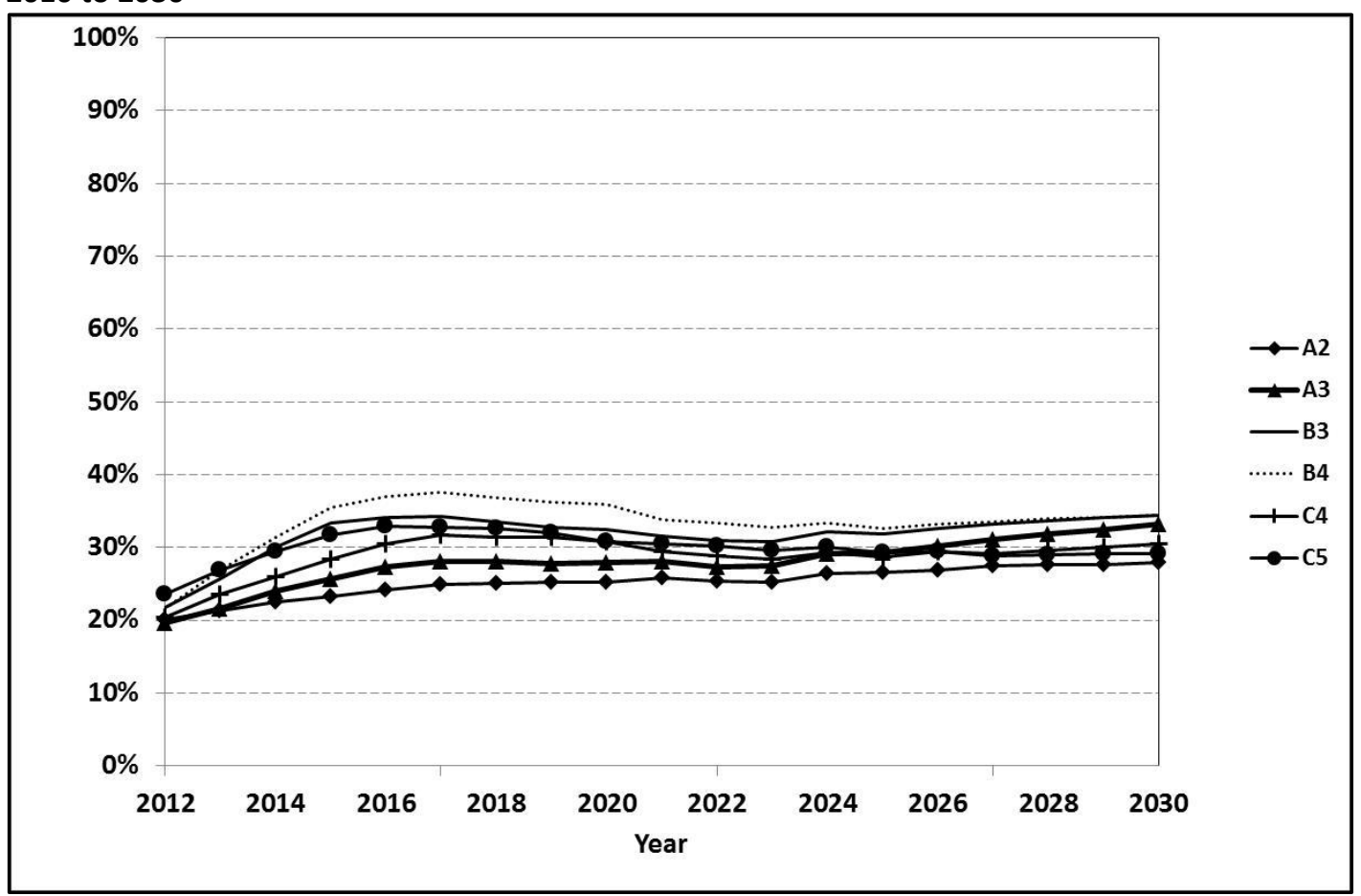

The stock of CEVs, in 2030, was projected to range from $371,204 \pm 4,431$ to 554,987 $\pm 4,609$ vehicles, or $26.4 \% \pm 0.3 \%$ to $34.4 \% \pm 0.4 \%$ of the EV fleet (Table 8.4 and Figure 8.12).

Table 8.4: City electric vehicles - stock and proportion of EV fleet in 2030

\begin{tabular}{|c|c|c|c|c|c|c|}
\hline VFM scenario & A2 & A3 & B3 & B4 & C4 & C5 \\
\hline Stock in 2030 & 371,204 & 402,972 & 498,821 & 551,370 & 452,423 & 554,987 \\
\hline \pm & 4,431 & 5,359 & 5,012 & 5,332 & 4,687 & 4,609 \\
\hline Proportion of EV fleet in 2030 & $26.4 \%$ & $29.7 \%$ & $32.8 \%$ & $34.4 \%$ & $29.9 \%$ & $30.4 \%$ \\
\hline \pm & $0.3 \%$ & $0.4 \%$ & $0.4 \%$ & $0.4 \%$ & $0.4 \%$ & $0.3 \%$ \\
\hline
\end{tabular}

$\pm \quad 95 \%$ confidence intervals 
Figure 8.12: Stock of city electric vehicles all vehicle fleet model scenarios: 2010 to 2030

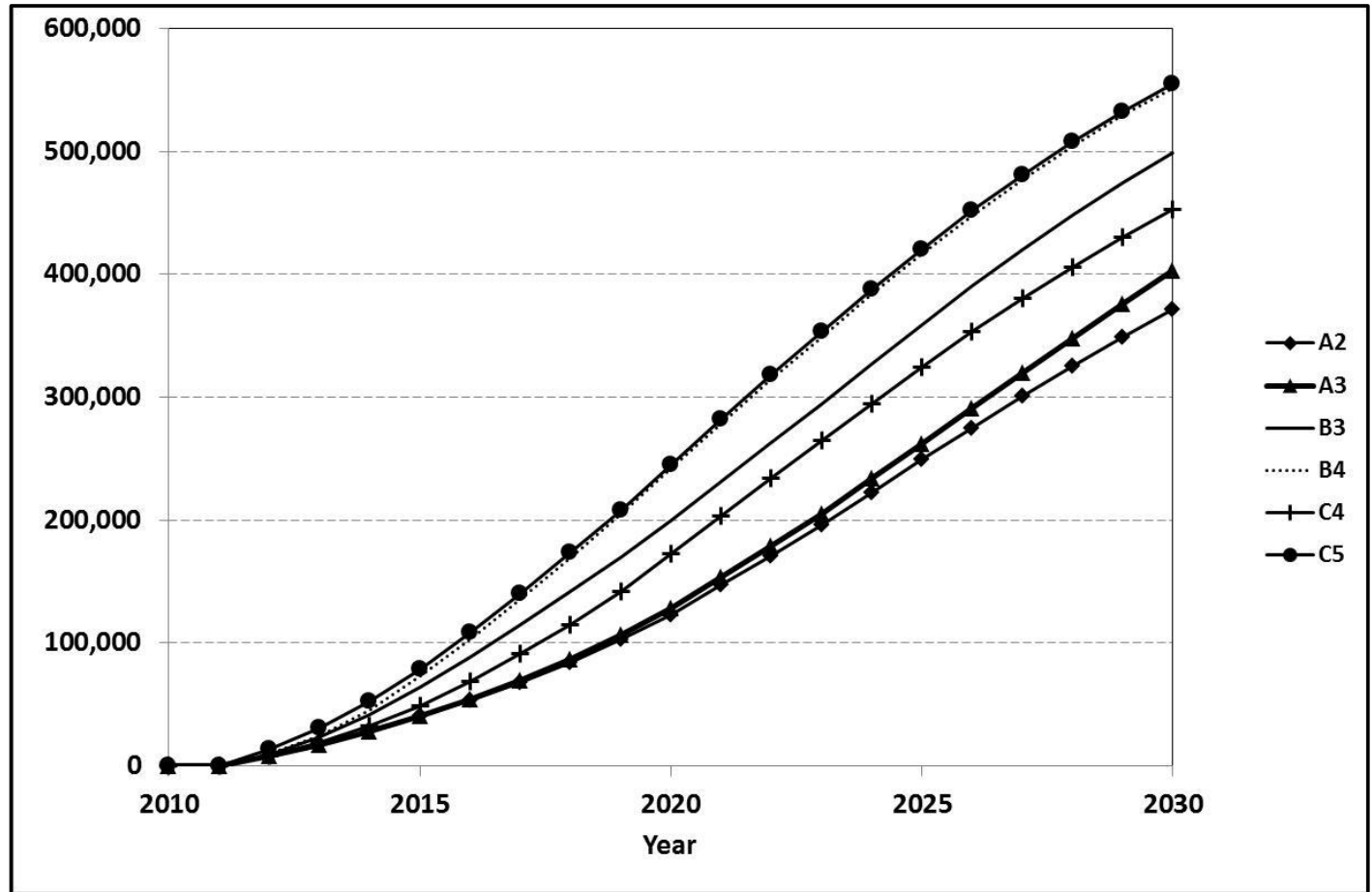

\section{GEV demand and vehicle stock}

GEVs were the least popular of the EVs across all the VFM scenarios due to their high purchase prices and limited driving range.

In the VFM scenarios where it was assumed that, over time, the driving ranges of the GEVs that entered the LPV fleet would increase their share of the EV market declined. The demand for greater driving range significantly increased the purchase price of GEVs due to the additional costs of larger batteries required. This loss of EV market share occurred even in scenario $\mathrm{C} 5$ where the price of battery cells was projected to decline by 2020 to NZ\$150 per kWh. However, the assumed low battery cell price resulted in the highest projected uptake of GEVs (Table 8.5 and Figure 8.14).

Sensitivity analysis indicated that approximately two-thirds of the GEVs' lost demand would be transferred to PHEVs, with the remainder distributed equally between ICEVs and CEVs.

Scenario A2 was the only VFM scenario containing the assumption that the electric driving range of GEVs would not increase over the modelling period. With the 
driving range of GEVs held constant, the battery size also remained constant. In this VFM scenario, the projected reductions in battery cell prices resulted in reductions in the price of a GEV's battery and purchase price. Consequently, in this scenario there was a steady increase in GEVs' market share throughout the modelling period (Figure 8.13).

Figure 8.13: General purpose electric vehicles as a percentage of EVs entering the light passenger vehicle fleet: 2010 to 2030

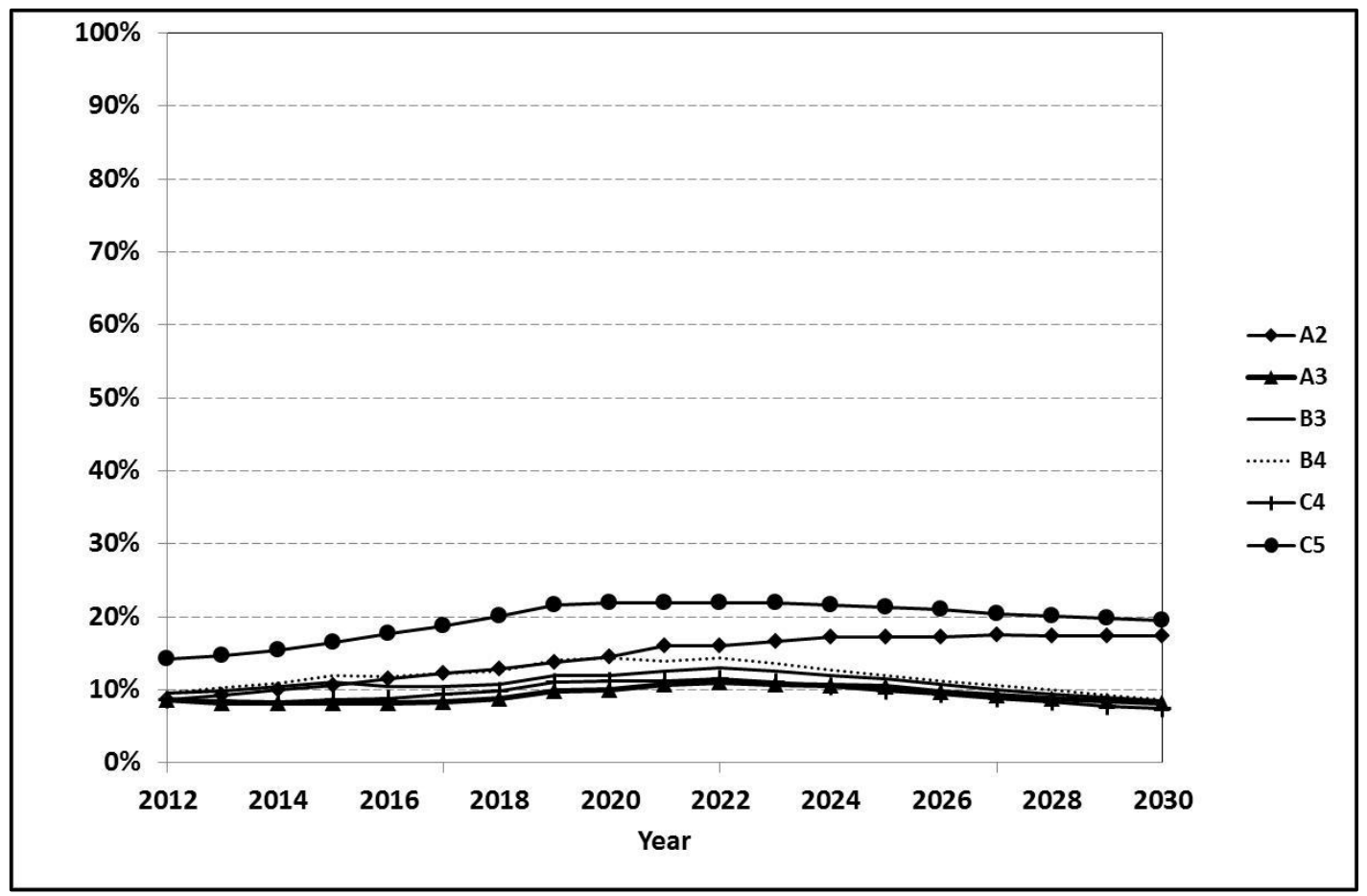

Table 8.5: General purpose electric vehicles - stock in 2030 and proportion of EV fleet

\begin{tabular}{|c|c|c|c|c|c|c|}
\hline VFM scenario & A2 & A3 & B3 & B4 & C4 & C5 \\
\hline Stock in 2030 & 220,118 & 127,431 & 162,057 & 187,113 & 142,925 & 368,988 \\
\hline \pm & 1,329 & 1,637 & 1,825 & 1,376 & 1,897 & 1,344 \\
\hline Proportion of EV fleet in $\mathbf{2 0 3 0}$ & $15.6 \%$ & $9.4 \%$ & $10.7 \%$ & $11.7 \%$ & $9.4 \%$ & $20.2 \%$ \\
\hline \pm & $0.1 \%$ & $0.1 \%$ & $0.1 \%$ & $0.1 \%$ & $0.1 \%$ & $0.1 \%$ \\
\hline
\end{tabular}


Figure 8.14: Stock of General purpose electric vehicles all vehicle fleet model scenarios: 2010 to 2030

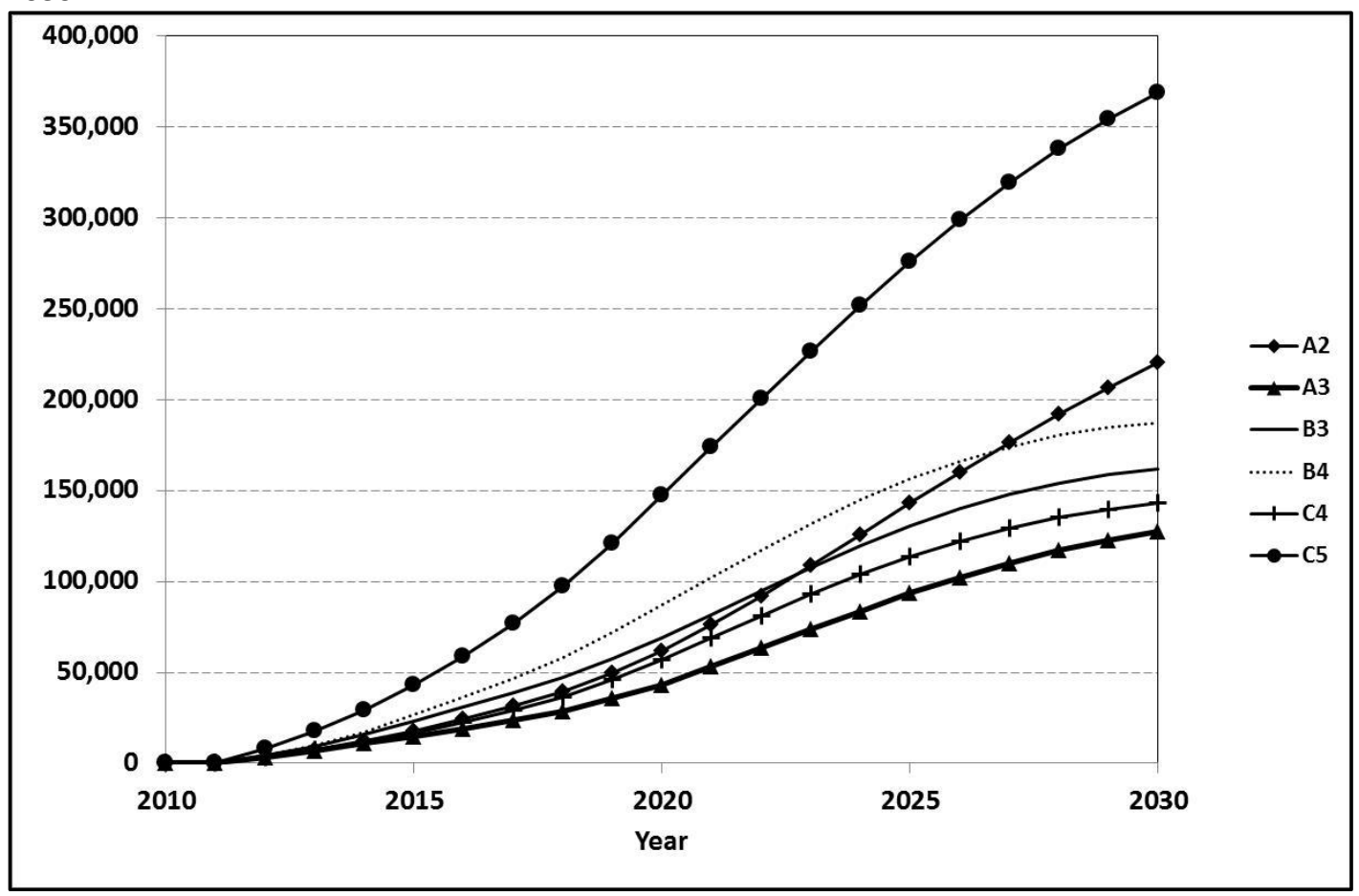

\subsubsection{Impact of used imported EVs on demand and vehicle stock}

Used imported LPVs are a significant component of the New Zealand vehicle market and it was reasonable to assume that, over time, used imported EVs from Japan will become an increasingly important component of the New Zealand LPV market in the future.

The output from the VFM indicated that, by 2030, when the supply of used imported EVs would be almost completely unconstrained, they would comprise between $56.3 \% \pm 0.7$ and $62.2 \% \pm 0.9$ of the EVs entering the New Zealand LPV fleet.

Lower rates of uptake of used imported EVs occurred in those VFM scenarios that contained assumptions which promoted the rapid uptake of EVs. These assumptions included high petroleum prices, as in the B scenarios, or rapidly declining EV prices, as in scenario C5 (Figure 8.15).

During the period when the supply of used imported EVs was constrained, the VFM reallocated the frustrated demand for used imported EVs to the other types of vehicle. A proportion of this frustrated demand transferred to new EVs and, as a result, the average age of the vehicles that entered the LPV fleet was younger than 
in a situation where the supply of used imported EVs was unconstrained (Figure 8.16).

Figure 8.15: Proportion of EVs entering the light passenger vehicle fleet as used imports

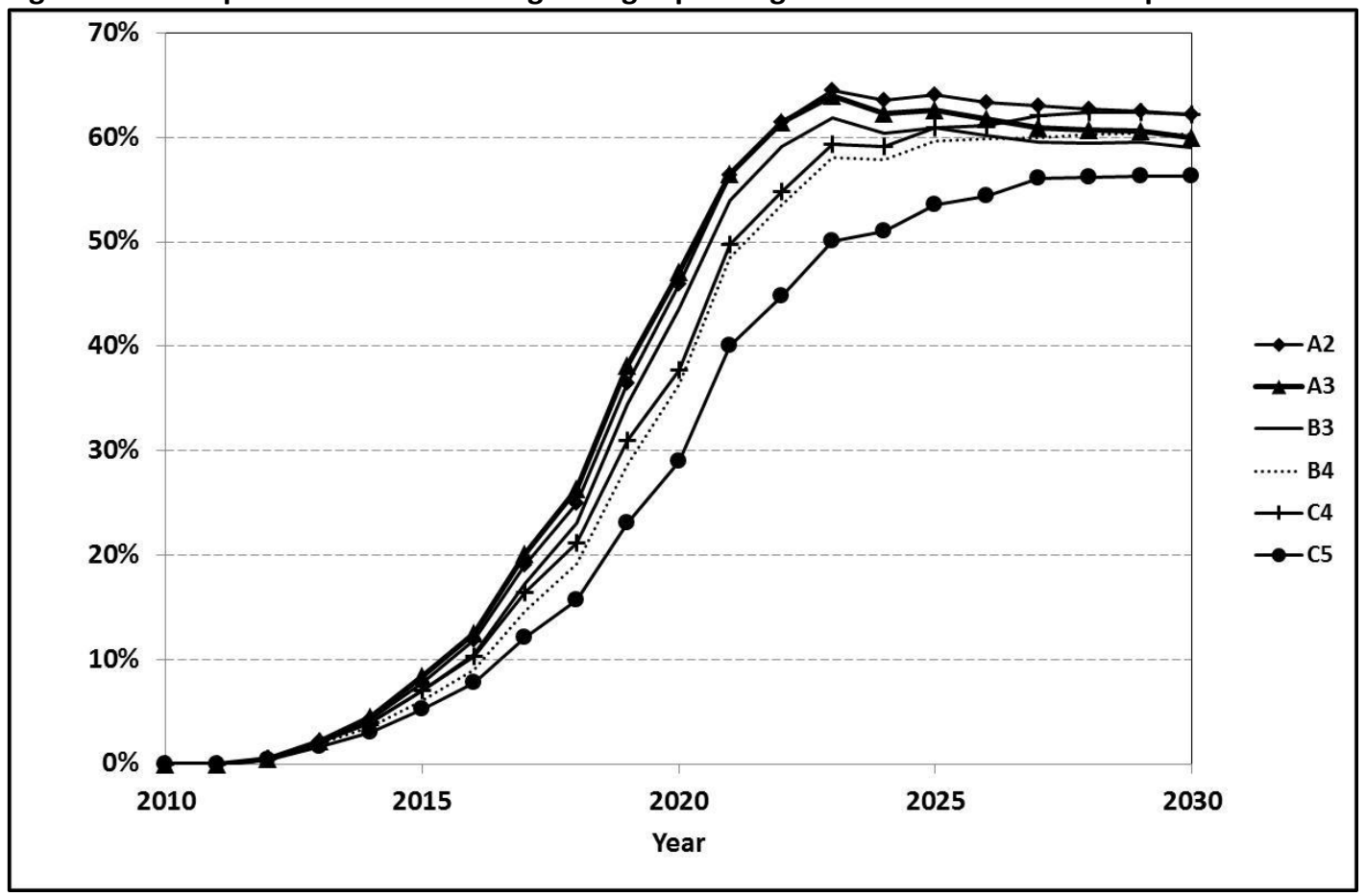

Figure 8.16: Proportion of new vehicles entering the light passenger vehicle fleet: 2010 to 2030

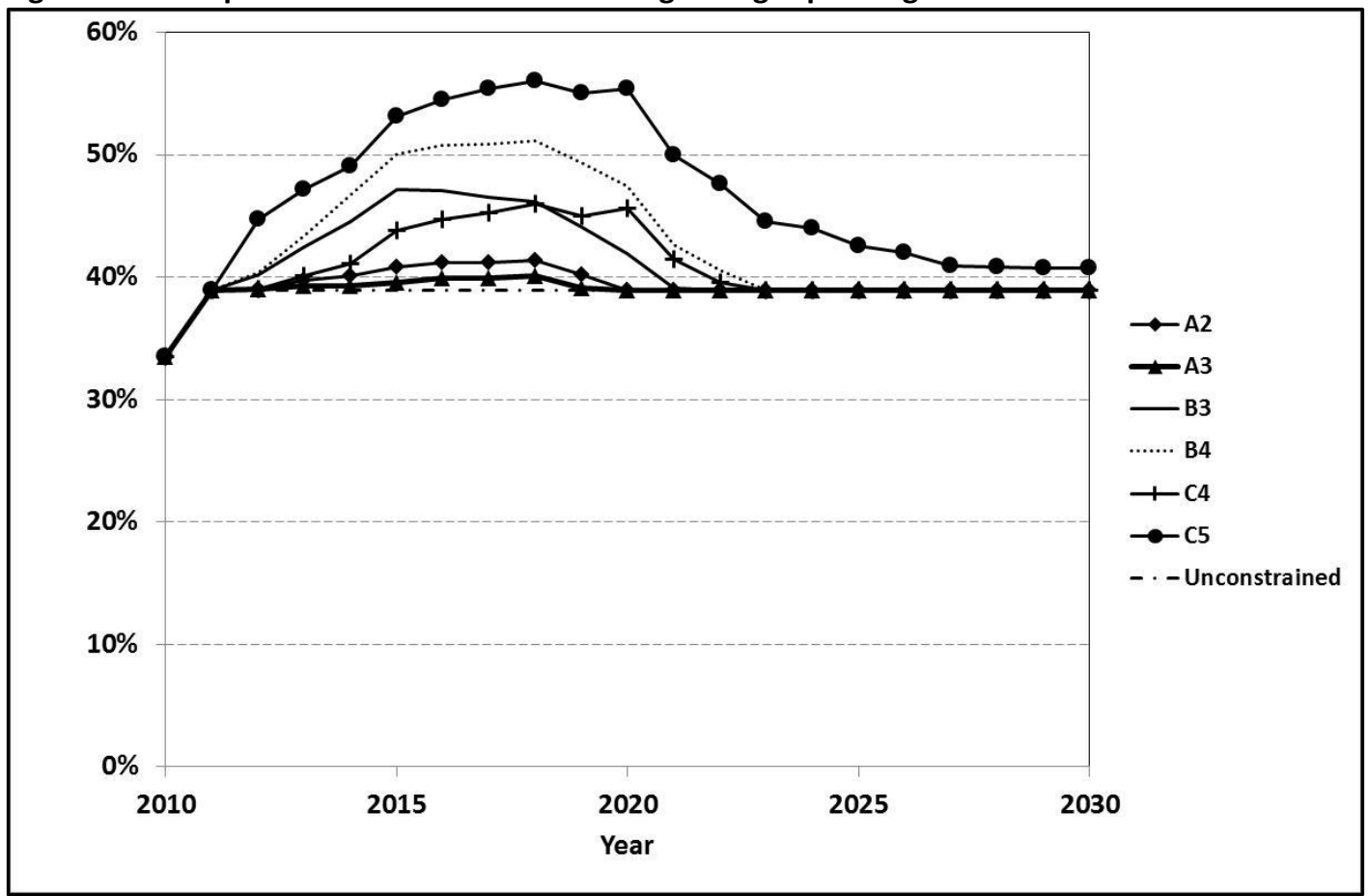

The results of a sensitivity analysis indicated that if used imported EVs were not available for purchase, the proportion of EVs in the LPV fleet in 2030, with the exception of scenario C5, would be between $16.1 \% \pm 0.1 \%$ and $25.4 \% \pm 0.1 \%$ lower 
than in a situation where they progressively became available. This decline in demand would reduce the stock of EVs in 2030 by between $258,000 \pm 11,500$ to $354,483 \pm 8,900$ vehicles.

The high demand for EVs in scenario C5 was, in large part, due to the early availability of very low priced used imports in the market. If these vehicles were not available for purchase, the proportion of EVs in the LPV fleet in 2030 would decline by $33.5 \% \pm 0.2 \%$, equivalent to $611,000 \pm 6,900 \mathrm{EVs}$.

For most VFM scenarios, the substitution patterns resulting from the removal of used imported EVs varied only in degree. Most of the demand that would have been allocated to these vehicles was transferred to used imported ICEVs. New PHEVs received the next largest transfer of demand. The amount of demand transferred from used imported EVs to new ICEVs and GEVs was not statistically significant.

The substitution patterns in scenario $\mathrm{C} 5$ differed, with most of the frustrated demand transferred to used imported ICEVs, followed by new ICEVs and then GEVs. The amount of demand transferred from used EVs to new CEVs and PHEVs was not statistically significant.

\subsection{VFM sensitivity analysis}

\subsubsection{Growth in GDP and rate of vehicle scrapping}

The sensitivity analysis indicated that, under the assumption of sustained high economic growth, car ownership levels quickly approached the saturation level. After this point, the growth of the LPV fleet proceeded at the same rate as population growth. By 2030, LPV ownership reached 615 per 1000 people and the LPV fleet contained $3,164,500$ vehicles, $0.9 \%$ higher than the number of vehicles in the default growth projection.

The low GDP growth path resulted in a much slower increase in vehicle ownership, increasing from the current level of 562.3 vehicles per 1000 people to 578.1 vehicles per 1000 people in 2020 . After this point, the GDP growth declined to $0.7 \%$ 
per year, which resulted in a decline in vehicle ownership by 2030 to 576 LPVs per 1000 people. Under these assumptions, the LPV fleet in 2030 would comprise 2,962,300 vehicles, 5.5\% lower than in the default growth projection (Figure 8.17).

Figure 8.17: Projected total light passenger vehicle ownership low, default, and high GDP growth assumptions

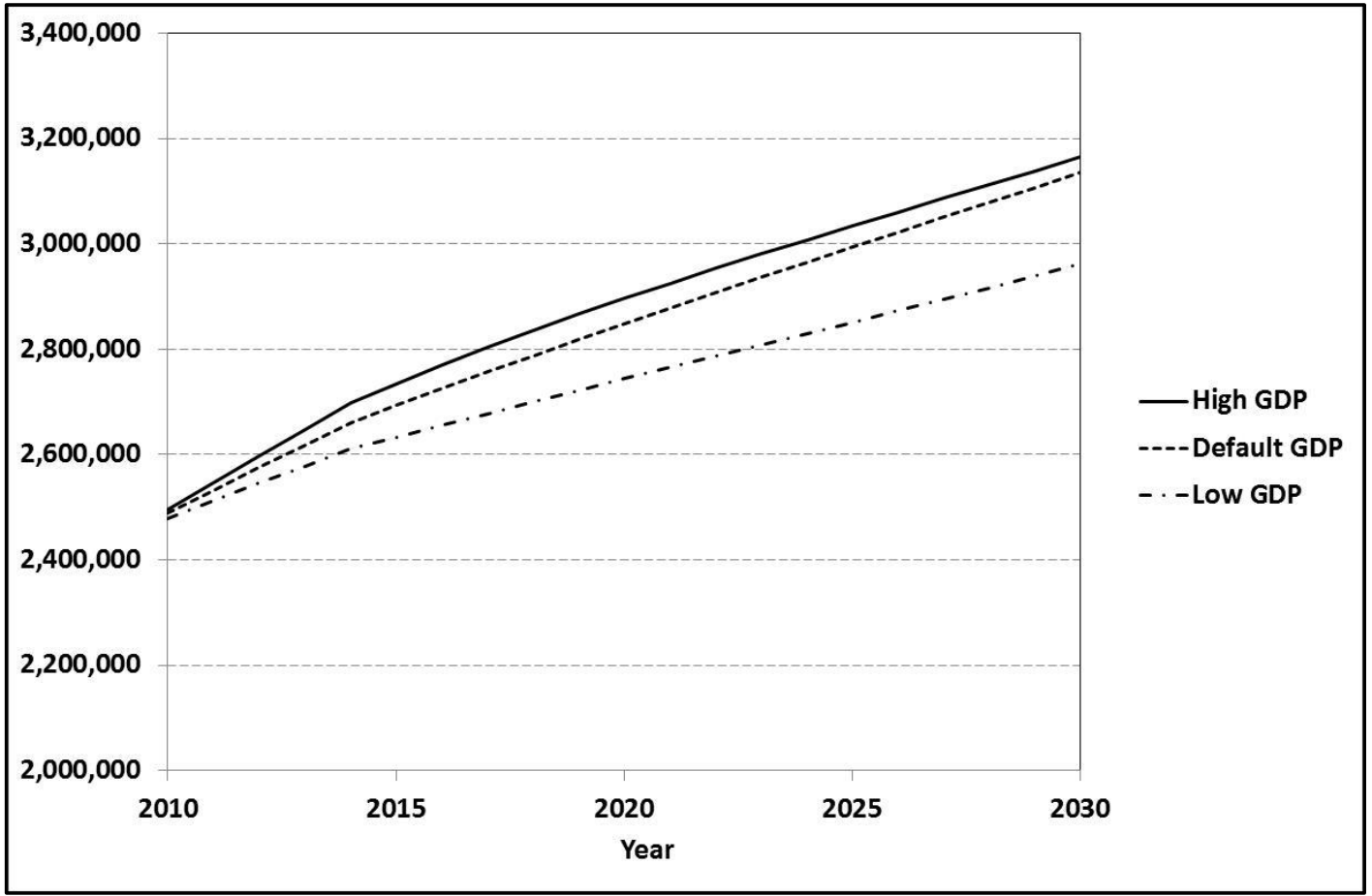

With lower GDP growth and lower rates of vehicle scrapping, the total number of LPVs that entered the LPV fleet over the modelling period declined by between 423,000 and 460,000 vehicles relative to the default GDP and scrapping assumptions. The high GDP and scrapping rates increased the number of LPVs that entered the LPV fleet by 213,500 and 235,000 vehicles relative to the default assumptions (Table 8.6). 
Table 8.6: Sensitivity analysis - impact of GDP growth and scrapping rates on the cumulative demand for light passenger vehicles and EVs entering the light passenger vehicle fleet: 2010 to 2030

\begin{tabular}{|c|c|c|c|c|c|c|c|c|c|c|}
\hline & \multicolumn{5}{|c|}{$\begin{array}{l}\text { Cumulative LPV demand } \\
2010 \text { to } 2030 \\
\text { (thousands) }\end{array}$} & \multicolumn{5}{|c|}{$\begin{array}{l}\text { Cumulative EV demand } \\
2010 \text { to } 2030 \\
\text { (thousands) }\end{array}$} \\
\hline & Default & Low & $\begin{array}{c}\text { Change } \\
\text { from } \\
\text { default }\end{array}$ & High & $\begin{array}{l}\text { Change } \\
\text { from } \\
\text { default }\end{array}$ & Default & Low & $\begin{array}{l}\text { Change } \\
\text { from } \\
\text { default }\end{array}$ & High & $\begin{array}{l}\text { Change } \\
\text { from } \\
\text { default }\end{array}$ \\
\hline A1 & $4,257.7$ & $\mid 3,797.0$ & -460.7 & $4,471.2$ & 213.5 & N/A & N/A & N/A & N/A & N/A \\
\hline A2 & $\begin{array}{c}4,210.8 \\
\pm 2.9\end{array}$ & \begin{tabular}{|c}
$3,778.4$ \\
\pm 2.5
\end{tabular} & \begin{tabular}{c|}
-432.4 \\
\pm 3.8
\end{tabular} & \begin{tabular}{|c}
$4,445.8$ \\
\pm 2.9
\end{tabular} & $\begin{array}{c}235.0 \\
\pm 4.1\end{array}$ & $\begin{array}{c}1,644.3 \\
\pm 1.9\end{array}$ & $\begin{array}{c}1,490.0 \\
\pm 0.6\end{array}$ & \begin{tabular}{|c|}
-154.3 \\
\pm 2.0
\end{tabular} & $\begin{array}{c}1,731.0 \\
\pm 0.7\end{array}$ & $\begin{array}{l}86.7 \\
\pm 2.0\end{array}$ \\
\hline B3 & $\begin{array}{c}4,165.1 \\
\pm 4.4\end{array}$ & \begin{tabular}{|c}
$3,741.7$ \\
\pm 3.0
\end{tabular} & \begin{tabular}{c|}
-423.4 \\
\pm 5.3
\end{tabular} & \begin{tabular}{|c}
$4,396.1$ \\
\pm 3.1
\end{tabular} & $\begin{array}{c}231.0 \\
\pm 5.4\end{array}$ & $\begin{array}{c}1,785.5 \\
\pm 2.7 \\
\end{array}$ & $\begin{array}{c}1,610.4 \\
\pm 0.6\end{array}$ & \begin{tabular}{|c|}
-175.1 \\
\pm 2.8 \\
\end{tabular} & \begin{tabular}{|c}
$1,872.7$ \\
\pm 2.1
\end{tabular} & $\begin{array}{l}87.2 \\
\pm 3.4\end{array}$ \\
\hline C4 & $\begin{array}{c}4,180.2 \\
\pm 3.7\end{array}$ & \begin{tabular}{|c}
$3,754.1$ \\
\pm 2.4
\end{tabular} & $\begin{array}{c}-426.10 \\
\pm 4.4\end{array}$ & \begin{tabular}{|c}
$4,413.6$ \\
\pm 3.7
\end{tabular} & $\begin{array}{c}233.4 \\
\pm 5.2\end{array}$ & $\begin{array}{c}1,764.9 \\
\pm 2.6\end{array}$ & $\begin{array}{c}1,594.7 \\
\pm 1.8\end{array}$ & $\begin{array}{c}-170.2 \\
\pm 3.2\end{array}$ & $\begin{array}{c}1,848.6 \\
\pm 2.4\end{array}$ & $\begin{array}{l}83.7 \\
\pm 3.5\end{array}$ \\
\hline
\end{tabular}

95\% confidence intervals

\subsubsection{EV demand}

The sensitivity analyses undertaken to assess the effect of changing key assumptions on the cumulative demand projections for EVs, over the 2010 to 2030 modelling period, indicated that changing the design of EVs to improve energy efficiency and the provision of public street recharging facilities would have the most impact for increasing the uptake of EVs. Changing the design of EVs to increase their energy efficiency had the effect of increasing the demand for GEVs and CEVs and reducing the demand for PHEVs and ICEVs. The higher energy efficiency assumption increased the cumulative sales of EVs, over the modelling period, by between $6.6 \%$ and $11.4 \%$ when compared to the default assumptions of EV energy efficiency (Table 8.7).

The presence of EV street vehicle recharging facilities had the effect of increasing the cumulative sales of EVs by between $8.5 \%$ and $9.2 \%$. This sensitivity analysis also indicated that the provision of street recharging would have more effect in promoting the uptake of EVs in a VFM scenario where the driving range of EVs remained limited, as in scenario $\mathrm{A} 2$. 
The availability of the Government subsidy on the purchase of EVs was the next most effective measure in promoting EVs with sales over the modelling period. This measure increased cumulative EV sales by between $5.2 \%$ and $5.6 \%$.

The sensitivity analysis indicated that the Government's current policy of exempting EVs from RUC would have an effect on the uptake of EVs when they became available. The analysis indicated that the removal of the exemption would reduce cumulative sales of EVs over the modelling period by between $1.6 \%$ and $2.2 \%$.

The sensitivity analysis indicated that the impact of the Government raising the price of carbon to $\$ 100$ per tonne would be to increase the cumulative demand for EVs over the modelling period by between $1.1 \%$ and $1.8 \%$.

The impact of different growth rates in electricity prices had a very small impact on the sales of EVs. In the case of scenario B3, changing the price of electricity resulted in no statistically significant difference in the cumulative number of EVs that entered the LPV fleet over the modelling period (Table 8.7). 
Table 8.7: Sensitivity analysis - effect of changed assumptions on the cumulative number of EVs entering the light passenger vehicle fleet

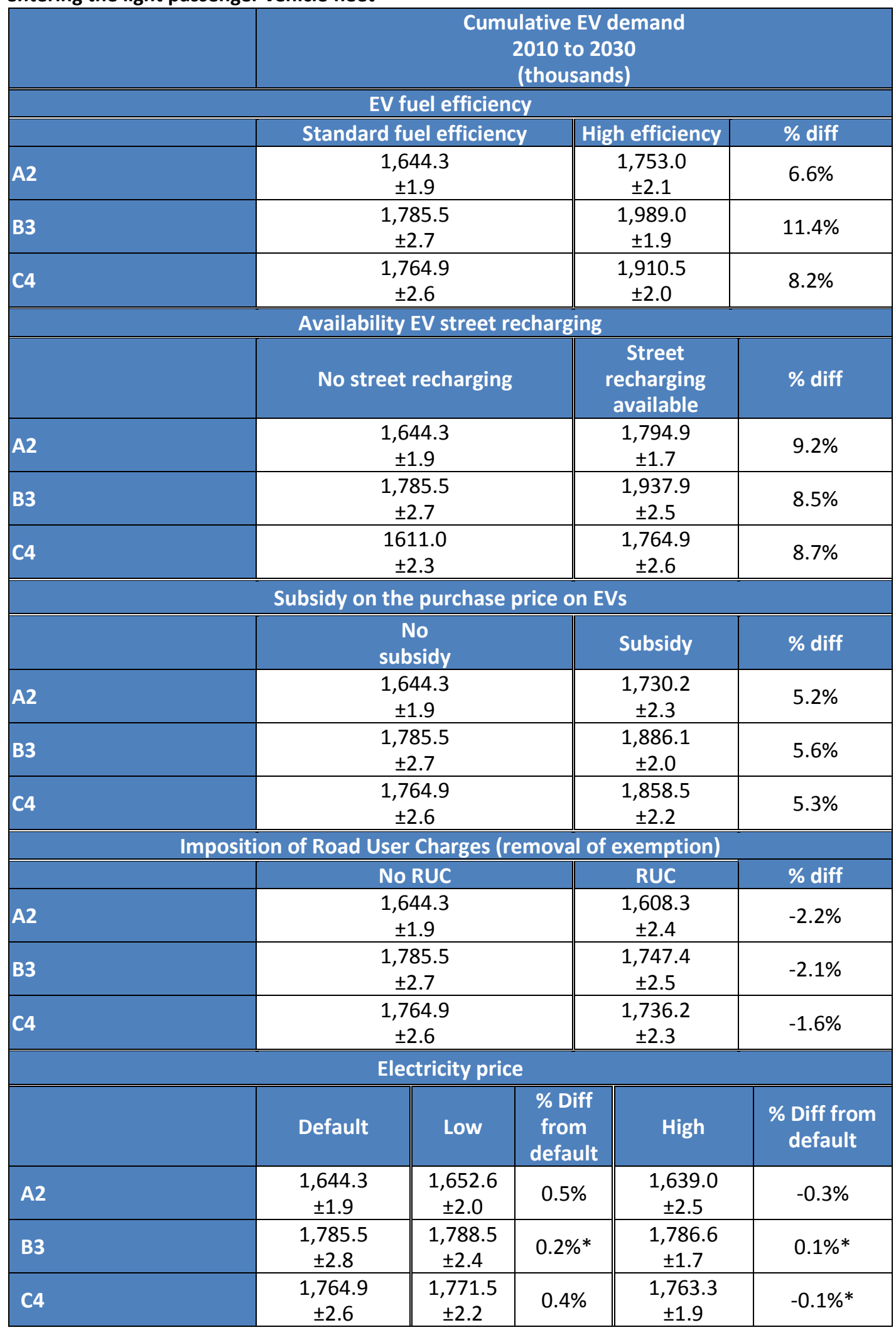

$\pm \quad 95 \%$ confidence intervals

* Difference not significant at $95 \%$ level of significance 


\subsection{Discussion}

\subsubsection{Future composition of the LPV fleet}

The choice simulator in the VFM was a demand model that did not take into account the effect of EV supply constraints on vehicle choice. In this study, it was assumed that there would be no constraints in the availability of new EVs from the first year of their introduction. This assumption resulted in a jump in the market share of EVs (Figure 8.6). As discussed in section 4.4, in practice the supply of EVs will remain limited and is unlikely to increase rapidly, as vehicle manufacturers are cautious about introducing new vehicle technologies in markets.

Other studies that have modelled the uptake of EVs into the New Zealand LPV fleet have used diffusion models and expert based market penetration scenarios (Dirr, 2008; Duncan et al., 2010), or a hybrid economic cost and market penetration scenario model (Baxter et al., 2009). These models implicitly incorporated the effect of supply constraints. Consequently, these studies projected lower numbers of EVs entering the LPV fleet during the period immediately following the introduction of EVs. However, by 2020, the rates of EV uptake in this study became comparable to those in the default uptake scenario in Baxter et al. (2009) and the upper case scenario in Duncan et al. (2010).

The conditional logit model contained in the UniSyD model did not take vehicle availability into account, but also resulted in very low projections of EV uptake. By 2030, EVs were projected to comprise less than $4 \%$ of the vehicle fleet. However hydrogen fuel cell vehicles (HFCVs) and hydrogen internal combustion engine vehicles (HICEVs) were projected to comprise over $60 \%$ of the fleet. This result was the direct effect of the assumption used in the study that EVs would all have a driving range of $320 \mathrm{~km}$. This assumption resulted in the purchase price of EVs remaining throughout the modelling period more than $\$ 10,000$ higher than the projected purchase price an HFCV and \$15,000 more than that of HICEV (Leaver and Gillingham, 2010). 
Factors such as the rate of increase in the driving range of BEVs, battery life, price of petroleum, and the availability of street recharging facilities all had an effect on the rate at which the level of EV uptake increased, but had little effect on the maximum rate of uptake. The VFM indicated that the maximum rate of EV uptake occurred when the average purchase prices of EVs entering the LPV market ceased to decline. The largest determinant of reductions in the price of EVs was the decline in the price of EV batteries.

In this study, the default assumption for the lowest battery cell price that would be achievable during the modelling period was NZ\$300 per $\mathrm{kWh}$. This assumption resulted in a maximum level of EV uptake of approximately $58 \%$ of all LPVs, which is lower than the $65 \%$ maximum level in Baxter et al. (2009). In scenario C5, it was assumed that the minimum achievable battery cell price would be NZ\$150 per kWh and that this level would be reached by 2020 . This assumption resulted in a higher maximum level of EV uptake of approximately $70 \%$.

In this study, the EV stock projections for 2030 ranged from 1.4 million in scenario A3 to 1.8 million for breakthrough scenario C5. These projections are higher than the default scenarios in Baxter et al. (2009) and Duncan et al. (2010). Baxter, et al's (2009) BAU EV demand model estimated a stock of EVs, in 2030, of approximately 900,000 to $1,000,000$ vehicles. Duncan et al's (2010) moderate projections estimated that the stock of EVs in 2030 would range from 600,000 to 800,000 . In this study, the projections of the 2030 stock of EVs are more consistent with the accelerated uptake scenarios in Baxter et al. (2009), which projected a stock of 1.3 million EVs in 2030, and the high market penetration diffusion scenario in Dirr (2008), which projected 1.4 million EVs.

The approach used in this study was to divide the EV market into CEVs, GEVs, and PHEVs. This allowed for a better understanding of the future composition of the LPV fleet and better estimation of future energy demand and GHG emissions. In the other New Zealand studies, CEVs and GEVs were not treated separately and PHEVs were treated separately from BEVs only in Baxter et al's (2009) study. They 
concluded that PHEVs would still be the dominant type of EV at the end of the study period in 2049 , comprising $60 \%$ of the EV fleet (Table 8.8 ).

Table 8.8: Comparison of New Zealand EV studies of EV stock in 2030

\begin{tabular}{|c|c|c|c|c|c|}
\hline & Clover & $\begin{array}{c}\text { Dirr } \\
(2008)\end{array}$ & $\begin{array}{l}\text { Baxter et al. } \\
\text { (2009) }\end{array}$ & $\begin{array}{l}\text { Duncan et al. } \\
\text { (2010) }\end{array}$ & $\begin{array}{c}\text { Leaver and } \\
\text { Gillingham } \\
\text { (2010) }\end{array}$ \\
\hline $\begin{array}{c}\text { EV as \% of LPV } \\
\text { stock in } 2030\end{array}$ & $\begin{array}{l}43 \% \text { to } 51 \% \\
\text { (61\% in the } \\
\text { high } \\
\text { efficiency } \\
\text { scenario) }\end{array}$ & $\begin{array}{c}23 \% \text { to } \\
60 \%\end{array}$ & $35 \%$ & $8 \%$ to $42 \%$ & $<4 \%$ \\
\hline $\begin{array}{l}\text { PHEVs as a \% of } \\
\text { EV stock in } \\
2030\end{array}$ & $\begin{array}{l}54 \text { to } 61 \% \\
\text { (50\% in the } \\
\text { high } \\
\text { efficiency } \\
\text { scenario) }\end{array}$ & $\mathrm{n} / \mathrm{a}$ & $60 \%$ & $\mathrm{n} / \mathrm{a}$ & $\mathrm{n} / \mathrm{a}$ \\
\hline
\end{tabular}

This study also concluded that PHEVs would be most popular type of EV in New Zealand. PHEVs would be more popular even though they cost more than CEVs to purchase and are more expensive than both CEVs and GEVs to run. This study indicated that the ability of PHEVs to operate over long ranges with lower fuel running costs than ICEVs and PHEV's ability to use public EV recharging facilities were significant factors in overcoming the negative incentive of the higher purchase price.

This study indicated that the family sized GEV type would be the least popular EV due to its high purchase price and limited driving range. Efforts to increase the EV market share of GEVs by increasing the driving range to match ICEVs and PHEVs would have the opposite effect due to the expense of the larger batteries. GEVs would be much more competitive if the price of batteries drops, at the cell level, to NZ\$150 per kWh (Figure 8.13).

\subsubsection{Impact of possible policy interventions}

The VFM provided an opportunity to assess the relative effectiveness of three policy interventions that are implementable by the New Zealand Government to promote the uptake of EVs. These comprised the promotion of a widely available street based EV recharging facility, subsidising New Zealand car buyers in their 
purchase of an EV, and the continuation of the Government's policy of exempting EVs from the RUC.

Of the three initiatives, the VFM indicated that the provision of a widely available street based EV recharging facility would be the most effective measure. The next most effective measure would be to have a $20 \%$ subsidy on the purchase price of EVs, capped at $\$ 7,500$ for new EVs, and $\$ 3,750$ for used imported EVs. The VFM analysis indicated that the removal of the Government's current exemption of EVs from RUC would have a negative effect on future uptake of EVs, but this was the least effective of the three measures. 


\section{Chapter 9: Results III: projected energy demand and GHG emissions}

\section{$9.1 \quad$ Introduction}

This chapter comprises three parts. Section 9.2 presents the projected energy demand and the greenhouse gas (GHG) emissions output from the light passenger vehicle (LPV) fleet produced by the VFM. Section 9.3 presents the results of the analysis of the impact of electric vehicles (EVs) on New Zealand's electricity sector, using the generation expansion model (GEM). Section 9.4 presents the projections of the combined GHG emissions from both the LPV fleet and electricity sectors.

\subsection{LPV fleet: projected energy demand and GHG emissions}

\subsubsection{Projected travel demand}

\section{Annual VKT per vehicle}

In all the VFM scenarios, changing the price of electricity over the range of values used in the modelling work had only a small effect on running costs and on the number of kilometres travelled by battery electric vehicles (BEVs) and plug-in hybrid electric vehicles (PHEVs). For BEVs, the vehicle use model (VUM) estimated that the annual vehicle kilometres travelled (VKT) per vehicle would decline by less than $0.5 \%$ due to the effects of increasing electricity prices from $13 \mathrm{c} / \mathrm{kWh}$ to $20 \mathrm{c} / \mathrm{kWh}$. The small increase in electricity prices was the result of the assumption that at least $70 \%$ of EV charging will occur on the cheaper night tariff. The effect of this tariff increase was to increase BEV fuel running costs by less than $\$ 1.00 / 100 \mathrm{~km}$ over the modelling period.

The annual VKT per vehicle of PHEVs was influenced by changes in the price of electricity and petroleum and the proportions of electric and non-electric driving. In scenario $A 2$, it was assumed that the electric driving range of PHEVs and the energy efficiency of an internal combustion engine (ICE) would remain at current levels. For this VFM scenario, the VUM projected that the annual VKT per vehicle of PHEVs would decline, over the modelling period, by $1.1 \%$ due to the effect of increasing petrol prices increasing from $\$ 2.30 / \mathrm{L}$ to $\$ 2.87 / \mathrm{L}$. 
In all the other VFM scenarios, it was assumed that the electric driving range of PHEVs would increase, which would improve their energy efficiency and lower fuelrunning costs. For these VFM scenarios, the VUM projected that the annual VKT per vehicle of PHEVs would increase by between $1.6 \%$ and $4.5 \%$.

The VUM estimated that changes to the annual VKT per vehicle of internal combustion engine vehicles (ICEVs) would be more significant due to the lower fuel efficiencies of these vehicles and the projected increases in the future price of petroleum. In the A scenarios, annual VKT per vehicle declined by $6.6 \%$ by 2030 and, in the $C$ scenarios, the annual VKT per vehicle declined by $4.8 \%$ (Figure 9.1).

In the B scenarios, which projected that the price of petroleum would increase to US\$300 per barrel by 2015, the VUM estimated that, for ICEVs, this would result in a reduction in the annual VKT per vehicle from $12,250 \mathrm{~km}$ in 2010 , to $10,270 \mathrm{~km}$ in 2015. After 2015, the price of petroleum was projected to decrease to US\$250 per barrel. Under these assumptions, the VUM estimated that, by 2030, the VKT per vehicle for ICEVs would then increase to $10,879 \mathrm{~km}$ per year. Over the entire period, 2010 to 2030 , the reduction in VKT per vehicle was projected to be $11.2 \%$ (Figure 9.1).

Figure 9.1: Annual VKT per vehicle for internal combustion engine vehicles: 2010 to 2030

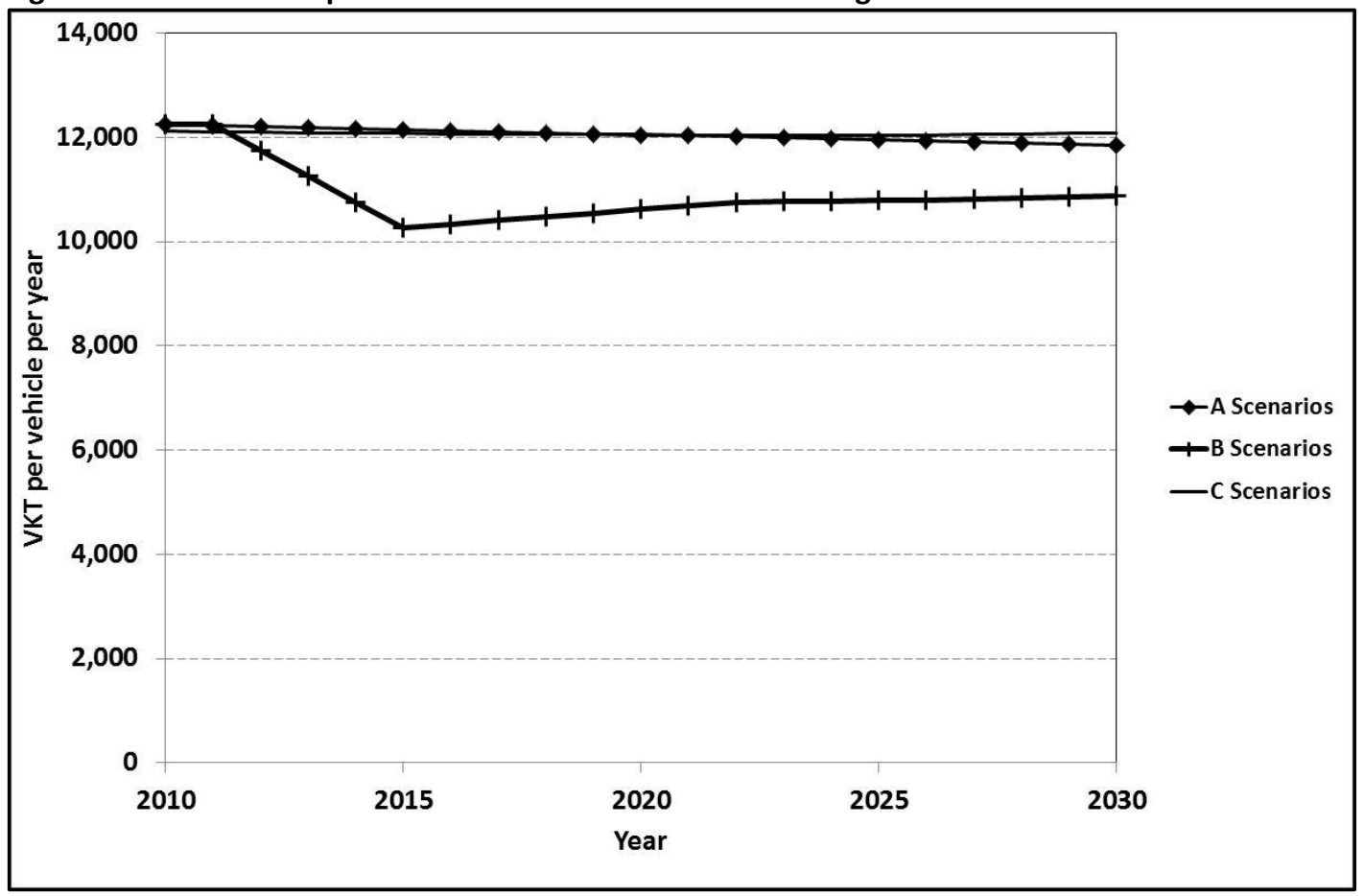




\section{Total annual VKT for ICEVs}

The sharp increase in the price of petroleum in the two B scenarios resulted in a sharp decline in the total annual VKT of the ICEV fleet. In both VFM scenarios, total VKT declined during the period 2011 to 2015 from approximately 31 billion kilometres in 2011 to 25 billion kilometres in 2015 (Figure 9.2).

With the introduction of EVs, the annual VKT using petrol was estimated to decline from 30.5 billion kilometres in 2010 to between $26.3 \pm 0.09$ billion kilometres in scenario $\mathrm{A} 2$ and $19.8 \pm 0.07$ billion kilometres in scenario $\mathrm{C} 5$.

Figure 9.2: Annual VKT for internal combustion engine vehicles - scenarios A2, B3, B4, and C5: 2010 to 2030

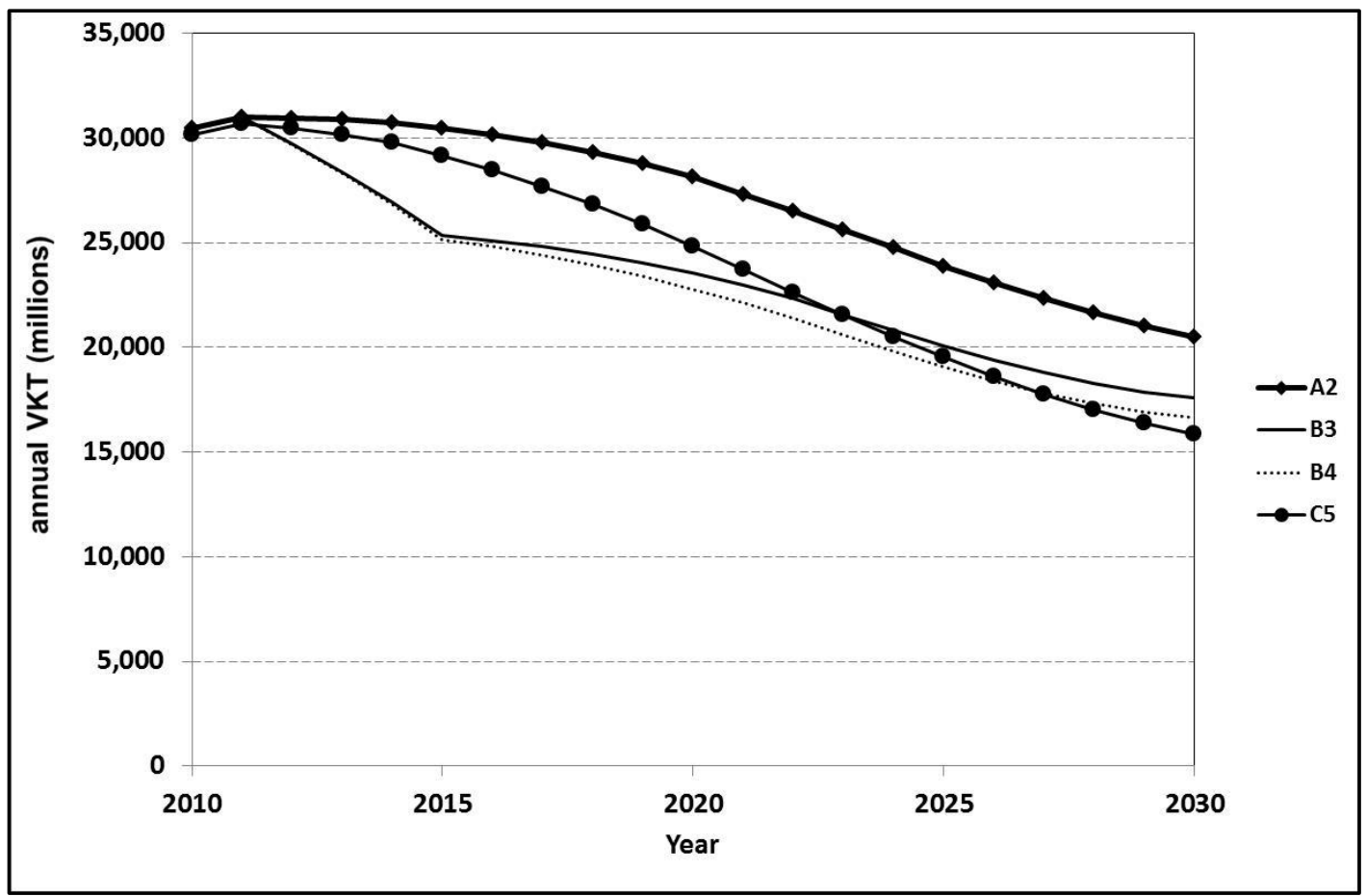

\section{VKT per year using electricity}

For BEVs and PHEVs operating on the electric drive cycle the total annual VKT was estimated to reach, in 2030, between $11.26 \pm 0.06$ billion kilometres, in scenario $A 2$, and $18.77 \pm 0.10$ billion kilometres in scenario C5 (Figure 9.3). 
Figure 9.3: Light passenger vehicle fleet annual electric powered VKT by vehicle fleet model scenario: 2010 to 2030

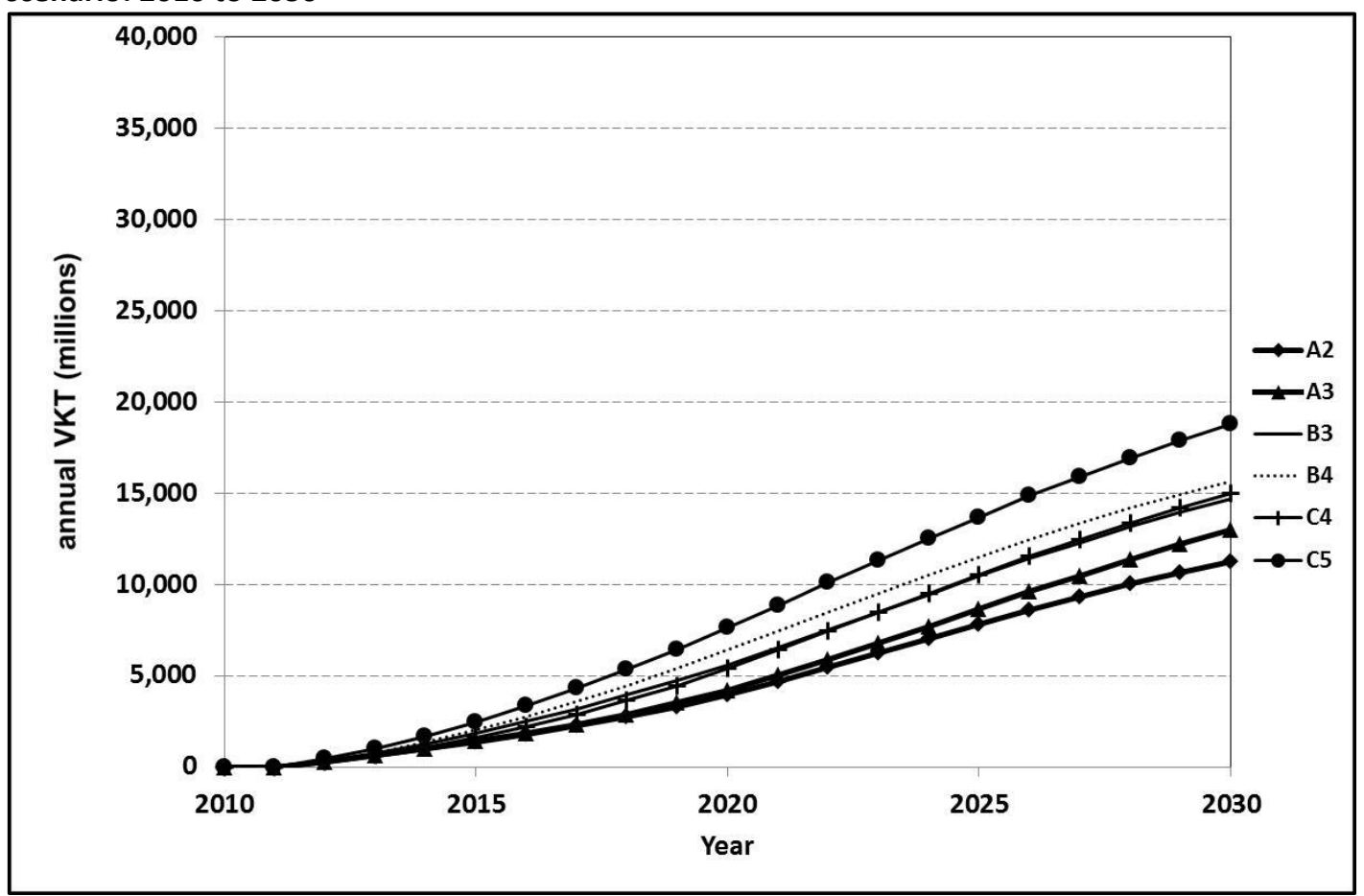

Total annual VKT for the LPV fleet

In total, the annual VKT for the LPV fleet was projected to increase from 30.49 billion kilometres, in 2010, to between $36.39 \pm 0.15$ billion kilometres and 38.67 \pm 0.16 billion kilometres in 2030 (Figure 9.4).

The variation in these projections was due to the effect of different levels of uptake of EVs, different assumptions regarding the energy efficiency of vehicles, and different assumptions about of the future price of fuel. Those VFM scenarios assuming greater improvements in the energy efficiency of ICEVs, increased electric driving ranges in PHEVs, lower EV cost, and lower petroleum prices resulted in higher estimates of total annual VKT. The effect of these assumptions was most clearly seen in scenarios C4 and C5. 
Figure 9.4: Light passenger vehicle fleet - total annual VKT by vehicle fleet model scenario: 2010 to 2030

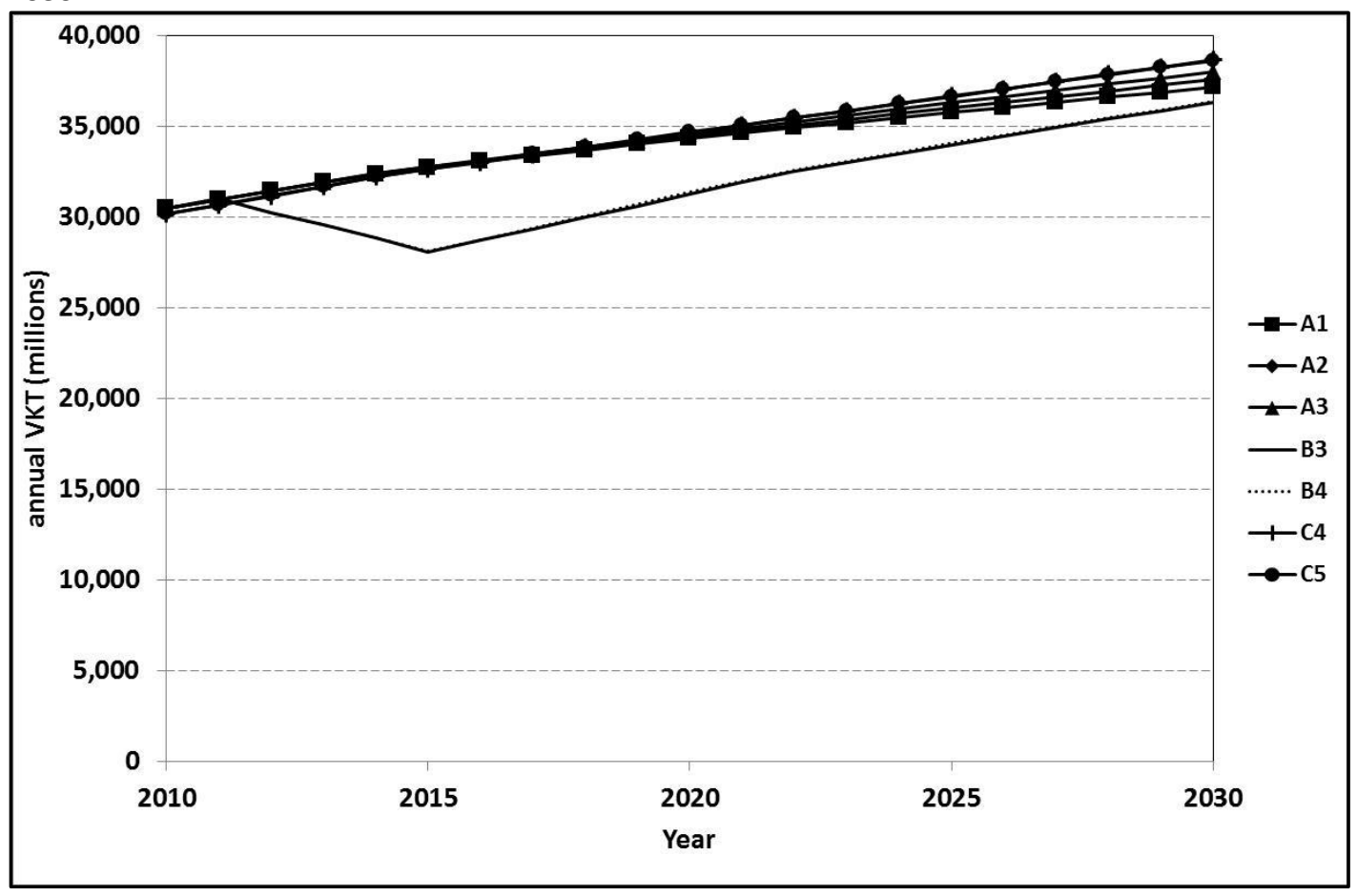

Total annual PHEV VKT

The annual VKT by PHEVs was estimated for both petroleum and electric powered driving cycles. These estimates were sensitive to the assumptions made about the electric driving range of these vehicles. In scenario A2, it was assumed that the average electric driving range of PHEVs that entered the LPV fleet over the modelling period would stay at $30 \mathrm{~km}$. In this VFM scenario, by $2030,57 \%$ of the $10.22 \pm 0.04$ billion kilometres travelled by PHEVs in 2030 was still being undertaken using petrol.

The other VFM scenarios assumed that the electric driving range of PHEVs would increase, which reduced the estimated amount of VKT using petrol in 2030 of approximately 4 billion kilometres (Figure 9.5). However, estimated travel using electricity varied depending on whether it was assumed whether there were one or two recharging events during the day. For VFM scenarios A3, B3, and B4, it was assumed that, in 2030, all PHEV users would recharge once a day. In these VFM scenarios, PHEV would travel between $6.83 \pm 0.06$ billion kilometres and $7.00 \pm 0.08$ billion kilometres using electric power in 2030 (Figure 9.6). In the two C scenarios, it was assumed that $30 \%$ of PHEVs users would undertake two charging events per 
day. This charging behaviour increased the amount of driving using electric power in these two VFM scenarios to $7.99 \pm 0.07$ billion kilometres and $8.08 \pm 0.04$ billion kilometres in 2030 (Figure 9.6).

Figure 9.5: Annual VKT of plug-in hybrid electric vehicles using petrol by vehicle fleet model scenario: 2010 to 2030

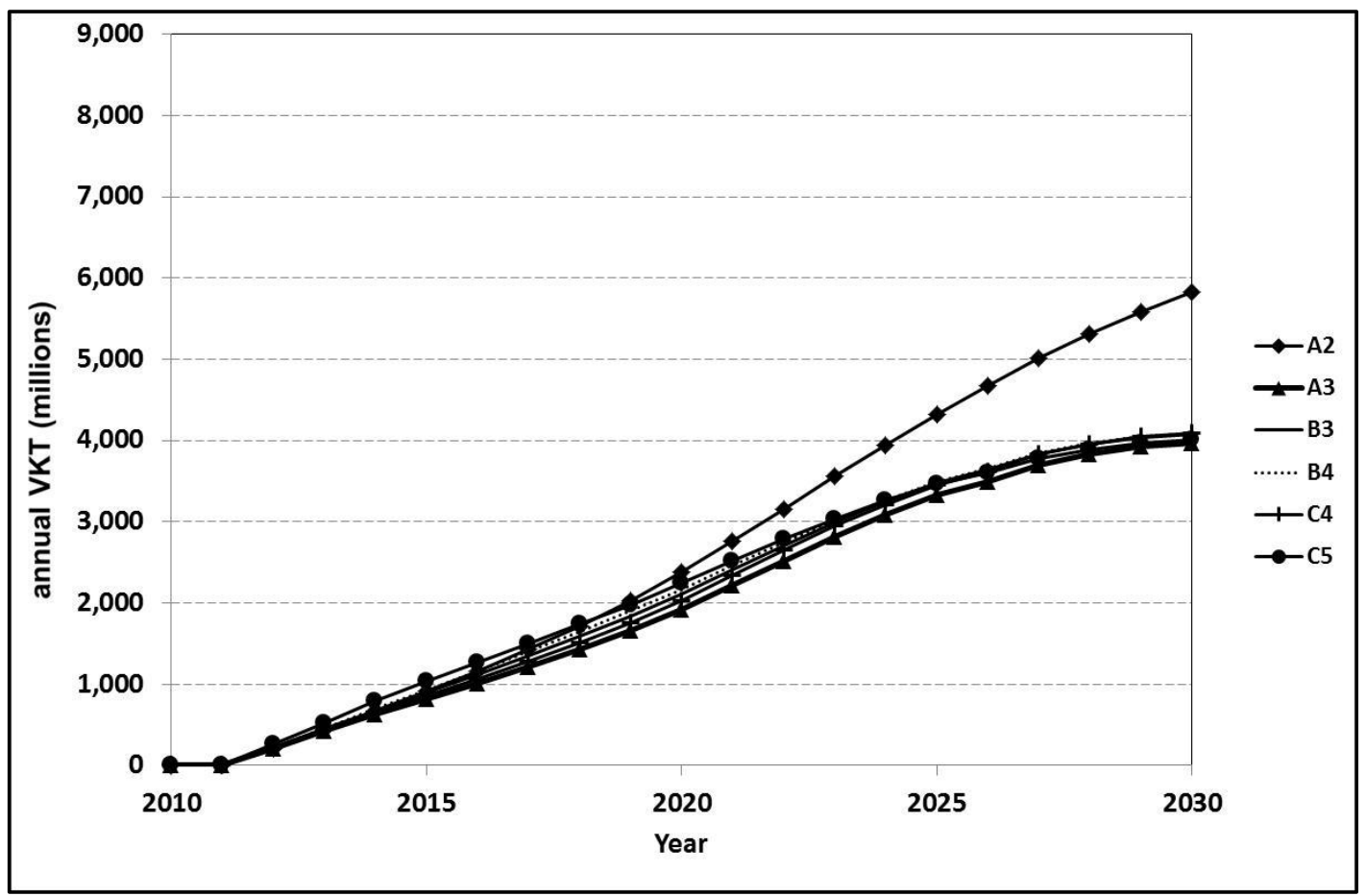

Figure 9.6: Annual VKT of plug-in hybrid electric vehicles using electricity by vehicle fleet model scenario: 2010 to 2030

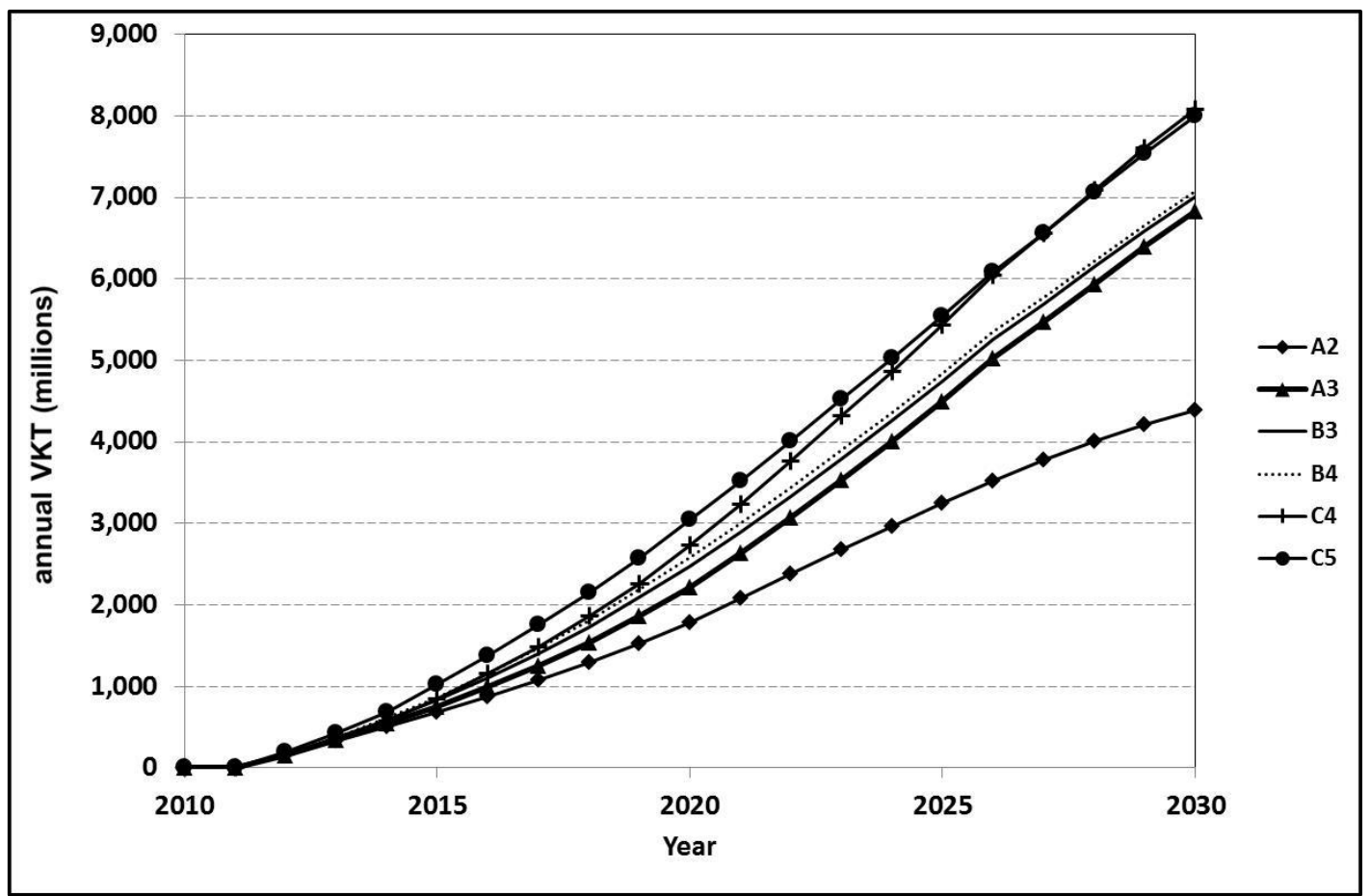




\section{VKT sensitivity to changes in the price of carbon}

Increasing the price of carbon from $\$ 25$ per tonne to $\$ 100$ per tonne added $17.6 \mathrm{c} / \mathrm{L}$ to the price of petrol and $20.3 \mathrm{c} / \mathrm{L}$ to that of diesel. The VUM estimated that these price increases had only a small effect on the total amount of VKT travelled by the LPV fleet. In scenario A1 (no-EV), where no EVs are available, the higher carbon price led to a decrease in total annual VKT of between $0.98 \%$ and $1.01 \%$. In other VFM scenarios the effect was even smaller and decreased with the size of the EV fleet. By 2030, the effect of the higher carbon price on annual VKT, in those VFM scenarios, was a reduction of between $0.24 \%$ and $0.66 \%$ depending on the level of EV uptake and the proportion of PHEVs in the EV fleet.

\subsubsection{LPV fleet energy efficiency}

The annual energy demand estimates for the LPV fleet were derived from the estimates of the annual VKT by fuel type, and the average energy efficiency for each vehicle type. The VFM took no account of the effect of declining energy efficiency due to the aging of vehicles.

For BEVs and the electric drive cycle of PHEVs, the average EV energy efficiency was found to vary very little across the modelling period (Table 9.1).

Table 9.1: Fleet average energy efficiency values of battery electric vehicles and the electric cycle of plug-in hybrid electric vehicles - selected years

\begin{tabular}{|c|c|c|c|c|c|c|c|c|c|c|c|c|}
\hline \multicolumn{13}{|c|}{ Wh/km } \\
\hline & $\begin{array}{c}\text { CEV } \\
\text { A2 }\end{array}$ & \pm & $\begin{array}{c}\text { CEV } \\
\text { A3, } \\
\text { B3, B4, } \\
\text { and C4 }\end{array}$ & \pm & $\begin{array}{c}\text { GEV } \\
\text { and } \\
\text { PHEV } \\
\text { electric } \\
\text { cycle } \\
\text { A2 }\end{array}$ & \pm & $\begin{array}{l}\text { GEV } \\
\text { and } \\
\text { PHEV } \\
\text { electric } \\
\text { cycle } \\
\text { A3, B3, } \\
\text { B4, } \\
\text { and C4 }\end{array}$ & \pm & $\begin{array}{c}\text { CEV } \\
\text { C5 }\end{array}$ & \pm & $\begin{array}{l}\text { GEV } \\
\text { and } \\
\text { PHEV } \\
\text { electric } \\
\text { cycle } \\
\text { C5 }\end{array}$ & \pm \\
\hline 2012 & 177 & 1 & 177 & 1 & 209 & 1 & 209 & 1 & 115 & 1 & 134 & 1 \\
\hline 2020 & 177 & 9 & 175 & 12 & 209 & 6 & 209 & 13 & 114 & 10 & 133 & 1 \\
\hline 2030 & 177 & 19 & 174 & 19 & 209 & 11 & 208 & 33 & 114 & 17 & 133 & 7 \\
\hline $\begin{array}{c}\% \\
\text { change } \\
2012 \text { to } \\
2030\end{array}$ & 0.0 & & -1.7 & & $0.0 \%$ & & $-0.5 \%$ & & $-0.9 \%$ & & $-0.8 \%$ & \\
\hline
\end{tabular}


The energy efficiency assumptions described in section 5.3.4 for ICEVs and PHEVs entering the LPV fleet in scenarios B and C resulted in an improvement in the average energy efficiency of the ICEVs in the LPV fleet. However, the rate of improvement in efficiency was moderated by the large stock of lower efficiency ICEVs already present in the LPV fleet, and the ongoing importation of older, less fuel efficient, used ICEVs (Table 9.2).

Table 9.2: Fleet average energy efficiency values for internal combustion engine vehicles and the petrol cycle of plug-in hybrid electric vehicles - selected years

\begin{tabular}{|c|c|c|c|c|c|c|c|c|c|c|c|c|c|c|c|c|}
\hline & \multicolumn{8}{|c|}{ ICEVs } & \multicolumn{8}{|c|}{ PHEV petrol cycle } \\
\hline & $\begin{array}{l}\text { A2 } \\
\text { and } \\
\text { A3 }\end{array}$ & \pm & $\begin{array}{l}\text { B3 } \\
\text { and } \\
\text { B4 }\end{array}$ & \pm & C4 & \pm & C5 & \pm & $\left|\begin{array}{c}\mathrm{A} 2 \\
\text { and } \\
\mathrm{A} 3\end{array}\right|$ & \pm 1 & $\begin{array}{c}\text { B3 } \\
\text { and } \\
\text { B4 }\end{array}$ & \pm & C4 & \pm & C5 & \pm \\
\hline & \multicolumn{16}{|c|}{ Wh/km } \\
\hline 2012 & 969 & 1 & 969 & 1 & 969 & 1 & 969 & 1 & 624 & 3 & 624 & 6 & 624 & 4 & 624 & 1 \\
\hline 2020 & 969 & 3 & 967 & 7 & 963 & 5 & 964 & 3 & 624 & 23 & 571 & 38 & 554 & 28 & 556 & 5 \\
\hline \multirow[t]{2}{*}{2030} & 969 & 9 & 918 & 15 & 894 & 11 & 908 & 6 & 624 & 42 & 509 & 46 & 466 & 34 & 470 & 12 \\
\hline & \multicolumn{16}{|c|}{$\mathrm{I} / 100 \mathrm{~km}$} \\
\hline 2012 & 9.9 & 0.0 & 9.9 & 0.0 & 9.9 & 0.0 & 9.9 & 0.0 & 6.4 & 0.0 & 6.4 & 0.1 & 6.4 & 0.0 & 6.4 & 0.0 \\
\hline 2020 & 9.9 & 0.0 & 9.9 & 0.1 & 9.8 & 0.1 & 9.8 & 0.0 & 6.4 & 0.2 & 5.8 & 0.4 & 5.6 & 0.3 & 5.7 & 0.1 \\
\hline 2030 & 9.9 & 0.1 & 9.4 & 0.2 & 9.1 & 0.1 & 9.3 & 0.1 & 6.4 & 0.4 & 5.2 & 0.5 & 4.7 & 0.3 & 4.8 & 0.1 \\
\hline $\begin{array}{c}\% \text { change } \\
2012 \text { to } 2030\end{array}$ & $0.0 \%$ & & $-5.3 \%$ & & $-7.7 \%$ & & $-6.3 \%$ & & $0.0 \%$ & & $-18.4 \%$ & & $-25.3 \%$ & & $-24.7 \%$ & \\
\hline
\end{tabular}

$\pm$

95\% confidence intervals

The overall effect of the introduction of EVs and improved ICE technology into the LPV fleet was that the average energy efficiency of the entire LPV fleet improved by approximately $30 \%$ for the A scenarios, and $40 \%$ to $42 \%$ for the two B scenarios and scenario C4. Modelling indicated that to obtain greater improvements in average energy efficiency of the LPV fleet would require the changes to EV design envisaged in scenario C5 (Table 9.3). 
Table 9.3: Light passenger vehicle fleet average energy efficiency by vehicle fleet model scenario selected years

\begin{tabular}{|l|c|c|c|c|c|c|c|c|c|c|c|c|c|}
\hline & \multicolumn{10}{|c|}{ Wh/ km } \\
\hline & A1 & A2 & \pm I & A3 & \pm & B3 & \pm & B4 & \pm & C4 & \pm & C5 & \pm \\
\hline 2010 & 969 & 969 & 0 & 969 & 0 & 969 & 0 & 969 & 0 & 969 & 0 & 969 & 0 \\
\hline 2012 & 969 & 959 & 3 & 959 & 10 & 958 & 7 & 958 & 4 & 959 & 4 & 954 & 1 \\
\hline 2020 & 969 & 857 & 26 & 855 & 38 & 802 & 44 & 781 & 36 & 819 & 33 & 753 & 12 \\
\hline $\begin{array}{l}2030 \\
\% \text { change } \\
2010 \text { to 2030 }\end{array}$ & 969 & 684 & 49 & 669 & 38 & 580 & 63 & 561 & 52 & 578 & 53 & 483 & 23 \\
\hline
\end{tabular}

$\pm \quad 95 \%$ confidence intervals

\subsubsection{Energy demand forecasts for the LPV fleet}

\section{Electricity demand from EVs}

The projected annual electricity demand from EVs, in 2030, ranged from 2,701.2 $\pm 13.5 \mathrm{GWh}$, in scenario A2, to 3,715.3 $\pm 19.9 \mathrm{GWh}$, in scenario B4 (Table 9.4).

Scenario C5 had the highest projected uptake of EVs, but due to the energy efficient design assumptions the electricity demand, in 2030 , was constrained to $2,892.3$ $\pm 14.9 \mathrm{GWh}$.

The electricity demand forecast, exclusive of the demand from EVs, was projected to be, in $2030,60,316 \mathrm{GWh}$. The modelling indicated that EVs would add between $4.5 \%$ and $6.2 \%$ to the annual demand forecast in 2030 (Table 9.4).

Table 9.4: Light passenger vehicle fleet annual electricity demand in 2030, and total electricity demand in 2030 (GWh)

\begin{tabular}{|c|c|c|c|c|}
\hline & $\begin{array}{c}\text { LPV fleet } \\
\text { annual demand } \\
2030\end{array}$ & \pm & $\begin{array}{l}\text { Total (all uses) } \\
\text { demand } 2030\end{array}$ & $\begin{array}{c}\text { Additional } \\
\text { demand (\%) } \\
\text { above BAU in } \\
2030\end{array}$ \\
\hline A1 & 0 & 0 & $60,316.0$ & 0 \\
\hline A2 & $2,701.2$ & 13.5 & $63,017.2$ & 4.5 \\
\hline A3 & $3,111.3$ & 19.2 & $63,427.3$ & 5.2 \\
\hline B3 & $3,494.1$ & 23.3 & $63,810.1$ & 5.8 \\
\hline B4 & $3,715.3$ & 19.9 & $64,031.3$ & 6.2 \\
\hline $\mathrm{C} 4$ & $3,597.4$ & 23.1 & $63,913.4$ & 6.0 \\
\hline $\mathrm{C} 5$ & $2,892.3$ & 14.9 & $63,208.3$ & 4.8 \\
\hline
\end{tabular}

$\pm \quad 95 \%$ confidence intervals 


\section{Petroleum demand}

The annual demand for petroleum energy by the LPV fleet under the A1 (no-EV) BAU scenario was projected to increase by $21.9 \%$ by 2030 , rising from $29,531.5$ GWh in 2010, to 35,988.6 GWh. Introducing EVs into the LPV fleet reduced the total annual petroleum demand in 2030 relative to 2010 by between $20.5 \%$, in scenario $A 2$, and $44.9 \%$, in the high efficiency scenario C5. However, when the cumulative demands for petroleum were compared, the greatest reduction occurred in scenario B4, with a $29.9 \%$ reduction, and scenario B3, with a $28.3 \%$ reduction. The reduction in petroleum demand was due to the high petroleum prices in these VFM scenarios depressing the demand for travel by ICEVs (Table 9.5 and Figure 9.7).

Table 9.5: Annual petroleum demand for the light passenger vehicle fleet in 2030 and total cumulative petroleum demand (GWh): 2010 to 2030

\begin{tabular}{|c|c|c|c|c|c|c|c|}
\hline & $\begin{array}{c}\text { LPV fleet } \\
\text { demand } \\
2030\end{array}$ & \pm & $\begin{array}{c}\% \text { diff } \\
\text { relative to } \\
2010\end{array}$ & $\begin{array}{c}\text { \% diff } \\
\text { relative } \\
\text { to } 2030 \\
\text { BAU }\end{array}$ & $\begin{array}{c}\text { Cumulative } \\
\text { demand } \\
2010 \text { to } \\
2030\end{array}$ & $\begin{array}{c}\% \text { diff } \\
\text { relative to } \\
\text { total } \\
\text { cumulative } \\
\text { demand BAU }\end{array}$ \\
\hline A1 & $35,988.6$ & & $21.9 \%$ & $0.00 \%$ & $694,954.1$ & & $0.0 \%$ \\
\hline A2 & $23,477.4$ & 83.9 & $-20.5 \%$ & $-34.8 \%$ & $583,826.2$ & 241.9 & $-16.0 \%$ \\
\hline A3 & $22,872.6$ & 128.9 & $-22.5 \%$ & $-36.4 \%$ & $581,477.5$ & 371.7 & $-16.3 \%$ \\
\hline B3 & $18,204.6$ & 130.2 & $-38.4 \%$ & $-49.4 \%$ & $498,602.1$ & 402.3 & $-28.3 \%$ \\
\hline B4 & $17,385.5$ & 103.5 & $-41.1 \%$ & $-51.7 \%$ & $487,221.2$ & 337.2 & $-29.9 \%$ \\
\hline C4 & $19,417.2$ & 110.7 & $-34.2 \%$ & $-46.0 \%$ & $548,954.2$ & 319.0 & $-21.0 \%$ \\
\hline C5 & $16,268.9$ & 61.8 & $-44.9 \%$ & $-54.8 \%$ & $510,015.4$ & 211.8 & $-26.6 \%$ \\
\hline
\end{tabular}


Figure 9.7: Annual petroleum demand for the light passenger vehicle fleet: 2010 to 2030

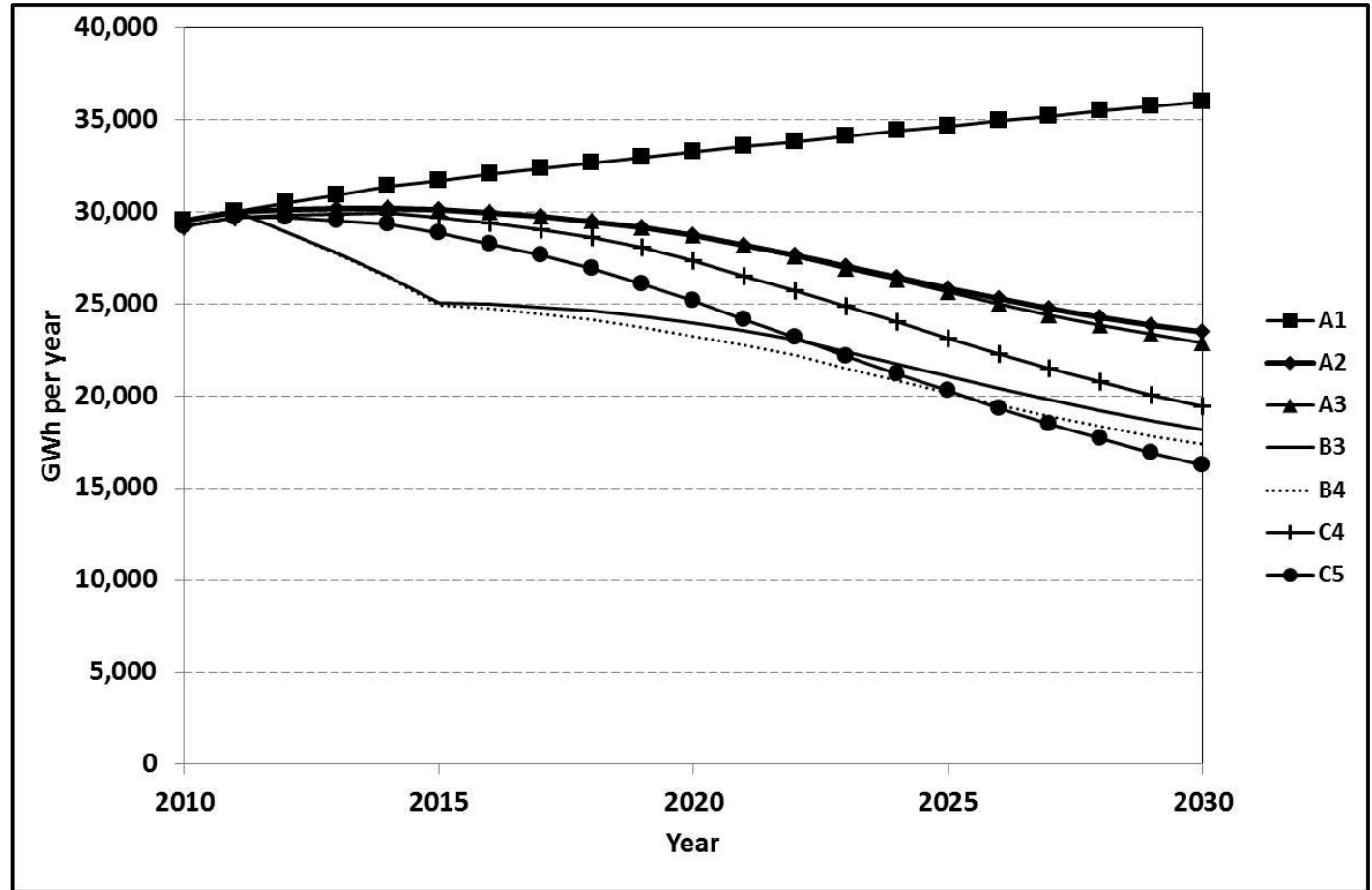

A sensitivity analysis of the two B scenarios was undertaken that removed the effect of the high petroleum prices on the VKT demand projections. This analysis indicated that, without the reductions in travel demand, the cumulative total energy and petroleum demand projections over 2010-2030 were comparable to those in scenario C4 (Table 9.6). 
Table 9.6: Vehicle fleet model scenarios B3 and B4 - sensitivity analysis of petroleum demand with no change in VKT (GWh)

\begin{tabular}{|c|c|c|c|c|c|c|c|}
\hline & $\begin{array}{l}\text { Petroleum } \\
\text { demand in } \\
2030\end{array}$ & \pm & $\begin{array}{l}\% \text { diff } \\
\text { relative } \\
\text { to } 2010\end{array}$ & $\begin{array}{l}\% \text { diff } \\
\text { relative } \\
\text { to } 2030 \\
\text { BAU }\end{array}$ & $\begin{array}{l}\text { Cumulative } \\
\text { demand } \\
2010 \text { to } \\
2030\end{array}$ & \pm & $\begin{array}{c}\% \text { diff } \\
\text { relative to } \\
\text { total } \\
\text { cumulative } \\
\text { demand } \\
\text { BAU }\end{array}$ \\
\hline $\begin{array}{c}\text { B3 } \\
\text { (with change in } \\
\text { VKT) }\end{array}$ & $18,204.6$ & 130.2 & $-38.4 \%$ & $-49.4 \%$ & $498,602.1$ & 402.3 & $-28.3 \%$ \\
\hline $\begin{array}{c}\text { B3 } \\
\text { (with no change } \\
\text { in VKT) }\end{array}$ & $19,865.9$ & 68.1 & $-32.7 \%$ & $-44.8 \%$ & $549,773.4$ & 222.8 & $-20.9 \%$ \\
\hline $\begin{array}{c}\text { B4 } \\
\text { (with change in } \\
\text { VKT) }\end{array}$ & $17,385.5$ & 103.5 & $-41.1 \%$ & $-51.7 \%$ & $487,221.2$ & 337.2 & $-29.9 \%$ \\
\hline $\begin{array}{c}\text { B4 } \\
\text { (with no change } \\
\text { in VKT) }\end{array}$ & 18773.9 & 79.2 & $-36.4 \%$ & $-47.8 \%$ & $535,265.3$ & 302.7 & $-23.0 \%$ \\
\hline
\end{tabular}

$\pm \quad 95 \%$ confidence intervals

The VUM also indicated that the total annual energy demand of the LPV fleet (both petroleum and electricity), by 2030 , could be reduced by between $11.4 \%$ and $35.1 \%$ assuming the introduction of EVs and measures to improve the energy efficiency of ICEVs (Table 9.7). The analysis also indicated that, under these assumptions, the cumulative energy consumed by the LPV fleet during the period 2010 to 2030 would be reduced by between $12.6 \%$ and $24.9 \%$. The vehicle design changes envisaged in scenario $\mathrm{C} 5$ resulted in the lowest energy demand projections that could be achieved without the reductions in VKT envisaged in the two B scenarios. 
Table 9.7: Total light passenger vehicle fleet petroleum and electricity annual demand in 2030 and cumulative energy demand 2010 to 2030 (GWh)

\begin{tabular}{|c|c|c|c|c|c|c|c|}
\hline & $\begin{array}{c}\text { Total } \\
\text { demand } \\
\text { in } 2030\end{array}$ & \pm & $\begin{array}{l}\% \text { diff } \\
\text { relative } \\
\text { to } 2010\end{array}$ & $\begin{array}{c}\% \text { diff } \\
\text { relative } \\
\text { to } 2030 \\
\text { BAU }\end{array}$ & $\begin{array}{l}\text { Total } \\
\text { cumuli- } \\
\text { tive } \\
\text { demand } \\
2010 \text { to } \\
2030\end{array}$ & \pm & $\begin{array}{l}\% \text { diff } \\
\text { relative } \\
\text { to total } \\
\text { cumuli- } \\
\text { tive } \\
\text { demand } \\
\text { BAU }\end{array}$ \\
\hline A1 & $35,988.6$ & & $21.9 \%$ & $0.0 \%$ & $694,954.1$ & & $0.0 \%$ \\
\hline A2 & $26,178.6$ & 85.0 & $-11.4 \%$ & $-27.3 \%$ & $607,451.7$ & 244.6 & $-12.6 \%$ \\
\hline $\mathbf{A 3}$ & $25,983.9$ & 130.3 & $-12.0 \%$ & $-27.8 \%$ & $607,598.3$ & 375.3 & $-12.6 \%$ \\
\hline B3 & $21,698.7$ & 132.2 & $-26.5 \%$ & $-39.7 \%$ & $530,043.7$ & 407.3 & $-23.7 \%$ \\
\hline B4 & $21,100.8$ & 105.4 & $-28.5 \%$ & $-41.4 \%$ & $521,655.6$ & 341.7 & $-24.9 \%$ \\
\hline $\mathrm{C} 4$ & $23,014.6$ & 113.1 & $-22.1 \%$ & $-36.1 \%$ & $580,437.7$ & 324.6 & $-16.5 \%$ \\
\hline $\mathrm{C} 5$ & $19,161.2$ & 63.6 & $-35.1 \%$ & $-46.8 \%$ & $536,673.2$ & 216.1 & $-22.8 \%$ \\
\hline
\end{tabular}

$\pm \quad 95 \%$ confidence intervals

A sensitivity analysis of scenario $C 5$, which replaced the high energy efficiency assumptions for EVs with the standard assumptions, resulted in more expensive EVs due to the need for larger batteries. This increase in price resulted in a $4.9 \%$ reduction in the stock of EVs in 2030. The standard EV energy efficiency assumptions also resulted in the cumulative demand for petroleum fuel, over the modelling period, increasing by $2.6 \%$, and the cumulative demand for electricity declining by $4.5 \%$. However, because scenario C5 still assumed lower cost batteries and the presence of EV purchase subsidies, this VFM scenario still had the highest uptake of EVs of all the scenarios (Table 9.8).

Table 9.8: Scenario C5 - sensitivity analysis, high and standard EV energy efficiency (GWh): 2010 to 2030

\begin{tabular}{|c|c|c|c|c|c|c|c|c|c|c|}
\hline & $\begin{array}{c}\text { Total } \\
\text { number of } \\
\text { EVs in } \\
2030\end{array}$ & \pm & \begin{tabular}{|} 
Cumula- \\
tive \\
petroleum \\
demand \\
2010 to \\
2030
\end{tabular} & \pm & $\begin{array}{l}\% \text { diff } \\
\text { relative } \\
\text { total } \\
\text { cumula- } \\
\text { tive } \\
\text { demand } \\
\text { BAU }\end{array}$ & $\begin{array}{l}\text { Cumula- } \\
\text { tive } \\
\text { electricity } \\
\text { demand } \\
2010 \text { to } \\
2030\end{array}$ & \pm & $\begin{array}{l}\text { Total } \\
\text { cumula- } \\
\text { tive } \\
\text { demand } \\
2010 \text { to } \\
2030\end{array}$ & \pm & $\begin{array}{l}\% \text { diff } \\
\text { relative } \\
\text { to total } \\
\text { cumula- } \\
\text { tive } \\
\text { demand } \\
\text { BAU }\end{array}$ \\
\hline C5 & $1,827,893$ & 5,411 & $510,015.4$ & 211.8 & $-26.6 \%$ & $26,657.8$ & 43.2 & $536,673.2$ & 216.1 & $-22.8 \%$ \\
\hline $\begin{array}{l}\text { C5 (with } \\
\text { standard } \\
\text { EV } \\
\text { efficiency) }\end{array}$ & $1,737,671$ & 5,783 & $523,527.2$ & 478.1 & $-24.7 \%$ & $37,459.0$ & 89.2 & $560,986.2$ & 368.1 & $-19.3 \%$ \\
\hline
\end{tabular}




\subsubsection{Direct GHG emissions from the LPV fleet}

This section summarises the GHG emissions directly produced by the LPV fleet. The indirect GHG emissions from the generation of the additional electricity required by EVs are discussed in section 9.3.

The GHG emissions from the LPV fleet, in 2010 , were estimated to be $7,216,000$ tonnes $\mathrm{CO}_{2} \mathrm{e}$. Under the $\mathrm{BAU}$ projection in scenario $\mathrm{A} 1$ (no-EV), the GHG emissions from the LPV fleet would increase by $22 \%$ to 8,794,000 tonnes $\mathrm{CO}_{2} \mathrm{e}$ in 2030.

The introduction of EVs had the effect of reducing the LPV fleet's projected annual GHG emissions, in 2030, from those in 2010 by between $1.48 \pm 0.01$ million tonnes $\mathrm{CO}_{2} \mathrm{e}$ and $3.26 \pm 0.01$ million tonnes $\mathrm{CO}_{2} \mathrm{e}$. These represent reductions of between $20.5 \%$ in scenario $\mathrm{A} 2$ and $45.1 \%$ in scenario $\mathrm{C} 5$. The reductions of the cumulative GHG emissions over the modelling period, relative to the BAU projection, were reduced by $27.16 \pm 0.04$ million tonnes $\mathrm{CO}_{2} \mathrm{e}$, in scenario $\mathrm{A} 2$, and $50.76 \pm 0.04$ million tonnes $\mathrm{CO}_{2} \mathrm{e}$ in scenario $\mathrm{B} 4$ (Table 9.9). Scenarios $\mathrm{B} 3$ and $\mathrm{B} 4$ had greater projected reductions in cumulative GHG emissions, but these reductions were, in large part, due to the reductions in VKT by ICEVs.

Table 9.9: Estimated light passenger vehicle fleet annual GHG emissions in 2030 and cumulative GHG emissions (thousand tonnes $\mathrm{CO}_{2} \mathrm{e}$ ): 2010 to 2030

\begin{tabular}{|c|c|c|c|c|c|c|c|c|c|c|}
\hline & \multicolumn{6}{|c|}{ Annual GHG emissions } & \multicolumn{4}{|c|}{$\begin{array}{c}\text { Cumulative GHG emissions from } 2010 \\
\text { to } 2030\end{array}$} \\
\hline & $\begin{array}{c}\text { GHG } \\
\text { emissions } \\
\text { in } 2030\end{array}$ & \pm & $\begin{array}{l}\text { Change } \\
\text { relative } \\
\text { to } 2010\end{array}$ & $\begin{array}{l}\% \text { diff } \\
\text { relative } \\
\text { to } 2010\end{array}$ & $\begin{array}{c}\text { Change } \\
\text { relative } \\
\text { to } 2030 \\
\text { BAU }\end{array}$ & $\begin{array}{c}\% \text { diff } \\
\text { relative } \\
\text { to } 2030 \\
\text { BAU }\end{array}$ & $\begin{array}{c}\text { Cumula- } \\
\text { tive GHG } \\
\text { emissions } \\
2010 \text { to } \\
2030\end{array}$ & \pm & $\begin{array}{c}\text { Change in } \\
\text { GHG } \\
\text { cumula- } \\
\text { tive GHG } \\
\text { emissions } \\
\text { from BAU }\end{array}$ & $\begin{array}{l}\text { \% diff } \\
\text { relative to } \\
\text { total } \\
\text { cumulative } \\
\text { GHG } \\
\text { emissions } \\
\text { BAU }\end{array}$ \\
\hline A1 & 8,794 & & 1,578 & $21.9 \%$ & 0 & $0.0 \%$ & 169,819 & & 0 & $0.0 \%$ \\
\hline A2 & 5,737 & 14 & $-1,479$ & $-20.5 \%$ & 3,057 & $-34.8 \%$ & 142,661 & 41 & 27,158 & $-16.0 \%$ \\
\hline A3 & 5,589 & 24 & $-1,627$ & $-22.6 \%$ & 3,205 & $-36.4 \%$ & 142,087 & 66 & 27,732 & $-16.3 \%$ \\
\hline B3 & 4,448 & 23 & $-2,768$ & $-38.4 \%$ & 4,346 & $-49.4 \%$ & 121,836 & 68 & 47,983 & $-28.3 \%$ \\
\hline B4 & 4,248 & 18 & $-2,968$ & $-41.1 \%$ & 4,546 & $-51.7 \%$ & 119,055 & 59 & 50,764 & $-29.9 \%$ \\
\hline $\mathrm{C} 4$ & 4,745 & 21 & $-2,471$ & $-34.2 \%$ & 4,049 & $-46.0 \%$ & 134,140 & 57 & 35,679 & $-21.0 \%$ \\
\hline $\mathrm{C5}$ & 3,959 & 11 & $-3,257$ & $-45.1 \%$ & 4,835 & $-55.0 \%$ & 124,470 & 40 & 45,349 & $-26.7 \%$ \\
\hline
\end{tabular}




\subsubsection{Sensitivity analysis - electricity demand and GHG emissions}

As noted in section 8.4, the growth of the LPV fleet, sales of LPVs and sales of EVs were sensitive to GDP growth and scrapping assumptions. The sensitivity analysis indicated that, under the low GDP and scrapping assumptions in scenario A1 (noEV), cumulative GHG emissions over the modelling period would be 5.9 million tonnes $\mathrm{CO}_{2} \mathrm{e}$ lower than the same VFM scenario using the default GDP and scrapping assumptions. If high GDP growth and scrapping rates were assumed, the projected cumulative GHG emissions in this VFM scenario increased by 2.2 million tonnes $\mathrm{CO}_{2}$ e compared to the same scenario using the default GDP growth and scrapping assumptions.

The VFM's estimates of the GHG emissions from the LPV fleet were largely a function of the size of the ICEV fleet, the average energy efficiency of the ICEV fleet, and the distance that these vehicles travel. PHEVs also contributed to the GHG emissions from the LPV fleet, but this contribution was much smaller. In scenario C4, PHEVs contributed to $4.5 \%$ of the cumulative GHG emissions over the modelling period and $6.3 \%$ in scenario A2. Due to the dominance of ICEVs, changing the GDP and scrapping rate assumptions had less effect on GHG emissions in those VFM scenarios where there was a greater uptake of EVs (Table 9.10). 
Table 9.10: Sensitivity analysis - impact of GDP growth and scrapping rates, cumulative electricity demand, cumulative petroleum demand, and GHG emissions: 2010 to 2030

\begin{tabular}{|c|c|c|c|c|c|c|c|c|c|c|c|c|c|c|c|}
\hline & & Imulativ & $\begin{array}{l}\text { electrici } \\
10 \text { to } 20 \\
\text { GWh }\end{array}$ & $\begin{array}{l}\text { y demar } \\
0\end{array}$ & & & Cumulativ & $\begin{array}{l}\text { petroleu } \\
\text { LO to } 20 \text { : } \\
\mathrm{GWh}\end{array}$ & demano & & & $\begin{array}{r}\text { Cumulat } \\
\text { (thouse }\end{array}$ & $\begin{array}{l}\text { e GHG } \\
10 \text { to } 20 \\
\text { d) tonr }\end{array}$ & $\begin{array}{l}\text { nissions } \\
\mathrm{O} \mathrm{CO}_{2} \mathrm{e}\end{array}$ & \\
\hline & Default & Low & $\begin{array}{c}\text { Change } \\
\text { from } \\
\text { default }\end{array}$ & High & $\begin{array}{l}\text { Change } \\
\text { from } \\
\text { default }\end{array}$ & Default & Low & $\begin{array}{c}\text { Change } \\
\text { from } \\
\text { default }\end{array}$ & High & $\begin{array}{c}\text { Change } \\
\text { from } \\
\text { default }\end{array}$ & Default & Low & $\begin{array}{c}\text { Change } \\
\text { from } \\
\text { default }\end{array}$ & High & $\begin{array}{c}\text { Change } \\
\text { from } \\
\text { default }\end{array}$ \\
\hline A1 & $\mathrm{N} / \mathrm{A}$ & $\mathrm{N} / \mathrm{A}$ & $\mathrm{N} / \mathrm{A}$ & $\mathrm{N} / \mathrm{A}$ & N/A & 694,954 & 670,704 & $-24,250$ & 704,041 & 9,087 & 169,819 & 163,893 & $-5,926$ & 172,018 & 2,199 \\
\hline A2 & $\begin{array}{c}23,625 \\
\pm 141 \\
\end{array}$ & $\begin{array}{c}21,613 \\
\pm 111 \\
\end{array}$ & $\begin{array}{c}-2,012 \\
\pm 179\end{array}$ & $\begin{array}{c}24,679 \\
\pm 179 \\
\end{array}$ & $\begin{array}{l}1,054 \\
\pm 228 \\
\end{array}$ & $\begin{array}{c}583,826 \\
\pm 242 \\
\end{array}$ & $\begin{array}{c}568,853 \\
\pm 183 \\
\end{array}$ & $\begin{array}{c}-14,973 \\
\pm 303 \\
\end{array}$ & $\begin{array}{c}587,721 \\
\pm 239 \\
\end{array}$ & $\begin{array}{l}3,895 \\
\pm 340 \\
\end{array}$ & $\begin{array}{c}142,661 \\
\pm 41 \\
\end{array}$ & $\begin{array}{c}139,002 \\
\pm 32 \\
\end{array}$ & $\begin{array}{c}-3,659 \\
\pm 52 \\
\end{array}$ & $\begin{array}{c}143,612 \\
\pm 40 \\
\end{array}$ & $\begin{array}{l}951 \\
\pm 57 \\
\end{array}$ \\
\hline B3 & $\begin{array}{c}31,442 \\
\pm 246\end{array}$ & $\begin{array}{c}29,872 \\
\pm 160 \\
\end{array}$ & $\begin{array}{c}-1,570 \\
\pm 293\end{array}$ & $\begin{array}{c}32,246 \\
\pm 178\end{array}$ & $\begin{array}{c}804 \\
\pm 304 \\
\end{array}$ & $\begin{array}{c}498,602 \\
\pm 402 \\
\end{array}$ & $\begin{array}{c}488,278 \\
\pm 277\end{array}$ & $\begin{array}{c}-10,324 \\
\pm 488\end{array}$ & $\begin{array}{c}501,163 \\
\pm 232\end{array}$ & $\begin{array}{l}2,561 \\
\pm 464 \\
\end{array}$ & $\begin{array}{c}121,836 \\
\pm 68\end{array}$ & $\begin{array}{c}119,314 \\
\pm 49\end{array}$ & $\begin{array}{c}-2,522 \\
\pm 84\end{array}$ & $\begin{array}{c}122,462 \\
\pm 41\end{array}$ & $\begin{array}{l}626 \\
\pm 79 \\
\end{array}$ \\
\hline $\mathrm{C} 4$ & $\begin{array}{c}31,484 \\
\pm 227\end{array}$ & $\begin{array}{c}28,751 \\
\pm 177\end{array}$ & $\begin{array}{c}-2,733 \\
\pm 288\end{array}$ & $\begin{array}{c}32,764 \\
\pm 204\end{array}$ & $\begin{array}{l}1,280 \\
\pm 305\end{array}$ & $\begin{array}{c}548,954 \\
\pm 319\end{array}$ & $\begin{array}{c}537,387 \\
\pm 173\end{array}$ & $\begin{array}{c}-11,567 \\
\pm 363\end{array}$ & $\begin{array}{c}552,157 \\
\pm 329\end{array}$ & $\begin{array}{l}3,203 \\
\pm 458\end{array}$ & $\begin{array}{c}134,140 \\
\pm 57\end{array}$ & $\begin{array}{c}131,314 \\
\pm 35\end{array}$ & $\begin{array}{c}-2,826 \\
\pm 67\end{array}$ & $\begin{array}{c}134,923 \\
\pm 59\end{array}$ & $\begin{array}{r}783 \\
\pm 82 \\
\end{array}$ \\
\hline
\end{tabular}


Of the assumptions tested in the sensitivity analysis, the high energy efficiency measures, as used in scenario C5, had the single greatest effect of reducing GHG emissions. Increasing the energy efficiency of EVs resulted in reductions in the cumulative GHG emissions of $2.5 \%$ to $5.2 \%$ depending on the VFM scenario (Table 9.11).

Of the measures available to the New Zealand Government to promote EVs and reduce GHG emissions, the analysis indicated that promoting widespread availability of EV charging facilities and the provision of a subsidy on the purchase of an EV had a similar effect on the GHG emissions produced by the LPV fleet with the cumulative GHG emissions over the modelling period being reduced by $1.4 \%$ to 1.9\%. Removing the current exemption of EVs from RUC had the smallest impact on GHG emissions, resulting in an increase in cumulative GHG emissions of just below 1.1 million tonnes (Table 9.11).

The sensitivity analysis that assessed the impact on electricity demand of owners of PHEVs undertaking a supplementary top-up recharging event between main charging events indicated that annual demand could vary by as much as $8.4 \%$. For scenario $\mathrm{C} 4$, by 2030 , this variation was equivalent to $300 \pm 30 \mathrm{GWh}$ and a variation in the cumulative demand of $2,900 \pm 80 \mathrm{GWh}$ over the modelling period. The impact of the different recharging behaviour on cumulative GHG emissions was estimated at $1.4 \pm 0.1$ million tonnes $\mathrm{CO}_{2} \mathrm{e}$ (1.1\% difference) (Table 9.11).

Changing the assumptions of the future growth rate of electricity prices was found to have no statistically significant impact on the output of the VFM (Table 9.12). 
Table 9.11: Sensitivity analysis - effect of changed assumptions on cumulative electricity demand, and GHG emissions, thousand tonnes $\mathrm{CO}_{2} \mathrm{e}: \mathbf{2 0 1 0}$ to 2030

\begin{tabular}{|c|c|c|c|c|c|c|}
\hline & \multicolumn{3}{|c|}{$\begin{array}{l}\text { Cumulative electricity demand } 2010 \text { to } \\
2030 \\
\text { GWh }\end{array}$} & \multicolumn{3}{|c|}{$\begin{array}{l}\text { Cumulative GHG emissions } \\
2010 \text { to } 2030 \\
\text { (thousand) tonnes } \mathrm{CO}_{2} \mathrm{e} \\
\end{array}$} \\
\hline \multicolumn{7}{|c|}{ EV energy efficiency } \\
\hline & $\begin{array}{l}\text { Standard } \\
\text { energy } \\
\text { efficiency }\end{array}$ & $\begin{array}{l}\text { High } \\
\text { efficiency }\end{array}$ & $\%$ diff & $\begin{array}{l}\text { Standard } \\
\text { energy } \\
\text { efficiency }\end{array}$ & $\begin{array}{c}\text { High } \\
\text { efficiency }\end{array}$ & $\%$ diff \\
\hline A2 & $\begin{array}{c}23,625 \\
\pm 141 \\
\end{array}$ & $\begin{array}{c}21,325 \\
\pm 141 \\
\end{array}$ & $-9.7 \%$ & $\begin{array}{c}142,661 \\
\pm 41 \\
\end{array}$ & $\begin{array}{c}139,084 \\
\pm 61 \\
\end{array}$ & $-2.5 \%$ \\
\hline B3 & $\begin{array}{c}31,442 \\
\pm 246 \\
\end{array}$ & $\begin{array}{c}30,070 \\
\pm 139 \\
\end{array}$ & $-4.4 \%$ & $\begin{array}{c}121,836 \\
\pm 68 \\
\end{array}$ & $\begin{array}{c}115,533 \\
\pm 39 \\
\end{array}$ & $-5.2 \%$ \\
\hline C4 & $\begin{array}{c}31,484 \\
\pm 227 \\
\end{array}$ & $\begin{array}{c}29,275 \\
\pm 177 \\
\end{array}$ & $-7.0 \%$ & $\begin{array}{c}134,140 \\
\pm 57 \\
\end{array}$ & $\begin{array}{c}130,205 \\
\pm 11 \\
\end{array}$ & $-2.9 \%$ \\
\hline \multicolumn{7}{|c|}{ Availability EV street recharging } \\
\hline & $\begin{array}{l}\text { No street } \\
\text { recharging }\end{array}$ & $\begin{array}{l}\text { Street } \\
\text { recharging } \\
\text { available } \\
\end{array}$ & diff\% & $\begin{array}{l}\text { No street } \\
\text { recharging }\end{array}$ & $\begin{array}{l}\text { Street } \\
\text { recharging } \\
\text { available }\end{array}$ & $\%$ diff \\
\hline A2 & $\begin{array}{c}23,625 \\
\pm 141 \\
\end{array}$ & $\begin{array}{c}25,565 \\
\pm 137 \\
\end{array}$ & $8.2 \%$ & $\begin{array}{c}142,661 \\
\pm 41 \\
\end{array}$ & $\begin{array}{c}140,438 \\
\pm 45 \\
\end{array}$ & $-1.6 \%$ \\
\hline B3 & $\begin{array}{c}31,442 \\
\pm 246\end{array}$ & $\begin{array}{c}33,915 \\
\pm 219\end{array}$ & $7.9 \%$ & $\begin{array}{c}121,836 \\
\pm 68\end{array}$ & $\begin{array}{c}119,566 \\
\pm 56\end{array}$ & $-1.9 \%$ \\
\hline C4 & $\begin{array}{c}28,946 \\
\pm 227 \\
\end{array}$ & $\begin{array}{c}31,484 \\
\pm 213 \\
\end{array}$ & $8.8 \%$ & $\begin{array}{c}136,621 \\
\pm 57 \\
\end{array}$ & $\begin{array}{c}134,140 \\
\pm 57 \\
\end{array}$ & $-1.8 \%$ \\
\hline \multicolumn{7}{|c|}{ Subsidy on the purchase price on EVs } \\
\hline & $\begin{array}{c}\text { No } \\
\text { subsidy }\end{array}$ & Subsidy & diff\% & No subsidy & Subsidy & $\%$ diff \\
\hline $\mathbf{A 2}$ & $\begin{array}{c}23,625 \\
\pm 141 \\
\end{array}$ & $\begin{array}{c}25,283 \\
\pm 170 \\
\end{array}$ & $7.0 \%$ & $\begin{array}{c}142,661 \\
\pm 41 \\
\end{array}$ & $\begin{array}{c}140,730 \\
\pm 58 \\
\end{array}$ & $-1.4 \%$ \\
\hline B3 & $\begin{array}{c}31,442 \\
\pm 246 \\
\end{array}$ & $\begin{array}{c}33,789 \\
\pm 171 \\
\end{array}$ & $7.5 \%$ & $\begin{array}{c}121,836 \\
\pm 68\end{array}$ & $\begin{array}{c}119,681 \\
\pm 50\end{array}$ & $-1.8 \%$ \\
\hline $\mathrm{C} 4$ & $\begin{array}{c}31,484 \\
\pm 227 \\
\end{array}$ & $\begin{array}{c}33,730 \\
\pm 218 \\
\end{array}$ & $7.1 \%$ & $\begin{array}{c}134,140 \\
\pm 57 \\
\end{array}$ & $\begin{array}{c}131,930 \\
\pm 20 \\
\end{array}$ & $-1.6 \%$ \\
\hline \multicolumn{7}{|c|}{ Imposition of Road User Charges } \\
\hline & No RUC & RUC & $\%$ diff & No RUC & RUC & $\%$ diff \\
\hline A2 & $\begin{array}{c}23,625 \\
\pm 141\end{array}$ & $\begin{array}{c}22,716 \\
\pm 206\end{array}$ & $-3.8 \%$ & $\begin{array}{c}142,661 \\
\pm 41\end{array}$ & $\begin{array}{c}143,592 \\
\pm 58\end{array}$ & $0.7 \%$ \\
\hline B3 & $\begin{array}{c}31,442 \\
\pm 246 \\
\end{array}$ & $\begin{array}{c}30,674 \\
\pm 238 \\
\end{array}$ & $-2.4 \%$ & $\begin{array}{c}121,836 \\
\pm 68\end{array}$ & $\begin{array}{c}122,565 \\
\pm 68\end{array}$ & $0.6 \%$ \\
\hline $\mathrm{C} 4$ & $\begin{array}{c}31,484 \\
\pm 227 \\
\end{array}$ & $\begin{array}{c}30,882 \\
\pm 204 \\
\end{array}$ & $-1.9 \%$ & $\begin{array}{c}134,140 \\
\pm 57\end{array}$ & $\begin{array}{c}134,765 \\
\pm 20\end{array}$ & $0.5 \%$ \\
\hline \multicolumn{7}{|c|}{ PHEV recharging } \\
\hline & & High & & & High & \\
\hline & ( $0 \%$ of PHEV) & $\begin{array}{c}\text { (100\% of } \\
\text { PHEVs fleet) }\end{array}$ & $\%$ diff & (0\% of PHEV) & $\begin{array}{c}(100 \% \text { of } \\
\text { PHEVs fleet })\end{array}$ & $\%$ diff \\
\hline C4 & $\begin{array}{c}30,853 \\
\pm 159 \\
\end{array}$ & $\begin{array}{c}33,759 \\
\pm 190 \\
\end{array}$ & $8.6 \%$ & $\begin{array}{c}134,310 \\
\pm 48 \\
\end{array}$ & $\begin{array}{c}132,911 \\
\pm 55\end{array}$ & $-1.1 \%$ \\
\hline
\end{tabular}

$95 \%$ confidence intervals 
Table 9.12: Sensitivity analysis - effect of changed assumptions of the rate of increase in electricity prices on cumulative electricity demand and GHG emissions: 2010 to 2030

\begin{tabular}{|c|c|c|c|c|c|c|c|c|c|c|}
\hline \multicolumn{11}{|c|}{ Electricity price } \\
\hline & $\begin{array}{l}\text { Cumula- } \\
\text { tive } \\
\text { electricity } \\
\text { demand } \\
2010 \text { to } \\
2030 \\
\text { GWh }\end{array}$ & \begin{tabular}{|} 
Cumula- \\
tive \\
electricity \\
demand \\
2010 to \\
2030 \\
GWh
\end{tabular} & $\begin{array}{c}\text { Diff } \\
\%\end{array}$ & $\mid \begin{array}{c}\text { Cumula- } \\
\text { tive } \\
\text { electricity } \\
\text { demand } \\
2010 \text { to } \\
2030 \\
\text { GWh }\end{array}$ & $\begin{array}{c}\text { Diff } \\
\%\end{array}$ & \begin{tabular}{|c|} 
Cumula- \\
tive GHG \\
emissions \\
2010 to \\
2030 \\
(thousand) \\
Tonnes \\
$\mathrm{CO}_{2} \mathrm{e}$ \\
\end{tabular} & $\begin{array}{l}\text { Cumula- } \\
\text { tive GHG } \\
\text { emissions } \\
2010 \text { to } \\
2030 \\
\text { (thousand) } \\
\text { Tonnes } \\
\mathrm{CO}_{2} \mathrm{e}\end{array}$ & $\begin{array}{c}\text { Diff } \\
\%\end{array}$ & \begin{tabular}{||c} 
Cumula- \\
tive GHG \\
emissions \\
2010 to \\
2030 \\
(thousand) \\
Tonnes \\
$\mathrm{CO}_{2} \mathrm{e}$
\end{tabular} & $\begin{array}{c}\text { Diff } \\
\%\end{array}$ \\
\hline & Default & Low & & High & & Default & Low & & High & \\
\hline A2 & $\begin{array}{c}23,625 \\
\pm 141 \\
\end{array}$ & $\begin{array}{c}23,720 \\
\pm 169 \\
\end{array}$ & $0.4 \% *$ & $\begin{array}{c}23,346 \\
\pm 196 \\
\end{array}$ & $-1.2 \% *$ & $\begin{array}{c}142,661 \\
\pm 41 \\
\end{array}$ & $\begin{array}{c}142,526 \\
\pm 54\end{array}$ & $-0.1 \% *$ & $\begin{array}{c}142,762 \\
\pm 75 \\
\end{array}$ & $0.1 \% *$ \\
\hline B3 & $\begin{array}{c}31,442 \\
\pm 246\end{array}$ & $\begin{array}{c}31,539 \\
\pm 217 \\
\end{array}$ & $0.3 \% *$ & $\begin{array}{c}31,403 \\
\pm 135 \\
\end{array}$ & $-0.1 \% *$ & $\begin{array}{c}121,836 \\
\pm 68 \\
\end{array}$ & $\begin{array}{c}121,747 \\
\pm 52 \\
\end{array}$ & $-0.1 \% *$ & $\begin{array}{c}121,711 \\
\pm 21\end{array}$ & $-0.1 \% *$ \\
\hline C4 & $\begin{array}{c}31,484 \\
\pm 227 \\
\end{array}$ & $\begin{array}{c}31,678 \\
\pm 200 \\
\end{array}$ & $0.6 \% *$ & $\begin{array}{c}31,297 \\
\pm 183\end{array}$ & $-0.6 \% *$ & $\begin{array}{c}134,140 \\
\pm 57\end{array}$ & $\begin{array}{c}134,120 \\
\pm 50\end{array}$ & $0.0 \% *$ & $\begin{array}{c}134,126 \\
\pm 54\end{array}$ & $0.0 \% *$ \\
\hline
\end{tabular}

$\pm \quad 95 \%$ confidence intervals

* Difference not significant at $95 \%$ level of confidence

\subsection{Impact of EVs on the electricity sector}

This section presents the results of the analysis, using the GEM, of the effect of the additional electricity demand from EVs on the GHG emissions from the electricity sector in the context of the EC's MDS1, MDS3, and MDS5 generation scenarios. To provide an estimate of the GHG emissions from the electricity sector in a policy environment that strongly promotes the reduction of GHG emissions the GEM was run using the electricity demand from scenario $\mathrm{C} 5$ in conjunction with the MDS1 scenario $^{68}$.

\subsubsection{Projected electricity demand and generation}

Table 9.13 and Figure 9.8 present the electricity demand projections from the A1 (no-EV), A2, C4, and C5 VFM scenarios.

\footnotetext{
${ }^{68}$ See sections 5.4 .4 and 6.5 .5 for the reasons for choosing these scenarios.
} 
Table 9.13: Vehicle fleet model scenarios forecast annual electricity demand in 2030, and cumulative demand (GWh): 2010 to 2030

\begin{tabular}{|c|c||c|c||c|c||c|c|}
\hline & A1 & $\begin{array}{c}\text { A2 } \\
\$ 25 \text { per } \\
\text { tonne }\end{array}$ & $\begin{array}{c}\text { Diff } \\
\text { from } \\
\text { BAU }\end{array}$ & $\begin{array}{c}\text { C4 } \\
\$ 100 \text { per } \\
\text { tonne }\end{array}$ & $\begin{array}{c}\text { Diff } \\
\text { from } \\
\text { BAU }\end{array}$ & $\begin{array}{c}\text { C5 } \\
\$ 100 \text { per } \\
\text { tonne }\end{array}$ & $\begin{array}{c}\text { Diff } \\
\text { from } \\
\text { BAU }\end{array}$ \\
\hline $\begin{array}{c}\text { Annual demand } \\
2010\end{array}$ & \multicolumn{6}{|c||}{43,141} \\
\hline $\begin{array}{c}\text { Annual demand } \\
2030\end{array}$ & 60,316 & 63,025 & 2,709 & 63,923 & 3,607 & 63,216 & 2,900 \\
\hline $\begin{array}{c}\text { Cumulative demand } \\
\text { 2010 to 2030 }\end{array}$ & $1,076,975$ & $1,100,642$ & 23,667 & $1,108,506$ & 31,531 & $1,103,679$ & 26,704 \\
\hline
\end{tabular}

Figure 9.8: Projected total electricity demand including demand from EVs

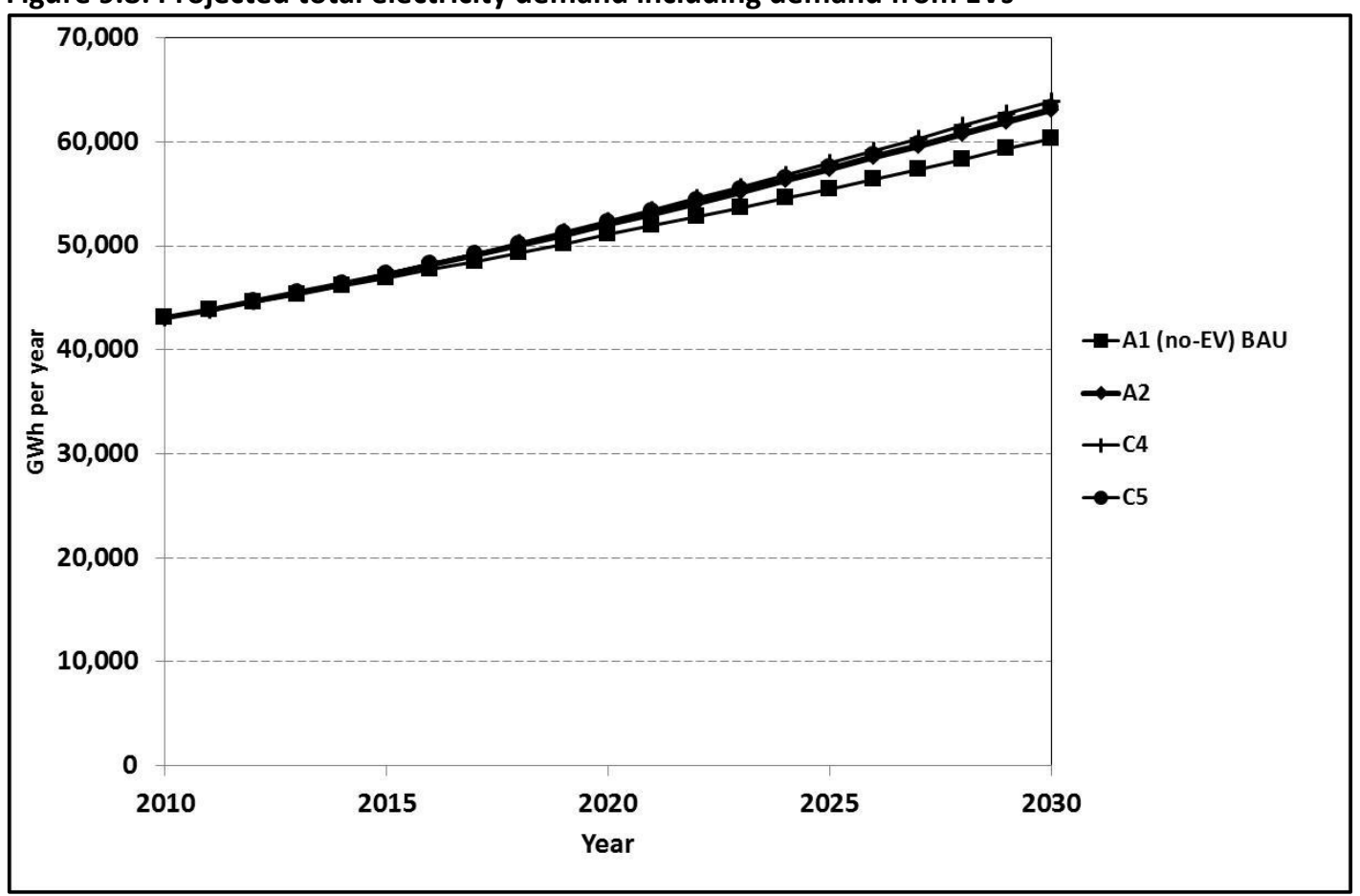

For this study, the distribution of the EV load was based on the current distribution of the New Zealand population, with $76 \%$ of the load occurring in the North Island, and $24 \%$ in the South Island. Each of the three generation scenarios produced its own configuration of generation plant in terms of location and resulting transmission losses. For each generation scenario, a given level of forecast electricity demand resulted in the dispatch of a different mix of electricity generation and DSM. The MDS1 scenario made greater use of the renewable resources in the South Island and had the highest transmission losses. The model runs based on the MDS1 scenario resulted in the dispatch of more electricity than in the MDS3 and MDS5 generation scenarios as these scenarios made more use of 
fossil-fuelled plants located closer to the major load centres in the North Island (Table 9.14).

Table 9.14: Forecast and dispatched electricity demand for MDS1, MDS3 and MDS5 scenarios annual 2030 and cumulative demand (GWh): 2010 to 2030

\begin{tabular}{|c|c|c|c|c|c|c|}
\hline & \multicolumn{3}{|c|}{ Annual electricity demand in 2030} & \multicolumn{3}{|c|}{ Cumulative demand 2010 to 2030} \\
\hline & Forecast & $\begin{array}{l}\text { Electricity } \\
\text { dispatched }\end{array}$ & $\begin{array}{l}\text { Difference } \\
\text { from } \\
\text { forecast }\end{array}$ & Forecast & $\begin{array}{l}\text { Electricity } \\
\text { dispatched }\end{array}$ & $\begin{array}{l}\text { Difference } \\
\text { from } \\
\text { forecast }\end{array}$ \\
\hline \multicolumn{7}{|c|}{ MDS1 (High use of renewables, restriction on coal and lignite fuels) } \\
\hline $\begin{array}{c}\text { A1 (no EV) } \\
\$ 25 \text { per tonne }\end{array}$ & 60,316 & 63,444 & 3,128 & $1,076,975$ & $1,125,421$ & 48,446 \\
\hline $\begin{array}{c}\text { A2 } \\
\$ 25 \text { per tonne }\end{array}$ & 63,025 & 66,267 & 3,242 & $1,100,642$ & $1,150,029$ & 49,387 \\
\hline $\begin{array}{l}\text { C4 } \\
\$ 100 \text { per } \\
\text { tonne }\end{array}$ & 63,923 & 67,102 & 3,179 & $1,108,506$ & $1,158,130$ & 49,624 \\
\hline $\begin{array}{c}\text { C5 } \\
\$ 100 \text { per } \\
\text { tonne }\end{array}$ & 63,216 & 66,433 & 3,217 & $1,103,677$ & $1,153,334$ & 49,657 \\
\hline \multicolumn{7}{|c|}{ MDS3 (Moderate access to hydro sites no restriction on the use of coal) } \\
\hline $\begin{array}{c}\text { A1 (no EV) } \\
\$ 25 \text { per tonne }\end{array}$ & 60,316 & 63,198 & 2,882 & $1,076,979$ & $1,124,331$ & 47,352 \\
\hline $\begin{array}{c}\text { A2 } \\
\$ 25 \text { per tonne }\end{array}$ & 63,025 & 65,957 & 2,932 & $1,100,642$ & $1,148,529$ & 47,887 \\
\hline $\begin{array}{c}\text { C4 } \\
\$ 100 \text { per } \\
\text { tonne }\end{array}$ & 63,923 & 66,860 & 2,937 & $1,108,508$ & $1,157,001$ & 48,493 \\
\hline \multicolumn{7}{|c|}{ MDS5 (High gas discovery) } \\
\hline $\begin{array}{c}\text { A1 (no EV) } \\
\$ 25 \text { per tonne }\end{array}$ & 60,316 & 62,893 & 2,577 & $1,076,979$ & $1,123,535$ & 46,556 \\
\hline $\begin{array}{c}\text { A2 } \\
\$ 25 \text { per tonne }\end{array}$ & 63,025 & 65,710 & 2,685 & $1,100,642$ & $1,148,200$ & 47,558 \\
\hline $\begin{array}{c}\text { C4 } \\
\$ 100 \text { per } \\
\text { tonne }\end{array}$ & 63,923 & 66,639 & 2,716 & $1,108,508$ & $1,156,569$ & 48,061 \\
\hline
\end{tabular}

The effect of the introduction of EVs was to increase the annual amount of electricty required to be generated in 2030 , depending on the generation scenario, by between $4.5 \%$ and $5.9 \%$. 


\subsubsection{Generation plant build, retirement, dispatch, and GHG emissions}

High use of renewables, restriction on coal and lignite fuels (MDS1)

\section{Low carbon price, scenario A2 EV electricity demand}

This model run assumed a low carbon price signal and a New Zealand Government ban on the building of new coal and lignite plants, but there was greater access to the use of hydro and wind sites and use of price-responsive load curtailment and interruptible load measures. This generation scenario assumed that two of the four $250 \mathrm{MW}$ coal fired units at Huntly, the $122 \mathrm{MW}$ Southdown combined cycle gas turbine (CCGT) plant, and the 380 MW Taranaki Combined Cycle gas plant would be retired before 2030 .

Without the additional electricity demand from EVs, this generation scenario projected that 9,342 MW of new generation and demand side measures (DSM) would be commissioned over the period 2010 to 2030 . At the same time, 1,770 MW of the existing generation capacity would be retired, which would result in a net increase in generation capacity of 7,572 MW.

When the additional load from EVs was included, the generation scenario resulted in the building of 9,196 MW of new generation plant and DSM, and the retirement of 1,440 MW existing capacity (Table 9.15). Less new generation was built in this model run when compared to the $\mathrm{A} 1$ (no-EV) model run, because the Otahuhu B 380 MW CCGT plant was not retired in 2024. The introduction of EVs resulted in a net increase in generation capacity of 7,756 MW. By 2030, this model run resulted in an additional $184 \mathrm{MW}$ of installed generation and DSM capacity when compared to the A1 (no-EV) model run (Figure 9.9).

For both the $A 1$ (no-EV) and A2 model runs using the MDS1 scenario, the building schedules resulted in the installation of large quantities of diesel and gas fired faststart peaking capacity, because of wind generation's limited peak generation capability.

In the $\mathrm{A} 1$ (no-EV) model run, the amount of renewable electricity, as a proportion of all electricity generated, increased from $71 \%$ in 2010 to $89.8 \%$ in 2027 , and then 
declined to $88 \%$ in 2030 . The increased electricity demand from EVs had no impact on the proportion of renewable electricity compared to the no EVs model run until after 2024. After this time, due to the continued use of the Otahuhu B plant the proportion of renewable electricity generated declined, by 2027 , by $3 \%$ relative to the no EVs model run (Figure 9.10).

In both the $\mathrm{A} 1$ (no-EV) run, and the $\mathrm{A} 2$ model run, annual GHG emissions increased from 7.4 million tonnes $\mathrm{CO}_{2} \mathrm{e}$, in 2010, to a peak, in 2017, of 9.0 million tonnes $\mathrm{CO}_{2} \mathrm{e}$ for the $\mathrm{A} 1$ (no-EV) and 9.4 million tonnes $\mathrm{CO}_{2} \mathrm{e}$ for the $\mathrm{A} 2$ model run. GHG emissions then declined in both model runs due to the retirement of a $250 \mathrm{MW}$ coal-fired unit at Huntly in 2017, the 122 MW Southdown CCGT plant in 2019, a second coal-fired 250 MW unit at Huntly in 2020, and in 2024 the Taranaki Combined Cycle $380 \mathrm{MW}$ plant. In the A1 (no-EV) model run, this caused GHG emissions from electricity generation to decline to 4.8 million tonnes $\mathrm{CO}_{2}$ e by 2027 . After 2027, GHG emissions increased slightly to reach 5.6 million tonnes $\mathrm{CO}_{2} \mathrm{e}$ by 2030 (Figure 9.11).

In the A2 model run, the annual GHG emissions after 2024 increased above those in the $A 1$ (no-EV) model run due to one of the two remaining coal units at Huntly operating more often. In 2025, due to the non-retirement of the $380 \mathrm{MW}$ Otahuhu B CCGT plant, annual GHG emissions then increased to 6.4 million tonnes of $\mathrm{CO}_{2} \mathrm{e}$ by 2030 (Figure 9.11).

\section{High carbon price, scenario C4 EV electricity demand}

The C4 model run resulted in only one CCGT plant being retired, the $122 \mathrm{MW}$ plant at Southdown in 2019. The Taranaki Combined Cycle plant would be retained, displacing some of the specialised Open Cycle Gas Turbine (OCGT) peaking plant capacity that was built in the no EVs model run (Table 9.15).

The effect of the higher carbon price in this model run did not change the retirement schedule of the two $250 \mathrm{MW}$ coal fired units at Huntly, but all the coal units were dispatched less often. To offset the reduced use of the Huntly coal units, the construction dates of some renewable plants were brought forward. The largest of these projects were: 
- $1000 \mathrm{MW}$ of wind projects in the Waikato region, brought forward from 2023 to 2018.

- $400 \mathrm{MW}$ of wind projects in the Otago region, brought forward from 2021 to 2019.

- A $280 \mathrm{MW}$ hydro project in the Otago region, brought forward from 2019 to 2016.

- A 100 MW Clutha project in the Otago region, brought forward from 2019 to 2014.

- A $180 \mathrm{MW}$ geothermal project, in the Waikato region, brought forward from 2015 to 2013.

The effect of refurbishing the Taranaki Combined Cycle plant was that less new capacity needed to be built, and, by the end of the modelling period, total installed capacity was only $85 \mathrm{MW}$ greater than in the no EVs model run and $99 \mathrm{MW}$ less than in the A2 model run (Table 9.15 and Figure 9.9).

The reduced use of the Huntly plants and the early commissioning of the renewable plants had the effect of increasing the proportion of renewable electricity above that of the no EV and $A 2$ model EV runs until 2023. After this time, the retention of both the Taranaki Combined Cycle plant and the Otahuhu B plant resulted in a decline in the proportion of renewable generation to $83.1 \%$ by 2030 (Figure 9.10).

The decreased use of Huntly and early commissioning of renewables plants resulted in a decline in annual GHG emissions from 7.4 million tonnes $\mathrm{CO}_{2}$ e to 5.2 million tonnes $\mathrm{CO}_{2} \mathrm{e}$ by 2020. After this time, the on-going use of the two non-retired CCGT plants caused GHG emissions to increase after 2020 and, by 2030, annual GHG emissions would reach 6.7 million tonnes $\mathrm{CO}_{2}$ e (Figure 9.11).

\section{High carbon price, scenario C5 EV electricity demand}

Even with the higher uptake of EVs in the C5 model run, the higher energy efficiency standards resulted in a similar cumulative electricity demand over the modelling period as the A2 model run with only an additional 3,305 GWh more electricity being dispatched over the modelling period. The A2 and C5 model runs resulted in the same plants being retired, but with different timings. In the $\mathrm{C} 5$ 
model run, the retirement of the gas plants was delayed by up to six years, while the retirement of the Wairakei and Ngawha geothermal plants occurred between 10 and 16 years earlier. The key differences between $\mathrm{C} 5$ and $\mathrm{A} 2$ model runs was that, in the C5 model run, $10 \mathrm{MW}$ less run-of-river hydro in the South Island, $46 \mathrm{MW}$ less DSM in the North Island, and $48 \mathrm{MW}$ more diesel plant in the North Island were commissioned and the renewable generation plant and DSM were commissioned earlier.

The higher carbon price in the $\mathrm{C} 4$ and $\mathrm{C} 5$ model runs resulted in similar renewable electricity profiles until 2022. After this time in the scenario C5 model run, the proportion of renewable electricity was slightly higher than in the C4 model run, peaking at $87.5 \%$ in 2026 and then declining to $85.6 \%$ in 2030 (Figure 9.10).

The GHG emission profile in the C5 model run closely followed the profile in the C4 model run, decreasing from 7.4 million tonnes in 2010 to 5.2 million tonne $\mathrm{CO}_{2} \mathrm{e}$ in 2020. In the C5 model run, but not in the C4 model run, the Taranaki Combined Cycle plant was retired in 2023, and this accounts for the difference in GHG emissions profiles of the two model runs until 2030. By 2030, annual GHG emissions in the $\mathrm{C} 5$ model run would increase to 6.4 million tonnes $\mathrm{CO}_{2} \mathrm{e}$. 
Table 9.15: MDS1: Retirements and new capacity installed (MW)

\begin{tabular}{|c|c|c|c|c|c|c|c|c|c|c|c|c|}
\hline \multirow{4}{*}{ Technology } & \multicolumn{12}{|c|}{ Retirements } \\
\hline & \multicolumn{12}{|c|}{ VFM scenario } \\
\hline & \multicolumn{3}{|c|}{$\begin{array}{l}\text { A1 (no-EV) } \\
\$ 25 \text { per tonne }\end{array}$} & \multicolumn{3}{|c|}{$\begin{array}{c}\text { A2 } \\
\text { EVs } \$ 25 \text { per tonne }\end{array}$} & \multicolumn{3}{|c|}{$\begin{array}{c}\text { C4 } \\
\text { EVs } \$ 100 \text { per tonne }\end{array}$} & \multicolumn{3}{|c|}{$\begin{array}{c}\text { C5 } \\
\text { EVs } \$ 100 \text { per tonne }\end{array}$} \\
\hline & North Island & South Island & Total & North Island & South Island & Total & North Island & South Island & Total & North Isle & Ith Island & Total \\
\hline Coal & 500 & 0 & 500 & 500 & 0 & 500 & 500 & 0 & 500 & 500 & 0 & 500 \\
\hline Combined cycle gas turbine & 882 & 0 & 882 & 502 & 0 & 502 & 122 & 0 & 122 & 502 & 0 & 502 \\
\hline Geothermal & 188 & 0 & 188 & 188 & 0 & 188 & 188 & 0 & 188 & 188 & 0 & 188 \\
\hline Open cycle gas turbine - gas & 45 & 0 & 45 & 95 & 0 & 95 & 95 & 0 & 95 & 95 & 0 & 95 \\
\hline Peaker, diesel-fired OCGT & 155 & 0 & 155 & 155 & 0 & 155 & 155 & 0 & 155 & 155 & 0 & 155 \\
\hline Total & 1,770 & 0 & 1,770 & 1,440 & & 1,440 & 1,060 & 0 & 1,060 & 1,440 & 0 & 1,440 \\
\hline
\end{tabular}


VFM scenario

\begin{tabular}{|c|c|c|c|c|c|c|c|c|c|c|c|c|}
\hline & \multicolumn{12}{|c|}{ Build } \\
\hline & \multicolumn{12}{|c|}{ VFM scenario } \\
\hline & \multicolumn{3}{|c|}{$\begin{array}{c}\text { A1 (no-EV) } \\
\$ 25 \text { per tonne }\end{array}$} & \multicolumn{3}{|c|}{$\begin{array}{c}\text { A2 } \\
\text { EVs } \$ 25 \text { per tonne }\end{array}$} & \multicolumn{3}{|c|}{$\begin{array}{c}\text { C4 } \\
\text { EVs } \$ 100 \text { per tonne }\end{array}$} & \multicolumn{3}{|c|}{$\begin{array}{c}\text { C5 } \\
\text { EVs } \$ 100 \text { per tonne }\end{array}$} \\
\hline Technology & North Island & $\begin{array}{l}\text { South } \\
\text { Island }\end{array}$ & Total & $\begin{array}{l}\text { North } \\
\text { Island }\end{array}$ & $\begin{array}{l}\text { South } \\
\text { Island }\end{array}$ & Total & $\begin{array}{l}\text { North } \\
\text { Island }\end{array}$ & $\begin{array}{l}\text { South } \\
\text { Island }\end{array}$ & Total & $\begin{array}{l}\text { North } \\
\text { Island }\end{array}$ & $\begin{array}{l}\text { South } \\
\text { Island }\end{array}$ & Total \\
\hline Coal & 0 & 0 & 0 & 0 & 0 & 0 & 0 & 0 & 0 & 0 & 0 & 0 \\
\hline Cogeneration, biomass-fired & 157 & 0 & $157^{*}$ & 157 & 0 & $157^{*}$ & 157 & 0 & $157^{*}$ & 157 & 0 & $157^{*}$ \\
\hline Cogeneration, gas-fired & 135 & 0 & $135^{*}$ & 85 & 0 & 85 & 85 & 0 & 85 & 85 & 0 & 85 \\
\hline Cogeneration, other & 80 & 0 & $80 *$ & 80 & 0 & $80 *$ & 80 & 0 & $80 *$ & 80 & 0 & $80 *$ \\
\hline Combined cycle gas turbine & 0 & 0 & 0 & 0 & 0 & 0 & 0 & 0 & 0 & 0 & 0 & 0 \\
\hline Geothermal & 1,065 & 0 & $1,065^{*}$ & 1,065 & 0 & $1,065^{*}$ & 1065 & 0 & $1065 *$ & 1065 & 0 & $1065^{*}$ \\
\hline Hydro, peaking & 150 & 1,098 & 1,248 & 150 & 1,098 & 1,248 & 150 & 1098 & 1248 & 150 & 1098 & 1248 \\
\hline Hydro, pumped storage & 300 & 0 & 300 & 300 & 0 & 300 & 300 & 0 & 300 & 300 & 0 & 300 \\
\hline Hydro, run-of-river & 323 & 545 & 867 & 323 & 633 & 956 & 323 & 590 & 913 & 323 & 623 & 946 \\
\hline Interruptible load & 100 & 0 & 100 & 100 & 0 & 100 & 100 & 0 & 100 & 100 & 0 & 100 \\
\hline Open cycle gas turbine - gas & 0 & 0 & 0 & 0 & 0 & 0 & 0 & 0 & 0 & 0 & 0 & 0 \\
\hline Peaker, diesel-fired OCGT & 1,330 & 0 & 1,330 & 1,180 & 0 & 1,180 & 820 & 0 & 820 & 1180 & 0 & 1180 \\
\hline Peaker, fast-start gas-fired & 610 & 0 & $610^{*}$ & 610 & 0 & $610 *$ & 610 & 0 & $610^{*}$ & 610 & 0 & $610 *$ \\
\hline $\begin{array}{l}\text { Price-responsive load } \\
\text { curtailment }\end{array}$ & 650 & 0 & 650 & 612 & 0 & 612 & 650 & 0 & 650 & 566 & 0 & 566 \\
\hline Reciprocating engine diesel & 146 & 0 & 146 & 38 & 0 & 38 & 23 & 0 & 23 & 86 & 0 & 86 \\
\hline Solar & 0 & 50 & 50 & 0 & 50 & 50 & 0 & 50 & 50 & 0 & 50 & 50 \\
\hline Wave & 38 & 76 & 114 & 38 & 76 & 114 & 38 & 76 & 114 & 38 & 76 & 114 \\
\hline Wind & 1932 & 558 & 2490 & 1932 & 670 & 2602 & 1932 & 570 & 2502 & 1932 & 665 & 2597 \\
\hline Total & 7016 & 2327 & 9342 & 6669 & 2527 & 9196 & 6,333 & 2,384 & 8,717 & 6,672 & 2,512 & 9,183 \\
\hline
\end{tabular}

GEM utilises $100 \%$ of the available generation resources 
Figure 9.9: MDS1 gross and net installed generation and demand side management capacity: 2010 to 2030

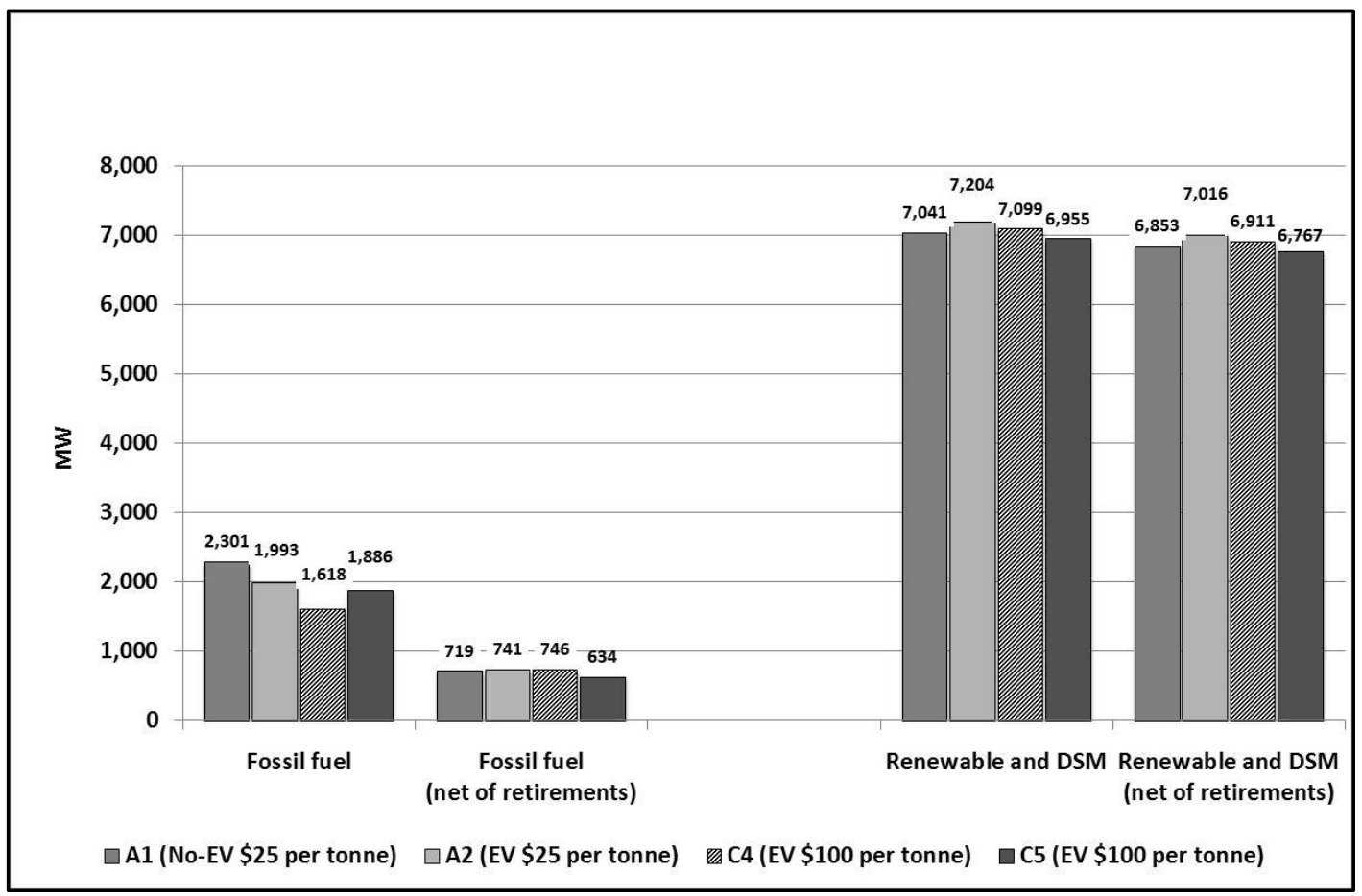

Figure 9.10: Proportion of renewable generation, MDS1 - A1 (no-EV), A2, C4, and C5 electricity demand

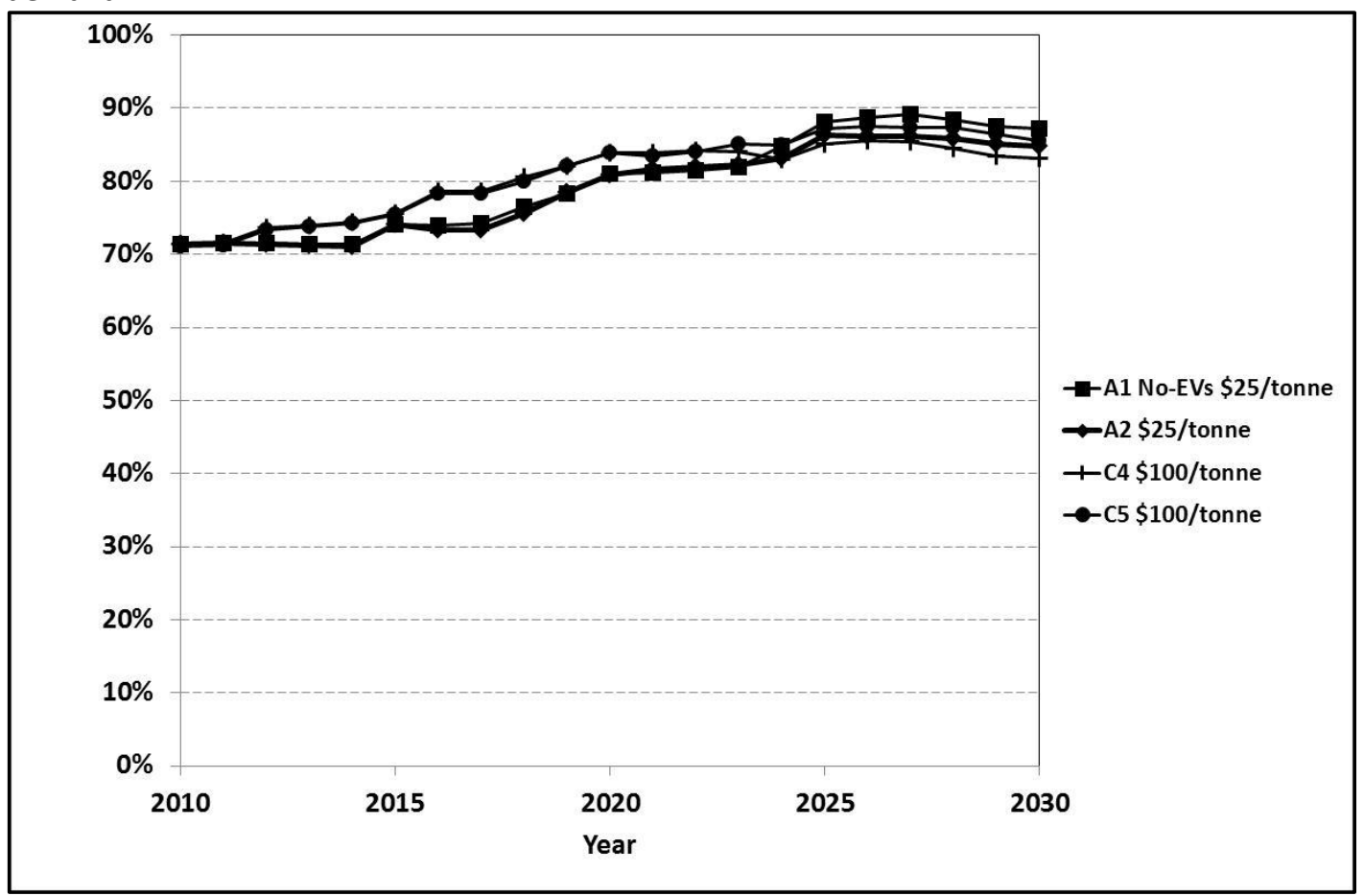


Figure 9.11: Annual GHG emissions, MDS1 - A1 (no-EV), A2, C4, and C5 electricity demand

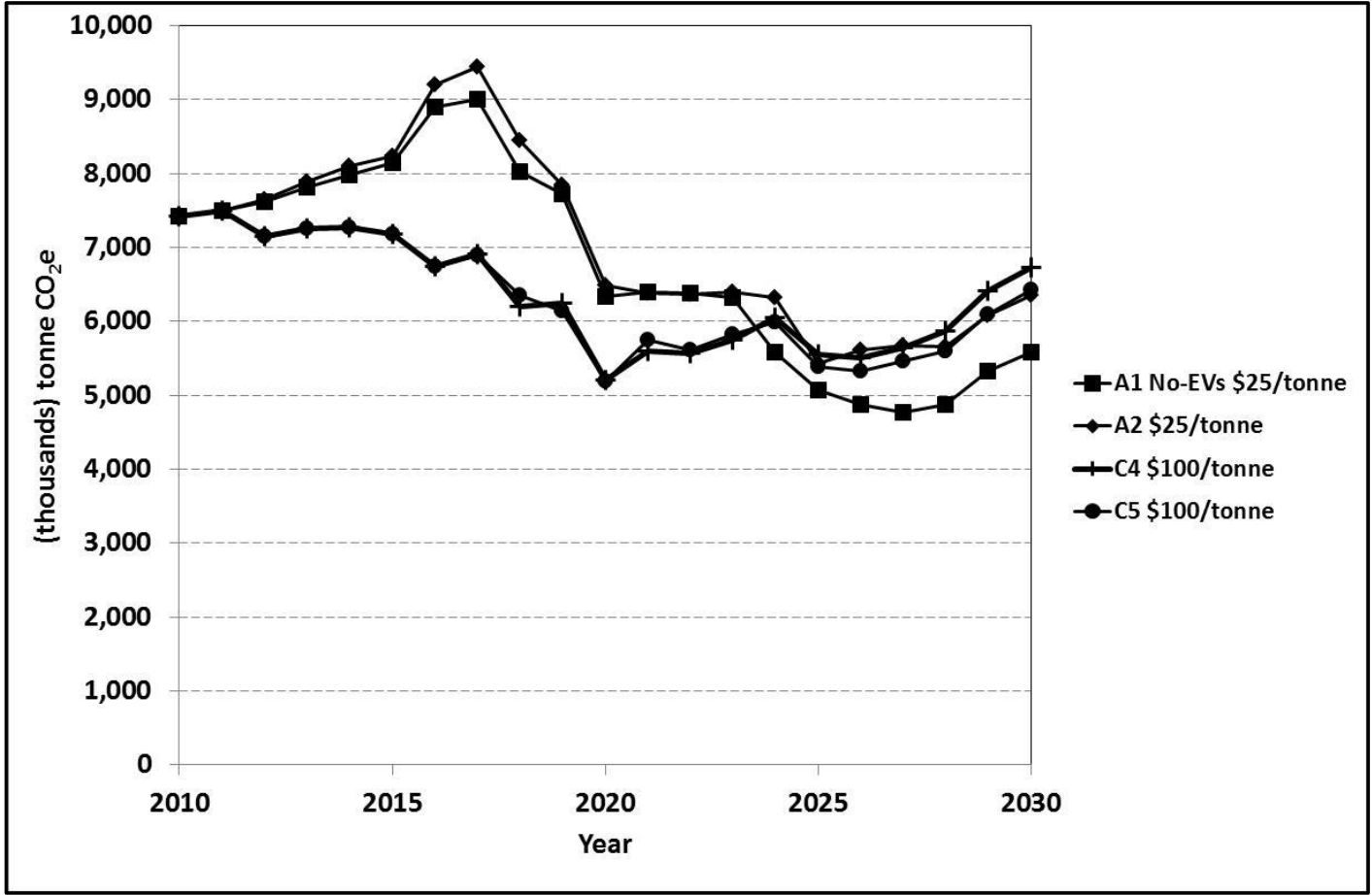

Moderate access to hydro sites and no restrictions on the use of coal (MDS3)

Low carbon price, scenario A2 EV electricity demand

In the A2 model run, the additional electricity demand due to the introduction of EVs and a carbon price of $\$ 25$ per tonne resulted in all four $250 \mathrm{MW}$ coal-fired units at Huntly being refurbished, rather than three units in the $A 1$ (no-EV) run. The nonretirement of the fourth Huntly unit reduced the need for some of the specialised peak generation capacity that was built in the $A 1$ (no-EV) model run. Due to the retention of the fourth coal unit at Huntly, 294 MW less new fossil-fuelled generation was built in the $A 2$ model run. In the $A 1$ (no-EV) and $A 2$ runs, 1,020 MW of new coal plant capacity was built.

The A2 model run resulted in the commissioning of an additional $171 \mathrm{MW}$ of renewable and demand side measures compared to the A1 (no-EV) run. By 2030, the installed generation and DSM capacity in the A2 model run was $127 \mathrm{MW}$ more than in the $A 1$ (no-EV) run (Table 9.16 and Figure 9.12). 
In the $\mathrm{A} 1$ (no-EV) model run, the proportion of renewable electricity increased from $72.1 \%$ to $75.9 \%$ by 2020 . However, by 2020 , three new coal plants were constructed in the North Auckland, Auckland, and Bay of Plenty regions. By 2030, these plants reduced the proportion of renewable electricity to $72.4 \%$. After 2020 , the additional demand from EVs resulted in the greater utilisation of the new coal plants, and four existing $250 \mathrm{MW}$ coal units at Huntly, which resulted in the proportion of renewable electricity generated by 2030 being $2 \%$ to $3 \%$ below the levels in the A1 (no-EV) model run (Figure 9.13).

In the $\mathrm{A} 1$ (no-EV) model run, annual GHG emissions from electricity generation increased by $79.7 \%$ from 7.4 million tonnes $\mathrm{CO}_{2} \mathrm{e}$, in 2010 , to 13.3 million tonnes $\mathrm{CO}_{2} \mathrm{e}$ in 2029. At that time, the $385 \mathrm{MW}$ e3p CCGT plant at Huntly was retired and GHG emissions declined to 12.9 million tonnes $\mathrm{CO}_{2} \mathrm{e}$. The three new coal plants accounted for $45 \%$ of the annual GHG emissions produced in 2030 (Figure 9.14).

The presence of EVs in this model run caused annual GHG emissions from electricity generation to increase by $109.5 \%$ to 15.5 million tonnes $\mathrm{CO}_{2} \mathrm{e}$ in 2029 compared to the A1 (no-EV) run. The electricity demand from EVs resulted in the greater use of the new coal plant in the Auckland region. This plant accounted for $59.1 \%$ of the additional cumulative GHG emissions over the modelling period due to the presence of EVs in this model run. The continued use of the fourth coal unit at Huntly accounted for another $30.5 \%$ of the additional cumulative GHG emissions. In 2030 , in both the $\mathrm{A} 1$ (no-EV) and A2 model runs, the 385 MW e3p CCGT plant at Huntly was retired and GHG emissions declined in that year to 14.7 million tonnes $\mathrm{CO}_{2} \mathrm{e}$. Overall, the presence of EVs resulted in an increase in the cumulative GHG emissions from the electricity sector over the modelling period of 23.9 million tonnes $\mathrm{CO}_{2} \mathrm{e}$.

\section{High carbon price, scenario C4 EV electricity demand}

Increasing the price of carbon to $\$ 100$ per tonne had a significant impact on the building and retirement schedules in the MDS3 scenario. The higher carbon price resulted in all four coal units at Huntly being retired between 2022 and 2025 and the reduction of the number of new coal plants built from three to two. Compared 
to the low carbon price $\mathrm{A} 2$ model run, the $\mathrm{C} 4$ model run had 1,400 MW less coal capacity by 2030 . The two new coal plants in the C4 model run were located in the North Auckland and Bay of Plenty regions (Table 9.16).

To offset the reduction in coal fired generation capacity in the $\mathrm{C} 4$ model run, an additional 2,680 MW of wind, $298 \mathrm{MW}$ of hydro, and $157 \mathrm{MW}$ of biomass cogeneration were installed. To provide back-up peaking generation for the wind generation, an additional $275 \mathrm{MW}$ of fast-start gas fired peaking plant was built. By 2030, the effect of these changes was an additional 2,334 MW installed in this model run when compared to the $\mathrm{A} 1$ (no-EV) model run (Table 9.16 and Figure 9.12).

The higher carbon price resulted in the proportion of renewable electricity generated increasing from $72.1 \%$ in 2010 , to $86.4 \%$ in 2025 . After 2025 , the proportion of renewable generation declined to $82.7 \%$ in 2030 due to the commissioning, in 2026, of a $300 \mathrm{MW}$ coal plant in the Bay of Plenty region, and a second 407 MW CCGT plant at Otahuhu. Most of the additional renewable generation in this model run, compared to the $\mathrm{A} 1$ (no-EV) model run and low carbon price A2 model run, was wind generation located in the North Island. These wind projects produced $73 \%$ of all the renewable electricity generated over the modelling period. The remaining renewable generation was sourced from South Island wind (15.3\%), hydro (5.9\%), and biogas congregation (6.6\%) (Figure 9.13).

The annual GHG emissions in this model run were significantly lower than in both the $\mathrm{A} 1$ (no-EV) model run and the low carbon price $\mathrm{A} 2$ model run. In the $\mathrm{C} 4$ model run, annual GHG emissions declined from 7.4 million tonnes $\mathrm{CO}_{2} \mathrm{e}$ in 2010 , to 5.2 million tonnes $\mathrm{CO}_{2} \mathrm{e}$ in 2025. After 2025, GHG emissions increased to 7.1 million tonnes $\mathrm{CO}_{2} \mathrm{e}$ by 2030 . This increase was due to the commissioning of new coal and CCGT plants in 2026. By 2030, there was an overall 3.9\% decline in annual GHG emissions compared to the levels in 2010 (Figure 9.14). 
Table 9.16: MDS3 medium renewables and no restriction on coal - New capacity installed and retirements (MW)

\begin{tabular}{|c|c|c|c|c|c|c|c|c|c|}
\hline & \multicolumn{9}{|c|}{ Retirements } \\
\hline & \multicolumn{9}{|c|}{ VFM scenario } \\
\hline & \multicolumn{3}{|c|}{$\begin{array}{l}\text { A1 (no EV) } \\
\$ 25 \text { per tonne }\end{array}$} & \multicolumn{3}{|c|}{$\begin{array}{c}\text { A2 } \\
\text { EVs } \$ 25 \text { per tonne }\end{array}$} & \multicolumn{3}{|c|}{ C4 } \\
\hline Technology & North Island & South Island & Total & North Island & South Island & Total & North Island & South Island & Total \\
\hline Coal & 250 & 0 & 250 & 0 & 0 & 0 & 1,000 & 0 & 1,000 \\
\hline Combined cycle gas turbine & 887 & 0 & 887 & 887 & 0 & 887 & 887 & 0 & 887 \\
\hline Geothermal & 163 & 0 & 163 & 163 & 0 & 163 & 163 & 0 & 163 \\
\hline Open cycle gas turbine - gas & 95 & 0 & 95 & 95 & 0 & 95 & 95 & 0 & 95 \\
\hline Peaker, diesel-fired OCGT & 155 & 0 & 155 & 155 & 0 & 155 & 155 & 0 & 155 \\
\hline Total & 1,550 & 0 & 1,550 & 1,300 & 0 & 1,300 & 2,300 & 0 & 2,300 \\
\hline
\end{tabular}


Table 9.16: (cont.)

\begin{tabular}{|c|c|c|c|c|c|c|c|c|c|}
\hline & & & & & Build & & & & \\
\hline & & & & & VFM scenarios & & & & \\
\hline & & $\begin{array}{c}\text { A1 (no EV) } \\
\$ 25 \text { per tonne }\end{array}$ & & & $\begin{array}{c}\text { A2 } \\
\text { /s } \$ 25 \text { per tonr }\end{array}$ & & & $\begin{array}{c}C 4 \\
\text { Is } \$ 100 \text { per ton }\end{array}$ & \\
\hline Technology & North Island & South Island & Total & North Island & South Island & Total & North Island & South Island & Total \\
\hline Coal & 1,020 & 0 & 1,020 & 1,020 & 0 & 1,020 & 620 & 0 & 620 \\
\hline Cogeneration, biomass-fired & 0 & 0 & 0 & 0 & 0 & 0 & 157 & 0 & $157^{*}$ \\
\hline Cogeneration, gas-fired & 0 & 0 & 0 & 85 & 0 & 85 & 85 & 0 & 85 \\
\hline Cogeneration, other & 80 & 0 & $80^{*}$ & 80 & 0 & $80^{*}$ & 80 & 0 & $80 *$ \\
\hline Combined cycle gas turbine & 407 & 0 & $407^{*}$ & 407 & 0 & $407^{*}$ & 407 & 0 & $407^{*}$ \\
\hline Geothermal & 1,065 & 0 & $1,065^{*}$ & 1,065 & 0 & $1,065^{*}$ & 1,065 & 0 & $1,065^{*}$ \\
\hline Hydro, peaking & 0 & 478 & 478 & 0 & 478 & 478 & 150 & 478 & 628 \\
\hline Hydro, pumped storage & 300 & 0 & 300 & 300 & & 300 & 300 & 0 & 300 \\
\hline Hydro, run-of-river & 60 & 158 & 218 & 80 & 158 & 238 & 92 & 274 & 366 \\
\hline Interruptible load & 100 & 0 & 100 & 100 & 0 & 100 & 100 & 0 & 100 \\
\hline Open cycle gas turbine - gas & 0 & 0 & 0 & 0 & 0 & 0 & 0 & 0 & 0 \\
\hline Peaker, diesel-fired OCGT & 545 & 100 & 645 & 500 & 100 & 600 & 400 & 0 & 400 \\
\hline Peaker, fast-start gas-fired peaker & 965 & 0 & 965 & 600 & 0 & 600 & 1,240 & 0 & 1,240 \\
\hline Price-responsive load curtailment & 277 & 0 & 277 & 300 & 0 & $300 *$ & 300 & 0 & $300^{*}$ \\
\hline Reciprocating engine diesel & 8 & 0 & 8 & 39 & 0 & 39 & 160 & 6 & 166 \\
\hline Wave & 0 & 0 & 0 & 0 & 0 & 0 & 38 & 15 & 53 \\
\hline Wind & 787 & 440 & 1,227 & 805 & 550 & 1,355 & 2,994 & 912 & 3,907 \\
\hline Total & 5,614 & 1,176 & 6,790 & 5,381 & 1,286 & 6,667 & 8,189 & 1,685 & 9,873 \\
\hline
\end{tabular}

GEM utilises $100 \%$ of the available generation resources 
Figure 9.12: MDS3 gross and net installed generation and demand side management capacity: 2010 to 2030

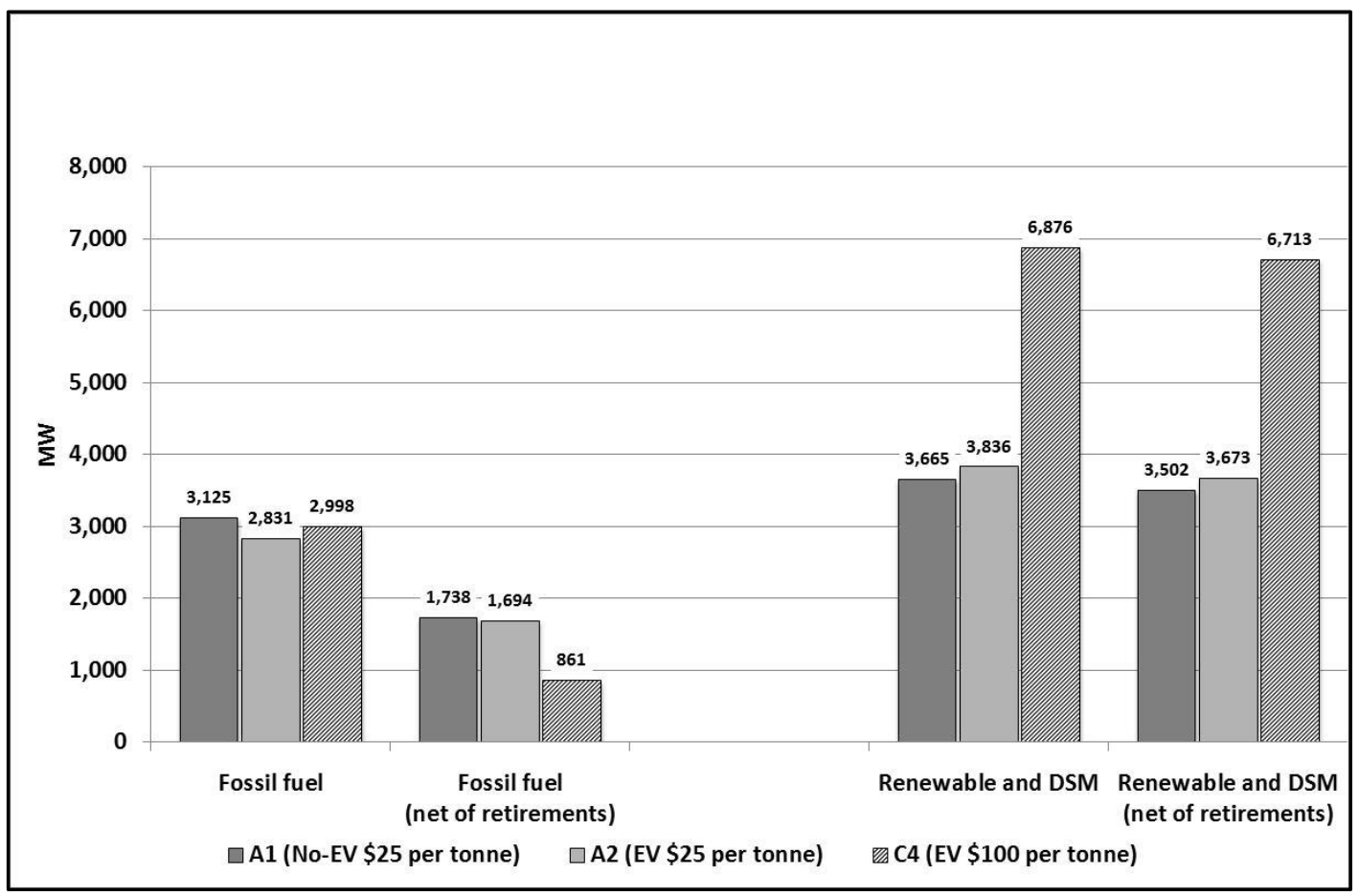

Figure 9.13: Proportion of renewable generation, MDS3 - A1 (no-EV), A2 and C4 electricity demand

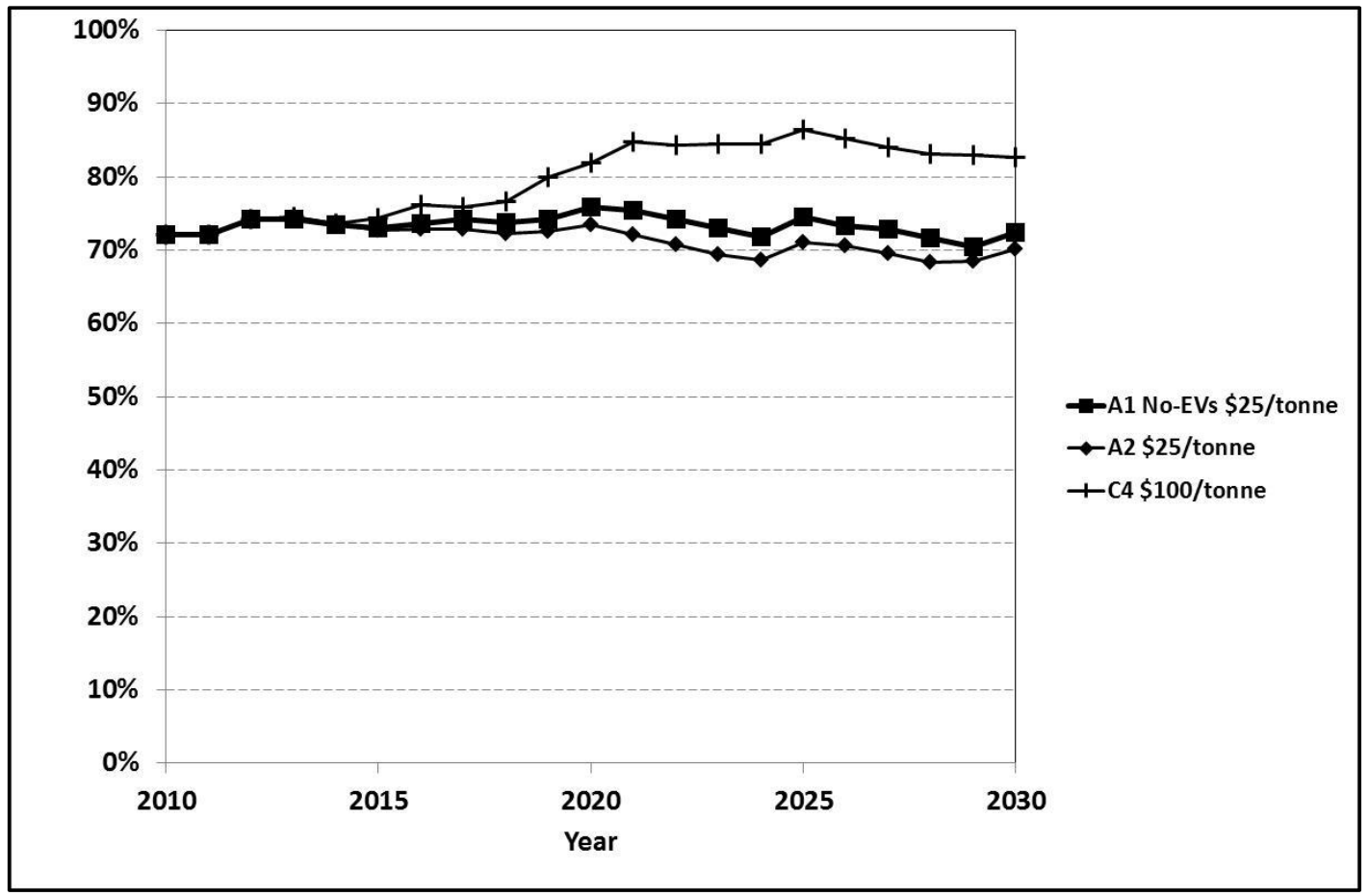


Figure 9.14: Annual GHG emissions, MDS3 - A1 (no-EV), A2, and C4 electricity demand: thousand tonnes $\mathrm{CO}_{2} \mathrm{e}$

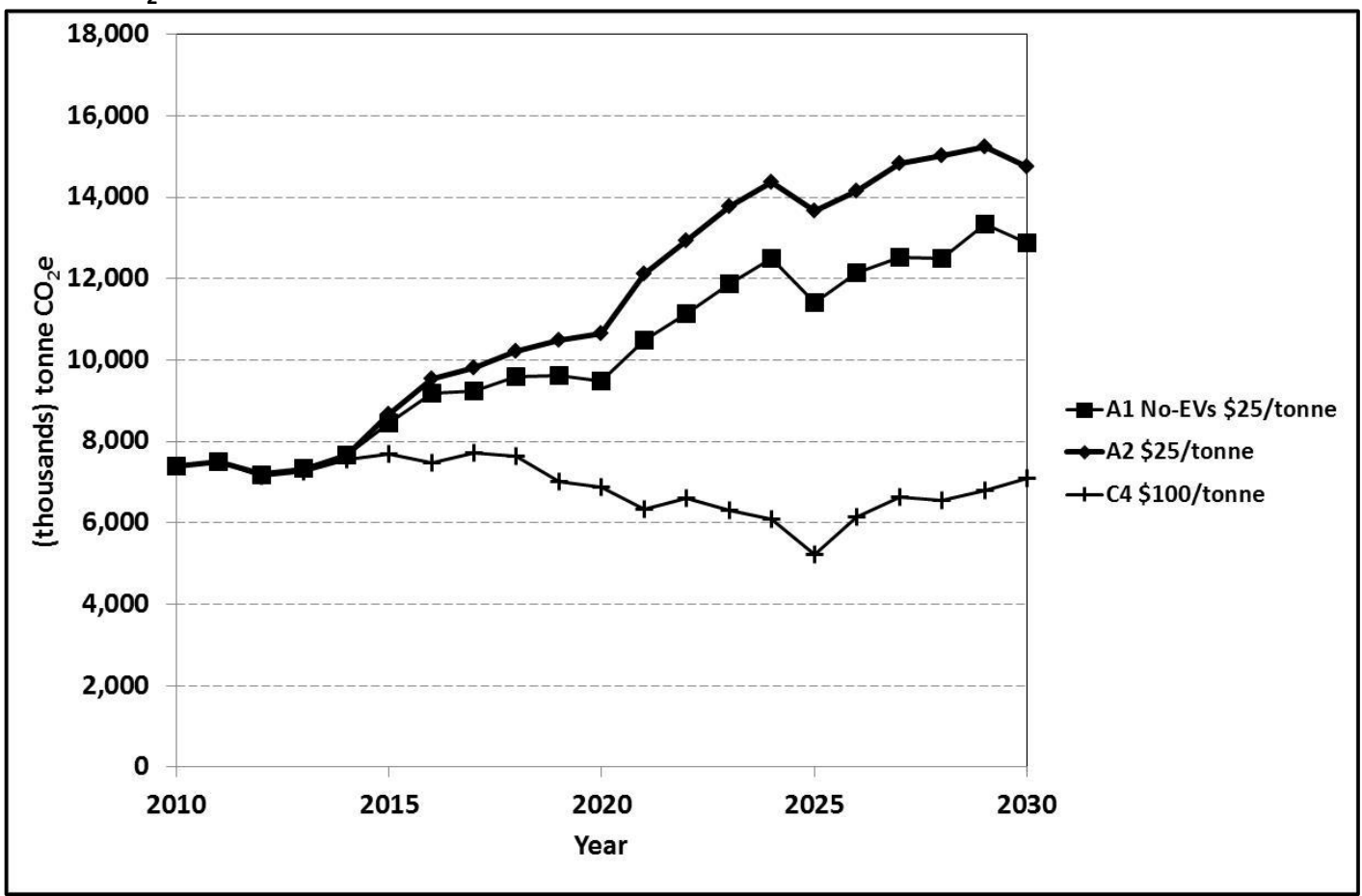

High gas discovery (MDS5)

Low carbon price, scenario A2 EV electricity demand

The MDS5 scenario is a natural gas dominated generation scenario based on the assumptions of a discovery of large quantities of low cost domestic natural gas and its availability for use in electricity generation. In this generation scenario, both the A1 (no-EV) and A2 model runs resulted in all four $250 \mathrm{MW}$ coal units at Huntly being retired. The e3p Huntly CCGT unit (385 MW), Otahuhu B CCGT (380 MW), and Taranaki Combined Cycle plant (380 MW) were also retired.

The building schedules for both the $A 1$ (no-EV) model run and $A 2$ model run were dominated by the commissioning of 2,467 MW of new CCGT plants. These plants were located in Auckland (817 MW), North Auckland (480 MW), and Taranaki $(1,170 \mathrm{MW})$. These plants were all of the CCGT projects available for the GEM to use in the MDS5 scenario.

In the $\mathrm{A} 2$ model run, the additional demand from EVs resulted in the building of an additional $380 \mathrm{MW}$ of peaking hydro capacity in the South Island. This capacity included the $280 \mathrm{MW}$ Waitaki North Bank Tunnel hydro peaking project, and 
$209 \mathrm{MW}$ of wind in the North Island (Table 9.17). To meet the additional demand from EVs in the South Island, a $150 \mathrm{MW}$ of diesel plant was also constructed, which resulted in the building of less fast-start gas-fired peaking capacity. Overall, the A2 model run resulted in the construction of $74 \mathrm{MW}$ less fossil-fuelled generation than the $A 1$ (no-EV) model run. In 2030, the effect of EVs on the total installed capacity was an additional $465 \mathrm{MW}$ in total installed capacity (Figure 9.15).

The proportion of renewable generation in these model runs declined in both the $\mathrm{A} 1$ (no-EV) and the A2 model run as the new gas generation plants came online and displaced the retired coal capacity. Although the same gas plants were built in both model runs, the timing of the construction of the 410 MW CCGT plant in the Auckland region differed. In the A1 (no-EV) model run, the plant was built in 2015 , but, in the A2 model run, it was not built until 2020. To offset the delay in construction of this plant, one of the coal units at Huntly was retired in 2020 instead of being retired in 2016. The additional demand from EVs had no effect on the timing of the retirements of the remaining three coal units at Huntly. In the A2 model run, the demand from EVs resulted in the increased use of the CCGT plants at Huntly and Otahuhu before their retirement. In the $A 2$ model run, after the retirement of the coal unit at Huntly, the three remaining units were used more often than in the A1 (no-EV) model run until they were retired in 2023 and 2024.

In both the $A 1$ (no-EV) and $A 2$ model runs, the proportion of renewable electricity declined from $72.0 \%$ in 2010 to $67.8 \%$ in 2024 . After 2024 , due to the commissioning of the Waitaki North Bank Tunnel hydro project, in the A2 model run the proportion of renewable electricity was $1 \%$ higher than in the $\mathrm{A} 1$ (no-EV) model run by 2030 (Figure 9.16).

Both the $A 1$ (no-EV) and $A 2$ model runs provided similar estimates of the annual GHG emissions in 2030. The A1 (no-EV) model run projected that annual GHG emissions would be 10.3 million tonnes $\mathrm{CO}_{2} \mathrm{e}$ in 2030 and the $\mathrm{A} 2$ model run projected that annual GHG emissions would be 10.4 million tonnes $\mathrm{CO}_{2} \mathrm{e}$. However, during the period 2016 to 2023, the A2 model run had higher annual GHG emissions due to the increased use of coal and gas at Huntly and the increased use of the 
Otahuhu CCGT plants. This increased use of these plants resulted in an additional 4.3 million tonnes $\mathrm{CO}_{2} \mathrm{e}$ being emitted over the modelling period. In both model runs, these two CCGT plants were retired in 2023 and 2024, but in the A2 model run the North Bank Tunnel hydro project came into operation. This hydro project had the effect of reducing the effect of the additional demand from EVs on the GHG emissions from the electricity sector. From 2025, the levels of GHG emissions in the two model runs run were very similar (Figure 9.17).

\section{High carbon price, scenario C4 EV electricity demand}

Using the MDS5 scenario, the imposition of a \$100 per tonne carbon charge was found to have no effect on which plants were retired, when compared to the low carbon price A2 model run. However, the timing of some of the retirements did change with two of the $250 \mathrm{MW}$ coal units at Huntly being retired earlier in 2017 instead of in 2023 and 2024.

In this generation scenario, due to the low price of gas, the high carbon price had no impact on the amount of CCGT capacity that was built and all the CCGT projects available for use by the GEM were utilised. However, the high carbon price prevented the building of $620 \mathrm{MW}$ of new coal plant.

To offset the use of coal plant in this model run, an additional 1,125 MW of wind capacity was installed compared to the $\mathrm{A} 2$ low carbon price model run, of which 97\% was located in the North Island. The high carbon price also resulted in the building of an additional $101 \mathrm{MW}$ of run-of-river hydro ( $80 \%$ in the South Island), and $157 \mathrm{MW}$ of biogas cogeneration. To provide back-up for the wind generation and offset the loss of peaking capability from the reduction in new coal capacity, an additional $480 \mathrm{MW}$ of fast-start gas-fired peaking plant was installed (Table 9.17). This shift to wind generation and supporting back-up capacity resulted in a net increase in renewable generation of 1,981 MW above that in the $\mathrm{A} 1$ (no-EV) model run and an increase in the total installed capacity of 1,512 MW by 2030 (Figure 9.15).

The high carbon price increased the proportion of renewable electricity in stages. The first stage increase occurred in 2017 when three coal units at Huntly were 
retired. The next stage increase occurred when the final unit at Huntly and the two CCGT plants were retired in 2024 and 2025. At the same time as these retirements, a number of small to medium renewable plants were commissioned. The combined effect of the retirements and the new renewable generation was to increase the proportion of renewable generation from $72.0 \%$ in 2010 , to $73.9 \%$ in 2017 , and then $76.1 \%$ in 2023 . After 2025 , the proportion of renewable generation declined to $72.8 \%$ by 2030 . This decline was due to the commissioning of a new CCGT plant in Rodney and the greater utilisation of the two new CCGT plants located in the Auckland region (Figure 9.16).

The decommissioning of three Huntly coal units in 2017 had a major impact on the annual GHG emissions produced by the electricity sector (Figure 9.17). In 2016, annual GHG emissions were 8.2 million tonnes $\mathrm{CO}_{2} \mathrm{e}$, but, in 2017 , they declined to 6.5 million tonnes $\mathrm{CO}_{2} \mathrm{e}$. After 2017, annual GHG emissions declined further to 6.1 million tonnes $\mathrm{CO}_{2} \mathrm{e}$ in 2025. After 2025, the annual GHG emissions increased and, by 2030 , reached 7.3 million tonnes $\mathrm{CO}_{2} \mathrm{e}$. The increased GHG emissions were due to the use of the five new CCGT plants that were commissioned after 2024; three located in Taranaki, and two in North Auckland (Figure 9.17). 
Table 9.17: MDS5 High gas discovery - New capacity installed and retirements (MW)

\begin{tabular}{|c|c|c|c|c|c|c|c|c|c|}
\hline & \multicolumn{9}{|c|}{ Retirements } \\
\hline & \multicolumn{9}{|c|}{ VFM scenario } \\
\hline & \multicolumn{3}{|c|}{$\begin{array}{c}\text { A1 (no EV) } \\
\$ 25 \text { per tonne }\end{array}$} & \multicolumn{3}{|c|}{$\begin{array}{c}\text { A2 } \\
\text { EVs } \$ 25 \text { per tonne }\end{array}$} & \multicolumn{3}{|c|}{$\begin{array}{c}\text { C4 } \\
\text { EVs } \$ 100 \text { per tonne }\end{array}$} \\
\hline Technology & North Island & South Island & Total & North Island & South Island & Total & North Island & South Island & Total \\
\hline Coal & 1,000 & 0 & 1,000 & 1,000 & 0 & 1,000 & 1,000 & 0 & 1,000 \\
\hline Combined cycle gas turbine & 1,145 & 0 & 1,145 & 1,145 & 0 & 1,145 & 1,145 & 0 & 1,145 \\
\hline Geothermal & 163 & 0 & 163 & 163 & 0 & 163 & 163 & 0 & 163 \\
\hline Open cycle gas turbine - gas & 95 & 0 & 95 & 95 & 0 & 95 & 95 & 0 & 95 \\
\hline Peaker, diesel-fired OCGT & 155 & 0 & 155 & 155 & 0 & 155 & 155 & 0 & 155 \\
\hline Total & 2,558 & 0 & 2,558 & 2,558 & 0 & 2,558 & 2,558 & 0 & 2,558 \\
\hline
\end{tabular}




\section{Table 9.17: (cont.)}

\begin{tabular}{|c|c|c|c|c|c|c|c|c|c|}
\hline & \multicolumn{9}{|c|}{ Build } \\
\hline & \multicolumn{9}{|c|}{ VFM scenario } \\
\hline & \multicolumn{3}{|c|}{$\begin{array}{c}\text { A1 (no EV) } \\
\$ 25 \text { per tonne }\end{array}$} & \multicolumn{3}{|c|}{ 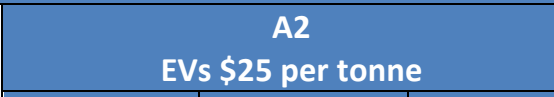 } & \multicolumn{3}{|c|}{$\begin{array}{c}\text { C4 } \\
\text { EVs } \$ 100 \text { per tonne }\end{array}$} \\
\hline Technology & North Island & South Island & Total & North Island & South Island & Total & North Island & South Island & Total \\
\hline Coal & 620 & 0 & 620 & 620 & 0 & 620 & 0 & 0 & 0 \\
\hline Cogeneration, biomass-fired & 0 & 0 & 0 & 0 & 0 & 0 & 157 & 0 & $157^{*}$ \\
\hline Cogeneration, gas-fired & 135 & 0 & $135^{*}$ & 135 & 0 & $135^{*}$ & 135 & 0 & $135 *$ \\
\hline Cogeneration, other & 80 & 0 & $80^{*}$ & 80 & 0 & $80^{*}$ & 80 & 0 & $80 *$ \\
\hline Combined cycle gas turbine & 2,467 & 0 & $2,467^{*}$ & 2,467 & 0 & $2,467^{*}$ & 2,467 & 0 & $2,467 *$ \\
\hline Geothermal & 830 & 0 & $830 *$ & 830 & 0 & $830 *$ & 830 & 0 & $830 *$ \\
\hline Hydro, peaking & 0 & 271 & 271 & 0 & 651 & 651 & 0 & 651 & 651 \\
\hline Hydro, run-of-river & 60 & 158 & 218 & 60 & 158 & 218 & 80 & 239 & 319 \\
\hline Interruptible load & 100 & 50 & $150 *$ & 100 & 0 & 100 & 100 & 0 & 100 \\
\hline Open cycle gas turbine - gas & 0 & 0 & 0 & 0 & 0 & 0 & 0 & 0 & 0 \\
\hline Peaker, diesel-fired OCGT & 600 & 150 & 750 & 800 & 150 & 950 & 700 & 100 & 800 \\
\hline Peaker, fast-start gas-fired peaker & 720 & 0 & 720 & 460 & 0 & 460 & 940 & 0 & 940 \\
\hline Price-responsive load curtailment & 300 & 0 & $300 *$ & 300 & 0 & $300^{*}$ & 300 & 0 & $300 *$ \\
\hline Reciprocating engine diesel & 39 & 30 & 69 & 55 & 0 & 55 & 0 & 0 & 0 \\
\hline Wind & 322 & 50 & 372 & 531 & 50 & 581 & 1,618 & 97 & 1,716 \\
\hline Total & 6,272 & 709 & 6,982 & 6,437 & 1,009 & 7,446 & 7,407 & 1,087 & 8,494 \\
\hline
\end{tabular}

GEM utilises $100 \%$ of the available generation resources 
Figure 9.15: MDS5 gross and net installed generation and demand side management capacity: 2010 to 2030

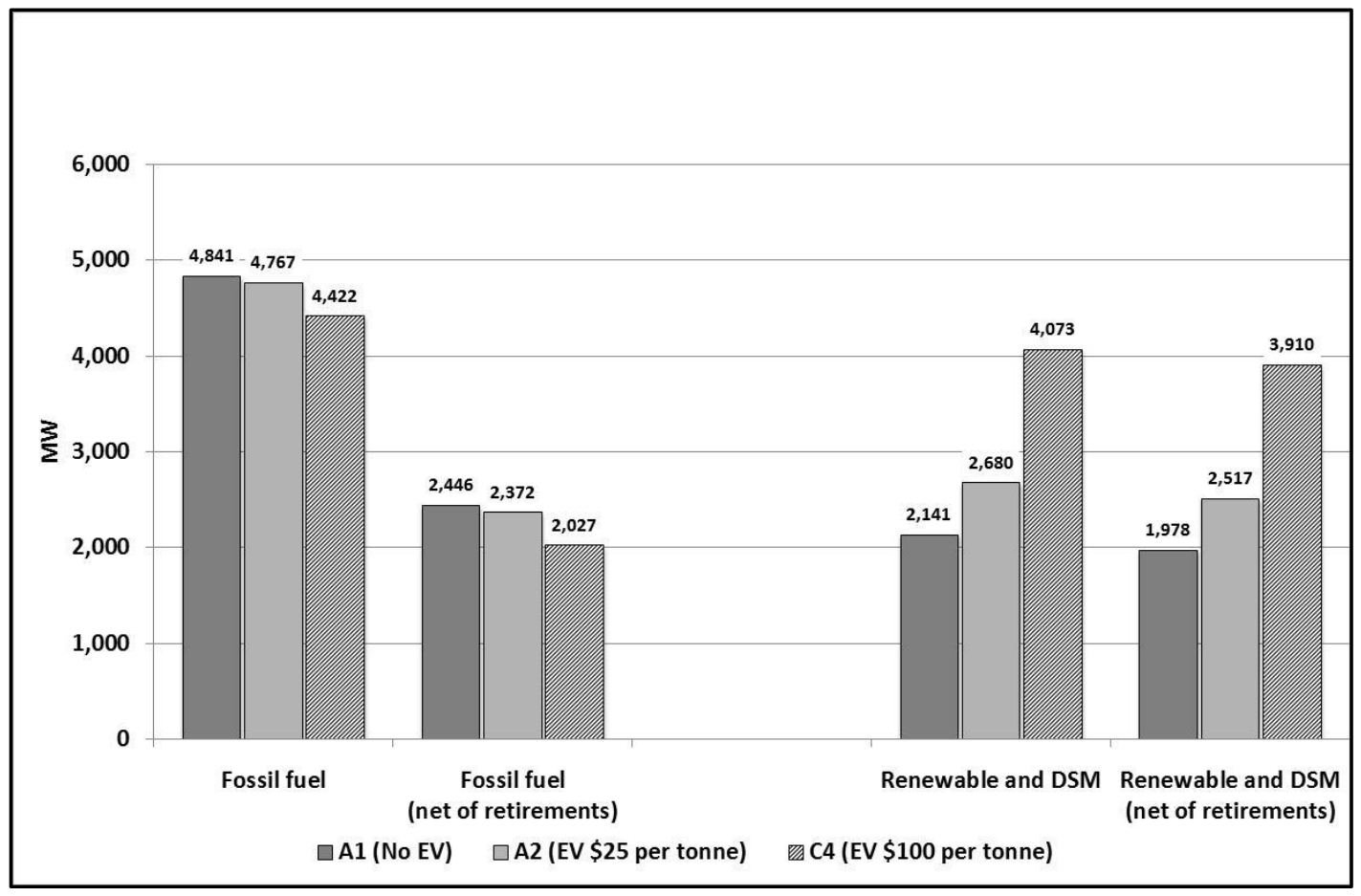

Figure 9.16: Proportion of renewable generation, MDS5 - A1 (no-EV) and A2 and C4 electricity demand

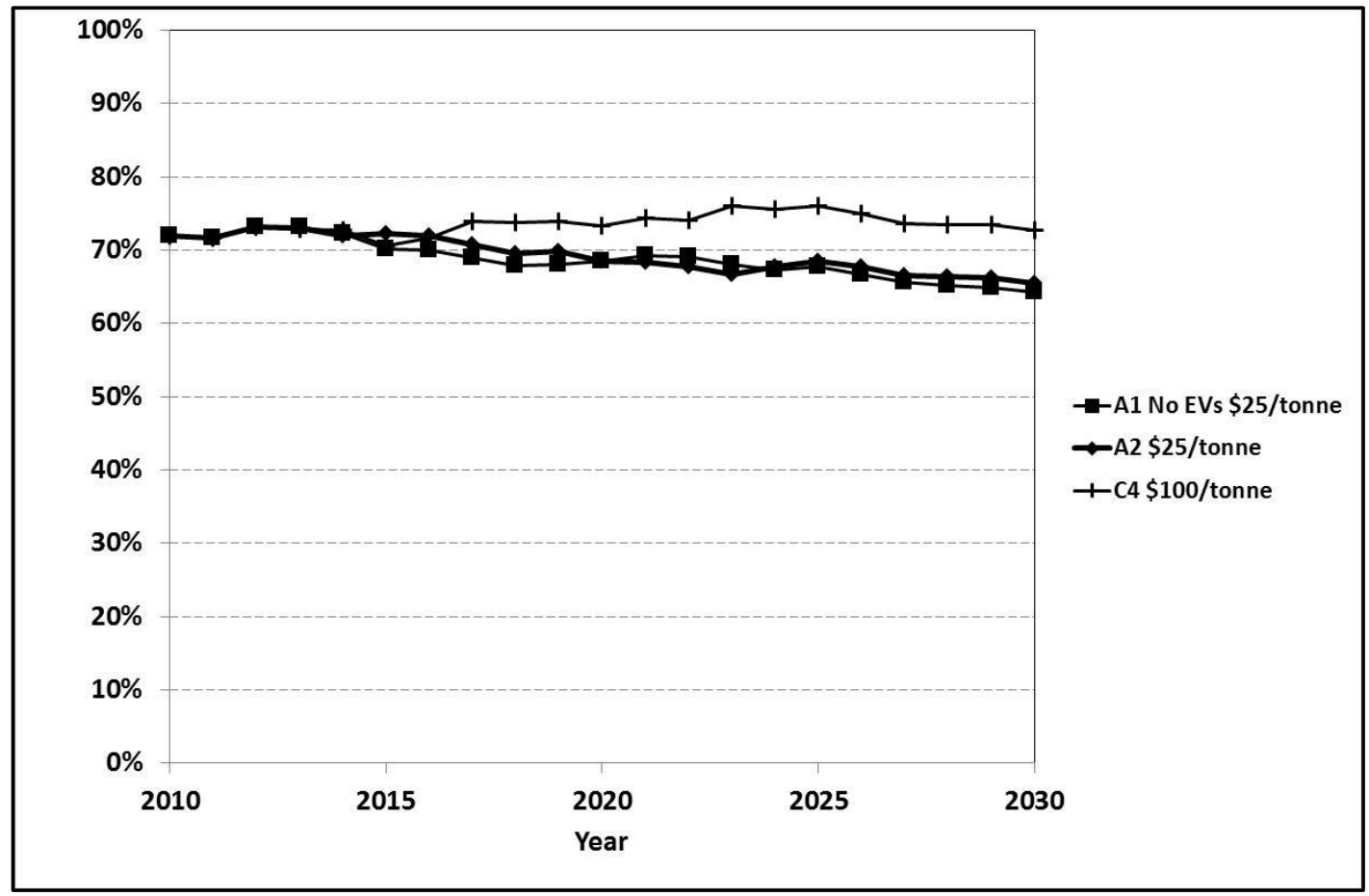


Figure 9.17: Annual GHG emissions, MDS5 - A1 (no-EV), A2 and C4 electricity demand: thousand tonnes $\mathrm{CO}_{2} \mathrm{e}$

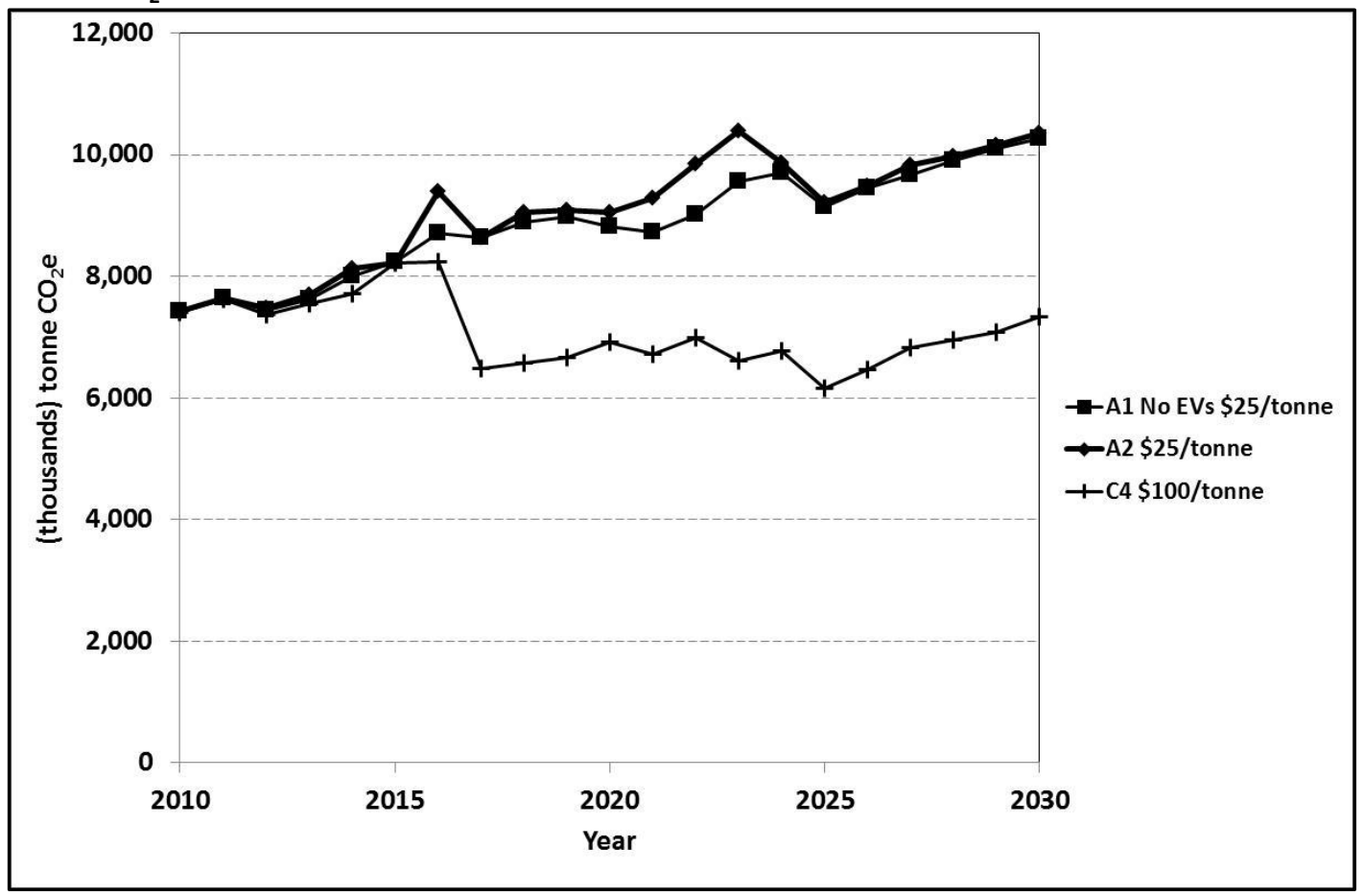

\subsubsection{The impact of EVs on installed capacity}

\section{Fully interruptible load}

The default assumption used in this analysis was that the system operator could, if required, interrupt the entire EV load on the electricity grid. Assuming no change in the price of carbon, the amount of additional generation and DSM capacity required to be in place in 2030 to meet the additional demand from EVs would be, at most, $729 \mathrm{MW}$ more than in the $\mathrm{A} 1$ (no-EV) model run (Table 9.18).

Increasing the price of carbon had the effect of reducing the reliance on coal plants and increasing the utilisation of wind generation. As wind generation technology, has a lower availability factor and less capacity to provide peaking generation than coal plants, more generation capacity and backup peaking plant would be required to meet the same level of demand. This occurred in the MDS3 and MDS5 generation scenarios where, in conjunction with increased wind capacity, there was increased installation of fast-start peaking plants. 
However, in the case of the hydro and DSM focused MDS1 scenario, increasing the carbon price had little effect on the amount of installed capacity due to the policy ban already in place in this generation scenario on new coal plant, which had already resulted in a high uptake of renewable capacity and the associated faststart peaking plants.

In the MDS1 scenario, under a high carbon price the impact of the additional electricity demand from EVs was the refurbishment of the Otahuhu B and Taranaki CCGT plants, which delayed the construction of wind generation. In 2015 , this delay had the effect of reducing the amount of installed capacity compared to the situation where there was no additional demand from EVs (Table 9.18) ${ }^{69}$.

\footnotetext{
${ }^{69}$ A possible reason why the GEM resulted in this solution was that most of the remaining unutilised renewable resources in the GEM database were located in the South Island and HVDC transmission constraints may have come into effect.
} 
Table 9.18 Total installed generation and demand side management capacity - fully interruptible (MW): selected years

\begin{tabular}{|c|c|c|c|c|c|c|}
\hline & & 2010 & 2015 & 2020 & 2025 & 2030 \\
\hline \multicolumn{7}{|c|}{ MDS1 } \\
\hline A1 & No EVs (BAU) & 9,420 & 10,682 & 12,329 & 14,883 & 16,642 \\
\hline \multirow{2}{*}{ A2 } & EV \$25 per tonne & 9,420 & 10,766 & 12,578 & 15,138 & 16,826 \\
\hline & Total difference from BAU & 0 & 84 & 249 & 255 & 184 \\
\hline \multirow{5}{*}{ C4 } & No EV \$100 per tonne & 9,420 & 10,958 & 12,807 & 15,033 & 16,378 \\
\hline & $\begin{array}{c}\text { Difference due to carbon } \\
\text { price }\end{array}$ & 0 & 276 & 478 & 150 & -264 \\
\hline & EV $\$ 100$ per tonne & 9,437 & 10,636 & 12,973 & 15,063 & 16,643 \\
\hline & Difference due to EVs & 17 & -322 & 166 & 30 & 265 \\
\hline & Total difference from BAU & 17 & -46 & 644 & 180 & 1 \\
\hline \multirow{5}{*}{ C5 } & No EV \$100 per tonne & 9,420 & 10,958 & 12,807 & 15,033 & 16,378 \\
\hline & $\begin{array}{c}\text { Difference due to carbon } \\
\text { price }\end{array}$ & 0 & 276 & 478 & 150 & -264 \\
\hline & C5 EV $\$ 100$ per tonne & 9,420 & 10,936 & 13,121 & 15,222 & 16,813 \\
\hline & Difference due to EVs & 0 & -22 & 314 & 189 & 435 \\
\hline & Total difference from BAU & 0 & 254 & 792 & 339 & 171 \\
\hline \multicolumn{7}{|c|}{ MDS3 } \\
\hline $\mathrm{A} 1$ & No EVs (BAU) & 9,437 & 10,478 & 11,858 & 13,091 & 14,310 \\
\hline \multirow{2}{*}{ A2 } & EV \$25 per tonne & 9,437 & 10,495 & 12,451 & 13,091 & 14,437 \\
\hline & Difference from BAU & 0 & 17 & 593 & 524 & 127 \\
\hline \multirow{5}{*}{ C4 } & No EV \$100 per tonne & 9,437 & 10,618 & 12,647 & 14,835 & 16,400 \\
\hline & $\begin{array}{c}\text { Difference due to carbon } \\
\text { price }\end{array}$ & 0 & 140 & 789 & 1,744 & 2,090 \\
\hline & EV \$100 per tonne & 9,437 & 10,636 & 12,974 & 15,063 & 16,644 \\
\hline & Difference due to EVs & 0 & 18 & 327 & 228 & 244 \\
\hline & Total difference from BAU & 0 & 158 & 1,116 & 1,972 & 2,334 \\
\hline \multicolumn{7}{|c|}{ MDS5 } \\
\hline $\mathrm{A1}$ & No EVs (BAU) & 9,420 & 10,684 & 11,422 & 12,402 & 13,494 \\
\hline \multirow{2}{*}{ A2 } & EV \$25 per tonne & 9,420 & 10,473 & 11,603 & 12,794 & 13,959 \\
\hline & Difference from BAU & 0 & -211 & 181 & 392 & 465 \\
\hline \multirow{5}{*}{ C4 } & No EV \$100 per tonne & 9,420 & 10,688 & 11,893 & 13,321 & 14,278 \\
\hline & $\begin{array}{c}\text { Difference due to carbon } \\
\text { price }\end{array}$ & 0 & 4 & 471 & 919 & 784 \\
\hline & EV \$100 per tonne & 9,420 & 10,713 & 11,865 & 13,819 & 15,007 \\
\hline & Difference due to EVs & 0 & 25 & -28 & 498 & 729 \\
\hline & Total difference from BAU & 0 & 29 & 443 & 1417 & 1,513 \\
\hline
\end{tabular}

\section{Impact of uninterruptible load from EVs on installed capacity}

If EV recharging was only partially interruptible or completely non-interruptible, then the recharging of EVs would add to the peak demand and this additional load would need to be met by installing additional fast-start peaking plants. 
The possibility of having up to $40 \%$ of the EV fleet recharging during an evening winter peak demand event resulted in between 1,344 MW and 2,158 MW of additional peaking plant having to be built by 2030 to ensure that the system operator could meet the $n-1$ security requirement.

If all EV recharging was uncontrolled by the system operator, and this resulted in the possibility of up to $80 \%$ of the EV fleet charging during an evening winter peak demand event, then an additional 3,783 MW to 4,436 MW of peak generation would be required to be built by 2030 (Table 9.19).

The amount of additional peak generation capacity required to be built to meet security requirement would vary depending on the amount of peaking and DSM capacity already in the system. The MDS1 scenario required less additional peaking capacity because this generation scenario already contained a large amount of diesel-fired OCGT, fast-starting gas-fired plant, and peaking hydro plant. 
Table 9.19: Generation and demand side management capacity (MW): selected years

\begin{tabular}{|c|c|c|c|c|c|c|}
\hline $\begin{array}{l}\text { Generation } \\
\text { scenario }\end{array}$ & EV recharging regime & 2010 & 2015 & 2020 & 2025 & 2030 \\
\hline \multirow{18}{*}{ MDS1 } & & \multicolumn{5}{|c|}{ A2 (\$25 per tonne) } \\
\hline & & \multicolumn{5}{|c|}{ Total capacity } \\
\hline & Fully interruptible & 9,420 & 10,766 & 12,57 & 15,138 & 16,826 \\
\hline & & \multicolumn{5}{|c|}{ Additional capacity } \\
\hline & Partially interruptible & 0 & 179 & 491 & 825 & 1,344 \\
\hline & Not interruptible & 0 & 461 & 1,268 & 1,685 & 3,225 \\
\hline & & \multicolumn{5}{|c|}{ C4 (\$100 per tonne) } \\
\hline & & \multicolumn{5}{|c|}{ Total capacity } \\
\hline & Fully interruptible & 9,437 & 10,636 & 12,97 & 15,063 & 16,643 \\
\hline & & \multicolumn{5}{|c|}{ Additional capacity } \\
\hline & Partially interruptible & -17 & 484 & 909 & 1,350 & 1,947 \\
\hline & Not interruptible & -17 & 847 & 1,608 & 2,648 & 3,783 \\
\hline & & \multicolumn{5}{|c|}{ C5 (\$100 per tonne) } \\
\hline & & \multicolumn{5}{|c|}{ Total capacity } \\
\hline & Fully interruptible & 9,420 & 10,936 & 13,12 & 15,222 & 16,813 \\
\hline & & \multicolumn{5}{|c|}{ Additional capacity } \\
\hline & Partially interruptible & 0 & 593 & 1,888 & 2,044 & 2,246 \\
\hline & Not interruptible & 0 & 908 & 2,787 & 3,640 & 4,702 \\
\hline \multirow{12}{*}{ MDS3 } & & \multicolumn{5}{|c|}{ A2 (\$25 per tonne) } \\
\hline & & \multicolumn{5}{|c|}{ Total capacity } \\
\hline & Fully interruptible & 9,437 & 10,521 & 11,93 & 12,975 & 14,437 \\
\hline & & \multicolumn{5}{|c|}{ Additional capacity } \\
\hline & Partially interruptible & 0 & 367 & 438 & 1,245 & 1,975 \\
\hline & Not interruptible & 0 & 490 & 1,366 & 2,755 & 4,169 \\
\hline & & \multicolumn{5}{|c|}{ C4 (\$100 per tonne) } \\
\hline & & \multicolumn{5}{|c|}{ Total capacity } \\
\hline & Fully interruptible & 9,437 & 10,636 & 12,97 & 15,063 & 16,644 \\
\hline & & \multicolumn{5}{|c|}{ Additional capacity } \\
\hline & Partially interruptible & 0 & 189 & 717 & 1,343 & 2,158 \\
\hline & Not interruptible & 0 & 620 & 1,820 & 3,300 & 4,436 \\
\hline \multirow{12}{*}{ MDS5 } & & \multicolumn{5}{|c|}{ A2 (\$25 per tonne) } \\
\hline & & \multicolumn{5}{|c|}{ Total capacity } \\
\hline & Fully interruptible & 9,420 & 10,473 & 11,60 & 12,794 & 13,959 \\
\hline & & \multicolumn{5}{|c|}{ Additional capacity } \\
\hline & Partially interruptible & 0 & 399 & 528 & 1,195 & 1,886 \\
\hline & Not interruptible & 0 & 608 & 1,329 & 2,985 & 4,307 \\
\hline & & \multicolumn{5}{|c|}{ C4 (\$100 per tonne) } \\
\hline & & \multicolumn{5}{|c|}{ Total capacity } \\
\hline & Fully interruptible & 9,420 & 10,713 & 11,86 & 13,819 & 15,007 \\
\hline & & \multicolumn{5}{|c|}{ Additional capacity } \\
\hline & Partially interruptible & 0 & 251 & 596 & 1,195 & 1,987 \\
\hline & Not interruptible & 0 & 427 & 1,437 & 2,778 & 4,206 \\
\hline
\end{tabular}




\subsubsection{Levelised cost of energy}

The levelised cost of energy (LCOE) measure was used to compare the costs of the alternative generation scenarios. The LCOE measure provides an estimate of the average price per kWh required for a generation project, or group of projects, to break even, given a specific discount rate. Investors will choose, all other things being equal, the generation and DSM project, or set of projects, that produces the lowest LCOE. The LCOE is not, however, a comprehensive measure of the cost and benefits because it omits the social benefits associated with those projects that reduce $\mathrm{GHG}$ emissions.

In this analysis, the LCOE was calculated using discount rates of $4 \%$ and $8 \%$ to assess the cost of the additional generation required to meet the growth in demand from 2010 to 2030, with and without the additional demand from EVs (Short et al., $1995)^{70}$. The LCOE analysis found that the use of a different discount rate had no effect on the relative LCOE values across the generation scenarios when meeting the same demand.

The LCOE also indicated that if there was the discovery of large quantities of low cost natural gas, the MDS5 scenario would be the least cost generation scenario even if the price of carbon reached $\$ 100$ per tonne.

If there were no large domestic natural gas discoveries, then the MDS3 scenario that allowed for moderate access to hydro sites and no restrictions on the use of coal generation scenario would be the next least cost option. The impact of raising the carbon price to $\$ 100$ per tonne reduced the difference in the LCOE estimates for the generation scenarios, but under both carbon prices, the MDS1 scenario had the highest LCOE (Table 9.20).

\footnotetext{
${ }^{70}$ It was assumed that the base demand would remain constant at 2009 levels, but the carbon price was set at the same level as used in the model run.
} 


\begin{tabular}{|c|c|c|c|}
\hline & MDS 1 & MDS 3 & MDS 5 \\
\hline \multicolumn{4}{|c|}{$\$ 25 /$ tonne } \\
\hline \multicolumn{4}{|c|}{$4 \%$} \\
\hline A1 & 7.82 & 7.03 & 6.58 \\
\hline $\mathrm{A} 2$ & 8.45 & 7.21 & 6.77 \\
\hline \multicolumn{4}{|c|}{$8 \%$} \\
\hline A1 & 7.70 & 6.94 & 6.52 \\
\hline A2 & 8.28 & 7.14 & 6.69 \\
\hline \multicolumn{4}{|c|}{$\$ 100 /$ tonne } \\
\hline \multicolumn{4}{|c|}{$4 \%$} \\
\hline A1 & 8.72 & 8.71 & 8.39 \\
\hline $\mathrm{C} 4$ & 9.31 & 9.23 & 8.58 \\
\hline \multicolumn{4}{|c|}{$8 \%$} \\
\hline A1 & 8.61 & 8.61 & 8.38 \\
\hline $\mathrm{C} 4$ & 10.45 & 10.28 & 9.88 \\
\hline
\end{tabular}

\subsection{Combined LPV fleet and electricity sector GHG emissions}

This section presents the estimates of the GHG emissions from both the LPV fleet and electricity sector.

For all scenarios, the base year (2010) GHG emissions for the LPV fleet and electricity sector combined were estimated to be 14.6 million tonnes $\mathrm{CO}_{2} \mathrm{e}$ (Table 9.21).

\subsubsection{BAU combined GHG emissions}

The business as usual (BAU) represents those futures where no EVs (scenario A1) would be introduced into the New Zealand LPV, but the development of the electricity sector could follow one of the three generation scenarios.

The choice of generation scenario had a significant influence on the BAU GHG emissions projections. The MDS3 scenario, which most closely reflects current government policy and industry practice, resulted in projected combined annual BAU GHG emissions, in 2030, rising to 21.6 million tonnes $\mathrm{CO}_{2} \mathrm{e}$, a $48 \%$ increase above 2010 levels (Figure 9.18 and Table 9.21). If the high gas discovery MDS5 scenario was assumed the most likely development path, the combined annual GHG emissions, in 2030, were projected to increase to 19.1 million tonnes $\mathrm{CO}_{2} \mathrm{e}$, or 
$30.2 \%$ above 2010 levels. Finally, if it was assumed that the policy settings in the MDS1 scenario were applied, then the combined annual GHG emissions, in 2030, were projected to decline marginally to 14.4 million tonnes $\mathrm{CO}_{2} \mathrm{e}$, a reduction of 1.8\% from levels in 2010 (Figure 9.18 and Table 9.21).

The cumulative BAU combined GHG emissions over the modelling period ranged from 383.2 million tonnes $\mathrm{CO}_{2} \mathrm{e}$ under the MDS3 scenario to 311.5 million tonnes $\mathrm{CO}_{2} \mathrm{e}$ under the MDS1 scenario (Table 9.22).

\subsubsection{Combined GHG emissions under a low carbon price regime}

\section{A2 model run}

Modelling indicates that the introduction of EVs under a low carbon price regime would have some moderating effect on the growth of the combined annual GHG emissions from the LPV fleet and electricity sector. However, if the development of the electricity sector followed a path similar to that of the MDS3 (coal permitted) or MDS5 (high gas discovery) generation scenarios, in absolute terms, the annual combined GHG emissions from these two sectors would continue to grow. In a future where the development of the electricity sector followed the MDS3 scenario, annual GHG emissions increased by $40 \%$ in 2030 to 20.5 million $\pm 14,000$ tonnes $\mathrm{CO}_{2} \mathrm{e}$ (Figure 9.20). If the development of the electricity sector followed the MDS5 scenario, the combined annual GHG emissions still increased, but only by $10 \%$, or to 16.1 million $\pm 14,000$ tonnes $\mathrm{CO}_{2} \mathrm{e}$, in 2030 (Figure 9.21).

Only in the MDS1 hydro and DSM scenario, under a low carbon price scenario, did the annual combined GHG emissions decrease. In this generation scenario, annual GHG emissions declined by $17.4 \%$, by 2030 , to 12.1 million $\pm 14,000$ tonnes $\mathrm{CO}_{2} \mathrm{e}$ (Figure 9.19).

In all three generation scenarios the introduction of EVs reduced the cumulative combined GHG emissions, over the modelling period, relative to the BAU projections. The extent to which EVs reduced New Zealand's cumulative GHG 
emissions varied depending on the additional GHG emissions produced from the generation of electricity needed to supply the EV fleet.

In scenario A2, the introduction of EVs was estimated to reduce the LPV fleets' cumulative $\mathrm{GHG}$ emissions by 27.2 million $\pm 41,000$ tonnes $\mathrm{CO}_{2} \mathrm{e}$ relative to the $\mathrm{BAU}$ future where no-EVs were introduced.

When the electricity sector followed the development path as described in the MDS3 scenario, the additional GHG emissions from the refurbished and new fossil fuels plants limited the reduction achieved by the LPV fleet to 3.2 million $\pm 41,000$ tonnes $\mathrm{CO}_{2} \mathrm{e}$, or by $88.2 \%$ (Figure 9.22). Overall, the cumulative GHG emissions in this future were only $0.8 \%$ below a future where $\mathrm{EV}$ s had not been introduced (Table 9.22).

The smallest offsetting effect to the cumulative GHG emissions reductions occurred in the MDS5 scenario where the GHG emissions savings achieved in the LPV fleet were reduced to 22.9 million $\pm 41,000$ tonnes $\mathrm{CO}_{2} \mathrm{e}$ (Figure 9.22).

\subsubsection{Combined GHG emissions with a high carbon price}

\section{C4 model run}

With the price of carbon set at $\$ 100$ per tonne, the projected combined annual GHG emissions from the LPV fleet and the electricity sector in 2030 was $17.4 \%$ to 21.6\% lower than the combined annual GHG emissions in 2010 (Table 9.21).

The projected combined annual GHG emissions in 2030, when compared to each generation scenario's respective low carbon price BAU model run, were between 2.9 million $\pm 21,000$ tonnes $\mathrm{CO}_{2} \mathrm{e}$ and 9.8 million $\pm 21,000$ tonnes $\mathrm{CO}_{2} \mathrm{e}$ lower (Table 9.21).

The effect of the introduction of EVs and the imposition of a higher carbon price was to reduce cumulative combined GHG emissions by between 43.6 million $\pm 57,000$ tonnes $\mathrm{CO}_{2} \mathrm{e}(\mathrm{MDS} 1)$, and 104.0 million $\pm 57,000$ tonnes $\mathrm{CO}_{2} \mathrm{e}$ (MDS3), relative to each generation scenario's low carbon price BAU (Table 9.22). 
Separating the influence of the increased price of carbon and the introduction of EVs indicated that the contribution of EVs versus the introduction of a high carbon price in reducing New Zealand's GHG emissions would be dependent on the future development path of the electricity sector. Where the use of fossil fuels was not precluded, as in the MDS3 and MDS5 generation scenarios, the increased price of carbon would have more of an effect in reducing GHG emissions than the introduction of EVs. If the construction of new fossil fuel plants were banned, as in MDS1, then the introduction of EVs had more of an effect in reducing GHG emissions than the imposition of a high carbon price (Table 9.23).

The imposition of a $\$ 100$ per tonne carbon price was effective in reducing the GHG emissions offsetting effect from electricity generation in the MDS3 scenario. In scenario C4, the cumulative GHG emissions reductions achieved in the LPV fleet due to the introduction of EVs were 35.7 million $\pm 57,000$ tonnes. With the high carbon price, the additional GHG emissions from electricity generation only offset the LPV fleet reductions by $29.4 \%$ to 25.2 million $\pm 57,000$ tonnes reduction in cumulative GHG emissions (Figure 9.22).

\section{C5 model run}

This model run resulted in the greatest reductions in annual and cumulative GHG emissions. In 2030, the combined annual GHG emissions from the LPV fleet and the electricity sector were projected to decline to 10.4 million $\pm 11,000 \mathrm{CO}_{2} \mathrm{e}, 27.8 \%$ lower than the estimated annual GHG emissions in 2010 (Table 9.21 and Figure 9.19).

The reductions in total cumulative combined GHG emissions in this model run were projected to be 54.4 million $\pm 40,000$ tonnes lower than those projected in the low carbon price BAU model run (Table 9.22). 
Figure 9.18: Combined annual GHG emissions from the light passenger vehicle fleet and electricity sector - A1 (no-EV)

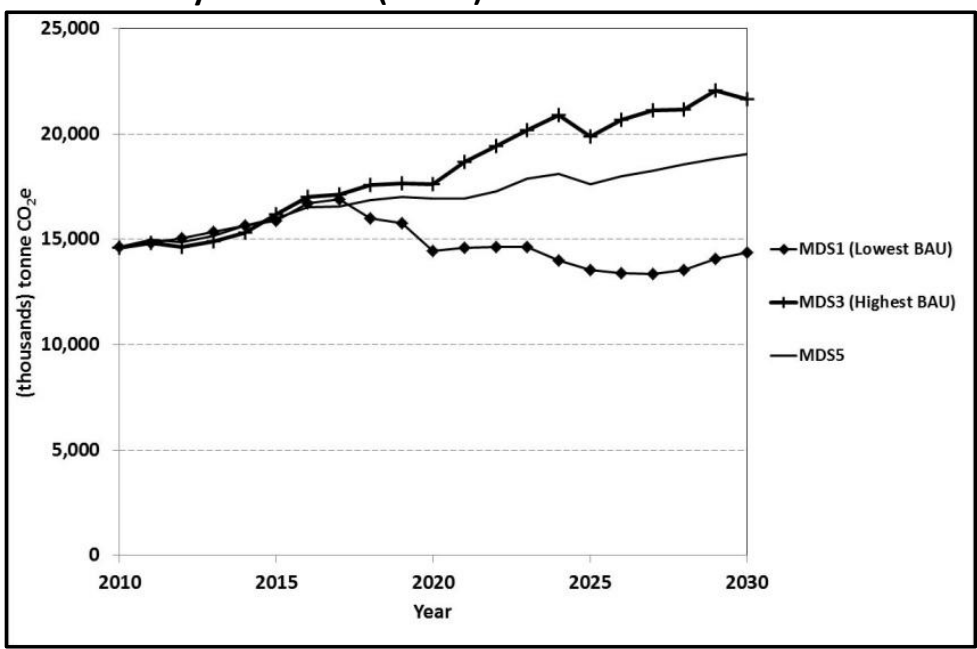

Figure 9.19: Combined annual GHG emissions from the light passenger vehicle fleet and electricity sector - MDS1

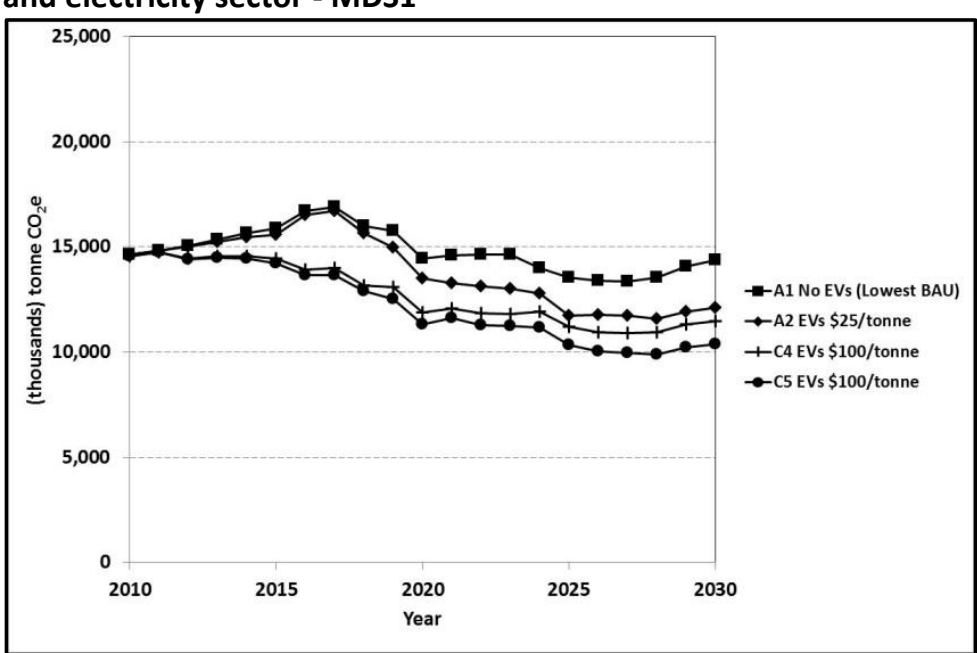

Figure 9.20: Combined annual GHG emissions from the light passenger vehicle fleet and electricity sector - MDS3

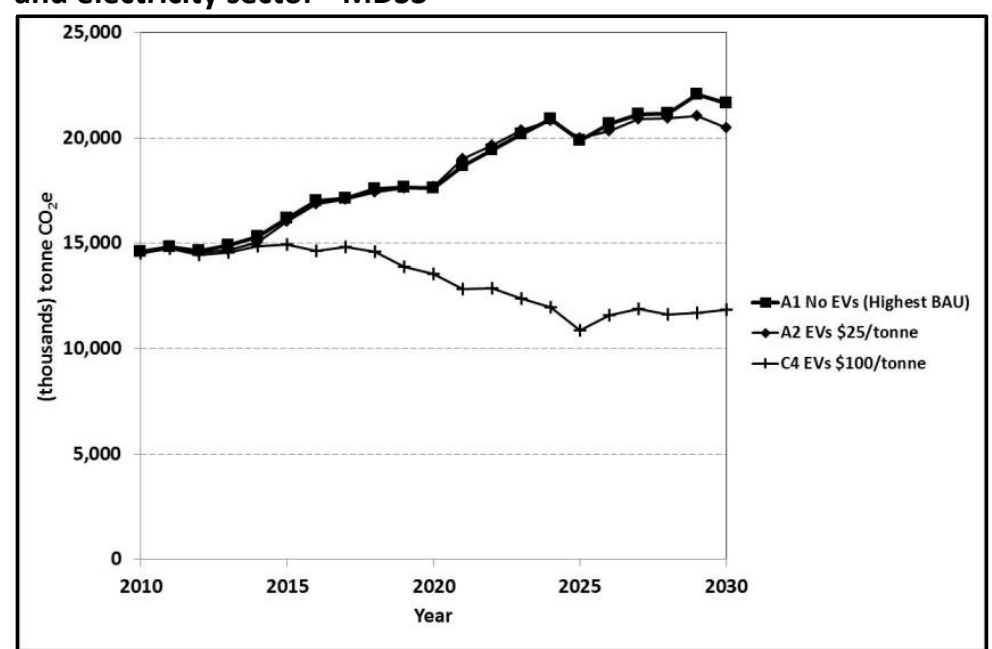

Figure 9.21: Combined annual GHG emissions from the light passenger vehicle fleet and electricity sector - MDS5

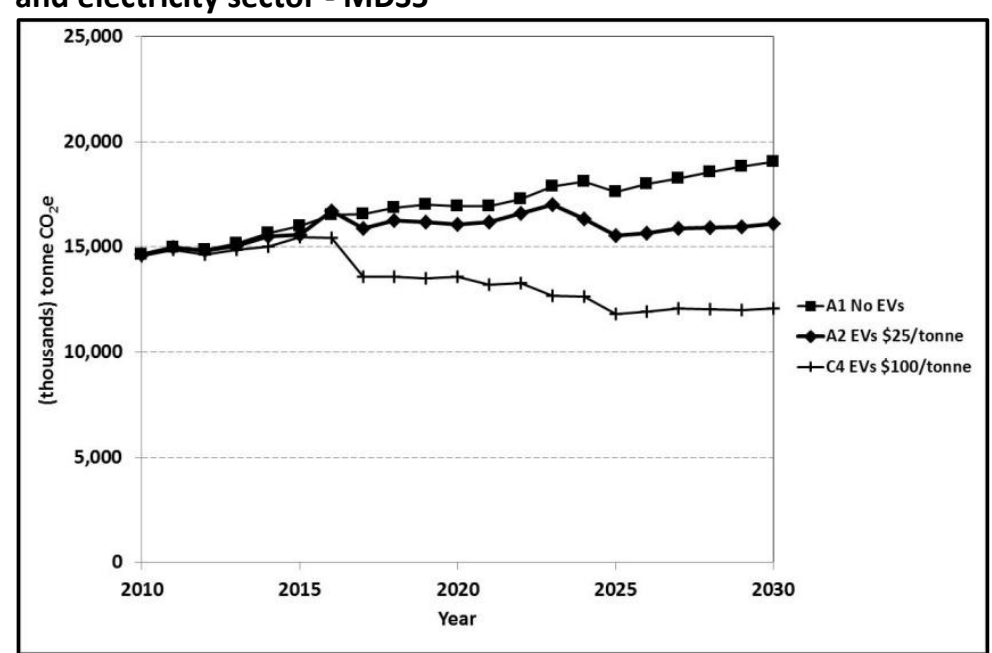


Table 9.21: Annual GHG emissions from the light passenger vehicle fleet and electricity sector (thousand tonnes $\mathrm{CO}_{2} \mathrm{e}$ )

\begin{tabular}{|c|c|c|c|c|c|c|c|c|c|c|c|c|c|c|c|}
\hline & \multirow[b]{2}{*}{$\begin{array}{c}\text { GHG } \\
\text { emissions } \\
\text { in } 2010\end{array}$} & \multicolumn{14}{|c|}{ GHG emissions in 2030} \\
\hline & & $\begin{array}{c}\text { A1 } \\
\$ 25 / \\
\text { tonne } \\
2030(B A U)\end{array}$ & $\begin{array}{l}\text { Diff } \\
\text { from } \\
2010\end{array}$ & $\begin{array}{c}\text { A2 } \\
\text { EV \$25/ } \\
\text { tonne } \\
2030\end{array}$ & $\begin{array}{l}\text { Diff } \\
\text { from } \\
2010\end{array}$ & $\begin{array}{c}\text { (A1-A2) } \\
\text { Change } \\
\text { due to EVs }\end{array}$ & $\begin{array}{l}\text { Diff } \\
\text { from } \\
2030 \\
\text { BAU }\end{array}$ & $\begin{array}{c}\text { C4 } \\
\text { EV \$100/ } \\
\text { tonne } \\
2030\end{array}$ & $\begin{array}{l}\text { Diff } \\
\text { from } \\
2010\end{array}$ & $\begin{array}{c}\text { (A1-C4) } \\
\text { Change due to } \\
\text { EVs and } \\
\text { increase in } \\
\text { carbon price }\end{array}$ & $\begin{array}{c}\text { Diff from } \\
2030 \\
\text { BAU }\end{array}$ & $\begin{array}{c}\text { C5 } \\
\text { EV } \$ 100 / \\
\text { tonne } \\
2030\end{array}$ & $\begin{array}{c}\text { Diff } \\
\text { from } \\
2010\end{array}$ & $\begin{array}{c}\text { (A1-C5) } \\
\text { Change due } \\
\text { to EVs and } \\
\text { increase in } \\
\text { carbon } \\
\text { price }\end{array}$ & $\begin{array}{l}\text { Diff } \\
\text { from } \\
2030 \\
\text { BAU }\end{array}$ \\
\hline MDS1 & 14,637 & 14,376 & $-1.8 \%$ & $\begin{array}{c}12,097 \\
\pm 14 \\
\end{array}$ & $-17.4 \%$ & $\begin{array}{c}2,279 \\
\pm 14 \\
\end{array}$ & $-15.8 \%$ & $\begin{array}{c}11,480 \\
\pm 21 \\
\end{array}$ & $-21.6 \%$ & $\begin{array}{c}2,896 \\
\pm 21 \\
\end{array}$ & $-20.1 \%$ & $\begin{array}{c}10,381 \\
\pm 11 \\
\end{array}$ & -28.7 & $\begin{array}{c}4,256 \\
\pm 11 \\
\end{array}$ & $-27.8 \%$ \\
\hline MDS3 & 14,637 & 21,655 & $48.0 \%$ & $\begin{array}{c}20,492 \\
\pm 14\end{array}$ & $40.0 \%$ & $\begin{array}{c}1,163 \\
\pm 14 \\
\end{array}$ & $-5.4 \%$ & $\begin{array}{c}11,844 \\
\pm 21 \\
\end{array}$ & $-19.1 \%$ & $\begin{array}{c}9,811 \\
\pm 21 \\
\end{array}$ & $-45.3 \%$ & $\mathrm{n} / \mathrm{a}$ & $\mathrm{n} / \mathrm{a}$ & $\mathrm{n} / \mathrm{a}$ & $\mathrm{n} / \mathrm{a}$ \\
\hline MDS5 & 14,637 & 19,057 & $30.2 \%$ & $\begin{array}{c}16,100 \\
\pm 14\end{array}$ & $10.0 \%$ & $\begin{array}{c}2,957 \\
\pm 14\end{array}$ & $-15.5 \%$ & $\begin{array}{c}12,085 \\
\pm 21\end{array}$ & $-17.4 \%$ & $\begin{array}{c}6,972 \\
\pm 21\end{array}$ & $-36.6 \%$ & $\mathrm{n} / \mathrm{a}$ & $\mathrm{n} / \mathrm{a}$ & $\mathrm{n} / \mathrm{a}$ & $\mathrm{n} / \mathrm{a}$ \\
\hline
\end{tabular}

$\pm \quad 95 \%$ confidence intervals

Table 9.22: Cumulative GHG emissions from the light passenger vehicle fleet and electricity sector (thousand tonnes $\mathrm{CO}_{2} \mathrm{e}$ ): 2010 to 2030

\begin{tabular}{|c|c|c|c|c|c|c|c|c|c|c|}
\hline & $\begin{array}{c}\text { A1 } \\
\text { \$25 per } \\
\text { tonne } \\
\text { (BAU) }\end{array}$ & $\begin{array}{c}\text { A2 } \\
\text { EV } \$ 25 \text { per } \\
\text { tonne }\end{array}$ & $\begin{array}{l}\text { (A1-A2) } \\
\text { Change due } \\
\text { to EVs }\end{array}$ & $\begin{array}{l}\text { Difference } \\
\text { from BAU }\end{array}$ & $\begin{array}{c}\text { C4 } \\
\text { EV } \$ 100 \text { per } \\
\text { tonne }\end{array}$ & $\begin{array}{l}\text { (A1-C4) } \\
\text { Change due } \\
\text { to EVs and } \\
\text { increase in } \\
\text { carbon price }\end{array}$ & $\begin{array}{l}\text { Difference } \\
\text { from BAU }\end{array}$ & $\begin{array}{c}\text { C5 } \\
\text { EV } \$ 100 \text { per } \\
\text { tonne }\end{array}$ & $\begin{array}{l}\text { (A1-C5) } \\
\text { Change due } \\
\text { to EVs and } \\
\text { increase in } \\
\text { carbon price }\end{array}$ & $\begin{array}{l}\text { Difference } \\
\text { from BAU }\end{array}$ \\
\hline MDS1 & 311,486 & $\begin{array}{c}291,185 \\
\pm 41 \\
\end{array}$ & $\begin{array}{c}20,301 \\
\pm 41 \\
\end{array}$ & $-6.5 \%$ & $\begin{array}{c}267,927 \\
\pm 57 \\
\end{array}$ & $\begin{array}{c}43,559 \\
\pm 57 \\
\end{array}$ & $-14.0 \%$ & $\begin{array}{c}257,077 \\
\pm 40 \\
\end{array}$ & $\begin{array}{c}54,409 \\
\pm 40 \\
\end{array}$ & $-17.5 \%$ \\
\hline MDS3 & 383,162 & $\begin{array}{c}379,950 \\
\pm 41\end{array}$ & $\begin{array}{c}3,212 \\
\pm 41 \\
\end{array}$ & $-0.8 \%$ & $\begin{array}{c}279,171 \\
\pm 57\end{array}$ & $\begin{array}{c}103,991 \\
\pm 57\end{array}$ & $-27.1 \%$ & $\mathrm{n} / \mathrm{a}$ & $\mathrm{n} / \mathrm{a}$ & $\mathrm{n} / \mathrm{a}$ \\
\hline MDS5 & 355,778 & $\begin{array}{c}332,882 \\
\pm 41\end{array}$ & $\begin{array}{c}22,896 \\
\pm 41\end{array}$ & $-6.4 \%$ & $\begin{array}{c}282,865 \\
\pm 57\end{array}$ & $\begin{array}{c}72,913 \\
\pm 57\end{array}$ & $-20.5 \%$ & $n / a$ & $n / a$ & $n / a$ \\
\hline
\end{tabular}

$\pm \quad 95 \%$ confidence intervals 


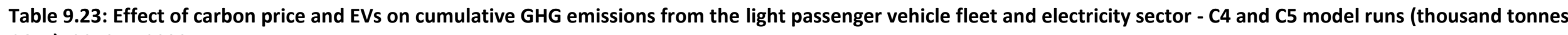
$\left.\mathrm{CO}_{2} \mathrm{e}\right): 2010$ to 2030

\begin{tabular}{|c|c|c|c|c|c|c|c|c|c|}
\hline \multicolumn{10}{|c|}{ Cumulative GHG emissions } \\
\hline & $\begin{array}{l}\text { No EVs } \\
\$ 25 \text { per } \\
\text { tonne }\end{array}$ & $\begin{array}{l}\text { No EVs } \\
\$ 100 / \\
\text { tonne }\end{array}$ & $\begin{array}{l}\text { Change due } \\
\text { to carbon } \\
\text { price }\end{array}$ & $\begin{array}{l}\text { EV } \$ 100 / \text { tonne } \\
\text { (C4 model run) }\end{array}$ & $\begin{array}{c}\text { Change } \\
\text { due to EVs }\end{array}$ & $\begin{array}{l}\text { Proportion of total } \\
\text { GHG reductions due } \\
\text { to EVs }\end{array}$ & $\begin{array}{l}\text { EV } \$ 100 / \text { tonne } \\
\text { (C5 model run) }\end{array}$ & $\begin{array}{c}\text { Change due } \\
\text { to EVs }\end{array}$ & $\begin{array}{l}\text { Proportion of total } \\
\text { GHG reductions due to } \\
\text { EVs }\end{array}$ \\
\hline MDS1 & 311,486 & 296,610 & $-14,876$ & $\begin{array}{c}267,927 \\
\pm 57\end{array}$ & $\begin{array}{c}-28,683 \\
\pm 57\end{array}$ & $65.8 \%$ & $\begin{array}{c}257,077 \\
\pm 40\end{array}$ & $\begin{array}{c}39,533 \\
\pm 40\end{array}$ & $72.7 \%$ \\
\hline MDS3 & 383,162 & 304,318 & $-78,844$ & $\begin{array}{c}279,171 \\
\pm 57 \\
\end{array}$ & $\begin{array}{c}-25,147 \\
\pm 57 \\
\end{array}$ & $24.2 \%$ & $\mathrm{n} / \mathrm{a}$ & $\mathrm{n} / \mathrm{a}$ & $\mathrm{n} / \mathrm{a}$ \\
\hline MDS5 & 355,778 & 312,027 & $-43,751$ & $\begin{array}{c}282,865 \\
\pm 57 \\
\end{array}$ & $\begin{array}{c}-29,162 \\
\pm 57\end{array}$ & $40.0 \%$ & $\mathrm{n} / \mathrm{a}$ & $\mathrm{n} / \mathrm{a}$ & $\mathrm{n} / \mathrm{a}$ \\
\hline
\end{tabular}


Figure 9.22: Reductions in cumulative GHG emissions from EVs - light passenger vehicle fleet only, and combined light passenger vehicle fleet and electricity sector: 2010 to 2030

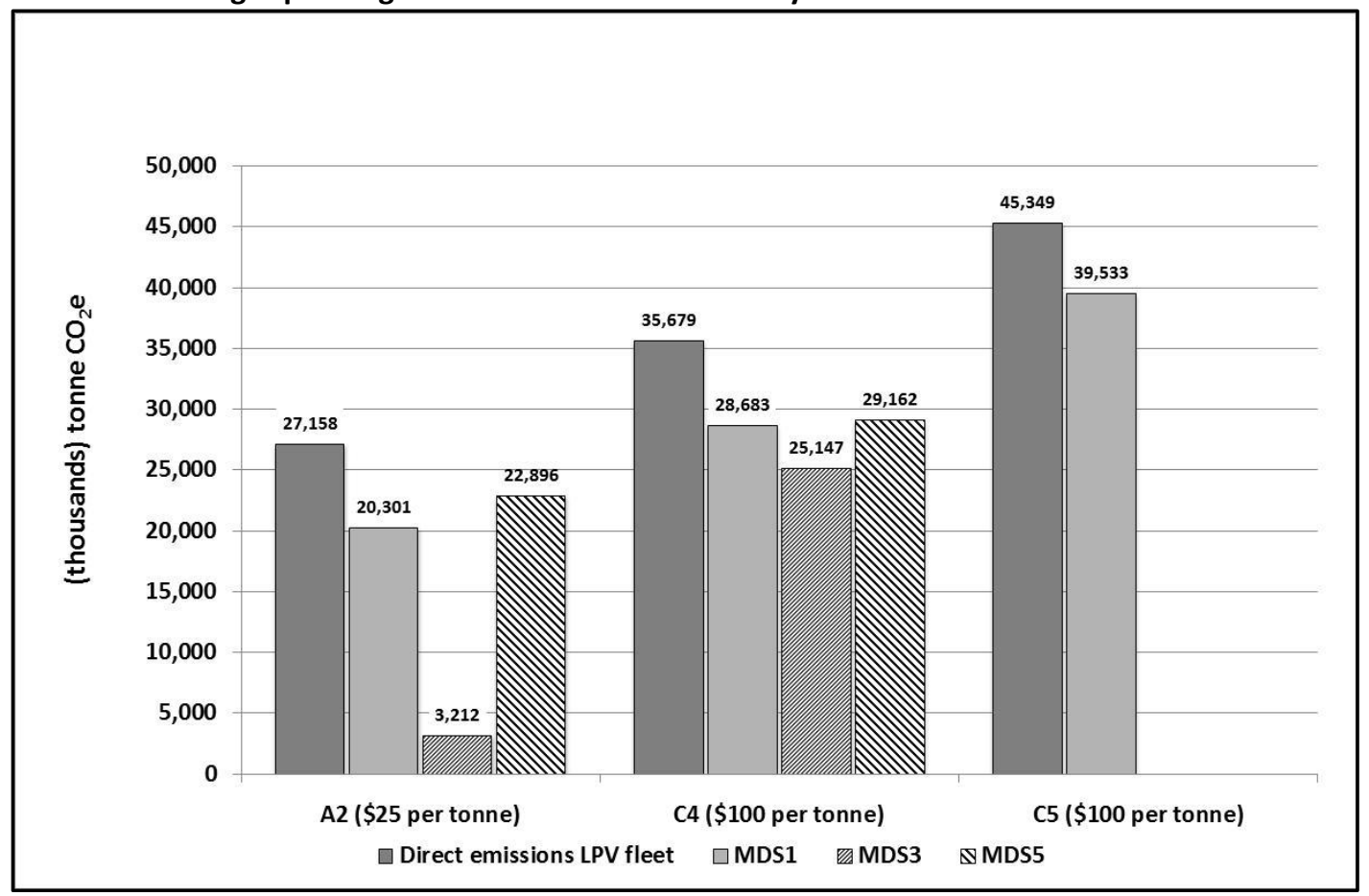

\subsection{Discussion}

\subsubsection{Impact of EVs on LPV fleet energy efficiency}

The projections of energy demand from the LPV fleet were sensitive to assumptions made about the energy efficiency of both EVs and ICEVs. The approach taken for EVs in this study was to recognise the differences in energy efficiency between small CEVs, GEVs, and PHEVs and to take account of the effects of real world driving behaviour.

Dividing the EV fleet into groups resulted in EV energy efficiency values that were not readily comparable to those used in other New Zealand studies. However, where they were comparable, the estimates in this study appeared to be higher than the estimates in most of the other studies with the exception of those used by Baxter et al. (2009) and Duke et al. (2009) (Table 9.24). 
Table 9.24: EV energy efficiency estimates used in New Zealand studies (Wh/km)

\begin{tabular}{|c|c|c|c|c|c|c|}
\hline & Clover & $\begin{array}{c}\text { Dirr } \\
(2008)\end{array}$ & $\begin{array}{c}\text { Leaver } \\
\text { and } \\
\text { Gillingham } \\
\text { (2010) }\end{array}$ & $\begin{array}{c}\text { Baxter } \\
\text { et al. } \\
\text { (2009) }\end{array}$ & $\begin{array}{c}\text { Duncan } \\
\text { et al. } \\
(2010)\end{array}$ & $\begin{array}{c}\text { Duke et al. } \\
\text { (2009) }\end{array}$ \\
\hline CEV & $\begin{array}{c}\text { 177-174 } \\
\text { (standard } \\
\text { efficiency) } \\
115-114 \\
\text { (high efficiency) }\end{array}$ & $\mathrm{n} / \mathrm{a}$ & $\mathrm{n} / \mathrm{a}$ & $\mathrm{n} / \mathrm{a}$ & $\mathrm{n} / \mathrm{a}$ & $\begin{array}{c}84.6 \\
\text { (high } \\
\text { efficiency) }\end{array}$ \\
\hline GEV & $\begin{array}{c}\text { 209-208 } \\
\text { (standard } \\
\text { efficiency) } \\
134-133 \\
\text { (high efficiency) }\end{array}$ & $\mathrm{n} / \mathrm{a}$ & $\mathrm{n} / \mathrm{a}$ & $\mathrm{n} / \mathrm{a}$ & $\mathrm{n} / \mathrm{a}$ & $\begin{array}{c}216 \text { (ICEV } \\
\text { EV } \\
\text { conversion) } \\
126 \\
\text { (high } \\
\text { efficiency) }\end{array}$ \\
\hline BEV & $\begin{array}{c}\text { Weighted } \\
\text { average } \\
188-182 \\
\text { (standard } \\
\text { efficiency) } \\
122-121 \\
\text { (high efficiency) }\end{array}$ & $\begin{array}{c}150 \\
(2010) \\
100 \\
(2030)\end{array}$ & $\begin{array}{l}150(2015) \\
130(2030)\end{array}$ & 204 & 175 & $\mathrm{n} / \mathrm{a}$ \\
\hline $\begin{array}{c}\text { PHEV } \\
\text { (electric } \\
\text { cycle) }\end{array}$ & $\begin{array}{c}\text { 209-208 } \\
\text { (standard } \\
\text { efficiency) } \\
134-133 \\
\text { (high efficiency) }\end{array}$ & $\mathrm{n} / \mathrm{a}$ & $\mathrm{n} / \mathrm{a}$ & 204 & 175 & $\mathrm{n} / \mathrm{a}$ \\
\hline $\begin{array}{c}\text { All EVs } \\
\text { (electric } \\
\text { cycle) }\end{array}$ & $\begin{array}{c}\text { Weighted } \\
\text { average } \\
199-195 \\
\text { (standard } \\
\text { efficiency) } \\
126-127 \\
\text { (high efficiency) }\end{array}$ & $\mathrm{n} / \mathrm{a}$ & $\mathrm{n} / \mathrm{a}$ & $\mathrm{n} / \mathrm{a}$ & $\mathrm{n} / \mathrm{a}$ & $\mathrm{n} / \mathrm{a}$ \\
\hline
\end{tabular}

\subsubsection{Projected electricity demand for the EV fleet}

In this study, the additional annual electricity demand in 2030 due to the introduction of EVs was estimated to be between 2,700 GWh and 3,700 GWh depending on the level of EV uptake, mix of EV types, and the energy efficiency of the EVs. When compared to the other New Zealand studies the estimated additional electricity demand in this study were broadly similar (Table 9.25) even though this study projected higher levels of EV uptake (see Table 8.8). A factor in the lower estimated additional demand relative to most studies was the separation 
of EVs into CEVs, GEVs, and PHEVs in this study. The greatest demand for EVs would be for PHEVs, which only use electricity part of the time and for the smaller, more energy efficient CEVs and as a result, even with the higher projected uptake of EVs, there was a lower overall additional demand for electricity.

Table 9.25: Comparison of New Zealand EV studies, EV stock and additional electricity demand in 2030

\begin{tabular}{|c|c|c|c|c|}
\hline & Clover & Dirr (2008) & $\begin{array}{c}\text { Baxter et al. } \\
\text { (2009) }\end{array}$ & $\begin{array}{c}\text { Duncan et al. } \\
\text { (2010) }\end{array}$ \\
\hline $\begin{array}{c}\text { Annual } \\
\text { electricity } \\
\text { Demand } \\
\text { (GWh) }\end{array}$ & $2,700-3,700$ & $1,000-4,000$ & 3,000 & $700-3,000$ \\
\hline
\end{tabular}

\subsubsection{Impact of EVs on the development of the electricity sector}

The use of the Electricity Commission's GEM allowed for the assessment of the extent to which the reductions, due to the introduction of EVs, in the GHG emissions from the LPV fleet would be offset by increased GHG emissions from the electricity sector. Three generation scenarios from the Electricity Commission's 2010 Statement of Opportunities report were adapted for use in this study.

The MDS1 and MDS3 generation scenarios were chosen as they represented the two primary alternative policy paths for the future development of the electricity generation sector. The MDS5 scenario was included in recognition of the important role that low cost natural gas has had in the generation of electricity in New Zealand and that new discoveries of low cost natural gas could occur in the future (Crown Minerals, 2010; Ministry of Business Innovation and Employment, 2012).

The analysis using the GEM indicated that future GHG emissions from the electricity sector would be most influenced by the extent to which coal would be used. The impact of coal generation in the production of GHG emissions from the electricity sector was demonstrated in the MDS3 scenario. In the A2 model run where it was assumed that there would be a low carbon price, some or all of the units at Huntly would be refurbished and three large new coal plants located in the northern half of the North Island would be constructed. This resulted in annual GHG emissions 
from the electricity sector doubling from 7.4 million tonnes $\mathrm{CO}_{2} \mathrm{e}$ in 2010 to 14.8 million tonnes $\mathrm{CO}_{2} \mathrm{e}$ in 2030.

In the C4 model run, it was assumed that there would be a high carbon price regime. In this model run, no Huntly units were refurbished and only two new coal plants were built, which were then used less often. By 2030, annual GHG emissions in this model run declined to 7.1 million tonnes $\mathrm{CO}_{2} \mathrm{e}$, a reduction from 2010 levels of 3.9\%. This reduction in GHG emissions occurred even though scenario C4 had a higher projected electricity demand from EVs.

The general effect of a higher carbon price in the GEM model runs was to bring forward the timing of the retirement of old gas and coal plants, and the construction of renewable generation and DSM projects.

The GEM analysis indicated that, if the system operator had the ability to interrupt the recharging of all EVs at times of peak demand, then the amount of additional capacity required to be installed to meet the demand from EVs would be less than $730 \mathrm{MW}$ in 2030 (Table 9.18).

However, if the system operator had only partial ability, or no ability, to interrupt the recharging of EVs at times of peak demand, the amount of installed capacity required to meet the $n-1$ security requirement would have to increase significantly. The analysis indicated that the amount of additional installed capacity would vary depending on the generation scenario, but ranged from 1,344 to 2,158 MW if EV recharging was partially interruptible and from 3,224 to 4,436 MW if EV recharging was completely uninterruptible by the system operator (Table 9.19). The additional generation capacity required to meet the expected contribution from EVs to peak demand will be largely determined by the degree that EV charging is interruptible.

The analysis was based on the assumption that EVs would be recharged using standard recharging units, which have a maximum demand of $5 \mathrm{kVA}$, and an average demand of $4 \mathrm{kVA}$. These EV recharging units would be able to operate within the present configuration of New Zealand's low voltage networks. If vehicle recharging units that draw higher loads were installed, this would require upgrading 
of the local networks to three phase supply, or the development of public fast recharging facilities. It is unlikely that such developments would occur without the requirement to incorporate fully interruptible control capability.

\subsubsection{Impact of EVs on New Zealand's GHG emissions}

This analysis indicated that if EVs were available for sale, there would be sufficient demand for EVs to yield significant reductions in the GHG emissions directly produced by the New Zealand LPV fleet.

The level of demand for EVs and thus the extent of the GHG emissions reductions would be dependent on the future development of the EV technology, the future price of oil, and the policies put in place to promote the demand for EVs. The LPV fleet use modelling indicated that reductions in the cumulative GHG emissions over the 2010 to 2030 modelling period of $16 \%$ to $27 \%$ would be achievable compared to a BAU future where no EVs were available for sale (Table 9.9). The VFM analysis indicated that greater reductions were possible in some VFM scenarios, but these were, in large part, due to the effect of high petroleum prices on the demand for travel by the users of ICEVs.

When the additional GHG emissions from the generation of electricity for the EV fleet were taken into account, the reductions in the combined cumulative GHG emissions from both the LPV fleet and electricity sector ranged from $0.8 \%$ to $27.1 \%$ when compared to each generation scenario's BAU GHG emissions projection.

This result demonstrates that the effectiveness of EVs in reducing New Zealand's GHG emissions is not dependent just on the development in the vehicle sector but also on the future development of New Zealand's electricity generation sector and the price that electricity generators would pay for using fossil fuels (Figure 9.20).

The GEM was run using the using the MDS1, MDS3, and MDS5 generation scenarios, but unless there were discoveries of large reserves of cheap natural gas, the most probable development path for the electricity sector would be one that was similar to that of the MDS3 scenario under a low carbon price regime. This 
generation scenario most closely reflects the Government's current policy and industry practice. The LCOE analysis also indicated that, in the absence of the gas discoveries envisaged in the MDS5 scenario, the MDS3 scenario had a lower LCOE than the MDS1 scenario. This implies that the suite of generation projects in the MDS3 scenario would, all else being equal, be more attractive to investors.

The MDS3 scenario, in combination with the output from VFM scenarios A1 (no-EV) and $A 2$, was considered to be the more probable of the futures investigated in this study, in the absence of a strong political will to place a high price on carbon emissions. The combination of the VFM scenario A1 (no-EV) and MDS3 scenario reflects a continuation of current conditions in the transport and electricity sectors, including the present limited supply of EVs in New Zealand's vehicle market and the current low carbon price regime.

Scenario A2 described a future where it was assumed that the introduction of EVs in New Zealand would be the result of the spillover of the efforts to promote EVs in other countries and not be due to efforts by the New Zealand Government. At present, the supply of EVs more closely reflects scenario A1 (no-EV), but with the recent availability of the Holden Volt PHEV, and the Mitsubishi i-MIEV and Nissan Leaf BEVs, there are early indications that vehicle manufacturers and importers are beginning to view EVs as a viable alternative for New Zealand car buyers. However, scenario A2 did assume a more aggressive position on the potential of EVs by vehicle manufacturers and importers both internationally and in New Zealand than is currently being taken. If this situation continues, then the A1 (no-EV)/MDS3 scenario combination is the more probable of these two futures.

The scenario C4 and MDS3 scenario combination under a high price on carbon emissions was considered the next most probable of the model runs. This combination represents a future where current international effort to implement policies to address climate change would be successful, including the imposition of a carbon price that more accurately reflects the real social cost of climate change. 
Scenarios $A 2, C 4$, and $C 5$, which occurred in conjunction with the MDS1 and MDS5 generation scenarios, were considered less probable of the futures. The MDS1 generation scenario relied on the assumption that there would be unfettered access to New Zealand's hydro sites. Hydro expansion on this scale seems very unlikely as New Zealand has a long history of environmental activism focused on the prevention of the development of hydro sites (Kelly, 2011; O'Brien, 2012), but there is substantial potential for further development of geothermal and wind technology under a high carbon price regime. By comparison, the MDS3 scenario contained a more limited, and perhaps more realistic, view for the potential development of new hydro sites.

VFM scenarios A2 and C4 in combination with the MDS5 scenario were also considered less probable futures than in combination with the MDS3 scenario. The MDS5 scenario was based on the assumption that there would be large quantities of low cost natural gas available until 2030. This was considered unlikely, because it would depend on the existence of a large amount of natural gas waiting to be found in New Zealand at a low cost. There is potential for the discovery of large amounts of gas in the unexplored deep-water offshore basins. However, the development of discoveries in these basins would require the development of costly new production, refining, and transport infrastructures with an associated impact on the cost of the natural gas produced (Ministry of Business Innovation and Employment, 2012). There is also the possibility that a sufficiently large offshore discovery, if found, would result in the development of a liquefied natural gas (LNG) export facility. Under these conditions, the domestic price of gas would increase to that of the international price (Hemery, 2009). 


\section{Chapter 10: Summary and conclusions}

\subsection{Summary of the study}

This study represents the first use of stated choice methods to assess the potential of light passenger electric vehicles (EVs) in assisting in the reduction of greenhouse gas (GHG) emissions from New Zealand's road transport sector. This is also the first study to combine stated choice methods with electricity generation expansion modelling to provide an integrated view of the impact of EV uptake on the future development of electricity generation and electricity sector GHG emissions.

The stated choice survey incorporates a number of unique design features. The design recognises that there two types of EV technology entering the market battery electric vehicles (BEVs) and plug-in hybrid electric vehicles (PHEVs) - which have different characteristics and will appeal to different segments of the car buying market. The survey design also recognises that BEV technology is particularly suited for use in urban environments and that consequently there will be a market for specialised small city EVs (CEVs).

This design also recognised that these different types of EVs have different energy requirements, which will have an impact on the GHG emissions that they directly or indirectly produce.

The option of purchasing a used imported vehicle from Japan is another key feature of the New Zealand car market and the design of the stated choice survey incorporates the option of a New Zealand car buyer choosing a used imported EV.

For this study, a vehicle fleet model (VFM) of light passenger vehicles (LPVs) was constructed. This model was used to simulate the stock of LPVs, travel demand, and LPV energy demand over a twenty-year period. The VFM utilised the discrete choice model's outputs to simulate the demand for ICEVs and the different types of EV, taking account of the effects of changing EV prices, improving EV technology, changing fuel costs, and the development of a public EV charging network. 
A critical issue in the evaluation of the ability of EVs to reduce GHG emissions is the impact of the additional electricity demand on the GHG emissions from electricity generation. The approach used in this study was to simulate the evolution of the electricity generation sector, and its GHG emissions, over the modelling period using the New Zealand Electricity Commission's generation expansion model (GEM). The GEM is a mixed integer programming optimisation model and is one of the most detailed generation forecast model for the New Zealand electricity system currently available. The GEM provided a means of modelling the GHG emissions from the New Zealand electricity sector with a level of "realism" not achievable using either life-cycle-analysis or other modelling approaches. The GEM was also used to provide insights of the impact of introducing EVs on GHG emissions in various policy environments - where the future development of fossil fuel generation plants range from permitted, to discouraged, or banned outright.

\subsection{Limitations of the study}

This study has limitations that are a consequence of the choice of methodology, resource constraints that affected the design and implementation of the stated choice survey, and constraints imposed by the design of the GEM.

When based on data from stated choice surveys, discrete choice models do not take into account the effects of limited availability of the product on the estimated market shares. These models will tend to overestimate the demand for a new product if there is a period of limited availability. Experience with the introduction of hybrid electric vehicles and current experience with EVs indicates that vehicle manufacturers will probably introduce new EV makes and models incrementally into the market. This implies an extended period when there could be a limited selection of EVs available for purchase in global markets.

Rather than anticipate the future market strategies of EV manufacturers, this study has addressed the question of whether New Zealand car buyers would buy EVs and what effect changing EV technology, prices, and policies would have on this demand. Thus for this study it was assumed that new EVs would be widely available 
for purchase in both New Zealand and overseas markets from the first year of introduction, which was assumed to be 2012. However, it was possible to forecast the availability of used imported EVs based on the current experience with the importation of used vehicles from Japan and the effect of the Japanese vehicletesting regime, which encourages Japanese car owners to replace their aging vehicles with newer vehicles.

Study resource constraints prevented the development and implementation of a separate survey to collect data from organisations that buy cars. These organisations are responsible for the purchase of the majority of new LPVs that enter New Zealand. By using the household car choice model as a proxy, it was possible to take account of these car buyers in this study. In this study, the household discrete choice model combines the new and used internal combustion engine vehicles (ICEVs) into one alternative and this simplification should be relaxed when designing a separate discrete model based on the observed car buying behaviour of organisations.

This study made use of an existing electricity generation expansion model (GEM), whose purpose was to help the Electricity Commission (now the Electricity Authority) in its regulatory role of evaluating the validity of Transpower's grid upgrade proposals. The GEM's focus is on the impact of the development of largescale grid linked generation projects on the transmission grid. Consequently, the GEM's database of potential generation and demand side management (DSM) projects excludes small-scale (less than $10 \mathrm{MW}$ ) distributed generation and DSM projects that may be implemented at the residential, business, or community scales, many of which will utilise emerging renewable generation and DSM technologies.

\subsection{Conclusions}

The results of this study indicate that given the current state of EV technology, and if CEVs, PHEVs, and the larger general-purpose electric vehicles (GEVs) were widely available for sale in New Zealand, these vehicles would comprise approximately 
$20 \%$ of the sales of all LPVs entering the LPV fleet in the first year of availability. This level of demand is much higher than is currently observed in the United States market. This result could imply that the preferences of United States car buyers are significantly different from those of New Zealand car buyers, or that the responses in the stated choice survey contained an element of hypothetical bias. However, the low uptake of EVs in the United States must in part be due to the limited range of makes and models of EVs that are currently available for sale in that market.

This study found that the demand for EVs is most sensitive to reductions in the purchase price of EVs, but reductions in the cost of replacement batteries, increased driving range for BEVs, increased ICEV fuel costs, and increasing availability of charging facilities also have the expected impact in increasing the demand for EVs.

The output from the VFM indicates that, if the improvements in EV technology and reductions in EV prices follow the projections in the 'no technology breakthrough' VFM scenarios, the combined uptake for all types of EVs entering the LPV fleet will reach a maximum level of $56 \%$ to $62 \%$ of all LPVs entering the LPV fleet. Only in the technology breakthrough VFM scenario, where the projected price of batteries declines to $\$ 150$ per $\mathrm{kWh}$, would the saturation rate reach the higher level of $70 \%$ of all LPVs entering the LPV fleet.

The degree to which EVs reduced GHG emissions over the modelling period depended on how long it took to reach the level of maximum EV demand relative to ICEVs. The VFM model shows that the demand for EVs is largely a function of the purchase price: the faster the price declines the faster the demand for EVs increases. The level of maximum EV demand was determined by the limit on the price ratio between EVs and ICEVs. In VFM scenarios $A 2$ and $A 3$, the decline in the price of EV technology occurs at a modest rate, with the prices reaching the minimum level considered achievable with current EV technology in 2030. This assumed slow rate of price decline resulted in the lowest projected uptake of EVs into the New Zealand LPV fleet of all the VFM scenarios modelled, which meant 
that in these two scenarios the projected reductions of annual GHG emissions from the LPV fleet were the smallest, at $21 \%$ and $23 \%$ below those of 2010.

VFM scenarios which assumed faster EV price reductions resulted in higher demand for EVs in the early years and larger GHG emissions reductions. In VFM scenario C4 (steady improvement in technology, accelerated decline in prices), the minimum price levels for EVs were achieved by 2023 and the annual GHG emissions from the LPV fleet in 2030 were projected to be $46 \%$ below the levels in 2010 . To achieve further reductions in GHG emissions would require either very high oil prices that increase the demand for EVs and reduce the vehicle kilometres travelled (VKT) by ICEVs - modelled in the VFM scenarios B3 (steady improvement in technology, steady decline in prices) and B4 (steady improvement in technology, accelerated decline in prices) - or lower minimum EV prices and very rapid price reductions as in VFM scenario C5 (breakthrough technology, accelerated decline in prices).

This study finds that the effectiveness of EVs in reducing New Zealand's GHG emissions is critically affected by how the electricity generation sector develops, with a key determinant being the future role of coal and lignite-fired generation.

If there are no constraints on the use of coal and lignite-fired generation, the increased GHG emissions from the electricity generated to meet the demand from EVs will almost completely offset the emissions reductions achieved by bringing EVs into the LPV fleet. In a future where the uptake of EVs follows the VFM scenario A2 (no improvement in EV technology, steady decline in EV prices) combined with the MDS3 generation scenario (which allows for the continued use of coal plants and the development of new coal plants), and there is a carbon price of $\$ 25$ per tonne, the 27.2 million tonnes $\mathrm{CO}_{2} \mathrm{e}$ of cumulative $\mathrm{GHG}$ emissions reductions achieved in the LPV fleet over the modelling period decreased by $88 \%$, to only 3.2 million tonnes $\mathrm{CO}_{2} \mathrm{e}$, from the increased $\mathrm{GHG}$ emissions from the electricity sector.

The MDS3 scenario, in conjunction with a low carbon price regime closely reflects the current policy and electricity sector settings and probably represents the business as usual (BAU) development path for the electricity generation sector. 
Maximising the GHG emissions reductions from the introduction of EVs into New Zealand therefore requires moving away from the BAU development path. This study assessed two possible measures for achieving this outcome: (1) imposing a ban on the use of coal and lignite; and (2) placing a high price on the use of carbonbased fuels.

A ban on the use of coal and lignite, as in the MDS1 scenario, reduced the offsetting effects of additional GHG emissions from the electricity sector. When the conservative VFM scenario A2 (no improvement in technology, steady decline in prices), was combined with the imposition of the ban on coal only $25 \%$ of the GHG emissions reductions attributable to EVs were offset by increased GHG emissions from electricity generation, with the result that cumulative emissions, over the modelling period, fell by 20.3 million tonnes $\mathrm{CO}_{2}$ e relative to the no-EV BAU projection.

The imposition of a carbon price of $\$ 100$ per tonne was also effective in reducing the offsetting effects of increased GHG emissions from electricity generation. When the output from VFM scenario C4 (steady improvement in technology, accelerated decline in prices) combined with the MDS3 scenario and with the high price on carbon the offset from increased electricity sector GHG emissions was reduced to $30 \%$, which brought the 35.7 million tonnes $\mathrm{CO}_{2}$ e of cumulative $\mathrm{GHG}$ emissions reductions achieved in the LPV fleet down to 25.1 million tonnes $\mathrm{CO}_{2} \mathrm{e}$.

Using both the coal ban and the high carbon price together further reduced the offsetting effects of increased GHG emissions from the electricity generation further. In the case of VFM scenario C4 (steady improvement in technology, accelerated decline in prices), the offset was only $20 \%$, resulting in cumulative GHG emissions reductions of 28.7 million tonnes.

This study found that PHEVs would be the most popular type of EV in New Zealand and remain so throughout the modelling period irrespective of improvements in EV technology or the reductions in prices that are considered achievable for EVs based on lithium-ion batteries. The VFM indicated that, at current EV prices and 
performance, PHEVs would comprise $70 \%$ of the EV demand and $14 \%$ of all the LPVs entering the fleet. Over time, projected improvements in BEV technology and declining battery costs would reduce the market share of PHEVs, but by 2030 , they would still constitute $51 \%$ to $62 \%$ of all EVs entering the LPV fleet. The popularity of PHEVs, when compared to the BEVs, indicates that the ability to drive the same distance as an ICEV offsets the disincentive effects of the higher purchase price and higher fuel costs.

The low demand for the GEV indicated that the high purchase price, in conjunction with restricted driving range, severely limits the appeal of these vehicles in the New Zealand market. In the first year of their introduction, they would constitute only $10 \%$ of the EV sales or $2 \%$ of the total LPV sales. Unless there are technological breakthroughs that significantly reduce the price of EV batteries, attempts to grow the GEVs' share of the EV market by increasing the driving range would not be effective due to the increased costs of the larger battery packs offsetting the effect of declining manufacturing costs.

The direct point elasticity estimates produced by the discrete choice model indicate that the demand for EVs is most sensitive to changes in the purchase price of these vehicles. Significant reductions in the purchase price of EVs would require reductions in the cost of EV lithium-ion batteries. These reductions could be the result of technological breakthroughs that reduce manufacturing costs, or through improvements in energy efficiency that allow for the sale of EVs with smaller cheaper batteries that could still achieve the same driving range.

Of the policy measures investigated in this study that the New Zealand Government might adopt to promote the demand for EVs, the provision of an extensive network of public street recharging stations had the greatest impact. The analysis indicates that this measure would be more effective than the Government providing a $20 \%$ subsidy on the purchase price of new and used imported EVs entering the fleet for the first time (capped at $\$ 7,550$ for new EVs and $\$ 3,750$ for used imported EVs). The analysis also indicated that the Government's current exemption from road 
user charge would increase the demand for EVs to some extent, but this measure was the least effective of the three investigated.

The default assumption used in this study is that over time used imported EVs from Japan will become a major component of the New Zealand LPV fleet. The availability of low cost used imported EV was one of the reasons why this study projects a high uptake of EVs. In the unlikely event that used Japanese EVs were not available for sale in New Zealand, the VFM analysis indicated that the projected stock of EVs in 2030, based on the standard price and technology assumptions, would decline from between $43 \%$ and $51 \%$ of the stock of LPVs to between $32 \%$ and $42 \%$. This reduction in demand results in the stock of EVs in 2030 being 1.01 to 1.22 million vehicles. These lower stock projections are consistent with the projections in the previous studies by Baxter et al. (2009) and Duncan et al. (2010) Depending on the rate of EV uptake, the projected additional electricity demand from the EV fleet would reach between 2,701 GWh and 3,715 GWh by 2030, or an additional $4.5 \%$ to $6.2 \%$ over the BAU forecast.

The impact of the additional demand on the amount of extra generation capacity required will be in large part determined by the management of the EV charging load. This study used a simplifying assumption that the management of the additional load from EVs resulted in most of the charging occurring during off-peak periods and only a small proportion occurring at times of peak demand. Another assumption was that the system operator could interrupt EV charging to ensure that during times of peak demand the system complies with the $n-1$ system security standard (section 6.5.6). Using these assumptions, the amount of additional generation capacity required to be in place by 2030 would not exceed $730 \mathrm{MW}$ (MDS5 scenario) when compared to the same generation scenario without the EV demand.

However, if the system operator has limited ability to interrupt EV charging during periods of maximum demand to maintain system security at the $n-1$ standard, the 
analysis indicates that significant amounts of standby fast-start peaking plant would be required.

\subsection{Further research}

There is considerable potential to expand and improve the models developed for this study by addressing some of the limitations imposed by resource constraints.

A priority area for further research is the development of a demand model of organisational car buyers. Other areas of improvement include the implementation of a pre-survey to collect data to understand better the characteristics of New Zealand car buyers within the general adult population, and additional techniques to help reduce the presence of hypothetical bias. These techniques could include the use of personal interviews and pivot designs. The potential to use personal interviews to reduce hypothetical bias could also enable the implementation of a choice experiment without a single opt-out alternative, which would allow the separation of the ICEV alternative into new and used options.

The current study's assessment of the impact of EV charging on vehicle choice has been limited to considering the availability of publicly accessible charging facilities. An issue for possible further research is the impact on vehicle choice of the time required to recharge an EV. During the design of the stated choice survey, the decision was made to simplify the design by not including an attribute for the length of charging time. The exclusion of this attribute made the web-based and postal survey less complex and was based on feedback from the reference group, which indicated that recharging time was not as important as the other attributes.

Another possible issue for further research is the impact of interrupted charging on the decision to purchase an EV. During the design of the stated choice experiment, it was not envisaged that interrupted charging could have an impact on EV purchase behaviour. However, the recent research by Parsons et al. (2011) indicates that owners of EVs value highly their convenience, suggesting that the system operator's ability to interrupt EV charging may be a disincentive to purchasing an EV. 
The high value placed on convenience by EV owners brings into question the assumption used in the study that most charging of EVs will occur during off-peak periods. If owners do value their convenience highly, then the incentive of a lower off-peak tariff may struggle to shift charging away from the evening peak period. With increasing numbers of EVs, peak-time charging would have implications for the future development path of the electricity system and the GHG emissions produced from electricity generation. Both the system operator and the line companies have strong incentives to minimise the amount of EV charging occurring during peak periods and understanding the factors that will influence EV owners' charging behaviour is potentially a fruitful area for further research.

The research by Parsons et al (2011) also points to potential difficulties with the implementation of vehicle-to-grid (V2G) arrangements by New Zealand electricity system operators. The exploration of the attitudes of New Zealand car buyers to the provision of V2G could also be a productive area of further research. 


\section{Appendix 1: Example of a choice task}

\begin{tabular}{|c|c|c|c|c|c|c|c|}
\hline & $\begin{array}{l}\text { City EV } \\
\text { (new) }\end{array}$ & $\begin{array}{l}\text { Plug-in } \\
\text { hybrid EV } \\
\text { (new) }\end{array}$ & $\begin{array}{l}\text { General } \\
\text { purpose } \\
\text { EV (new) }\end{array}$ & $\begin{array}{l}\text { Plug-in } \\
\text { hybrid EV } \\
\text { (second- } \\
\text { hand) }\end{array}$ & $\begin{array}{c}\text { General } \\
\text { purpose } \\
\text { EV } \\
\text { (second- } \\
\text { hand) }\end{array}$ & $\begin{array}{l}\text { City EV } \\
\text { (second- } \\
\text { hand) }\end{array}$ & $\begin{array}{c}\text { Petrol or } \\
\text { diesel } \\
\text { vehicle } \\
\text { (new or } \\
\text { used) }\end{array}$ \\
\hline Purchase price & $\$ 45,000$ & $\$ 100,000$ & $\$ 95,000$ & $\$ 50,000$ & $\$ 7,500$ & $\$ 22,500$ & $\begin{array}{c}\text { Same } \\
\text { range of } \\
\text { prices as } \\
\text { now }\end{array}$ \\
\hline Total Range km & 150 & 500 & 500 & 500 & 500 & 100 & 500 \\
\hline $\begin{array}{l}\text { Fuel cost per } \\
100 \mathrm{~km}\end{array}$ & $\$ 4$ & $\$ 2$ & $\$ 2$ & $\$ 20$ & $\$ 2$ & $\$ 1$ & $\$ 60$ \\
\hline Top speed km/hr & 140 & $130+$ & $130+$ & $130+$ & $130+$ & 70 & $130+$ \\
\hline $\begin{array}{l}\text { Cost of replacing } \\
\text { the battery }\end{array}$ & $\$ 4,000$ & $\$ 8,750$ & $\$ 130,000$ & $\$ 8,750$ & $\$ 70,000$ & $\$ 17,000$ & \\
\hline $\begin{array}{l}\text { Time to replacing } \\
\text { the battery (years) }\end{array}$ & 5 & 12.5 & 5 & 10 & 0 & 20 & \\
\hline $\begin{array}{c}\text { Availability of on- } \\
\text { street EV charging } \\
\text { posts }\end{array}$ & $\begin{array}{l}\text { Town } \\
\text { centre }\end{array}$ & $\begin{array}{l}\text { Town } \\
\text { centre } \\
\text { and inner } \\
\text { suburbs }\end{array}$ & $\begin{array}{c}\text { Town } \\
\text { centre } \\
\text { and inner } \\
\text { suburbs }\end{array}$ & $\begin{array}{l}\text { Town } \\
\text { centre } \\
\text { and inner } \\
\text { suburbs }\end{array}$ & $\begin{array}{l}\text { Town } \\
\text { centre }\end{array}$ & None & \\
\hline $\begin{array}{c}\text { Choose one vehicle } \\
\text { type: }\end{array}$ & & & & & & & \\
\hline
\end{tabular}

The design of stated choice experiments requires the manipulation of the attribute levels across the choice tasks in a systematic manner to allow for the determination of the independent influence of the attributes on choice. In many cases, this manipulation results in combinations of attribute levels within, and between, the alternatives that will not occur in reality. 


\section{Appendix 2: The supporting questions used in the survey}

8. If you purchased a vehicle would you:

Make the decision on your own

Make the decision jointly with someone else?

9. Did you complete the scenarios

Jointly with someone else

On your own, but taking into account another person's views

On your own and not taking into account another person's views.

10. What is your age?

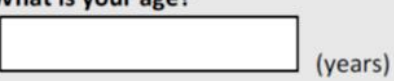

11. Are you female or male? [If a couple answer the questions, it is the person first contacted.]

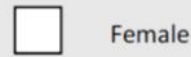

Male

12. What is your estimated household income (before tax) for the year 2009?

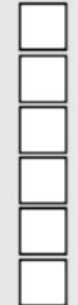

$$
\begin{aligned}
& \text { Less than }<\$ 20,000 \\
& \$ 20,001-\$ 40,000 \\
& \$ 40,001-\$ 60,000 \\
& \$ 60,001-\$ 80,000 \\
& \$ 80,001-\$ 100,000 \\
& \$ 100,001 \text { or more }
\end{aligned}
$$

13. Do you live in a:

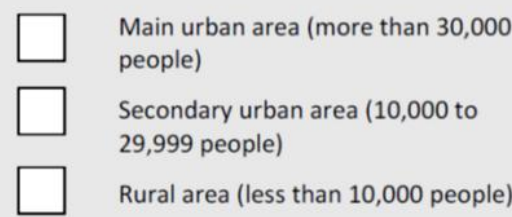

14. Could you recharge an electric vehicle where you live?

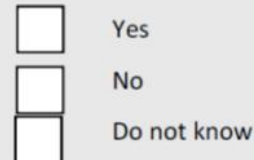

15. Have you ever previously owned a car?

\begin{tabular}{ll}
$\square$ & Yes \\
\hline & No (go to question 17)
\end{tabular}

16. If you answered YES in question $\mathbf{1 5}$ was the last car you bought:

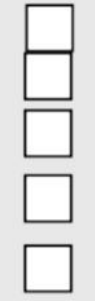

New

Used, but arrived in NZ new

A used import and the first NZ owner

A used import, but not the first NZ owner

Used, but don't know about its previous ownership

17. What will your next car purchase mainly be

\begin{tabular}{|c|}
\hline Strongly agree \\
\hline Agree \\
\hline Have no opinion \\
\hline Disagree \\
\hline Strongly disagree \\
\hline
\end{tabular}
used for?

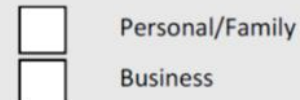

Here are three statements about road transport in New Zealand.

18. The era of cheap oil is over. Do you:

19. There is an urgent need for policies to reduce greenhouse gas emissions from road transport in New Zealand. Do you:

\begin{tabular}{ll}
\hline & Strongly agree \\
\hline & Agree \\
\hline$\square$ & Have no opinion \\
$\square \quad$ Disagree \\
$\square \quad$ Strongly disagree
\end{tabular}

20. In aiming to reduce greenhouse gas emissions, the Government should not try to influence the purchase decisions of private car buyers. Do you:

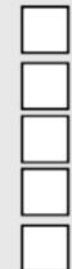

Strongly agree

Agree

Have no opinion

Disagree

Strongly disagree 


\section{Appendix 3: Supporting information provided in the survey}

\section{Information about electric vehicles (EVs)}

\section{Types of EVs}

Electric cars (commonly known as electric vehicles or EVs) are not a new technology. However, within the next few years a new generation of EVs, based on the Lithium-ion (Li-ion) family of battery technologies, will become available in the New Zealand market. This generation of EVs is seen as a potential replacement for petrol and diesel ICEVs and a means of reducing reliance on imported petroleum and greenhouse gas emissions. It is this generation of EVs that is the focus of this survey.

EVs will be introduced into the market in two forms:

- Full-time EVs

- Plug-in hybrid electric vehicles (PHEVs).

Both types will plug into the electricity grid to charge their batteries. However, fulltime EVs rely solely on electricity; whereas PHEVs will first use electric power and then, when the battery is discharged, will run on petrol or diesel.

\section{Driving range}

\section{Full-time EV driving range}

Both types of EV will use Li-ion batteries to store the electric energy. At this time, Liion batteries do not have the same ability to carry energy as petrol, diesel, or biofuels. This will mean that, when EVs are first introduced, they will have a shorter driving range than petrol or diesel vehicles, which, on a full tank, can travel between 450 to 600 kilometres.

At present, the driving range for most full-time EVs varies from 120 to 160 kilometres. There are full-time EVs that have greater driving range, but these come at considerably greater cost. As with ICEVs, EV driving range will be reduced by aggressive driving habits and hilly terrain. 


\section{PHEV driving range}

PHEVs will have smaller batteries so they can only travel a short distance on electric power. Once the battery is discharged, an on-board petrol or diesel-powered generator will start and recharge the batteries while driving. This will give a combined driving range that is at least as great as that of petrol or diesel cars. The electric only distance of PHEVs varies, and these vehicles are classified by this distance (For example a PHEV 30 can travel 30 kilometres using its battery).

\section{The purchase price of EVs}

Initially, EVs will cost significantly more than petrol and diesel ICEVs. However, over time, these costs are expected to decrease due to:

- Improvements in battery technology

- Increased production volumes of EVs and PHEV and EV batteries

- The importation of second-hand EVs from Japan.

\section{EV batteries}

The capabilities of EV battery technology largely determine the performance and cost of EVs.

Li-ion batteries represent the "state of the art" in EV batteries. There are a number of different types of Li-ion batteries; each with different energy storage, power output, and battery life capabilities. Most of the research effort and current progress are with Li-ion batteries.

\section{EV battery life}

Batteries need to be replaced after a certain number of recharges. The life of a battery varies depending on the type of Li-ion battery and how it is used. Under normal personal operating conditions, a Li-ion battery should last for at least 5 years. Some types of Li-ion batteries are expected to last up to 20 years.

The life of the battery will be reduced if it is fully discharged too often, or if it is charged using a faulty charger. 


\section{Battery recharging and recharging facilities}

Batteries take longer to fill-up (recharge) than filling a car's fuel tank. Using household current, it will take around 7 hours to fully recharge the battery of a fulltime EV with a range of 160 kilometres.

Battery recharging facilities that provide high voltage and electric current can significantly reduce recharging time. With suitable recharging facilities, some types of Li-ion batteries can be charged to $80 \%$ of full charge in 15 minutes.

It is expected that, as EVs become common, two types of charging facilities will be available.

There will be charging stations similar to service stations where fast charging can occur, and, for some EVs, where depleted battery packs can be swapped out for a fully charged one.

There will also be charging posts, similar to parking meters, located on the sides of streets and in car parking buildings. These charging posts will allow people to charge up while at work, or shopping. For people who live in town centres or the inner suburbs, these charging posts will be able used to recharge overnight.

\section{Fuel costs}

EVs have the advantage of being much more fuel efficient than petrol or diesel vehicles. An average sized petrol or diesel ICEV (i.e. 1500 to $3000 \mathrm{cc}$ ), at current fuel prices, will cost around $\$ 15$ to drive 100 kilometres and, assuming a total driving range of 500 kilometres, will cost about $\$ 75$ to fill-up the tank. A small petrol or diesel vehicle (less than $1350 \mathrm{cc}$ ) will cost around $\$ 10$ to drive a 100 kilometres or around $\$ 50$ to fill-up the tank.

In comparison, at current domestic electricity prices, a small city sized EV will cost around $\$ 2$ to $\$ 3$ to drive 100 kilometres or $\$ 12$ to drive 500 kilometres. A larger family-sized EV will cost about $\$ 4$ to $\$ 6$ per 100 kilometres to run, depending on the size and weight of the vehicle, or $\$ 20$ to $\$ 30$ to drive 500 kilometres. 
The fuel costs for plug-in hybrid EVs vary depending on the amount of driving that is done on electric power and how much is done using the petrol or diesel engine. If only used in electric mode, PHEVs are as inexpensive as an equivalent full-time electric vehicle. If they are driven long distances between recharging, they will be become almost as expensive to run as a comparable ICEV. Over time, new models of plug-in hybrid EVs are expected to become available that will have longer electric driving ranges.

\section{Future cost of fuel}

There is considerable uncertainty about what the price of electricity, petrol, diesel, and biofuels will be in the future; but there is general acceptance that the future price of these fuels will be greater than now. Some of the impact of these future price increases will be off-set by new petrol and diesel technologies that are expected to reduce average fuel consumption by up to a half.

\section{Speed and Acceleration}

Most new generation EVs are designed to be 'highway capable' and have top speeds and acceleration similar to ICEVs.

There are a number of EVs that are designed for urban only use and some of these have top speeds that are less than the open road speed limit.

\section{Other costs}

EVs' insurance costs are not expected to differ from an equivalent priced petrol and diesel vehicle.

At present, EVs do not have to pay any form of road user charges; but this policy may change.

As full-time EVs have less moving parts than petrol and diesel vehicles, maintenance costs (excluding battery replacement) are expected to be lower. PHEVs have both electric and normal vehicle components and are more complicated than standard EVs, which could result in maintenance costs the same or higher as petrol and diesel vehicles. 


\section{Vehicle emissions}

When operating on electric power, EVs produce no direct emissions and will be beneficial in reducing local air pollution where this is a concern.

EVs can also have greenhouse gas emission benefits if the electricity used by them is generated from renewable resources. Even when the electricity is produced using natural gas, EVs still produce less greenhouse gas emissions than equivalent petrol or diesel ICEVs produce. It is only when they rely totally on electricity generated from coal that the greenhouse gas emissions are similar, or slightly higher, than that of ICEVs.

New Zealand is fortunate in that it produces between $60 \%$ and $70 \%$ of its electricity from renewable sources such as hydro, geothermal, and wind. New Zealand is also fortunate in that it has significant unused renewable generation resources. 


\section{Appendix 4: Results of vehicle fleet model choice simulator verification test}

\begin{tabular}{|c|c|c|c|c|c|c|c|c|c|c|c|c|c|c|c|c|c|c|c|c|}
\hline & \multicolumn{4}{|c|}{ Trial 1} & \multicolumn{4}{|c|}{ Trial 2} & \multicolumn{4}{|c|}{ Trial 3} & \multicolumn{4}{|c|}{ Trial 4} & \multicolumn{4}{|c|}{ Trial 5} \\
\hline & NLOGIT & $\begin{array}{l}\text { VFM } \\
\text { (mean) }\end{array}$ & SD & T-test & NLOGIT & $\begin{array}{l}\text { VFM } \\
\text { (mean) }\end{array}$ & SD & T-test & NLOGIT & $\begin{array}{l}\text { VFM } \\
\text { (mean) }\end{array}$ & SD & T-test & NLOGIT & $\begin{array}{l}\text { VFM } \\
\text { (mean) }\end{array}$ & SD & T-test & NLOGIT & $\begin{array}{l}\text { VFM } \\
\text { (mean) }\end{array}$ & SD & T-test \\
\hline & \multicolumn{20}{|c|}{ All alternatives } \\
\hline ICEV & $61.59 \%$ & $62.01 \%$ & $0.70 \%$ & -1.50 & $42.72 \%$ & $42.57 \%$ & $0.56 \%$ & 0.85 & $37.84 \%$ & $37.74 \%$ & $0.44 \%$ & 0.73 & $37.46 \%$ & $37.29 \%$ & $0.63 \%$ & 0.87 & $27.05 \%$ & $26.81 \%$ & $0.46 \%$ & 1.65 \\
\hline New CEV & $2.14 \%$ & $2.15 \%$ & $0.09 \%$ & -0.11 & $3.33 \%$ & $3.33 \%$ & $0.14 \%$ & 0.04 & $5.23 \%$ & $5.21 \%$ & $0.12 \%$ & 0.61 & $6.09 \%$ & $6.18 \%$ & $0.19 \%$ & -1.42 & $7.62 \%$ & $7.77 \%$ & $0.25 \%$ & -1.91 \\
\hline Used CEV & $5.76 \%$ & $5.63 \%$ & $0.18 \%$ & 2.09 & $9.62 \%$ & $9.62 \%$ & $0.18 \%$ & 0.06 & $1.35 \%$ & $11.49 \%$ & $0.20 \%$ & -2.06 & $11.91 \%$ & $11.79 \%$ & $0.22 \%$ & 1.68 & $12.42 \%$ & $12.55 \%$ & $0.21 \%$ & -2.00 \\
\hline New GEV & $0.88 \%$ & $0.87 \%$ & $0.02 \%$ & 2.17 & $1.31 \%$ & $1.32 \%$ & $0.03 \%$ & -0.72 & $2.00 \%$ & $2.01 \%$ & $0.02 \%$ & -1.38 & $1.10 \%$ & $1.08 \%$ & $0.03 \%$ & 1.94 & $4.22 \%$ & $4.23 \%$ & $0.05 \%$ & -0.62 \\
\hline Used GEV & $1.53 \%$ & $1.54 \%$ & $0.03 \%$ & -1.25 & $6.89 \%$ & $6.84 \%$ & $0.10 \%$ & 1.55 & $8.35 \%$ & $8.39 \%$ & $0.09 \%$ & -1.60 & $3.67 \%$ & $3.65 \%$ & $0.03 \%$ & 1.77 & $10.15 \%$ & $10.08 \%$ & $0.10 \%$ & 2.01 \\
\hline New PHEV & $7.83 \%$ & $7.82 \%$ & $0.17 \%$ & 0.04 & $5.68 \%$ & D. & $0.10 \%$ & (.) & 然 & $6.43 \%$ & $0.08 \%$ & 1.00 & 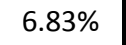 & $6.83 \%$ & $0.11 \%$ & -0.17 & 年 & $8.38 \%$ & $0.12 \%$ & -1.89 \\
\hline \multirow[t]{2}{*}{ Used PHEV } & $20.28 \%$ & $19.98 \%$ & $0.36 \%$ & 1.78 & $30.45 \%$ & $30.61 \%$ & $0.25 \%$ & -2.10 & $28.74 \%$ & $28.72 \%$ & $0.18 \%$ & 0.32 & $32.94 \%$ & $33.18 \%$ & $0.37 \%$ & -2.02 & $30.23 \%$ & $30.17 \%$ & $0.26 \%$ & 0.66 \\
\hline & \multicolumn{20}{|c|}{ New EVs only } \\
\hline ICEV & $73.86 \%$ & $73.82 \%$ & $0.72 \%$ & 0.17 & $63.75 \%$ & $63.50 \%$ & $0.42 \%$ & 1.85 & $56.32 \%$ & $56.28 \%$ & $0.34 \%$ & 0.39 & $56.61 \%$ & $56.21 \%$ & $0.62 \%$ & 2.03 & $41.01 \%$ & $40.81 \%$ & $0.35 \%$ & 1.87 \\
\hline New CEV & $4.51 \%$ & $4.52 \%$ & $0.15 \%$ & -0.21 & $10.21 \%$ & $10.33 \%$ & $0.21 \%$ & -1.74 & $14.46 \%$ & 14. & $0.27 \%$ & 0.82 & $3 \%$ & $16.61 \%$ & $0.49 \%$ & -1.20 & $\%$ & $19.41 \%$ & $0.07 \%$ & -2.12 \\
\hline New GEV & $2.22 \%$ & $2.20 \%$ & $0.04 \%$ & 1.89 & $4.81 \%$ & $4.87 \%$ & $0.09 \%$ & -2.16 & $6.92 \%$ & $6.97 \%$ & $0.10 \%$ & -1.80 & $3.16 \%$ & $3.13 \%$ & $0.06 \%$ & 1.75 & $13.12 \%$ & $13.17 \%$ & $0.10 \%$ & -1.66 \\
\hline New PHEV & $19.41 \%$ & $19.47 \%$ & $0.60 \%$ & -0.30 & $21.23 \%$ & $21.30 \%$ & $0.32 \%$ & -0.71 & $22.30 \%$ & $22.36 \%$ & $0.31 \%$ & -0.59 & $23.80 \%$ & $24.05 \%$ & $0.52 \%$ & -1.48 & $26.50 \%$ & $26.61 \%$ & $0.23 \%$ & -1.39 \\
\hline
\end{tabular}




\section{Appendix 5: Method used to estimate PHEV electric and non-electric travel}

This section was developed in conjunction with Professor Euan Smith.

Data are sourced from the annual New Zealand Household Travel Survey. For the period 2003/04 to 2007/08 (inclusive), the sample comprised 2,200 households per year. Since $2008 / 09,4,600$ households have been invited to take part in the annual survey (Ministry of Transport, 2010c). Over seven years during 2003-2010 the full response rate was $63-70 \%$, with another $10 \%$ partial response.

The survey is of 4-wheeled vehicles and includes cars, vans, utilities, and SUVs, but not heavy vehicles ( 3.5 tonnes or more) and motorcycles. The survey uses data on reported travel by household members over a 2-day period (travel days), which are distributed across the year (L. Povey; Ministry of Transport, personal communication, August 17, 2010).

The Ministry of Transport uses the Statistics New Zealand geographical classification: (1) main urban areas (population of 30,000 or more); (2) secondary urban areas (population 10,000 to 29,999); (3) rural and small towns (population under 10,000).

Table A5.1 provides a summary of the data provided by the Ministry of Transport.

Table: A5.1 Summary of daily travel distance data provided by the Ministry of Transport: annual New Zealand household travel survey

\begin{tabular}{|c|c|c|c|c|c|c|c|c|c|c|c|c|c|c|}
\hline & \multirow{3}{*}{$\begin{array}{l}\text { Vehicles in } \\
\text { sample }\end{array}$} & \multirow{3}{*}{$\begin{array}{l}\text { Number of } \\
\text { responses }\end{array}$} & \multicolumn{12}{|c|}{ km/day } \\
\hline & & & \multirow{2}{*}{ Mean } & \multirow{2}{*}{ Median } & \multicolumn{10}{|c|}{ Percentiles } \\
\hline & & & & & Min & p1 & p5 & p10 & p25 & p75 & p90 & p95 & p99 & $\max$ \\
\hline All NZ & 10,635 & 18,437 & 37.9 & 22.1 & 0.0 & 1.2 & 3.0 & 4.8 & 10.1 & 45.1 & 82.4 & 124.0 & 266.8 & 759.3 \\
\hline $\begin{array}{l}\text { Major urban } \\
30000+\end{array}$ & 6,803 & 11,928 & 34.2 & 20.8 & 0.0 & 1.4 & 3.2 & 5.1 & 10.4 & 40.5 & 68.7 & 104.0 & 254.2 & 746.5 \\
\hline $\begin{array}{c}\text { Secondary } \\
\text { urban and } \\
\text { rural } \\
\text { under } 30000\end{array}$ & 3,832 & 6,509 & 47.3 & 27.6 & 0.0 & 0.9 & 2.3 & 4.0 & 9.2 & 62.8 & 109.9 & 157.8 & 290.7 & 759.3 \\
\hline
\end{tabular}

Note: The reason that the number of responses is higher than the number of vehicles in the survey is due to a vehicle being driven by more than one person in many households.

For each data subset, an empirical cumulative distribution curve was constructed by assigning to each distance in the sample, sorted into ascending order, a probability 
equal to its weight divided by the sum of all the weights in that subset (curves shown in Figure A5.1).

Figure A5.1 shows the fits to the model for the four geographic categories. The fits for 'Main urban' and, consequently 'All NZ' data are excellent, but the fit for the $<30,000$ data is not as good because the fit of 'Rural' data is less satisfactory. This result may indicate that rural daily kilometres are differently distributed from those of other regions in New Zealand.

Figure A5.1: Daily distance driven cumulative survey data distributions (solid) for the four geographic sets described, and a best-fitting log-normal model (dashed)

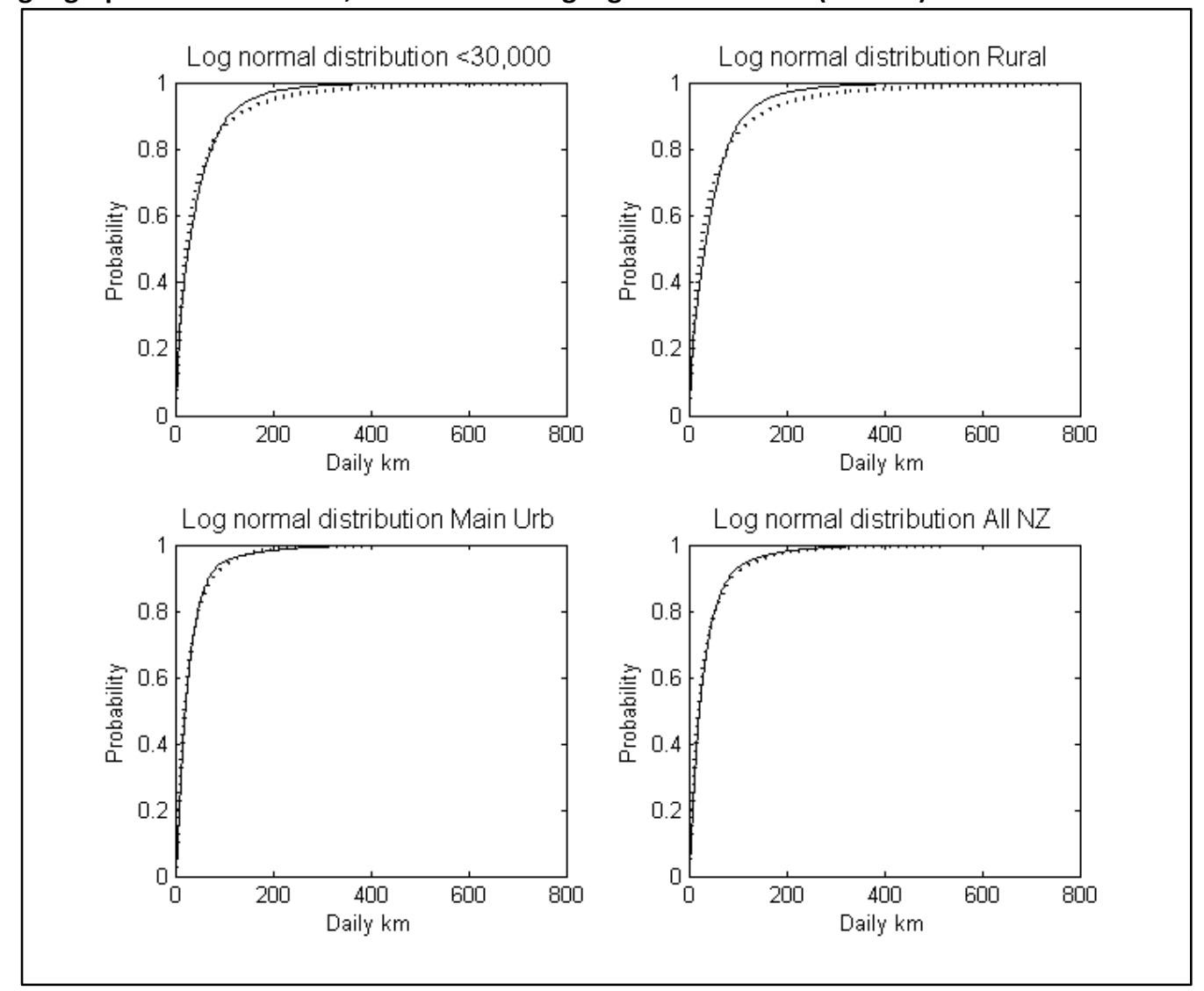

A log-normal distribution was fitted by MLE to the data ${ }^{71}$. Table A5.2 shows the estimated log-normal mean and log-normal standard deviation for the four geographical categories for the best fitting models.

\footnotetext{
${ }^{71}$ We are indebted to Prof. Estate Khmaladze for suggesting the use of the log-normal distribution.
} 
Assuming that PHEVs will be predominantly recharged overnight and therefore, only recharged once per day, and that the all-electric range cycle is used, the lognormal model offers a ready calculation of the distance travelled on electric power, $\mathrm{S}\left(\mathrm{e}_{\mathrm{r}}\right)$, where $\mathrm{e}_{\mathrm{r}}$ is the electric range; and hence the petroleum-powered distance that could be 'saved', i.e. the number of kilometres of ICE travel avoided.

It can readily be shown that the partial average $p\left(e_{r}\right)$ of a log-normal distribution with parameters log-mean $=\mu$ and log-standard deviation $=\sigma$ over the range 0 to $e_{r}$ is given by:

$\mathrm{p}\left(\mathrm{e}_{\mathrm{r}}\right)=\exp \left(\mu+\frac{1}{2} \sigma^{2}\right) \int_{0}^{\log _{\mathrm{e}}\left(\mathrm{e}_{\mathrm{r}}\right)} 1 / \sqrt{ }\left(2 \pi \sigma^{2}\right) \exp \left\{-1 /\left(2 \sigma^{2}\right)\left(\mathrm{z}-\left(\mu+\sigma^{2}\right)\right)^{2}\right\} \mathrm{dz}(1)$ where the integrand is a normal probability density function with mean $\left(\mu+\sigma^{2}\right)$ and standard deviation $\sigma$, and $z$ is the natural logarithm of $e_{r}$.

Therefore, the saving for a vehicle with electric range $e_{r}$ is given by $p\left(e_{r}\right)$ plus the saving for the rest of the population, that travels further than $e_{r}$ whose savings will be just $e_{r}$. So the sum of distances is:

$\mathrm{S}\left(\mathrm{e}_{\mathrm{r}}\right)=\mathrm{p}\left(\mathrm{e}_{\mathrm{r}}\right)+\mathrm{e}_{\mathrm{r}}\left(1-\int_{0}^{\log _{\mathrm{e}}\left(\mathrm{e}_{\mathrm{r}}\right)} 1 / \sqrt{ }\left(2 \pi \sigma^{2}\right) \exp \left\{-1 /\left(2 \sigma^{2}\right)(z-\mu)^{2}\right\} d z\right)$

This can be conceptually simplified as follows. Write $\mathrm{N}(\mathrm{x}, a, b)$ for the cumulative Normal (Gaussian) probability distribution with mean $a$ and standard deviation $b$.

Then:

$$
p\left(e_{r}\right)=\exp \left(\mu+1 / 2 \sigma^{2}\right) N\left(\log \left(e_{r}\right),\left(\mu+\sigma^{2}\right), \sigma\right)
$$

and:

$$
S\left(e_{r}\right)=p\left(e_{r}\right)+e_{r}\left[1-N\left(\log \left(e_{r}\right), \mu, \sigma\right]\right.
$$

The results of the fitted log-normal model are shown against the actual New Zealand household travel survey data in Table A5.2. 
Table: A5.2: Estimated log-normal mean distance travelled, log-normal standard deviation, and maximum probability model

\begin{tabular}{|c|c|c|c|c|}
\hline & $\begin{array}{c}\text { Rural areas and } \\
\text { towns with } \\
\text { population } \\
<10,000\end{array}$ & $\begin{array}{c}\text { Rural areas and } \\
\text { towns with } \\
\text { population } \\
<30,000\end{array}$ & $\begin{array}{c}\text { Cities and } \\
\text { towns with } \\
\text { population } \\
30,000 \text { or more }\end{array}$ & All NZ \\
\hline Mean (km) & 51.03 & 47.30 & 34.23 & 37.94 \\
\hline $\begin{array}{c}\text { Mean of } \\
\text { log (data) }\end{array}$ & 3.255 & 3.150 & 2.993 & 3.038 \\
\hline $\begin{array}{c}\text { Standard } \\
\text { deviation of } \\
\text { log (data) }\end{array}$ & 1.331 & 1.322 & 1.057 & 1.141 \\
\hline $\begin{array}{c}\text { Maximum } \\
\text { probability } \\
\text { misfit (\%) }\end{array}$ & $7.3 \%$ & $5.4 \%$ & $2.2 \%$ & $2.2 \%$ \\
\hline
\end{tabular}

Using equations (3) and (4), it is possible to estimate the fossil fuelled distance 'savings' for a PHEV with a specified all-electric range. The results are displayed in Figure A5.2. As can be seen, the 'savings' become asymptotically close to the overall data mean of each geographical category (the dotted line) as the electric range increases.

Figure A5.2: Mean fossil-fuelled distance 'savings' $(\mathrm{km})$ as a function of plug-in hybrid electric vehicle electric driving range for the four geographical categories. Dotted line is at the mean for each set.
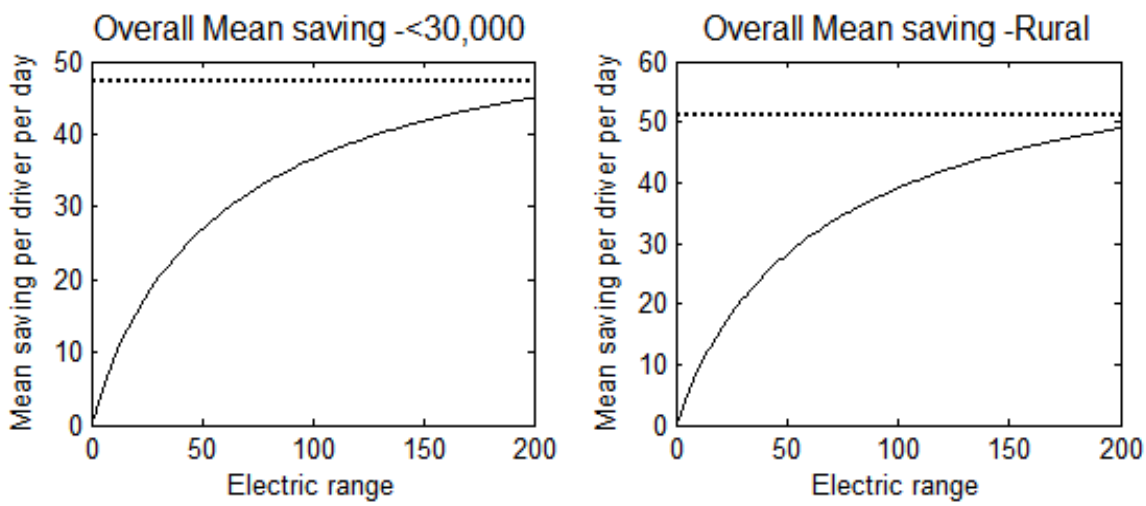

Overall Mean saving -Main Urb
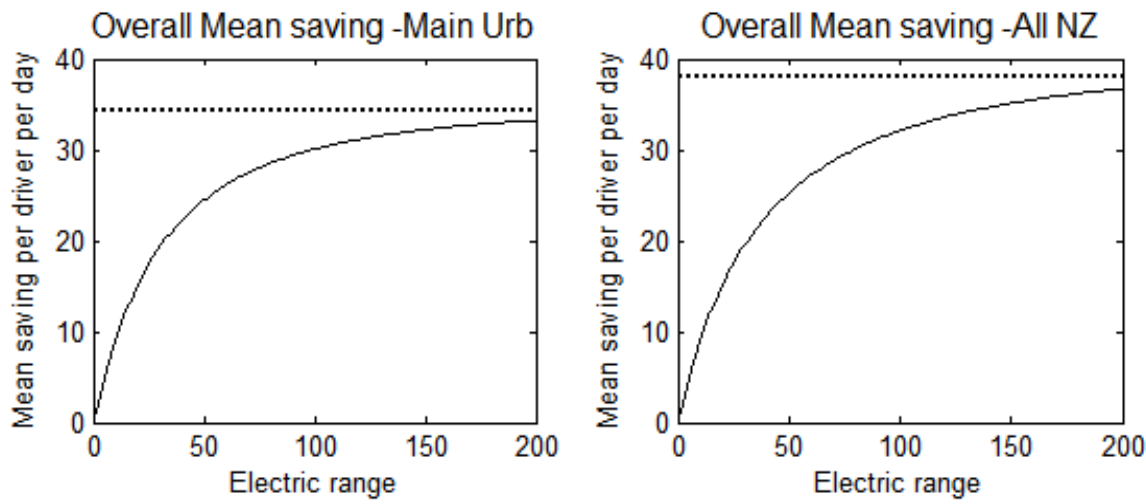
Figure A5. 3 consolidates the results from Figure A5.2 and shows the proportion of electric driving that is estimated to occur given a specified all-electric driving range for a PHEV in the four geographic categories.

Figure A5.3: Proportion of driving that will occur as electric given a specified all-electric driving range, in the four geographic categories

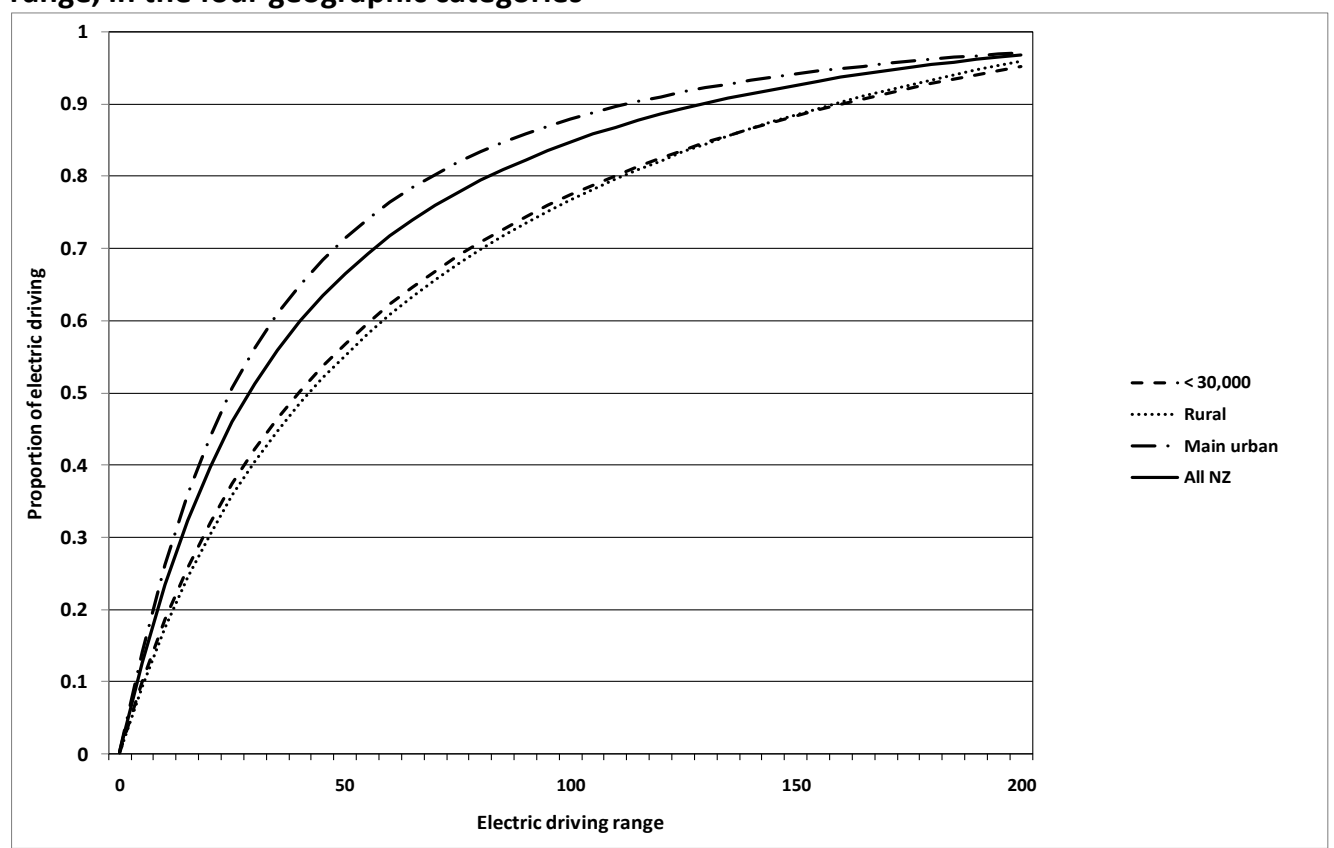




\section{Appendix 6: VFM scenario data tables}

\section{A scenarios: Internal combustion engine vehicle inputs}

Table: A6.1 Internal combustion engine vehicles

\begin{tabular}{|c|c|c|c|c|c|c|}
\hline & $\begin{array}{c}\text { Crude oil } \\
\text { price } \\
\text { US\$/bbl. }\end{array}$ & $\begin{array}{c}\text { Carbon } \\
\text { charge } \\
\mathbf{\$} / \text { tonne } \\
\text { Carbon }\end{array}$ & $\begin{array}{c}\text { Combined } \\
\text { Fuel price \$/L }\end{array}$ & $\begin{array}{c}\text { Petrol Fuel } \\
\text { efficiency } \\
\text { I/100 } \mathbf{k m}\end{array}$ & $\begin{array}{c}\text { Diesel Fuel } \\
\text { efficiency } \\
\text { //100 km }\end{array}$ & $\begin{array}{c}\text { Fuel cost } \\
\mathbf{\$} / 100 \text { km }\end{array}$ \\
\hline 2010 & 100 & 12.5 & 2.30 & 9.92 & 9.42 & 22.70 \\
\hline 2011 & 102 & 12.5 & 2.32 & 9.92 & 9.42 & 22.93 \\
\hline 2012 & 103 & 25 & 2.38 & 9.92 & 9.42 & 23.46 \\
\hline 2013 & 105 & 25 & 2.40 & 9.92 & 9.42 & 23.70 \\
\hline 2014 & 106 & 25 & 2.43 & 9.92 & 9.42 & 23.94 \\
\hline 2015 & 108 & 25 & 2.45 & 9.92 & 9.42 & 24.18 \\
\hline 2016 & 109 & 25 & 2.48 & 9.92 & 9.42 & 24.43 \\
\hline 2017 & 111 & 25 & 2.50 & 9.92 & 9.42 & 24.68 \\
\hline 2018 & 113 & 25 & 2.53 & 9.92 & 9.42 & 24.94 \\
\hline 2019 & 114 & 25 & 2.55 & 9.92 & 9.42 & 25.20 \\
\hline 2020 & 116 & 25 & 2.58 & 9.92 & 9.42 & 25.46 \\
\hline 2021 & 118 & 25 & 2.61 & 9.92 & 9.42 & 25.73 \\
\hline 2022 & 120 & 25 & 2.63 & 9.92 & 9.42 & 26.00 \\
\hline 2023 & 122 & 25 & 2.66 & 9.92 & 9.42 & 26.28 \\
\hline 2024 & 123 & 25 & 2.69 & 9.92 & 9.42 & 26.56 \\
\hline 2025 & 125 & 25 & 2.72 & 9.92 & 9.42 & 26.84 \\
\hline 2026 & 127 & 25 & 2.75 & 9.92 & 9.42 & 27.13 \\
\hline 2027 & 129 & 25 & 2.78 & 9.92 & 9.42 & 27.42 \\
\hline 2028 & 131 & 25 & 2.81 & 9.92 & 9.42 & 27.72 \\
\hline 2029 & 133 & 25 & 2.84 & 9.92 & 9.42 & 28.02 \\
\hline 2030 & 135 & 25 & 2.87 & 9.92 & 9.42 & 28.33 \\
\hline
\end{tabular}

These values apply to ICEVs in all the scenarios in the A family. 


\section{A2 scenario: EV inputs}

Table: A6.2 City electric vehicles

\begin{tabular}{|c|c|c|c|c|c|c|c|c|c|c|c|c|c|}
\hline \multicolumn{8}{|c|}{ New } & \multicolumn{6}{|c|}{ Used imports } \\
\hline & $\begin{array}{c}\text { Vehicle } \\
\text { price \$ }\end{array}$ & $\begin{array}{l}\text { kWh in } \\
\text { battery }\end{array}$ & $\begin{array}{c}\text { Vehicle } \\
\text { weight kg }\end{array}$ & $\begin{array}{l}\text { Time to } \\
\text { battery } \\
\text { replace- } \\
\text { ment } \\
\text { (years) }\end{array}$ & $\begin{array}{c}\text { Expected } \\
\text { battery } \\
\text { price in } \\
\text { year of } \\
\text { replace- } \\
\text { ment } \$ \\
\end{array}$ & $\begin{array}{c}\text { Max range } \\
\text { km }\end{array}$ & $\begin{array}{l}\text { Fuel cost } \\
\$ \text { per } \\
100 \mathrm{~km} \\
\text { (incl } \\
\text { charging } \\
\text { losses) }\end{array}$ & $\begin{array}{l}\text { Vehicle } \\
\text { Price \$ }\end{array}$ & $\begin{array}{c}\text { Value of } \\
\text { battery on } \\
\text { import- } \\
\text { ation \$ }\end{array}$ & $\begin{array}{c}\text { Average } \\
\text { year of } \\
\text { manu- } \\
\text { facture }\end{array}$ & $\begin{array}{l}\text { Time to } \\
\text { battery } \\
\text { replace- } \\
\text { ment } \\
\text { (years) }\end{array}$ & $\begin{array}{c}\text { Expected } \\
\text { battery } \\
\text { price in } \\
\text { year of } \\
\text { replace- } \\
\text { ment } \$\end{array}$ & $\begin{array}{c}\text { Max range } \\
\text { km }\end{array}$ \\
\hline 2012 & 60,700 & 20 & 1116 & 8 & 20,700 & 150 & 2.89 & 40,000 & 20,734 & 2012 & 7 & 20,700 & 150 \\
\hline 2013 & 57,700 & 20 & 1116 & 8 & 19,200 & 150 & 2.92 & 40,000 & 18,142 & 2012 & 7 & 20,700 & 146 \\
\hline 2014 & 54,900 & 20 & 1116 & 8 & 17,800 & 150 & 2.95 & 40,000 & 15,550 & 2012 & 6 & 20,700 & 143 \\
\hline 2015 & 52,200 & 20 & 1116 & 8 & 16,500 & 150 & 2.98 & 40,000 & 12,959 & 2012 & 5 & 19,200 & 139 \\
\hline 2016 & 49,600 & 20 & 1116 & 8 & 15,300 & 150 & 3.01 & 35,100 & 12,017 & 2013 & 5 & 17,800 & 139 \\
\hline 2017 & 47,200 & 20 & 1116 & 8 & 14,200 & 150 & 3.05 & 29,600 & 9,613 & 2013 & 4 & 16,500 & 135 \\
\hline 2018 & 44,900 & 20 & 1116 & 8 & 13,200 & 150 & 3.08 & 27,200 & 7,210 & 2013 & 3 & 15,300 & 131 \\
\hline 2019 & 42,800 & 20 & 1116 & 8 & 12,200 & 150 & 3.12 & 22,800 & 4,807 & 2013 & 2 & 14,200 & 128 \\
\hline 2020 & 40,700 & 20 & 1116 & 8 & 11,300 & 150 & 3.15 & 18,900 & 4,457 & 2014 & 2 & 13,200 & 128 \\
\hline 2021 & 38,800 & 20 & 1116 & 8 & 10,500 & 150 & 3.18 & 15,500 & 2,229 & 2014 & 1 & 12,200 & 124 \\
\hline 2022 & 37,000 & 20 & 1116 & 8 & 9,800 & 150 & 3.22 & 15,200 & 2,067 & 2015 & 1 & 11,300 & 124 \\
\hline 2023 & 35,200 & 20 & 1116 & 8 & 9,000 & 150 & 3.25 & 12,600 & 1,917 & 2016 & 1 & 10,500 & 124 \\
\hline 2024 & 33,600 & 20 & 1116 & 8 & 8,400 & 150 & 3.29 & 13,800 & 1,777 & 2017 & 1 & 9,800 & 124 \\
\hline 2025 & 32,000 & 20 & 1116 & 8 & 7,800 & 150 & 3.33 & 12,200 & 1,648 & 2018 & 1 & 9,000 & 124 \\
\hline 2026 & 31,100 & 20 & 1116 & 8 & 7,800 & 150 & 3.36 & 13,600 & 1,528 & 2019 & 1 & 8,400 & 124 \\
\hline 2027 & 30,200 & 20 & 1116 & 8 & 7,800 & 150 & 3.40 & 12,100 & 1,417 & 2020 & 1 & 7,800 & 124 \\
\hline 2028 & 29,400 & 20 & 1116 & 8 & 7,800 & 150 & 3.44 & 12,700 & 1,314 & 2021 & 1 & 7,800 & 124 \\
\hline 2029 & 28,600 & 20 & 1116 & 8 & 7,800 & 150 & 3.48 & 12,500 & 1,219 & 2022 & 1 & 7,800 & 124 \\
\hline 2030 & 27,800 & 20 & 1116 & 8 & 7,800 & 150 & 3.51 & 12,200 & 1,130 & 2023 & 1 & 7,800 & 124 \\
\hline
\end{tabular}


Table A6.3 General purpose electric vehicles

\begin{tabular}{|c|c|c|c|c|c|c|c|c|c|c|c|c|c|}
\hline & \multicolumn{7}{|l|}{ New } & \multicolumn{6}{|c|}{ Used imports } \\
\hline & $\begin{array}{l}\text { Vehicle } \\
\text { price \$ }\end{array}$ & $\begin{array}{l}\mathrm{KWh} \text { in } \\
\text { battery }\end{array}$ & $\begin{array}{c}\text { Vehicle } \\
\text { weight kg }\end{array}$ & $\begin{array}{c}\text { Time to } \\
\text { battery } \\
\text { replace- } \\
\text { ment } \\
\text { (years) }\end{array}$ & $\begin{array}{c}\text { Expected } \\
\text { battery } \\
\text { price in } \\
\text { year of } \\
\text { replace- } \\
\text { ment } \$\end{array}$ & $\begin{array}{c}\text { Max range } \\
\text { km }\end{array}$ & $\begin{array}{l}\text { Fuel cost } \\
\$ \text { per } \\
100 \mathrm{~km} \\
\text { (incl } \\
\text { charging } \\
\text { losses) }\end{array}$ & $\begin{array}{l}\text { Vehicle } \\
\text { price \$ }\end{array}$ & $\begin{array}{c}\text { Value of } \\
\text { battery on } \\
\text { import- } \\
\text { ation \$ }\end{array}$ & $\begin{array}{c}\text { Average } \\
\text { year of } \\
\text { manu- } \\
\text { facture }\end{array}$ & $\begin{array}{c}\text { Time to } \\
\text { battery } \\
\text { replace- } \\
\text { ment } \\
\text { (years) }\end{array}$ & $\begin{array}{c}\text { Expected } \\
\text { battery } \\
\text { price in } \\
\text { year of } \\
\text { replace- } \\
\text { ment } \$\end{array}$ & $\begin{array}{c}\text { Max range } \\
\text { km }\end{array}$ \\
\hline 2012 & 79,100 & 28 & 1589 & 8 & 29,100 & 180 & 3.41 & 61,600 & 29,098 & 2012 & 7 & 29,100 & 180 \\
\hline 2013 & 76,400 & 28 & 1589 & 8 & 27,000 & 180 & 3.45 & 56,700 & 25,460 & 2012 & 7 & 27,000 & 176 \\
\hline 2014 & 73,800 & 28 & 1589 & 8 & 25,000 & 180 & 3.49 & 46,200 & 21,823 & 2012 & 6 & 25,000 & 171 \\
\hline 2015 & 71,400 & 28 & 1589 & 8 & 23,200 & 180 & 3.53 & 39,100 & 18,186 & 2012 & 5 & 23,200 & 167 \\
\hline 2016 & 69,100 & 28 & 1589 & 8 & 21,500 & 180 & 3.56 & 35,100 & 16,864 & 2013 & 5 & 21,500 & 167 \\
\hline 2017 & 66,900 & 28 & 1589 & 8 & 20,000 & 180 & 3.60 & 29,200 & 13,492 & 2013 & 4 & 20,000 & 162 \\
\hline 2018 & 64,900 & 28 & 1589 & 8 & 18,500 & 180 & 3.64 & 23,800 & 10,119 & 2013 & 3 & 18,500 & 158 \\
\hline 2019 & 63,000 & 28 & 1589 & 8 & 17,200 & 180 & 3.68 & 18,900 & 6,746 & 2013 & 2 & 17,200 & 153 \\
\hline 2020 & 61,200 & 28 & 1589 & 8 & 15,900 & 180 & 3.72 & 17,500 & 6,256 & 2014 & 2 & 15,900 & 153 \\
\hline 2021 & 59,500 & 28 & 1589 & 8 & 14,800 & 180 & 3.76 & 13,500 & 3,128 & 2014 & 1 & 14,800 & 149 \\
\hline 2022 & 57,900 & 28 & 1589 & 8 & 13,700 & 180 & 3.81 & 14,100 & 2,900 & 2015 & 1 & 13,700 & 149 \\
\hline 2023 & 56,300 & 28 & 1589 & 8 & 12,700 & 180 & 3.85 & 11,500 & 2,690 & 2016 & 1 & 12,700 & 149 \\
\hline 2024 & 54,900 & 28 & 1589 & 8 & 11,800 & 180 & 3.89 & 13,000 & 2,494 & 2017 & 1 & 11,800 & 149 \\
\hline 2025 & 53,500 & 28 & 1589 & 8 & 10,900 & 180 & 3.93 & 10,900 & 2,313 & 2018 & 1 & 10,900 & 149 \\
\hline 2026 & 52,900 & 28 & 1589 & 8 & 10,900 & 180 & 3.98 & 11,500 & 2,145 & 2019 & 1 & 10,900 & 149 \\
\hline 2027 & 52,400 & 28 & 1589 & 8 & 10,900 & 180 & 4.02 & 11,300 & 1,989 & 2020 & 1 & 10,900 & 149 \\
\hline 2028 & 51,900 & 28 & 1589 & 8 & 10,900 & 180 & 4.06 & 11,100 & 1,844 & 2021 & 1 & 10,900 & 149 \\
\hline 2029 & 51,400 & 28 & 1589 & 8 & 10,900 & 180 & 4.11 & 10,500 & 1,710 & 2022 & 1 & 10,900 & 149 \\
\hline 2030 & 50,900 & 28 & 1589 & 8 & 10,900 & 180 & 4.15 & 10,100 & 1,586 & 2023 & 1 & 10,900 & 149 \\
\hline
\end{tabular}


Table: A6.4 Plug-in hybrid electric vehicles

\begin{tabular}{|c|c|c|c|c|c|c|c|c|c|c|c|c|c|c|}
\hline & \multicolumn{7}{|l|}{ New } & \multicolumn{7}{|c|}{ Used imports } \\
\hline & $\begin{array}{c}\text { Vehicle } \\
\text { price \$ }\end{array}$ & $\begin{array}{c}\text { Max } \\
\text { electric } \\
\text { range km }\end{array}$ & $\begin{array}{l}\mathrm{kWh} \text { in } \\
\text { battery } \\
\text { (Total) }\end{array}$ & $\begin{array}{c}\text { Vehicle } \\
\text { weight kg }\end{array}$ & $\begin{array}{c}\text { Time to } \\
\text { battery } \\
\text { replace- } \\
\text { ment } \\
\text { (years) }\end{array}$ & $\begin{array}{c}\text { Expected } \\
\text { battery } \\
\text { price in } \\
\text { year of } \\
\text { replace- } \\
\text { ment } \$ \\
\end{array}$ & $\begin{array}{c}\text { Fuel cost \$ } \\
\text { per } \\
100 \mathrm{~km} \\
\text { (incl } \\
\text { charging } \\
\text { losses) }\end{array}$ & $\begin{array}{l}\text { Vehicle } \\
\text { price \$ }\end{array}$ & $\begin{array}{c}\text { Value of } \\
\text { battery } \\
\text { on } \\
\text { import- } \\
\text { ation \$ }\end{array}$ & $\begin{array}{l}\text { Average } \\
\text { year of } \\
\text { manu- } \\
\text { facture }\end{array}$ & $\begin{array}{c}\text { Time to } \\
\text { battery } \\
\text { replace- } \\
\text { ment } \\
\text { (years) }\end{array}$ & $\begin{array}{c}\text { Expected } \\
\text { battery } \\
\text { price in } \\
\text { year of } \\
\text { replace- } \\
\text { ment \$ } \\
\end{array}$ & $\begin{array}{c}\text { Max } \\
\text { electric } \\
\text { range km }\end{array}$ & $\begin{array}{c}\text { Fuel cost } \\
\$ \text { per } \\
100 \mathrm{~km} \\
\text { (incl } \\
\text { charging } \\
\text { losses) } \\
\end{array}$ \\
\hline 2012 & 69,000 & 30 & 7.4 & 1,620 & 8 & 8,974 & 10.16 & 48,000 & 8,974 & 2012 & 8 & 4,908 & 30 & 10.16 \\
\hline 2013 & 67,000 & 30 & 7.4 & 1,620 & 8 & 8,322 & 10.27 & 45,300 & 7,853 & 2012 & 7 & 4,908 & 29 & 10.27 \\
\hline 2014 & 66,000 & 30 & 7.4 & 1,620 & 8 & 7,717 & 10.37 & 36,000 & 6,731 & 2012 & 6 & 4,908 & 29 & 10.37 \\
\hline 2015 & 64,000 & 30 & 7.4 & 1,620 & 8 & 7,157 & 10.48 & 30,700 & 5,609 & 2012 & 5 & 4,908 & 28 & 10.48 \\
\hline 2016 & 63,000 & 30 & 7.4 & 1,620 & 8 & 6,636 & 10.59 & 27,000 & 5,201 & 2013 & 5 & 4,551 & 28 & 10.67 \\
\hline 2017 & 62,000 & 30 & 7.4 & 1,620 & 8 & 6,154 & 10.70 & 22,900 & 4,161 & 2013 & 4 & 4,551 & 27 & 10.79 \\
\hline 2018 & 60,000 & 30 & 7.4 & 1,620 & 8 & 5,707 & 10.81 & 19,500 & 3,121 & 2013 & 3 & 4,551 & 26 & 10.90 \\
\hline 2019 & 59,000 & 30 & 7.4 & 1,620 & 8 & 5,292 & 10.92 & 16,700 & 2,081 & 2013 & 2 & 4,551 & 26 & 11.01 \\
\hline 2020 & 58,000 & 30 & 7.4 & 1,620 & 8 & 4,908 & 11.04 & 15,300 & 1,929 & 2014 & 2 & 4,220 & 26 & 11.22 \\
\hline 2021 & 57,000 & 30 & 7.4 & 1,620 & 8 & 4,551 & 11.15 & 13,400 & 965 & 2014 & 1 & 4,220 & 25 & 11.34 \\
\hline 2022 & 55,000 & 30 & 7.4 & 1,620 & 8 & 4,220 & 11.27 & 14,200 & 895 & 2015 & 1 & 3,914 & 25 & 11.56 \\
\hline 2023 & 54,000 & 30 & 7.4 & 1,620 & 8 & 3,914 & 11.39 & 11,300 & 830 & 2016 & 1 & 3,629 & 25 & 11.68 \\
\hline 2024 & 53,000 & 30 & 7.4 & 1,620 & 8 & 3,629 & 11.51 & 13,200 & 769 & 2017 & 1 & 3,365 & 25 & 11.90 \\
\hline 2025 & 52,000 & 30 & 7.4 & 1,620 & 8 & 3,365 & 11.64 & 10,800 & 713 & 2018 & 1 & 3,365 & 25 & 12.14 \\
\hline 2026 & 51,000 & 30 & 7.4 & 1,620 & 8 & 3,365 & 11.76 & 11,300 & 662 & 2019 & 1 & 3,365 & 25 & 12.37 \\
\hline 2027 & 51,000 & 30 & 7.4 & 1,620 & 8 & 3,365 & 11.89 & 11,600 & 613 & 2020 & 1 & 3,365 & 25 & 12.51 \\
\hline 2028 & 50,000 & 30 & 7.4 & 1,620 & 8 & 3,365 & 12.02 & 11,200 & 569 & 2021 & 1 & 3,365 & 25 & 12.75 \\
\hline 2029 & 49,000 & 30 & 7.4 & 1,620 & 8 & 3,365 & 12.15 & 10,600 & 528 & 2022 & 1 & 3,365 & 25 & 12.89 \\
\hline 2030 & 48,000 & 30 & 7.4 & 1,620 & 8 & 3,365 & 12.28 & 10,400 & 489 & 2023 & 1 & 3,365 & 25 & 13.03 \\
\hline
\end{tabular}




\section{A3 scenario: EV inputs}

Table: A6.5 City electric vehicles

\begin{tabular}{|c|c|c|c|c|c|c|c|c|c|c|c|c|c|}
\hline & \multicolumn{7}{|l|}{ New } & \multicolumn{6}{|c|}{ Used imports } \\
\hline & $\begin{array}{l}\text { Vehicle } \\
\text { price \$ }\end{array}$ & $\begin{array}{l}\text { kWh in } \\
\text { battery }\end{array}$ & $\begin{array}{l}\text { Vehicle } \\
\text { weight kg }\end{array}$ & $\begin{array}{l}\text { Time to } \\
\text { battery } \\
\text { replace- } \\
\text { ment } \\
\text { (years) }\end{array}$ & $\begin{array}{c}\text { Expected } \\
\text { battery } \\
\text { price in } \\
\text { year of } \\
\text { replace- } \\
\text { ment \$ } \\
\end{array}$ & $\begin{array}{c}\text { Max } \\
\text { range km }\end{array}$ & $\begin{array}{l}\text { Fuel cost } \\
\$ \text { per } \\
100 \mathrm{~km} \\
\text { (incl } \\
\text { charging } \\
\text { losses) } \\
\end{array}$ & $\begin{array}{l}\text { Vehicle } \\
\text { Price \$ }\end{array}$ & $\begin{array}{c}\text { Value of } \\
\text { battery } \\
\text { on } \\
\text { import- } \\
\text { ation \$ }\end{array}$ & $\begin{array}{c}\text { Average } \\
\text { year of } \\
\text { manu- } \\
\text { facture }\end{array}$ & $\begin{array}{c}\text { Time to } \\
\text { battery } \\
\text { replace- } \\
\text { ment } \\
\text { (years) }\end{array}$ & $\begin{array}{c}\text { Expected } \\
\text { battery } \\
\text { price in } \\
\text { year of } \\
\text { replace- } \\
\text { ment \$ } \\
\end{array}$ & $\begin{array}{l}\text { Max range } \\
\text { km } \\
\text { (adjusted) }\end{array}$ \\
\hline 2012 & 60,700 & 20 & 1,116 & 8 & 20,700 & 150 & 2.89 & 40,000 & 20,700 & 2012 & 8 & 20,700 & 150 \\
\hline 2013 & 58,000 & 20 & 1,102 & 9 & 19,500 & 153 & 2.91 & 40,000 & 18,100 & 2012 & 7 & 19,200 & 147 \\
\hline 2014 & 55,400 & 21 & 1,091 & 9 & 18,300 & 156 & 2.93 & 35,100 & 15,600 & 2012 & 6 & 17,800 & 145 \\
\hline 2015 & 52,900 & 21 & 1,080 & 10 & 17,300 & 158 & 2.96 & 29,600 & 13,000 & 2012 & 5 & 16,500 & 143 \\
\hline 2016 & 50,500 & 21 & 1,072 & 11 & 16,200 & 161 & 2.98 & 27,200 & 12,800 & 2013 & 6 & 15,600 & 146 \\
\hline 2017 & 48,300 & 21 & 1,064 & 11 & 15,300 & 164 & 3.01 & 22,800 & 10,500 & 2013 & 5 & 14,400 & 145 \\
\hline 2018 & 46,100 & 22 & 1,057 & 12 & 14,400 & 167 & 3.04 & 18,900 & 8,300 & 2013 & 4 & 13,400 & 143 \\
\hline 2019 & 44,100 & 22 & 1,051 & 13 & 13,500 & 169 & 3.07 & 15,500 & 6,000 & 2013 & 3 & 12,400 & 141 \\
\hline 2020 & 42,100 & 22 & 1,045 & 13 & 12,700 & 172 & 3.10 & 15,200 & 6,600 & 2014 & 3 & 11,700 & 144 \\
\hline 2021 & 40,300 & 23 & 1,040 & 14 & 12,000 & 175 & 3.13 & 12,600 & 4,600 & 2014 & 2 & 10,800 & 141 \\
\hline 2022 & 38,500 & 23 & 1,036 & 15 & 11,300 & 178 & 3.16 & 13,800 & 5,200 & 2015 & 3 & 10,200 & 143 \\
\hline 2023 & 36,800 & 23 & 1,032 & 15 & 10,600 & 181 & 3.19 & 12,200 & 5,600 & 2016 & 4 & 9,600 & 144 \\
\hline 2024 & 35,200 & 24 & 1,029 & 16 & 10,000 & 183 & 3.22 & 13,600 & 5,800 & 2017 & 4 & 9,000 & 145 \\
\hline 2025 & 33,700 & 24 & 1,026 & 17 & 9,400 & 186 & 3.25 & 12,100 & 6,000 & 2018 & 5 & 8,500 & 147 \\
\hline 2026 & 32,900 & 24 & 1,022 & 17 & 9,600 & 189 & 3.29 & 12,700 & 6,100 & 2019 & 6 & 8,600 & 148 \\
\hline 2027 & 32,100 & 25 & 1,019 & 18 & 9,700 & 192 & 3.32 & 12,500 & 6,000 & 2020 & 6 & 8,700 & 148 \\
\hline 2028 & 31,400 & 25 & 1,011 & 19 & 9,800 & 194 & 3.35 & 12,200 & 6,000 & 2021 & 7 & 8,900 & 149 \\
\hline 2029 & 30,700 & 25 & 1,009 & 19 & 9,900 & 197 & 3.39 & 11,600 & 5,900 & 2022 & 8 & 9,000 & 150 \\
\hline 2030 & 30,000 & 26 & 1,007 & 20 & 10,000 & 200 & 3.42 & 11,200 & 5,800 & 2023 & 8 & 9,100 & 151 \\
\hline
\end{tabular}


Table: A6.6 General purpose electric vehicles

\begin{tabular}{|c|c|c|c|c|c|c|c|c|c|c|c|c|c|}
\hline & \multicolumn{5}{|c|}{ New } & \multicolumn{8}{|c|}{ Used imports } \\
\hline & $\begin{array}{l}\text { Vehicle } \\
\text { price \$ }\end{array}$ & $\begin{array}{l}\mathrm{kWh} \text { in } \\
\text { battery }\end{array}$ & $\begin{array}{c}\text { Vehicle } \\
\text { weight kg }\end{array}$ & $\begin{array}{l}\text { Time to } \\
\text { battery } \\
\text { replace- } \\
\text { ment } \\
\text { (years) }\end{array}$ & $\begin{array}{c}\text { Expected } \\
\text { battery } \\
\text { price in } \\
\text { year of } \\
\text { replace- } \\
\text { ment } \$\end{array}$ & $\begin{array}{l}\text { Range km } \\
\text { (Max) }\end{array}$ & $\begin{array}{l}\text { Fuel cost } \\
\$ \text { per } \\
100 \mathrm{~km} \\
\text { (incl } \\
\text { charging } \\
\text { losses) }\end{array}$ & $\begin{array}{l}\text { Vehicle } \\
\text { Price \$ }\end{array}$ & $\begin{array}{l}\text { Value of } \\
\text { battery } \\
\text { on } \\
\text { import- } \\
\text { ation }\end{array}$ & $\begin{array}{l}\text { Average } \\
\text { year of } \\
\text { manu- } \\
\text { facture }\end{array}$ & $\begin{array}{l}\text { Time to } \\
\text { battery } \\
\text { replace- } \\
\text { ment } \\
\text { (years) }\end{array}$ & $\begin{array}{c}\text { Expected } \\
\text { battery } \\
\text { price in } \\
\text { year of } \\
\text { replace- } \\
\text { ment \$ }\end{array}$ & $\begin{array}{c}\text { Range km } \\
\text { (Max) } \\
\text { (adjusted) }\end{array}$ \\
\hline 2012 & 79,100 & 28 & 1589 & 8 & 29,100 & 180 & 3.41 & 61,600 & 29,100 & 2012 & 8 & 29,100 & 180 \\
\hline 2013 & 79,000 & 31 & 1593 & 9 & 29,600 & 198 & 3.45 & 56,700 & 25,500 & 2012 & 7 & 27,000 & 183 \\
\hline 2014 & 78,900 & 34 & 1598 & 9 & 30,100 & 216 & 3.48 & 46,200 & 21,800 & 2012 & 6 & 25,000 & 188 \\
\hline 2015 & 78,300 & 36 & 1601 & 10 & 30,100 & 233 & 3.52 & 39,100 & 18,200 & 2012 & 5 & 23,200 & 196 \\
\hline 2016 & 77,600 & 39 & 1603 & 11 & 30,000 & 251 & 3.56 & 37,600 & 19,400 & 2013 & 6 & 23,600 & 207 \\
\hline 2017 & 77,000 & 42 & 1606 & 11 & 30,000 & 269 & 3.60 & 31,600 & 15,900 & 2013 & 5 & 21,900 & 218 \\
\hline 2018 & 76,000 & 45 & 1608 & 12 & 29,600 & 287 & 3.64 & 26,200 & 12,500 & 2013 & 4 & 20,300 & 224 \\
\hline 2019 & 74,900 & 47 & 1610 & 13 & 29,100 & 304 & 3.68 & 21,300 & 9,100 & 2013 & 3 & 18,800 & 231 \\
\hline 2020 & 73,900 & 50 & 1612 & 13 & 28,700 & 322 & 3.72 & 21,900 & 10,700 & 2014 & 3 & 19,100 & 240 \\
\hline 2021 & 72,700 & 53 & 1614 & 14 & 28,000 & 340 & 3.75 & 17,900 & 7,500 & 2014 & 2 & 17,700 & 237 \\
\hline 2022 & 71,500 & 56 & 1615 & 15 & 27,300 & 358 & 3.79 & 20,200 & 9,000 & 2015 & 3 & 17,800 & 241 \\
\hline 2023 & 70,300 & 59 & 1617 & 15 & 26,700 & 376 & 3.84 & 19,100 & 10,300 & 2016 & 4 & 17,700 & 244 \\
\hline 2024 & 69,000 & 61 & 1618 & 16 & 25,900 & 393 & 3.88 & 22,000 & 11,500 & 2017 & 4 & 17,700 & 247 \\
\hline 2025 & 67,600 & 64 & 1619 & 17 & 25,000 & 411 & 3.92 & 20,900 & 12,300 & 2018 & 5 & 17,400 & 249 \\
\hline 2026 & 68,200 & 67 & 1620 & 17 & 26,200 & 429 & 3.96 & 22,400 & 13,000 & 2019 & 6 & 18,500 & 251 \\
\hline 2027 & 68,800 & 70 & 1621 & 18 & 27,300 & 447 & 4.01 & 22,900 & 13,600 & 2020 & 6 & 19,700 & 252 \\
\hline 2028 & 69,300 & 73 & 1622 & 19 & 28,300 & 464 & 4.05 & 23,200 & 14,000 & 2021 & 7 & 20,700 & 254 \\
\hline 2029 & 70,000 & 76 & 1623 & 19 & 29,500 & 482 & 4.10 & 23,000 & 14,300 & 2022 & 8 & 21,800 & 255 \\
\hline 2030 & 70,500 & 78 & 1623 & 20 & 30,500 & 500 & 4.14 & 23,000 & 14,500 & 2023 & 8 & 22,900 & 256 \\
\hline
\end{tabular}


New

\begin{tabular}{|c|c|c|c|c|c|c|c|c|c|c|c|c|c|c|}
\hline \multicolumn{8}{|c|}{$\overline{\text { New }}$} & \multicolumn{7}{|c|}{ Used imports } \\
\hline & $\begin{array}{l}\text { Vehicle } \\
\text { price \$ }\end{array}$ & $\begin{array}{c}\text { Max } \\
\text { electric } \\
\text { range km }\end{array}$ & $\begin{array}{c}\text { kWh in } \\
\text { battery } \\
\text { (Total) }\end{array}$ & $\begin{array}{c}\text { Vehicle } \\
\text { weight } \\
\text { kg }\end{array}$ & $\begin{array}{c}\text { Time to } \\
\text { battery } \\
\text { replace- } \\
\text { ment } \\
\text { (years) }\end{array}$ & $\begin{array}{c}\text { Expected } \\
\text { battery } \\
\text { price in } \\
\text { year of } \\
\text { replace- } \\
\text { ment \$ }\end{array}$ & $\begin{array}{l}\text { Fuel cost } \\
\$ \text { per } \\
100 \mathrm{~km} \\
\text { (incl } \\
\text { charging } \\
\text { losses) }\end{array}$ & $\begin{array}{l}\text { Vehicle } \\
\text { price \$ }\end{array}$ & $\begin{array}{l}\text { Value of } \\
\text { battery } \\
\text { on } \\
\text { import- } \\
\text { ation }\end{array}$ & $\begin{array}{c}\text { Average } \\
\text { year of } \\
\text { manu- } \\
\text { facture }\end{array}$ & $\begin{array}{c}\text { Time to } \\
\text { battery } \\
\text { replace- } \\
\text { ment } \\
\text { (years) }\end{array}$ & $\begin{array}{l}\text { Expected } \\
\text { battery } \\
\text { price in year } \\
\text { of replace- } \\
\text { ment \$ }\end{array}$ & $\begin{array}{c}\text { Max } \\
\text { electric } \\
\text { range km } \\
\text { (adjusted) }\end{array}$ & $\begin{array}{c}\text { Fuel cost } \\
\$ \text { per } \\
100 \mathrm{~km} \\
\text { (incl } \\
\text { charging } \\
\text { losses) }\end{array}$ \\
\hline 2012 & 69,000 & 30 & 7 & 1,620 & 8 & 8,960 & 10.16 & 48,000 & 8,960 & 2012 & 8 & 8,960 & 30 & 10.16 \\
\hline 2013 & 66,500 & 34 & 8 & 1,617 & 9 & 9,040 & 9.85 & 45,300 & 7,840 & 2012 & 7 & 8,310 & 31 & 10.27 \\
\hline 2014 & 64,400 & 38 & 9 & 1,618 & 9 & 9,390 & 9.57 & 36,000 & 6,720 & 2012 & 6 & 7,700 & 32 & 10.37 \\
\hline 2015 & 62,200 & 42 & 10 & 1,618 & 10 & 9,640 & 9.31 & 30,700 & 5,600 & 2012 & 5 & 7,140 & 34 & 10.48 \\
\hline 2016 & 60,100 & 46 & 11 & 1,619 & 11 & 9,810 & 9.08 & 27,700 & 5,910 & 2013 & 6 & 7,210 & 37 & 10.48 \\
\hline 2017 & 57,900 & 49 & 12 & 1,619 & 11 & 9,900 & 8.87 & 23,600 & 4,870 & 2013 & 5 & 6,680 & 39 & 10.59 \\
\hline 2018 & 55,200 & 53 & 13 & 1,610 & 12 & 9,120 & 8.68 & 20,200 & 3,820 & 2013 & 4 & 5,710 & 41 & 10.70 \\
\hline 2019 & 53,200 & 57 & 14 & 1,611 & 13 & 9,100 & 8.50 & 17,400 & 2,780 & 2013 & 3 & 5,290 & 42 & 10.81 \\
\hline 2020 & 51,200 & 61 & 15 & 1,612 & 13 & 9,020 & 8.34 & 16,700 & 3,350 & 2014 & 3 & 5,500 & 44 & 10.79 \\
\hline 2021 & 51,100 & 65 & 16 & 1,604 & 14 & 8,260 & 8.19 & 14,800 & 2,350 & 2014 & 2 & 4,740 & 44 & 10.90 \\
\hline 2022 & 51,500 & 69 & 17 & 1,604 & 15 & 8,130 & 8.05 & 16,200 & 2,890 & 2015 & 3 & 4,860 & 45 & 10.78 \\
\hline 2023 & 51,900 & 73 & 18 & 1,605 & 15 & 7,980 & 7.93 & 13,800 & 3,370 & 2016 & 4 & 4,950 & 45 & 10.64 \\
\hline 2024 & 52,200 & 77 & 19 & 1,605 & 16 & 7,800 & 7.82 & 16,300 & 3,780 & 2017 & 4 & 4,990 & 46 & 10.48 \\
\hline 2025 & 52,500 & 81 & 19 & 1,604 & 17 & 7,480 & 7.71 & 13,900 & 3,800 & 2018 & 5 & 5,000 & 46 & 10.44 \\
\hline 2026 & 52,900 & 84 & 20 & 1,604 & 17 & 7,860 & 7.62 & 14,700 & 4,070 & 2019 & 6 & 5,370 & 46 & 10.38 \\
\hline 2027 & 53,200 & 88 & 21 & 1,605 & 18 & 8,230 & 7.53 & 15,200 & 4,290 & 2020 & 6 & 5,750 & 47 & 10.32 \\
\hline 2028 & 53,600 & 92 & 22 & 1,605 & 19 & 8,610 & 7.45 & 14,800 & 4,130 & 2021 & 7 & 6,110 & 47 & 10.46 \\
\hline 2029 & 54,000 & 96 & 23 & 1,606 & 19 & 8,980 & 7.38 & 14,300 & 4,250 & 2022 & 8 & 6,490 & 47 & 10.50 \\
\hline 2030 & 54,400 & 100 & 24 & 1,606 & 20 & 9,360 & 7.31 & 14,300 & 4,340 & 2023 & 8 & 6,860 & 48 & 10.56 \\
\hline
\end{tabular}


B scenario: Internal combustion engine vehicle inputs

Table: A6.8: Internal combustion engine vehicles

\begin{tabular}{|c|c|c|c|c|c|c|}
\hline & $\begin{array}{c}\text { Crude oil } \\
\text { price } \\
\text { US\$/bbl }\end{array}$ & $\begin{array}{l}\text { Carbon } \\
\text { charge } \\
\$ / \text { tonne } \\
\text { Carbon } \\
\end{array}$ & $\begin{array}{c}\text { Combined } \\
\text { Fuel price } \\
\$ / L\end{array}$ & $\begin{array}{l}\text { Petrol Fuel } \\
\text { efficiency } \\
\text { 1/100 km }\end{array}$ & $\begin{array}{l}\text { Diesel Fuel } \\
\text { efficiency } \\
\text { 1/100 km }\end{array}$ & $\begin{array}{l}\text { Fuel cost } \\
\$ / 100 \mathrm{~km}\end{array}$ \\
\hline 2010 & 100 & 12.5 & 2.30 & 9.9 & 9.4 & 22.70 \\
\hline 2011 & 100 & 12.5 & 2.30 & 9.9 & 9.4 & 22.70 \\
\hline 2012 & 150 & 25 & 3.10 & 9.9 & 9.4 & 30.61 \\
\hline 2013 & 200 & 25 & 3.87 & 9.9 & 9.4 & 38.22 \\
\hline 2014 & 250 & 25 & 4.64 & 9.9 & 9.4 & 45.83 \\
\hline 2015 & 300 & 25 & 5.41 & 9.9 & 9.4 & 53.44 \\
\hline 2016 & 293 & 25 & 5.30 & 9.6 & 9.1 & 52.35 \\
\hline 2017 & 286 & 25 & 5.19 & 9.3 & 8.8 & 51.27 \\
\hline 2018 & 279 & 25 & 5.08 & 8.9 & 8.5 & 50.13 \\
\hline 2019 & 271 & 25 & 4.97 & 8.6 & 8.2 & 49.05 \\
\hline 2020 & 264 & 25 & 4.86 & 8.3 & 7.8 & 47.96 \\
\hline 2021 & 257 & 25 & 4.75 & 8.0 & 7.5 & 46.83 \\
\hline 2022 & 250 & 25 & 4.64 & 7.6 & 7.2 & 45.70 \\
\hline 2023 & 250 & 25 & 4.65 & 7.3 & 6.9 & 45.62 \\
\hline 2024 & 250 & 25 & 4.65 & 7.0 & 6.6 & 45.53 \\
\hline 2025 & 250 & 25 & 4.65 & 6.6 & 6.3 & 45.41 \\
\hline 2026 & 250 & 25 & 4.65 & 6.3 & 6.0 & 45.24 \\
\hline 2027 & 250 & 25 & 4.65 & 6.0 & 5.6 & 45.07 \\
\hline 2028 & 250 & 25 & 4.65 & 5.7 & 5.3 & 44.81 \\
\hline 2029 & 250 & 25 & 4.66 & 5.3 & 5.0 & 44.46 \\
\hline 2030 & 250 & 25 & 4.66 & 5.0 & 4.7 & 44.07 \\
\hline
\end{tabular}




\section{B3 scenario: EV inputs}

For CEVs and GEVs refer to the input tables for scenario A3.

Table: A6.9 Plug-in hybrid electric vehicles

\begin{tabular}{|c|c|c|c|c|c|c|c|c|c|c|c|c|c|c|}
\hline \multicolumn{8}{|c|}{ New } & \multicolumn{7}{|c|}{ Used imports } \\
\hline & $\begin{array}{c}\text { Vehicle } \\
\text { price \$ }\end{array}$ & $\begin{array}{c}\text { Max } \\
\text { electric } \\
\text { range km } \\
\text { (adjusted) }\end{array}$ & $\begin{array}{l}\text { kWh in } \\
\text { battery } \\
\text { (Total) }\end{array}$ & $\begin{array}{l}\text { Vehicle } \\
\text { weight kg }\end{array}$ & $\begin{array}{c}\text { Time to } \\
\text { battery } \\
\text { replace- } \\
\text { ment } \\
\text { (years) }\end{array}$ & $\begin{array}{c}\text { Expected } \\
\text { battery } \\
\text { price in } \\
\text { year of } \\
\text { replace- } \\
\text { ment \$ }\end{array}$ & $\begin{array}{l}\text { Fuel cost } \\
\$ \text { per } \\
100 \mathrm{~km} \\
\text { (incl } \\
\text { charging } \\
\text { losses) }\end{array}$ & $\begin{array}{l}\text { Vehicle } \\
\text { price \$ }\end{array}$ & $\begin{array}{l}\text { Value of } \\
\text { battery } \\
\text { on } \\
\text { import- } \\
\text { ation }\end{array}$ & $\begin{array}{l}\text { Average } \\
\text { year of } \\
\text { manu- } \\
\text { facture }\end{array}$ & $\begin{array}{c}\text { Time to } \\
\text { battery } \\
\text { replace- } \\
\text { ment } \\
\text { (years) }\end{array}$ & $\begin{array}{c}\text { Expected } \\
\text { battery } \\
\text { price in } \\
\text { year of } \\
\text { replace- } \\
\text { ment } \$\end{array}$ & $\begin{array}{c}\text { Max } \\
\text { electric } \\
\text { range km } \\
\text { (adjusted) }\end{array}$ & $\begin{array}{l}\text { Fuel cost } \\
\$ \text { per } \\
100 \mathrm{~km} \\
\text { (incl } \\
\text { charging } \\
\text { losses) }\end{array}$ \\
\hline 2012 & 69,000 & 30 & 7 & 1,620 & 8 & 8,960 & 12.81 & 48000 & 8,960 & 2012 & 8 & 8,960 & 30 & 12.81 \\
\hline 2013 & 66,500 & 34 & 8 & 1,617 & 9 & 8,620 & 14.63 & 45300 & 7,840 & 2012 & 7 & 8,310 & 31 & 15.65 \\
\hline 2014 & 64,400 & 38 & 9 & 1,618 & 9 & 8,540 & 16.11 & 36000 & 6,720 & 2012 & 6 & 7,700 & 32 & 18.49 \\
\hline 2015 & 62,200 & 42 & 10 & 1,618 & 10 & 8,370 & 17.31 & 30700 & 5,600 & 2012 & 5 & 7,140 & 34 & 21.32 \\
\hline 2016 & 60,100 & 46 & 11 & 1,619 & 11 & 8,120 & 15.94 & 27700 & 5,910 & 2013 & 6 & 7,210 & 37 & 20.27 \\
\hline 2017 & 57,900 & 49 & 12 & 1,619 & 11 & 7,820 & 14.72 & 23600 & 4,870 & 2013 & 5 & 6,680 & 39 & 19.90 \\
\hline 2018 & 55,200 & 53 & 13 & 1,610 & 12 & 6,870 & 13.61 & 20200 & 3,820 & 2013 & 4 & 5,710 & 41 & 19.53 \\
\hline 2019 & 53,200 & 57 & 14 & 1,611 & 13 & 6,540 & 12.63 & 17400 & 2,780 & 2013 & 3 & 5,290 & 42 & 19.16 \\
\hline 2020 & 51,200 & 61 & 15 & 1,612 & 13 & 6,190 & 11.74 & 16700 & 3,350 & 2014 & 3 & 5,500 & 44 & 18.14 \\
\hline 2021 & 51,100 & 65 & 16 & 1,604 & 14 & 6,110 & 10.94 & 14800 & 2,350 & 2014 & 2 & 4,740 & 44 & 17.79 \\
\hline 2022 & 51,500 & 69 & 17 & 1,604 & 15 & 6,490 & 10.22 & 16200 & 2,890 & 2015 & 3 & 4,860 & 45 & 16.66 \\
\hline 2023 & 51,900 & 73 & 18 & 1,605 & 15 & 6,860 & 9.75 & 13800 & 3,370 & 2016 & 4 & 4,950 & 45 & 15.89 \\
\hline 2024 & 52,200 & 77 & 19 & 1,605 & 16 & 7,240 & 9.31 & 16300 & 3,780 & 2017 & 4 & 4,990 & 46 & 15.14 \\
\hline 2025 & 52,500 & 81 & 19 & 1,604 & 17 & 7,480 & 8.91 & 13900 & 3,800 & 2018 & 5 & 5,000 & 46 & 14.60 \\
\hline 2026 & 52,900 & 84 & 20 & 1,604 & 17 & 7,860 & 8.54 & 14700 & 4,070 & 2019 & 6 & 5,370 & 46 & 14.05 \\
\hline 2027 & 53,200 & 88 & 21 & 1,605 & 18 & 8,230 & 8.21 & 15200 & 4,290 & 2020 & 6 & 5,750 & 47 & 13.53 \\
\hline 2028 & 53,600 & 92 & 22 & 1,605 & 19 & 8,610 & 7.90 & 14800 & 4,130 & 2021 & 7 & 6,110 & 47 & 13.32 \\
\hline 2029 & 54,000 & 96 & 23 & 1,606 & 19 & 8,980 & 7.62 & 14300 & 4,250 & 2022 & 8 & 6,490 & 47 & 12.95 \\
\hline 2030 & 54,400 & 100 & 24 & 1,606 & 20 & 9,360 & 7.36 & 14300 & 4,340 & 2023 & 8 & 6,860 & 48 & 12.63 \\
\hline
\end{tabular}




\section{B4 scenario: EV inputs}

Table: A6.10 City electric vehicles

\begin{tabular}{|c|c|c|c|c|c|c|c|c|c|c|c|c|c|}
\hline \multicolumn{8}{|c|}{ New } & \multicolumn{6}{|c|}{ Used imports } \\
\hline & $\begin{array}{l}\text { Vehicle } \\
\text { price \$ }\end{array}$ & $\begin{array}{l}\text { kWh in } \\
\text { battery }\end{array}$ & $\begin{array}{c}\text { Vehicle } \\
\text { weight } \\
\text { kg }\end{array}$ & $\begin{array}{c}\text { Time to } \\
\text { battery } \\
\text { replace- } \\
\text { ment } \\
\text { (years) }\end{array}$ & $\begin{array}{c}\text { Expected } \\
\text { battery } \\
\text { price in } \\
\text { year of } \\
\text { replace- } \\
\text { ment \$ }\end{array}$ & $\begin{array}{c}\text { Max range } \\
\text { km }\end{array}$ & $\begin{array}{c}\text { Fuel cost \$ } \\
\text { per } \\
100 \mathrm{~km} \\
\text { (incl } \\
\text { charging } \\
\text { losses) }\end{array}$ & $\begin{array}{l}\text { Vehicle } \\
\text { Price \$ }\end{array}$ & $\begin{array}{c}\text { Value of } \\
\text { battery on } \\
\text { import- } \\
\text { ation \$ }\end{array}$ & $\begin{array}{c}\text { Average } \\
\text { year of } \\
\text { manu- } \\
\text { facture }\end{array}$ & $\begin{array}{c}\text { Time to } \\
\text { battery } \\
\text { replace- } \\
\text { ment } \\
\text { (years) }\end{array}$ & $\begin{array}{l}\text { Expected } \\
\text { battery } \\
\text { price in year } \\
\text { of replace- } \\
\text { ment \$ }\end{array}$ & $\begin{array}{c}\text { Max range } \\
\text { km } \\
\text { (adjusted) }\end{array}$ \\
\hline 2012 & 60,700 & 20 & 1,116 & 8 & 20,730 & 150 & 2.89 & 40,000 & 20,730 & 2012 & 8 & 20,730 & 150 \\
\hline 2013 & 55,300 & 20 & 1,102 & 9 & 18,600 & 153 & 2.91 & 40,000 & 18,140 & 2012 & 7 & 18,340 & 147 \\
\hline 2014 & 50,300 & 21 & 1,091 & 9 & 16,700 & 156 & 2.93 & 35,100 & 15,550 & 2012 & 6 & 16,230 & 145 \\
\hline 2015 & 45,800 & 21 & 1,080 & 10 & 14,990 & 158 & 2.96 & 29,600 & 12,960 & 2012 & 5 & 14,350 & 143 \\
\hline 2016 & 41,700 & 21 & 1,072 & 11 & 13,450 & 161 & 2.98 & 26,400 & 12,160 & 2013 & 6 & 12,880 & 146 \\
\hline 2017 & 38,000 & 21 & 1,064 & 11 & 12,070 & 164 & 3.01 & 22,000 & 10,020 & 2013 & 5 & 11,390 & 145 \\
\hline 2018 & 34,600 & 22 & 1,057 & 12 & 10,840 & 167 & 3.04 & 18,100 & 7,870 & 2013 & 4 & 10,080 & 143 \\
\hline 2019 & 31,500 & 22 & 1,051 & 13 & 9,720 & 169 & 3.07 & 14,800 & 5,720 & 2013 & 3 & 8,920 & 141 \\
\hline 2020 & 28,700 & 22 & 1,045 & 13 & 8,730 & 172 & 3.10 & 14,200 & 5,960 & 2014 & 3 & 8,000 & 144 \\
\hline 2021 & 28,900 & 23 & 1,040 & 14 & 8,850 & 175 & 3.13 & 11,800 & 4,170 & 2014 & 2 & 8,000 & 141 \\
\hline 2022 & 29,000 & 23 & 1,036 & 15 & 8,980 & 178 & 3.16 & 12,600 & 4,500 & 2015 & 3 & 8,120 & 143 \\
\hline 2023 & 29,100 & 23 & 1,032 & 15 & 9,100 & 181 & 3.19 & 10,500 & 4,620 & 2016 & 4 & 8,240 & 144 \\
\hline 2024 & 29,300 & 24 & 1,029 & 16 & 9,300 & 183 & 3.22 & 11,400 & 4,620 & 2017 & 4 & 8,360 & 145 \\
\hline 2025 & 29,400 & 24 & 1,026 & 17 & 9,420 & 186 & 3.25 & 9,700 & 4,510 & 2018 & 5 & 8,480 & 147 \\
\hline 2026 & 29,600 & 24 & 1,022 & 17 & 9,550 & 189 & 3.29 & 9,800 & 4,350 & 2019 & 6 & 8,600 & 148 \\
\hline 2027 & 29,700 & 25 & 1,019 & 18 & 9,680 & 192 & 3.32 & 9,200 & 4,140 & 2020 & 6 & 8,730 & 148 \\
\hline 2028 & 29,800 & 25 & 1,011 & 19 & 9,790 & 194 & 3.35 & 9,200 & 4,430 & 2021 & 7 & 8,850 & 149 \\
\hline 2029 & 29,900 & 25 & 1,009 & 19 & 9,920 & 197 & 3.39 & 9,000 & 4,690 & 2022 & 8 & 8,980 & 150 \\
\hline 2030 & 30,000 & 26 & 1,007 & 20 & 10,050 & 200 & 3.42 & 9,000 & 4,950 & 2023 & 8 & 9,100 & 151 \\
\hline
\end{tabular}


Table: A6.11 General purpose electric vehicles

\begin{tabular}{|c|c|c|c|c|c|c|c|c|c|c|c|c|c|}
\hline \multicolumn{8}{|c|}{ New } & \multicolumn{2}{|c|}{ Used imports } & \multirow[b]{2}{*}{$\begin{array}{l}\text { Average } \\
\text { year of } \\
\text { manu- } \\
\text { facture }\end{array}$} & \multirow[b]{2}{*}{$\begin{array}{l}\text { Time to } \\
\text { battery } \\
\text { replace- } \\
\text { ment } \\
\text { (years) }\end{array}$} & \multirow[b]{2}{*}{$\begin{array}{c}\text { Expected } \\
\text { battery } \\
\text { price in } \\
\text { year of } \\
\text { replace- } \\
\text { ment \$ }\end{array}$} & \multirow[b]{2}{*}{$\begin{array}{l}\text { Max range } \\
\text { km } \\
\text { (adjusted) }\end{array}$} \\
\hline & $\begin{array}{c}\text { Vehicle } \\
\text { price \$ }\end{array}$ & $\begin{array}{l}\text { kWh in } \\
\text { battery }\end{array}$ & $\begin{array}{c}\text { Vehicle } \\
\text { weight kg }\end{array}$ & $\begin{array}{l}\text { Time to } \\
\text { battery } \\
\text { replace- } \\
\text { ment } \\
\text { (years) }\end{array}$ & $\begin{array}{c}\text { Expected } \\
\text { battery } \\
\text { price in } \\
\text { year of } \\
\text { replace- } \\
\text { ment \$ }\end{array}$ & $\begin{array}{c}\text { Max } \\
\text { range km }\end{array}$ & $\begin{array}{l}\text { Fuel cost } \\
\$ \text { per } \\
100 \mathrm{~km} \\
\text { (incl } \\
\text { charging } \\
\text { losses) }\end{array}$ & $\begin{array}{l}\text { Vehicle } \\
\text { price \$ }\end{array}$ & $\begin{array}{l}\text { Value of } \\
\text { battery } \\
\text { on } \\
\text { import- } \\
\text { ation } \$\end{array}$ & & & & \\
\hline 2012 & 79,100 & 28 & 1,589 & 8 & 29,100 & 180 & 3.41 & 61,600 & 29,100 & 2012 & 8 & 29,100 & 180 \\
\hline 2013 & 76,900 & 31 & 1,593 & 9 & 28,240 & 198 & 3.45 & 56,700 & 25,500 & 2012 & 8 & 25,700 & 183 \\
\hline 2014 & 74,700 & 34 & 1,598 & 9 & 27,370 & 216 & 3.48 & 46,200 & 21,800 & 2012 & 8 & 22,800 & 188 \\
\hline 2015 & 72,200 & 36 & 1,601 & 10 & 26,170 & 233 & 3.52 & 39,100 & 18,200 & 2012 & 8 & 20,100 & 196 \\
\hline 2016 & 69,600 & 39 & 1,603 & 11 & 24,880 & 251 & 3.56 & 36,600 & 18,500 & 2013 & 9 & 19,500 & 207 \\
\hline 2017 & 67,200 & 42 & 1,606 & 11 & 23,670 & 269 & 3.60 & 30,700 & 15,200 & 2013 & 9 & 17,300 & 218 \\
\hline 2018 & 64,600 & 45 & 1,608 & 12 & 22,290 & 287 & 3.64 & 25,400 & 11,900 & 2013 & 9 & 15,300 & 224 \\
\hline 2019 & 62,100 & 47 & 1,610 & 13 & 20,920 & 304 & 3.68 & 20,700 & 8,700 & 2013 & 9 & 13,500 & 231 \\
\hline 2020 & 59,700 & 50 & 1,612 & 13 & 19,650 & 322 & 3.72 & 20,800 & 9,800 & 2014 & 9 & 13,100 & 240 \\
\hline 2021 & 60,700 & 53 & 1,614 & 14 & 20,710 & 340 & 3.75 & 17,000 & 6,800 & 2014 & 9 & 13,100 & 237 \\
\hline 2022 & 61,800 & 56 & 1,615 & 15 & 21,770 & 358 & 3.79 & 18,800 & 7,900 & 2015 & 10 & 14,200 & 241 \\
\hline 2023 & 62,900 & 59 & 1,617 & 15 & 22,920 & 376 & 3.84 & 17,000 & 8,600 & 2016 & 11 & 15,200 & 244 \\
\hline 2024 & 64,000 & 61 & 1,618 & 16 & 23,980 & 393 & 3.88 & 19,100 & 9,000 & 2017 & 11 & 16,400 & 247 \\
\hline 2025 & 65,000 & 64 & 1,619 & 17 & 25,040 & 411 & 3.92 & 17,300 & 9,300 & 2018 & 12 & 17,400 & 249 \\
\hline 2026 & 66,200 & 67 & 1,620 & 17 & 26,190 & 429 & 3.96 & 18,100 & 9,400 & 2019 & 13 & 18,500 & 251 \\
\hline 2027 & 67,300 & 70 & 1,621 & 18 & 27,250 & 447 & 4.01 & 18,000 & 9,300 & 2020 & 13 & 19,700 & 252 \\
\hline 2028 & 68,300 & 73 & 1,622 & 19 & 28,310 & 464 & 4.05 & 18,800 & 10,400 & 2021 & 14 & 20,700 & 254 \\
\hline 2029 & 69,500 & 76 & 1,623 & 19 & 29,460 & 482 & 4.10 & 19,400 & 11,400 & 2022 & 15 & 21,800 & 255 \\
\hline 2030 & 70,500 & 78 & 1,623 & 20 & 30,520 & 500 & 4.14 & 20,200 & 12,500 & 2023 & 15 & 22,900 & 256 \\
\hline
\end{tabular}


Table: A6.12 Plug-in hybrid electric vehicles

\begin{tabular}{|c|c|c|c|c|c|c|c|c|c|c|c|c|c|c|}
\hline \multicolumn{8}{|c|}{ New } & \multicolumn{7}{|c|}{ Used imports } \\
\hline & $\begin{array}{c}\text { Vehicle } \\
\text { price \$ }\end{array}$ & $\begin{array}{c}\text { Max } \\
\text { electric } \\
\text { range km }\end{array}$ & $\begin{array}{l}\text { kWh in } \\
\text { battery } \\
\text { (Total) }\end{array}$ & $\begin{array}{l}\text { Vehicle } \\
\text { weight kg }\end{array}$ & $\begin{array}{l}\text { Time to } \\
\text { battery } \\
\text { replace- } \\
\text { ment } \\
\text { (years) }\end{array}$ & $\begin{array}{c}\text { Expected } \\
\text { battery } \\
\text { price in } \\
\text { year of } \\
\text { replace- } \\
\text { ment } \$\end{array}$ & $\begin{array}{l}\text { Fuel cost } \\
\$ \text { per } \\
100 \mathrm{~km} \\
\text { (incl } \\
\text { charging } \\
\text { losses) }\end{array}$ & $\begin{array}{l}\text { Vehicle } \\
\text { price \$ }\end{array}$ & $\begin{array}{l}\text { Value of } \\
\text { battery } \\
\text { on } \\
\text { import- } \\
\text { ation }\end{array}$ & $\begin{array}{l}\text { Average } \\
\text { year of } \\
\text { manu- } \\
\text { facture }\end{array}$ & $\begin{array}{l}\text { Time to } \\
\text { battery } \\
\text { replace- } \\
\text { ment } \\
\text { (years) }\end{array}$ & $\begin{array}{c}\text { Expected } \\
\text { battery } \\
\text { price in } \\
\text { year of } \\
\text { replace- } \\
\text { ment } \$\end{array}$ & $\begin{array}{c}\text { Max } \\
\text { electric } \\
\text { range km } \\
\text { (adjusted) }\end{array}$ & $\begin{array}{l}\text { Fuel cost } \\
\$ \text { per } \\
100 \mathrm{~km} \\
\text { (incl } \\
\text { charging } \\
\text { losses) }\end{array}$ \\
\hline 2012 & 69,000 & 30 & 7 & 1,620 & 8 & 8,960 & 12.81 & 48,000 & 8,960 & 2012 & 8 & 8,960 & 30 & 12.81 \\
\hline 2013 & 66,500 & 34 & 8 & 1,617 & 9 & 8,620 & 14.63 & 45,300 & 7,840 & 2012 & 7 & 7,920 & 31 & 15.65 \\
\hline 2014 & 64,400 & 38 & 9 & 1,618 & 9 & 8,540 & 16.11 & 36,000 & 6,720 & 2012 & 6 & 7,010 & 32 & 18.49 \\
\hline 2015 & 62,200 & 42 & 10 & 1,618 & 10 & 8,370 & 17.31 & 30,600 & 5,600 & 2012 & 5 & 6,200 & 34 & 21.32 \\
\hline 2016 & 60,100 & 46 & 11 & 1,619 & 11 & 8,120 & 15.94 & 27,300 & 5,640 & 2013 & 6 & 5,970 & 37 & 20.27 \\
\hline 2017 & 57,900 & 49 & 12 & 1,619 & 11 & 7,820 & 14.72 & 23,200 & 4,640 & 2013 & 5 & 5,280 & 39 & 19.90 \\
\hline 2018 & 55,200 & 53 & 13 & 1,610 & 12 & 6,870 & 13.61 & 19,700 & 3,650 & 2013 & 4 & 4,300 & 41 & 19.53 \\
\hline 2019 & 53,200 & 57 & 14 & 1,611 & 13 & 6,540 & 12.63 & 17,000 & 2,650 & 2013 & 3 & 3,810 & 42 & 19.16 \\
\hline 2020 & 51,200 & 61 & 15 & 1,612 & 13 & 6,190 & 11.74 & 16,100 & 3,050 & 2014 & 3 & 3,770 & 44 & 18.14 \\
\hline 2021 & 51,100 & 65 & 16 & 1,604 & 14 & 6,110 & 10.94 & 14,200 & 2,140 & 2014 & 2 & 3,500 & 44 & 17.79 \\
\hline 2022 & 51,500 & 69 & 17 & 1,604 & 15 & 6,490 & 10.22 & 15,400 & 2,510 & 2015 & 3 & 3,880 & 45 & 16.66 \\
\hline 2023 & 51,900 & 73 & 18 & 1,605 & 15 & 6,860 & 9.75 & 12,700 & 2,790 & 2016 & 4 & 4,250 & 45 & 15.89 \\
\hline 2024 & 52,200 & 77 & 19 & 1,605 & 16 & 7,240 & 9.31 & 14,800 & 2,990 & 2017 & 4 & 4,630 & 46 & 15.14 \\
\hline 2025 & 52,500 & 81 & 19 & 1,604 & 17 & 7,480 & 8.91 & 12,200 & 2,860 & 2018 & 5 & 5,000 & 46 & 14.60 \\
\hline 2026 & 52,900 & 84 & 20 & 1,604 & 17 & 7,860 & 8.54 & 12,700 & 2,930 & 2019 & 6 & 5,370 & 46 & 14.05 \\
\hline 2027 & 53,200 & 88 & 21 & 1,605 & 18 & 8,230 & 8.21 & 12,900 & 2,940 & 2020 & 6 & 5,750 & 47 & 13.53 \\
\hline 2028 & 53,600 & 92 & 22 & 1,605 & 19 & 8,610 & 7.90 & 12,600 & 3,060 & 2021 & 7 & 6,110 & 47 & 13.32 \\
\hline 2029 & 54,000 & 96 & 23 & 1,606 & 19 & 8,980 & 7.62 & 12,300 & 3,390 & 2022 & 8 & 6,490 & 47 & 12.95 \\
\hline 2030 & 54,400 & 100 & 24 & 1,606 & 20 & 9,360 & 7.36 & 12,500 & 3,730 & 2023 & 8 & 6,860 & 48 & 12.63 \\
\hline
\end{tabular}




\section{C scenario: Internal combustion engine vehicle inputs}

Table: A6.13 Internal combustion engine vehicles

\begin{tabular}{|c|c|c|c|c|c|c|}
\hline & $\begin{array}{l}\text { Crude } \\
\text { oil price } \\
\text { US\$/bbl }\end{array}$ & $\begin{array}{l}\text { Carbon } \\
\text { charge } \\
\$ / \text { tonne } \\
\text { Carbon }\end{array}$ & $\begin{array}{c}\text { Combined } \\
\text { Fuel price } \\
\$ / L\end{array}$ & $\begin{array}{l}\text { Petrol Fuel } \\
\text { efficiency } \\
\text { I/100 km }\end{array}$ & $\begin{array}{l}\text { Diesel Fuel } \\
\text { efficiency } \\
\text { I/100 km }\end{array}$ & $\begin{array}{l}\text { Fuel cost } \\
\$ / 100 \mathrm{~km}\end{array}$ \\
\hline 2010 & 100 & 12.5 & 2.51 & 9.9 & 9.4 & 24.76 \\
\hline 2011 & 101 & 12.5 & 2.52 & 9.9 & 9.4 & 24.85 \\
\hline 2012 & 101 & 100 & 2.53 & 9.7 & 9.2 & 24.95 \\
\hline 2013 & 102 & 100 & 2.54 & 9.4 & 8.9 & 25.04 \\
\hline 2014 & 102 & 100 & 2.55 & 9.1 & 8.7 & 25.11 \\
\hline 2015 & 103 & 100 & 2.56 & 8.9 & 8.4 & 25.21 \\
\hline 2016 & 104 & 100 & 2.57 & 8.6 & 8.2 & 25.30 \\
\hline 2017 & 104 & 100 & 2.58 & 8.4 & 7.9 & 25.38 \\
\hline 2018 & 105 & 100 & 2.59 & 8.1 & 7.7 & 25.48 \\
\hline 2019 & 106 & 100 & 2.60 & 7.8 & 7.4 & 25.55 \\
\hline 2020 & 106 & 100 & 2.61 & 7.6 & 7.2 & 25.63 \\
\hline 2021 & 107 & 100 & 2.62 & 7.3 & 6.9 & 25.69 \\
\hline 2022 & 108 & 100 & 2.63 & 7.1 & 6.7 & 25.75 \\
\hline 2023 & 108 & 100 & 2.64 & 6.8 & 6.4 & 25.79 \\
\hline 2024 & 109 & 100 & 2.65 & 6.6 & 6.2 & 25.82 \\
\hline 2025 & 110 & 100 & 2.67 & 6.3 & 5.9 & 25.80 \\
\hline 2026 & 110 & 100 & 2.68 & 6.0 & 5.7 & 25.76 \\
\hline 2027 & 111 & 100 & 2.69 & 5.8 & 5.4 & 25.69 \\
\hline 2028 & 112 & 100 & 2.70 & 5.5 & 5.2 & 25.60 \\
\hline 2029 & 112 & 100 & 2.72 & 5.3 & 4.9 & 25.45 \\
\hline 2030 & 113 & 100 & 2.73 & 5.0 & 4.7 & 25.24 \\
\hline
\end{tabular}




\section{C4 scenario: EV inputs}

For CEVs and GEVs refer to the input tables for scenario B4.

Table: A6.14 Plug-in hybrid electric vehicles

\begin{tabular}{|c|c|c|c|c|c|c|c|c|c|c|c|c|c|c|}
\hline \multicolumn{8}{|c|}{ New } & \multicolumn{7}{|c|}{ Used imports } \\
\hline & $\begin{array}{l}\text { Vehicle } \\
\text { price \$ }\end{array}$ & $\begin{array}{c}\text { Max } \\
\text { electric } \\
\text { range km }\end{array}$ & $\begin{array}{l}\mathrm{kWh} \text { in } \\
\text { battery } \\
\text { (Total) }\end{array}$ & $\begin{array}{c}\text { Vehicle } \\
\text { weight kg }\end{array}$ & $\begin{array}{c}\text { Time to } \\
\text { battery } \\
\text { replace- } \\
\text { ment } \\
\text { (years) }\end{array}$ & $\begin{array}{c}\text { Expected } \\
\text { battery } \\
\text { price in } \\
\text { year of } \\
\text { replace- } \\
\text { ment \$ } \\
\end{array}$ & $\begin{array}{c}\text { Fuel cost } \\
\$ \text { per } \\
100 \mathrm{~km} \\
\text { (incl } \\
\text { charging } \\
\text { losses) } \\
\end{array}$ & $\begin{array}{l}\text { Vehicle } \\
\text { price \$ }\end{array}$ & $\begin{array}{c}\text { Value of } \\
\text { battery } \\
\text { on } \\
\text { import- } \\
\text { ation }\end{array}$ & $\begin{array}{c}\text { Average } \\
\text { year of } \\
\text { manu- } \\
\text { facture }\end{array}$ & $\begin{array}{c}\text { Time to } \\
\text { battery } \\
\text { replace- } \\
\text { ment } \\
\text { (years) }\end{array}$ & $\begin{array}{c}\text { Expected } \\
\text { battery } \\
\text { price in } \\
\text { year of } \\
\text { replace- } \\
\text { ment } \$ \\
\end{array}$ & $\begin{array}{c}\text { Max } \\
\text { electric } \\
\text { range km } \\
\text { (adjusted) }\end{array}$ & $\begin{array}{c}\text { Fuel cost } \\
\$ \text { per } \\
100 \mathrm{~km} \\
\text { (incl } \\
\text { charging } \\
\text { losses) } \\
\end{array}$ \\
\hline 2012 & 69,000 & 30 & 7 & 1,620 & 8 & 8,960 & 11.06 & 48,000 & 8960 & 2012 & 8 & 8,960 & 30 & 11.06 \\
\hline 2013 & 66,500 & 34 & 8 & 1,617 & 9 & 8,620 & 10.52 & 45,300 & 7840 & 2012 & 7 & 7,920 & 31 & 11.12 \\
\hline 2014 & 64,400 & 38 & 9 & 1,618 & 9 & 8,540 & 10.03 & 36,000 & 6720 & 2012 & 6 & 7,010 & 32 & 11.17 \\
\hline 2015 & 62,200 & 42 & 10 & 1,618 & 10 & 8,370 & 9.59 & 30,600 & 5600 & 2012 & 5 & 6,200 & 34 & 11.23 \\
\hline 2016 & 60,100 & 46 & 11 & 1,619 & 11 & 8,120 & 9.20 & 27,300 & 5640 & 2013 & 6 & 5,970 & 37 & 10.99 \\
\hline 2017 & 57,900 & 49 & 12 & 1,619 & 11 & 7,820 & 8.84 & 23,200 & 4640 & 2013 & 5 & 5,280 & 39 & 11.04 \\
\hline 2018 & 55,200 & 53 & 13 & 1,610 & 12 & 6,870 & 8.51 & 19,700 & 3650 & 2013 & 4 & 4,300 & 41 & 11.10 \\
\hline 2019 & 53,200 & 57 & 14 & 1,611 & 13 & 6,540 & 8.22 & 17,000 & 2650 & 2013 & 3 & 3,810 & 42 & 11.16 \\
\hline 2020 & 51,200 & 61 & 15 & 1,612 & 13 & 6,190 & 6.70 & 16,100 & 3050 & 2014 & 3 & 3,770 & 44 & 10.90 \\
\hline 2021 & 51,100 & 65 & 16 & 1,604 & 14 & 6,110 & 6.64 & 14,200 & 2140 & 2014 & 2 & 3,500 & 44 & 10.96 \\
\hline 2022 & 51,500 & 69 & 17 & 1,604 & 15 & 6,490 & 6.59 & 15,400 & 2510 & 2015 & 3 & 3,880 & 45 & 10.63 \\
\hline 2023 & 51,900 & 73 & 18 & 1,605 & 15 & 6,860 & 6.55 & 12,700 & 2790 & 2016 & 4 & 4,250 & 45 & 10.29 \\
\hline 2024 & 52,200 & 77 & 19 & 1,605 & 16 & 7,240 & 6.51 & 14,800 & 2990 & 2017 & 4 & 4,630 & 46 & 9.96 \\
\hline 2025 & 52,500 & 81 & 19 & 1,604 & 17 & 7,480 & 6.48 & 12,200 & 2860 & 2018 & 5 & 5,000 & 46 & 9.73 \\
\hline 2026 & 52,900 & 84 & 20 & 1,604 & 17 & 7,860 & 6.46 & 12,700 & 2930 & 2019 & 6 & 5,370 & 46 & 9.49 \\
\hline 2027 & 53,200 & 88 & 21 & 1,605 & 18 & 8,230 & 6.44 & 12,900 & 2940 & 2020 & 6 & 5,750 & 47 & 7.61 \\
\hline 2028 & 53,600 & 92 & 22 & 1,605 & 19 & 8,610 & 6.43 & 12,600 & 3060 & 2021 & 7 & 6,110 & 47 & 7.68 \\
\hline 2029 & 54,000 & 96 & 23 & 1,606 & 19 & 8,980 & 6.42 & 12,300 & 3390 & 2022 & 8 & 6,490 & 47 & 7.70 \\
\hline 2030 & 54,400 & 100 & 24 & 1,606 & 20 & 9,360 & 6.41 & 12,500 & 3730 & 2023 & 8 & 6,860 & 48 & 7.73 \\
\hline
\end{tabular}




\section{C5 scenario: EV inputs}

Table: A6.15 City electric vehicles

\begin{tabular}{|c|c|c|c|c|c|c|c|c|c|c|c|c|c|}
\hline \multicolumn{8}{|c|}{ New } & \multicolumn{6}{|c|}{ Used imports } \\
\hline & $\begin{array}{l}\text { Vehicle } \\
\text { price \$ }\end{array}$ & $\begin{array}{l}\text { kWh in } \\
\text { battery }\end{array}$ & $\begin{array}{l}\text { Vehicle } \\
\text { weight kg }\end{array}$ & $\begin{array}{l}\text { Time to } \\
\text { battery } \\
\text { replace- } \\
\text { ment } \\
\text { (years) }\end{array}$ & $\begin{array}{c}\text { Expected } \\
\text { battery } \\
\text { price in } \\
\text { year of } \\
\text { replace- } \\
\text { ment } \$ \\
\end{array}$ & $\begin{array}{c}\text { Max range } \\
\text { km }\end{array}$ & $\begin{array}{c}\text { Fuel cost \$ } \\
\text { per } \\
100 \mathrm{~km} \\
\text { (incl } \\
\text { charging } \\
\text { losses) } \\
\end{array}$ & $\begin{array}{l}\text { Vehicle } \\
\text { Price \$ }\end{array}$ & $\begin{array}{c}\text { Value of } \\
\text { battery on } \\
\text { import- } \\
\text { ation \$ }\end{array}$ & $\begin{array}{l}\text { Average } \\
\text { year of } \\
\text { manu- } \\
\text { facture }\end{array}$ & $\begin{array}{l}\text { Time to } \\
\text { battery } \\
\text { replace- } \\
\text { ment } \\
\text { (years) }\end{array}$ & $\begin{array}{c}\text { Expected } \\
\text { battery } \\
\text { price in } \\
\text { year of } \\
\text { replace- } \\
\text { ment \$ } \\
\end{array}$ & $\begin{array}{c}\text { Max range } \\
\text { km } \\
\text { (adjusted) }\end{array}$ \\
\hline 2012 & 47,100 & 13 & 860 & 8 & 14,580 & 150 & 2.33 & 36,800 & 14,600 & 2012 & 8 & 14,600 & 150 \\
\hline 2013 & 41,400 & 13 & 840 & 10 & 12,200 & 156 & 2.35 & 34,000 & 12,800 & 2012 & 7 & 11,800 & 149 \\
\hline 2014 & 36,400 & 13 & 830 & 11 & 10,290 & 163 & 2.37 & 26,700 & 10,900 & 2012 & 6 & 9,600 & 148 \\
\hline 2015 & 32,000 & 14 & 820 & 13 & 8,670 & 169 & 2.39 & 22,000 & 9,100 & 2012 & 5 & 7,800 & 149 \\
\hline 2016 & 28,400 & 14 & 810 & 14 & 7,240 & 175 & 2.41 & 18,800 & 8,300 & 2013 & 7 & 6,500 & 155 \\
\hline 2017 & 25,300 & 14 & 810 & 16 & 5,660 & 181 & 2.44 & 15,300 & 7,100 & 2013 & 6 & 4,900 & 157 \\
\hline 2018 & 22,800 & 14 & 800 & 17 & 4,750 & 188 & 2.46 & 12,800 & 5,800 & 2013 & 5 & 4,000 & 157 \\
\hline 2019 & 20,600 & 14 & 800 & 19 & 3,960 & 194 & 2.48 & 10,900 & 4,500 & 2013 & 4 & 3,200 & 158 \\
\hline 2020 & 18,700 & 15 & 790 & 20 & 3,320 & 200 & 2.51 & 10,300 & 4,700 & 2014 & 5 & 2,700 & 163 \\
\hline 2021 & 18,700 & 15 & 790 & 20 & 3,320 & 200 & 2.54 & 9,100 & 3,700 & 2014 & 4 & 2,700 & 161 \\
\hline 2022 & 18,700 & 15 & 790 & 20 & 3,320 & 200 & 2.57 & 9,500 & 3,800 & 2015 & 6 & 2,800 & 164 \\
\hline 2023 & 18,700 & 15 & 790 & 20 & 3,320 & 200 & 2.59 & 7,600 & 3,600 & 2016 & 7 & 2,900 & 166 \\
\hline 2024 & 18,700 & 16 & 790 & 20 & 3,320 & 200 & 2.62 & 7,900 & 3,100 & 2017 & 9 & 3,000 & 168 \\
\hline 2025 & 18,700 & 16 & 790 & 20 & 3,320 & 200 & 2.65 & 6,400 & 2,800 & 2018 & 10 & 3,100 & 170 \\
\hline 2026 & 18,700 & 16 & 790 & 20 & 3,320 & 200 & 2.68 & 6,300 & 2,500 & 2019 & 12 & 3,200 & 171 \\
\hline 2027 & 18,700 & 16 & 790 & 20 & 3,320 & 200 & 2.71 & 5,800 & 2,200 & 2020 & 13 & 3,300 & 172 \\
\hline 2028 & 18,700 & 17 & 790 & 20 & 3,320 & 200 & 2.74 & 5,500 & 2,200 & 2021 & 13 & 3,300 & 172 \\
\hline 2029 & 18,700 & 17 & 790 & 20 & 3,320 & 200 & 2.77 & 5,200 & 2,200 & 2022 & 13 & 3,300 & 172 \\
\hline 2030 & 18,700 & 17 & 790 & 20 & 3,320 & 200 & 2.80 & 5,000 & 2,200 & 2023 & 13 & 3,300 & 172 \\
\hline
\end{tabular}


Table: A6.16 General purpose electric vehicles

\begin{tabular}{|c|c|c|c|c|c|c|c|c|c|c|c|c|c|}
\hline \multicolumn{8}{|c|}{ New } & \multicolumn{6}{|c|}{ Used imports } \\
\hline & $\begin{array}{l}\text { Vehicle } \\
\text { price \$ }\end{array}$ & $\begin{array}{l}\text { kWh in } \\
\text { battery }\end{array}$ & $\begin{array}{c}\text { Vehicle } \\
\text { weight kg }\end{array}$ & $\begin{array}{c}\text { Time to } \\
\text { battery } \\
\text { replace- } \\
\text { ment } \\
\text { (years) }\end{array}$ & $\begin{array}{c}\text { Expected } \\
\text { battery } \\
\text { price in } \\
\text { year of } \\
\text { replace- } \\
\text { ment } \$ \\
\end{array}$ & $\begin{array}{c}\text { Max range } \\
\text { km }\end{array}$ & $\begin{array}{l}\text { Fuel cost \$ } \\
\text { per } 100 \mathrm{~km} \\
\text { (incl } \\
\text { charging } \\
\text { losses) }\end{array}$ & $\begin{array}{l}\text { Vehicle } \\
\text { price \$ }\end{array}$ & $\begin{array}{c}\text { Value of } \\
\text { battery on } \\
\text { import- } \\
\text { ation \$ }\end{array}$ & $\begin{array}{l}\text { Average } \\
\text { year of } \\
\text { manu- } \\
\text { facture }\end{array}$ & $\begin{array}{c}\text { Time to } \\
\text { battery } \\
\text { replace- } \\
\text { ment } \\
\text { (years) }\end{array}$ & $\begin{array}{c}\text { Expected } \\
\text { battery } \\
\text { price in } \\
\text { year of } \\
\text { replace- } \\
\text { ment } \$ \\
\end{array}$ & $\begin{array}{c}\text { Max range } \\
\text { km } \\
\text { (adjusted) }\end{array}$ \\
\hline 2012 & 61,100 & 18 & 1,234 & 8 & 18,600 & 180 & 2.71 & 47,400 & 18,600 & 2,012 & 8 & 18,600 & 180 \\
\hline 2013 & 59,600 & 22 & 1,240 & 10 & 18,470 & 220 & 2.74 & 43,700 & 16,300 & 2012 & 7 & 15,100 & 193 \\
\hline 2014 & 57,500 & 26 & 1,234 & 11 & 17,680 & 260 & 2.77 & 34,600 & 14,000 & 2012 & 6 & 12,200 & 208 \\
\hline 2015 & 55,000 & 30 & 1,237 & 13 & 16,560 & 300 & 2.80 & 28,800 & 11,600 & 2012 & 5 & 9,900 & 233 \\
\hline 2016 & 52,500 & 34 & 1,240 & 14 & 15,230 & 340 & 2.83 & 27,000 & 12,600 & 2013 & 7 & 9,900 & 258 \\
\hline 2017 & 49,800 & 38 & 1,242 & 16 & 13,820 & 380 & 2.86 & 22,500 & 10,700 & 2013 & 6 & 8,000 & 289 \\
\hline 2018 & 47,200 & 42 & 1,243 & 17 & 12,390 & 420 & 2.89 & 18,500 & 8,700 & 2013 & 5 & 6,500 & 308 \\
\hline 2019 & 44,600 & 46 & 1,245 & 19 & 11,010 & 460 & 2.92 & 15,100 & 6,800 & 2013 & 4 & 5,300 & 331 \\
\hline 2020 & 42,200 & 50 & 1,246 & 20 & 9,710 & 500 & 2.95 & 15,300 & 8,000 & 2014 & 5 & 5,000 & 351 \\
\hline 2021 & 42,200 & 50 & 1,246 & 20 & 9,710 & 500 & 2.98 & 13,300 & 6,400 & 2014 & 4 & 5,000 & 349 \\
\hline 2022 & 42,200 & 50 & 1,246 & 20 & 9,710 & 500 & 3.01 & 14,600 & 7,300 & 2015 & 6 & 5,800 & 357 \\
\hline 2023 & 42,200 & 50 & 1,246 & 20 & 9,710 & 500 & 3.05 & 12,800 & 7,600 & 2016 & 7 & 6,600 & 363 \\
\hline 2024 & 42,200 & 50 & 1,246 & 20 & 9,710 & 500 & 3.08 & 14,100 & 7,600 & 2017 & 9 & 7,400 & 367 \\
\hline 2025 & 42,200 & 50 & 1,246 & 20 & 9,710 & 500 & 3.11 & 12,300 & 7,300 & 2018 & 10 & 8,200 & 370 \\
\hline 2026 & 42,200 & 50 & 1,246 & 20 & 9,710 & 500 & 3.15 & 12,500 & 6,800 & 2019 & 12 & 8,900 & 373 \\
\hline 2027 & 42,200 & 50 & 1,246 & 20 & 9,710 & 500 & 3.18 & 12,000 & 6,300 & 2020 & 13 & 9,700 & 375 \\
\hline 2028 & 42,200 & 50 & 1,246 & 20 & 9,710 & 500 & 3.22 & 11,800 & 6,300 & 2021 & 13 & 9,700 & 375 \\
\hline 2029 & 42,200 & 50 & 1,246 & 20 & 9,710 & 500 & 3.25 & 11,400 & 6,300 & 2022 & 13 & 9,700 & 375 \\
\hline 2030 & 42,200 & 50 & 1,246 & 20 & 9,710 & 500 & 3.29 & 11,300 & 6,300 & 2023 & 13 & 9,700 & 375 \\
\hline
\end{tabular}


Table: A6.17 Plug-in hybrid electric vehicles

\begin{tabular}{|c|c|c|c|c|c|c|c|c|c|c|c|c|c|c|}
\hline \multicolumn{8}{|c|}{ New } & \multicolumn{7}{|c|}{ Used imports } \\
\hline & $\begin{array}{l}\text { Vehicle } \\
\text { price \$ }\end{array}$ & $\begin{array}{c}\text { Max } \\
\text { electric } \\
\text { range } \mathrm{km}\end{array}$ & $\begin{array}{l}\text { kWh in } \\
\text { battery } \\
\text { (Total) }\end{array}$ & $\begin{array}{c}\text { Vehicle } \\
\text { weight kg }\end{array}$ & $\begin{array}{c}\text { Time to } \\
\text { battery } \\
\text { replace- } \\
\text { ment } \\
\text { (years) }\end{array}$ & $\begin{array}{c}\text { Expected } \\
\text { battery } \\
\text { price in } \\
\text { year of } \\
\text { replace- } \\
\text { ment \$ }\end{array}$ & $\begin{array}{c}\text { Fuel cost } \\
\$ \text { per } \\
100 \mathrm{~km} \\
\text { (incl } \\
\text { charging } \\
\text { losses) }\end{array}$ & $\begin{array}{l}\text { Vehicle } \\
\text { price \$ }\end{array}$ & $\begin{array}{l}\text { Value of } \\
\text { battery } \\
\text { on } \\
\text { import- } \\
\text { ation }\end{array}$ & $\begin{array}{c}\text { Average } \\
\text { year of } \\
\text { manu- } \\
\text { facture }\end{array}$ & $\begin{array}{c}\text { Time to } \\
\text { battery } \\
\text { replace- } \\
\text { ment } \\
\text { (years) }\end{array}$ & $\begin{array}{c}\text { Expected } \\
\text { battery } \\
\text { price in } \\
\text { year of } \\
\text { replace- } \\
\text { ment \$ }\end{array}$ & $\begin{array}{c}\text { Max } \\
\text { electric } \\
\text { range km } \\
\text { (adjusted) }\end{array}$ & $\begin{array}{c}\text { Fuel cost } \\
\$ \text { per } \\
100 \mathrm{~km} \\
\text { (incl } \\
\text { charging } \\
\text { losses) }\end{array}$ \\
\hline 2012 & 58,500 & 30 & 5 & 1,287 & 8 & 5,760 & 10.41 & 41,000 & 5,760 & 2012 & 8 & 5,760 & 30 & 10.41 \\
\hline 2013 & 56,500 & 39 & 6 & 1,283 & 10 & 5,880 & 9.29 & 38,700 & 4,670 & 2012 & 7 & 4,670 & 33 & 10.46 \\
\hline 2014 & 54,500 & 48 & 7 & 1,282 & 11 & 5,920 & 8.38 & 29,800 & 3,790 & 2012 & 6 & 3,790 & 37 & 10.51 \\
\hline 2015 & 51,500 & 56 & 9 & 1,281 & 13 & 5,610 & 7.64 & 24,900 & 3,070 & 2012 & 5 & 3,070 & 42 & 10.56 \\
\hline 2016 & 49,500 & 65 & 10 & 1,282 & 14 & 5,310 & 7.02 & 21,900 & 3,140 & 2013 & 7 & 3,140 & 48 & 10.04 \\
\hline 2017 & 47,500 & 74 & 11 & 1,281 & 16 & 4,830 & 6.51 & 18,200 & 2,550 & 2013 & 6 & 2,550 & 55 & 10.09 \\
\hline 2018 & 44,500 & 83 & 13 & 1,275 & 17 & 4,060 & 6.07 & 15,100 & 1,900 & 2013 & 5 & 1,900 & 59 & 10.14 \\
\hline 2019 & 42,500 & 91 & 14 & 1,275 & 19 & 3,620 & 5.70 & 13,200 & 1,540 & 2013 & 4 & 1,540 & 64 & 10.19 \\
\hline 2020 & 40,500 & 100 & 15 & 1,270 & 20 & 3,000 & 4.61 & 12,600 & 1,440 & 2014 & 5 & 1,440 & 68 & 9.66 \\
\hline 2021 & 40,500 & 100 & 15 & 1,270 & 20 & 3,000 & 4.65 & 11,400 & 1,440 & 2014 & 4 & 1,440 & 68 & 9.71 \\
\hline 2022 & 40,500 & 100 & 15 & 1,270 & 20 & 3,000 & 4.68 & 12,300 & 1,680 & 2015 & 6 & 1,680 & 70 & 9.04 \\
\hline 2023 & 40,500 & 100 & 15 & 1,270 & 20 & 3,000 & 4.72 & 10,100 & 1,960 & 2016 & 7 & 1,960 & 71 & 8.47 \\
\hline 2024 & 40,500 & 100 & 15 & 1,270 & 20 & 3,000 & 4.75 & 11,500 & 2,210 & 2017 & 9 & 2,210 & 72 & 7.88 \\
\hline 2025 & 40,500 & 100 & 15 & 1,270 & 20 & 3,000 & 4.79 & 9,400 & 2,480 & 2018 & 10 & 2,480 & 72 & 7.51 \\
\hline 2026 & 40,500 & 100 & 15 & 1,270 & 20 & 3,000 & 4.83 & 9,600 & 2,720 & 2019 & 12 & 2,720 & 73 & 7.13 \\
\hline 2027 & 40,500 & 100 & 15 & 1,270 & 20 & 3,000 & 4.87 & 9,500 & 3,000 & 2020 & 13 & 3,000 & 73 & 5.66 \\
\hline 2028 & 40,500 & 100 & 15 & 1,270 & 20 & 3,000 & 4.90 & 9,200 & 3,000 & 2021 & 13 & 3,000 & 73 & 5.71 \\
\hline 2029 & 40,500 & 100 & 15 & 1,270 & 20 & 3,000 & 4.94 & 8,700 & 3,000 & 2022 & 13 & 3,000 & 73 & 5.70 \\
\hline 2030 & 40,500 & 100 & 15 & 1,275 & 20 & 3,000 & 4.99 & 8,600 & 3,000 & 2023 & 13 & 3,000 & 73 & 5.70 \\
\hline
\end{tabular}




\section{References}

ADAMOWICZ, W., LOUVIERE, J. \& WILLIAMS, M. 1994. Combining Revealed and Stated Preference Methods Valuing Environmental Amenities. Journal of Environmental Economics and Management, 26, 271-292.

AGUIRRE, K., EISENHARDT, L., LIM, C., NELSON, B., NORRING, A., SLOWIK, P. \& TU, N. 2012. Lifecycle Analysis Comparison of a Battery Electric Vehicle and a Conventional Gasoline Vehicle. Los Angeles, CA: Institute of the Environment and Sustainability, UCLA.

AJANOVIC, A. 2011. Biofuels versus food production: Does biofuels production increase food prices? Energy, 36, 2070-2076.

AJZEN, I., BROWN, T. \& CARVAJAL, F. 2004. Explaining the Discrepancy Between Intentions and Actions: The Case of Hypothetical Bias in Contingent Valuation. Personality and Social Pschology Bulletin, 1109-1121.

AMINE, K., LU, L., ABOUIMRANE, A., WU, H., ZHANG, Z., DU, P., BELHAROUAK, I., YANG, J., SUN, Y. K. \& CURTISS, L. 2012. Next generation Lithium lon and Beyond lithium lon. Beyond Lithium Ion V, 5 June 2012 - 7 June 2012 Berkley, CA: Lawrence Berkeley National Laboratory.

ANDERSSON, S., ELOFSSON, A., GALUS, M., GORANSSON, L., KARLSSON, S., JOHNSSON, F. \& ANDERSSON, G. 2010. Plug-in hybrid electric vehicles as regulating power providers: Case studies of Sweden and Germany. Energy Policy, 38, 2751-2762.

ARGOTE, L. \& EPPLE, D. 1990. Learning Curves in Manufacturing. Science, 247, 920-924.

ARROW, K. 1962. The Economic Implications of Learning by Doing. The Review of Economic Studies, 29, 155-173.

AUTOMOTIVE MANAGEMENT ONLINE. 2011. Nissan addresses long term viability of Leaf battery [Online]. Peterborough: Bauer Media. Available: http://www.amonline.com/news/2011/9/26/nissan-addresses-long-term-viability-of-leafbattery/29731/ [Accessed 3 October 2011 2011].

AXEN, J. \& KURANI, K. 2008. The Early U.S. Market for PHEVs: Anticipating Consumer Awareness, Recharge Potential, Design Priorities and Energy Impacts. Available: http://escholarship.org/uc/item/4491w7kf.

BAIER, S., CLEMENTS, M., GRIFFITHS, C. \& IHRIG, J. 2009. Biofuels Impact on Crop and Food Prices: Using an Interactive Spreadsheet. International Finance Discussion Papers: Number 967. Washington, D.C.: Board of Governors of the Federal Reserve System.

BALDUCCI, P. 2008. Plug-in Hybrid Electric Vehicle Market Penetration Scenarios. Richland, Washington: Pacific Northwest National Laboratory.

BANDIVADEKAR, A., BODEK, K., CHEAH, L., EVANS, C., GROODE, T., HEYWOOD, J. B., KASSERIS, E., KROMER, M. A. \& WEISS, M. 2008. On the Road in 2035: Reducing Transportation's Petroleum Consumption and GHG Emissions. Cambridge, Massachusetts: Massachusetts Institute of Technology.

BAPTISTA, P., ROLIN, C. \& SILVA, C. 2012. Plug-In Vehicle Acceptance and Probable Utilization Behaviour. Journal of Transportation Technologies, 2, 67-74.

BAPTISTA, P., TOMAS, M. \& SILVA, C. 2010. Plug-in hybrid fuel cell vehicles market penetration scenarios. International Journal of Hydrogen Energy, 35, 10024-10030.

BASS, F. 1969. A new product for model consumer durables. Management Science, 15, 215227.

BATEMAN, I., CARSON, R., DAY, B., HANEMANN, M., HANLEY, N., HETT, T., JONES-LEE, M., LOOMES, G., MOURATO, S., OZDEMIROGLU, E., PEARCE, D., SUGDEN, R. \& SWANSON, J. 2002. Economic Valuation with Stated Preference Techniques: A Manual, Cheltenham, Edward Elgar.

BAXTER, R., FLACK, N., PARKER, C., BOWMAN, A., CHADWICK, D. \& MONEY, C. 2009. Meridian Energy Ltd and Contact Energy Ltd - National cost-benefit assessment of 
the early uptake of electric vehicles in New Zealand: Methodology, assumptions and results. Wellington: Hyder Consulting (NZ) Limited.

BEGGS, S., CARDELL, S. \& HAUSMAN, J. 1981. Assessing the Potential Demand for Electric Cars. Journal of Econometrics, 16, 1-19.

BERTRAM, G. 2006. Restructuring the New Zealand Electricity sector 1984-2005. In: SIOSHANSI, F. \& PFAFFENBERGER, V. (eds.) Electricity Market Reform: An International Perspective. Kidlington, UK: Elsevier.

BERTRAM, G. \& CLOVER, D. 2009. Kicking the Fossil-Fuel Habit: New Zealand's Ninety Percent Renewable Target for Electricity. In: SIOSHANSI, F. (ed.) Generating Electricity in a Carbon Constrained World. Burlington: Academic Press.

BHAT, C., ELURU, N. \& COPPERMAN, R. 2008. Flexible model structures for discrete choice analysis In: HENSHER, D. \& BUTTON, K. (eds.) Handbook of Transport Modelling. 2 ed. Oxford Amsterdam: Elsevier.

BISHOP, P. 2007. GEM: A brief description. Wellington: Electricity Commission.

BISHOP, P. 2010. GEM Input Data. http://www.ea.govt.nz/document/13511/download/industry/ecarchive/soo/2010-soo/: Electricity Authority.

BLIEMER, M. \& ROSE, J. 2009. Efficiency and Sample Size Requirements for Stated Choice Experiments. Transportation Research Board 88th Annual Meeting. Washington D.C.: Transportation Research Board

BLIEMER, M. \& ROSE, J. 2010. Construction of Experimental Designs for Mixed Logit Models Allowing for Correlation Across Choice Observations. Transportation Research Part $B, 44,710-734$.

BLIEMER, M., ROSE, J. \& COLLINS, A. 2011. Ngene 1.1 User Manual \& Reference Guide. Melbourne: Choicemetrics.

BOLLARD, A. \& PICKFORD, M. 1998. Deregulation and Competition Policy in the Transport Sector in New Zealand. Journal of Transport Economics and Policy, 32, 267-276.

BONE, I. 2003. Smartgrowth - Car Ownership Forecasting. www.smartgrowthbop.org.nz: Smartgrowth.

BOSSEL, U. 2003. Efficiency of Hydrogen Fuel Cell, Diesel-SOFC-Hybrid and Battery Electric Vehicles. European Fuel Cell Forum 2003. Oberrohrdorf, Switzerland: European Fuel Cell Forum.

BRADY, J. \& O'MAHONY, M. 2011. Travel to work in Dublin. The potential impacts of electric vehicles on climate change and urban air quality. Transportation Research Part D, 16, 188-193.

BROWN, S., PYKE, D. \& STEENHOF, P. 2010. Electric vehicles: The role and importance of standards in an emerging market. Energy Policy, 38, 3797-3806.

BROWN, S. \& YUCEL, M. 2007. What Drives Natural Gas Prices? Research Department Working Paper. Dallas: Federal Reserve Bank of Dallas.

BROWNSTONE, D., BUNCH, D. \& TRAIN, K. 2000. Joint mixed logit models of stated and revealed preferences for alternative-fuel vehicles. Transportation Research Part $B$, 34, 315-338.

BROWNSTONE, D. \& TRAIN, K. 1999. Forecasting new product penetration with flexible substitution patterns. Journal of Econometrics, 89, 109-129.

BUCHANAN, C., COOPER, G., MACEWEN, A., CROMPTON, D., CROW, G., MICHELL, G., DALLIMORE, D., HILLS, P. \& BURTON, D. 1963. Traffic in Towns, London, HMSO.

BUCHMANN, I. 2011. Batteries in a Portable World: A Handbook on Rechargable Batteries for Non-Engineers, 3rd Edition, Vancouver, Cadex Electronics Inc.

BULL, B. 2008. Regional Peak Demand Forecast from 2007 - an update. Wellington: Electricity Commission. 
BUNCH, D., BRADLEY, M., GOLOB, T., KITAMURA, R. \& OCCHIUZZO, G. 1992. Demand for a Clean-Fuel Personal Vehicles in California: A Discrete-Choice Stated Preference Survey. Irvine, CA: Institute of Transportation Studies, University of California, Irvine.

BUREAU OF TRANSPORT STATISTICS 2011. 2009/10 Household Travel Survey: Summary Report 2011 Release. Sydney: NSW Government.

BURGE, P., MUNRO, C., READ, P. \& HEYWOOD, C. 2007. Investigating the Likely Behavioural Responses to Alternative Congestion Charge Schemes in London. Proceedings of the European Transport Conference 2007 Held 17-19 October 2007, Leiden, the Netherlands.

BURKE, A. \& MILLER, M. 2009. Performance Characteristics of Lithium-ion Batteries of Various Chemistries for Plug-in Hybrid Vehicles. EVS24 International Battery, Hybrid and Fuel Cell Electric Vehicle Symposium. Stavanger: escholarship.org University of California.

CALEF, D. \& GOBLE, R. 2007. The allure of technology: How France and California promoted electric and hybrid vehicles to reduce urban air pollution. Policy Science, 40, 1-34.

CALFEE 1985. Estimating the demand for electric automobiles using fully disaggregated probabilistic choice analysis. Transportation Research Part B, 19B, 287-301.

CAMERER, C. \& LOEWENSTEIN, G. 2004. Behavioral Economics: Past, Present, Future. In: CAMERER, C., LOEWENSTEIN, G. \& RABIN, M. (eds.) Advances in Behavioral Economics. Princeton and Oxford: Princeton University press.

CAMPBELL, A. 2010. Acceptance of the Use of Biodiesel Fuels in Vehicle and Engine Applications in New Zealand. Wellington: Fuel Technology Limited: prepared for EECA.

CAMPBELL, C. \& LAHERRERE, J. 1998. The End of Cheap Oil. Scientific American, March $1998,6$.

CAO, X. \& MOKHTARIAN, P. 2004. The Future Demand for Alternative Fuel Passenger Vehicles: A Diffusion of Innovation Approach. Davis CA: Department of Civil and Environmental Engineering, University of California, Davis.

CARLSON, R. \& UMBLE, M. 1980. Statistical Demand Functions for Automobiles and Their Use for Forecasting in an EnergyCrisis. The Journal of Business, 53, 193-204.

CARLSSON, F. \& MARTINSSON, P. 2001. Do Hypothetical and Actual Marginal Willingness to Pay Differ in Choice Experiments? Journal of Environmental Economics and Management, 41, 179-192.

CARLSSON, F. \& MARTINSSON, P. 2003. Design Techniques for Stated Preference Methods in Health Economics. Health Economics, 12, 281-294.

CARROLL, S. 2010. The smart move trial: description and initial results. Loughborough University Leicestershire, U.K.: CENEX.

CASTLE, C. \& CROOKS, A. 2006. Principles and Concepts of Agent-Based Modelling for Developing Geospatial Simulations. Working Paper Series. London: Centre for Advanced Spatial Analysis, University College London.

CENTRAAL BUREAU VOOR DE STATISTIEK 2012. Dutch population mobility, design, mode of transport, 2007. 3 June 2010 ed. Statline.

CENTRE OF ADVANCED ENGINEERING 2004. Energy Supply in the Post-Maui Era: An Investigation into Thermal Fuels Options and Their Contribution to Energy Security CAE Comments 04. Christchurch: Centre of Advanced Engineering.

CENTRONE, F., GOIA, R. \& SALINELLI, E. 2007. Demographic processes in a model of innovation diffusion with a dynamic market. Technological Forecasting \& Social Change, 74, 247-266. 
CHAMP, P. \& WELSH, M. 2006. Survey Methodologies for Stated Choice Studies. In: KANNINEN, B. (ed.) Valuing Environmental Amenities Using Stated Choice Studies. AA Dordrecht: Springer.

CHANG, J. B., LUSK, J. \& NORWOOD, F., B 2009. How closely do hypothetical surveys and laboratory experiments predict field behavior? American Journal of Agricultural Economics, 91, 518-534.

CHEVROLET.COM. 2012. 2012 Volt Model \& Specs [Online]. Detroit. MI: General Motors. Available: http://www.chevrolet.com/volt-electric-car/features-specs/trims.html [Accessed 25 September 2012].

CHINNECK, J. 2010. Practical Optimization: A Gentle Introduction. Carleton University.

CHRISTENSSON, J. 2008. Forecasting the Sales of Light Trucks in the United States. 4th Annual Symposium: Graduate Research and Scholarly Projects. Wichita State University, Wichita, KS: Wichita State University.

CHRISTIDIS, P., HIDALGO, I. \& SORIA, A. 2003. Dynamics of the Introduction of New Passenger Car Technologies-The IPTS Transport Technologies Model. In: EUROPEAN COMMISSION JOINT RESEARCH CENTRE, S. (ed.).

CHRUN, K. (24 May 2010, personal communication). RE: Licensed vehicles as at June of each year from 1962 to 2008, New Zealand Transport Agency

COHEN, G., JOUTZ, F. \& LOUNGANI, P. 2011. Measuring Energy Security: Trends in the Diversification of Oil and Natural Gas Supplies. IMF Working Paper. Washington: International Monetary Fund.

COHEN, M. 2012. The future of automobile society: a socio-technical transitions perspective. Technology Analysis \& Strategic Management, 24, 377-390.

COLEGRAVE, F. \& DENNE, T. 2006. Socio-economic impacts of emissions standards on used imported vehicles; prepared for the Ministry of Transport. Auckland: COVEC.

COLLANTES, G. 2005. Foreseeing the Market for Hydrogen Fuel-Cell Vehicles: Stakeholders' Perspectives and Models of New Technology Diffusion. University of California at Davis: Institute of Transportation Studies.

COLLINS, K. 2008. The Role of Biofuels and Other Factors in Increasing Farm and Food Prices: A Review of Recent Development with a Focus on Feed Grain Markets and Market Prospects,. Supporting Material for a Review Conducted by Kraft Foods Global, Inc. Centreville, VA: Keith J. Collins LLC.

COMMITTEE ON CLIMATE CHANGE 2008. Building a low-carbon economy-the UK's contribution to tackling climate change. The first report of the Committee on Climate Change. London: UK Government.

COMMONWEALTH OF AUSTRALIA 2011. Draft Energy White Paper 2011: Strengthening the foundations for Australia's energy future. Canberra ACT: Department of Resources, Energy and Tourism.

CONDER, T. 2009. Development and application of a New Zealand car ownership and traffic forecasting model. Wellington: New Zealand Transport Agency.

CONLISK, J. 1996. Why Bounded Rationality? Journal of Economic Literature, XXXIV, 669700.

COWAN, R. \& HULTEN, S. 1996. Escaping Lock-In: The Case of the Electric Vehicle. Technological Forecasting and Social Change, 53, 61-79.

CROWN MINERALS 2010. New Zealand Petroleum Basins. Wellington: Crown Minerals, Ministry of Economic Development.

CUI, X., LIU, C., KIM, H., KAO, S.-C., TUTTLE, M. \& BHADURI, B. Multi Agent-Based Framework for Simulating Household PHEV Distribution and Electric Distribution Network Impact. Transportation Research Board 90th Annual Meeting Electric Distribution Network 2011 Washington D.C., Paper 11-2036. Paper 11-2036. 
DALKEY, N. \& HELMER, O. 1963. An Experimental Application of the Delphi Method to the Use of Experts. Management Science, 9, 458-467.

DARGAY, J., GATELY, D. \& SOMMER, M. 2007. Vehicle ownership and income growth, worldwide: 1960-2030. Energy Journal, 28, 143-170.

DAVEY, J. 2007. Older people and transport: coping without a car. Ageing and Society, 27, 49-65.

DAVIS, S., ANDERSON-TEIXEIRA, K. \& DELUCIA, E. 2008. Life-cycle analysis and the ecology of biofuels. Trends in Plant Science, 14, 140-146.

DE CEUSTER, G., VAN HERBRUGGEN, B., IVANOVA, O., CARLIER, K., MARTINO, A. \& FIORELLO, D. 2007. TREMOVE: Service contract for the further development and application of the transport and environmental TREMOVE model Lot 1 (Improvement of the data set and model structure). Brussels: European Commission: Directorate General Environment.

DE HAAN, P., MUELLER, M. \& PETERS, A. 2006. Does the hybrid Toyota Prius lead to rebound effects? Analysis of size and number of cars previously owned by Swiss Prius buyers. Ecological Economics, 58, 592-605.

DE JONG, G., FOX, F., DALY, A., PIETERS, M. \& SMIT, R. 2004. Comparison of Car Ownership Models. Transport Reviews, 24, 379-408.

DE PONT, J. 2006. Enabling Biofuels: Risks to vehicles and other engines Auckland: Transport Engineering Research New Zealand Limited: prepared for the Ministry of Transport.

DE PONT, J. 2009. Low-emission fuel-efficient light vehicles. NZ Transport Agency research report 391. Wellington: NZ Transport Agency.

DENNE, T. \& COLEGRAVE, F. 2005. Review of Policy Instruments to Reduce Greenhouse Gas Emissions in the Transport Sector: Improving Fuel Economy of the Fleet Auckland: Covec.

DENNIS, K. \& URRY, J. 2009. After the Car, Cambridge, Polity Press.

DEPARTMENT FOR TRANSPORT 2011a. National Travel Survey: 2010, Statistical Release. London: Department for Transport.

DEPARTMENT FOR TRANSPORT. 2011b. Plug-in car grant [Online]. Department for Transport. Available: http://www.dft.gov.uk/topics/sustainable/olev/plug-in-cargrant/ [Accessed 23 July 2011 2011].

DEPARTMENT OF MOTOR VEHICLES 2011. Statistics for Publication. Sacramento: State of California.

DEUTCH, J. \& MONIZ, E. 2010. Electrification of the Transportation System: An MIT Energy Initiative Symposium. Cambridge, MA: MIT Energy Intiative.

DILLMAN, D., PHELPS, G., TORTORA, R., SWIFT, K., KOHRELL, J., BERCK, J. \& MESSER, B. 2009a. Response Rate and Measurement Differences in Mixed-mode Surveys Using Mail, Telephone, Interactive Voice Response (IVR) and the Internet. Social Science Research, 28, 1-18.

DILLMAN, D., SMYTH, J. \& CHRISTIAN, L. 2009b. Internet, Mail, and Mixed-Mode Surveys: The Tailored Design Method, Hoboken NJ, John Wiley \& Sons, Inc.

DIRR, N. 2008. A Feasibility Assessment for Electric Vehicles and the New Zealand Grid. MSc Master of Science, University of Otago.

DOEFF, M. 2011. Batteries: Overview of Battery Cathodes. http://escholarship.org/uc/item/1n55870s: Lawrence Berkeley National Laboratory.

DONOVAN, S., GENTER, J., PETRENAS, B., MUMBY, N., HAZLEDINE, T., LITMAN, T., HEWISON, G., GUIDERA, T., O'REILLY, L., GREEN, A. \& LEYLAND, G. 2008. Managing transport challenges when oil prices rise. NZ Transport Agency Research Report 357. Wellington: NZ Transport Agency. 
DOSI, G. \& NELSON, R. 1994. An introduction to evolutionary theories in economics. Journal of Evolutionary Economics, 4, 153-172.

DUKE, M., ANDREWS, D. \& ANDERSON, T. 2009. The feasibility of long range battery electric cars in New Zealand. Energy Policy, 37, 3455-3462.

DUNCAN, J., HALLIBURTON, T., HEFFERNAN, B., HARDIE, S., WATSON, N. \& COATES, G. 2010. Electric Vehicles: Impacts on New Zealand's Electricity System - Technical report. Christchurch: New Zealand Centre for Advanced Engineering (CAENZ).

EADS, G. 2011. 50by50: Prospects and Progress. Global Fuel Economy Initiative.

EBERHARD, M. \& TARPENNING, M. 2006. The 21st Century Electric Car, 6 October 2006. Palo Alto, CA: Tesla Motors Inc.

EBERLE, U., MÜLLER, B. \& HELMOLT, R. 2012. Fuel cell electric vehicles and hydrogen infrastructure: status 2012. Energy \& Environmental Science, 8780-8798.

ECKERMANN, E. 2001. World History of the Automobile, Warrendale PA, Society of Automotive Engineers, Inc.

EGBUE, O. \& LONG, S. 2012. Barriers to wide spread adoption of electric vehicles: Ananalysis of consumer attitudes and perceptions. Energy Policy, 48, 717-72.

ELECTRIC VEHICLES INITIATIVE OF THE CLEAN ENERGY MINISTERIAL \& INTERNATIONAL ENERGY AGENCY 2013. Global EV Outlook: Understanding the Electric Vehicle Landscape to 2020. Paris: International Energy Agency, OECD.

ELECTRICITY AUTHORITY 2010. Energy Forecast Input Assumptions. Electricity Authority.

ELECTRICITY AUTHORITY 2011. Electricity in New Zealand. Wellington: Electricity Authority.

ELECTRICITY AUTHORITY 2012. Centralised dataset - web interface. Wellington: Electricity Authority.

ELECTRICITY COMMISSION 2009. Response to submissions on draft 2010 Statement of Opportunities. http://www.ea.govt.nz/document/11389/download/industry/ecarchive/soo/2010-soo/: Electricity Authority.

ELECTRICITY COMMISSION 2010. 2010 Statement of Opportunities. Wellington: Electricity Commission.

ELECTRICITY NETWORK ASSOCIATION. 2012. Consumer Info: Local Electricity Networks [Online]. Wellington: Electricity Network Association. Available: http://www.electricity.org.nz/Site/Consumer/electricity_networks.aspx [Accessed 13 July 2012].

ELEMENT ENERGY LIMITED 2012. Cost and performance of EV batteries: Final report for The Committee on Climate Change. Cambridge: Element Energy Limited.

ENERGY EFFICIENCY AND CONSERVATION AUTHORITY 2007. Energy end use database. Energy Efficiency and Conservation Authority.

ENERGY SAFETY SERVICE 2004. New Zealand electrical code of practice for homeowner/occupier's electrical wiring work in domestic installations Ministry of Economic Development.

ENG, G. 2008. A Formula for LNG Pricing - An Update.

http://www.med.govt.nz/upload/65505/Formula_for_LNG_Pricing.pdf: Ministry of Economic Development.

ENG, G., BYWATER, I. \& HENDTLASS, C. 2008. New Zealand Energy Information Handbook Third Edition, Christchurch, New Zealand centre for Advanced Engineering.

EPPSTEIN, M., GROVER, D., MARSHALL, J. \& RIZZO, D. 2011. An agent-based model to study market penetration of plug-in hybrid electric vehicles. Energy Policy, 39, 37893802.

EUROPEAN AUTOMOBILE MANUFACTURERS' ASSOCIATION 2011. Overview of Purchase and Tax Incentives for Electric Vehicles in the EU. http://www.acea.be/images/uploads/files/20110330_EV_tax_overview.pdf: European Automobile Manufacturers' Association. 
EUROPEAN COMMISSION 2011. A Roadmap for moving to a competitive low carbon economy in 2050. In: COMMUNICATION FROM THE COMMISSION TO THE EUROPEAN PARLIAMENT, THE EUROPEAN ECONOMIC AND SOCIAL COMMITTEE AND THE COMMITTEE OF THE REGIONS (ed.). Brussels: European Commission.

FRANKE, T., NEUMANN, I., BÜHLER, F., COCRON, P. \& KREMS, J. 2012. Experiencing Range in an Electric Vehicle: Understanding Psychological Barriers. Applied Psycology: An International Review, 61, 368-391.

GEELS, F. 2004. Understanding system innovations: A critical literature review and a conceptual synthesis. In: ELZEN, B., GEELS, F. \& GREEN, K. (eds.) System Innovation and the Transition to Sustainability: Theory, Evidence and Policy. Cheltenham: Edward Elgar Publishing.

GEELS, F. 2010. Ontologies, socio-technical transitions (to sustainability), and the multilevel perspective. Research Policy, 38, 495-510.

GEELS, F., HEKKERT, M. P. \& JACOBSSON, S. 2008. The dynamics of sustainable innovation journeys. Technology Analysis \& Strategic Management, 20, 521-536.

GHASSEMIEH, E. 2008. Materials in Automotive Application, State of the Art and Prospects. In: CHIABERGE, M. (ed.) New Trends and Developments in Automotive Industry. Online: INTECH.

GIFFI, C., VITALE, J., DREW, M., KUBOSHIMA, Y. \& SASE, M. 2011. Unplugged: Electric vehicle realities versus consumer expectations. Deloitte Survey. Deloitte Touche Tohmatsu Limited.

GLAISTER, S. 2011. Evidence to the Transport Select Committee Inquiry on Transport and the Economy, Third session 2010 -11, Question 439. London: House of Commons.

GLOBAL CLIMATE AND ENERGY PROJECT 2006. A Technical Assessment of High-Energy Batteries for Light-Duty Electric Vehicles. http://gcep.stanford.edu: Stanford University.

GOLOB, T., BROWNSTONE, D., BUNCH, D. \& KITAMURA, R. 1996. Forecasting Electric Vehicle Ownership and use in the California South Coast Air Basin. Irvine, CA: Institute of Transportation Studies, University of California, Irvine.

GOLOMB, D., H \& BUNCH, H. 1979. Stochastic Analysis of Future Vehicle Populations. In: HIGHWAY SAFETY RESEARCH INSTITUTE (ed.). Ann Arbor: University of Michigan.

GONDER, J. \& MARKEL, T. 2007. Energy Management Strategies for Plug-In Hybrid Electric Vehicles. 2007 SAE World Congress 2007. Detroit, Michigan: SAE International.

GOODWIN, P., DARGAY, J. \& HANLY, M. 2004. Elasticities of Road Traffic and Fuel Consumption with Respect to Price and Income: A Review. Transport Reviews, 24, 275-292.

GORDON-BLOOMFIELD, N. 2010. Electric Vehicle Range, In the Real World: What You Need to Know [Online]. Palo Alto: High Gear Media. Available:

http://www.allcarselectric.com/blog/1044871_electric-vehicle-range-in-the-realworld-what-you-need-to-know [Accessed 12 September 2010 2010].

GORDON, D., GREENE, D., ROSS, M. \& WENZEL, T. 2006. Sipping Fuel and Saving Lives: Increasing Fuel Economy Without Sacrificing Safety. eScolarship; University of California: Lawrence Berkeley National Laboratory.

GRAHAM, D. \& GLAISTER, S. 2004. Road Traffic Demand Elasticity Estimates: A Review. Transport Reviews, 24, 261-274.

GRAY, A. \& SUNTHERALINGAM, R. 2007. Feasibility of using samples of telephone numbers for Tier 1 Official Statistical Household Surveys. Official Statistical Research Series Vol 1. www.statisphere.govt.nz/osresearch. Statistics New Zealand.

GREENE, D. 2001. TAFV Alternative Fuels and Vehicles Choice Model Documentation. Oak Ridge: Oak Ridge National Laboratory. 
GREENE, D. 2010. Why the Market for New Passenger Cars Generally Undervalues Fuel Economy, . Discussion Paper No. 2010-6. Paris: International Transport Forum OECD.

GREENE, W. 2002. Econometric Analysis, Upper Saddle River, New Jersey, Prentice Hall.

GREENING, L., GREENE, D. \& DIFFIGLIO, C. 2000. Energy efficiency and consumption - the rebound effect - a survey. Energy Policy, 28, 389-401.

GREENSPAN, A. \& COHEN, D. 1999. Motor vehicle stock, scrappage and sales. The Review of Economics and Statistics, 81, 369-383.

GREMBAN, R. 2007. PHEVs: the Technical Side. EET-2007 European Ele-Drive Conference. Brussels, Belgium: Unpublished.

GRÜBLER, A. \& GRITSEVSKYI, A. 1997. A Model of Endogenous Technological Change Through Uncertain Returns on Learning (R\&D and Investments). International Workshop on Induced Technical Change and the Environment. 26-27, June, International Institute for Applied Systems Analysis, Laxenburg, Austria: International Institute for Applied Systems Analysis.

GRÜBLER, A., NAKIĆENOVIĆ, N. \& VICTOR, D., G. 1999. Modelling Technological Change: Implications for the Global Environment. . Annu Rev. Energy Environ. , 24, 545-569

GUILFORD, M., HALL, C., O'CONNOR, P. \& CLEVELAND, C. 2011. A new long term assessment of energy return on investment (EROI) for U.S. oil and gas discovery and production. Sustainability, 3, 1866-1887.

HADLEY, S. \& TSVETKOVA, A. 2009. Potential Impacts of Plug-in Hybrid Electric Vehicles on Regional Power Generation. The Electricity Journal, 22, 56-68.

HAMILTON, J. 2009. Causes and Consequences of the Oil Shock of 2007-08. Brookings Papers on Economic Activity, 1, 215-261.

HAMILTON, J. 2012. Oil prices, exhaustible resources, and economic growth. NBER Working paper No. 17759. Cambridge, MA: National Bureau of Economic Research.

HAN, S. \& HAN, S. 2013. Economic Feasibility of V2G Frequency Regulation in Consideration of Battery Wear. Energies, 6, 748-765.

HANSEN, J., SATO, M., RUEDY, R., KHARECHA, P., LACIS, A., MILLER, R., NAZARENKO, L., LO, K., SCHMIDT, G., RUSSELL, G. \& ALEINOV, I. 2007. Dangerous human-made interference with climate: a GISS modelE study. Atmos. Chem. Phys, 7, 2287-2312.

HÅRD, M. \& KNIE, A. 2001. The Cultural Dimension of Technology Management: Lessons from the History of the Automobile. Technology Analysis \& Strategic Management, 13, 91-103.

HARRIS, C. 2007. Lost City: Forgotten Plans for an Alternative Auckland. 2nd International Conference on Sustainability Engineering and Science. Auckland: New Zealand Society for Sustainability Engineering and Science.

HARRISON, G. 2006a. Hypothetical Bias Over Uncertain Outcomes In: LIST, J. (ed.) Using Experimental Methods In Environmental And Resource Economics. Cheltenham UK: Edward Elgar Publishing

HARRISON, G. 2006b. Making Choice Studies Incentive Compatible. In: KANNINEN, B. (ed.) Valuing Environmental Amenities Using Stated Choice Studies. AA Dordrecht: Springer.

HELMS, H., PEHNT, M., LAMBRECHT, U. \& LIEBICH, A. 2010. Electric vehicle and plug-in hybrid energy efficiency and life cycle emissions. 18th International Symposium Transport and Air Pollution. Dübendorf, Switzerland.

HEMERY, E. 2009. Gas for electricity generation: availability and price forecasts. http://www.ea.govt.nz/industry/ec-archive/gpas/: Electricity Commission.

HEMERY, E. \& SMITH, B. 2008. Impact of Plug-in Hybrid Vehicles on the NZ Electric Grid. Grid Planning Assumptions workshop. Electricity Commission, ASB Tower, Level 7, 2 Hunter St, Wellington: Electricity Commission. 
HENDRY, I. 2011. Chevrolet Volt and Camaro Pricing Announced For UK, Launch Spring 2012 [Online]. Gmotors.co.uk: Gmotors.co.uk. Available:

http://www.gmotors.co.uk/news/chevrolet-volt-and-camaro-pricing-announcedfor-uk-launch-spring-2012/ [Accessed 15 August 2011].

HENRY, J. 2011. Chevy Volt Battery Fires Threaten All Electric Vehicle Makers, Not Just GM. Forbes.

HENSHER, D. 1982. Functional measurement, individual preference and discrete-choice modelling: theory and application. Journal of Economic Psychology, 2, 323-335.

HENSHER, D. 2006a. Attribute Processing. In: KANNINEN, B. (ed.) Valuing Environmental Amenities Using Stated Choice Studies. AA Dordrecht: Springer.

HENSHER, D. 2006b. Reducing sign violation for VTTS distributions through recognition of an individual's atrribute processing strategy. Sydney: Institute of Transport and Logistics Studies, University of Sydney.

HENSHER, D. 2009. Hypothetical bias, choice experiments and willingness to pay. Sydney: Institute of Transport and Logistics Studies, University of Sydney.

HENSHER, D., BECK, M. \& ROSE, J. 2011. Accounting for preference and scale heterogeneity in establishing whether it matters who is interviewed to reveal household automobile purchase preferences. Environ Resource Econ, 49, 1-22.

HENSHER, D. \& GREENE, W. 2003. The Mixed Logit Model: The State of Practice. Transportation, 30, 17.

HENSHER, D., ROSE, J. \& BLACK, I. 2008. Interactive agency choice in automobile purchase decisions: the role of negotiation in determining equilibrium choice outcomes. Journal of Transport Economics and Policy, 42, 269-296.

HENSHER, D., ROSE, J. \& GREENE, W. 2005. Applied Choice Analysis: A Primer, Cambridge, Cambridge University Press.

HENSLEY, R., NEWMAN, J. \& ROGERS, M. 2012. Battery technology charges ahead. McKinsey Quarterly. New York: McKinsey \& Company.

HIRSCH, R., BEZDEK, R. \& WENDLING, R. 2005. Peaking of world oil production: impact, mitigation \& risk management. Washington: Department of Energy.

HOLDWAY, A., WILLIAMS, A., INDERWILDI, O. \& KING, D. 2010. Indirect emissions from electric vehicles: emissions from electricity generation. Energy \& Environmental Science, 3, 1825-1832.

HOLZMAN, D. 2011. Vehicle Motion Alarms: Necessity, Noise Pollution, or Both? Environmental Health Perspectives, 119, A30-A33.

HOUSE OF COMMONS: ENERGY AND CLIMATE CHANGE COMMITTEE 2011. UK Energy Supply: Security or Independence? London: The Stationery Office Limited.

HUBBERT, M. 1956. Nuclear energy and the fossil fuels. Spring meeting of the Southern District, Division of Production, American Petroleum Institute. San Antonio: Shell Development Company.

HUGHES, J. 2013. Energy: A reality check on the shale revolution. Nature, 494, 307.

HUGHES, L. \& RUDOLPH, J. 2011. Future world oil production: growth, plateau, or peak? Current Opinion in Environmental Sustainability, 3, 225-234.

HUME, D. \& CHIU, J. 2008. Transmission to Enable Renewables Project: Transmission Network Reinforcement Inputs for GEM. Wellington: Electricity Commission.

I-GO. 2012. I-GO Car Sharing Chicago [Online]. Available: www.igocars.org/ [Accessed 20 September 2012.

IHS CAMBRIDGE ENERGY RESEARCH ASSOCIATES. 2010. 'Peak Oil' Postponed Again, Part 1: Liquids Production Capacity to 2030 [Online]. Cambridge, MA: IHS Cambridge Energy Research Associates. [Accessed 10 November 2010.

INGRAM, G. \& LIU, Z. 1999. Determinants of Motorization and Road Provision. Policy Research Working Paper. Washington D.C.: World Bank. 
INLAND REVENUE SERVICE. 2009. Internal Revenue Bulletin: 2009-48: New Qualified Plugin Electric Drive Motor Vehicle Credit [Online]. IRS.gov: IRS. Available: http://www.irs.gov/irb/2009-48_IRB/ar09.html [Accessed 13 March 2011 2011].

INTERNATIONAL ENERGY AGENCY 2000. Experience Curves for Energy Technology Policy. Paris: International Energy Agency.

INTERNATIONAL ENERGY AGENCY 2008. Energy Technology Perspectives, 2008: Scenarios and Strategies to 2050. Paris: International Energy Agency.

INTERNATIONAL ENERGY AGENCY 2010. World Energy Outlook 2010. World Energy Outlook. Paris: IEA.

INTERNATIONAL ENERGY AGENCY 2011. Technology Roadmap: Biofuels for Transport. Paris: International Energy Agency.

INTERNATIONAL ENERGY AGENCY 2012. World Energy Outlook 2012. World Energy Outlook. Paris: IEA.

INTERNATIONAL TRANSPORT FORUM 2010. Reducing transport greenhouse gas emissions: trends and data. Paris: International Transport Forum of the OECD.

IPCC 2007a. Climate Change 2007: Impacts, Adaptation and Vulnerability. Contribution of Working Group II to the Fourth Assessment Report of the Intergovernmental Panel on Climate Change. In: M. PARRY, O. C., J. PALUTIKOF, P. VAN DER LINDEN, AND C. HANSON (ed.). Cambridge, Cambridge University Press.

IPCC 2007b. Climate Change 2007: The Physical Science Basis. Contribution of Working Group I to the Fourth Assessment Report of the Intergovernmental Panel on Climate Change. In: SOLOMON, S., QIN, D., MANNING, M., CHEN, Z., MARQUIS, M., AVERYT, K., TIGNOR, M. \& MILLER, H. (eds.). Cambridge, United Kingdom and New York: Cambridge University Press.

JACCARD, M., MURPHY, R. \& RIVERS, N. 2004. Energy-environment policy modeling of endogenous technological change with personal vehicles: combining top-down and bottom-up methods. Ecological Economics 51, 31-46.

JENKINS, S., ROSSIMAIER, J. \& FERDOWSI, M. 2008. Utilization and Effect of Plug-In Hybrid Electric Vehicles in the United States Power Grid. IEEE Vehicle Power and Propulsion Conference (VPPC), 2008. Harbin, China: IEEE.

JOHANSSON, O. \& SCHIPPER, L. 1997. Measuring the Long-run Fuel Demand of cars. Journal of Transport Economics and Policy, 31, 277-292.

JOHNSON, F., KANNINEN, B., BINGHAM, M. \& OZDEMIR, S. 2006. Experimental Design for Stated Choice Studies. In: KANNINEN, B. (ed.) Valuing Environmental Amenities Using Stated Choice Studies. AA Dordrecht: Springer.

J $\varnothing$ RGENSEN, J., SØRENSEN, S., BEHNKE, K. \& ERIKSEN, P. EcoGrid EU - A prototype for European Smart Grids. Power and Energy Society Meeting, 2011 IEEE, 2011 Detroit, MI. IEEE, 7.

KAHN RIBEIRO, S., KOBAYASHI, S., BEUTHE, M., GASCA, J., GREENE, D., LEE, D. S., MUROMACHI, Y., NEWTON, P. J., PLOTKIN, S., SPERLING, D., WIT, R. \& ZHOU, P. J. 2007. Transport and its infrastructure. In: B. METZ, O.R. DAVIDSON, P.R. BOSCH, R. DAVE \& MEYER, L. A. (eds.) In Climate Change 2007: Mitigation. Contribution of Working Group III to the Fourth Assessment Report of the Intergovernmental Panel on Climate Change. Cambridge: Cambridge University Press.

KALHAMMER, F., KAMATH, H., DUVALL, M. \& ALEXANDER, M. 2009. Plug-In Hybrid Electric Vehicles: Promise, Issues and Prospects. EVS24 - International Battery, Hybrid and Fuel Cell Electric Vehicle Symposium. Stavanger, Norway: World Electric Vehicle Association.

KALHAMMER, F., KOPF, B., SWAN, D., ROAN, V. \& WALSH, M. 2007. Status and Prospects for Zero Emissions Vehicle Technology: Report of the ARB Independent Expert 
Panel 2007. Sacramento: State of California Air Resources Board Sacramento, California.

KALVELAGEN, E. 2004. Model Building with GAMS: Chapter 3: Building Integer Programming Models. Washington D.C.: GAMS Development Corporation.

KEANE, A. 2012. GM's Chevy Volt Used as 'Political Punching Bag,' Akerson Says. Bloomberg.com.

KEETER, S., KENNEDY, C., CLARK, A., TOMPSON, T. \& MOKRZYCKI, M. 2007. What's Missing from National Landline RDD Surveys? Public Opinion Quarterly, 71, 772-792.

KELLY, G. 2011. History and Potential of Renewable Energy Development in New Zealand. Renewable and Sustainable Energy Reviews, 15, 2501-2509.

KEMP, R. \& LOORBACH, D. 2006. Transition management: a reflexive governance approach. Reflexive Governance. Cheltenham: Edward Elgar.

KEMPTON, W. \& LETENDRE, S. 1997. Electric Vehicles as a New Power Source for Electric Utilities. Transportation Research Part D, 2, 157-175.

KEMPTON, W. \& TOMI'C, J. 2005a. Vehicle-to-grid power fundamentals: Calculating capacity and net revenue. Journal of Power Sources, 144, 268-279.

KEMPTON, W. \& TOMI'C, J. 2005b. Vehicle-to-grid power implementation: From stabilizing the grid to supporting large-scale renewable energy. J. Power Sources, 144, 280294.

KENNEDY, D. \& WALLIS, I. 2007. Impacts of fuel price changes on New Zealand transport. Land Transport New Zealand Research Report 331. Land Transport New Zealand.

KENNEDY, E., BECKLEY, T., MCFARLANE, B. \& NADEAU, S. 2009. Why We Don't "Walk the Talk": Understanding the Environmental Values/Behaviour Gap in Canada. Human Ecology Review, 16, 151-160.

KENNEDY, P. 2008. A Guide to Econometrics, Malden, MA, Blackwell Publishing. KERR, P. 2009. MIA NZTA Sales Data: Jan 02 - Sep 09. Motor Industry Association,. KESHAVARZIAN, M., KAMALI ANARAKI, S., ZAMANI, M. \& ERFANIFARD, A. 2012. Projections of oil demand in road transportation sector on the basis of vehicle ownership projections, worldwide: 1972-2020. Economic Modelling, 29, 1979-1985.

KESSELS, R., GOOS, P. \& VANDEBROEK, M. 2006. A Comparison of Criteria to Design Efficient Choice Experiments. Journal of Marketing Research, 43, 409-419.

KHARECHA, P. \& HANSEN, J. 2008. Implications of "peak oil" for atmospheric $\mathrm{CO}_{2}$ and climate. Global Biogeochemical Cycles, 22, GB3012.

KIM, D. S., EMERSON, R., NAGHSHINEH, K., PLISKOW, J. \& MYERS, K. 2012. Impact of adding artificially generated alert sound to hybrid electric vehicles on their detectability by pedestrians who are blind. Journal of Rehabilitation Research and Development, 49, 381-394.

KINTNER-MEYER, M., SCHNEIDER, K. \& PRATT, R. 2007a. Impact assessment of plug-in hybrid vehicles on electric utilities and regional U.S. power grids. Part 1: technical analysis. Richland, WA: Pacific Northwest National Laboratory.

KINTNER-MEYER, M., SCHNEIDER, K. \& PRATT, R. 2007b. Impacts assessment of plug-in hybrid vehicles on electric utilities and regional U.S. power grids - Part 1: technical analysis. Richland, WA: Pacific Northwest National Laboratory, DOE.

KIRTLAN, B. 2008. Electricity Demand Forecast: Review February 2008. Electricity Commission.

KIRTLAN, B. 2009. 2009 Electricity Demand Forecasts. Electricity Commission.

KIVILUOMA, J. \& MEIBOM, P. 2011. Methodology for modelling plug-in electric vehicles in the power system and cost estimates for a system with either smart or dumb electric vehicles. Energy, 36, 1758-1767.

KOCKELMAN, K. \& ZHAO, Y. 2000. Behavioral distinctions: the use of light-duty trucks and passenger cars. Journal of Transportation and Statistics, 3, 47-60. 
KOPPELMAN, F. \& BHAT, C. 2006. A self instructing course in mode choice modeling: multinomial and nested logit models. Prepared for U.S. Department of Transportation Federal Transit Administration. www.ce.utexas.edu/prof/bhat/.../LM_Draft_060131Final-060630.pdf: University of Texas.

KOWALEWICZ, A. \& WOJTYNIAK, M. 2005. Alternative fuels and their application to combustion engines. Proceedings of the Institution of Mechanical Engineers, 219, 103-125.

KOYANAGI, F. \& URIU, Y. 1997. Modeling Power Consumption, by Electric Vehicles and Its Impact on Power Demand. Electricial Engineering in Japan, 120, 41-46.

KROMER, M. A. \& HEYWOOD, J. B. 2007. Electric Powertrains: Opportunities and Challenges in the U.S. Light-Duty Vehicle Fleet. Cambridge Massachusetts: Sloan Automotive Laboratory, Laboratory for Energy and the Environment, Massachusetts Institute of Technology.

KROMER, M. A. \& HEYWOOD, J. B. 2009. A Comparative Assessment of Electric Propulsion Systems in the 2030 US Light-Duty Vehicle Fleet. SAE International Journal of Engines, 1, 372-390.

KRUGER, P., BLAKELEY, J. \& LEAVER, J. 2003. Potential in New Zealand for use of hydrogen as a transportation fuel. International Journal of Hydrogen Energy, 28, $795-802$.

KRUPNICK, A. \& ADAMOWICZ, W. 2006. Supporting Questions in Stated Choice Studies. In: KANNINEN, B. (ed.) Valuing Environmental Amenities Using Stated Choice Studies. AA Dordrecht: Springer.

KURCZY, S. 2010. International Energy Agency says 'peak oil' has hit. Crisis averted? The Christian Science Monitor. Boston: The Christian Science Publishing Society.

LADENBURG, J., OLSEN, S. \& NIELSEN, R. 2007. Reducing hypothetical bias in Choice Experiments - testing an Opt-Out Reminder. EAERE Annual Conference. University of Macedonia, Thessaloniki, Greece, 27-30 June 2007.

LANCASTER, K. 1966. A New Approach to Consumer Theory. The Journal of Political Economy, 74, 132-157.

LANDRY, C. \& LIST, J. 2007. Using ex ante approaches to obtain credible signals for value in contingent markets: evidence from the field. American Journal of Agricultural Economics, 89, 420-429.

LANE, B. \& POTTER, S. 2007. The adoption of cleaner vehicles in the UK: exploring the consumer attitude-action gap. Journal of Cleaner Production, 15, 1085-1092.

LARMINIE, J. \& LOWRY, J. 2003. Electric Vehicle Technology Explained, Chichester, John Wiley \& Sons Ltd.

LAVE, L., SAVITZ, M., BERRY, R., BROWN, M., COHEN, L., CRAFORD, M., DECOTIS, P., DEGRAFFENREIDT, J., GELLER, H., GOLDSTEIN, D., MACLACHLAN, A., POWERS, W., ROSENFELD, A. \& SPERLING, D. 2010. Real Prospects for Energy Efficiency in the United States. Washington D.C.: National Academy of Sciences; National Academy of Engineering; National Research Council.

LEAVER, J. \& GILLINGHAM, K. 2010. Economic impact of the integration of alternative vehicle technologies into the New Zealand vehicle fleet. Journal of Cleaner Production, 18, 908-916.

LEE, S. W., MCDOWELL, M., CHOI, J. W. \& CUI, Y. 2011. Anomalous Shape Changes of Silicon Nanopillars by Electrochemical Lithiation. Nano Letters, 11, 3034-3039.

LEIBY, P. \& RUBIN, J. 2004. Understanding the Transition to New Fuels and Vehicles: Lessons Learned from Analysis and Experience of Alternative Fuel and Hybrid Vehicles. In: SPERLING, D. \& CANNON, J. (eds.) The Hydrogen Energy Transition. Burlington: Elsevier Academic Press. 
LETENDRE, S. \& WATTS, R. 2009. Effects of Plug-In Hybrid Electric Vehicles on the Vermont Electric Transmission System. Transportation Research Board Annual Meeting, 1115 January 2009. Washington D.C.

LIQUID BIOFUELS INTEREST GROUP. 2012. Liguid Biofuels in New Zealand [Online]. Wellington: Bioenergy Association of New Zealand. Available: http://www.liquidbiofuels.org.nz/index.asp [Accessed 30 October 2012.

LIST, J. \& GALLET, C. 2001. What Experimental Protocol Influence Disparities Between Actual and Hypothetical Stated Values? Environmental and Resource Economics, 20, 241-254.

LIST, J., SINHA, P. \& TAYLOR, M. 2006. Using Choice Experiments to Value Non-Market Goods and Services: Evidence from Field Experiments. Advances in Economic Analysis \& Policy, 6, 1132-1132.

LOPES, J. 2009. Identifying Management Procedures to Deal with Connection of Electric Vehicles in the Grid. IEEE Bucharest Power Technology Conference, 28 June -2 July 2009. Bucharest: IEEE.

LOUVIERE, J., FLYNN, T. \& CARSON, R. 2010. Discrete Choice Experiments Are Not Conjoint Analysis. Journal of Choice Modelling, 3, 57-63.

LOUVIERE, J. \& HENSHER, D. 1983. Using discrete choice models with experimental design data to forecast consumer demand for a unique cultural event. Journal of Consumer Research, 10, 348-361.

LOUVIERE, J., HENSHER, D. \& SWAIT, J. 2000. Stated choice methods: Analysis and application, Cambridge, Cambridge University Press.

LOUVIERE, J., MEYER, R., BUNCH, D., CARSON, R., DELLAERT, B., MICHAEL HANEMANN, W., HENSHER, D. \& IRWIN, J. 1999. Combining Sources of Preference Data for Modeling Complex Decision Processes. Marketing Letters, 10, 205-217.

LOVEDAY, E. 2010. Concerns continue to swirl over "true" range of electric vehicles [Online]. autobloggreen.com. Available: http://green.autoblog.com/2010/06/13/concernscontinue-to-swirl-over-true-range-of-electric-vehicle/ [Accessed 12 September 2010 2010].

LOVINS, A., DATTA, E., BUSTNES, O., KOOMEY, J. \& GLASGOW, N. 2005. Winning the Oil Endgame: Innovation for Profits, Jobs, and Security, Snowmass, CO, Rocky Mountain Institute.

LUCAS, K. 2012. Transport and social exclusion: Where are we now? Transport Policy, 20, 105-113.

LUCE, D. 1959. Individual Choice Behavior, New York, John Wiley and Sons.

LUE, A., COLORNI, A., NOCERINO, R. \& PARUSCIO, V. Green Move: an innovative electric vehicle-sharing system. Procedia - Social and Behavioral Sciences, 48, 2978- 2987.

LUSK, J. 2003. Willingness to pay for golden rice. American Journal of Agricultural Economics, 85, $840-856$.

LUSK, J. \& SCHROEDER, T. 2004. Are Choice Experiments Incentive Compatible? A Test with Quality Differentiated Beef Steaks. American Journal of Agricultural Economics, 86, 467-482.

LUX RESEARCH. 2012. Li-ion Battery Costs Fall to $\$ 397 / \mathrm{kWh}$ in 2020; Not Enough for Mass Adoption of Electric Vehicles. Press release, 27 March 2012.

MA, H., BALTHASAR, F., TAIT, N., RIERA-PALOU, X. \& HARRISON, A. 2012. A new comparison between the lifecycle greenhouse gas emissions of battery electric vehicles and internal combustion vehicles. Energy Policy, 44, 160-173.

MACKAY, D. 2009. Sustainable energy: without the hot air, Cambridge, Cambridge, England: UIT Cambridge Ltd.

MACY, M. \& WILLER, R. 2002. From Factors to Actors: Computational Sociology and AgentBased Modeling. Annual Review of Sociology, 28, 143-166. 
MAIRESSE, O., MACHARIS, C., LEBEAU, K. \& TURCKSIN, L. 2012. Understanding the attitudeaction gap: functional integration of environmental aspects in car purchase intentions. Psicológica, 33, 547-574.

MAK, H., RONG, Y. \& SHEN, Z. 14 March 2012. Infrastructure Planning for Electric Vehicles with Battery Swapping. Available at SSRN: http://ssrn.com/abstract $=2022651$

MANSFIELD, C. \& PATTANAYAK, S. 2006. Getting Started. In: KANNINEN, B. (ed.) Valuing Environmental Amenities Using Stated Choice Studies. AA Dordrecht: Springer.

MARKARD, J., RAVEN, R. \& TRUFFLER, B. 2012. Sustainability transitions: An emerging field of research and its prospects. Research Policy, 41, 955-967.

MARSCHAK, J. 1960. Binary choice constraints on random utility indications. In: ARROW, K. (ed.) Stanford Symposium on Mathematical Methods in the Social Sciences. Stanford, CA: Stanford University Press.

MATHEWS, K., FREEMAN, M. \& DESVOUSGES, W. 2006. How and How Much? In: KANNINEN, B. (ed.) Valuing Environmental Amenities Using Stated Choice Studies. AA Dordrecht: Springer.

MATTHEW, C. \& ROBERTSON, S. 2010. The Dog and Lemon Guide, Auckland, Publications (2008) Limited.

MCDONALD, A. \& SCHRATTENHOLZER, L. 2001. Learning rates for energy technologies. Energy Policy, 29, 255-261.

MCFADDEN, D. 1974. Conditional logit analysis of qualitative choice behavior. In: ZAREMBKA, P. (ed.) Frontiers in Econometrics. New York: Academic Press.

MCFADDEN, D. \& TRAIN, K. 2000. Mixed MNL Models for Discrete Response. Journal of Applied Econometrics, 15, 447-470.

MCMANUS, W. \& SENTER, R. 2009. Market Models for Predicting PHEV Adoption and Diffusion. Technical Challenges of Plug-In Hybrid Electric Vehicles and Impacts to the U.S. Power System. Ann Arbor, Michigan: University of Michigan Transportation Research Institute.MEES, P. \& DODSON, J. 2006. Backtracking Auckland: Bureaucratic rationality and public preferences in transport planning. Urban Research Program: Issues Paper 5. Brisbane: Griffith University.

MELAINA, M. \& BREMSON, J. 2008. Refueling Availability for Alternative Fuel Vehicle Markets: Sufficient Urban Station Coverage. Energy Policy, 36, 3223-3231.

METCALFE, J. 1994. Evolutionary Economics and Technology Policy. The Economic Journal, 104, 931-944.

METZ, D. 2010. Saturation of Demand for Daily Travel. Transport Reviews, 30, 659-674.

METZ, D. 2012. Demographic determinants of daily travel demand. Transport Policy, 21, 2025.

MIAOU, S. P. 1995. Factors associated with aggregate car scrappage rate in the United States: 1966-1992. Transportation Research Record: Journal of the Transportation Research Board, 1475 3-9.

MILLARD-BALL, A. \& SCHIPPER, L. 2011. Are we reaching peak travel? Trends in passenger transport in eight Industrialized countries. Transport Reviews, 31, 357-378.

MILLENNIUM ECOSYSTEM ASSESSMENT 2005. Ecosystems and Human Well-being: Scenarios, Volume 2, Washington D.C., Island Press.

MINISTRY FOR THE ENVIRONMENT 2011. New Zealand's greenhouse gas inventory 19902009. Wellington: Ministry for the Enviornment.

MINISTRY OF BUSINESS INNOVATION AND EMPLOYMENT 2012. Economic contribution and potential of New Zealand's oil and gas industry. Occasional Paper 12/07. Wellington: Ministry of Business Innovation and Employment.

MINISTRY OF ECONOMIC DEVELOPMENT 2010a. New Zealand Energy Data File: 2009 Calendar Edition. Wellington: Ministry of Economic Development. 
MINISTRY OF ECONOMIC DEVELOPMENT 2010b. New Zealand's Energy Outlook 2010. Wellington: Ministry of Economic Development,.

MINISTRY OF ECONOMIC DEVELOPMENT. 2011a. Carbon Capture and Storage in New Zealand [Online]. Ministry of Economic Development. Available: http://www.med.govt.nz/templates/ContentTopicSummary__38284.aspx [Accessed 5 September 2011].

MINISTRY OF ECONOMIC DEVELOPMENT 2011b. New Zealand Energy Strategy: Developing our Energy Potential. Wellington: Ministry of Economic Development.

MINISTRY OF ECONOMIC DEVELOPMENT 2011c. New Zealand's Energy Outlook 2011: Reference Scenario and Sensitivity Analysis. Wellington: Ministry of Economic Development,.

MINISTRY OF ECONOMIC DEVELOPMENT 2012. New Zealand Energy Data File: 2011 Calendar Edition. Wellington: Ministry of Economic Development.

MINISTRY OF ECONOMIC DEVELOPMENT: ELECTRICITY TECHNICAL ADVISORY GROUP 2009. Summary note on recommendations taking account of submissions. Ministry of Economic Development.

MINISTRY OF TRANSPORT 1998. Vehicle fleet emissions model (VFEM), New Zealand Fleet Database and Model Development-Technical Report. Ministry of Transport.

MINISTRY OF TRANSPORT 2007. Land Transport Rule: Vehicle Exhaust Emissions 2007. New Zealand Transport Agency.

MINISTRY OF TRANSPORT 2008. Comparing travel modes - Household travel survey: version 1.4. Ministry of Transport.

MINISTRY OF TRANSPORT 2009a. Driver travel - Household Travel Survey, version 2. Ministry of Transport.

MINISTRY OF TRANSPORT 2009b. The New Zealand Light Vehicle Fleet Light Fleet Statistics 2009: Statistical Report. Ministry of Transport.

MINISTRY OF TRANSPORT 2010a. The New Zealand Vehicle Fleet: Data Spreadsheet: version 2. Ministry of Transport.

MINISTRY OF TRANSPORT. 2010b. Road User Charges [Online]. Wellington: Ministry of Transport. Available:

http://www.transport.govt.nz/ourwork/land/roadusercharges/ [Accessed 4 November 2010].

MINISTRY OF TRANSPORT. 2010c. Travel Survey - Information for researchers [Online]. Wellington: Ministry of Transport. Available:

http://www.transport.govt.nz/research/Pages/TravelSurvey-Method.aspx [Accessed 2 September 2010].

MINISTRY OF TRANSPORT 2011. The New Zealand Vehicle Fleet: Data Spreadsheet: version 2. Ministry of Transport.

MINISTRY OF TRANSPORT 2012a. Comparing travel modes. New Zealand Household Travel Survey 2008-2011, March 2012. Wellington: Ministry of Transport.

MINISTRY OF TRANSPORT 2012b. The New Zealand Vehicle Fleet: Annual Fleet Statistics 2011. Wellington: Ministry of Transport.

MINISTRY OF TRANSPORT. 2012c. Summary of the Transport Climate Change Work programme [Online]. Ministry of Transport. Available: http://www.transport.govt.nz/ourwork/climatechange/ [Accessed 25 July 2012.

MINISTRY OF TRANSPORT 2012d. Transport Monitoring Indicator Framework. Wellington: Ministry of Transport.

MITCHELL, D. 2008. A Note on Rising Food Prices. Policy Research Working Paper 4682. Washington, D.C.: World Bank.

MITSUBISHI MOTORS AUSTRALIA LIMITED. 2011. Mitsubishi announces pricing on its i-MiEV electric vehicle [Online]. http://www.mitsubishi-motors.com.au/about- 
mitsubishi/latest-information/news-releases/corporate-news/view/mitsubishiannounces-pricing-on-its-i-miev-electric-vehicle. [Accessed 15 August 2011].

MITSUBISHI MOTORS IN THE UNITED KINGDOM. 2011. i MIEV [Online]. Mitsubishi Motors UK. Available: http://www.mitsubishi-cars.co.uk/imiev/ [Accessed 15 August 2011].

MITSUBISHI MOTORS NORTH AMERICA INC. 2011. All-New 100\% Electric-Powered Mitsubishi i Earns Impressive EPA Rated 62 Mile/98 Mile LA4 Mode Range and 112 MPGe Rating [Online]. Available:

http://www.mitsubishicars.com/MMNA/jsp/media.do?sID=0\&alD=5 [Accessed 15 August 2011].

MOAWAD, A., SINGH, G., HAGSPIEL, S., FELLAH, M. \& ROUSSEAU, A. 2009. Impact of real world drive cycles on PHEV fuel efficiency and cost for different powertrain and battery characteristics. EVS24. Stavanger, Norway: Argonne National Laboratory.

MORROW, K., KARNER, D. \& FRANCFORT, J. 2008. Plug-in Hybrid Electric Vehicle Charging Infrastructure Review: Final Report INL/EXT-08-15058. Idaho Falls: Idaho National Laboratory.

MULLER, J. 2010. Electric Car Warning: Actual Mileage May Vary [Online]. New York, New York: Forbes.com. Available:

http://blogs.forbes.com/energysource/2010/06/11/warning-your-mileage-mayvary/ [Accessed 12 September 2010 2010].

MURPHY, J., ALLEN, P., STEVENS, T. \& WEATHERHEAD, D. 2005a. A Meta-Analysis of Hypothetical Bias in Stated Preference Valuation. Environmental and Resource Economics, 30, 313-325.

MURPHY, J., STEVENS, T. \& WEATHERHEAD, D. 2005b. Is Cheap Talk Effective at Eliminating Hypothetical Bias in a Provision Point Mechanism? Environmental and Resource Economics, 30, 327-343.

MURRAY, J. \& KING, D. 2012. Oil's tipping point has passed. Nature, 481, 433-435.

MUSTI, S. \& KOCKELMAN, K. 2009. Evolution of the household vehicle fleet: anticipating fleet composition, PHEV adoption and GHG emissions in Austin, Texas. 12th International Association of Travel Behavior Research. Jaipur, India.

NAIK, S., GOUD, V., ROUT, P. \& DALAI, A. 2010. Production of first and second generation biofuels: A comprehensive review. Renewable and Sustainable Energy Reviews, 14, 578-597.

NAKIĆENOVIĆ, N. 2002. Technological Change and Diffusion as a Learning Process. In: GRÜBLER, A., NAKICENOVIC, N. \& NORDHAUS, W. D. (eds.) Technological Change and the Environment. Washington: Resources for the Future.

NEL, W. \& COOPER, C. 2009. Implications of fossil fuel constraints on economic growth and global warming. Energy Policy, 37, 166-180.

NEUBAUER, J. \& PEARSAN, A. 2011. The ability of battery second use strategies to impact plug-in electric vehicle prices and serve utility energy storage applications. Journal of Power Sources, 196, 10351-10358.

NEW ZEALAND CUSTOMS SERVICE. 2011. Types of duties, fees, and charges [Online]. http://www.customs.govt.nz/features/charges/feetypes/Pages/default.aspx?s=22. [Accessed 25 February 2011 2011].

NEW ZEALAND GEOTHERMAL ASSOCIATION. 2012. New Zealand Geothermal Fields [Online]. Wellington: New Zealand Geothermal Association. Available: http://www.nzgeothermal.org.nz/nz_geo_fields.html [Accessed 7 October 2012].

NEW ZEALAND GOVERNMENT 1986. Commerce Act, s52R. Wellington.

NEW ZEALAND INSTITUTE OF ECONOMIC RESEARCH 2009. Review of Electricity Demand Forecast Model. Wellington: NZIER.

NEW ZEALAND TRANSPORT AGENCY 2006. New Zealand motor vehicle registration statistics 2005. Palmerston North: NZ Transport Agency. 
NEW ZEALAND TRANSPORT AGENCY 2010. New Zealand motor vehicle registration statistics 2010. NZ Transport Agency.

NEW ZEALAND TRANSPORT AGENCY 2011. New Zealand motor vehicle registration statistics 2010. Palmerston North: NZ Transport Agency.

NEW ZEALAND TRANSPORT AGENCY 2013. New Zealand motor vehicle registration statistics 2012. Palmerston North: NZ Transport Agency.

NEW ZEALAND WIND ENERGY ASSOCIATION. 2012. Wind farms operating and under construction [Online]. Wellington: New Zealand Wind Energy Association. Available: http://windenergy.org.nz/nz-wind-farms/operating-wind-farms [Accessed 7 October 2012].

NEWMAN, P. 2006. The environmental impact of cities. Environment and Urbanization, 18, 275-295.

NEWMAN, P. 2009. Beyond Peak Oil: Will Our Cities Collapse? Journal of Urban Technology, $14,15-30$.

NEWMAN, P. \& KENWORTHY, J. 2011. 'Peak Car Use': Understanding the Demise of Automobile Dependence. World Transport, Policy \& Practice, 17, 31-42.

NGUYEN, T., WARD, J. \& JOHNSON, K. 2013. Well-to-Wheels Greenhouse Gas Emissions and Petroleum Use for Mid-Size Light-Duty Vehicles. Program Record (Offices of Bioenergy Technologies, Fuel Cell Technologies \& Vehicle Technologies). Washington D.C.: U.S. Department of Energy.

NISSAN MOTOR COMPANY. 2012. Product information: LEAF [Online]. Nishi-ku, Yokohama: Nissan Motor Company. Available: http://www.nissanglobal.com/EN/NISSAN/LEAF/ [Accessed 15 June 2012.

NISSAN MOTOR MANUFACTURING UK. 2011. Nissan Leaf [Online]. Available: http://www.nissan.co.uk/vehicles/electric-vehicles/electricleaf/leaf.html\#vehicles/electric-vehicles/electric-leaf/leaf/pricing-andspecifications/prices-and-government-incentives [Accessed 20 August 2011 2011].

NISSAN NORTH AMERICA, I. 2011. Nissan Leaf packages and features [Online]. Available: http://www.nissanusa.com/leaf-electric-car/estimator/index\#/leaf-electriccar/feature/pricing_information [Accessed 15 August 2011].

NORGROVE, L. 2010. Impacts of Biofuel Production on Food Security. IUFoST Scientific Information Bulletin. Oakville, Ontario: International Union of Food Science and Technology.

NORTON, J. \& BASS, F. 1987. A Diffusion Theory Model of Adoption and Substitution for Sucessive Generations of High-Technology Products. Management Science, 33, 1069-1086.

O'BRIEN, T. 2012. Environmental protest in New Zealand (1997-2010). British Journal of Sociology, 63, 641-661.

OBAMA, B. 2011. Blueprint for a Secure Energy Future. Washington D.C.: The White House.

OFFICE OF INTEGRATED ANALYSIS AND FORECASTING 2009. The National Energy Modeling System: An Overview 2009. Washington D.C.: U.S. Department of Energy.

OGDEN, J. \& YANG, C. 2009. Build-up of a hydrogen infrastructure in the US. In: BALL, M. \& WIETSCHEL, M. (eds.) The Hydrogen Economy: Opportunities and Challenges. Cambridge: Cambridge University Press.

OGDEN, J., YANG, C., CUNNINGHAM, J., JOHNSON, N., LI, X., NICHOLAS, M., PARKER, N. \& SUN, Y. 2011. The Hydrogen Fuel Pathway. In: J. OGDEN, A. L. A. (ed.) Sustainable Transportation Energy Pathways: A research summary for decision makers. University of California, Davis: Institute of Transportation Studies.

OGILVY, J. \& SCHWARTZ, P. 1998. Plotting Your Scenarios. In: FAHEY, L. \& RANDALL, R. (eds.) Learning from the Future. New York: John Wiley \& Sons. 
PACKEY, D. 1993. Market Penetration of New Energy Technologies Golden, Colorado: National Renewable Energy Laboratory.

PAINE, M. 2011. Safety Requirements for Small Motorised Alternative Vehicles. 22nd International Technical Conference on the Enhanced Safety of Vehicles (ESV). Washington D.C.: National Highway Traffic Safety Adminstration.

PARKS, K., DENHOLM, P. \& MARKEL, T. 2007. Costs and Emissions Associated with Plug-In Hybrid Electric Vehicle Charging in the Xcel Energy Colorado Service Territory. http://www.nrel.gov/docs/fy07osti/41410.pdf: National Renewable Energy Laboratory.

PARKS, R. W. 1977. Determinants of scrapping rates for postwar vintage automobiles. Econometrica, 45, 1099-1115.

PARSONS BRINCKERHOFF ASSOCIATES LTD 2006. Electricity Generation Database Statement of Opportunities: Update 2006. Wellington: Electricity Commission.

PARSONS, G., HIDRUE, M., KEMPTON, W. \& GARDNER, M. 2011. Can vehicle-to-grid revenue help electric vehicles on the market? Working paper series. University of Delaware: Alfred Lerner College of Business \& Economics.

PERUJO, A. \& CUIUFFO, A. 2010. The introduction of electric vehicles in the private fleet: Potential impact on the electric supply system and on the environment. A case study for the Province of Milan, Italy. Energy Policy, 38, 4549-4561.

PESARAN, A., MARKEL, T., TATARIA, H. \& HOWELL, D. 2009. Battery Requirements for PlugIn Hybrid Electric Vehicles - Analysis and Rationale. 23rd International Electric Vehicle Symposium (EVS-23). Anaheim, California: National Renewable Energy Laboratory.

PETERSON, S., WHITACRE, J. \& APT, J. 2010a. The economics of using plug-in hybrid electric vehicle battery packs for grid storage. Journal of Power Sources, 195, 2377-2384.

PETERSON, S., WHITACRE, J. \& APT, J. 2010b. Lithium-ion battery cell degradation resulting from realistic vehicle and vehicle-to-grid utilization. Journal of Power Sources, 195, 2385-2392.

PHILPOT, E. \& SHAW, G. 2006. Emissions Tail Pipe Test Trial: Final Report. Auckland: Japan Export Vehicle Inspection Center Company New Zealand Limited.

PLOTKIN, S. 2000. Technologies and Policies for Controlling Greenhouse Gas Emissions from the U.S. Automobile and Light Truck Fleet. Lemont, IL: Argonne National Laboratory

PORTER, M. 1979. How Competitive Forces Shape Strategy. Harvard Business Review, 57, 137-145.

POTOGLOU, D. \& KANAROGLOU, P. 2006. Household Demand and Willingness to pay for Alternative Fuelled Vehicles. Working Paper Series. Hamilton, ON: Centre for Spatial Analysis, McMaster University.

PUTRUS, G. 2009. Impact of Electric Vehicles on Power Distribution Networks. IEEE Vehicle Power and Propulsion Conference (VPPC), 7-10 Sept. 2009. Dearborn, MI: IEEE.

RAMBERG, D. 2010. The Relationship between Crude Oil and Natural Gas Spot Prices and its Stability Over Time. Technology and Policy MSc, Massachusetts Institute of Technolgy.

REVELT, D. \& TRAIN, K. 1988. Mixed logit with repeated choices. Review of Economics and Statistics, 80, 647-657.

ROGERS, E. 1995. Diffusion of Innovations: Fourth Edition, The Free Press.

ROSE, E., WITTEN, K. \& MCCREANOR, T. 2009a. Transport related social exclusion in New Zealand: evidence and challenges. Kotuitui: New Zealand Journal of Social Sciences Online, 4, 191-203.

ROSE, J. \& BLIEMER, M. 2008. Stated Preference Experimental Design Strategies. In: HENSHER, D. \& BUTTON, K. (eds.) Handbook of Transport Modelling. Oxford and Amsterdam: Elsevier. 
ROSE, J. \& BLIEMER, M. 2009. Constructing Efficient Stated Choice Experimental Designs. Transport Reviews, 29, 587-617.

ROSE, J., BLIEMER, M., HENSHER, D. \& A, C. 2008. Designing efficient stated choice experiments in the presence of reference alternatives. Transportation Research Part B, 42, 395-406.

ROSE, J., SCARPA, R. \& BLIEMER, M. 2009b. Incorporating model uncertainty into the generation of efficient stated choice experiments: A model averaging approach. The University of Sydney: Institute of Transport and Logistics Studies.

SAMARAS, C. \& MEISTERLING, K. 2008. Life Cycle Assessment of Greenhouse Gas Emissions from Plug-in Hybrid Vehicles: Implications for Policy. Environmental Science \& Technology, 42, 3170-3176.

SANDOR, Z. \& WEDEL, M. 2001. Designing Conjoint Choice Experiments Using Managers' Prior Beliefs. Journal of Marketing Research, 38, 430-444.

SANKEY, P., CLARK, D. \& MICHELOTO, S. 2010. The End of the Oil Age 2011 and beyond: a reality. Global Markets Research: Deutsche Bank.

SANTINI, D. 2010. Cost-Effective PHEV Range: Battery Costs Versus Charging Infrastructure Costs. Transportation Research Board Annual Meeting. Washington D.C.: Argonne National Laboratory.

SAVIAK, J. 2007. An Investigation into the Predictors of Adoption and Utilization of Information-Sharing Networks by Local Law Enforcement in Three States. PhD, University of Central Florida.

SCHADE, W. \& KRAIL, M. 2006. Modeling and calibration of large scale system dynamics models: the case of the ASTRA model. In: GRÖßLER, A., ROUWETTE, E., LANGER, R., ROWE, J. \& YANNI, J. (eds.) 24th International Conference of the System Dynamics Society. Nijmegen, The Netherlands, 23-27th July 2006: The System Dynamics Society.

SCHARLEMANN, J. \& LAURANCE, W. 2008. How Green Are Biofuels? Science, 319, 43-44.

SCHEY, S., SCOFFIELD, D. \& SMART, J. 2012. A First Look at the Impact of Electric Vehicle Charging on the Electric Grid in the EV Project. EVS26 International Battery, Hybrid and Fuel Cell Electric Vehicle Symposium. Los Angeles: Idaho National Laboratory.

SCHILL, W.-P. 2011. Electric vehicles in imperfect electricity markets: The case of Germany. Energy Policy, 39, 6178-6189.

SCHIPPER, L. 2011. Automobile use, fuel economy and $\mathrm{CO}_{2}$ emissions in industrialized countries: Encouraging trends through 2008? Transport Policy, 18 358-372.

SCHIPPER, L. \& TAX, W. 1994. New car test and actual fuel economy: yet another gap? Transport Policy, 1, 257-265.

SCHOT, J., HOOGMA, R. \& ELZEN, B. 1994. Strategies for Shifting Technological Systems: The case of the automobile system. Futures, 26, 1060-1076.

SCHWOON, M. 2006a. Learning-by-doing, Learning Spillovers and the Diffusion of Fuel Cell Vehicles. FNU Working Paper Number 112 Hamburg: Research Unit Sustainability and Global Change, University of Hamburg.

SCHWOON, M. 2006b. Simulating the Adoption of Fuel Cell Vehicles. Journal of Evolutionary Economics, 16 435-472.

SHACKLEY, S. \& GREEN, K. 2007. A conceptual framework for exploring transitions to decarbonised energy systems in the United Kingdom. Energy, 32, 221-236.

SHAHABUDDIN, S. 2009. Forecasting automobile sales. Management Research News, 32, 670-682.

SHAHEEN, S., SPERLING, D. \& WAGNER, C. 1998. Carsharing in Europe and North America: Past, Present, and Future. Transportation Quarterly, 52, 35-52.

SHELLER, M. 2003. Automotive emotions: Feeling the car. Department of Sociology, Lancaster University. 
SHELLER, M. \& URRY, J. 2000. The City and the Car. International Journal of Urban and Regional Research, 24, 737-757.

SHORT, W., PACKEY, D. \& HOLT, T. 1995. Manual for the Economic Evaluation of Energy Efficiency and Renewable Technologies. Golden, Colorado: National Renewable Energy Laboratory.

SIMPSON, A. G. 2004. Full-cycle assessment of alternative fuels for light-duty road vehicles in Australia Proceedings of the 2004 World Energy Congress-Youth Symposium, 5-9, 2004. Sydney: World Energy Congress.

SIMS, R., MABEE, W., SADDLER, J. \& TAYLOR, M. 2010. An overview of second generation biofuel technologies. Bioresource Technology, 101, 1570-1580.

SKREBOWSKI, C. 2011. A brief economic explanation of Peak Oil. ODAC Newsletter [Online]. Available: http://www.odac-info.org/newsletter/2011/09/16.

SLOANE, A. 2012. Holden embraces bio fuel [Online]. Auckland: The New Zealand Herald. Available:

http://www.nzherald.co.nz/motoring/news/article.cfm?c_id=9\&objectid=1081844 5 [Accessed 12 July 2012.

SMALL, K. \& VAN DENDER, K. 2007. Fuel Efficiency and Motor Vehicle Travel: The Declining Rebound Effect. The Energy Journal, 28, 27.

SMART, J., DAVIES, J., SHIRK, M., QUINN, C. \& KURANI, K. 2010. Electricity Demand of PHEVs Operated by Private Households and Commercial Fleets: Effects of Driving and Charging Behavior. EVS-25. Shenzhen, China: World Electric Vehicle Association.

SMIL, V. 2006. Peak oil: a catastrophist cult and complex realities. World Watch. Washington D.C.: World Watch Institute.

SMIT, R. \& CAMPBELL, A. 2007. Cost and Impacts of a Transition to Hydrogen Fuel in New Zealand. Wellington: CRL Energy Ltd.

SOUCHE, S. 2010. Measuring the structural determinants of urban travel demand. Transport Policy, 17, 127-134.

SOVACOOL, B. \& HIRSH, R. 2009. Beyond batteries: An examination of the benefits and barriers to plug-in hybrid electric vehicles (PHEVs) and a vehicle-to-grid (V2G) transition. Energy Policy, 37, 1095-1103.

SPENCE, M. 1981. The Learning Curve and Competition. The Bell Journal of Economics, 12, 49-70.

SRINIVASAN, V. Batteries for Vehicular Applications. In: HAFEMEISTER, D., LEVINE, M. \& SCHWARTZ, P., eds. PHYSICS OF SUSTAINABLE ENERGY: Using Energy Efficiently and Producing It Renewably, 2008 Berkeley, CA. American Institute of Physics, 283-296.

STATISTICS NEW ZEALAND 2006a. Internal Migration: Home ownership. Wellington: New Zealand Government.

STATISTICS NEW ZEALAND 2006b. New Zealand Census. Wellington: New Zealand Government.

STATISTICS NEW ZEALAND 2009. National Population Projections: 2009 (base)-2061. Wellington: Statistics New Zealand.

STATISTICS NEW ZEALAND 2010a. Household Economic Survey. 25 November 2010 ed. Wellington: Statistics New Zealand.

STATISTICS NEW ZEALAND 2010b. Infoshare database: Motor Vehicles Currently Licensed by Type (Qrtly-Mar/Jun/Sep/Dec) Statistics New Zealand.

STATISTICS NEW ZEALAND 2011. National Accounts - SNA 1993 - SNC: Prodn A/C, GDP \& GDE, Market Price, Seas. Adj. Quarterly (Qrtly-Mar/Jun/Sep/Dec) Statistics New Zealand Infoshare Database.

STERN, N. H. 2006. The Economics of Climate Change: The Stern Review [Online]. Available: http://www.hm- 
treasury.gov.uk/independent_reviews/stern_review_economics_climate_change/s tern_review_report.cfm (accessed January 2007).

STRUBEN, J. \& STERMAN, J. 2007. Transition challenges for alternative fuel vehicle and transportation systems. Working Paper. MIT Sloan School of Management.

SUITS, D. 1958. The demand for new automobile in the United States, 1926-1956. Review of Economics and Statistic, 40, 273-80.

SULLIVAN, J., SALMEEN, I. \& SIMON, C. 2009. PHEV Marketplace Penetration: An Agent Based Simulation. University of Michigan: Transportation Research Institute.

SWART, R., RASKIN, P. \& ROBINSON, J. 2004. The problem of the future: sustainability science and scenario analysis. Global Environmental Change, 14, 137-146.

SWEDISH INSTITUTE FOR TRANSPORT AND COMMUNICATIONS ANALYSIS 2007. RES 2005 2006, The National Travel Survey. Östersund, Sweden.

TATE, E., HARPSTER, M. \& SAVAGIAN, P. 2008. The Electrification of the Automobile: From Conventional Hybrid, to Plug-in Hybrids, to Extended-Range Electric Vehicles. 2008 World Congress. Detroit, Michigan: SAE International.

TAYLOR, D. 2009. The Differences and Similarities between Plug-in Hybrid EVs and Battery EVs. EVS24 International Battery, Hybrid and Fuel Cell Electric Vehicle Symposium. Stavanger, Norway: World Electric Vehicle Association.

TECHNICAL UNIVERSITY OF DENMARK 2012. The Danish National Travel Survey. Lyngby, Denmark: Department of Transport.

TESLA MOTORS. 2012. Model S/ Options and Pricing [Online]. Palo Alto, CA: Tesla Motors. Available: http://www.teslamotors.com/models/options [Accessed 15 June 2012.

THE INDEPENDENT. 2011. Leased batteries offer better electric vehicle value, says research. The Indendent.

THE REDBOOK 2010. RedBook.co.nz.

THE TREASURY. 2010. Cost Benefit Analysis: including Public Sector Discount Rates [Online]. The Treasury website: The Treasury. Available:

http://www.treasury.govt.nz/publications/guidance/planning/costbenefitanalysis [Accessed 7 August 2011].

THOMAS, C. 2009. Fuel Cell and Battery Electric Vehicles Compared. International Journal of Hydrogen Energy, 34, 6005-6020.

THURSTONE, L. 1927. A Law of Comparative Judgement. Psychological Review, 34, 273-286.

TOMI'C, J. \& KEMPTON, W. 2007. Using fleets of electric-drive vehicles for grid support. Journal of Power Sources, 168, 459-468.

TOYOTA. 2012. Prius Plug-in Hybrid (Prius PHV) [Online]. Torrance, CA: Toyota U.S.A Inc. Available: http://www.toyota.com/prius-plug-in/trims-prices.html [Accessed 25 September 2012.

TRAIN, K. 1980. The Potential Market for Non-Gasoline-Powered Automobiles. Transportation Research Part A, 14A, 405-414.

TRAIN, K. 1986. Qualitative Choice Analysis, Cambridge, MA and London U.K., MIT Press.

TRAIN, K. 1999. Halton sequences for mixed logit. Technical paper. Berkeley: University of California.

TRAIN, K. 2003. Discrete Choice Methods with Simulation, Cambridge, Cambridge University Press.

TRANSPOWER 2012. Annual Planning Report 2012. Wellington: Transpower.

TRANSPOWER. 2013. New HVDC Pole 3 Commissioned [Online]. Wellington: Transpower. Available: www.transpower.co.nz/news/new-hvdc-pole-3-commissioned [Accessed 14 October 2013].

TRIGG, T. 2010. The transport sector chapter. Workshop on a Manual on Energy Statistics for Energy Efficiency Indicators, 29 November 2010. Paris: International Energy Agency. 
TROSTLE, R., MARTI, D., ROSEN, S. \& WESTCOTT, P. 2011. Why Have Food Commodity Prices Risen Again? A Report from the Economic Research Service, WRS-1103. Washington, D.C.: United States Department of Agriculture.

TSANG, F., PEDERSEN, J., WOODING, S. \& POTOGLOU, D. 2012. Bringing the electric vehicle to the mass market. Cambridge U.K.: RAND Europe.

TURRENTINE, T. \& KURANI, K. 2007. Car buyers and fuel economy? Energy Policy, 35, 12131223.

TUTTLE, D. \& BALDICK, R. 2012. The Evolution of Plug-In Electric Vehicle-Grid Interactions. IEEE Transactions on Smart Grid, 3, 500-505.

U.S. ADVANCED BATTERY CONSORTIUM. 2012. USABC Goals for Advanced Batteries for EVs [Online]. Available: http://www.uscar.org/guest/article_view.php?articles_id=85 [Accessed 30 May 2013].

U.S. DEPARTMENT OF ENERGY 2001. Homogeneous Charge Compression Ignition ( $\mathrm{HCCl}$ ) Technology: A Report to the U.S. Congress. Washington D.C.: Energy Efficiency and Renewable Energy Office of Transportation Technologies.

U.S. DEPARTMENT OF ENERGY 2009. Targets for Onboard Hydrogen Storage Systems for Light-Duty Vehicles. Office of Energy Efficiency and Renewable Energy and The FreedomCAR and Fuel Partnership. Washington D.C.: U.S. Department of Energy.

U.S. DEPARTMENT OF ENERGY 2010. On-Board Hydrogen Storage Systems - Projected Performance and Cost Parameters. Hydrogen and Fuel Cells Program. Washington D.C.: U.S. Department of Energy.

U.S. DEPARTMENT OF ENERGY 2011a. Fuel Cell Technologies Program Record: Fuel Cell Stack Durability. Hydrogen and Fuel Cells Program. Washington D.C.: U.S. Department of Energy.

U.S. DEPARTMENT OF ENERGY 2011b. Fuel Cell Technologies Program Record: Fuel Cell System Cost - 2011. Hydrogen and Fuel Cells Program. Washington D.C.: U.S. Department of Energy.

U.S. DEPARTMENT OF ENERGY. 2013. www.fueleconomy.gov [Online]. Washington D.C.: U.S. Department of Energy. [Accessed 31 May 2013].

U.S. DEPARTMENT OF TRANSPORTATION 2010. Transportation's Role in Reducing U.S. Greenhouse Gas Emissions: Volume 1: Synthesis Report. Washington D.C.: U.S. Department of Transportation.

UK INDUSTRY TASKFORCE ON PEAK OIL \& ENERGY SECURITY 2010. The Oil Crunch: A wakeup call for the UK economy. In: ROBERTS, S. (ed.). London: UK Industry Taskforce on Peak Oil \& Energy Security.

UNRUH, G. 2000. Understanding carbon lock-in. Energy Policy, 28, 817-830.

URRY, J. 2004. The 'System' of Automobility. Theory Culture Society, 21, 25-39.

URRY, J. 2006. Inhabiting the car. Sociological Review, 54, 17-31.

URRY, J. 2008. Climate change, travel and complex futures. The British Journal of Sociology, 59, 261-279.

VERBRUGGEN, A. \& MARCHOHI, M. 2010. Views on peak oil and its relation to climate change policy. Energy Policy, 38, 5572-5581.

VERHELST, S. \& WALLNER, T. 2009. Hydrogen-fueled internal combustion engines. Progress in Energy and Combustion Science, 35, 490-527.

VILLAR, J. \& JOUTZ, F. 2006. The Relationship Between Crude Oil and Natural Gas Prices. EIA manuscript. Washington: Energy Information Administration, Office of Oil and Gas.

VYAS, A., NG, H., SANTINI, D. \& ANDERSON, J. 1997. Electric and Hybrid Electric Vehicles: A Technology Assessment Based on a Two-Stage Delphi Study. Argonne, Illnois: Argonne National Laboratory.

VYAS, A., SANTINI, D., DUOBA, M. \& ALEXANDER, M. 2007. Plug-In Hybrid Electric Vehicles: How Does One Determine Their Potential for Reducing U.S. Oil Dependence? EVS23 
International Battery, Hybrid and Fuel Cell Electric Vehicle Symposium. Anaheim, California: WEVA.

WARD'S AUTOMOTIVE GROUP 2011. Vehicles in Operation by Country 15 August 2011 ed. Wardsauto.com: Penton Media Inc.

WEILLER, C. 2011. Plug-in hybrid electric vehicle impacts on hourly electricity demand in the United States. Energy Policy, 39, 3766-3778.

WELLS, P. \& NIEUWENHUIS, P. 2012. Transition failure: Understanding continuity in the automotive industry. Technological Forecasting \& Social Change, In Press.

WEMBRIDGE, N. 2011. 2011 NZ Generation Data Update. Wellington: Ministry of Economic Development,.

WEMBRIDGE, N., BURBIDGE, D. \& WILDES, R. 2009a. Coal Price and Availability Study: Report for the Electricity Commission. http://www.ea.govt.nz/ourwork/consultations/modelling/09-gpa/: Parsons Brinckerhoff

WEMBRIDGE, N., POLONI, L., MCGIMPSEY, N. \& HALL, N. 2009b. Thermal Power Station Advice: Report for the Electricity Commission. Auckland: Parsons Brinckerhoff.

WERPY, M., SANTINI, D., BURNHAM, A. \& MINTZ, M. 2009. White Paper on Natural Gas Vehicles: Status, Barriers, and Opportunities: A Discussion Paper for Clean Cities Coalitions and Stakeholders to Develop Strategies for the Future. Lemont, IL: Argonne National Laboratory

WHITEHEAD, J., PATTANAYAK, S., VAN HOUTVEN, G. \& GELSO, R. 2008. Combining Revealed and Stated Preference Data to Estimate the Nonmarket Value of Ecological Services: Assessment of the State of the Science. Journal of Economic Surveys, 22, 872-908.

WILLIAMS, A. 2012. Silent But Deadly, electric and hybrid vehicles: Guide Dogs calls for urgent action to prevent road accidents [Online]. Reading, U.K.: The Guide Dogs for the Blind Association. Available: http://www.guidedogs.org.uk/news/silent-butdeadly-electric-and-hybrid-vehicles/ [Accessed 25 September 2012.

WILLIAMS, B. 2011. Plug-In-Vehicle Battery Second Life: the Effect of Post-Vehicle, Distributed-Grid Energy Storage Value on Battery-Lease Payments. Berkeley, CA: Transportation Sustainability Research Center, University of California, Berkeley.

WILLIAMS, B. \& LIPMAN, T. 2010. Strategies for Transportation Electric Fuel Implementation in California: Overcoming Battery First-Cost Hurdles. California Energy Commission, PIER Transportation Program. CEC-500-2009-091.

WILSON, C. \& DOWLATABADI, H. 2007. Models of Decision Making and Residential Energy use. Annual Review of Environment and Resources, 32, 169-203.

WILSON, C. \& GRUBLER, A. 2011. Lessons from the history of technological change for clean energy scenarios and policies. Natural Resources Forum, 35, 165-184.

WIPKE, K., SPRIK, S., KURTZ, J., RAMSDEN, T., AINSCOUGH, C. \& SAUR, G. 2012. National Fuel Cell Electric Vehicle Learning Demonstration Final Report. Golden, Colorado: National Renewable Energy Laboratory.

WORLD BANK 2012. World Bank Open Data: Passenger cars (per 1,000 people), Motor vehicles (per 1,000 people). World Bank.org.

WRIGHT, J. \& BAINES, J. 1990. Transport fuels in New Zealand after Maui- lignite on the back burner. Lincoln University: Centre for Resource Management, Lincoln University and University of Canterbury.

YELLE, L. 1979. The Learning Curve: Historical Review and Comprehensive Survey. Decision Sciences, 10, 302-328.

ZECCA, A. \& CHIARI, L. 2010. Fossil-fuel constraints on global warming. Energy Policy, 38, 13. 
ZELLNER, M. 2008. Embracing Complexity and Uncertainty: The Potential of Agent-Based Modeling for Environmental Planning and Policy. Planning Theory \& Practice, 9, 437-457.

ZHANG, T., GENSLER, S. \& GARCIA, R. 2011. A Study of the Diffusion of Alternative Fuel Vehicles: An Agent-Based Modeling Approach. Journal of Product Innovation Management, 28, 152-168. 